University of the Pacific Scholarly Commons

1753

\title{
Theoria motus lunae exhibens omnes eius inaequalitates
}

Leonhard Euler

Follow this and additional works at: https://scholarlycommons.pacific.edu/euler-works

Part of the Mathematics Commons

Record Created:

2018-09-25

\section{Recommended Citation}

Euler, Leonhard, "Theoria motus lunae exhibens omnes eius inaequalitates" (1753). Euler Archive - All Works. 187. https://scholarlycommons.pacific.edu/euler-works/187

This Book is brought to you for free and open access by the Euler Archive at Scholarly Commons. It has been accepted for inclusion in Euler Archive All Works by an authorized administrator of Scholarly Commons. For more information, please contact mgibney@pacific.edu. 


\section{BEST COPY AVAILABLE}

\section{TH E O R I A MOTUS LUNAE E $X$ III B E N 3 OMNES EIUS INAEQUALITATES \\ I N \\ ADDITAMENTO} HOC IDEM ARGUMENTUM ALITER TRACTATUR SIMULEUE OSTENDITUR SIMULEUE OSTENDITUR
QUEMADMODUM MOTUS LUNAE CUM OMNIBUS INAEQUARITATIBUS

INNUMERIS ALIIS MODIS REPRAESENTARI

ATQUE AD GALCULUM REUOCARI POSSIT $\triangle$ UCTORE

L. E U L E R O

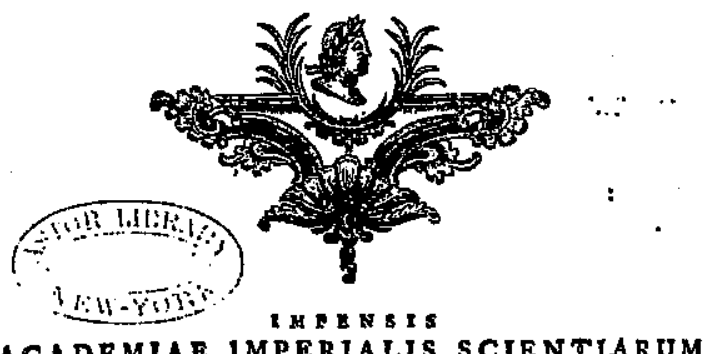
CADBMIAE 1MPERIALIS SCIENTIARUM PBTROPOLITANAE

$$
\text { ANNO 1253. }
$$



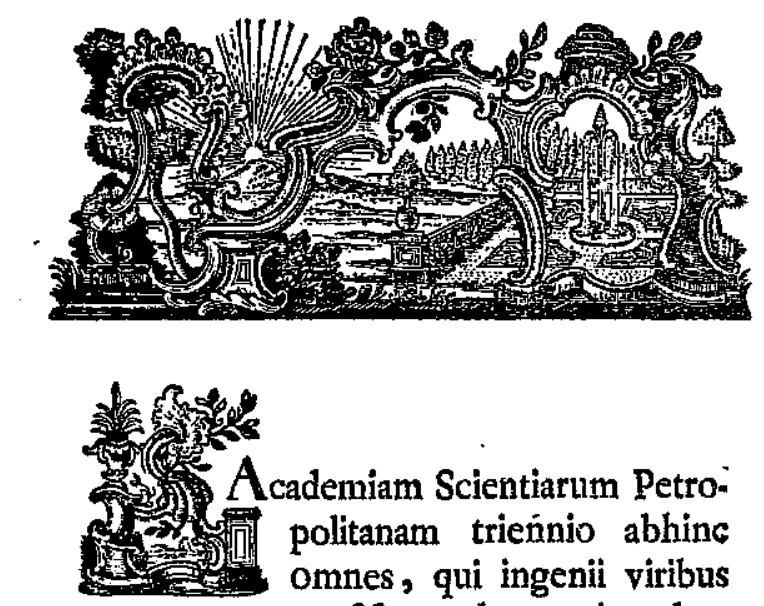

cademiam Scientiarum Petro. politanam trieñnio abhinc omnes, qui ingenii viribus confifi, ad examinandam

Neutonianam de motu Lunae Theoriam, animum applicare vellent, inuitafle, atque ei, qui in hac parte tenuiffet primas, prae. mii loco propofuiffe nummos aureos cen. tum; poltmodum Celeberrimum Clairautum huius certaminis exftitife victorem, publico Academicorum; qui Petropoli agunt, iudicio diuulgatum.

* 2

Cele- 
Celeberrimus Eulerus, Academiae Petropolitanae membrum honorarium, officii fui effe exiftimauit, ferre vna cum ceteris de illa Clairanti differtatione iudicium. Transmifit ergo huc ad nos una cum fila fententia ampliffimam de eodem argumento differtationem; quae quo celerius innotefceret, vifum eft Academiae Praefidi, minoris Ruffiae Hetmano Illuftriflimo Cyrillo Gregoridae, Comiti Rafumouio, eam tradere Academicis in folenni conuentu examinandam, ea fini, vt fi fuffragio Academicorum comprobata, dignaque indicata foret, quae orbis eruditi proponeretur theatro, ea praelo quam maturrime fubiiceretur; quandoquidem ille mos iam indè a principio obtinuit, $v t$, quae in publico coetu praeleguntur differtationes, eae vel ante folennem actum, vel paruulo intermiffo fpatio typis diuulgentur poftea.

\section{察 櫒 㶅}

Ceterum differtatio illa conftat magnam partem calculis, veris quidem illis et omnibus numeris abfolutis, fed propter nimiam fui molem atque difficultatem auditu moleftiffimis: quae fi recitarentur publice, periculum erat, ne auditorum animi aufcultandis iis deficerent, neue Aca. demia Summorum patientia. Virorum ab$v t i$, caeterosque enicare odio videretur. Praeuidit hoc incommodum, prouiditque Illuftriffimi Praefidis fapientia. Mandavit Aftronomiae Profeffori V. C. Nicetae Popouio, cuius id temporis dicendi erat prouincia, vt prolixam illam Euleri disfertationem, omiffis calculis, redigeret in compendium, et quae inde excepiffet, auditorum caufa recitaret publice. Quo quidem facto et auribus hominum confuluit, et tamen rerum capitum participes eos facere aequabili temperamento inflituit: at- 
VI

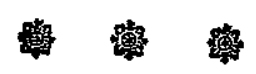

que differtationem, ne quid forte naeuorum obreperet, ipfo auctore coram typis excudi aequum cenfuit.

Iam qui illo tempore interfierant conuentui Academicorum folenni auditores, aequis nimis auribusque aufculta. runt excerpta recitantem Euleriana Popouium: eft ergo quod fperare liceat, et iis, quorum intereft, vt qui hoc genere ftudiorum maxime delectantur, factum iri fatis ipfa differtatione. Datum Petropoli Nov. 1752.

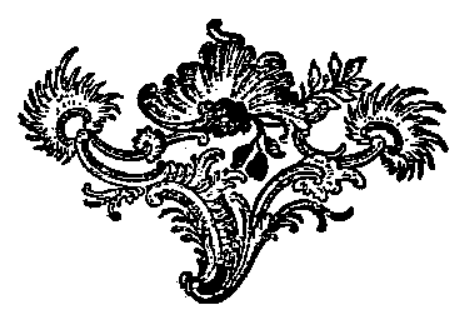

需 路

VII

\section{INDEX CAPITUM.}

INTRODUCTIO

Caput L, De motu corporis a virius - - pag. coris a viribus quibuscungue follici.

Caput II. Inueftigatio virium Lsnam $\overrightarrow{-}-\overrightarrow{-}-9$

Caput Ill. Introduttio anomaliae verae Lumee in proecedevtes aequationts

Caput IV. quae eariato distur inasqualitat is Lunae alfolutae,

$-49$

Caput V. Inuefigatio inaequalitatum Lunae ab eius ex. centricitats fimplici fotum pendentiam -

Caput VI. Inueftigatio inaequalitatum Lutzae a quadrato excentricitatis ipfous ortarum - -

Capuc VIl. Correctio inaequatitatum Lunge hactenus insventorum

Caput VIll. De Motu Apogei Znase $\quad-\quad$ - 87

Caput IX. Inuefigatio inaequatitotum Lnnce a fola ex. centricitane orbitae folis pendentium -

Caput X. Invefigatio inaequalitatum Lunae ab vtriss. que orbitae exceiurricitate fimul pesdentium - 132

Caput XI. Inuefigatio inoequalitatum Lunae a parallaxi folis pendentium -

Caput XI. Inuefigatio insequalitatum mosam linese no. derum affeintion 
VIII

\section{触 路}

Caput XIII. Isseefigatio inclizationis orbitae lunaris od eclipticam cum eius variatione

Caput XIV. Inuefigatio inaiqualitatum Lunae ob eius 190 inclinatione ad eclipticam oriundarum - 199

Caput XV. Accuratior inseffigatio inaequalisatum LWnae ab inclinatione eius orbitae perdentium - 208

Caput XVI. Expoftio inaequalitatum Lamae hatesut inuentarum - - - - 224

Capue XVII. Inuefigatio Elementorum motus Lunoe 231

Caput XVIII. Confizutio Elementorum pro tabulis Livwaribus - $\quad$ - $\quad$ - $\quad$ - 266

Additamentum centiness alias methodos inueffigandi mosus Lunae inaequalisates
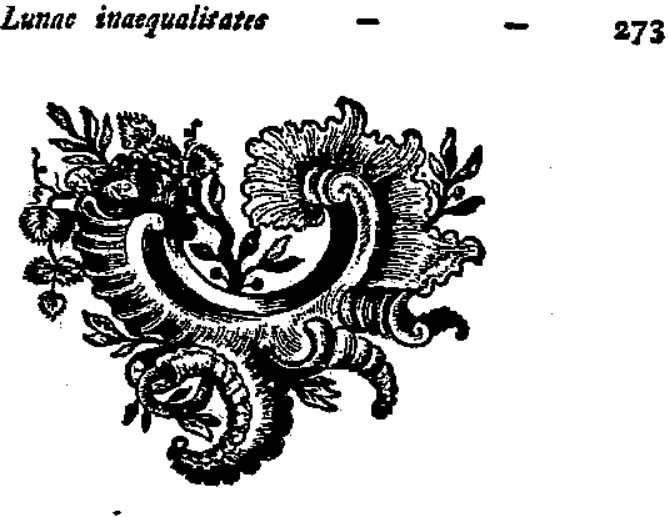

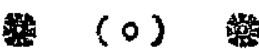

\section{INTRODUCTIO.}

199

-

208

224

231

-

266

273

TRO.
$\mathrm{C}$

am nullum fit dubium, quin fummi Newtoni Theo. ria, qua motum Planetarum felicifimo cum fursceffu cercis legibus adftrinxit, plurimum ad motum Lunae accuratius determinandum contulerit, maximi fane in Aftronomia momenti eft quaeftio: verum haec Newtoni Theoria omnino fit fufficiens omnibus motus inaequalitatibus, quae in Luna obferuantur, exactifime explicandis nec ne? Quanquam autem a Newtoni affeclis plerumque affirmarl foler, nullam in motu Lunae obferuari inaequalitatem, cuius ratio in ifta Theoria non continescur; tamen tantum abeft, ve hic confenfus a quoquam perfpicue fit monftratus, vt potius applicatio huius Theoriae ad Lunam tantis implicetur calculi difficultatibus, quibus penims euoluendis vires ingenii humani vix fufficere viden tur. Plurimae quidem. adhuc prodierune Tabulae Lunares, quae ex Theoria Newtoniana deductae perhibentur, fed praeterquam gnod faepius ultra $5^{\prime}$ ab obletuationibus difcrepent, earum conuenientia cum Theoria ipfa neutiquam eft euieta; quin potius pleraeque Tabulae inaequalitatum non tam Theoriae quam obferuationibus funt fuperttructae. Huiusmodi ergo tabularum fiue confenfus fiue diffenfus cum obferuationibus neque ad Theoriam Newtonianam plenifime confirmandam, neque ad eam infringendam alle.

INTRO. 


$$
\text { 整 ( } 0 \text { ) }
$$

gari poteft : nam quatenus iftae tabulae obferuationibus fatisfaciunt, hoc non foli Theoriae eft tribuendum; qua. tenus autem cum obferuationibus minus conueniunt, hoc ne Theoriae quidem imputari poterit, propterea quod iftae Tabulae non foli Theoriae innituntur.

Quaeftio itaque, cujus mentionem feci, rete enodari nequit, nifi ante eiusmodi Tabulae exhibeantur, quae ex fola Theoria, nullis obferuationibus: in fubfidium vocatis, fint formatae; tum enim demum ex huiusmodi Tabularum collatione cum ingenti obferuationum fummo fudio infttutarum copia diiudicare licebit, vtrum Theoria omnibus obferuationibus refpondeat, an vero correctione quapiam indigeat. Non diffile quidem eft ex principiis mechanicis motum Lunae aequationibus analyticis complecti; quoniam autem hae aequationes plures variabiles inter fe permixtas continent, atque adeo differentialibus fecundi ordinis implicantur, earum refolutio maximis difficultatibus eft obnoxia ; et quoniam alio modo nifi per approximationem fufcipi non poteft, vtcunque inftituatur, femper non leue dubium remaner, vtrum partes, quae in calculo funt neglectae et praetermiffae, nihil, quod in motu Lunae effet notabile, efficere potuerint. Hoc modo explicatio motuum Lunae tota ad folam Analyfin transfertur, ac difficultates, quibus premitur, inde oriuntur, quod Analyfis nondum fatis eft exculta.

Cum Igitur Theoria Newtoniana hoc principio latisfime patente innitatur, quod omnia corpora coeleftia fe mutuo attrahant in ratione reciproca duplicata diftantia-

rum,
整 (0)

rum, fi motum Lunae fecundum hane Theoriam definire velimus, vires erunt fpectandae omnes, quibus Luna follicitatur. Atque inter has vires primaria eft ea, qua ad terram vrgetur, quae fi fola adeffet, terraque quiefceret, Iuna in ellipfi perfecta fecundum regulas Keplerianas motum fuum circa terram abfolueret. At cum Luna praeterea aeque ac terra ipfa etiam ad folem trahatur, hac vi motus ille regularis non mediocriter perturbabitur: atgue haec vis a fole profeeta omnium difficultatum, quae in determinatione motus Lunae offenduntur, caufa eft exiftimanda. Reliquae enim vires, quibus forte Luna fe. cundum Theoriam Newtoni ad reliquos planetas vrgeri deberet, tam funt exiguae, vt effectus inde oriundus me. rito pro nihilo haberi queat.

Solas ergo vires folis ac terrae in computum duci oportet, fi motum Lunae fecundum Theoriam definire velimus, atque cum ex his viribus formulae analyticae fuerint erutae, quae motum Lunae complętantur, ornne ftudium in his formulis ita euoluendis erit impendendum, ve inde ad quoduis tempus propofitum locus Lunae affegnari, ac more apud Aftronomos folito fecundum longitudinem et latitudinem definiri queat. Hinc porro Tabulse Aftronomicae pro mota Lunae erunt condendae, quibus omnes inaequalitates tam in longitudine quam in latitudine exhibeantur, ex quibus fi pro cujusuis obferuationis mo. mento locus Lunae computetur, confenfus vel diffenfus calculi ab obferuacionibus Theoriam vel confirmabir, vel ejus defectum declarabit. Neque tamen Theoria fola hujusmodi Tabulis conftruendis fufficir, fed quaedam ele-

A 2

menta 
mienta extrinfecas ab obferuationibus affumi oportet, quae funt $1^{\circ}$. Excentricitas orbitae Lunaris, quae falua theoria vel major vel minor effe potuiffet; pendet enim a mota Lunae primitus imprefo, quem Theoria non determinat, fed tanquam cognitum affurnit. $2^{\circ}$. Locus Lunae medius pro quapiam Epocha propofita, qui pariter ex obferuationibus eft concludendus. $3^{\circ}$. Locus Apogei orbitae Lunasis pro Epocha quadam data. $4^{\circ}$. Tempus periodicum Lunae fecundum motum medium, quod pendet a diftantia Lunae media a Terra, ideoque ex fola 'Theoria definiri nequit. $5^{\circ}$. Locus nodorum Lunae pro Epocha quadam data : et $6^{\text {to }}$ denique inclinatio media orbicae Lunaris ad planum Eclipticae.

His autem fex elementis 'per obferuationes definitis reliqua omnia, quibus ad locum Lunae pro quouis tempore affignandum opus eft, ex fola Theoria funt petenda; quae primo ad guoduis tempus locum Apogei eiusque ideo motum verum praebere debet, vt inde ex loco Lunae medio eius anomalia media colligi queat. Deinde Theoria quoque omnes correctiones feu Proftaphaerefes, quae loco Lunae medio vel addicae vel fublatae eius locum verum exhibeant, inppeditare debet; atque iftae correctiones, quae motus inaequalitates appellari folent, partim ab Anomalia media Lunae, partim ab eius Phafi feu elongationea fole, partimque ab Anomalia folis media pendenc, ex quo triplici fonte numerus inaequalicatum in immenfum augetur. Porro etiam Theoria motum nodi eiusque omnes inaequalitates indicare tenetur, ac denique etiam pro quouis tempore orbitae Lunaris veram inclinationem ad eclipticam, ve inde eius latitudinem veram evuere liceat.

Cum antem, ve iam innut, nemo adhuc ommes inaequalitates, quae in motu Lunae reperiunture, ex Theoria elicuerit, vt ex is iudicium ferri poffic, quantum haec Theoria cum obferuationibus conueniat; etfi nullum eft dubium quin difcrimen, fi quod deprehenderetur, admo. dum paruum fit futurum: iam pridem haec quaeftio ex folo motu Apogei dirimi eft coepta, dum aliis motus Apogei ex obferuationibus cognitus magnopere a Theoria difcrepaxe eft vifas, alii autem etiam hoc loco pulcerrimuso confenfum Theoriae es veritatis iactauerunt. Mirum autem eft, ipfum Newtonum nihil circa motum Apogei ex Theoria ftatuiffe, fed eum ex folis obfermationibus in calculum tran-ftulife, cum tamen motum nodorum fumma fagacitate ex Theoria elicuiffet, atque veritati confentaneuin oftendiffet. Cur igitur motum Apogei plane filentio praeterierit, nulla alia ratio fabeffe videtur, nif, quod animaduerterit hune motum, prouti ex Theoria prodiret, oblertationibus parum fore conformem. Ex îs enim, quạe Newtonus in fio immortali opere de mont abfidum in genere tradidit, non admodum difficile videtur motum apogei Lunise definire: verum hic praeter expedationem exenit, vt motus apogei annuus vix $20^{\circ}$ fuperans reperiatur, cum tamen ex obfer. tationibus confete, Apogeum Lunae interuallo units anni vlera $40^{\circ}$ promoueri.

Siue autem ifta motus Apogel quantitas $20^{\circ}$ legitime fit ex Theoria deriuara, fire minus; confideratio Apogei tucifiminn prabet remedium quaeftionem de fufticientia A 3

Theo- 


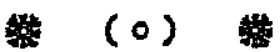

Theoriae Newtonianae decidendi. Quamuis enim ex Theoria inaequalisas quaepiam in ipfo motu Lunae aliquor minutis fecundis vel etiam primis maior minorue prodirer, quam experientia monfraret, tamen tantilla differentia merito vel leui cuipiam errori in obferuationibus, vel vicie in approximatione commiffo tribueretur; quandoquidem aliunde certum eft Theoriam Newtonianam non admodum a veritate receders At longe aliter eft comparata ratio motus Apogei: quodfi enim vires Lunam follicitantes tantillum a Theoria Newtoniana difcrepent, vt ex is in iplo motu Lunae vix perceptibile difcrimen nafceretur, tamen inde in motu Apogei annuo differentia plurrium graduum oriri poterit. Quae tanta differentia cum nulli errori vel obferuationum vel ipfius calculi, fiquidem omni cura inftituatur, tribui queat, inueftigatio motus apogei certifimum fuppeditat criterium iudicandi, vtrum quaepiam Theoria veritati fit confentanea nec ne?

Quodfi ergo calculo rite adminiftrato Theoria Newtoni reperiatar tantum Apogei Lunaris motum exhibere; quantus per obferuationés deprehenditur, fcilicet ulera $40^{\circ}$ quotannis; fortius certe argumentum, quo veritas hujus Theoriae indubie demonftretur, defiderari nequic. Sin autem contra eueniat; vt progrefio Apogei annua ex Theoria rite deriuata notabiliter a $40^{\circ}$ deficiat, hine certo erit concludendum Theorian Newtonianam correctione quapian indigere, negue vires, quibus Luna re. nera follicieatur, exaCtifime huic Theoriae effe conformes.

Verum haec ipla quaeftio, vtrum Theoria Newtoniana ad verum apogei Eurise motum perducat nec ne? eft pro-

(0)

$\mathrm{m}$ ex

liquot

נdiret,

rentia

1 vitio

jidem

odum

1 ratio

is tan-

n ipfo

tamen

duum

ori vel

ra in-

ertiffi-

epiam

New:

uibere;

i ulcra

veritas

requit.

nua ex

hine

a cor-

una re.

ormes.

oniana

if $\mathrm{pro}$

fune fundiffimae indaginis, atque fummam in calculo circuma fpectionem ac follertiam requirit. Quanquam enim exs principiis generalibus, vnde vulgo motus abfidum dexinaixi folet, fatis luculenter femiflis tantum pro motu Apogei Lus nae elicitur; tamen quonian in calculo plures termini, gas in determinationem motus Lunae ingrediuntur, ob paruitatem funt reiecti, mexito dubitatio fuboritur, num hi ipli termini, fi eorum ratio effet habiea, non iftum defeetum compenfare valuerint? Quin etiam non defuere Geomea trae, qui confenfum huius, Theoriae cunivero Apogei, gnotu demonftrare funt conati: yerum pleramque non difficile erat paralogifmum in ipforum ratiociniis deprehendere. Maximam autem hoc loco attentionem meretur indicium profundiffimi Geometrae Glairaut, qui cum primum valio difimis argumentis ftatuifet, Newtoni Theoriam non vlo tra dimidium veri apogei.Lunaris motus fuppeditare, fubito in contrariam abiic fententiam fatzens hanc Theoriara elegantifime cum veritate confpirare : neque certe tantae perfpicaiciáe Vir a priftina fententia; quam omni fudio prọ: pugnauerat, : treceffife: ent pitandus, nifi firmifimis argumentis- éo effet adactus.

Cum autem omnesirationes, quae Ipfum ad hanc rem traetationen impalézims nondurir publice expofuerit, licen at inilhi quidem, qui femper contraraie fententiae fui addio Etus, tanififer arduam haric quaeftionem cançuan nondum decifam fpeetare, donec per propriam inueftigationem in uenero; guid de cá fit ftatuendum. Poftquam enim iam

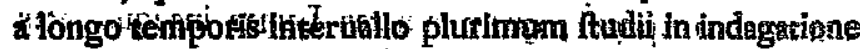
motus Lunae confumffem, ac varis medhodis infiften

femper 
(emper conciufionem Theoriae Newtonianae minus fauen=. tem effem adeptus; quam tamen pro rite demonfrata venditare non eram aufus, propterea quod approximatione eflem vfis, ac femper fufpicio quaedam ratione terminorum praetermifforim remaneret: nuper in aliam incidi viam hane inueftigationem fufcipiendi, quae mihi multo certior videtur, ita ve per eam nulla dabitatione interięta ad veritatem penetrare confidam. Ne autem fi-forte Theoriam Newtonianam minus fufficientem in-

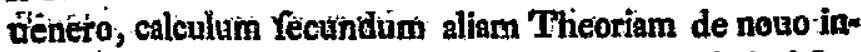
ftituere cogar, ftatim meam inueftigationem in latiori fen. fi exordiar, viresque guibus Luna ad terram folliciratur, non exaÈe fed proxime tantum quadratis diftantiarum reciproce proportionales frumaim : deinceps feilicet innotefcet, vitruin haec abeturatio a regula Newtoniana locum habeat nec ne? Galeulum autem icka adornabo, ve quicquid cueñerit, non folum verum apogei motum affequar, fed etiatin omnes Lunae inaequalizates inde elicere valeam, quas deincieps Aftronomorum mare tabulis completti ticebit.

$\because$ Primum exgo problema in latiftimo fignificatu concipiam, vt corporis a viribus quibuscúnque follicitati mocum fim inueftigaturus; deinde vires; quibus Luna altu vigeri cenfenda eft; inçalculum iptroducam; etaequationncist unat motum deterninantes exhibabo. Has porro Eequationes variis modis in slias formas eransmutabo, donfe eas'eo perduxero, vbi ad finem propofitum maxime accommodatae videbuntur: quo cum peruenero, tandem

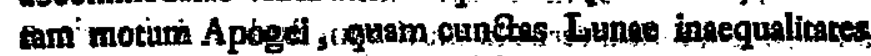

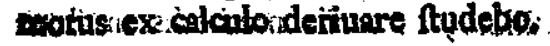
$\because$.

CAPUT

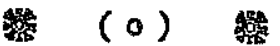

\section{A P U T}

DE MOTU CORPORIS A VIRIBUS QUIBUSCUNQUE SOLLIGITATZ

am in-

: mihi

acione

autem

sm in-

110 in-

ri fen.

icasur,

am re-

$\because$ inno-

locum

icquid

ar, fed

n, quas:

sebit.

a con-

ati mo.

na actu

quatio-

i porro

0 , do-

raxime

andem

alisates

\section{I.}

Q

aoniam corpus a viribus quibuseunque follicitasa ponimus, fieri poteft, vz eius motus non in eo. dem plano abfoluatur. Hinc ad ejus monum per calculum ita repraefentandum, vt ad quoduis temo pus verus locus, in quo corpus verfabitur, affignar? queat, conueniet corporis momm ad planum qtoddam fixum pro lubitu aftumtum referri. Exhibeat igitur $T_{2}$ bula hoc planum, atque corpus iam verfetur extra hoe planum in puneto $\mathbf{L}$, vnde ad planum detrittatur per. pendiculum $\mathbf{L} \mathbf{M}$; eritque punetum $\mathbf{M}$ locus corporis ad planum relatus. Quod fi ergo ad quoduis tempus propofitum hune corporis locum relatum $M$, fimulque eius a plano diftantiam $\mathbf{L} M$ indicare valeamus, verus corporis loctis $\mathbf{L}$ ad hoc tempus innotefcet.

5. 2. Ad locum sutem pundi $\mathbf{M}$ commodius dè terminandum, affumamus in plano reêtam quandam

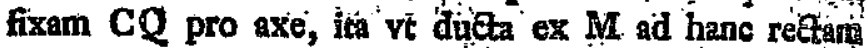
perpendiculari $M$ P, locus punat $M$ more apud Geo. metras recepto per coordinatas orttiogonales definiatutio Afumto ergo porro in axe punkto quodam fixo $\mathrm{C}_{8}$ vnde abfeiffae C:P computenuzr, erir PM dpplicatis punkto $M$ refporidens, \& ipfam punctúm $\cdot L$ detetmina bitur per tres coordisatas jnter fe normales $C P_{\text {, }}$ PMig: 


\section{so}

$C A P U T$ I.

M L. Cum igitur praefenti temporis momento corpus in $L$ verfari ponatur, vocentur ịtae tres soordinatae eo fpectantes:

$$
\mathrm{CP}=p ; \mathrm{PM}=q \text { et } \mathrm{ML}=r
$$

elapfo autem temporis elemento, quod per $d t$ indicemus, coordinatae ternae tum locum corporis indicantes erunt :

$$
p+d p ; q+d q ; \text { et } r+d r
$$

- ฐ. 3. Quạeçunque nune fuerint vires; quibus corpus follicitatuf, eae femper per cognitam virium refolutionem reduci poterunt ad ternas vires fecundum directiones ternarum coordinatarum vrgentes.. Ponamus ergo vim $=\mathbf{P}$, qua corpus in $\mathbf{L}$ fecundum disectionem infi $P$ C parallelam trahinur : eam porro vim

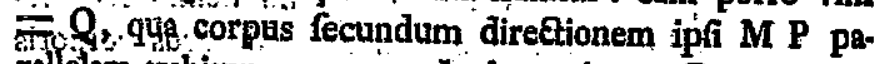
rallelam trahitur: earnque denique vim $=R$, qua cor - pus fecundum direetionem $\mathbf{L} \mathbf{M}$ follicitatur. Has feilicet vires in diregtas cencipio, yt fi corpus eqrum altioภi libere obediret, palores coordinatarum $p, q,{ }^{\prime}$ inde diminuerentur. His pofitis, ex principiis, Mechanicae conftat, fi elementulín temporis $d t$ pro conftanti afti. thatur, motum sprporis his tribius formulitis differentio differentialibus deterininarit

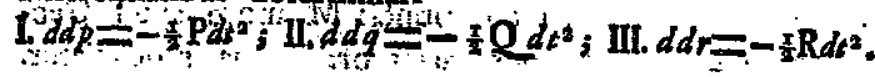
.. S. 4. Verum hae coordinatae ad vfum aftronomicum; $_{*}$ ad quern hic potifimum refpicimus, non fatis funt arcommodacat. Nam fi Tpectatorem in $\mathbf{C}$ confticutum

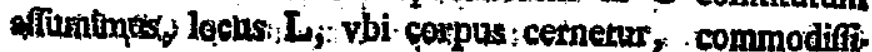

\section{$C A P U T A$}

II

me per quantitatem reftre $\mathrm{CM}$, et anguhum $\mathrm{QCM}$ vna cum angulo $M C L$ repraefentacur: atque fi tabula planum eclipticae referat, rettaque $\mathrm{CQ}$ ad principium arietis fit directa, angulus Q CM in Aftronomia vocari folet fideris longitudo, angulus M C L vero eins latituio do, et recta C M eius diftantia çurtata. Vocemus ergo porro:

1. Diftantiam curtatam fea reEtam $\mathrm{CM}=s$

II. Longitudinem fen angulum QC $M=\emptyset$ III. Latimudinem feu angulum $\mathrm{M} \mathbf{C} \mathrm{L}=\psi$ ac pofito conftanter finu toto $=I$, erunt valores cooso dinatarum ante adhibitarum:

$\mathrm{CP}=p=x \operatorname{cor} \varphi ; \mathrm{PM}=q=x \operatorname{fin} \varphi \& \mathrm{M} \mathrm{L}=r=x \mathrm{sing} \psi$ atque diftantia comporis vera puneto C exic C L $=$ $*$ fec. $\psi=\frac{\pi}{\operatorname{cor} \psi}$.

6. 5. Sumtis nune differentialibus grore : confueteo obtinebimus :

$d p=d x \operatorname{cor} \phi-x d \varphi$ fin $\phi ; d q=d x$ fin $\phi+x d \phi \operatorname{cor} \phi$

$$
\text { et } d r=d \text { tang } \psi+\frac{x d \psi}{\operatorname{cor} \psi^{2}}
$$

atque hinc denuo differentialibus fumendị reperietur,

$d d p=d d x \operatorname{cor} \varphi ;-2 d x d \phi \sin \varphi-* d d \phi$ fin $\phi-x d \phi^{2} \operatorname{cof} \varphi$

$d d q=d d x \sin \varphi+2 d x d \varphi \operatorname{co}\left[\varphi+x d d \varphi \operatorname{cor} \varphi-x d \varphi^{2} \cdot \operatorname{fin} \varphi\right.$

$d d r=d d x \operatorname{cog} \psi+\frac{2 d s d \psi}{\operatorname{cor} \psi^{2}}+\frac{x d d \psi}{\cos \psi^{2}}+\frac{2 x d \psi^{2} f i n \psi}{\operatorname{cor} \psi^{3}}$ 
Binae priores formulae rite combinatae fuppeditabunt fequentes multo concinniores

$$
\begin{aligned}
& d d p \operatorname{cor} \varphi+d d q \text { fin } \varphi=d d x-x d \phi^{2} \\
& d d q \cos \varphi-d d p \text { fin } \varphi=2 d x d \varphi+x d d \varphi
\end{aligned}
$$

ficque habebitur :

$$
\begin{aligned}
& d d x-x d \phi^{2}=-\frac{x}{2} d t^{2}(\mathrm{P} \operatorname{col} \varphi+Q \text { in } \varphi) \\
& 2 d x d \varphi+x d \phi \varphi=-\frac{3}{2} d t^{2}(Q \cos \varphi-\mathrm{P} \text { (in } \varphi)
\end{aligned}
$$

Tertiam vero aequationem deinceps magis tractabilem efficiemus.

S. 6 Manifertum autem eft formularn $P$ cor $\varphi+Q$ fin $\emptyset$ praebere vim ex viribis $P$ et $Q$ compofitam, qua corpus in $\mathrm{L}$ fecundum directionem rectae $\mathrm{MC}$. vrgetur, formulam vero alseram $Q \operatorname{co} \varphi-P$ fin $\varphi$ exprimere vim ex eadem refolutione fecundum directionem $M Q$ adMC normalem direttam. Cum igitur hae duae vires aflum. tis binis $\mathbf{B}$ et $\mathbf{Q}$ aequiualeant, ponamus effe

I. Vim corpus $\mathbf{L}$ fecundum $M C$ trahentem $=V$ r. II Vim corpus $\mathrm{L}$.ècundur MQ trahentem $=T$ manente tertia ,vi corpus ad planum normaliter fecundum LM ,vrgente $=\mathbf{R}$. Atque fequentes habebimus aequationes:

I. $2 d x d \varphi+x d d \varphi=-\frac{x}{2} \mathbf{T} d t^{2}$

II. $d d x-x d \varphi^{2}=-\frac{x}{2} \mathrm{~V} d t^{2}$

IIIddztang $\psi+\frac{2 d x d \psi}{\operatorname{cor} \psi^{2}}+\frac{2 d d \psi}{\operatorname{col} \psi^{2}}+\frac{2 x d \psi^{2} \sin \psi}{\operatorname{cor} \psi^{3}}=-\frac{x}{3} R d t^{2}$

6. 7. Quo autem effectum terciae vis $R$ commodius ad calculum retrovemus, tiore apud Aftronomos recepto conternplemur planum, in quo corpus durance elemento

$$
C A P U T A
$$

elemento temporis $d t$ mouetur, et quod fimul per pun Eum , C tranfeat. Hoc igitur planum cum plano affum to interfeCtionem alicubi formabit, quae fit recta $\subset \Omega$, ac linea nodorum appellari folet; ficque eric $\Omega \mathbb{C L}$ planum orbitae, in qua corpus $\mathbb{L}$ praefenti inftanti movetur, et angulus, quo hoc planum $\Omega \mathrm{CL}$ ad planum fixum QCM inclinatur, vocatur inclinatio orbitz ad eclipticam pro tempore praefenti. Cum jgitur ex his duabus rebus latitudo fideris definiri foleat, ponamus.

Longitudinem nodi afcendentis feu angulum $\mathrm{QC} \Omega=\pi$ ac inclinationem orbitae $\Omega$ CL ad éclipticam $=\rho$ atque loco latitudinis $\psi$ has duas quantitates, et $\boldsymbol{\rho}$ definire oportebir.

5. 8. Tertia ergo aequatio in duas difpertietur; ad quas inueniendas ex $M$ et $L$ ad lineam nodorum $C \Omega$ ducantur normales $M N$ et $L N$, eritque angulus LN.M imenfura inclinationis orbitae ad eclipticam! ideoque $L \mathrm{~N} \mathrm{M}=$ s. Tam vero ob angulum $\Omega \mathrm{CM}$ $=\emptyset-\pi$ et $\mathbf{C} M=x$ erit :

$$
\mathrm{CN}=x \operatorname{cof}(\varphi-\pi) \text { et } \mathrm{MN}=x \operatorname{lin}(\varphi-\pi)
$$

hinc elicietur $\mathrm{ML}=x \operatorname{tang}_{\rho}$ fin $(\varphi-\pi)$, vnde prodic tang $\mathbf{M ~ C ~ L ~}=\operatorname{tang} \psi=\operatorname{tang} \rho$ fin $(\varphi-\pi)$, quae formela inferuit latitudini $\psi$ ex cognita inclinatione $\rho$ et loco nodi eiusue longitudine $z$ inueniendae, fi quidem iam cognita fuerit longitudo fideris $\varphi$. Quoniam ausem fidus elemenso temporis $d t$ in . eodem plano manet, in differentiatione formulae tang $\psi=\operatorname{tang} \rho$ fin $(\varphi-\pi)$, $B_{3}$ quantio 
quantitates $\pi$ et $\rho$ tanquam conitantes fpectari poterunt, eritque idcirco.

$$
\frac{d \psi}{\operatorname{cor} \psi^{2}}=d \varphi \operatorname{tang} \xi \operatorname{cor}(\varphi-\pi)
$$

6. 9. Interim tamen nihil impedit, quominus in cadem differentiatione quantikates $\boldsymbol{z}$ et $\rho$ tanquam va riabiles tractemus, quales reuera effe poffunt fucceflu temporis; vnde orietur haec aequatio:

$$
\frac{d \psi}{\operatorname{cor} \psi^{2}}=\frac{d \rho}{\operatorname{cor} \rho^{2}} \text { fin }(\varphi-\pi)+(d \varphi-d \pi) \text { tang } \rho \operatorname{cor}(\varphi-\pi)
$$

erit :

$$
\frac{d d \psi}{\operatorname{cor} \psi^{2}}+\frac{2 d \psi^{2} \sin \psi}{\operatorname{cor} \psi^{3}}=d d \varphi \text { tang } \rho \operatorname{cof}(\varphi-\pi)
$$$$
+\frac{d \phi d \pi \operatorname{tang} \rho \operatorname{cof}(\varphi-\pi)^{2}}{\text { fin }(\varphi-\pi)}=d \phi(d \varphi-d \pi) \operatorname{tang} \rho \text { fin }(\varphi-\pi)
$$

feu $\frac{d d \psi}{\operatorname{cor} \psi^{2}}+\frac{2 d \psi^{2} \sin \psi}{\operatorname{cor} \psi^{3}}$

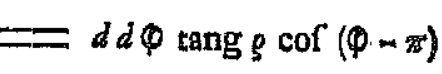

$$
+\frac{d \varphi d \pi \operatorname{tang} \rho}{\text { fin }(\varphi-\pi)}-d \varphi^{2} \text { tang } f(n)(\varphi-\pi)
$$

qui valores pro $\psi$ in tertia aequatione fuperiori fabftio turi fuppeditabunt:

$d d x \operatorname{tang} \rho(\operatorname{in}(\varphi-\pi)+2 d x d \phi$ tang $\rho \operatorname{cor}(\varphi-\pi)+x d d \phi$ tang $\rho \operatorname{col}(\varphi-\pi)$

$$
+\frac{x d \phi d \pi \operatorname{tang} \rho}{\operatorname{fin}(\varphi-\pi)}-x d \varphi^{2} \text { tang } \rho \operatorname{fin}(\varphi-\pi)=-\frac{\pi}{2} \mathrm{R} d t^{2}
$$

quae transmutatur in hanc:

$\left(d d x-x d \varphi^{2}\right)$ tang $\rho$ lin $\left(\varphi_{-\pi}\right)+i\left(2 d x d \varphi+{ }^{\prime} x d d \varphi\right)$ tang $\rho \operatorname{cof}\left(\varphi_{m} x\right)$

$$
+\frac{x d \phi d \pi \text { tange }}{\operatorname{fin}(\varphi-\pi)}=-\frac{\pi}{2} R d s^{3}
$$

\$. In. Commode, hic euenit vt in ifta formula illae ipfae expreffiones differentio-differentiales $d d x-x d \varphi^{2}$ et $2 d x d \varphi+x d d \varphi$ occurrant, quae ex actione duarum reliquarum virium funt enatae : vnde fi formularum hav rum valores aequilualentes $-\frac{x}{2} \mathrm{~V} d t^{2}$ et $-\frac{x}{2} \mathrm{~T} d t^{2}$ fub tituamus, impetrabimus

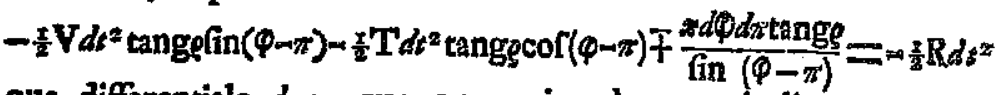
qua differentiale $d \pi$, quo promotio elementaris linese nodorum indicatur, ita determinabitur, vt fit

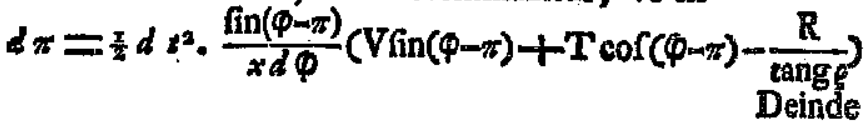

$$
\begin{aligned}
& \text { 5. 10. Differentiemus formulam primo inuentam } \\
& \frac{d \psi}{\operatorname{cof} \psi^{2}}=d \varphi \text { tang } \rho \operatorname{cor}(\varphi-\pi)
\end{aligned}
$$

iterum, et cum fit d: tang $\rho=\frac{d \pi \text { tang } \rho \operatorname{co} \Gamma(\varphi-\pi)}{\text { fin }(\varphi-\pi)}$ 
Deinde cum fit $d, l$ tang $\rho=\frac{d \pi}{\operatorname{tang}(\varphi-\pi)}$; exit $d . l$ tang $\rho=\frac{x}{2} d t^{2} . \frac{\operatorname{cor}(\varphi-\pi)}{x d \phi}\left(V \operatorname{lin}(\varphi-\pi)+\operatorname{Tco}(\varphi-\pi)-\frac{\mathbf{R}}{\operatorname{tang} \varphi}\right)$ Duas ergo has aequationes loco fuperioris tertiae, ex qua latitudo $\psi$ inueniri debebat, in calculum introduci conveniet; inuentis enim $\pi$ et $\rho$ erit tang $\psi=$ rang $\rho$ fin $(\varphi-\pi)$.

§. 12. Hine pater lineam nodorum nunquam effe mobilem, quin fimul inclinatio variationi fit obnoxia. Eadem enim vis $V$ fin $(\varphi-\pi)+T \operatorname{cor}(\varphi-x)-\frac{R}{\operatorname{cang} e^{3}}$ quac lineae nodorum motum imprimit eius longitudinem $x$ immutando, fimul in inclinatione $\rho$ variationem generat. Nulli autem plane immutationi cam linea nodorum, quam inclinatio erunt fubjectae, fi vis illa eue. nefcat, quod euenit fi media direltio omnjum virium - corpus L follicisantium in ipfum planum $\& \mathbf{C L}$, in quo corpus femel moueri coepit, perpetuo incidat; hicque eft cafus, quo corpus continuo in eodem plano moueri pergit. Generatim ergo corporis a tribus viribus $V_{3} \mathbf{T}, \mathbf{R}$ follicitati motus quatuor fequentibus aequationibus determinatur.
I. $2 d x d \varphi+x d d \varphi=-T d t^{2}$
II. $\quad d d x-x d \varphi^{2}=-\frac{x}{2} \mathrm{~V} d t^{*}$
III. $d \pi=\frac{x}{2} d t^{2}, \frac{\text { fin }(\varphi-\pi)}{x d \varphi}\left(\operatorname{Vin}\left(\varphi_{m \pi}\right)+T \operatorname{cor}\left(\varphi_{m} \pi\right)-\frac{R}{\operatorname{Rong} \rho}\right)$
IV. $d, l$ tang $\rho=\frac{d \sigma}{\operatorname{tang}(\varphi-\pi)}$

quas ergo quouis cafu oblato refolui oportet.

CAPUT

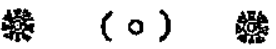

xy

\section{CA P UT II. INUESTIGATIO VIRIUM LUNAM SOLLICITANTIUM. \\ §. 13.}

Cum Lunae motus, qualis ex centro terrae fpectare. tur, definiri debeat, fit $\mathbf{C}$ terrae centrum, ad Fig. \&. quod etiam praecipua vis, qua Luna virgetur, dìrecta concipitur; atque tabula exhibear planum eclipti. cae, in quo nune quidem Sol exiftat in $S$, Luna vero fupra hoc planum verfetur in $L$ latitudinem habens bo. realem, vnde ad planum eclipticae perpendiculum demittatur LM. Hinc ductis reetis $\mathrm{CL}, \mathrm{CM}, \mathrm{CS}$, itemque $C Q$ initium arietis verfus, vnde longitudines computari folent, fiant fequentes denominationes.

I. Longitudo Solis feu angulus A C S $=0$

2. Longisudo Lunae feu angulus $\mathbf{A C M}=\emptyset$

3. Laticudo Lunae feu angulus $M C L=\psi$

4. Diftantia Solis a Terra C S $=y$

5. Diftantia Lunae a Terra curtata $\mathrm{CM}=$.

§. I4. Sit iam AM proieCtio orbitae lunaris in pla. num eclipticae; ac planum, in quo Luna nunc moue. cur, per centrum terrae ductum, planum eclipticae interfecet fecundum rectam $C \Omega$, quae lineam nodorum pro tempore praefenti exhibebir: ac terminus $\Omega$ quidem nodum afcendentem referet, fiquidem lunam fe. cundum regionem AM promoueri ponamus. Quod fi ergo porro vocemus
C
6. Lon- 
6. Lorgindinem nodi afc: AC $\Omega=\pi$

7. Incl. orbitae Lunae ad eclipticam $=\rho$

hinc latitudo Lunae geocentrica ita definietur, vt fic tang $\psi=$ tang. fin $(\varphi-\pi)$. Vnde incrementum latitu= dinis $d \psi$ commode affignabitur, cum fic, ve fupra vidimus $d$. tang $\psi=\frac{d \psi}{\operatorname{cof} \frac{\psi^{2}}{2}}=d \varphi \operatorname{tang} \rho \operatorname{cof}(\varphi-\pi):$ ac praeterea ex motu nodi cognito variatio inclinationis ita definietur, vt fit $d . \operatorname{tang} \rho=\frac{d \pi \text { tang } \rho}{\operatorname{tang}(\varphi-\pi)}$, reu $d \rho=\frac{d \pi \text { fin } \rho \operatorname{cor} \rho}{\tan g(\varphi-\pi)}$.

§. 15. Cum nune primum Luna al centrum terrae C fecundum directionem LC attrahatur, fit haec vis $=\mathrm{M}$. Deinde fit vis, gua Luna ad folem $\mathrm{S}$ vrgetur fecundum $L S=N$; atque his duabus viribus Luna proprie vrgeri cenfenda.ef. Praeterea vero cum Terra ipfa, - ad guam motum Lunae referimus, in motu verfetur, ut eam tanquam quiefcentem confiderare queamus, non fo. lum motum Terrae, fed etiam vires, quibus Terra vrgetur, toti mundo fecundum plagas oppofitas imprimi concipiamus. Sit igitur vis qua Terra ad Solem vrgetur $=\mathrm{s}$, \& vis qua ad Lunam trahitur $=\mathrm{L}$, his viribus contrario modo in lunam translatis, Lana fequentibus viribus follicitata habebitur

I. Secundum direationem $\mathbf{L}$. vi $=\mathbf{M}+\mathbf{L}$

2. Secundum directionem L $\mathrm{S}$ vi $=\mathbf{N}$

3. Secundum directionem $\mathbf{M} \mathbf{R}$ ipfi

$S C$ parailelam vi $=S$.
$C A P U T H H_{0}$

19.

§. 16. Nune primo has vires ad ternas directio nes fupra affumtas $M C, M Q$ et $L M$ reducananus; ac primo quidem vis $M+L$ dabit

pro direetione $\mathrm{MC}$ vim $=(\mathrm{M}-\mathrm{f}-\mathrm{L}) \operatorname{cor} \psi$

pro direCtione $L M$ vim $=(M+L)$ fin $\psi$

Secunda vis $\mathbf{N}$ vero dabit

pro diretzione $L N i$ vim $=\frac{L M}{L S} \cdot N$

pro directione MS vim $=\frac{M S}{L S}, N$

at haec viterius refoluta ducta $\mathrm{Mr}$. ipfi $\mathrm{CS}$ parallela dabit:

pro directione MC vim $=\frac{M C}{L S} . N$

pro dirętione $\mathrm{M} r \operatorname{vim}=\frac{\mathrm{CS}}{\mathrm{LS}} \cdot \mathrm{N}$

Haec poftrema a vi tertia $S$ fubtracta relinquet vimi S- $\frac{\text { CS }}{\mathrm{LS}}$. N, qua Luna fecundum direationem MR follicitatur, quae ob angulum CMR $=S C M=-$ dabit

$$
\begin{aligned}
& \text { pro direQtione } M C \text { vim }=\left(S-\frac{C S}{L S} \cdot N\right) \operatorname{co}(\phi-\theta) \\
& \text { pro directione } M_{q} \text { vim }=\left(S-\frac{C S}{L S} \cdot N\right) \sin \left(\varphi_{-} \theta\right)
\end{aligned}
$$

vbi direCtio $M_{q}$ contraria eft direCione $M Q$.

6. 17. His iam viribus cum ternis initio affum tis $\mathbf{V}, \mathbf{T}$ et $\mathbf{R}$ comparandis, inueniemus pro his viribus fequentes valores:

$\mathbf{C}_{2}$
1. pro-

\$. ร6. Hune 
2. pro diretione $\mathbf{M} \mathbf{C} \operatorname{vim} \mathbf{V}=$

$$
(\mathrm{M}+\mathrm{L}) \cos \psi+\frac{\mathrm{MC}}{\overline{\mathrm{LS}}} \cdot \mathrm{N}+\left(\mathrm{S}-\frac{\mathrm{CS}}{\overline{\mathrm{LS}}} \cdot \mathrm{N}\right) \operatorname{cor}(\varphi-\theta)
$$

2. pro directione $M Q \operatorname{vim} T=-\left(S-\frac{C S}{L S} \cdot N\right)$ fin $(\varphi-\theta)$ 3. pro direlione $L M \operatorname{vim} R=(M+L) \operatorname{lin} \psi+\frac{L M}{L S} \mathbf{N}$. Cum nune fit $\mathrm{CM}=x, \mathrm{CS}=y$, et angulus $\mathrm{SCM}=\varnothing-\theta$; erit MS $=V(x x-2 x y \operatorname{cof}(\varphi-\theta)+y y)$, et ob LM $=x$ tang $\psi$ erit L $S=V\left(y y-2 x y \operatorname{cof}(\varphi-\theta)+x x f e c, \psi^{2}\right)$, guae diftantia Solis a Lun LS brenitatis gratia ponatur $=u$, vt fit $u=V\left(y y-2 x y \operatorname{cof}(\varphi-\theta)+x+\right.$ fec. $\left.\psi^{2}\right)$. His ergo va. loribus introduetis erunt vires noltrae:

1. $\mathrm{V}=(\mathrm{M}-\mathrm{L}+\mathrm{L}) \operatorname{cor} \psi+\frac{\mathrm{Nx}}{\mathrm{s}}+\mathrm{S} \operatorname{cof}(\varphi-\theta)-\frac{\mathrm{N} y}{4} \operatorname{cor}(\varphi-\theta)$

$$
\text { 2. } T=-S \text { fin }(\varphi-\theta)+\frac{N y}{u} \operatorname{fin}(\varphi-\theta) \text {. }
$$

. 3. $R=-(M-L)$ fin $\psi+\mathbf{N} x \operatorname{tang} \psi$

8. 18. Quia nunc eft tang $\psi=\operatorname{tang}^{2} \rho$ fin $(\varphi-\pi)$ et fin $\psi=$ tang $\psi$ cor $\psi$ erit:

$\frac{R}{\operatorname{tang} \rho}=(M+L) \cos \psi$ fin $(\varphi-\pi)+\frac{N x \sin (\varphi-\pi)}{\psi}$

tum vero habebitur

$V \operatorname{fin}(\varphi-\pi)+T \operatorname{cor}(\varphi-\pi)=(M+L) \operatorname{cof} \psi \operatorname{fin}(\varphi-\pi)+N x \operatorname{Nin}(\varphi-\pi)$

$$
+S \operatorname{cor}(\varphi-\theta) \operatorname{fin}(\varphi-\pi)-\frac{N y \operatorname{cor}(\varphi-\theta) \operatorname{fin}(\varphi-\pi)}{\mu}
$$

$-\mathrm{S}$ fin $(\varphi-\theta) \operatorname{cor}(\varphi-\pi)+\frac{N y \sin (\varphi-\theta) \operatorname{cof}(\varphi-\pi)}{\omega}$

quae ob $\operatorname{cor}(\varphi-\theta) \operatorname{fin}(\varphi-\pi)-\operatorname{fin}(\varphi-\theta) \operatorname{co}(\varphi-x)=\operatorname{fin}(\varphi-x)$, dat

\section{$C A U T$ UR}

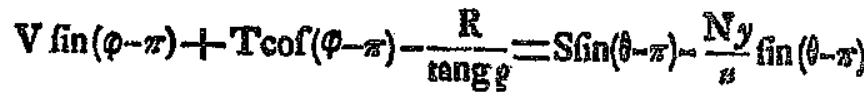
ex quo aequationes motum Lunae continentes erunt

I. $2 d x d \varphi+x d d \varphi=-\frac{x}{x} d t^{2}\left(\frac{N y}{t}-S\right)$ fin $(\varphi-\theta)$

II. $d d x \rightarrow x d \varphi^{2}=-\frac{1}{2} d t^{2}\left((M+L) \operatorname{co}\left(\psi+\frac{N_{x}}{w}-\left(\frac{N_{y}}{z}-S\right) \operatorname{co}\left(\left(\varphi_{-\infty}\right)\right)\right.\right.$

III. $d \pi=-\frac{\pi}{2} d t^{2} . \frac{\operatorname{lin}(\varphi-\pi) \operatorname{fin}(\theta-\pi)}{x d \varphi}\left(\frac{N y}{y}-\mathbb{S}\right)$

IV. $d . t$ tang $\rho=\frac{d x}{\operatorname{tang}(\varphi-\pi)}$

vbi $\theta-\pi$ exprimit angulum $\Omega$ CS fen diftantiam Solis a nodo afcendènte.

§. 19. Jam fecundum Theoriam Newtoni, fi masfam Terrae ponamus $=\delta$ ac Lunae $=\hat{a}$, ob diftantiam

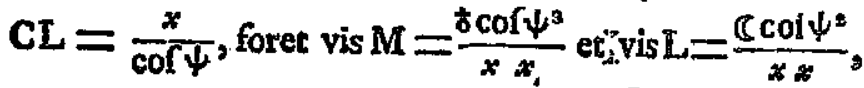
ficque vis tota $M+L=(t+C) \cdot \frac{\operatorname{cor} \psi^{2}}{x *}$. Quo aus tem, fi force haec Theoria infufficiens deprehendatur rem generalius compleetariur, ponamus hane vim:

$\mathrm{M}+\mathrm{L}=(t+c) \cos \psi^{2}\left(\frac{\mathrm{I}}{x^{2}}-\frac{\mathrm{I}}{b b}\right)$

vbi terminus $\frac{1}{b b}$ defectum huius vis a Theoria New toniana exhibeat; qui cum fit minimus, pro conftanci haberi poterit falcem pro exigua variabilitate, quam diftantis $x$ fubit. Vim autem Solis exacte Theoriae Newtonianae conformem aftumere poterimus; quoniam etiomfi inde recederet, differentia non folum foret C 3 quass 
quam minima; fed quia pro Luna aeque difcreparet ac pro Term, in noftris formulis nullius plane effet momenti.

5. 20. Pofits ergo Solis mafta $=0$, erit vis, qua Terram ad fe attrahit $S=\frac{0}{y y}$, vis autem qua Lunam ad fe trahit $\mathrm{N}=\frac{0}{4 *}$. His ergo valoribus virium in calculurr inductis, morus lunae ex quatuor fequentibus aequationibus determinari debet:

$$
\begin{aligned}
& 1.2 d x d \varphi+x d d \varphi=-\frac{3}{2} d t^{2}\left(\frac{Q y}{\|^{3}}-\frac{\Theta}{y y}\right) \text { fin }(\varphi-\theta) \\
& \text { II. } d d x-x d \varphi^{3}=-\frac{x}{2} d t^{2}(t+C) \cos \psi^{3}\left(\frac{1}{x+}-\frac{1}{b b}\right) \\
& -\frac{x}{2} d t^{2}\left(\frac{Q^{x}}{u^{3}}-\frac{O y}{u^{3}} \operatorname{col}(\varphi-\theta)+\frac{Q}{y y} \operatorname{cor}(\varphi-\theta)\right) \\
& \text { III. } d \pi=-\frac{x}{2} d t^{2} \cdot \frac{\text { fin }(\varphi-x) \text { fin }(\theta-x)}{x d \varphi}\left(\frac{O y}{n^{3}}-\frac{\theta}{y y}\right)
\end{aligned}
$$$$
\text { IV. } d . t \operatorname{tang} \varphi=\frac{d \pi}{\operatorname{tang}(\varphi-\pi)}
$$

Hic iam primum curandum eft, vt elementum tempo. poris, quod eft quantitss heterogenea, ex calculo eliminemus; id quod commodifime fiet, fi motum me dium folis vtpote tempori proportionalem, loco tem. poris in calculum introducemus.

6.21. Cum igitur etiam motus Solis in his aequationibus fit ratio habenda, eum prius inueftigemus: et quoniam pro terra quielcente Sol a fola vi $\frac{0}{j y}$ ad ter- ram follicitari concipiendus eft, fi formulas pro luna inuentas ad folem accommodemus, obtinebimus:

$$
\begin{aligned}
& 2 d y d \theta+y d d \theta=0 \\
& d d y-y d \theta^{2}=-\frac{x}{2} d t^{2} \cdot \frac{O}{y y}
\end{aligned}
$$

fi iam diftantiam Solis a terra mediam ponamus $=$ ejusque anomaliam mediam $=q$; cafu quo excentricitas orbitae folaris effet nulla, foret femper $y=\tilde{\delta} \&$ $d \theta=d q$ : vnde altera aequatio dabit $-b d q^{2}=$ $\frac{s}{2} d t^{2} \cdot \frac{O}{b b}$. Quare loco elementi temporis $d t$ elemen. tum anomaliae mediae folis ita in calculum introduci debee, ve vbique loco $\frac{x}{2} d t^{2}$ feribatur $\frac{b^{3} d q^{2}}{0}$, id quod tam in his formulis pro Sole, quam in fuperioribus pro Luna fieri poterit.

5. 22. Cum iam $b$ denotet diftantiam folis a terra mediam, fit eius vera diftantia $y=b \mathrm{co}$, et anomalia eius vera $=s$, erit $d \theta=d s$, quandoquidem a motu apogei folis animum abftrahimus. Hine itaque erit

$$
\begin{aligned}
& 2 d \omega d s+\omega d d s=0 \\
& d d \omega-\omega d s^{2}=-\frac{d q^{2}}{\omega \omega},
\end{aligned}
$$

quarum prior integrata dat $\omega \omega d s=\mathrm{C} d q$ ob $d q$ conftans, ideoque $\omega d s^{2}=\frac{C C d q^{2}}{\omega^{3}}$; qui valor in altera aequatione fubftitutus praebet,

$$
d d \omega=\frac{\mathrm{CC} d q^{2}}{\omega^{3}}-\frac{d q^{2}}{\omega \omega} \text {. }
$$


quae per $2 d$ io multiplicata et integrata dat:

$$
\frac{d \omega^{2}}{d q^{2}}=\mathrm{D}-\frac{\mathrm{CC}}{\omega \omega}+\frac{2}{\omega}
$$

vnde fit $d q=\frac{\omega d \omega}{V(\mathrm{D} \omega \omega+2 \omega-\mathrm{CC})}$

ac proinde $d s=\frac{\mathrm{C} d \omega}{\omega V(\mathrm{D} \omega \omega t+2 \omega}$

$$
\begin{aligned}
& 2 w-\mathrm{CC} \\
& \mathrm{C} d w \\
& w+2 \omega-\mathrm{CC})
\end{aligned}
$$

§. 23. Quanquam autem hine valores finiti haud difficulter deduci poffent, tamen alia vtar methodo, quae in motu Lunae . maiorem praeftabit vilitatem. Inuento autem $\omega \omega d s=\mathbf{C} d q$, alteram aequationem ita transformo, vt elementi conftantis $d q$ racio non am. plius habeatur:

$$
\text { . } \quad d q . d \cdot \frac{d \omega}{d q}-\omega d s^{2}=m \frac{d q^{2}}{\omega \omega}
$$

Sit nunc $u=\frac{\mathrm{r}}{z}$, vt habeat $d s=\mathrm{C} w \| d q$, et $d \ddot{w}$ $=-\frac{d u}{u x}$, et ob $d q=\frac{d s}{\mathrm{C} u u}$ erit $\frac{d \omega}{d q}=-\frac{\mathrm{C} d u}{d s} ;$ hine

fumto iam elemento $d s$ conftante, eric

$$
\begin{gathered}
\frac{-d^{4} s}{\mathrm{C} u w} \cdot \frac{\mathrm{C} d d u}{d s}-\frac{d s^{2}}{u}=-\frac{d s^{2}}{\mathbf{C C} u z} \mathrm{feu} \\
d d u+w d s^{z}=\frac{d s^{2}}{\mathbf{C C}}
\end{gathered}
$$

vnde ftatim elicitur $u=\frac{x-e \text { cors }}{\mathrm{C} \mathrm{C}}$; vbi $e$ excentricitatem orbitae folaris indicabit.

\$.24. Hinc porro habebitur $\omega=\frac{\mathbf{C ~ C}}{I-e \operatorname{cof} s^{5}}$, et,$=\frac{\mathbf{C C} b}{\mathrm{I}-\mathrm{Cofs^{2 }}}$ anomalia vera $s$ ab apogeo computata; vnde diftantia apogei

apogei a terra pofito $s=0$ erit $=\frac{\mathrm{CC} b}{\mathrm{I}-\mathrm{e}}$, ec diftankia pe. rigei potito $s=180^{\circ}$ prodit $=\frac{\mathrm{CC} b}{x+e} ;$ ficque diftancia me media fiet $=\frac{\mathrm{CC} b}{\mathrm{I}-e^{e}}$, quae cum per hypothefir segualis effe debeat ipfi 6 , ftatui oportet $\mathrm{CC}=\mathrm{x}$ mee: hisacque exic

$$
y=\frac{b(1-c e)}{x \rightarrow e \operatorname{cof} s} \text { et } \omega=\frac{1-s e}{i-e \operatorname{cor} s}
$$

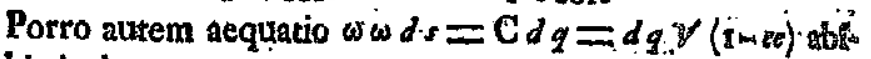
bit in hane:

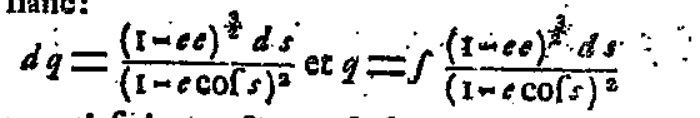

ex qua, vti facis conftat, vel data anomalia vera s inue: tiri poteft anomialia media qs vel vicifim. His itaque formulis motum Solis continentibus in determination motus Lunae vtamur.

f. 25. Primo ergo loco $\frac{5}{2} d t^{2}$ ybique feribamus $\frac{t^{3} d q^{2}}{0}$ et $6 \omega$ loço $y$, quo facto noftrae aequationes fient 1. $2 d x d \varphi+x d d \varphi=-b^{3} d q^{2}\left(\frac{b \omega}{b^{2}}-\frac{1}{b b \omega \omega}\right) \operatorname{cin}(\varphi-\theta)$

II. $d d x-x d \varphi^{2}=\frac{(\dot{t}+C) b^{3} d_{g^{2}}^{2}}{Q} \operatorname{cor} \psi^{3}\left(\frac{I}{x x^{2}}-\frac{1}{b b}\right)$

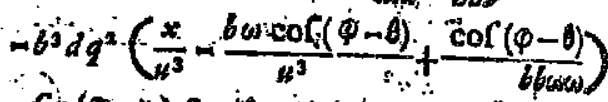

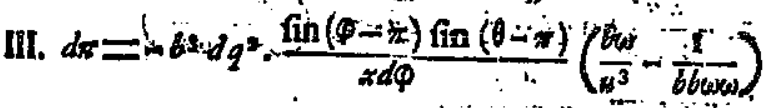

Ponatur porro $u=6 v$, adque in caictum quogue intro $\because \quad D$ D dica. 
duccatur diftantia media lunae a terra, quae fit=A, pofitoque $x=a z$, prodibis:

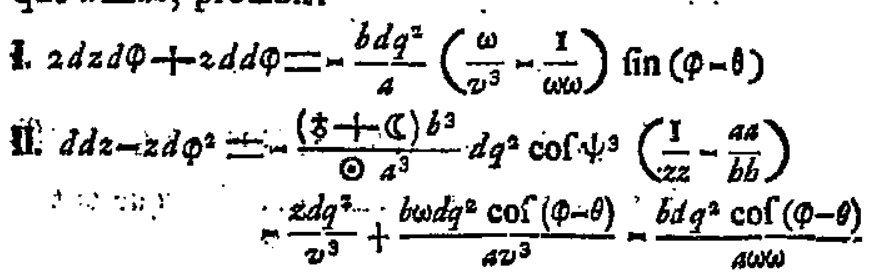

㫮 $d \pi=m \frac{b d q^{2}}{d z d \phi}$ fin $(\varphi-\pi)$ fin $(\theta m \pi)\left(\frac{\omega}{v^{3}}-\frac{1}{\omega \omega}\right)$

§. 26. Ponamus nune ad abbreuiandum: $\frac{(z+c) b^{3}}{O a^{3}}=m ; \frac{(\delta+\varepsilon) b^{3}}{0 a b b}=\mu$, feu $\mu=\frac{m a a}{b b}$

guarum litcerarum valores $m$ et $\mu$ per obferuationes defi thiri debent; 'tum vero fit $\frac{b}{b}=y$, quae eft quantitas valde parua a parallaxi folis pendens. Hisque valoribus introductis, sequationes noftrae fequentes induent formas; i. $2 d z d \varphi+z d d \varphi=-\frac{1}{y} d q^{2}\left(\frac{\omega}{v^{3}}-\frac{1}{\omega x+1}\right)($ in $\varphi-\theta)$

II. $d d z \rightarrow z d \varphi^{3}=-\frac{d q^{2} \operatorname{cor} \psi^{3}}{z 2}+\mu d q^{2} \operatorname{cor} \psi^{3}$

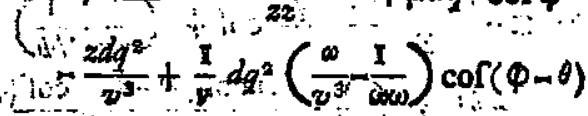

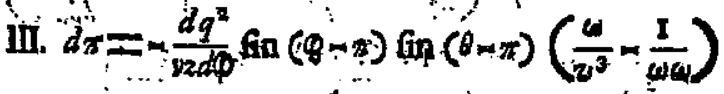

IV. $d . l_{\text {tang } \rho}=\frac{d \pi}{\operatorname{tang}\left(\varphi_{-\pi}\right)} \quad \therefore$

\$. 27.

$a$, pofito-

.8)

$\left.\frac{a b}{b b}\right)$

$\cot (\varphi-\theta)$

)

i
t
$t$
$\vdots$
$p$
e
$q$
$=$
$q$
$f i$
$f$
$c$
$t$
$g$
it

ones defi.

tcitas val.

ribus in-

$t$ formas:

$-\theta)$

$\cdots$
§. 27. Cum iam pofuerimus:

$$
x=a z ; y=b \omega ; \quad z=\frac{b}{b} \quad y=\frac{b}{b}
$$

erit $\quad u=V\left(b b \omega \omega-2 a b \omega z \operatorname{col}^{2}(\varphi-\theta)+a B z z\right.$ fec. $\left\{\begin{array}{l}2 \\ 2\end{array}\right)$ atque $v=V\left(\omega \omega-2 y \omega z \operatorname{cor}(\varphi-\theta)+v y z z\right.$ fec. $\left.\psi^{2}\right)$ vbi notandum eft quantitates $\omega, q$ et $\&$ ex mota folis isa inter fe pendere, $v t$ fit

$$
w=\frac{1-e e}{1-e \operatorname{cor} s} \text { et } d q=\frac{(1-e e)^{\frac{3}{2}} d s}{(x-e \operatorname{cof} s)^{2}}=\frac{1 e \cot d s}{V(I-e t)}
$$

ita ve huic ad datum quoduis tempus tam yalor ipfus quam anomaliae verae $s$ definiri poffit. - Motium autem has formulas ad calculum reuocandi hic non trado, quia eum alias fuibus iam expofut: hoc folum hic notari con. venier, excentricitatis orbitae folaris valorem ex obferruga tionibus colligi $c=0$, 01680 .

$\therefore$ 5. 28. Nunc antequam vlterius progred gàeamus, valorem irrationalem ipfius $v$ tolli conueniet, quod facile per feriem praeftabitur maxime conuergenseth, ob; $v$ fra. Etionem valde paruam; fumta enim parallaxi foliṣ $=12^{d /,}$ quia parallaxis lunae media eft $=3380 !$, ,

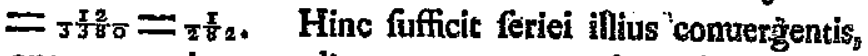
quam reperiemus, aliquot taneum terminos ab initio as fumfifie; quia reliqui ob paruitatem continuo magiș cre fcentem tuto omitti poterunt. Cam autem añgulus $\varphi-\theta$, qui diftantiam folis a luna fecundum longitudinem deno. tat, in hac refolutione frequentifime occurret, breuicatis gratia ponamus $\quad \phi-\theta=\eta$

ita ve pro $v$ fequentem habeamus valorem irrationalem $y=V\left(\omega \omega-2 \nu \omega z \operatorname{col} \eta+y y z s f\left(e c . \psi^{2}\right)\right.$.
$\mathrm{D}_{2}$ \$. 29. 
§. 29. Quoniam ergo in noftris formulis occürrit

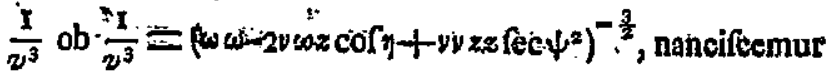

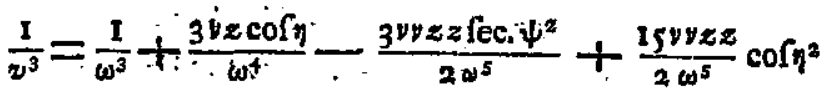
toi terminos altioies ipfius y poteftates inuoluentes fine haefitatione reiicere poflumus; in ipfis aequationibus au= tem tantum in prima ipfius y poteftate fubfiftemus. Ha. bebimus ergo:

$\frac{i}{v}\left(\frac{\omega}{v^{3}}-\frac{I^{\prime}}{\omega \omega}\right)=\frac{3 z \operatorname{cor} \eta}{\omega^{3}}+\frac{3^{y z z}}{2 \omega^{4}}\left(5 \operatorname{cor} \eta^{2}-f e c \psi_{3}^{3}\right)$ feu $\frac{1}{y}\left(\frac{\omega}{v^{3}}-\frac{1}{\omega \omega}\right)=\frac{3 z \operatorname{col} \eta}{\omega^{3}}+\frac{3 \nu z z}{4 \omega^{4}}\left(5+5 \operatorname{cof} 2 \eta-2 \operatorname{fec} \cdot \psi^{2}\right)$ hincque porro:

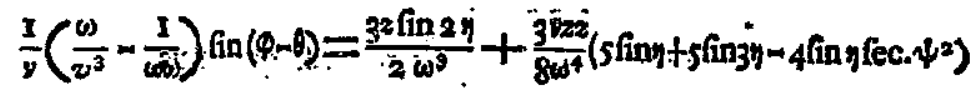
$\frac{x}{\nu}\left(\frac{\omega}{z^{3}}-\frac{1}{\omega \omega}\right) \operatorname{cof}(\varphi-\theta)=\frac{3 z}{2 \omega^{3}}(1+\operatorname{cor} 2 \eta)+\frac{3 y z z}{8 \omega^{4}}\left(15 \operatorname{cor} \eta+5 \operatorname{cof} 3 \psi 4 \operatorname{cof} f\left(e c \cdot \psi^{2}\right)\right.$ grque $\frac{z}{v^{3}}=\frac{\pi}{\omega^{3}}+\frac{3 y 2 z}{\omega^{4}}$ cof $\eta$

$\therefore$ 5o: Subltitianus hos valores in noftris aequa: tionibus atgue obtinebimus:

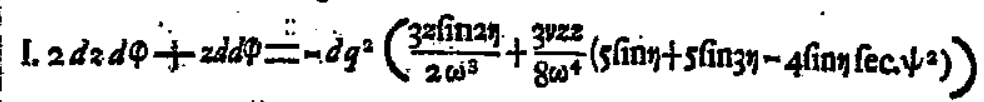
11. $d d z-z d \varphi^{2}=-\frac{m d q^{2} \operatorname{co}\left(\psi^{3}\right.}{z z}+\mu d q^{2} \operatorname{cor} \psi^{3}+\frac{z d q^{2}}{2 \omega^{3}}+\frac{3 z d q^{2}}{2 \omega^{3}} \operatorname{cof} 2 \eta$ $+\frac{3 y z z d g^{2}}{8 \omega^{4}} \cdot\left(7 \cos y+5 \cos 3 \pi-4 \cos y \cdot\left(\operatorname{cec} \psi^{2}\right)\right.$.

ill. $d \pi$

CAPUT HA

is occurrit

incifcemur $\frac{v z z}{x^{5}} \operatorname{cof} \eta^{2}$

ientes fine

onibus all-

nus. Ha.

:c. $\psi$ ? feu

$-2\left(e^{2} . \psi^{2}\right)$

$1 \eta\left(\operatorname{lec} . \psi^{2}\right)$

offifec. $\left.\psi^{2}\right)$

is aequa:

(yfec, $\left.\psi^{2}\right)$

$\frac{d g^{2}}{w^{3}} \cos 2 y$

ec, $\left.\psi^{2}\right)$

III. $d x$

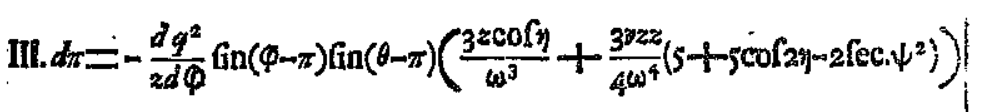

IV. $d_{0} l$ tang $\rho=\frac{d \pi}{\operatorname{tang}(\varphi-\pi)}$.

Hic iam obfervare licet, cum angulus $\psi$ nunquam fere $5^{\circ}$. fuperet; eiusque fecans nonnifi in terminis iam per multiplicatis, ac propterea refpeetu reliquorim valde parvis occurrat, fine villus erroris fenfibilis mesu in his terminis poni poffe fec. $\psi=\mathrm{I}$

5. 35. Deinde vt etiam ex maioribus terminis cof $\psi$ eliminemus; confideremus formulam tang $\psi=$ tang. $\rho$ fin $(\varphi-\pi)$, eritque fec. $\psi=\frac{I}{\operatorname{cof} \psi}=V\left(I+\operatorname{tang} e^{2} \operatorname{fin}(\varphi-\pi)^{3}\right)$ Hine ergo habebimus :

$$
\operatorname{cor} \psi^{3}=\left(1+\operatorname{tang} \rho^{x} . \operatorname{fin}(\varphi-x)^{2}\right)_{i}^{-\frac{3}{4}}
$$

et cuim tang $\rho^{2}$ nunquam fere fractionem $r^{x} x^{\circ}$ foperet eric fatis exacte:

$\operatorname{cor} \psi^{3}=x-\frac{3}{2} \operatorname{tang} \rho^{2}$. fin $(\varphi-\pi)^{2}-$ vel etiam $\operatorname{cor} \psi^{3}=x-\frac{3}{4}$ tang $\rho^{2}+\frac{3}{4} \operatorname{tang} \rho^{2} \operatorname{cor} 2(\varphi-\pi)$

qui valor pro cor $\psi^{3}$ in termino maiore $\frac{m d q^{2} \operatorname{cor} \psi^{3}}{2 z}$ fubfitui poreft: in altero autem termino $\mu d q^{2}$ cof $\psi^{3}$ quia per fe eft valde paruus, atgue adeo fecundum Theoriam Newtoni euanefceret, nihil impedit, quo minus loco $\operatorname{cor} \psi^{3}$ foribamus vnitatem.

D 3 
oftras a

fequen-

§. 32. Hoc ergo modo fi aequationes noftras a confideratione latitudinis Lunae $\psi$ liberemus ad fequentes perueniemus aequationes:

1. $2 d z d \varphi+z d d \phi=-d q^{2}\left(\frac{3 z(\mathrm{in} 2 \eta}{2 \omega^{3}}+\frac{3 y z z}{8 \omega^{4}}(\right.$ (in $y+5$ fin $\left.3 \eta)\right)$

11. $d d z-2 d \varphi^{2}=m \frac{m d q^{2}}{z z}\left(1-\frac{3}{4} \operatorname{tang} \rho^{2}+\frac{3}{4} \operatorname{tang} \rho^{2} \cot \left(\varphi_{m}-\pi\right)\right)+\mu d q^{2}$

$$
+\frac{z d q^{2}}{2 w^{3}}+\frac{3 z d q^{2}}{2 u^{3}} \operatorname{cor} 2 y+\frac{3^{y z z d q^{2}}}{8 w^{4}}(3 \operatorname{cor} y+5 \operatorname{cor} 3 \eta)
$$

III. $d \pi=-\frac{d q^{2}}{z d \phi} \operatorname{fin}(\phi-\pi) \operatorname{lin}(\theta-\pi)\left(\frac{3 z \operatorname{cof} \pi}{\omega^{3}}+\frac{3^{v z z}}{4^{2} \omega^{4}}(3+5 \operatorname{cor} 2 \pi)\right)$

IV. $d . l \operatorname{tang} \rho=\frac{d \pi}{\operatorname{tang}(\phi-\pi)}$.

Nunc igitur in hoc erit incumbendum; vt ex his quatuor aequationibus omnia motus phaenomena, quae in Luna fecundum Theoriam adeffe debent, follicite eru. antur, atque tum cum obferuationibus conferantur. (in 31$)$

1) $+\mu d q^{2}$

$-5 \operatorname{co}(37)$

sorin)

is qua.

quae in

ise eru.

ur.

APUT
繁.$(0)$ 路

\section{C $\triangle \mathrm{P}$ U T T}

IN'TRODUCTIO ANOMALIAE VERAE LUNAE IN PRAECEDENTES AEQUATIONES.

\section{33.}

noniam noftra quaeftio circa Lunam verfatur, 10 \% Q. co anomaliae mediae folis, quam pro tempore in calculum introduximus, magis conueniet mo. tu Lunae medio vti, qui itidem tempori eft proportio nalis. Verum ex fequentibus patebit calculum commodiorem reddi, if loco motus medii adhibeamus anoma liam Lunae mediam, cuius incrementa itidem tempor funt proportionalia. Sit itaque ad datum tempus ano malia media Lunae $=p$; et cum eius incremeneus: $d p$ ad incrementum. anomalias mediae folis coders tempufculo acceptum datum ac per obferuationes cog. nitam teneat rationem, ponamus $d p=n d q$. Tabulge autem Aftronomicae pro interuallo 365 dierum praebent: Motumanomaliae mediae Șolis $1 \mathrm{I}^{\prime}, 29^{\circ}, 44^{\prime}, 39^{\prime \prime}=1295079^{\prime l}$ Motumanomaliaémediae Lunae

$$
13^{\mathrm{Kov}}, 22^{s}, 28^{\circ}, 43^{\prime} \mathrm{R} 3^{t i}=17167393^{\text {tS }}
$$
vinde fit $a=\frac{17167393}{1295079^{9}} \div 13,25586$

' 5. 34. Pofito ergo $\frac{d p}{n}$ loco $d q$, aequationes noftrae erunt

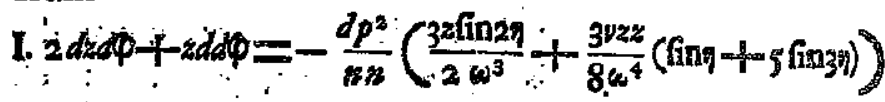

洫. $d d z$ 
II. $d d z-z d \varphi^{2}=-\frac{m d p^{2}}{n n 2 \sigma^{2}}\left(1-\frac{3}{4} \tan g \rho^{3}+\frac{3}{4} \operatorname{tang} \rho^{2} \operatorname{cof}(2(\varphi-\pi))+\frac{\dot{\mu} d p^{2}}{n \pi}\right.$

$$
+\frac{z d p^{2}}{2 n^{2} k^{3}}+\frac{3 z d p^{2}}{2 n^{2} \omega^{3}} \cos 2 \eta+\frac{3 y z z d p^{2}}{8 n n \omega^{4}}(3 \cos \eta+5 \cos 3 \eta)
$$

III. $d \pi=\frac{-d}{m n: d \varphi} \operatorname{lin}(\varphi-\pi)$ fin $(\theta-\pi)\left(\frac{3^{2} \operatorname{col} \eta}{\omega^{3}}+\frac{3 v e z}{4 \omega^{4}}(3+5 \operatorname{col}(2 \eta))\right.$ iv. $d . t \operatorname{tang} \rho=\frac{d x}{\operatorname{tang}(\varphi=\pi)}$

atque hic elementum $d p$ aftumtum ef conftans: fimul aurem patet terminos, qui per as fint diuifi, prae ceteris fatis effe paruos, cum fit $n=175,71795$. Quae circumitantia fequentes approximationes non mediocricer ađiuuabit.

\$. 35. Nune antequam vlterius progrediamur; ae: quationem primam per $z$ multiplieemus, atque integra. tione in priori parte inftituta obtinebimus

$$
\approx z d \varphi=\mathrm{C} d p-\frac{d p}{m} \int d p\left(\frac{3 \xi^{2} \text { fin } 2 \eta}{2 \omega^{3}}+\frac{3 y z^{3}}{8 w^{4}}(\text { fin } y+s \text { (in } 3 \eta)\right)
$$

ponamus breuitatis gratia hoc miembrum integrale.

$$
\operatorname{rdp}_{p}\left(\frac{3 x z}{2 \omega^{4}} \operatorname{fin} 21+\frac{3+z^{31}}{8 \omega^{4}}(\sin +\sin 3 \eta)\right)=s
$$

tgeod integrale, ne introductio conftantis incertitudinem pariat, ita capi affumo, ve nullua terminum mere conftantem contineat, quippe qui iam in $\mathbf{C}$ effet compre. henfus. Hoc ergo circa deterninationem integrationis probe obferuato, exit $z 2 d \dot{\varphi}=d p\left(\mathbf{C}-\frac{S}{n n}\right)$ : vbi ter. minus $S$ aequabilem arearum defcriptionem, quam Re. gula Kepleri in planetis ptimariis infert, perturbac; eft
Quae

gdiocri-

ar; ae:

ntegra.

(in34) )

e

$=s$

Idinem

e con.

impre.

ationis

ji ter-

$n R_{e}$ ; eft enim

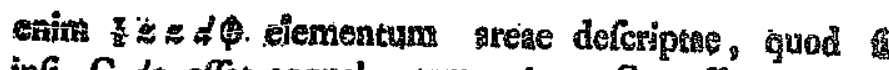
ipfi $\mathbf{C}$ do effet aequale, temposi exalte effet proposs tionale.

\$. 36. Cum igitur fit $d \varphi=\frac{d p}{2 z}\left(\mathrm{C}-\frac{S}{883}\right)$, erip $\varepsilon d \varphi^{2}=\frac{d p^{2}}{z^{3}}\left(\mathrm{CC}-\frac{2}{n^{2}} \mathrm{CS}+\frac{1}{n^{4}} \mathrm{SS}\right)$, quo valo re fubftituto reliquae noltrae aequationes fequentes igo duent formas:

$$
\begin{aligned}
& \text { II. } d d z=\frac{d p^{2}}{z^{3}}\left(\mathrm{CC}-\frac{2}{x_{13}} \mathrm{CS}+\frac{1}{x^{4}} \mathrm{~S} \mathrm{~S}\right) \\
& -\frac{m d p^{2}}{m n 2 z}\left(1-\frac{3}{4} \operatorname{tang} \rho^{2}+\frac{3}{4} \operatorname{tang} \rho^{2} \operatorname{co}(2(\varphi-\pi))+\frac{\dot{\mu} d p^{2}}{3 n}\right. \\
& +\frac{z d p^{2}}{2 n n \omega^{3}}+\frac{3 z d p^{2}}{2 n n u^{3}} \operatorname{cof} 2 \eta+\frac{3 v z z d p^{2}}{8 n n u^{4}}(3 \operatorname{cof} \eta+5 \operatorname{co} 3 \eta ! \\
& \text { III, } d \pi=-\frac{z d p}{\operatorname{Cos} m-S} \operatorname{fin}(\varphi-\pi) \text { fin }(\theta-\pi)\left(\frac{32 \operatorname{cof} \eta}{a^{3}}+\frac{3 y 2 z}{4 \omega^{2}}(3+5 \operatorname{co}(2 y))\right. \\
& \text { et quaxta manet } d_{e} l \text { tang } \rho=\frac{d \pi}{\operatorname{cang}(\varphi-\pi)} \text { vt ante. }
\end{aligned}
$$

Eo igitur pertigimus, vi inueftigari oporteat quantitates $z$, $\pi$ et $\rho$, quibus inuentis obtinebitur $\varphi$ ex formula primum eruta. Cum autem fit $d \eta=d \varphi-d \theta$, ob $d \theta=d s$ $=\frac{d q V(\mathrm{I}-e t)}{\omega \omega}=\frac{d p V(\mathrm{I}-e q)}{n \omega \omega} ;$ erit $d y=\frac{d p}{z e}\left(\mathrm{C}-\frac{\mathrm{S}}{n}\right)$ $-\frac{d p V(I-e c)}{n \omega \omega}$. Tum vero eft vii vidimus $u=\frac{i-e \varepsilon}{I-e \cot s^{3}}$ vnde et huius differentiale ad $d p$ reduci poterit.

4. 37. Si hunc caleulum profequi vellemus, tok: inneftigatio tandem to sediret, vt definiretur quancung E 
Iongitudo Lunae vera ab eius longitudiné media; quae ex anomalia media p haberetur, difcreparec: boc autem difcrimen nonnunquam vltra 8 gradus exfurgere posfet, ideoque correctiones admodum notabiles requireret. Vt igikur nobis guam minimae correctiones inueftigandae relinquantur, expediet differentiam inter locum Luinae verum, et locum corporis quod fecundum regulas Kepleri in ellipfi circa Terram reuoluerecur, ita tanen mobili, vt eius motus abfidum cum motu apogei Lunae per obferuationes cognito conueniret. Seu quod codem redit, quaeramus primo ex anomalia Lunae me: dia $p$ fecundum regulas Kepleri anomaliam eius veram quae fit $=r$, vade fi longitudo apogei fuerit $=n$, guantita, $+r$ nunquam multum vitra gradum a longitudine Lunae vera differet: vnde difcrimen ærulto facilius inueniri poterit, fi guidem debita orbitae luna: ris excentricitas in calculum inducatur. Hinc loco anomaliae Lunae mediae $p$ eius anomaliam veram, quae fcilicet mediae pro excentricitate rite affumta conueniat; in aequationes noftras inferamus.

5: 38. Tabülae quidem attronomicae excentricita: tem orbitae lunaris plerumque variabilem fatuunt; fed cum hic non de vera hujus orbitae excentricitate quaeftio fir, quam de excentricitate illius- orbitae ellipticue mo: bilis, in qua corpus motum proxime. motum Lunae jeferat; huís excentricitas media erit ftatuenda inter szaximam ac minimam, quae vulgo orbitae lunari tribuuntur: vnde ifta excentricitas media colligitur $=0$, 05445. Ne autem huic conclufioni timium fidamus generi- generatim hanc éxcentricitatem ponamus $=k$; atque anomalia vera per median ita determinabitus, vt fat $d p=\frac{(\mathrm{I}-k k)^{\frac{3}{2}} d r}{(\mathrm{I}-k \operatorname{cof} r)^{2}}$, vel fit brevitatis gratia $\frac{1-k k}{1-k \operatorname{cof} \rho}=k$, ve fit $d p=\frac{t t d r}{V(i-k k)^{\circ}}$. Pozro autem reliqua differen. tialia ita ad elementum $d r$ reuocabuntur, vt fie :

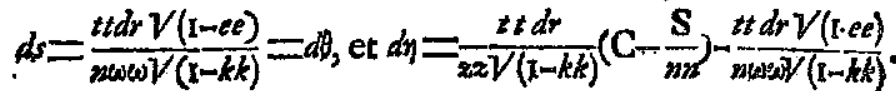
§, 39. Si motus Lunae cum motu huius corpo. ris, quod imaginamur, perfecte conueniret, tum ybigue foret $z=\frac{1-k k}{1-k \operatorname{col} r}$ fea $z=t$ : quoniam ausem hi duo motus intèr fe non conueniunt, non erit $z=b_{*}$ Ponamus ergo effe:

$$
z=t u=\frac{(1-k k) u}{1-k \operatorname{cof} r} \text { feu } x=\frac{(1-k k) a u}{1-k \operatorname{co} r r}
$$

vbi primum obferuo, quantitatem $u$ valde parum ab vaitate recedere. Erit autem quantitas variabilis, quae alium terminum conftantem praeter vnitatem non inuolo vet: nam fi alium terminum conftantem contineret, is in a poffet comprehendi, idque indicio effet diftantiam imediam a non relte effe affurntam. Habebit ergo $u$ huiusmodi formam $I+Z$, vbi $Z$ ex cerminis nonnifi variabilibus conftabit: Praeterea autem animaduerto, hanc quantitatem $\mathrm{Z}$ nullum terminum huius formae * col $r$ compleeti debere; quoniam hoc indicio effet excentricitatem $k$ non rele effe affumtam, fed eam vel maiorem vel minorem accipi oportuiffe.

$$
\text { E } 2
$$


§. 40. His igitur notatis, quod quantitas " primo terminum conftantem $=1$ contineat, tum vero nullum terminum formae $\alpha$ cof $r$ inuoluat, ftacuamus $z=t *$ feu $z=\frac{(\mathrm{I}-k k) u}{1-k \operatorname{so} \tilde{r} r}$ pofito breuitatis gratia $t=\frac{1-k k}{I-k \operatorname{col} r}$. Atque cum fupra elementisn d $p$ conPtans pofuiffemus, hac condicione exuenda erit $d d z=$ $d p d . \frac{d z}{d p}$, et $\frac{d d z}{d p^{2}}=\frac{1}{d p} d \cdot \frac{d z}{d p}$ Divifa ergo fecunda aequatione per $d p^{2}$, eric:

II. $\frac{\dot{I}}{d p} d \cdot \frac{d z}{d \dot{p}}=\frac{\mathbf{C}}{t^{3} u^{3}}-\frac{2 \mathrm{CS}}{m t^{3} z^{3}}+\frac{\text { S S }}{n^{4} t^{3} u^{3}}$

$-\frac{n}{n t w u}$ (1- $-\frac{3}{4}$ tang $\rho^{2}+\frac{3}{4}$ tang $\left.\rho^{2} \operatorname{cof}(\varphi-x)\right)+\frac{\mu}{n n}+\frac{n n}{2 n n \omega^{3}}$

$$
+\frac{3 t s \operatorname{cor} 2 \eta}{2 n n \omega^{3}}+\frac{3 v^{\circ} t t u n}{8 \pi n \omega^{4}}(3 \operatorname{cof} \eta+5 \operatorname{cor} 3 \eta)
$$

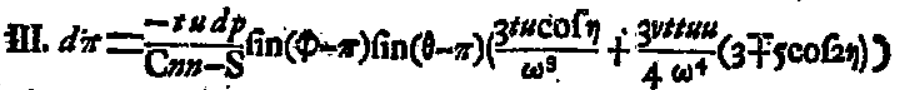
vbi nane nullum differentiale aftumtum eft conftans, fed Jàn p̀ro lubitu quoduis differentiale conttans aflumi poteric.

5. 4. Pofito autem $x=t w$ et $d_{i p} \equiv \frac{t t d r}{\sqrt{(I-k R)}}$

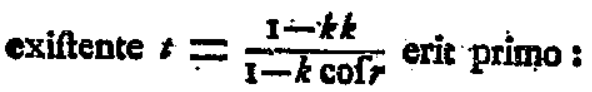

$S=\int \frac{t d r}{V(i-k k)}\left(\frac{3 t t x t}{2 w^{3}}\right.$ fina $y+\frac{3 t^{3} u^{3}}{8 w^{4}}($ fin $\eta+5$ fin $\left.3 \eta)\right)$ feu

$S=f \frac{d r}{V(I-k K)}\left(\frac{3^{4} w a}{2 \omega^{3}}\right.$ fin $2 y+\frac{3 y t^{5} z^{3}}{8 \omega^{4}}($ (finy + fin3y) $)$
Hinc fiet $d \varphi=\frac{d r}{m b V(I-k k)}\left(\mathrm{C}-\frac{\mathrm{S}}{\sin }\right)$ atque

$$
d \eta=\frac{d r}{u n V\left(\mathrm{I}-k_{k}\right)}\left(\mathrm{C}-\frac{S}{n n}\right)-\frac{t z d n V(\mathrm{I}-e e)}{n \omega \omega V(\mathrm{I}-k k)}
$$

Porro autem ob $d z=t d u+u d t$, erie $\frac{d z}{d p}=\frac{t d u+u d t}{t s d r} V(n \rightarrow k)_{j}$ at eft $d t=-\frac{(\mathrm{I}-k k) k d r \text { fin } r}{(\mathrm{I}-k \operatorname{cof} r)^{2}}=-\frac{k t t d r \text { fin } r}{\mathrm{I}-k k}$; fieque fiet $\frac{d z}{d p}=\frac{d u V(\mathrm{I}-k k)}{d r}-\frac{k u \text { fin } r}{V(\mathrm{I}-k k)} ;$

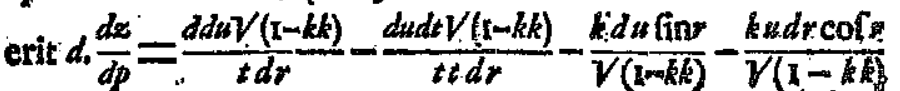

hincque ob $\frac{d t}{t t}=-\frac{k d r \text { fin } r}{1-k k}$ habebitur :

$$
\text { d. } \frac{d z}{d p}=\frac{d d n V(1-k k)}{d r}-\frac{k u d r c o[r}{V(1-k k)} \text {. }
$$

\$. 42. Hinc iam porro obtinemus pro fecunda aequatione $\frac{x}{d p} d \cdot \frac{d x}{d p}=\frac{(1-k k) d d u}{z^{3} d r^{2 k}} \div \frac{k u \operatorname{cor} r}{t t}$

qui valox fubftitutus in acquatione per $\frac{b^{3}}{\text { In } k^{2}}$ multiplick ta orietur haec aequatio:

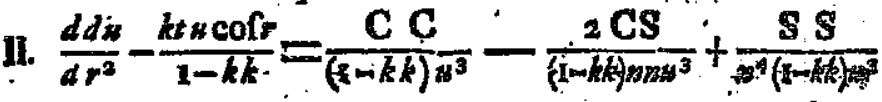

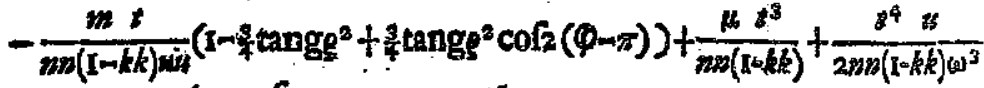

$$
\begin{aligned}
& +\frac{3 t^{4} u \operatorname{cof} 2 \eta}{2 m n(I-k k) \omega^{3}}+\frac{3 t^{5} u n}{8 m n \omega^{4}(1-k k)}(3 \operatorname{cof} \eta+5 \cos (2 n)
\end{aligned}
$$

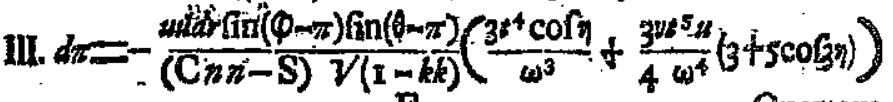


Quartam aequationem $\dot{d} . l$ tang $\rho=\frac{d \cdot \pi}{\operatorname{cang}} \frac{\pi-\pi)}{(\varphi-\text { cum }}$

cum

ntinuo

nullam mutationem fubeat, fuperfluum foret continuo repetere.

9. 43. Conueniet autem quantitates conftantes ' $\mathbf{C}$ et $m$, quarum valores nondum nouimus, faltem vero proxime indagare, qui fạcilius" deinceps iplam-aequatio: num refolutionem dirigere queamus. Perficuum autem eft, fomnes quantitates a fitu folis pendentes ex calculo deleantur, tum vique fieri debere $u=1$. Cum ifirur pritmúm $\mathbf{S}$ ab angulo y.pendeat, terminos tam $\mathbf{S}$ quiam $\eta$ inuolizentes omittamus, ac pro $w$ quidem fcribamus I; quia tantum determinationem ad verum acce. dentem requirimus, quem in finem quoque inclinationem orbitae negliganius. .. Fine aequetio lecunda dabit:

$\sin \frac{k . t \operatorname{cof} r}{I-k k}=\frac{C C}{I-k k}-\frac{m t}{m n(I-k k)}+\frac{\mu i^{3}}{n^{2}(1-k k)}+\frac{t^{4}}{2 n n(1-k k)}$ fiue $\mathbf{C C}=\frac{m t}{m n}+\frac{\mu^{3}}{m m}=-k t \operatorname{cor} r-\frac{t^{4}}{2 m n} \cdots \quad .$.

Cuin autern valde paruum habebitur.

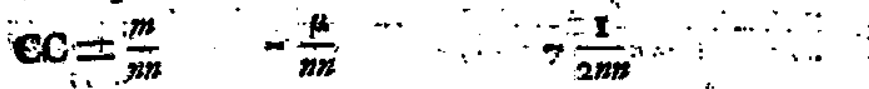

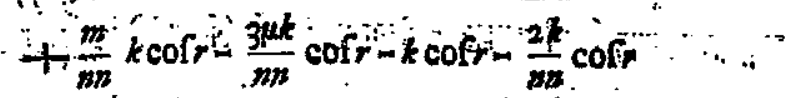

Ynde" peripiciuìn effe oportere.

$\Rightarrow \frac{m}{m}=1+\frac{2+34}{6 m}$ et $\mathrm{CC}=1+\frac{3+4}{2 m m}$

\$. 44. His tes: $: \mathbf{C}$

. vero

juatio:

$m$ au-

tes: ex

Cum

tam S

a fcri-

acce.

inatio-

dabit:

five

:

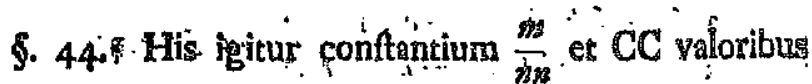
proximis inuentis ponamus effe reuera:

$$
\frac{m}{m y}=1+\frac{2+3 \mu+\gamma}{m} \text { ect } \mathrm{CC}=1+\frac{3+4 \mu+8}{2 m}=\lambda \mathrm{x}
$$

fcribanus enim $\boldsymbol{\lambda}$ pro $C$, quìa litteris viaiusculis $A$, $C, D$ etcideinceps in operationibus fequentibus vtemur: ficque fiet

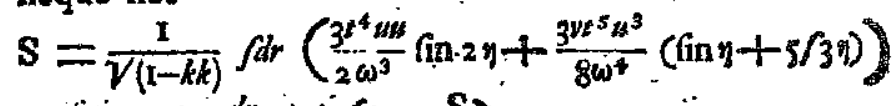

$$
\begin{aligned}
& d \phi=\frac{i d r}{u n v(1-k k)}\left(\lambda-\frac{S}{n n}\right) \\
& d \eta=\frac{d r}{u u V(I-k k)}\left(\lambda-\frac{S}{n n}\right)-\frac{t t d r V}{n \omega \omega V} \frac{(1-e e)}{(I-k k)} \\
& \text { II. } \frac{(1-k k) d d u}{d r^{2}}=k t u \operatorname{cor} r+\frac{\lambda \lambda}{z^{3}}-\frac{2 \lambda S}{m m w^{3}}+\frac{S S}{n^{4} z^{3}}+\frac{\mu^{3}}{n n}+\frac{t^{4} z^{2}}{2 m n \omega^{3}} \\
& \text { - } \frac{m t}{m n u t}\left(1-\frac{3}{4} \operatorname{tang} \rho^{2}+\frac{3}{4} \operatorname{tang} \rho^{2} \operatorname{cor} 2(\varphi-x)\right)
\end{aligned}
$$

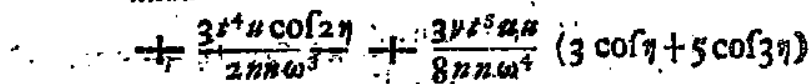

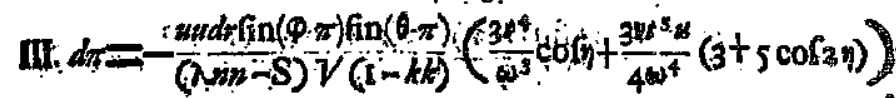

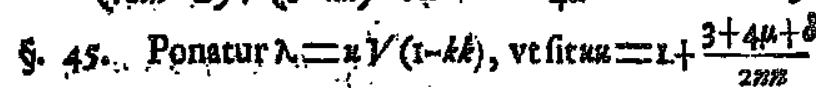
defectum enim in ermina indefinito \& compleat licet, exiftente $m=m+2+3 \mu+y$; tum vero ponarup $S=(I-k k)^{\frac{1}{2}} \int R d r$, vt fit $R=\frac{d S}{d r V(I-k k)} ;$ acfi pro tetes valores rettituarnius, qui erạne,

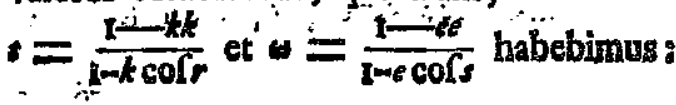


$\mathrm{B}=\frac{3(1-k k)^{3} \cdot(1-e \operatorname{col} s)^{3}}{2(1-i t)^{3} \cdot(1-k \operatorname{col} r)^{4}}$ we fin 23

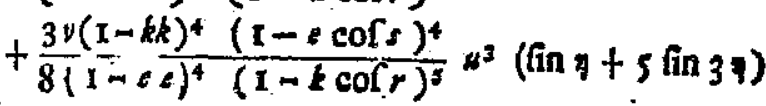

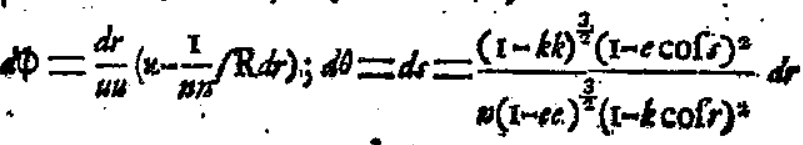

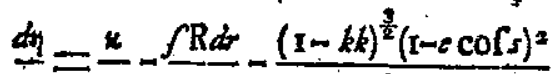

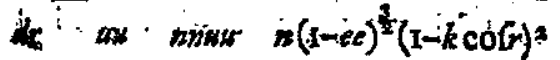

\$. 46. Aequatio autern fecunda facta hac fubtitus. tione, fi per I - kk diuidarur, abibit in fequentem:

II. $\frac{d d u}{d r^{2}}=\frac{k u \operatorname{cor} r}{1-k \operatorname{col} r}+\frac{k k}{u^{3}}-\frac{2 k / R d r}{n n z^{3}}+\frac{\left(\int \mathrm{R} d r\right)^{2}}{n^{4} u^{3}}+\frac{\mu(1-k k)^{2}}{n s(1-k \operatorname{col} r)^{3}}$

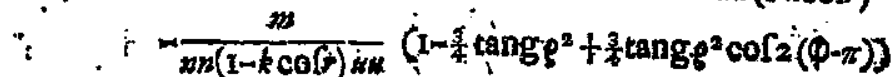

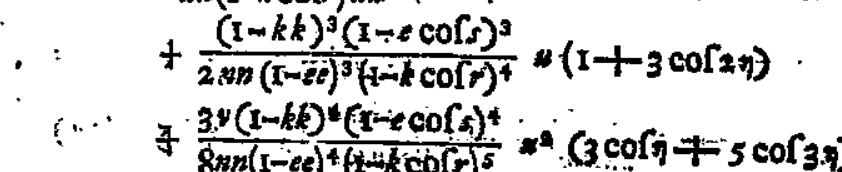

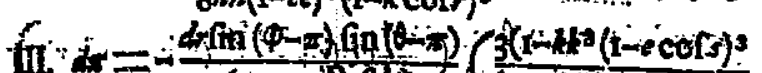

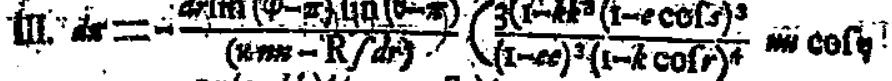

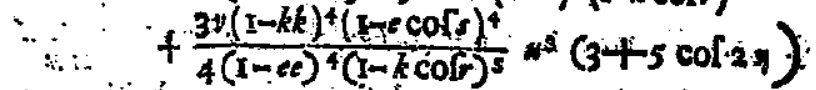

Ac fi denotet inclinationehin median or bitae Iunarts,

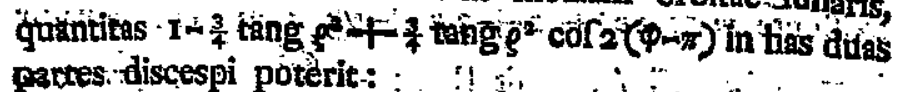
partes discespi potêrit:-

( $1-\frac{3}{4}$ rang $\left.\varepsilon^{2}\right)+\frac{3}{4}\left(\right.$ tang $\varepsilon^{2}-\operatorname{tang} \rho^{2}+\operatorname{tang} \rho^{2} \cos (2(\varphi-\pi))$ quarum illa eft conftans, thâc verto propre a nodo et inclinatione pondet $1:-1, \ldots$
$C A P O T M$

5. 47. Euoluamus autem prodicta illa ex $s$ et to orta, et quoniam excentricitates $k$ et $e$ funt valde par vae, fufficit ad cos vsque terminos tantum progredi, qui coefficientes habeant $k k$, $e k$ et $e e$, eosque qui per altio. res poteftates fint multiplicati omittere. Hinc erit:

$\frac{1}{I-k \operatorname{cof} r}=1+\frac{r}{2} k k+k \operatorname{cor} r+\frac{1}{2} k^{2} \operatorname{cof} 2 r$

$\frac{k \operatorname{cof} r}{x-k \operatorname{cof} r}=\frac{\pi}{2} k k+k \operatorname{cor} r+\frac{\pi}{2} k^{2} \operatorname{cor} 2 r$

$(\mathrm{I}-k k)^{2}$

bititu.

m:

$-k k)^{2}$

$\left(\frac{\cos (r))^{3}}{3}\right.$

$(\$-\pi))$

$\frac{(1-k k)}{(1-k \operatorname{cof} r)^{3}}=1+3 k \operatorname{cor} r$, quia hic terminus per $\mu$

$(1-k k)^{\frac{3}{2}}$ multiplicatur.

$\frac{(1-k k)^{\frac{3}{2}}}{(1-k \operatorname{cof} r)^{2}}=\mathrm{x}+2 k \operatorname{cor} r+\frac{3}{2} k k \operatorname{cor} 2 r$

$\frac{(\mathrm{I}-k k)^{3}}{(\mathrm{I}-k \cos r)^{4}}=\mathrm{I}+2 k k+4 k \operatorname{cof} r+5 k k \operatorname{cor} 2 r$

$\frac{\left(\mathrm{I}-k(k)^{4}\right.}{(\mathrm{I}-k \operatorname{cor} r)^{5}}=\mathrm{I}+5 k \operatorname{cor} r$, quia hic terminus iam per $v$ eft mulkiplicatus.

(3.)

coris

\$. 48. Porro vero pro terminis ex $\omega$ enatis eft: $\left(\operatorname{line\mathrm {cof}s)^{2}}\right.$

$\frac{(\mathrm{I}-e \mathrm{cof} s)^{2}}{(\mathrm{I}-e e)^{\frac{3}{2}}}=\mathrm{x}+2 e e-2 e \operatorname{cor} s+\frac{x}{2} e e \operatorname{col} 2 s$

$(\mathrm{I}-\mathrm{ecols})^{3}$

$\frac{(I-e \cos s)^{3}}{(I-e e)^{3}}=\mathrm{I}+\frac{3}{2} e e-3 e \operatorname{cof} s+\frac{3}{2} e e \operatorname{cof} 2 s$

$\frac{(1-c o f s)^{4}}{(1-c e)^{4}}=1-4 e \operatorname{cof} 4$, quia hic factor tantun in

Inaris;

; duas minimis terminis occurris.

Hine ergo colliginus:

$(i-\pi)$ )

lo et

6. 47.

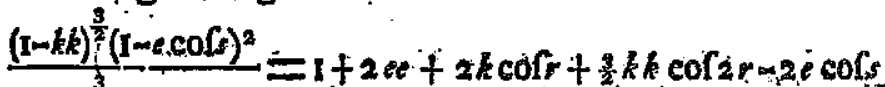
$(\mathrm{I}-\epsilon \epsilon)^{\frac{3}{2}}(\mathrm{I}-k \mathrm{coin})^{2}-2 e k \operatorname{cor}(r+s)-2 e k \operatorname{cor}(r-s)+\frac{1}{2} e c \cos 2 s$

E

(lawee) 
$(\mathrm{I}-k k)^{3}(1-e \operatorname{cof} s)^{3}=\mathrm{I}+2 k k+\frac{\rho}{2} e \epsilon+4 k \operatorname{cor} r+5 k k \operatorname{cof} 2 r$

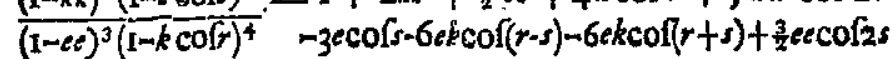
$\frac{(\mathrm{I} m k k)^{4}(\mathrm{I}-\mathrm{ecof} s)^{4}}{(1-e c)^{4}\left(\mathrm{I}-k \operatorname{col}(r)^{5}\right.}=\mathrm{x}+5 k \operatorname{cor} r-4 e \operatorname{cor} s$,

arque hinc fiet: $\alpha \varphi=\frac{d r}{u m}\left(x-\frac{1}{n n} / R d r\right)$ atque

$$
\frac{d s}{d r}=\frac{1+2 e e}{n}+\frac{2 k}{n} \cos r-\frac{2 e}{n} \operatorname{cof} s-\frac{2 e k}{n}(\operatorname{col} r-s)
$$$$
+\frac{3 k k}{2 n} \operatorname{col} 2 r+\frac{e c}{2 n} \operatorname{col} 2 s-\frac{2 e k}{\pi} \operatorname{cor}(r+s)
$$

$\frac{d n}{d r}=\frac{\pi}{u k}-\frac{\sqrt{R} d r}{m n n}-\frac{1-2 e t}{n}-\frac{2 k}{n} \operatorname{cof}+r \frac{2 e}{n} \operatorname{cor} s+\frac{2 e k}{n} \operatorname{cof}(r-s)$

$$
\frac{3^{k k}}{2 n} \operatorname{coc} 2 r-\frac{e \epsilon}{2 n} \operatorname{cor} 2 s+\frac{2 e k}{n} \operatorname{col}(r+s)
$$

6. 40. Introductis nune his valoribus enolutis in formulas noftras, iisque, qui per finum cofinumue alterius anguli funt multiplicati, pariter fecundum fimplices angulos explicatis, obtinebimus primum valorem ipfius $\mathbf{R}$, qui erit:

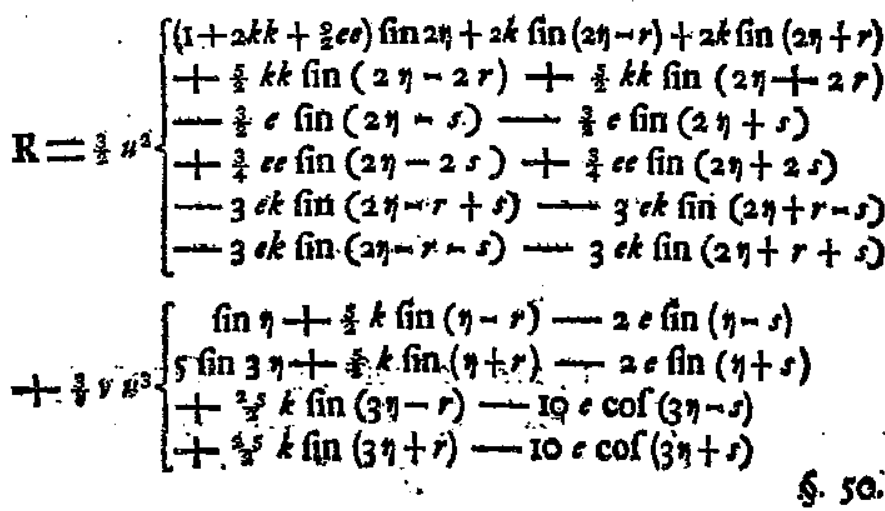

$\operatorname{cof} 2 r$ ecofiss ,

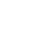

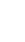
$f(r-s)$ $((r+s)$ ttis in $\because$ alteimplilorem $2 \eta+r)$ $(-2 r)$ )
\$. 50. A Aequatio autem fecunda priacipalis fequeng tem induet formam:

$$
\begin{aligned}
& \text { II. } \frac{d d u}{d r^{2}}=\frac{u t}{u^{3}}-\frac{2 u \int \mathrm{R} d p}{m m u^{3}}+\frac{\left(\int \mathrm{R} d r\right)^{3}}{m^{4} u^{3}} \\
& +\frac{3 m \operatorname{tang} \rho^{2}}{4 n n u u}(1-\cos 2(\varphi-\pi))(1+k \operatorname{cor} r) \\
& -\frac{m}{n n m i s}\left(\mathrm{I}+\frac{\pi}{2} k k+k \operatorname{co}\left[r+\frac{x}{2} k^{2} \operatorname{cof} 2 r\right)+\frac{\mu}{m n}(\mathrm{r}+3 k \operatorname{col} r)\right. \\
& +u\left(\frac{\pi}{2} k k+k \operatorname{col} r+\frac{\pi}{2} k k \operatorname{col} 2 r\right) \\
& +u\left\{x+2 k k+\frac{9}{2} e e+4 k \operatorname{cof} r-3 e \operatorname{cof} s-6 e k \operatorname{cof}(r-s)\right. \\
& +\frac{\pi}{2 m n}+5 k k \operatorname{col} 2 r+\frac{3}{2} e e \operatorname{col} 2 s-6 e k \operatorname{col}(r+s)
\end{aligned}
$$

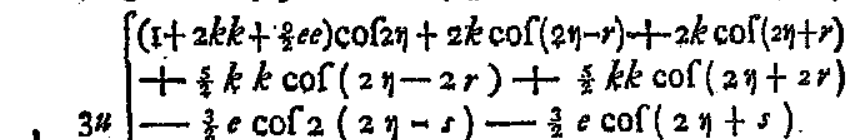

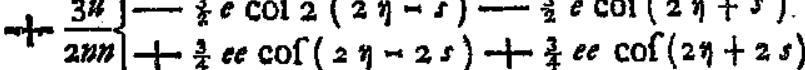

$$
\begin{aligned}
& -3^{e k} \operatorname{cor}(2 y-r+s)-3^{e k} \operatorname{cor}(2 y+r-s) \\
& -3 e k \operatorname{cof}(2 \eta-r-s)-3^{e k} \operatorname{col}(2 y+r+s)
\end{aligned}
$$

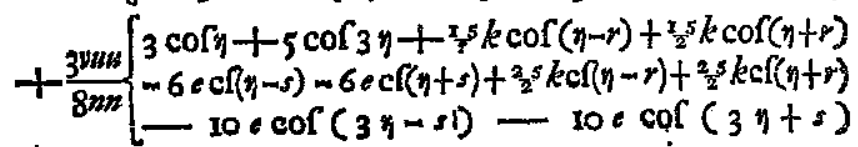

vbi terminos, qui adhue vlteriori euolutione indigent, primo loco pofui, et cum terminus tang $e^{2}$ implicans jam fit valde paruus, in eius multiplicatore fecundam ipfius $k$ poteftatem omifi : fin autem alicuius momenti videantur, loco $1+k$ cof $r$ fcribi poterit $I+\frac{1}{2} k k$ $+k \operatorname{cof} r+\frac{x}{2} k k \cos 2 r$.

§. 51. Pro longitudine vero nodi inuenienda ae quatio fequens prodibit refoluenda :

$\mathbf{F}_{2}$

$2 \pi=$ 


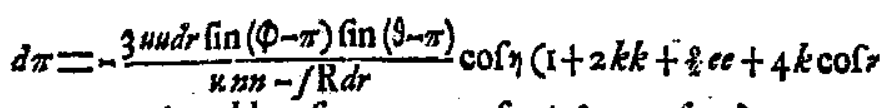
$\left.+5 k k \operatorname{col} 2 r-3 e \operatorname{col} s+\frac{3}{2} e e \cos 2 s\right)$ $\frac{3^{2 / k^{3}} d r \operatorname{fin}(\phi-\pi) \operatorname{fin}(\theta-\pi)}{4(x+\pi)-\sqrt{R} d r)}(3+5 \operatorname{co} 2 \eta j)(1+5 k \operatorname{cor} r)$ At eft fin $(\varphi-\pi)$ fin $(\theta-\pi)=\frac{\pi}{2} \operatorname{cof} y-\frac{\pi}{2}(\phi+\theta-2 \pi)$; vnde fin $(\varphi-\pi)$ fin $(\theta-\pi) \operatorname{cf} y=\frac{x}{4}+\frac{x}{4} \operatorname{cf} 2 y-\frac{x}{4} \operatorname{cf} 2(\varphi-\pi)-\frac{x}{4} \operatorname{cl} 2(\theta-\pi)$ et $\operatorname{fin}(\varphi-\pi) \sin (\theta-\pi) \operatorname{co}\left(2 y=\frac{\pi}{4} \cos \eta+\frac{\pi}{4} \operatorname{cof} 3 \eta\right.$

$$
-\frac{\pi}{4} \cot (3 \varphi-\theta-2 \pi)-\frac{\pi}{4} \cot (3 \theta-\varphi-2 \pi)
$$

Tum vero ob $J \mathbb{R} d r$ valde paruum prae $k m$, erit fatis exale $\frac{1}{x n n-\int R d r}=\frac{1}{x m n}+\frac{\int R d r}{x x n^{4}}+\frac{\left(\int R d r\right)^{2}}{x^{3} n^{6}}$ vbi quiden poftremus terminus tuto omitti poteft.

8. 52. Praeterea vero ponatur $u=1+\frac{v}{w}$, vt fit $d d u=\frac{d d v}{m}$, et reiectis terminis per $n^{4}$ diuifis, qui iam $+\frac{6 k v}{m m}$ fin $(2 \eta-r)+\frac{6 k v}{n n}$ fin $(2 y+r)$ $-\frac{9^{e v}}{2 m n}$ fin $(2 \eta-s)-\frac{9^{e v}}{2 n m}$ fin $(2 \eta+s)$ $+\frac{3}{y} y$ fin $y+\frac{\pi}{x} \frac{5}{y} y k$ fin $(\eta-r)-\frac{3}{4} y e$ fin $(y-s)$ $+\frac{3}{5} y y$ in $(3 y-r)-\frac{35}{4} y e$ fin $(3 y-s)$

$+\frac{x}{8} y \operatorname{lin} 3 \eta+\frac{x}{x} \frac{s}{y} y k$ tin $(\eta+r)-\frac{3}{4} \nu e$ fin $(\eta+s)$

$$
+\frac{x}{x} v k \text { in }(3 y+s)-\frac{i s}{4} y \in \text { fin }(3 y+s)
$$

8. 53. Ipfa vero aequatio fecunda per hanc fubftitutionem, poftquam per $n n$ fuerit multiplicata, in for mam fequentem abibit.

$$
\begin{aligned}
& \text { Il. } \frac{d d v}{d r^{2}}=x x n n-3 x x v+\frac{6 x k v v}{m z}-2 x \int \mathbb{R} d z \\
& +\frac{6 n v}{m m} \rho \mathrm{R} d r+\frac{\mathrm{I}}{w n}\left(\int \mathrm{R} d r\right)^{2} \\
& +\frac{3 m \operatorname{tang} \rho^{2}}{4}(I-\operatorname{col} 2(\varphi-\pi))\left(\mathrm{I}+\frac{\pi}{2} k k+k \operatorname{cof} r+\frac{\pi}{2} k k \operatorname{cof} 2 r\right) \\
& -\frac{3 m o \operatorname{tang} \rho^{2}}{2 m m}(1-\operatorname{cor} 2(\varphi-\pi))(1+k \operatorname{cor} r)
\end{aligned}
$$$$
\frac{d \varphi}{d r}=x-\frac{2 k v}{n n}+\frac{3 n v^{2}}{n^{4}}-\frac{\int \mathrm{R} d r}{n n}+\frac{2 v \int \mathrm{R} d r}{n^{4}}
$$$$
\frac{d n}{d r}=x-\frac{1-2 e \epsilon}{n}-\frac{2 k}{n} \operatorname{cor} r+\frac{2 e}{n} \cos s-\frac{3 k k}{2 n} \cos 2 r-\frac{e e}{2 n} \operatorname{col} 2 s
$$$$
+\frac{2 e k}{n} \operatorname{col}(r-s)+\frac{2 e k}{n} \operatorname{cof}(r+s)
$$$$
-\frac{2 k v-\int \mathrm{R} d r}{2 n}+\frac{3 k v^{2}+2 v / \mathrm{R} d r}{n^{4}} \quad \text { atane }
$$$$
\mathbf{R}=\frac{3}{2}\left(\mathrm{x}+2 k k+\frac{2}{2} e c\right) \text { fin } 2 \eta+3 k \text { fin }(2 y-r)-\frac{2}{2} e \text { in }(2 y-s)
$$$$
+3 v \text { t } 3 k \text { fin }(2 \eta+r)-\frac{a}{4} e \operatorname{fin}(2 y+s)
$$

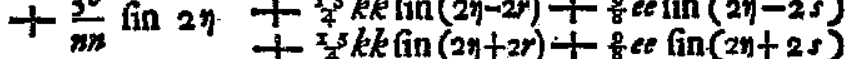

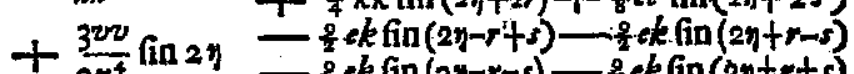


$+\frac{15}{7} k k \operatorname{cor}(2 y-2 r)+8 e t \operatorname{cor}(2 y-2 x)$

$+\frac{53}{4} k k \operatorname{cor}(2 \eta+2 r)+\frac{2}{8} e c \operatorname{cor}(2 \eta+2 s)$

- $\frac{s}{2} e k \operatorname{col}(2 \eta-r+s)-\frac{2}{2} e k \operatorname{col}(2 \eta+r-s)$

- $\frac{2}{2} e k \operatorname{cor}(2 y-r-s)-\frac{s}{2} e k \operatorname{cor}(2 y+r+s)$

$+3 v \int \cos 2 \eta+2 k \operatorname{col}(2 \eta-r)+2 k \operatorname{cof}(2 \eta+r)$

$+\frac{2 m}{2 m}\left\{-\frac{3}{2} e \operatorname{cor}(2 \eta-s)-\frac{3}{2}<\operatorname{cof}(2 \eta+s)\right.$

$\left\{+3 \cos \eta+\frac{x}{2} k \operatorname{cor}(\eta-r)-6 e \operatorname{cor}(\eta-s)\right.$

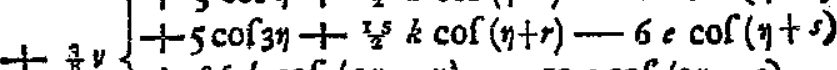

$\left\{\begin{array}{l}+\frac{25}{2} k \operatorname{col}(3 y-r)-10 e \operatorname{cof}(3 y-s) \\ +25 k \operatorname{cof}(3 y+r)-10 e \operatorname{col}(3 y+5)\end{array}\right.$

$+\frac{3^{n v}}{4^{m n}}(3 \cos \eta+5 \cos 3 \eta)$

\$. 54. Cum autem fit $m=n n+2+3 \mu+\gamma$ etu $x=$ $x+\frac{3+4 \mu+\delta}{2 \pi n}$, fi hi valores fubftituantur, plures - termini fe mutuo deftruent, aequatioque prodibit fequenti forma coneinniori contenta : vbi quidem in terminis per fe.minimis loso $m$ feribi licebit $n n$, et 1 loco zix vel $x$.

\section{AEquatio.}

$\frac{d d v}{d r^{2}}=\frac{\pi}{2} \delta-y+\frac{2}{4} e c-y k \operatorname{col} \dot{r}+-\frac{3}{2} k k \operatorname{cor} 2$.

$-2\left(1+\frac{3+4 \mu+\delta}{4^{n n}}\right) \int \mathrm{R} d r+\frac{1}{m n}(\gamma \mathrm{R} d r)^{2}$

$-v\left(1-\frac{3}{2} k k-3 k \cos r-\frac{3}{2} k k \operatorname{cof} 2 r\right)$

$+\frac{v v}{p n}(3-3 k \operatorname{col} r)-\frac{3}{2} e \operatorname{col} s+\frac{3}{4} e e \operatorname{co}[2 s-3 e k \operatorname{cor}(r-s)$

$-3 e k \operatorname{cor}(r+s)+\frac{3}{2}\left(1+2 k k+\frac{g}{2} e c\right) \operatorname{cor} 2 \eta$

$+3^{k} \operatorname{cor}(2 y-r)+\frac{\pi}{4} k k \operatorname{cor}(2 y-2 r)-\frac{3}{4} \operatorname{cof}(2 y-s)$ $+3^{k} \operatorname{cor}(2 \eta+r)+{ }_{4}^{5} k k \operatorname{col}(2 \eta+2 r)-\frac{3}{4} e \operatorname{cor}(2 \eta-s)$ $+2 e e \operatorname{cor}(2 \eta-2 s)-\frac{2}{2} e k \operatorname{col}(2 \eta-r+s)-\frac{0}{2} e k \operatorname{col}(2 \eta-r-s)$ $+\frac{0}{8} e e \operatorname{cor}(2 \eta+2 s)-\frac{0}{2} e k \operatorname{cor}(2 \eta+r-s)-\frac{2}{2} e k \operatorname{cor}(2 \eta+r+s)$ $\int 2 \gamma-\frac{3}{2} \delta+6 k \operatorname{cof} r+2(3 \mu+\gamma) k \operatorname{col} \gamma+6 \int R d \gamma$ $+\frac{v}{m n}-\frac{3}{2} e \operatorname{col} s+\frac{3}{2} \operatorname{col} 2 \eta+3 k \operatorname{cor}(2 \eta-r)+3^{k} \operatorname{cof}(2 \eta+r)$

$\int 3 \operatorname{cor} \eta+\frac{\pi}{2} k \operatorname{col}(\eta-r)-6 e \operatorname{col}(\eta-s)$

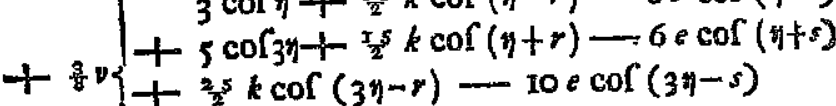
$+\frac{25}{2} k \operatorname{cor}(3 \eta-r)-10 e \operatorname{cor}(3 \eta-s)$
$+\frac{25}{3} k \operatorname{cor}(3 \eta+r)-10 e \operatorname{col}(3 \eta+s)$

$+\frac{3^{v 0}}{4^{n n}}(3 \cos \eta+5 \cos 3 \eta)$

$+\frac{3}{4}(m n+2+3 \mu+\gamma) \frac{(1-2 v)}{m n} \operatorname{tang} \rho^{2}(1-\operatorname{col} 2(\varphi-\pi))$ $\left(x+\frac{\pi}{2} k k+k \cos r+\frac{\pi}{2} k k \operatorname{cor} 2 r\right)$

5. 55. Pro loco nodi autem inveniendo prodibis fequens aequatio.

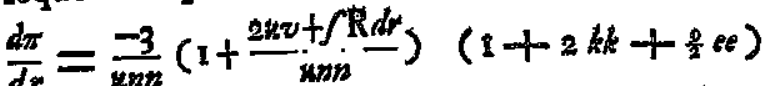

$$
\begin{aligned}
& \frac{\pi}{4}+\frac{\pi}{4} \cos 2 y-\frac{x}{4} \cot 2(\phi-\pi)-\frac{1}{4} \cos 2(\theta-\pi) \\
& +k \operatorname{cof} r+\frac{1}{2} k \operatorname{cof}(2 \pi-r)-\frac{t}{3} k \operatorname{cof}(2 \phi-2 \pi-r) \\
& \text { - } 3 e \operatorname{cor} s+\frac{\pi}{2} k \operatorname{cor}(2 \pi+r)-\frac{\pi}{2} k \operatorname{cor}(2 \varphi-2 \pi+t) \\
& \text { - } 3<\operatorname{cof}(2 y-s)-\frac{x}{2} k \operatorname{cor}(2 \theta-2 \pi-r) \\
& -\frac{3}{8} e \operatorname{cof}(2 \pi+r)-\frac{\pi}{2} k \operatorname{cof}(2 \theta-2 \pi+r) \\
& \text { 3y }\left\{\frac{\pi x}{7} \operatorname{cor} \eta+\frac{5}{4} \operatorname{col} 3 \eta-\frac{3}{2} \operatorname{col}(3 \varphi+\theta-2 \pi)\right. \\
& 4^{\sin n}\left\{-\frac{5}{4} \operatorname{cor}(3 \phi-\theta-2 \pi)-\frac{5}{4} \operatorname{cor}\left(3^{\theta}-\phi-2 \pi\right)\right.
\end{aligned}
$$


At pro inclinatione orbitae habebitur:

$$
\begin{aligned}
& \frac{d . l \operatorname{tang} \rho}{d r}=\frac{-3}{x m n}\left(I+\frac{2 k v+\int \mathrm{R} d r}{\sin m}\right)\left(1+2 \cdot k k+\frac{2}{2} e e\right)
\end{aligned}
$$

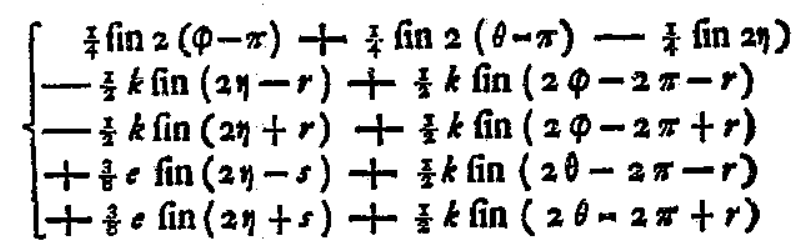

$$
\begin{aligned}
& -\frac{3 y}{4^{x} n n}\left(-\frac{\pi}{4} \text { fin } y-\frac{s}{4} \operatorname{fin} 3 y+\frac{3}{2} \text { fin }(\varphi+\theta-2 \pi)\right. \\
& +\frac{5}{4} \sin (3 \varphi-\theta-2 \pi)+\frac{3}{4} \operatorname{fin}(3 \theta-\varphi-2 \pi)
\end{aligned}
$$

Quomodo igitir his aequationibus ad motum Lunae cognofcendum vi conueniat, in fequentibus cepitibus videamus.
INUESTIGATIO INAEQUALITATIS LUNAE ABSOLUTAE, QUAE VARIATIO DICITUR.

\section{\$. 33.}

$\mathrm{E}$

Ix his aequationibus perfpicitur in determinationem motus Lunae plarimorum angulorum vel finus vel cofinus ingredi, qui anguli formantur per v2riam combinationem fequentium 4 angulorum:

1. ex diftantia Solis a Luna, quem angulum pofuimus $=$ y 2. ex anomalia Lunae vera $=r$

3. ex anomalia Solis vera $=s$

4. ex diftantia Lunae a nodo afcendente $=\emptyset-\mathrm{m}$. $\mathrm{Ne}$ igitur a tanta angulorum multitudine obruamur, a cafbus fimplicioribus ordiamur: ac primo quidem in eas tantum motus inaequalitates inquiramus, quae a folo angulo in pendeant, neque idcirco excentricitatem vel Solis vel Lunae implicent, neque ab orbitae luna. ris inclinatione ad eclipticam afficiantur.

6. 57. Has igitur inaequalitates, quae a folo fitu Solis refpedu Lunae nafcuntur, atque ab Aftronomis fub nomine variationis comprehendi folent, ex praecedentibus aequationibus eliciemus, fi tam excentricitatem Lunae $k$ quam folis $a$ pro nihilo habeamus, ato tem Lunationem orbitae lunaris ad eclipticam etranes centem ftacuamus, ita vt fit $k=0, e=0$ et tang $\varrho=0$. Sic enim obtinebimus eas inaequalitates Lunae, quae ab his elementis non pendent, ideoque tantum per angu-

$$
\text { G }
$$


Ium if determinantur; quae cum vnica tabula comprehendi queant, haec tabula variationem Lunae indicare dicitur. Interim tamen hic animaduerti oportet, partem guandam exiguam variationis quoque ab excentricitate orbitae Lunae $k$ pendere, quam partem deinceps fupplebimus, cum huius excentricitatis rationem fumus habituri.

§. 58. Reiectis ergo terminis $k, c$, et tang $\varrho$ continentibus, habebimus :

$$
\begin{aligned}
& \frac{d \varphi}{d r}=x-\frac{2 x v-\int \mathrm{R} d^{n} r}{n n}+\frac{3 k v^{2}+2 v \int \mathrm{R} d r}{n^{4}} \\
& \frac{d \eta}{d r}=n-\frac{1}{n}-\frac{2 k v-\int \mathrm{R} d r}{n n}+\frac{3 k v^{2}+2 v \int \mathrm{R} d r}{n^{4}} .
\end{aligned}
$$

$\mathrm{R}=\frac{3}{2} \operatorname{fin} 2 y+\frac{3 v}{n z} \operatorname{fin} 2 y+\frac{3}{8} v$ fin $\eta+\frac{r}{8} y$ fin $3 y$, ac denique $\frac{d d v}{d r^{2}}=\frac{1}{2} \delta-\gamma-s\left(1+\frac{3+4 \mu+\delta}{4 m n}\right) / \mathrm{R} d r+\frac{\mathrm{x}}{m n}(/ \mathrm{R} d r)^{2}-v^{2}+\frac{3 v v}{m n}$

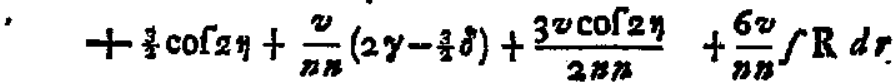
$+\frac{2}{8} \operatorname{cor} y+\frac{\pi}{8} y \operatorname{cor} 3 y$

Hic autem notandum eft effe $x=\gamma\left(x+\frac{3+4 \mu+\delta}{2 n z}\right)$; quoniam vero valores litterarum $\mu$ et demum cum per confenfum obferuatiomam, tum per indolem calculi definire inftieuimus, hic ex obferuationibus petamus valores ipfius $x$; cum enim fit $x: I=d \varphi: d r$, hoc eft $v t$ motus Lunae medius ad motum anomaliae, erit $*=$ I, 0085272 . Fieri quidem poteft, ve hic valor aliquantulum a vero differat, fed errorem fi quis lateat infra detegemus, facillimeque emendabimus.

5. 59.

$C A D U D D P$

compreindicare , partem ntricitate eps fup1 foumus

ontinen-

deniqūe $. v+\frac{3 v \pi}{m n}$ $\int \mathbf{R} d r$ $\left.\frac{\mu+\delta}{n z}\right)$; n cum calculf lus va: eft vt $: x=$ liquaninfra 5.59.
5. 59. Cum igitur iam fupra inuenerimus effe $n=13,25586$ ac proinde $n z=175,71795$ erit $\frac{x}{n}=0,075438$, ideoque $n-\frac{I}{n}=0,933089$ Hic autem numerus, qui iam quafi medium valorem rationis $\frac{d \eta}{d r}$ exprimit, in omnibus operationibus, quae fequuntur, frequentiflime occurret, hineque bretuitatis gratia ponamus

$$
n-\frac{1}{n}=a, \text { vt fit } a+\frac{1}{n}=V\left(+\frac{3+4 n+8}{2 n}\right)
$$

eritque ergo $\alpha=0,933089$, qui valor quam minime a vero difcrepat, vti mox patebile. Quod autem verus ipfius a valor aliquantulum diuerius effe pofic, inde primo pates, quod minutias, quae ex terminis $\frac{3 k v^{2}+2 v / \mathrm{R} d s}{z^{4}}$ quantitati conftanti accrefcere potuiffent, hic neglexio mus; tum vero fieri poteft, vt ratio media differencialiums $d \eta$ ad $d r$ alin fit atque quanticatum finitarum $\eta$ et $r$.

5. 60. Si has formulas attenze contemplemur; mox deprehendemus valorem integralis $\int \mathrm{R} d r$ conftare ex cofinibus angulorum $2 \eta, \eta, 3 \eta$, et $4 \eta$. Quanquam enim -altiora quoque mulkipla huius anguli ingredientur, tamen facile patet, coefficientes eorum continuo fieri minores, ita ve in quadruplo tuto fubfiftere poffimus:

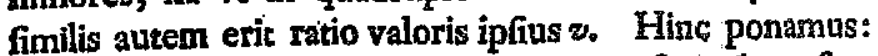
$r R d r=\dot{A} \operatorname{cor} 2 \eta+\$ 2 \operatorname{cor} 4 \eta+a v \operatorname{cor} y+b v \operatorname{cof} 3 y$ $v=A \operatorname{cor} 2 \eta+B \operatorname{cor} 4 \eta+a y \operatorname{col} \eta+b y \operatorname{cor} 3 \eta$ atque hos valores fieticios in formulis noftris fubftiruaG 2 mus: 
mus, vt inde valores iftorum coefficientium affumtorum determinare poffimus : quippe qui modus aptiffmus videtur ad cognitionem integralium perueniendi. Quia autem eft circiter $\nu=\frac{x}{282}$, patet terminos per $y$ multiplicatos prae religuis tam effe exiguos, vt eos qui multo fuerint minores, fine haefitatione praetermittere poffimus.

§. 61. Per hos ergo valores aftumtos confequemur:

$$
\begin{aligned}
& \frac{d \varphi}{d r}=x-\frac{(2 x \mathrm{~A}+\mathfrak{A})}{n n} \cos 2 \pi-\frac{(2 u \mathrm{~B}+\mathfrak{B})}{n n} \operatorname{cor} 4 \pi
\end{aligned}
$$

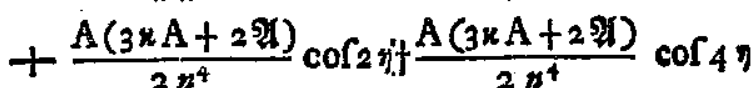

$$
\begin{aligned}
& -\frac{(2 \mu a+a)}{n z} y \operatorname{cor} \eta-\frac{(2 x b+b)}{n z} y \cos 3 \eta
\end{aligned}
$$

atque. ob $x-\frac{1}{x}=a$ erit minimis terminis omiffis, quia hi in operatione multo magis diminuerentur:

$$
\begin{aligned}
\frac{d y_{7}}{d r}= & a-\frac{(2 x A+x)}{n z} \cos 2 \eta-\frac{(2 \times B+B)}{n n} \cos 4 \eta \\
& -\frac{(2 x a+a)}{n n} y \cos \eta-\frac{(2 x b+b)}{n z} y \cos 3 \eta
\end{aligned}
$$

His pofitis erit :

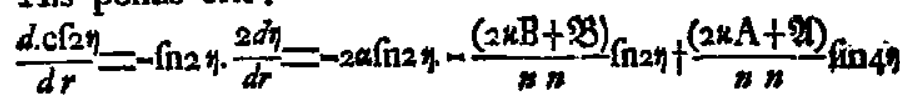

$$
\begin{aligned}
& +\frac{(2 x a+\mathfrak{a})}{n x} y \text { fin } n+\frac{(2 x a+\mathfrak{a})}{n x} y \text { in } 3 \text { 。 } \\
& -\frac{(2 \times b+b)}{n n} y \sin ?
\end{aligned}
$$

$\frac{d . \operatorname{cof} 4 n}{d r}=-\operatorname{fin} 4 n \cdot \frac{4 d n}{d r}=-4 \alpha \sin 4 n+\frac{2(2 x \mathrm{~A}+5)}{n z} \operatorname{fin} 2 n$ $\frac{d \cdot \operatorname{cof} \eta}{d r}=m$ fin $\eta_{0} \frac{d \eta}{d r}=-\alpha$ fin $\eta$ $\frac{d \cdot \cos 3 \eta}{d r}=-\operatorname{fin} 3 \% \frac{3^{d r}}{d r}=-3^{\sin 3 \eta}$

\$. 62. Quod fi iam fecundum has formulai quan. titas integralis $f \mathrm{R} d r$ differentietur, obtinebitur:

$\mathrm{R}=\left(-2 \alpha \mathfrak{A}-\frac{\mathfrak{A}(2 \times \mathrm{B}+\mathfrak{B})}{n B}+\frac{2 \mathfrak{B}(2 x \mathrm{~A}+\mathfrak{A})}{n n}\right) \mathrm{fin} 2 n$

$+\left(\frac{\mathfrak{A}(2 x A+2)}{n}-4 a .3\right)$ fin $4 n^{\circ}$

$+\left(\frac{2(2 k a+\mathfrak{a})}{n}+\frac{\mathfrak{A}(2 x b+b)}{n n}-a \mathfrak{a}\right) \times$ fin

$+\left(\frac{3(2 x a+a)}{n}-3 * b\right) \vee$ fin $3 \pi$

Cum iam fit per hypothefin

$$
\begin{aligned}
& \mathrm{R}=\frac{\sin 2 \pi}{2} \frac{+3 A}{2 n n} \sin 4 y+\frac{3}{8} y \text { fin }+\frac{25}{8} y \text { fin } 3 y \\
& -\frac{3 \mathbf{B}}{2 n z} \cdots \cdots+\frac{3^{a} y}{2 n m} y+\frac{3^{a}}{2 m m} y \\
& -\frac{3^{b}}{273 n} y
\end{aligned}
$$

prodibit terminis homogeneis comparandis :

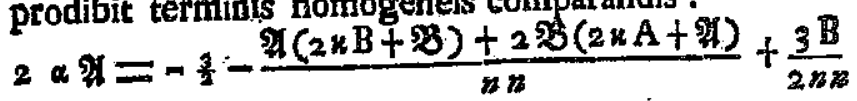$$
4 \cdot B=-\frac{3 A}{2 n n}+\frac{\pi(2 \times A+2)}{n * n}
$$$$
a \mathfrak{a}=-\frac{3}{b}-\frac{3(a-b)}{2 n z}+\frac{4(2 a a+a)-3(2 a b+b)}{n k}
$$

$3 * b=-\frac{x}{8}-\frac{3 k}{2 n n}+\frac{2(2 x a+a)}{n n}$

G 3 
8. 63. Aequatio autem noftra differentio-differentialis, fi pro $f \mathrm{R} d r$ et $v$ valores aflumti fubftituantur, fequentem induet formam :

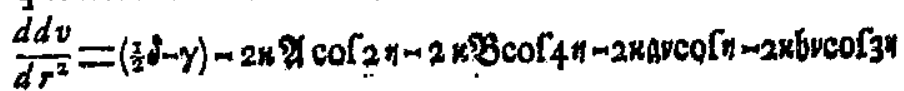

$$
\begin{aligned}
& +\frac{4 \pi}{2 \pi x}+\frac{2 \pi x}{2 \pi n} \\
& +\frac{3 A A}{2 n n} . A \quad \frac{2 n n}{2 n n}-A V-b V \\
& +4
\end{aligned}
$$

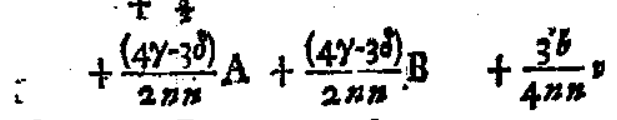

$$
\begin{aligned}
& +\frac{3 A}{4 n n}+\frac{3 B}{4 m n}+\frac{3 A}{4 n n} \cdot \because+\frac{3 A}{4 m n}+\frac{3 A}{4 m n} i \\
& +\frac{3 A A}{=0} \quad+\frac{3 A A}{3 n}+\cdots+1
\end{aligned}
$$

vibi quidem peripicuum eft, quinam termini refpectu reliquorum tam fint parpi, $v t$ fine errore dejeri queant.

\$. 64. Quaeramus ergo primum differentiale $\frac{d o}{d r}$ ac reperietur:

$$
\begin{aligned}
& \left(-2 A a-\frac{A(2 \times B+B)}{n}+\frac{2 B(2 \times A+2)}{x n}\right) \operatorname{lin} 2
\end{aligned}
$$

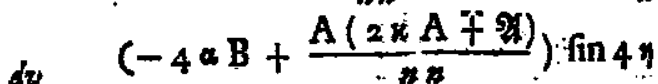

$$
\begin{aligned}
& \frac{d v}{d r}=\left(-a A+\frac{A(2 x a+a)}{n}-\frac{A(2 \times b+b)}{m}\right) v \text { fin } \\
& \left(-3+b+\frac{A(2 x a+4)}{2 \text { fin } 3 !} .\right. \\
& \text { pona- }
\end{aligned}
$$

$$
C A P U \cdot T \text { IP. }
$$

ponatur autem breuitatis ergo:

$\frac{d v}{d r}=-A^{\prime} \operatorname{fin} 2 \eta-B^{\prime} \operatorname{fin} 4 \eta-a^{\prime} v \operatorname{fin} \eta-b^{\prime} v \operatorname{fin} 3 \eta$

vt fit:

$A^{\prime}=2 \alpha A+\frac{A(2 n B+B)}{n B}-\frac{2 B(2 n A+3)}{m n}$

$B^{\prime}=4 \alpha B-\frac{A(2 n A+2)}{n n}$

$a^{\prime}=\alpha a-\frac{A(2 u a+a)}{m}+\frac{A(u b+b)}{m}$

$t^{\prime}=326-\frac{A(2 n a+a)}{m}$

§. 65. Hinc cum fit :

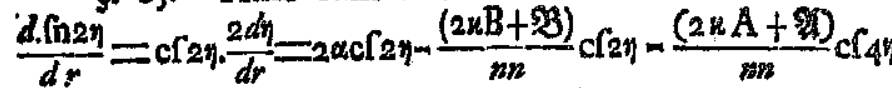

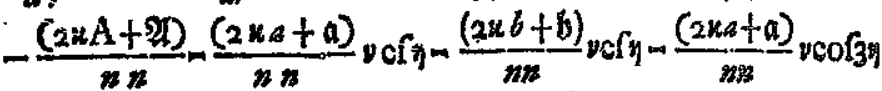

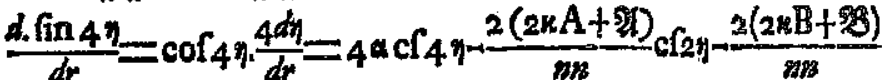
$\frac{d \cdot \operatorname{fin} \eta}{d r}=\operatorname{cof} \eta \cdot \frac{d i}{d r}=\alpha \operatorname{cor} \eta ;$ et $\frac{d \operatorname{fin} 3 \eta}{d r}=\operatorname{cor} 3 \eta \cdot \frac{3^{d} \pi}{d r}=3^{\omega} \operatorname{cor} 3^{\eta}$ prodibit

$$
\begin{aligned}
& +\frac{A^{\prime}(2 x A+2)}{x y}+\frac{2 B^{\prime}(2 x B+3)}{B} \\
& \left(-2 \pi A^{\prime}+\frac{A^{\prime}(2 n B+5)}{m}+\frac{2 B^{\prime}(2 n A+5)}{m}\right) \cos 2 n \\
& \frac{d d v}{d r^{2}}=\left(-4 A B^{\prime}+\frac{A^{\prime}(2 n A+2}{n s}\right) \cos 4 \pi^{n} \\
& \left(-a a^{\prime}+\frac{A^{\prime}(2 u a+a)}{s z}, \cos \right. \text {. } \\
& \left(-3 \omega b 1+\frac{A(2 m a+a}{m}\right) \times \cos 3 a
\end{aligned}
$$




\section{$C A P U T H$}

fen fubftitutis fuperioribus valoribus :

$$
+\frac{2 \mathrm{~A} \alpha(2 \kappa \mathrm{A}+\mathrm{A})}{m n}+\frac{8 \alpha \mathrm{B}(2 \times \mathrm{B}+\mathfrak{B})}{n n}
$$

$\left(-4 \alpha \alpha A+\frac{12 \alpha B(2 x A+\mathscr{A})}{a n}\right) \operatorname{co} 2 y$

$\frac{d d v}{d r^{2}}=\left(-16 \alpha \alpha \mathrm{B}+\frac{8 \alpha \mathrm{A}(2 * \mathrm{~A}+\mathfrak{a})}{m}\right) \operatorname{cor} 4 \pi$

$$
\begin{aligned}
& \left(-\alpha a a+\frac{3 \alpha A(2 \times a+a)}{n n}-\frac{a A(2 \times b+b)}{n n}\right) y \cos n \\
& \left(-9 \alpha a b+\frac{5 \alpha A(2 x a+a)}{n n}\right) \times \cos 3 n
\end{aligned}
$$

\$ 66. Hi iam termini fingulatim illis, qui $\$ .63$. fint exhibiti, sequales ftatuantur, atque fequentes prodibunt determinationes,

$\frac{x}{2} \delta-\gamma+\frac{3 \mathrm{AA}+6 \mathrm{~A} A+94}{2 m x}+\frac{3 \mathrm{~A}}{4 x}=\frac{2 \alpha \mathrm{A}(2 x \mathrm{~A}+2 \mathrm{~A})}{m n}+\frac{8 \alpha \mathrm{B}(2 x \mathrm{~B}+3)}{m}$

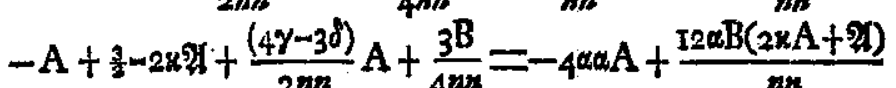

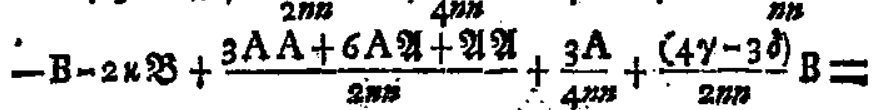
$-16 \pi a \mathrm{~B}+8 a \mathrm{~A}\left(2 \dot{\mathrm{x}} \mathrm{A}+\mathrm{A}^{2}\right)$

$-a+\frac{g}{8}-2 x a+\frac{3 a+3 b}{4 m}=-\alpha a a+\frac{3 a A(2 k a+a)}{m}-\frac{\alpha A(2 x b+b)}{m}$ $-b+\frac{x 5}{8} \div 2 \times b+\frac{3 a}{4 m n}=-9 a \alpha b+\frac{5 a A(2 x a+a)}{m}$

vnde primum quaeri debent valores vero proxirii, qui funt :

$$
\begin{aligned}
& \mathfrak{A}=-\frac{3}{4 \alpha} ; \quad \mathfrak{A}=-\frac{3}{8 \alpha} ; \quad \mathfrak{b}=-\frac{5}{8 \alpha} ;
\end{aligned}
$$

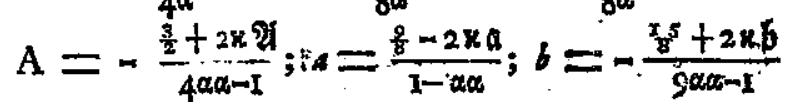

6. 67. Calculus ergo fequenti modo inftituatur:

$$
\begin{aligned}
& a=0,933089 ; \quad l a=9,969923 \\
& x=1,008527 ; l u=0,003687 \\
& \text { lam } \equiv 0,304717 \\
& \int / 3=0,477+21 \\
& \text { fubtr.a } \quad\left\{\begin{array}{l}
3=0,477121 \\
l / 5=0,698970
\end{array}\right. \\
& n=m 0,402 \text {; erie } \overline{l a}=9,604108 \\
& \mathfrak{b}=-0,635 ; \quad L \mathfrak{b}=9,825957 \\
& \text { ม् }=-0,804 ; \quad \text { L }=9,905138
\end{aligned}
$$

atque hinc conficietur:

$A=-\frac{3,121}{4 a \alpha-1} ; a=+\frac{x, 936}{1-\alpha a} ; b=-\frac{3,856}{90 a-1}$

quarum ergo litterarum valores proximi funt

6. 68. Quaeramus hinc primum valores litrerarum $\mathfrak{B}$ et $B$.

$2 * \mathrm{~A}+\mathscr{A}=-3,34 \mathrm{I}$; vnde colligitur

$$
\begin{aligned}
& 4 \propto \mathfrak{B}=+\frac{4,573}{m} \quad l 4,573=0,66020 \mathrm{x} \\
& \text { ln } n=\frac{2,244816}{8,415385} \\
& 14 a=0,571983
\end{aligned}
$$

$\mathfrak{B}=+0,00697 \quad l, 3=7,843402$

Deinde eft $(16 \alpha a-1) B=2 k 3-\frac{3 A}{4 m}-\frac{3 A A-6 A-4-2 A}{2 n}+\frac{8 \alpha A(2 n A+9)}{2}$ feu $B=+\frac{0,16819}{1600-1}$; vnde seperitur : $\quad \ldots .:$ : ;

$\mathrm{B}=+0,012792$ et $t \mathrm{~B}=8,106947 \%$
H

\$. 69
$A=-1,2583 ; a=+14,968 ; b=-0,4613$

$\mathfrak{A}=-0,804 ; l-\mathfrak{A}=9,905 \pi 38$ 
6. 69. His iarn valoribus proxime veris inịentis quaerantur exąti, ac primo quidem

$$
2 a \mathfrak{A}=-\frac{3}{2}+\frac{3 \mathrm{~B}-\mathfrak{M}(2 \times \mathrm{B}+\mathfrak{B})+2 \mathfrak{B}(2 \times \mathrm{A}+\mathfrak{A})}{n n}
$$

vnde reperitur vt ante:

$$
\mathfrak{A}=-0,80378 \ldots . \quad 1-\mathfrak{A}=9,905138
$$

$a \mathfrak{a}=-\frac{3}{8}-\frac{3(a-b)+\mathfrak{A}(2 x a+a)-\mathfrak{A}(2 \times b+b)}{n x}=-0,6536 r$

$\mathfrak{A}=-0,70048 \quad \therefore \quad l-\mathfrak{A}=9,845396$

$3 \backsim b=-\frac{25}{B}-\frac{3+2(2 k 4+a)}{n}=-2.13900$

$b=-0,76413 \cdot \cdots \cdot l-b=9,883167$ $\left(4 \alpha \alpha^{2}-1\right) A=-\frac{3}{2}+2 x 24-\frac{3 \cdot 3+12 \alpha B(2 x A+24)}{m}=-3,12379$

$A=-1,25826 \ldots, l-A=0,099771$ $(I-\alpha a) A=8-2 \times a+\frac{\frac{3}{3}(a+b)-3 \alpha A(2 k a+a)+a A(2 \times b+b)}{n}$ vel $\left(x-a a-\frac{3}{4 n s}+\frac{(6 a x A)}{\sin i}=\frac{8}{8}=2 x a\right.$

$$
+\frac{3 b-3 a A a+a A(2 \times b+b)}{m}=-2,53335
$$

hinc $A=+30,989$ ef $\quad l a=1,491207$

Vnde patet valorem ipfius a ante inuentum non fatis effe exactum, exactior ergo prodibit ex hac formula

$\left(a-\frac{A}{m}\right) a=-\frac{3}{8}-\frac{3(a-b)+2(2 k a-2 \times b-b)}{m}=-0,9370 g$ hine $\mathfrak{a}=-0,399939$. et $\cdot \ln \mathfrak{a}=9,999735$ vnde etiam exactius valor ipfius o reperitur, ex quo denuo denuo valor ipfius a corrigecur, ficque candem fatis ex. alte obtinebitur

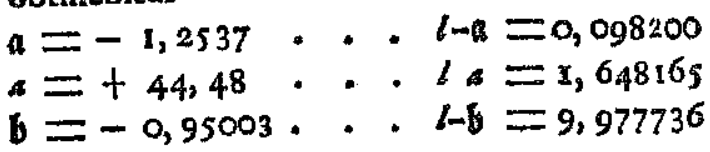

§. 7o. Hinc iam accuratius quaeramus valorem ipfius $b$

$$
\begin{aligned}
& \text { ipfius b } \\
& (9 \alpha a-x) b=-\frac{\pi}{8}+2 a b-\frac{3 a+5 \alpha A(24 a+a)}{m 3} \\
& \quad b=-1,0146 . . \cdot b-b=0,006314
\end{aligned}
$$

vode $f$ denur praecedentes valores corrigantur, fiet

$$
\begin{aligned}
& a=-1,830, \cdot 1-a=0,101403 \\
& b=-0.9500 . .1-b=9,977736 \\
& a=+44,325 \text {. . . la }=1,648604 \\
& b=-1,015 \text {. . l-b }=0,006400 \\
& \text { }=-0,80378 \cdots, L-\mathfrak{A}=9,905138 \\
& \Re=+0,00697 \ldots . \quad 15=7,843402 \\
& A=-1 ; 25826 \text {. . . } M A=0,099771 \\
& B=+0,01279 \cdots \cdot / B=8,106947
\end{aligned}
$$

His autem valoribus inuentis colligitur fore

$$
\frac{x}{2} \delta-\gamma=+0,01742
$$

Hic altem valor parcem infuper accipit cum ab excentricitate vtriusque orbitae, tum ab inclinatione criundam, quam deinceps determinabimus.

$$
\begin{aligned}
& \text { §. 7t. Ex his ergo valoribus habebimus: } \\
& f R d r=-0,80378 \operatorname{cof} 2 \eta+0,00697 \operatorname{cof} 47 \\
& 9,905138 \quad 7,843402 \\
& -1,2630 \vee \operatorname{col} y-0,9500 v \operatorname{col} 3 \% \\
& 0,101493 \\
& \mathrm{H}_{2} \\
& \text { 9, } 977.736 \text {. } \\
& v=
\end{aligned}
$$




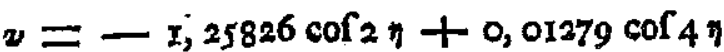 $0,099771 \quad 8,100647$ $+44,525 y \operatorname{cor} \eta-1,015 y \operatorname{cor} 3 \pi$ $1,648604 \quad 0,006400$ hincque porro $d \phi=x+0,019015 \operatorname{col} 2 \eta-0,0000762 \operatorname{cof} 4 \eta$ 9,702270 8,232184

at eft $\frac{d h}{d r}=\frac{d \varphi}{d r}-\frac{1}{n}$, pofuimusque $x-\frac{1}{n}=\alpha$, exi(tente $x=V\left(1+\frac{3+4 \hat{\alpha}+\delta}{2 m n}\right)$

8. 72. Pònatur breuitatis gratia $\frac{d \Phi}{d r}=D+\Im \cos 2 \eta-\mathfrak{Q} \cos 4 \eta-\Re v \cos \eta+S v \operatorname{cof} 3 \eta$ erit $\frac{d y}{d r}=\dot{a}+9 \cos 2 y-\infty \cos 4 y-q u \cos \eta+S v \cos 3 \eta$ fitque ad integrandum:

$\varphi=y r+q$ fin $2 \eta-q \cdot \operatorname{fin} 4 \eta-p y$ fin $\eta+\$ y \cdot$ fin $3 \eta$ vnde per differentiationem elicitur :

$\frac{d p}{d r}=0+2 a p \operatorname{col} 2 \eta-4 \alpha q \operatorname{cor} 4 \eta-a t v \operatorname{cor} \eta+3 a b u \cos 3 \eta$

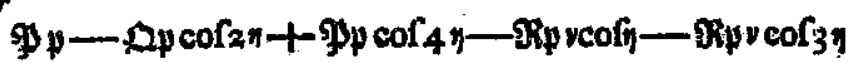
$+2 \mathrm{SO}_{q}-2 \mathrm{~S}_{q} \mathrm{Cor} 2 \mathrm{c}$ +2 Sqqy col3? hinc ergo fit:

$v=\mathfrak{D}-9 p-2 p q=x+0,0001103-9 p-3 p q$ $(2 \alpha-\mathcal{Q}) \mathfrak{p}=\mathfrak{P}+299 q ; 4 q=\mathcal{Q}+\mathfrak{P}_{p ;}$

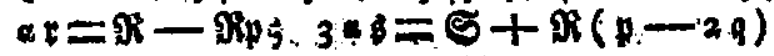

$C A P U T \quad I P$

62

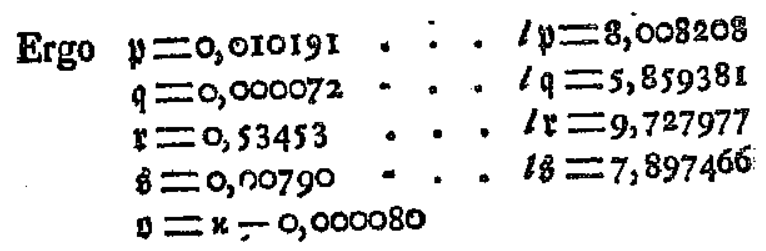

\$. 73. Longitudo igitur lunae $\phi$ guatenus pendet a fola diftantia lunae a fole erit.

$\phi=(u-0,000080) r+0,01019$ I fin $2 y-0,53453 v$ fin $-0,000072$ fin 4 \% $+0,00790$ vin $3 \eta$

Simili modo cum diftantia lunae a terra-pofita fit $=$ $\frac{a(1-k k) u}{z-b \operatorname{co}[r}$, ob $u=1+\frac{v}{n n}$, quatenus valor ipfins $z$ : la phafi lunae pendet, erit

la phafi lunae pendet, erie $\eta+0,00716 \operatorname{cof} 2 \eta+0,00287 v \operatorname{cof} \eta$ $+0,00007 \operatorname{cor} 4 \eta-0,00009$ v cof $3 \eta$

$5 v \operatorname{col} 37$

jucofin

Verum tamen hic valor litterae $\mathfrak{a}$ ac praecipue ipfius $\mathfrak{a}$ Verum tamen hic valor literae as a terminis neglectis non admodum certus videtur, cum a termitis queat. Hic enim pro $\alpha$ non folum $x-\frac{1}{x}$ fed $x-\frac{1}{x}+0,0001103$ accipi debuiffet; quare cum valorem ipfius a propiws cognofcimus, hanc determinationem repeti conuenies. 


\section{CAPUT V.}

\section{INUESTIGATIO INAEQUALITATUM LUNAE AB EIUS EXCENTRICYTATE SIMPLICI SOLUM PEDENTIUM.}

\section{\$. 74.}

$\mathrm{Q}$ uemadmodum in praecedenti capite inaequalitas abfoluta feu variatio duabus partibus conftans eft inuenta, quarum pofterior a littera y feu a pao rallaxi folis pendebat, ac maiorem curam requirebat; ita etiam inaequalitates, quas hoc capite fcrutamur, partes continent ab eadem parallaxi folis pendentes; quarum indagatio quoque accuraciorem cognitionem quorundam elementorum exigit. Hancobrem et praecedentis capitis et huius partes, quae litteram y inuoluunt deinceps, - cum reliquas inaequalitates, a parallaxi folis non pendentes determinauerimus, feorfim inueftigabimus, atque titulo inaequalitatum parallacticarum complectemur.

5. 75. In hoc ergo capite ac fequentibus, donec ad parallaxin folis perueniamus, terminos formularum noftrarum per $y$ mulciplicatos tantisper remouebimus; et quoniam hoc loco tantum propofitum eft in motus lunae inaequalitates a fola excentricitate orbitae lunaris ortas inquirere, eos terminos qui vel excentricicatem folis $e$ vel inclinationem $\rho$ continent, praetermittemus. Cum autem in formulis noftris duplicis generis termini relinquantur, quorum alteri per $k$, alteri per $k k$ funt af fedi, inaequalitates ab excentricitare lunae $k$ pendentes in duas partes diftribui conueniet; quarum altera excentricitatem tantum fimplicem $k$ implicet, cui hoc ca. put deftinatur, altera vero excentricitatis huius quadrato $k k$ afficiatur, de quo in requenti capite agemus.

\$. 76. Verum tam in huius generis inaequalitates, quam in fequentes, omnes inaequalitates abfolutae in praecedenti capite erutae praesipue ingrediuntur; ex quo eas quoque in calculum introduci oportebit. Retinendae ergo erunt in calculo litterae $\mathscr{A}_{2} \mathfrak{B}$ et $A, B$, quarum valores cum iam conftent, calculus vehementer contrahetur: imprimis autem quia valores littera. rum $\boldsymbol{B}$ et $\mathbf{B}$ per fe funt admodum parui, quatenus illi in valores fequentium terminorum influanc; effectum pro nibilo habendum praeftabunt. Inueftigationem ergo notram ita incipiemus, ve pro $\int \mathrm{R} d r$ et $\nu$ valores fietos affumamus, et quoniam $\int R d r$ nullum terminuma conftantem, $\dot{w}$ vero neque conftantem neque terminum huius formae a cof $r$ continere debet, ponamus :

$$
\begin{aligned}
& f \mathrm{R} d r=\mathfrak{A} \operatorname{col} 2 \eta+3 \operatorname{cor} 4 \eta+\mathbb{C} k \operatorname{cor} r \\
& +\mathfrak{D} k \operatorname{cof}(2 \eta-r)+\delta k \operatorname{col}(4 \eta-r) \\
& +\varepsilon k \operatorname{cor}(2 \eta+r)+0 k \operatorname{cor}(4 \eta+r) \\
& =A \cos 2 \eta+B \cos 4 \eta \\
& +\mathrm{D} k \operatorname{col}(2 \eta+r)+\mathrm{F} k \operatorname{cor}(4 \eta-r) \\
& +\mathrm{E} k \operatorname{cor}(2 \eta+r)+\mathrm{G} k \operatorname{cor}(4 \eta+r)
\end{aligned}
$$

vbi quidem facile colligere licet, coefficientes $\$, G, F$ et $G$ fore minimos.

\$. $7 \%$ 
§. 77. Ex his autem valoribus aftumtis obtinebimus ex (\$. 52.) lequentes expreffiones.

$$
\begin{aligned}
& x+\frac{A\left(3^{x A}+2 A\right)}{2 m^{4}}-\frac{(2 x A+A)}{* n} \cos 2 n \\
& \left(-\frac{(2 x \mathrm{~B}+B)}{3}+\frac{\mathrm{A}(3 x \mathrm{~A}+2 \mathrm{~A})}{2 \pi^{4}}\right) \cos 4 n \\
& d \varphi=\left(-\frac{C}{n *}+\frac{3 \times A D}{z^{4}}+\frac{A D+2 D}{n^{4}}\right) k \operatorname{col} r \\
& \frac{d \varphi}{d r}=-\frac{(2 \times \mathrm{D}+D)}{n n} k c 0 \mathrm{f}(2 \eta-r)-\frac{(2 \times \mathrm{E}+(\mathbb{E})}{n n} k \operatorname{cor}(2 n+r) \\
& -\frac{(24 \mathrm{E}+\mathrm{g})}{n n} k \operatorname{cof}(4 y-r)-\frac{(2 k \mathrm{G}+\mathrm{O})}{n n} k \operatorname{cof}(4 y+r) \\
& +\frac{(3 x A D+A D+2(D)}{n^{4}} k \operatorname{cor}(4 \eta-r)
\end{aligned}
$$

$\operatorname{CAPUT}$

cunt, qui ex calculo fine ersore expungi poserunt: atque ob hanc canfam in expreffione valoris $\frac{d \eta}{d r}$, fatim terminos prae reliquís admodum paruos praetermitere vifum eft.

§. 78. Valorem autem ipfius $\mathrm{R}$ atque $\frac{d d v}{d r^{2}}$ accura. tiffime exhiberi oportet, propterea quod his exprefionibus totus calculus praecipue innititur, dum valor $\frac{d \eta}{d r}$ formulam tantum fubfidiariam fuppeditat. Erit ergo

$$
\begin{aligned}
& \mathrm{R}=\frac{3}{2} \sin 2 \eta+\frac{3 \mathrm{~A}}{2 n z} \text { fin } 4 y+3 k \sin (2 y-r)+3 k \sin (2 y-r) \\
& +\frac{3 A}{n n} k \text { fin }(4 y-r)+\frac{3 A}{n n} k \text { fin }(4 y+r) \\
& +\frac{3 D}{2 n n} k \text { fin } r \quad-\frac{3 E}{2 n n} k \text { fin } r \\
& +\frac{3 \mathrm{D}}{2 n n} k \mathrm{fn}(4 y--r)+\frac{3 \mathrm{E}}{2 n n} k \sin (4 y+r) \\
& -\frac{3 \mathrm{~A}}{n n} k \text { fin } r \quad+\frac{3 \mathrm{~A}}{n n} k \text { fin } r
\end{aligned}
$$

vbi quidem terminos ab $k$ non pendentes onittere pos$-\frac{I}{n}$ feu $x+0,000$ rio $-\frac{I}{x}=a$ erit :

$$
\begin{aligned}
\frac{d \eta}{d r} & =a-\frac{(2 \times \mathrm{A}+\mathfrak{A})}{n n} \cos 2 n-\frac{2 k}{n} \operatorname{cor} r \\
& -\frac{(2 \times \mathrm{D}+\mathfrak{D})}{n n} k \operatorname{cor}(2 \eta-r)-\frac{(2 \times \mathrm{E}+\mathrm{E})}{n n} k \operatorname{col}(2 n+r)
\end{aligned}
$$

fur fumus, quia illorum iam habuimus rationem, ita vt fit

$$
\begin{aligned}
& R \doteq \ldots+\frac{3(D-E)}{2 n z} k f i n r+3 k f(2 y-r)+3 k f(2 y)(2 y+r) \\
& +\frac{3 D}{2 m n} k \text { fin }(4 n-r)+\frac{3 E}{2 m n} k \text { fin }(4 y+r) \\
& +\frac{3 A}{n n} k \text { fin }(4 y-r)+\frac{3 A}{n z} k \text { fin }(4 y+r)
\end{aligned}
$$


\$. 79. Simili modo terminis a $k$ non pendentibus omittendis habebitur:

$\frac{d d v}{d r^{2}}=-\gamma k \mathrm{c}(r+3 k \mathrm{c}(2 y-r)+3 k \mathrm{c}(2 y+r)-2 k f \xi \mathrm{c}\{(4 y-r)-2 x(S k \mathrm{c}(4 y+r)$

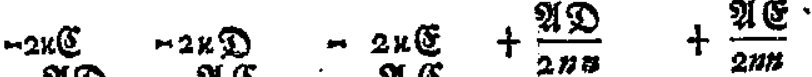

$$
\begin{aligned}
& +\frac{\mathscr{H D}}{2 n n}+\frac{\mathscr{A E}}{2 n n}+\frac{\mathscr{A C}}{2 n n} \\
& +\frac{\mathscr{A E}}{2 n \theta^{2}}-\mathbf{D}-\mathbf{E}-\mathbf{F} \quad-\mathbf{G} \\
& +\frac{3 A D}{n n}+\frac{3}{2} A+\frac{3}{2} A+3 B+3 B \\
& +\frac{3 \mathrm{AE}}{n n}+\frac{\left(2 \gamma-\frac{3}{2} \delta\right)}{n n} \mathrm{D}+\frac{\left(2 y-\frac{3}{2} \delta\right)}{n n} \mathrm{E}+\frac{3 \mathrm{AD}}{n n}+\frac{3 \mathrm{AE}}{n n} \\
& -\frac{3 \mathrm{AA}}{2 n n}+\frac{(3+3 \mu+\gamma)}{n n} \mathrm{~A}+\frac{(3+3 \mu+\gamma)}{n} \mathrm{~A}-\frac{3 \mathrm{AA}}{4 n n}-\frac{3 \mathrm{AA}}{43 n} \\
& +\frac{3 A D}{n n} \quad+\frac{3 A D}{n n}+\frac{3 A E}{n n} \\
& +\frac{3 A E}{n n} \\
& +\frac{3 \mathfrak{A D}}{m B}+\frac{3 \mathfrak{A E}}{m} \\
& +\frac{3 \mathfrak{A D}}{n n} \text {. } \\
& +\frac{3 \mathfrak{A E}}{n n}
\end{aligned}
$$

Hic fcilicet plures terminos, qui nullius futüri eflent momenti, omilimus, ne caleulus nimium implicaretur: notandum autem eft effex=V $\left(1+\frac{3+4 \mu+\delta}{2 n n}\right)=1+\frac{3+4 \mu+\delta}{4 n n}$ proxime; vnde $\mu=(x-1)=x-\frac{3-\delta}{4}$ et $\frac{3+3 \mu+\gamma}{4 n n}$ $=3(x-1)+\frac{3-3 \delta+4 y}{4 n n}$

\$. 80.
5. 80. Quaeramus nunc quoque ex forma pro $/ \mathbb{R} d r$ fieta valorem ipfius $R$, atque exclufis terminis ab $k$ non pendentibus reperiemus :

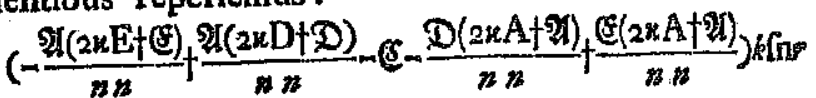

$$
\begin{aligned}
& \left(+\frac{2 \mathfrak{A}}{r}-(2 \alpha-1) D\right) k \operatorname{fin}(2 \eta-r) \\
& \mathrm{R}=\left(+\frac{2 \pi}{n}-(2 x+1) \Leftrightarrow\right) k \sin (2 \eta+\dot{y})
\end{aligned}
$$

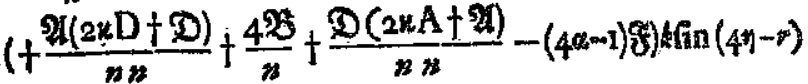

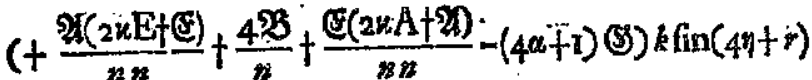
atque inftituta comparatione inuenietur:

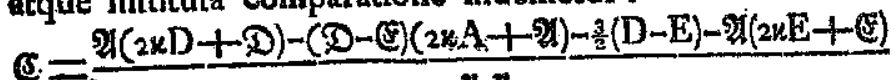
$(2 \alpha-1) \mathfrak{D}=\frac{2 \pi}{n}-3:(2 \alpha+r) E=\frac{2 \pi}{n}-3 ;$ $(4 \alpha-1) . F=\frac{4 \mathfrak{B}}{n}+\frac{\mathscr{P}(2 x \mathrm{D}+\mathcal{D})+\mathcal{D}(2 x \mathrm{~A}+\mathfrak{P})-\frac{3}{2}(2 A+D)}{3 n}$ $(4 \alpha+1) B=\frac{4 \mathscr{B}}{n}+\frac{\mathscr{A}(x E+E)-\frac{3}{2}(2 \mathscr{A}+E)+\mathcal{C}(2 x A+\mathfrak{X})}{n z}$ 5. 8I. Pro differentiali $\frac{d v}{d r}$ inueniendo, praeter terminos fupra inventos habebimus:

$i$ effent aagetur:

$3+4 \mu+\delta$

$4 n n$

$\mu+\gamma$ $\frac{d v}{d r}=-\mathrm{A}^{\prime}$ fin $2 \eta-\mathrm{A}$ fin 4

$\left(+\frac{\mathrm{A}(2 x \mathrm{D}+\mathrm{D})}{n n}-\frac{\mathrm{A}(2 x \mathrm{E}+\mathrm{E})}{n n}-\frac{\mathrm{D}(2 \times \mathrm{A}+2 \pi)}{n n}+\frac{\mathrm{E}(2 n \mathrm{~A}+2)}{n n}\right) n$ fin $\left(+\frac{2 A}{n}-(2 \alpha-1) D\right) k\left(i n(2 \eta-r)+\left(\frac{2 A}{n}-(2 x+1) E\right) k(\operatorname{in}(2 n+r)\right.$

s. 80.
I 2 
68

$$
C A P U \boldsymbol{T}
$$

$\left.C+\frac{A(2 x D+D)}{n}+\frac{4 B}{n}+\frac{D(2 x A+2 A)}{n \pi}-(4 \alpha-1) F\right) k \sin (4 \eta-r)$

$\left(+\frac{A(2 \times E+E)}{n n}+\frac{4 B}{n}+\frac{E(2 n A+2)}{n n}-(4 \alpha+1) G\right) k \sin \left(4^{n}+r\right)$

Ponatur antem breutitatis gratia:

$\frac{d v}{d r}=-A^{\prime}$ fnn $_{2 y}-B^{\prime} \operatorname{fin}_{4} y-\mathrm{C}^{\prime} k$ fin $r-D^{\prime} k \sin (2 \eta-r)$ $-\mathrm{E}^{\prime} k \sin (2 \eta+r)-\mathrm{F}^{\prime} k$ fin $(4 \eta-r)-\mathrm{G}^{\prime} k$ fin $(4 \eta+r)$

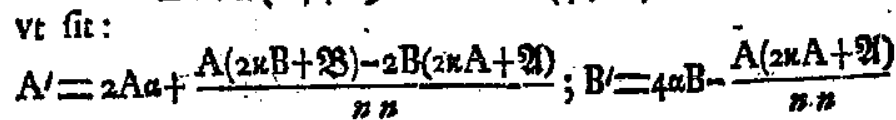

$C=\frac{-A(2 \times D+D)+A(2 \times E+C)+(D-E)(2 \times A+2 D)}{n}$

five $\mathbf{C}=-\frac{A(D-C)+2(D-E)}{n}$

$D^{\prime}=(2 \alpha-1) D-\frac{2 A}{n} ; \quad E^{\prime}=(2 \alpha+1) E-\frac{2 A}{n}$

$F^{\prime}=(4 \alpha-1) F-\frac{4 B}{n}-\frac{A(2 x D+9)-D(2 x A+2)}{n n}$

$\mathrm{G}^{\prime}=(4 \alpha+1) \mathrm{G}-\frac{4 \mathrm{~B}}{3}-\frac{\mathrm{A}(2 x \mathrm{E}+(0)-\mathrm{E}(2 x \mathrm{~A}+4)}{3}$

§. 82. Hinc denuo differentiando obtinebitur terminis tantum per $k$ mulciplicatis fcribendis :

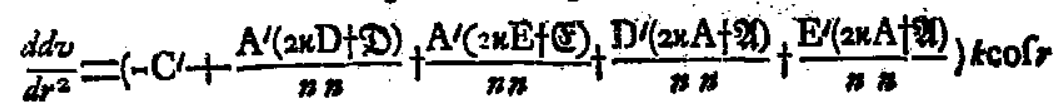
$\left(+\frac{2 A^{\prime}}{n}-(2 \omega-I) D \prime\right) k \cos (2 \eta-r)$ $\left(+\frac{2 A !}{n}-(2 a+1) E^{\prime}\right) * \operatorname{cor}(2 y+r)$
$C A P U T P$

$\left(+\frac{4 \mathrm{~B}^{\prime}}{n}+\frac{\mathrm{A}(2 \mathrm{kD}+\mathfrak{D})}{n n}+\frac{\mathrm{D}^{\prime}(2 n \mathrm{~A}+2)}{n n}-(4 a-1) \mathrm{F}^{2}\right) k \cos (4 n-r)$ $\left(+\frac{4 \mathrm{~B}}{n}+\frac{\mathrm{A}^{\prime}(2 \mu \mathrm{E}+(\mathrm{E})}{n n}+\frac{\mathrm{E}^{\prime}(2 x \mathrm{~A}+2 n)}{n n}-(4 \omega+n) \mathrm{G}\right) k \operatorname{cor}(4 \eta+n)$ vnde comparatione inftituta orietur::

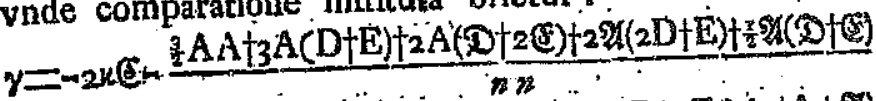
$\mathrm{A}^{\prime}\left(2 n \mathrm{D}+\mathfrak{D}^{2}\right)-\mathrm{A}^{\prime}\left(2 u \mathrm{E}+(\mathrm{E})-\left(\mathrm{D}^{\prime}+\mathrm{E}^{\prime}\right)\left(2 \mathrm{~A}^{2}+9\right)\right.$

$\eta-r)$

$(n+r)$

$1+(2)$

- - $n$

$(2 \alpha-1)^{2} \cdot \mathrm{D}-\frac{2(2 \alpha-1)}{\pi} \mathrm{A}-\frac{2 \cdot \mathrm{A}}{\pi}+3-2 x D-D$ $+\frac{3}{2} A+\frac{x^{2} \pi C+\left(2 y-\frac{3}{2} y\right) D+(3+3 \mu F y) A}{n n}=$

$(2 \alpha+1))^{2} \mathrm{E}-\frac{2(2 \alpha+1)}{\pi} A-\frac{2 A}{n}+3-2 H-E$

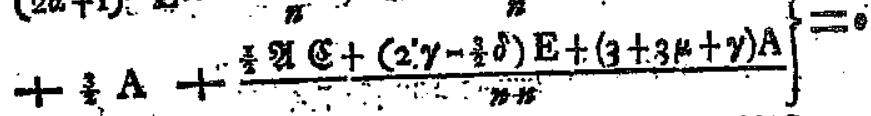

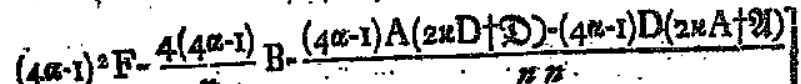
$-\frac{4^{\prime}}{n}-\mathrm{A}^{\prime}(2 \times \mathrm{D}+\mathrm{D})-\mathrm{D}^{\prime}(2 x \mathrm{~A}+2)-\mathrm{m}-\mathrm{F}=$ $+7 \mathrm{~B}+\frac{39+3 A D-3 A A+3 A D+3 P D}{* n}$

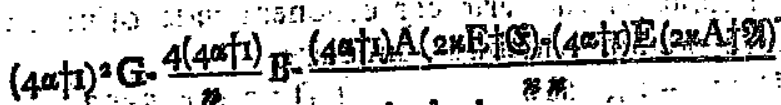

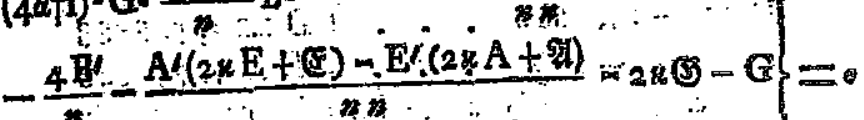

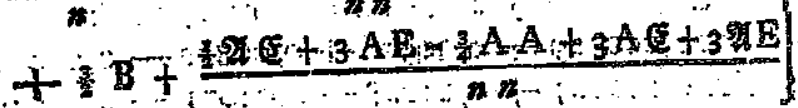
13 
§. 83. Incipiamus a coefficientibus $D$, $C$, et $D, E$; et quia $\mathcal{C}$ eft quanticas admodum exigua, erit:

$(2 \alpha-1) D=-3+\frac{2 A}{n} ;(2 \alpha+1) C=-3+\frac{2 A}{n}$ $\left((2 \alpha-1)^{2}-1+\frac{2 \gamma-\frac{3}{2} \delta}{n x}\right) D=-3-\frac{3}{2} A+2 x D$ $+2 \frac{(2 \alpha-1)}{x} \mathrm{~A}+\frac{2 \mathrm{~A}^{\prime}}{x}-\left(3 x-3+\frac{3-38+4 \gamma}{4 x}\right) \mathrm{A}$ $\left((2 a+1)^{2}-1+\frac{2 y-3}{m}\right) \mathrm{E}=-3-\frac{3}{2} \mathrm{~A}+2 x \Subset$

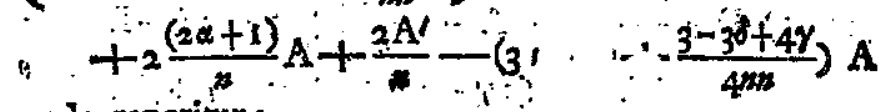
ynde reperitur:

$D=-3,6035 \therefore \therefore t-D=0,556724$

CU-

ac porro

$\left.\left(-0,24973+\frac{(2 \gamma-1)}{42}\right) D=-1,40048-7,4315\right]$

$\left.\left(+7,21497+\frac{\left(2 y-\frac{3}{2} d\right)}{m n}\right) E=-1,40048-2,7403\right\}+\frac{\left(2 y-\frac{3}{2} \theta\right)}{w 0,629}$

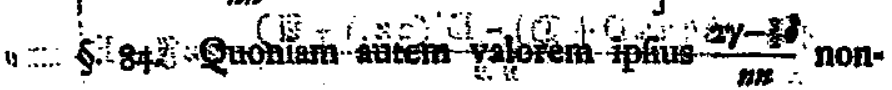
dum hoụinusg, hunc terminum; cum certo fit valde par. vus, reiiciamus. Poftmadumm vero cuin iftum terminum cognougerimus, facile erit correctionem inde oriundam,

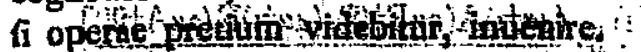

$$
\begin{aligned}
& \mathrm{D}=+35,3662, \cdot i \mathrm{D}=\mathrm{x}, 548588
\end{aligned}
$$

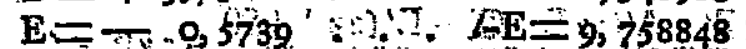

Porro autem litterae $\delta$ et 0 ita elieientur, vt fit; $(4 \alpha-1) y=6,000529-0,30976+0,06852 \div 0,28042$ $\left(4^{a}+1\right)\left(\hat{S}=0,000529+0,01028+0,0207 x+0,02 \sigma_{38}\right.$
CAPUT $Y$

$$
f=-0,1907 \cdot \cdots \quad b-\mathfrak{F}=9,280416
$$

$\mathrm{G}=+0,0122 \cdot . \cdot 1808,087607$

Praeterea autem colligimus fore

$$
c=-0,67465 \text {. . . } l-C=9,829072
$$

vnde exit proxime $\frac{\mathscr{A} \in}{2 n m}=0,00154$, ex que accuratius concluditur fore

$$
\begin{aligned}
& \mathrm{D}=+35,3724: \quad \cdot l \mathrm{D}=1,548664 \\
& \mathrm{E}=-.0,5741 . \quad \cdot \mathrm{L}=9,758988
\end{aligned}
$$

5. 85. Reliquae aequationes nobis praebebunt

$$
\begin{aligned}
6,4655 \mathrm{~F}+3,67820 & =0 \\
21,3946 \mathrm{G}-0,29574 & =0
\end{aligned}
$$

vnde obtinebitur

$$
\begin{array}{lll}
\mathrm{F}=-0,56890 & ; & l-\mathrm{F}=9,755033 \\
\mathrm{G}=+0,01382 & \cdot \quad l \mathrm{G}=8,140620
\end{array}
$$

ac denique $y=1,40673$.

Supra autem iam inuenimus $\frac{2}{2} \delta-\gamma=0,01742$, vnde ambas iftas quantitates $\gamma$ let $\&$, quas initio ad veros vallo res conftantium litterarum $m$ et $n$ determinandos affum fimus, nune cognitas habemus; erit enim:

$$
\delta=2,84830 \text {; et } 2 \gamma-\frac{3}{2} \delta=-1,45899
$$

ac propterea particulae illius $\frac{2 \gamma-\frac{3}{2} \delta}{n}$ haçtenus neglectae valor erit $\frac{2 \gamma-\frac{3}{2} \delta}{200}=-0,00832$, cuius ope iam literae D et $\mathbf{E}$ accuratius definiri poterunt.
5. 86 
8. 86. Hinc autem potifimum valor ipfius D mutationem patiur, fier enim re vera

$$
\begin{aligned}
& \text {-0,25805 D }=-8,83698 \text { feu } \\
& \mathrm{D}=34,24520 \ldots \text {. . . } l \mathrm{D}=1,534600 \\
& 7,20665 \mathrm{E} \equiv-4,14578 \text { feu } \\
& E=-0,57527: \ldots: E=9,759874
\end{aligned}
$$

et quoniam $D$ parte fua tricefima diminuittr, in eadem fere ratione diminuentur valores litterarum $\mathbb{E}$ ex $y$, ita vt exactius fit:

$$
\begin{aligned}
& C=-0 ; 65217 . \because \cdot 4 C=9,814361 \\
& \gamma=+1,35984 . \cdots l \gamma \equiv 0,133490 \\
& =72,75336 . .8=0,439863 \\
& \text { et } \frac{2 \gamma-\frac{3}{2}}{2 y}=-0,00804
\end{aligned}
$$

Deinceps autem operae eric pretium in hos valores ad. huc diligentius inquirere.

5. 87. Cum igitur finxerimus fequentes valores:

$\int \mathrm{R} d r=2 \operatorname{col} 2 \eta+2 \operatorname{col} 4 \eta+c k \operatorname{cor} r$

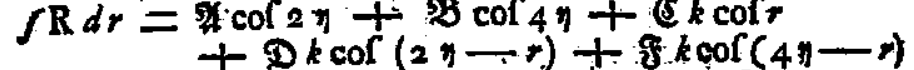

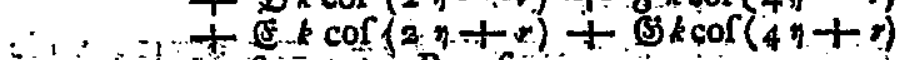

$$
\begin{aligned}
& \therefore=A \operatorname{cor} 2 \pi+B_{1} \cos 4
\end{aligned}
$$

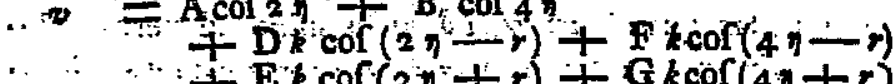$$
\therefore \quad \therefore \quad \mathbf{E} k \text { cor }(2 \eta+r)
$$

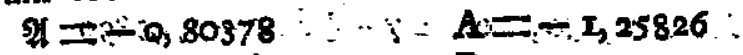

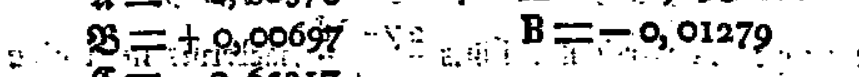

$$
\begin{aligned}
& \text { C }=-0,65217 \text {. } \\
& D=-3,60350 \quad D=+34,24520 \\
& \text { EE- } 1,08900 \quad \mathrm{E}=-0,57527 \\
& f=-0,19070 \ldots . . .5 \pm-0,56890 \\
& \therefore \quad B=+0,01220 \quad \mathrm{G}=+0,01382
\end{aligned}
$$

ynde pro diftantia lunae a terta $x=\frac{(1-k k) a k}{\mathrm{I}-k \operatorname{col} r}$ tit $u=1-0,007161 \operatorname{col} 2 \eta \quad+0,000073 \operatorname{cof} 4 \eta$ $+0,194888 k \operatorname{cor}(2 y-r)-0,003274 k \operatorname{cor}(2 y+r)$ $-0,003238 k \operatorname{cof}(4 \eta-r)+0,000078 k \operatorname{cor}\left(4^{n}+r\right)$

\$. 88. His valoribus in \$. 77. fubftituris obtinebimus: $d \varphi=* \quad+0,019015 \operatorname{cof} 2 n-0,001255 k \operatorname{cof} r$ $\frac{d \varphi}{d r}={ }_{+0,0001103-0,000076 \mathrm{col} 4 \eta}$ $-0,38410 k \operatorname{cof}(2 \eta-r)+0,01278 k \operatorname{col}(2 \eta+r)$ $+0,002647 k \operatorname{co}\left(4^{y-r}\right)-0,000229 k \operatorname{cof}(4 n+r)$ ad cuius. integrale inueniendum ponamus: $\phi=O r+\mathfrak{A}^{\prime}$ fin $2 \eta+\mathfrak{B}^{\prime}$ fin $4 \eta+(\mathbb{y} / k$ fin $r$

$+D^{\prime} k \operatorname{col}(2 n-r)+\mathbb{C}^{\prime} k \operatorname{col}(2 n+r)$ $+g^{\prime} k \operatorname{cor}(4 \eta-r)+\sigma^{\prime} k \operatorname{col}(4 i+r)$

eritque differentiando et terminis iam cognitis omittendis.

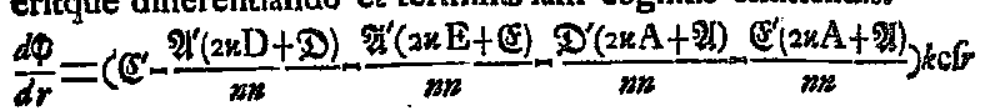

$$
\begin{aligned}
& \left(-\frac{2 \Omega i}{n}+(2 \alpha-r) D\right) k \cot (2 n-r) \\
& \left(-\frac{2 \pi}{n}+(2 \theta+1) e j\right) k \operatorname{cor}(2 n+r) \\
& \left(-\frac{4 \mathfrak{B}^{\prime}}{n}-\frac{\mathfrak{A}(2 x \mathrm{D}+\mathfrak{D})}{m}-\frac{\mathfrak{D}^{\prime}(2 x \mathrm{~A}+\mathfrak{A})}{n}+(4 x-1) F^{n}\right) k \operatorname{cor}(4 y-r)
\end{aligned}
$$

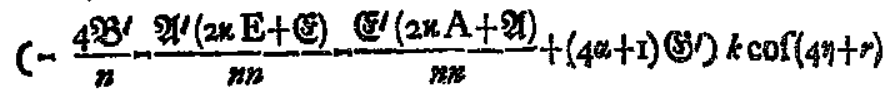

Pro terminis autem iam inuentis eft

$$
\mathbf{O}=x-0,000080 ; \quad W^{\prime}=0,010191 ; \quad B^{\prime}=-0,000072
$$

$$
\text { lश्रि'=8,008208; } l \mathfrak{B}^{\prime}=-5,85938 \mathrm{z}
$$

K

ร. 890 
\$. 89. Comparatione iam inftituta fiet:

$$
\begin{aligned}
& (2 \alpha-1) D^{\prime}=-0,38410+\frac{2 \mu^{\prime}}{n} ;(2 \alpha+1) \text { éf }=+0,01278+\frac{2 \mu^{\prime \prime}}{n}
\end{aligned}
$$

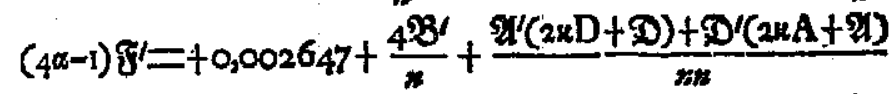

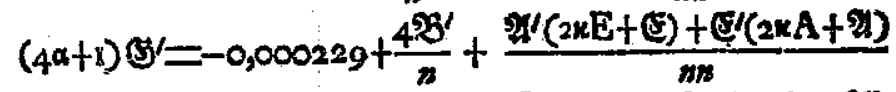

$$
\begin{aligned}
& \mathfrak{G}^{\prime}=-0,001255+\frac{\mathscr{W}^{\prime}(2 \times D+\mathfrak{D})+\mathfrak{D}^{\prime}(2 x \mathbf{A}+\mathfrak{W})}{m} \\
& +\mathscr{W}^{\prime}(2 x E+\mathbb{E})+\mathbb{C}^{\prime}(2 x A+\mathscr{A})
\end{aligned}
$$

rnde colligitur tore

$$
\begin{aligned}
& E^{\prime}=+0,01083 \\
& \mathfrak{D}^{\prime}=-0,44167 \text {. . } L \mathfrak{D}^{\prime}=9,645092
\end{aligned}
$$

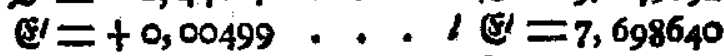

$$
\begin{aligned}
& g^{\prime}=+0,00546 \ldots g^{\prime \prime}=7,737733 \\
& \mathrm{~S}^{\prime}=-0,00010 \text {. . H } \mathrm{H}^{\prime}=6,002537
\end{aligned}
$$

in ve fit

$\varphi=(x-0,000080 r)+0,01019$ I fin $2 \eta+0,01083 k$ fin

$$
0,000072 \text { fin } 41
$$

$-0,44167 k$ fin $(2 \eta-r)+0,00499 k$ in $(2 \eta+r)$

$+0,00546 k$ fin $(4 \eta-r)-0,00010 k$ fin $(4 \eta+r)$ vnde ex comparatione motus medii ad modum ano. maliae exit $x=1,008607$, it $a=0,933279$, qui valores jam propius ad veritatem accedunt, quam haEenus vfurpati.

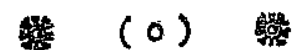 \\ C A P U T VI.}

\section{INUESTIGATIO INAEQUALITATUM LUNAE A QUADRATO EXCENTRIGITATHS TPSIUS ORTARUM:}

§. 90.

\section{$\mathbf{P}$}

Theruenimus nunc ad alteram partem inaequalitartata in motu Lunae, quae ab eius excentricitate $k$ peq. dent, einsque quadratum inuoluunt, ita ve hic nonnifi eos terminos fimus contemplaturi, qui per quadfatum excentricitatis lunae $k k$ funt multiplicati. Hic autem tam in valorem ipfius $\int \mathrm{R} d r$, quam ipfips $v$ termi xi formae $k k$ col $2 \eta$ et $k k$ cof $4 \eta$ ingredientur, qui poltquam fuerint inuenti, terminis huivs generis iama quam ruerint indici debent: praeterea vero vtringue etiam termini formae $k k$ cor $2 \%$ accedene. Hine ponamus ;

Rdr $=x \operatorname{cor} 2 \eta+a k k \operatorname{cor} 24+3 \operatorname{cor} 4 \eta+b k \operatorname{cor}_{4} \eta$ $\int R d r=\mathbb{A} \operatorname{cor} 2 \eta+a k k \operatorname{cor}(2 n-r)+\mathbb{C} k \operatorname{cor}(2 \eta+r)$

$+8 k k \cos (4 \eta-r)+8 k \operatorname{col}(4 \eta+r)$

$(2 n+r)$

$(4 n+r)$

$m$ ano-

9, qui

iam ha-

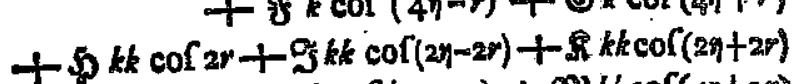
$+\{k k \operatorname{col}(4 \eta-2 r)+29 l k k \operatorname{cor}(4 \eta+2 r)$

$y=\mathrm{A} \operatorname{col} 2 \eta+a k k \operatorname{col} 2 \eta+\mathrm{B} \operatorname{cor} 4 \eta+b k k \operatorname{col} 4 y$ $+\mathrm{D} k \operatorname{cor}(2 \eta-r)+\mathrm{E} k \operatorname{col}(2 \eta+r)$ $+\mathrm{F} k \operatorname{cor}(4 y-r)+\mathrm{G} k \operatorname{col}(4 \eta+r)$ $+\mathrm{H} k k \operatorname{cof} 2 r+\mathrm{J} k k \operatorname{col}(2 \eta-2 r)+\mathrm{K} k k \operatorname{cof}(2 \eta+2 r)$ $+\mathrm{L} k k \operatorname{cor}(4 \eta-2 r)+\mathrm{M} k k \operatorname{cor}(4 \eta+3 r)$

K 2 S. 9l.

K 2 9. 
\$. 91. Nunc ad terminos, quibus ante valorem

valorem

per $k k$ ipfius $\frac{d \varphi}{d r}$ exprimi inuenimus, infisper fequentes per $k k$ multiplicati accedent :

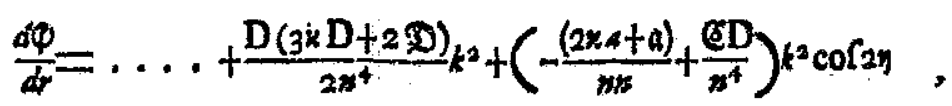
$\left(-\frac{(2 x b+b)}{m}+\frac{\mathrm{D}(3 x \mathrm{E}+2 \mathrm{E})}{2 n^{4}}+\frac{\mathrm{E}(3 x \mathrm{D}+2 D)}{2 n^{4}}\right) k^{2} \operatorname{cof} 1 n$

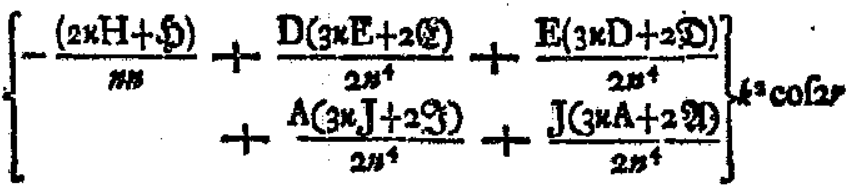
$\left(-\frac{(2 x J+9)}{m s}+\frac{\Lambda(3 \times H+2 \Phi)}{2 n^{4}}+\frac{H(3 x \Lambda+29)}{2 x^{4}}+\frac{(2 D}{n^{4}}\right) k^{2} \operatorname{col}(2 y-2 r)$ $\left(-\frac{(2 \times K+\beta)}{m}+\frac{A(3 x H+2 \xi)}{2 m^{4}}+\frac{H(3 x \Lambda+24)}{2 m^{2}}\right) k c(42 y+2 r)$ $\left(-\frac{(2 x L+2)}{x x}+\frac{A(3 x J+2 g)}{2 x^{4}}+\frac{J(3 x \Lambda+224)}{2 x^{4}}+\frac{D(3 x D+2 D)}{2 x^{4}}\right) x^{2} \mathrm{cl}\left(4 y^{2}-2 r\right)$ $-\frac{(2 \times M+m)}{2} k^{2} \cos (4 n+2 r)$

vbi quidem terminos, quos minimos fore freile eft praeuidere, omifimus.

5. 92. Terminus antem conftans $\frac{D(3 x D+29) k t}{2 x^{4}}$ seperitur $=0,000175$, vnde polito $x+0,000285$ $-\frac{1}{m}=\infty$, quonism valorem ipfius $\frac{d y}{d}$ non opus eft tam exgle noffe, fiomamus:

of $(2 y-2 r)$

$42 y+2 r)$

$[(4)-2 r)$

cile eft

29) kk

000285

ins eft

$$
\begin{aligned}
& \frac{d \eta}{d r}=\alpha-\frac{(2 \pi A+2 n)}{n n} \cos 2 n-\left(\frac{2 k}{n}+\frac{(5 k}{m}\right) \cos r \\
& -\frac{(2 x D+\mathfrak{D})}{m} k \operatorname{cor}(2 x-r)-\left(\frac{3 k k}{2 m}+\frac{(2 u H+\mathfrak{S})}{m}\right) \cos 2 \pi \\
& -\frac{(2 x)+9)}{y n} k^{2} \operatorname{cof}(2 y-2 r)
\end{aligned}
$$

Deinde vero praeter terminos iam traetatos habebitur:

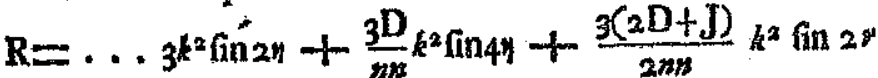
$+\left(\frac{x}{4} k^{2}+\frac{3(\mathrm{H}-\mathrm{L})}{2 n z}\right)$ (in $\left.(2 y)-2 r\right)+\left(\frac{3 \pi}{4} k^{2}+\frac{3 \mathrm{H}}{2 n n}\right) \operatorname{lin}(2 n+2 r)$

$$
+\frac{3(2 \mathrm{D}+J)}{2 m} k^{2} \text { fin }(4 y-2 r)
$$

atg̨ue fimili modo:

$\frac{d}{d r}$
$+\left(3+\frac{3}{2}(A+D+E)-a-2 x a\right) k k \operatorname{cor} 2 \eta$ $+\left(\frac{3}{2}(B+F+G)-6-2 \times b\right) k k \operatorname{col} 4$ 해

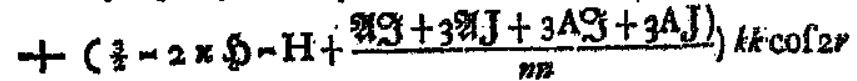
$+\left(\frac{x}{4}-2 x g-J+3 A+\frac{3}{2}\right.$ D) $k k \operatorname{cof}(2 n-2 r)$ $+\left(x \frac{x}{4}-2 x 3-\mathrm{K}+\frac{3}{4} \mathrm{~A}+\frac{3}{2} \mathrm{E}\right) k^{2} \cos (2 \pi+2 r)$ $+\left(-2 \times 2-\mathbf{L}+3 \mathbf{B}+\frac{3}{2} \mathbf{F}\right) k k \operatorname{col}(4 n-2 r)$ $+\left(-2 \pi m-M+\frac{3}{4} \dot{B}+\frac{3}{2} G\right) k^{2} \operatorname{col}(4 x+2 r)$ $+\frac{\left.249+39 \mathcal{A}+3 A J+\frac{1}{2} D D+3 D D+3 D D\right)}{m} k k \operatorname{cor}(4 \eta-2 r)$ 6. 93. Eliciamus' nunc quoque valoren ipfus $\mathrm{R}$ per differentiationem ex formula $\int \mathrm{R} d r$, ac terminis apte difpoficis habebimus

K 3

$\mathbf{R}=$

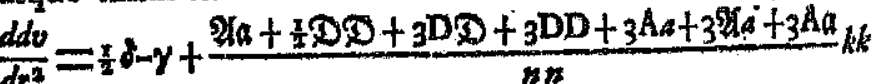




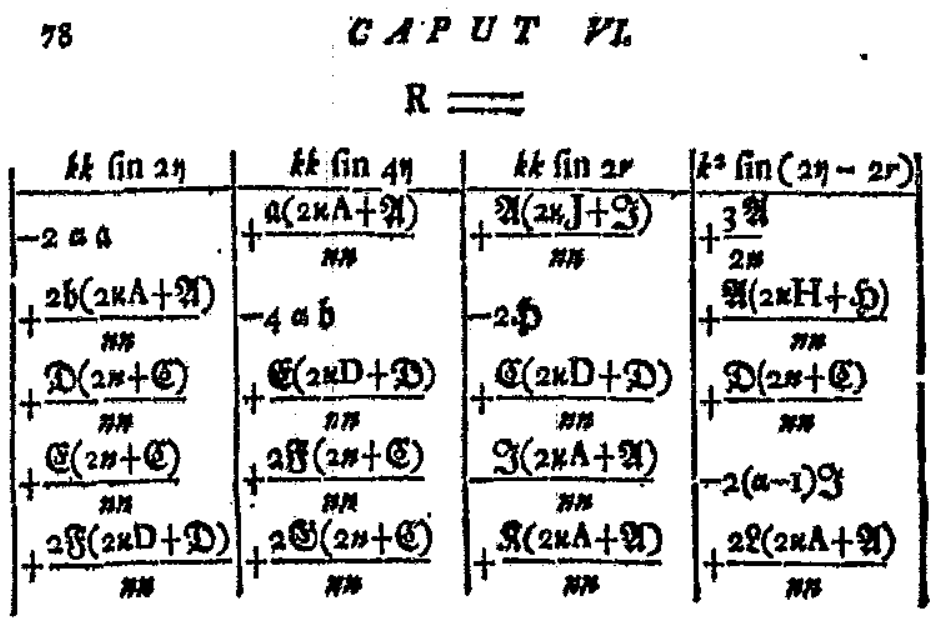

\section{$C A P U D$}

79

vade oriuntur fequentes determinationes:

$3=-2 w a+\frac{2 b(2 x A+D)+(D+C)(2 n+E)+2 \delta(2 w D+D)}{m}$

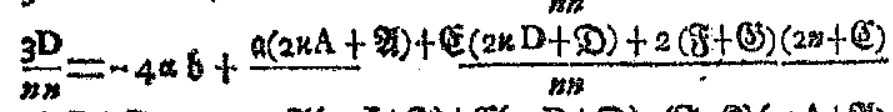

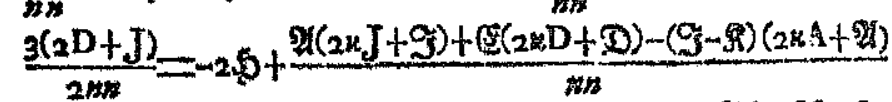

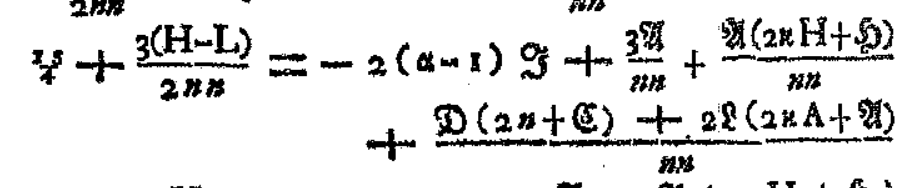
$z+\frac{3 H}{2 n n}=2(a+x) h+\frac{3 \mathfrak{H}}{2 n}+\frac{9(2 k \cdot \mathrm{H}+b)}{m n}$ $\pm 23(2 k J+3)+2 B(2 x D+D)+C(2 n+C)+2 M(2 x A+N)$ $+\frac{3(2 D+J)}{2 n n}=-2(2 x-1) x+\frac{393}{n}+\frac{\pi(2 u J+9)}{n}$

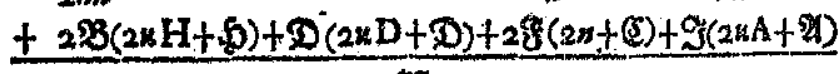
$0=-2(2 n+1) m+\frac{3 B}{m n}+2 B(2 x H+\mathfrak{D})+2 G(2 n+C)$ $+\$(2 n A+n)$

9. 94. Deinde fimili modo fi ponatur:

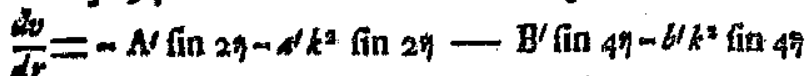
$-\mathrm{C}^{\prime} k$ fin $r-\mathrm{D}^{\prime} k$ fin $(2 y-r)-\mathrm{F}^{\prime} k$ in $(4 y-r)$ $-\mathrm{E}^{\prime} k \operatorname{fin}(2 y+r)-\mathrm{G}^{\prime} k$ fin $(2 y+r)$ $-H H^{2} \operatorname{fin} 2 r-J^{\prime k^{2}}$ fin $(2 \eta-2 r)-L / k^{2}$ (in $(4 \eta-2 r)$ $-K / k^{2} \mathrm{fin}(2 y+2 r)-M^{\prime} k^{2}$ fin $(4 y+2 r)$ 
80

$$
\operatorname{cov} \boldsymbol{P} \boldsymbol{T}
$$

erit pracher valores 5. 84 datos:

$d=2-2 b(2 x A+2)-(D+E)(2 x+2)-2 F(2 \times D+D))$

$H=4 \times b-\frac{(2 \times A+4)=E(2 \times D+D)}{n}$ $-2(F+G)(2 *+C)-D(2 x E+C)$ $n *$

$H^{\prime}=2 H-\frac{\Lambda(2 \times J+9)-E(2 \times D+D)}{N}$ $\frac{ \pm(I-K)(2 \times A+2 A)+D(2 \times E+E)}{n}$

$J^{\prime}=2(\alpha-1) J-\frac{3 A}{2}-\frac{A(2 \times H+\Phi)-D(2 x+B)-2 L(2 x A+2)}{N}$

$K^{\prime}=2(u+1) \mathrm{K}-\frac{3 \mathrm{~A}}{2 *}-\frac{\mathrm{A}(2 \times \mathrm{H}+\Phi)-2 \mathrm{~B}(2 \times]+0)-2 \mathrm{G}(2 \times \mathrm{D}+D)}{n}$

$$
-\frac{E(2 n+C)-2 M(2 n A+2 \pi)}{n}
$$

$\mathrm{L} /=2(2 x-1) \mathrm{L}-\frac{3 \mathrm{~B}}{\mathrm{~A}}-\frac{\mathrm{A}(2 \times \mathrm{J}+9)-2 \mathrm{~B}(2 \times \mathrm{H}+\mathrm{D})-\mathrm{D}(2 \times \mathrm{D}+\mathrm{D})}{n}$

$$
-\frac{2 \mathrm{~F}(2 x+\mathrm{C})-\mathrm{J}(2 x A+2)}{n}
$$

$M^{\prime}=2(2 x+1) M-\frac{3 B}{n}-\frac{2 B(2 x H+5)-G(2 x+6)-K(2 x A+2)}{x}$

vbi quidem plures terminos, quos admodum paruos fore praeuidimus, omifimus.
$C A P U T$

$8 \mathrm{r}$

\begin{tabular}{|c|c|c|c|}
\hline$k k$ & $k k \cos 2 \eta$ & $k k \operatorname{col} 4$ & $k k \operatorname{col} 2$, \\
\hline$A^{\prime}(2 \pi \mathrm{A}+4)$ & $7 \%$ & $A^{\prime}(2 \mu \mathrm{A}+9)$ & $A^{\prime}(2 x J+9)$ \\
\hline$D^{\prime}(2 \times D+D)$ & $2 b(2 k A+m)$ & & $\begin{array}{c}n n \\
E /(2 n \mathrm{D}+5)\end{array}$ \\
\hline$n \pi$ & $n n$ & & $n n$ \\
\hline & $D^{\prime}(2 n+C)$ & $\mathrm{D}^{\prime}(2 x \mathrm{D}+0)$ & $2 H^{\prime \prime}$ \\
\hline & $\mathrm{D}\left(2^{n}+6\right)$ & ${ }_{2} F^{n n}(2 n+(C)$ & $J^{\prime}(2 x A+\mathscr{A})$ \\
\hline & 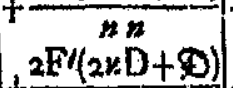 & $2 \mathrm{G}^{n n}(2 n+(\mathrm{C})$ & $\frac{n n}{K^{\prime}(2 n A+9)}$ \\
\hline & $\# *$ & $n n$ & $9 n$ \\
\hline$k k \operatorname{cor}(2 \eta-2 r)$ & $k k \operatorname{col}(2 \eta+2 r)$ & $\operatorname{cor}(4 \eta-2 r)$ & $\mid k k \operatorname{cor}(4 y)+2 r)$ \\
\hline $3 A^{\prime}$ & $+3 A^{\prime}$ & $A^{\prime}(2 u)+S$ & \\
\hline$A^{2}(2 \times H+B)$ & $\mathrm{A}^{2 \pi}(2 \mathrm{KH}+\mathrm{W})$ & $3 B^{\prime \prime \prime}$ & $3 \mathrm{~B} /$ \\
\hline$D^{n n}(2 n+C)$ & ${ }_{2} \mathrm{G}^{n n}(2 \times \mathrm{D}+9)$ & $2 \mathrm{~B}^{\prime \prime}(2 \mathrm{H}+5)$ & $\int^{+} \frac{n}{2 \mathrm{~B}^{\prime}(2 n \mathrm{H} \mathrm{H}+5)}$ \\
\hline$\left[\begin{array}{c}\pi * \\
-2(\alpha-1)\end{array}\right.$ & $\mid \begin{array}{c}\pi x \\
-2(a+1) k\end{array}$ & $\mathrm{D}(2 \times \mathrm{D}+\mathrm{D})$ & $\begin{array}{c}m n \\
2 G^{\prime}(2 n+6)\end{array}$ \\
\hline $2 L(2 \times A+5)$ & $2 M^{\prime}(2 x A+5 i)$ & $2{ }^{m}(2 m+c)$ & $n n$ \\
\hline$n n$ & $7 n$ & $n *$ & $2(2 a+x) M$ \\
\hline & & $J^{\prime}(2 x A+F)$ & \\
\hline & & $\mathrm{I}$ & \\
\hline
\end{tabular}

\$. 95. Hinc auten denuo differentiando obtinemus

valorem ipfius $\frac{d d v}{d r^{3}}$

ङ. 95.

5. 95.
L

vndè 
vnde tandem gancifcimur has determinationes:

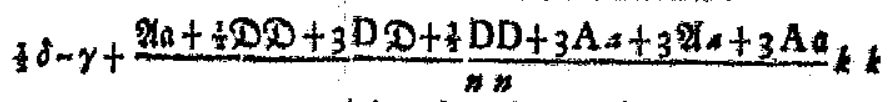

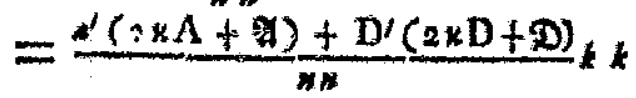

$-2 \times D n-M+3 B+\frac{3}{2} G=-2(2 \alpha+1) M^{\prime}+\frac{3 B^{\prime}}{n}+\frac{2 B^{\prime}(2 u H+S)}{n n}$

$$
+\frac{2 \mathrm{G}^{\prime}(2 n+\mathrm{C})+\mathrm{K}^{\prime}(2 n \mathrm{~A}+9)}{n n}
$$

\$. 96. Primum autem valoribus iam cognitis fub$3+4(A+D+E)-A-2 x A=-2 * \alpha^{\prime}+\frac{2 b^{\prime}(2 x \Lambda+A)}{*}$

$+(B+F+Q)-b-2 \times b=-4 a y+\frac{d(2 \times A+Q)}{n n}$ Atituendis, reperitur :

$2 a_{4}=-3,837-0,038 b$ et $4 a b=-x_{0}, 073-0,019 a$ hineque $a=-2,051$ et $b=-0,277$

ex quibus porro elicimus : $a=-12,595$ et $b=m 0,086$ et $A^{\prime}=-23,510$. Deinde pro reliquis litteris

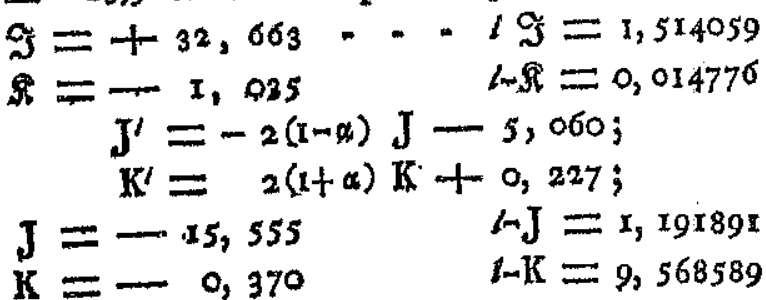

Porro $q=-1,453$. . $\operatorname{lm} q=0,162070$

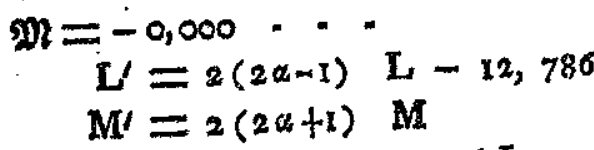

Denique $\mathfrak{S}=-0, x_{23}$ et $H=-i, 033$ atque $\frac{x}{2} \delta-\gamma=-7,459 \mathrm{kk}$

6. $9 \%$ His igitur valoribus inuentis innotefcet primum diftantia Lunae a terra curtata, quatenus a fola 
excentriaiste orbitae lunaris $k$ pendet. Cum enim

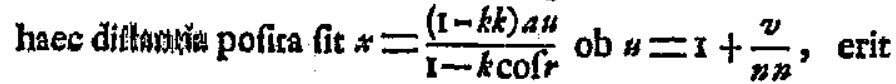

$y=1-0,0716 x \operatorname{cor} 2 \eta-0,0719 k \operatorname{cof} 2 \eta$ H* $000073 \operatorname{cof}_{4} \eta-0,0005 k k \operatorname{cof} 4 \eta$ +t. $194888 k \operatorname{cof}(2 y-r)-0,003274 k \operatorname{cof}(2 y+r)$ $-w_{j} 003238 k \operatorname{cof}(4 \eta-r)+0,000078 k \operatorname{cor}(4 \eta+r)$ $-\infty, 0059 k \operatorname{cof} 2 r$

$-m, \psi_{1} 0889 k k \operatorname{cor}(2 \eta-2 r)-0,002 x k k \operatorname{cor}(2 \eta+2 r)$ $+0,0357 k k$ cof $(4 \eta-2 r)$

At pro lamgittudine Lunae, quatenus a fola excentricitate $k$ pendat, prodibit $\frac{d \varphi}{d r}$

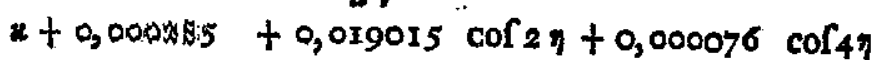
$+0,1562 k k \operatorname{cor} 2 \eta+0,0008 k k \operatorname{cor} 4 \eta$

$m 0,00125, k$ kicof $=0,38410 k \operatorname{cof}(2 y-r)+0,002647 k$ ef $(4 y-r)$ $+0,01278 k \operatorname{col}(2 y+r)-0,000229 k c(4 y+r)$

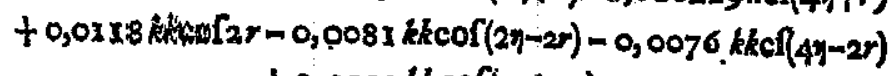
$+0,0102 k k \operatorname{cof}(2 \eta+2 r)$

\$. 9tir Ponatur nunc:

$\varphi=0$ rt 2 fin $2 \eta+a^{\prime} k k$ fin $2 \eta+B^{\prime} / \operatorname{fin} 4 \eta+b / k k$ fin $4 \eta$

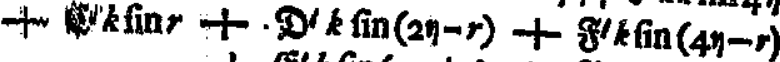

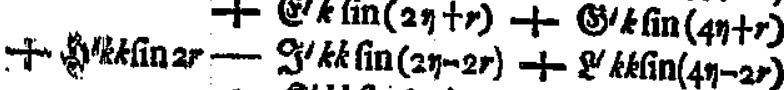

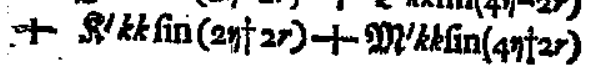

atque differentiando orientur fequentes comparationes

$x+0,000285=0-\frac{a^{\prime}(2 n A+9)-D^{\prime}(2 a D+D)}{n z} k k+0,000190$

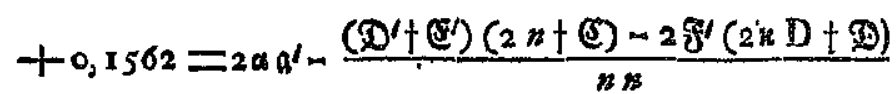

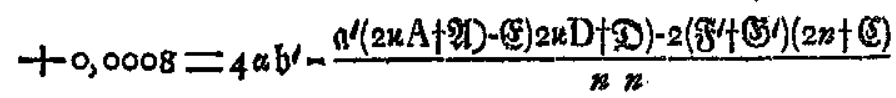

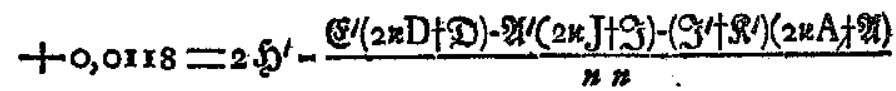

$-0,008 x=2(a-1) \mathfrak{S}^{\prime}-\frac{3 \mathscr{A}}{2}-\frac{\mathfrak{D}^{\prime}(2 n+\mathbb{C})-\mathfrak{W}^{\prime \prime}(2 n \mathrm{H}+\mathfrak{S})}{n}$

$-\frac{2 S^{\prime}(2 x+A+S)}{3}$

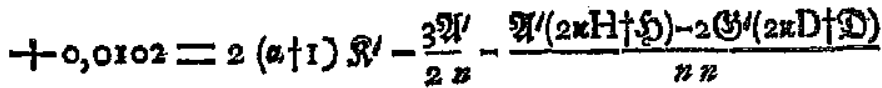

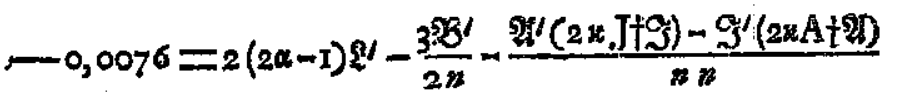

$$
-\frac{D^{\prime}(2 k D+D)-2 g(2 n+D)}{n}
$$

$=2(2 \alpha+1) \operatorname{mp}-\frac{3 \mathfrak{B}^{\prime}}{n}-\frac{2 \Theta f(2 n+\mathcal{E})}{n n}$

5. 99. Ex his comparationibus elicimus:

$$
\begin{array}{ll}
\mathfrak{a}^{\prime}=+0,0509 ; & W^{\prime}=0,0008 \\
\mathfrak{S}^{\prime}=+0,5385 ; & W^{\prime}=0,0028 \\
\mathfrak{W}^{\prime}=-0,1055 ; & \mathbb{W}^{\prime}=0,0000 \\
\mathfrak{W}^{\prime}=+0,0021 ; & \text { et } 0=k=0,000429
\end{array}
$$

L 3 
pofito $k=0,05445$. Hinc autem erit $\frac{x}{2} \delta-\gamma=-0,02302$ Cum autem iam ante inuentum effet $\frac{x}{2} \delta-\gamma=-0$, or 742 erit reuera $\frac{x}{2} \delta-\gamma=-0,00560$. Tum vero inueniemus: $y=1,40673$, vnde erit $\frac{x}{2} \delta=1,401_{13}$, et $\delta=2,80226$ hincque $2 \gamma-\frac{3}{2} \delta=-1,38993$ et $\frac{2 \gamma-\frac{3}{2} \delta}{n n}=-0,00794$. 0,01742

Verum ex cognita ratione motus medii ad motum anomaliae eft $\boldsymbol{O}=I, 008527^{2}$, vnde $x=I, 0089562$ Verum efle debet $x=\mathrm{I}+\frac{3+4 \mu \pm \delta}{4 n n}$; vnde foret $0,0089562=0,008289+\frac{\mu}{n n} ;$ ideoque $\frac{\mu}{n n}=0,000667$ quĩ valor cum fit tam exiguus, merito dubitamus, num $\mu$ non prorfus fit $\rightleftharpoons$.

, 00794 .

im ano-

2089562

foret

000667

sitamus,

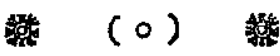

\section{CAPUT VII.}

CORRECTIO INAEQUALITATUM LUNAE, ANTE INUENTARUNE

\$. 100.

uoniam nunc quidem valores litterarum $y$ et $\delta$ Q. ita inuenimus, vt eos pro proxime veris habere queamus, ex is coefficientes terminorum, quibus inaequalitates lunae continentur, accuratius definire poterimus. Cum enim fit $\gamma=1,40573$ et $\delta=2,80226$, colligamus hic in vnum omnes formulas, quas hactenus pro inueniendis coefficientibus affumtis elicuimus. Pofueramus autem :

$\int R d r=2 \mathbb{A} \operatorname{cor} 2 y+a k k \operatorname{cof} 2 \eta+3 \operatorname{cor} 4 \eta+b k k \operatorname{cor} 4 \eta$ $+\mathbb{C} k \operatorname{col} r+\mathfrak{D} k \operatorname{cor}(2 \pi-r)+\mathfrak{f} k \operatorname{col}(4 y-r)$

+E $k \operatorname{cor}(2 \eta+r)+, \Leftrightarrow k \operatorname{cor}(4 \eta+r)$

$+\mathfrak{b} k k \operatorname{col} 2 r+\mathfrak{\$} k^{2} \operatorname{col}(2 \eta-2 r)+2 k^{2} \operatorname{cof}(4 \eta-2 r)$

$+\Re k^{2} \operatorname{cor}(2 \eta+2 r)+2 m k^{2} \operatorname{cor}(4 \eta+2 r)$

$v=\mathrm{A} \operatorname{cor} 2 \eta+a k k \operatorname{col} 2 \eta+\mathrm{B} \operatorname{col} 4 \eta+b k k \operatorname{cor} 4 \eta$

- $\quad+\mathrm{D} k \operatorname{col}(2 \eta-r)+\mathrm{F} k \operatorname{cor}(4 y-r)$

$+\mathrm{E} k \operatorname{cor}(2 \eta+r)+\mathrm{G} k \operatorname{cor}(4 \eta+r)$

o+ $\mathrm{H} k k \operatorname{cof} 2 r+\mathrm{J} k k \operatorname{cof}(2 \eta-2 r)+\mathrm{L}, k k \operatorname{cor}(4 \eta-2 r)$

$+\mathrm{K} k k \operatorname{cor}(2 y+2 r)+\mathrm{M} k k \operatorname{cor}(4 y+2 r)$

6. 1010 
§. 1or. Hine polito $x=V\left(x+\frac{3+4 \dot{\mu}+\delta}{2 m s}\right)$ collegimus fore

$$
\begin{aligned}
& \frac{d \Phi}{d r}=x+\frac{\mathrm{A}\left(3^{x} \mathrm{~A}+22 \mathrm{2}\right)}{2 n^{4}}+\frac{\mathrm{D}(3 x \mathrm{D}+2 D)}{2 n^{4}} k k+\frac{\mathrm{A}(3 u x+a)}{2 n^{4}} k k \\
& +\frac{a(3 x \mathrm{~A}+2 \mathfrak{2})}{2 n^{4}} k k-\frac{(2 x \mathrm{~A}+2 \mathrm{~A})}{m n} \operatorname{cor} 2 y-\frac{(2 \times \mathrm{B}+2)}{m} \operatorname{cof} 4 n \\
& +\frac{A(3 k A+2 \pi)}{2 n^{4}} \operatorname{cor} 4 \eta-\frac{\mathfrak{C}}{m n} k \operatorname{cor} r-\frac{(2 m a+a)}{m n} k^{2} \operatorname{cor} 2 \eta \\
& -\frac{(2 x b+b)}{m m} k^{3} \cos 4 y+\frac{\mathrm{D}(3 x \mathrm{~A}+2 \mathrm{2} 2)+\mathrm{A}(3 x \mathrm{D}+2 D)}{2 n^{4}} k \operatorname{cof} \\
& -\frac{(2 x \mathrm{D}+\mathcal{D})}{n n} k \operatorname{cor}(2 \eta-r)-\frac{(2 x \mathrm{~F}+\mathscr{P})}{m z} k \cos (4 \eta-r) \\
& +\frac{\mathrm{D}(3 x \mathrm{~A}+2 \Re)+\mathrm{A}(3 x \mathrm{D}+2 D)}{2 n^{4}} k \operatorname{cor}(4 \eta-r) \\
& \text { - }-\frac{(2 x \mathrm{E}+(\vec{F}))}{m} k \operatorname{cor}(2 n+r)-\frac{(2 \times \mathrm{G}+(\mathbb{H})}{m n} k \operatorname{cor}(4 n+r) \\
& -\frac{(2 x J+g)}{m m} k k \operatorname{cor}(2 \eta-2 v)-\frac{(2 x \mathrm{H}+5)}{m x} k k \operatorname{cor} 2 r \\
& +\frac{\mathrm{J}(3 * \mathrm{~A}+2 \mathrm{~A})+\mathrm{A}(3 \times \mathrm{J}+2 \Im)}{2 x^{4}} k^{2} \operatorname{col} 2 r \\
& -\frac{(2 x \mathrm{~K}+\Omega)}{m n} k k \operatorname{cor}(2 \eta+2 r)-\frac{(2 x \mathrm{~L}+\xi)}{n n} k^{2} \operatorname{cor}(4 r-2 r) \\
& -\frac{\mathrm{D}(3 x \mathrm{D}+2 \mathrm{D})+\mathrm{J}(3 x \mathrm{~A}+22 \mathrm{x})+\mathrm{A}\left(3 x \mathrm{~J}+2 \mathrm{~J}^{2}\right)}{2 n^{4}} k^{2} \operatorname{cof}(4 y-2 r) \\
& -\frac{(2 \times M+\mathfrak{M})}{k^{2}} \operatorname{cor}(4 \eta+2 r)
\end{aligned}
$$

5. 102. Si iam ponamus

$$
\begin{aligned}
& z+\frac{A(3 x A+2 A)}{2 n^{4}}+\frac{D(3 x D+2 D)}{2 n^{4}} k k \\
& +\frac{\mathrm{A}(3 x a+2 \mathfrak{a})}{2 m^{4}} k k+\frac{a(3 x \mathrm{~A}+2 \mathbb{2})}{23^{4}} k k \\
& -\frac{1}{n}=\alpha ; \text { vt fit negleetis terninis admodum exiguis } \\
& \frac{d \eta}{d r}=a-\frac{(2 x \mathrm{~A}+\mathfrak{Q})}{n \pi} \operatorname{cor} 2 \eta-\frac{(2 n+\mathfrak{C})}{n z} k \operatorname{cor} \Rightarrow \\
& -\frac{(2 x \mathrm{D}+D)}{m n} k \operatorname{cor}(2 y-r)-\frac{(2 x \mathrm{E}+\mathbb{E})}{m z s} k^{2} \operatorname{cor}(2 y+r)
\end{aligned}
$$

ex fuperioribus capitibus repetimus has determinationes:

$$
2 \alpha \mathfrak{Q}=-\frac{3}{2}
$$

${ }_{4} \alpha \mathfrak{B}=-\frac{3 \mathrm{~A}}{2 m n}+\frac{\mathfrak{2}\left(3^{\alpha} \mathrm{A}+2\right)}{m m}$

$\mathbb{C}=\frac{2(2 n \mathrm{D}+\mathrm{D})}{m n}-\frac{(\mathrm{D}-\mathrm{C})(2 n \mathrm{~A}+2)}{n n}-\frac{2(2 n \mathrm{E}+\mathrm{E})}{n m}-\frac{3(\mathrm{D}-\mathrm{E})}{2738}$

$(2 \alpha-1) D=-3+\frac{(2 n+\mathbb{E})}{n B}$

$(2 \alpha+1) E=-3+\frac{(2 n+b)}{n} \pi$

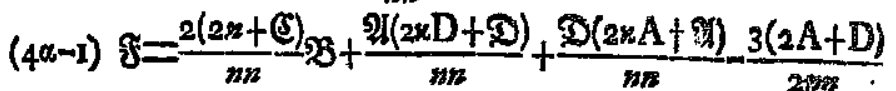

$(4 \alpha+1) \quad B=\frac{2(2 n+C)}{m}-B+\frac{\pi(2 x E+C)}{m}+C(2 n A+2)-\frac{3(2 A+E)}{2 n}$

$2 \alpha \mathfrak{a}=-\frac{x}{4}+\frac{(D+C)(2 n+C)}{m}+\frac{2 b(2 x A+2)}{n m}$

$$
+\frac{2 q(2 \times 1)+D)}{M}+\frac{D(2 m E+q)}{n}
$$

§. I02. 


$$
\begin{aligned}
& 4 a b=-\frac{3 D}{n m}+\frac{2(g+B)(2 n+C)}{n n}+\frac{E(2 x D+D)}{n n} \\
& +\frac{a(2 \times A+\mathscr{A})}{m}+\frac{\mathfrak{D}(2 \times E+\mathbb{E})}{m m}
\end{aligned}
$$

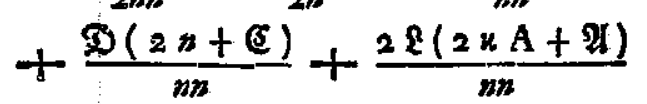

$2(a+1) \mathfrak{A}=-\frac{\pi 5}{4}-\frac{3 \mathrm{H}}{2 n n}+\frac{3 \pi}{2 n}+\frac{2(2 n}{n+\mathfrak{H}+\mathfrak{S})}$

$$
+\frac{E(2 n+C)}{m}+\frac{2 \Theta(2 n \mathrm{D}+\mathfrak{D})}{m}
$$

$2(2 a-1) \&=-\frac{3(2 \mathrm{D}+\mathrm{J})}{2 m}+\frac{3 \mathfrak{B}}{n}+\frac{2 \mathfrak{B}(2 n \mathrm{H}+\mathfrak{G})}{m n}+\frac{2 \mathfrak{f}(2 n+\mathrm{C})}{m}$

$$
+\frac{\mathfrak{N ( 2 x J}+\mathfrak{D})}{m}+\frac{\mathfrak{D}(2 x \mathrm{D}+\mathfrak{D})}{m}+\frac{\mathfrak{I}(2 x \mathrm{~A}+\mathfrak{A})}{m}
$$

$2(2 a+1) M=\ldots \frac{3 \mathfrak{B}}{n}+\frac{2 \mathfrak{B}(2 x \mathrm{H}+\mathfrak{W})}{n n}+\frac{2(3(2 n+())}{n n}+\frac{\Re(2 x \mathrm{~A}+\mathscr{N})}{m}$

6. 103. Antequam vlterius progrediamur, fequentes notandae funt nouse denominationes

$$
\begin{aligned}
& A=2 \alpha A+\frac{A(2 n B+B)}{n n}-\frac{B(2 x A+\pi)}{n n} \\
& B^{\prime}=4 B-\frac{A(2 \times A+m)}{m} \\
& C=\mathbb{A}(D-E)-A(D-E) \\
& D^{\prime}=(2 \pi-I) D-\frac{(2 n+C)}{A}
\end{aligned}
$$

$C A P U T V I U_{0}$

$$
\mathbf{E}^{\prime}=(2 a+1) \mathbf{E}-\frac{(2 x+\mathfrak{C})}{m z} \mathbf{A}
$$$$
F^{\prime}=(4 \alpha-1) F-\frac{4 B}{A}-A(2 u D+D)-D(2 u A+\infty)
$$$$
\mathbf{G}^{\prime}=(4 \alpha+1) \mathbf{G}-\frac{4 \mathrm{~B}}{n}-\frac{\mathrm{A}(2 x \mathrm{E}+\mathrm{E})-\mathrm{E}(2 \mathrm{~B}+2 \pi)}{m}
$$

$2 x \mathrm{H}+\mathfrak{S P}$

$n n$

: $u A+(2)$

$n n$

$\underline{\underline{k}+\underline{H}+\mathfrak{S})}$

$n n$

$2 x \mathrm{D}+D)$

$2 \approx(2 n+())$

$m n$

$\underline{(2 x A+2)}$

$\mathbb{S ( 2 x A + 2 )}$

$m n$

is fequen-

$+($ (i)

$a^{\prime}=2 \alpha A-\frac{2 b(2 n A+M)}{n n}-\frac{(D+E)(2 n+\mathbb{C})}{n n}$ $-\frac{2 F(2 \times D+D)}{m}+\frac{D(2 x}{m}$ $b=4 \alpha b-\frac{s(2 n A+2)}{m n}-\frac{A(2 n a+a)}{m}-\frac{D(2 n E+C)}{m m}$ $-\frac{E(2 \times D+D)}{m}-\frac{2(F+G)(2 n+C)}{m}$

$H^{\prime}=2 H+\frac{D E-D E-A(\mathfrak{S}-\mathfrak{R})+\mathscr{A}(\mathrm{J}-\mathrm{K})}{(2)}$

$J^{\prime}=2 \cdot(a-i) J+\frac{3 A}{2 n}-\frac{A(2 n H+\mathfrak{W})}{m n}-\frac{D(2 n+C)}{n n}$ $-\frac{2 q(2 x A+2)}{n x}+\frac{A(2 x L+q)}{n z}$

$\mathrm{K}^{\prime}=2(\alpha+1) \mathrm{K}-\frac{3 \mathrm{~A}}{n}-\frac{\mathrm{A}(2 n \mathrm{H}+\mathfrak{W})}{m}-\frac{\mathrm{E}(2 n+\mathrm{C})}{m}-\frac{2 \mathrm{G}(2 x \mathrm{D}+\mathrm{D})}{m}$ $L^{\prime}=(2 a-1) L-\frac{3 B}{n}-\frac{2 B(2 x H+5)}{m n}-\frac{2 E(2 n+C)}{n B}$

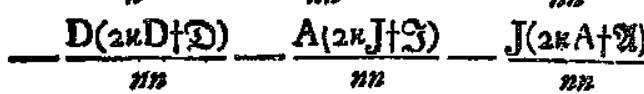
$M^{\prime}=2(2 \alpha+1) M-\frac{3 B}{n}-\frac{2 B(2 n H+5)}{m m}-\frac{2 G(2 n+C)}{n m}-\frac{E(2 n E+G)}{m m}$ - §. 104. Nune vt terminos completos obtineamus, faltem eos qui angulos $2 \eta$ et $r$ inuoluunt, nutandum 
eft in noftris aequationibus fin $2 \eta$ et $\operatorname{col} 2 \eta$ non per $\frac{3}{2}(1+2 k k)$ fed per $\frac{3}{2}\left(1+2 k k+\frac{3}{2} e e\right)$ effe multiplicatos. Hinc cum fit fere $\frac{f}{4} e t=\frac{x}{4} k k$, loco $k k$ hic fcribi oportebit $k k k$, vnde in valore ipfius a pro 3 fcripfi $3 . \frac{5}{4}$ feu $x_{4}^{5}$. Deinde $v e$ in his terminis quoque rationem habeamus inclinationis orbitae, cuius medius valor fit $=\varepsilon$, ponamus $\frac{3}{4}(n z+2+3 \mu+\gamma)$ tang $\varepsilon^{2}=f=$ $\frac{3}{4}\left(\frac{3}{3} x * n n-\frac{x}{2} n n-\frac{x}{4}+\gamma-\frac{3}{4} \delta\right)$ tang $s^{2}$ ob $\mu=$ $\frac{x}{2}(x, x-1) n n-\frac{3}{4}-\frac{1}{4} \delta$, erisque noftra aequatio:

$\frac{d d v}{d r^{2}}=\frac{x}{2} \delta-\gamma+\frac{x}{4} k k-\gamma k \operatorname{cof} r+\frac{3}{2} k k \operatorname{co} 2 r+\frac{3}{2} \operatorname{co}\left\{2 y+\frac{x}{4} k k \operatorname{co} \int 2 \eta\right.$ $+f+\frac{x}{2} f k k+f k \operatorname{co}\left[r+\frac{x}{2} f k k \operatorname{cof} 2 r\right.$

$+3 k \mathrm{cf}(2 \eta-r)+3 k \mathrm{c}(2 \eta+r)+\frac{\pi}{4} k k \mathrm{c}\left\{(2 \eta-2 r)+\frac{r}{4} k k \mathrm{cf}(2 \eta \dagger 2 r)\right.$ $m 2 k / R d r-v\left(1-\frac{3}{2} k k+\frac{2 f}{m m}+\frac{f k k}{m m}-\frac{\left(2 \gamma-\frac{3}{2} \delta\right)}{m n}\right)$ $4 v\left(3 x x+\frac{3}{2 m n}-\frac{2 f}{m m}+\frac{\left(2 \gamma-\frac{3}{2} d\right)}{m}\right) k \operatorname{cor} r$ $+v\left(\frac{3}{2}-\frac{f}{n+s}\right) k^{2} \operatorname{cor} 2 r$ $+v\left(\frac{3}{2 m x} \operatorname{col}\left(2 y+\frac{3 k}{m} \operatorname{cof}(2 y-r)+\frac{3 k}{m m} \operatorname{col}(2 y+r)\right)\right.$ $+\frac{1}{n n}\left(\int \mathrm{R} d r\right)^{2}+\frac{6 v}{n x} / \mathrm{R} d r+\frac{3 v v}{n n}-\frac{3 v v}{n n} k \operatorname{col} r$

5. 105. Sit breuitatis gratia:

$1+\frac{8 f}{z n}-\frac{\left(2 \gamma-\frac{3}{2} \delta\right)}{n z}=g ; \quad 3 x x+\frac{3}{2 n n}-\frac{2 f}{n n}+\frac{(2 y-3 d \theta)}{n n}=6$ ct $1-\frac{3}{3} k k+\frac{2 f}{n n}+\frac{f k k}{n n}-\frac{\left(2 \gamma-\frac{3}{2} d\right)}{n n}=6$, quo termino

CAPUT RIS.

non per

iplicatos.

bi opor-

$3 \cdot \frac{5}{4}$ feu

a habea.

it $=\varepsilon$,

$f=$

$\mu=$

0 :

$: k \operatorname{co}[2 y$

(2if $2 r)$

$\left.\frac{\left.-\frac{3}{2} d\right)}{3}\right)$

) $k \operatorname{cor} r$

$(n+r))$

ikcor

$2=6$

, quo

rmino termino in angulis ex $2 \%$ et $F$ compotitis vtenut: eritgue $\frac{d d w}{d r^{2}}=$

$\frac{z}{2} 8-\gamma+\frac{x}{4} k k+f+\frac{x}{2} f k k+\frac{3 A}{4 m m}+\frac{3 k k k}{4328}$ $+\frac{3 \mathrm{D} k k}{2 n n}+\frac{3 \mathrm{E} k k}{2 n n}+\frac{2 n}{2 m n}+\frac{G C k k}{2 n n}+\frac{D D k k}{2 n n}+\frac{\text { GEkk }}{2 n n} \frac{3 \mathrm{AD} k}{2 n z}$

$+\frac{3 A x}{n n}+\frac{3 \mathrm{DD} k k}{n k}+\frac{3 \mathrm{AA}}{2 n n}+\frac{3 \mathrm{DD} k k}{2 n n}+\frac{3 \mathrm{EEkk}}{2 n k}$

$+\cos 2 \eta\left(\frac{3}{2}-2 \pi\right)(-6 A)$

$+k k \operatorname{co} 2 \eta\left\{\frac{\pi s}{4}-2 x a-6 a+\frac{\pi}{2} b D+\frac{x}{2} 6 \mathrm{E}+\frac{C D}{n n}+\frac{3 C E}{8 k}\right.$

$+\cos 4 x\left\{-2 \times B-6 \mathrm{~B}+\frac{3 \mathrm{~A}}{4 m n}+\frac{2 \pi}{2 n n}+\frac{3 \mathrm{~A} A}{n n}+\frac{3 \mathrm{AA}}{288 B}\right.$

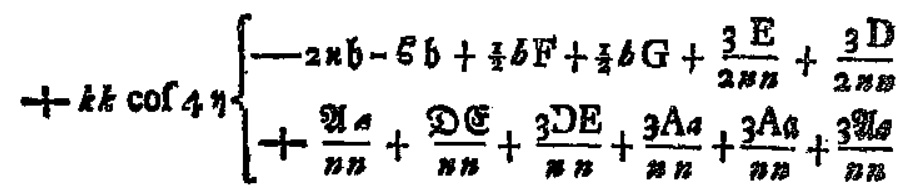

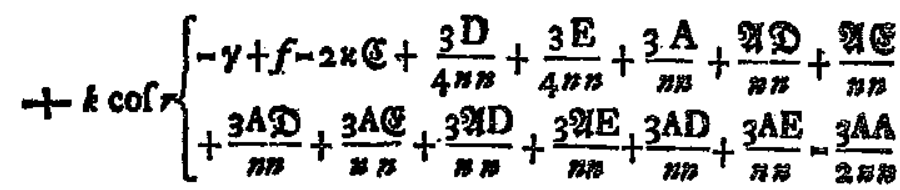

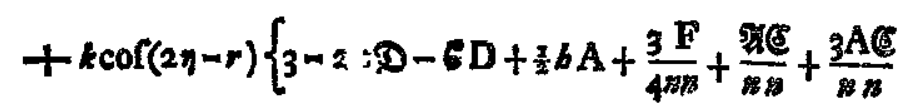

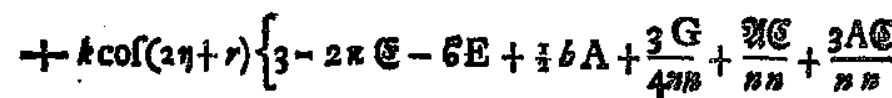

$\mathrm{M}_{3}$ 
lix

$-\frac{3 D}{2 n}$

I.

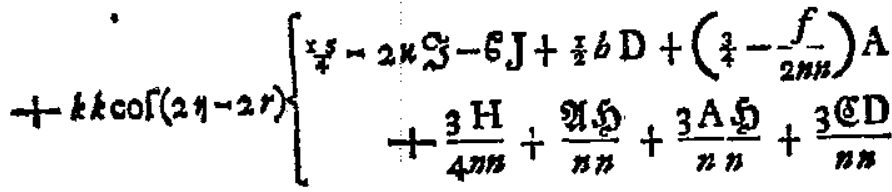

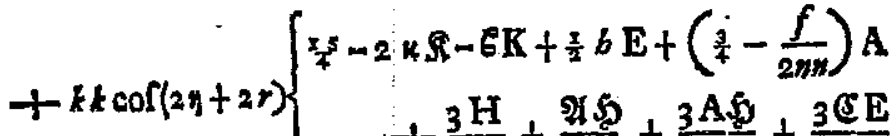
$+\frac{3 \mathrm{H}}{4 m}+\frac{\mathfrak{A H E}}{n z}+\frac{3 \mathrm{~A}}{n n}+\frac{3 \mathfrak{C E}}{n n}$

$+k k \operatorname{cof}(4 n-2 r)\left\{\begin{array}{l}-2 x \&-6 \mathrm{~L}+\left(\frac{3}{4}-\frac{f}{2 m n}\right) \mathrm{B} \\ +\frac{3 \mathrm{~J}}{4 m n}+\frac{3 \mathrm{D}}{2 m}+\frac{D D}{2 n n}+\frac{3 \mathrm{DD}}{2 m}+\frac{x}{2} 6 \mathrm{~F}\end{array}\right.$

$+k k \operatorname{col}(\eta 4+2 r)\left\{\begin{array}{l}-2 x \mathrm{M}-6 \mathrm{M}+\left(\frac{3}{4}-\frac{f}{2 m n}\right) \mathrm{B} \\ +\frac{3 \mathrm{~K}}{4 m}+\frac{3 \mathrm{E}}{2 m}+\frac{\mathrm{EC}}{2 m}+\frac{3 \mathrm{EE}}{2 n n}+\frac{1}{2} b \mathrm{G}\end{array}\right.$

$+k \operatorname{cor}(4 n-r)\left\{\begin{array}{l}-2 \times f-6 \mathrm{~F}+\frac{i}{2} k \mathrm{~B}+\frac{3 \mathrm{D}}{4 m}+\frac{3 \mathrm{~A}}{2 n n} \\ +\frac{29 D}{n n}+\frac{3 \mathrm{AD}}{n n}+\frac{32 \mathrm{D}}{n n}+\frac{3 \mathrm{AD}}{n n}-\frac{3 \mathrm{AA}}{4 n n}\end{array}\right.$

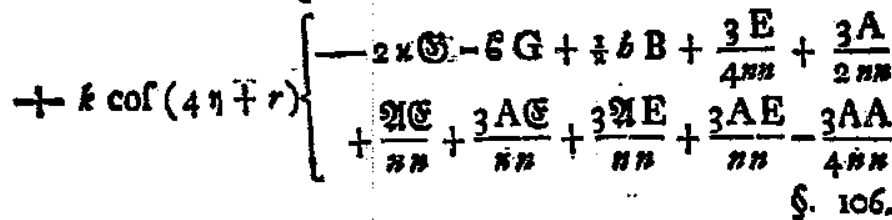
litates.

6. 106. Hinc denique nafcentur fequentes aequa.

I. $\quad \frac{x}{2} \delta-y+\frac{x}{4} k k+\frac{x}{x} f k k+\frac{3 A}{4 n n}+\frac{292}{2 n n}+\frac{3 A}{n z}+\frac{3 \cdot A A}{2 \pi n}$ $+\frac{3(\mathrm{D}+\mathrm{E})}{n n} k k-\frac{3 \mathrm{AD} k k}{2 n n}+\frac{(\mathrm{ECkk}}{2 n n}+\frac{(\mathrm{DD}+\mathbb{E})}{2 n k} k$ $+\frac{3 \mathrm{DD}}{n z} k k+\frac{3(\mathrm{DD}+\mathrm{EE})}{2 n n} k+\frac{9}{n} k k$ $+\frac{3 \mathrm{~A} a}{n n} k k+\frac{324 a}{n n} k k+\frac{3 \mathrm{Aa}}{n n} k k=\frac{\mathrm{A}^{\prime}(2 n \mathrm{~A}+9)}{n n}+\frac{a^{\prime}(2 n \mathrm{~A}+\mathrm{M})}{n n} k k$ $+\frac{D^{\prime}(2 u D+D)}{n n} k k+\frac{A^{\prime}(2 u a+a)}{n k} k k$

-11. $\quad 3-2 x \Re-6 A=-2 \alpha A^{\prime}+\frac{A^{\prime}(2 x B+5 B}{n n}+\frac{2 B^{\prime}(2 x A+92)}{n n}$

III. $-2 n 3-6 \mathrm{~B}+\frac{3 \mathrm{~A}}{4 n n}+\frac{9}{2 n n}+\frac{3 \mathrm{~A} N}{n n}+\frac{3 \mathrm{AA}}{2 n 88}$

$$
=-4 a B^{\prime}+\frac{A^{\prime}(2 x A+F)}{4}
$$

IV. $\quad \frac{35}{4}-2 x a-6 a+\frac{3}{2} b(D+E)+\frac{3 C(D+E)}{n}=-2 \pi a^{t}$ $+\frac{\left(D^{\prime}+E^{\prime}\right)(2 n+C)}{2 b^{\prime}(2 x A+2)}+\frac{2 F^{\prime}(2 x D+D)}{2}$

v. $-2 x b-66+\frac{2}{2} b(\mathrm{E}+\mathrm{G})+\frac{3(\mathrm{D}+\mathrm{E})}{n n}+\frac{3 \mathrm{D}(\mathrm{E}+\mathrm{J})}{n n}+\frac{3 A a}{38 n}=4 a^{b^{\prime}}$

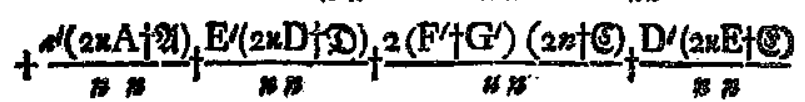


96:

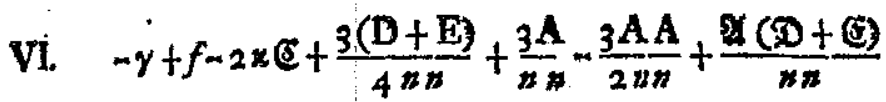
$+\frac{3 A(P)+C)}{n n}+\frac{3 A(D+E)}{n n}+\frac{3 n(D+E)}{n n}=$ $-C^{\prime}+\frac{A^{\prime}(2 \times D+9)}{n \pi}+\frac{A^{\prime}(2 \times E+C)}{n x}+\frac{\left(D^{\prime}+E^{\prime}\right)(2 \times A+9 D)}{n}$

vil. $3-2 \times \mathfrak{O}-6 \mathrm{D}+\frac{x}{2}+\frac{3 \mathrm{~F}}{4 * 3 x}+\frac{\mathfrak{C}(\mathfrak{A}+3 \mathrm{~A})}{n z}=$

$$
-(2 *-1) D^{\prime}+\frac{A^{\prime}(2 x+E)}{n}
$$

Viii. $3-2 x \mathbb{C}-6 \mathrm{E}+\frac{\pi}{2} b \mathrm{~A}+\frac{3 \mathrm{G}}{4 m}+\frac{E(\ddot{A}+3 \mathrm{~A})}{\Delta \pi}=$ $-(2 x+x) E^{\prime}+\frac{A^{\prime}(2 x+C)}{n}$.

'IX. $-3 \times 8-6 F+\frac{x}{2} 6 B+\frac{3 A}{2 m n}+\frac{3 D}{4 m}+\frac{3 D}{n n}+\frac{3 A D}{n n}$ $+\frac{32 \mathrm{~A}}{n B}+\frac{3 \mathrm{AD}}{3 i \mathrm{~B}}-\frac{3 \mathrm{AA}}{4 m}=-(4 \alpha-\mathrm{I}) \mathrm{F}+\frac{4 \mathrm{~B}}{3}$ $+\frac{A^{\prime}(2 \times D+D)}{n}+\frac{D^{\prime}(2 \times A+\pi)}{n}$

x. $\quad-2 * 0-G+\frac{2}{2} B+\frac{3 A}{2 m}+\frac{3 E}{4 m}+\frac{2 H E}{3 n}$ $+\frac{A C}{m i}+\frac{32 E}{m \pi}+\frac{3 A E}{m i}-\frac{3 A A}{4 m}=m(4 \alpha+x) G+\frac{4 B^{\prime}}{n}$ $+\frac{A^{\prime}(2 x E+Q)}{n}+\frac{E^{\prime}(2 n A+2)}{n}$
$C A P \cup \mathrm{T}$ VII.

XI. $+\frac{\pi}{2} f-2 x \mathfrak{s}-6 \mathrm{H}+\frac{3(\mathrm{D}+\mathrm{E})}{2 n}+\frac{3(\mathrm{~J}+\mathrm{K})}{4}$ $-\frac{3 \mathrm{AD}}{2 n n}+\frac{6 \mathrm{C}}{2 n}-\frac{3 \mathrm{AE}}{2 n n}+\frac{D C}{n n}+\frac{39 \mathrm{DE}}{n n}+\frac{3 \mathrm{DC}}{n n}+\frac{3 \mathrm{DE}}{n n}$

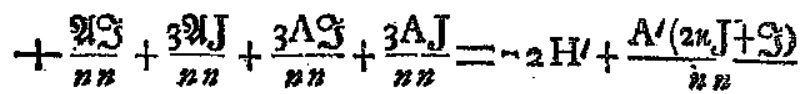

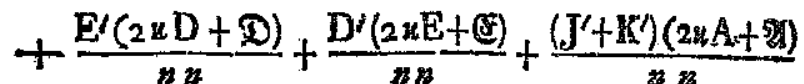

Xil. $\quad \frac{x}{4} \sin 2 x-6 \mathrm{~J}+\frac{x}{2} b \mathrm{D}+\left(\frac{3}{4}-\frac{f}{2 \pi n}\right) \mathrm{A}+\frac{3 \mathrm{H}}{4 m}$ $+\frac{\mathfrak{S}(2)+3 A)}{n n}+\frac{3(C D}{n n}=-2(n-1) J^{\prime}+\frac{3 A^{\prime}}{2 n}$ $+\frac{\mathrm{D}^{\prime}(2 n+\mathbb{C})}{n n}+\frac{\mathrm{A}^{\prime}(2 x \mathrm{H}+\mathfrak{B})}{n}+\frac{2 \mathrm{~L}(2 n \mathrm{~A}+\mathrm{A})}{3 n}$

XIII. $\frac{x}{4}-2 \times 3-6 \mathrm{~K}+\frac{\pi}{2} 6 \mathrm{E}+\left(\frac{3}{4}-\frac{f}{223}\right) \mathrm{A}+\frac{3 \mathrm{H}}{4 \cdot n 8}$ $+\frac{\underline{Q}(2)+3 A)}{n n}+\frac{36 E}{n}=-2(u+1) K\left(+\frac{3 A}{2 n}\right.$ $+\frac{\mathrm{E}^{\prime}(2 n+\mathcal{C})}{n n}+\frac{\mathrm{A}^{\prime}(2 n \mathrm{H}+\mathfrak{S})}{n n}+\frac{2 \mathrm{G}^{\prime}(2 n \mathrm{D}+\mathrm{D})}{n n}$

$\mathrm{XV}:-2 x \mathrm{C}-6 \mathrm{~L}+\frac{x}{2} 6 \mathrm{~F}+\left(\frac{3}{4}-\frac{f}{2 n n}\right) \mathrm{B}-\frac{3 \mathrm{AD}}{2.338}$

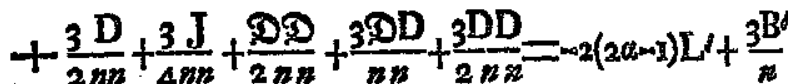
$+\frac{2 F^{\prime}(2 n+D)}{n n}+\frac{A^{\prime}(2 u J+9)}{n n}+\frac{J^{\prime}(2 n A+2)}{n n}+\frac{D(2 u D+D)}{n n}$ 
XV. $-2 \times m-6 \mathrm{M}+\frac{x}{2} b \mathrm{G}+\left(\frac{3}{7}-\frac{f}{2 n n}\right) \mathrm{B}-\frac{3 \mathrm{AE}}{2 n n}$ $+\frac{3 \mathrm{E}}{2 m n}+\frac{3 \mathrm{~K}}{4 m}+\frac{E E}{2 n n}+\frac{3 E \mathrm{E}}{n n}+\frac{3 \mathrm{EE}}{2 n n}=$ $-2(2 \alpha+1) M^{\prime}+\frac{3 B^{\prime}}{z}+\frac{2 G^{\prime}(2 n+\mathcal{O})}{n n}$.

6. 107. Nune antequam hos valores inuenire quea mus, verus valor ipfius a inueltigari debet: quod fiet ex valore integrali ipfius $\phi$, qui fi vti \$. 98. ponatus $\varphi=0 r+4$ fin $2 \eta+$ etc. obtinebitur. + alkkfin 21

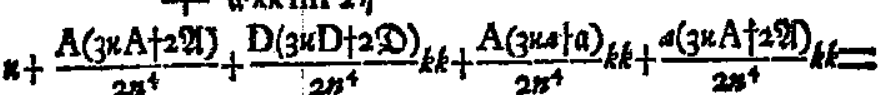

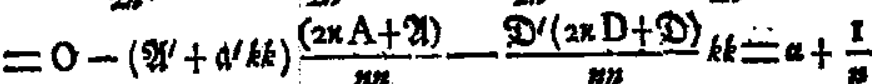

- vbi ex obferuationibus conftat effe $O=1,0085272$ Proxime autem effe fupra inuenimus effe:

$$
\begin{array}{l|l|l}
\mathfrak{H}^{\prime} \equiv 0,01 & \mathfrak{A} \equiv-0,80 & \mathrm{~A} \equiv-\mathrm{1}, 25 \\
\mathfrak{a}^{\prime} \equiv 0,05 & \mathfrak{a} \equiv-2,05 & \hat{D} \equiv-12,60 \\
\mathfrak{D}^{\prime}=-0,44 & \mathfrak{D} \equiv-3,60 & \mathbf{D}=34,25
\end{array}
$$

atque $x=1,0085 ; \quad k k=0,003 ; \quad n=175,71795$ vade inuenimus $0+0,000649=\alpha+\frac{1}{n}=x+0,000285$

§. 108. Cum nurde fit $0=1,0085272$, erit $\alpha+\frac{1}{n}=$ $x, 009176$, et ob $\frac{x}{8}=0,075438$, habebitur vrues valor:

$$
a=0,933738 \text { ot } l a=9,9702255
$$

atgue

$$
x=1,008991 \text { et } l x=0,0038874
$$

CAPUT HIS.

Hinc iam primo obsinemus:

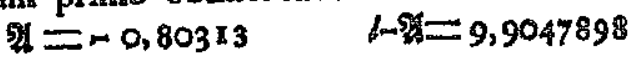

Deinde cum fit fatis prope $C=-0,67465$, erie

$$
\begin{aligned}
& \frac{(2 n+C)}{n n}=0,147037 \text { et } l \frac{2 n+6}{n n}=9,1674260 \\
& D=-3,5,93620 \ldots A D=0,5555310 \\
& \mathbb{E}=-x, 087320 \ldots l \in=0,0363580
\end{aligned}
$$

atque porro ex valore ipfius $A$ proxime cognito erit

$$
\mathfrak{B}=+0,006967 \ldots / \mathfrak{B}=7,8430540
$$

et quia eft fatis prope $B=0,0128$, erit $A=2 a A-0,000720$ et $B^{\prime}=4 \times B-0,023926$, vnde fit;

$1+1,62172-6 \mathrm{~A}=\approx \frac{1}{2}=\alpha \mathrm{A}+0,00144 \alpha+0,000374 \mathrm{~A}$

$+0,01247-6 \mathrm{~B}=-16 a \alpha \mathrm{B}+0,09570 a-0,038032 \mathrm{~A}$

109. Nunc primum guaeri debent valores litte um $f, b$ et $b:$ et cum fit $=5^{\alpha}, 9^{\prime}$ et $2 \gamma-\frac{3}{2} \delta=$ $-1,3899$ proxime, reperietur

$$
\begin{aligned}
& f=1,093757 \text { et } t f=0,0389208 \\
& b=3,0423 . \text {. } t b=0,4832020 \\
& b=1,0159 x \text {. . } t b=0,0068560
\end{aligned}
$$
bincque erit$$
12,9369 \quad B=+0,0768.4-0,0355 A
$$
unde concluditur fore:

$$
\begin{aligned}
& A=-1,262463: \because \ell A=0,1012186 \\
& B=+0,009404 \therefore N_{2}=7,9733114 \\
& \text { Porro }
\end{aligned}
$$

$$
2,4720 A=-3,71947-0,142 \quad B
$$

Hine 
Porro vero eit

§. IIx. Ex his iam porro inuenitur

$$
\begin{aligned}
& D^{\prime}=0,867676 \mathrm{D}+0,185628 \\
& \mathrm{E}^{\prime}=2,867676 \mathrm{E}+0,185628
\end{aligned}
$$

ct $\quad A^{\prime}=-2,35859 \cdot .4 N^{\prime}=0,3726530$

it VIII

\$. no. Ex his valoribus aequationes VII et VIII induent has formas,

$3+7,25185-6 D-1,92040-0,00244+0,01704=$ $-0,75286 \mathrm{D}-0,16106-0,34680$

$3+2,19420-6 \mathrm{E}-\mathrm{x}, 92040+0,00006+0,0 \times 704=$ $-8,22357 \mathrm{E}-0,53232-0,34680$

vnde prodibit

$$
\begin{aligned}
& \mathrm{D}=+33,6600 \text {. . / } \mathrm{D}=\mathrm{x,5271130} \\
& \mathrm{E}=-0,5785 \cdot \cdot 4 \mathrm{E} \equiv 9,7623410 \\
& D^{\prime}=29,39153 \\
& \mathbf{E}=-\hat{\mathbf{i}}, 47347
\end{aligned}
$$$$
\text { ergo }
$$

Ex his rancifcemur féquentes formulas pro calculo fe: quenti

$$
\begin{aligned}
& \frac{2 x \dot{A}+\dot{A}}{m}=-0,01884-\mu \frac{(2 x \dot{A}+2)}{m}=8,275051 \\
& \frac{2 x B+B}{m n}=+0,000148 \quad l \frac{2 \times B+x}{2}=6,770269 \\
& \frac{2 k \mathrm{D}+\mathrm{P}}{m n}=+0,366 \mathrm{iz} \cdot \frac{2 k \mathrm{D}+\mathrm{Q}}{m}=9,563604
\end{aligned}
$$

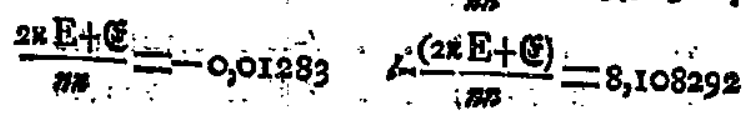

5. Irr.

$$
\begin{aligned}
& c=-0,64383 \cdot . \cdot b=9,60877 \mathrm{~B} \\
& \text { atque } \\
& C^{\prime}=-0,13847
\end{aligned}
$$

Porro valores litterarum $\mathfrak{F}$ et $\mathcal{O}$ determinabuntue per has aequationes

$$
\begin{array}{r}
(40-1) F=0,002049-0,294032+0,067699 \\
+0,021554-0,287335 \\
\left(4^{0}+1\right) B=0,002049+0,010306+0,020484 \\
+0,021554+0,004939
\end{array}
$$

ex quibus reperitur

$$
\begin{aligned}
& f=-0,17957 \quad \therefore \quad l-\xi=9,25424 \mathrm{I} . \\
& \mathrm{S}=+0,01253 . . \quad 15=8,097936
\end{aligned}
$$
atque

$$
\begin{aligned}
\mathrm{F}^{\prime}= & (4 \omega-1) \mathrm{F}-0,00283+0,46219+0,634 \mathrm{II}= \\
& (4 \omega-1) \mathrm{F}+1,00347 \\
\mathrm{G}^{\prime}= & \left(4^{\alpha+1}\right) \mathbf{G}-0,00283-0,01620-0,01090= \\
& (4 \alpha+1) \mathrm{G}-0,02993
\end{aligned}
$$

vnde aequationes IX et $X$ prodibunt.

$+0,36238-1,01591 \mathrm{~F}+0,00353-0,00680-1,00349=$

$$
m(40 x-1)^{2} \text { F-2,984.15-0,86349+0,00337-0,55370] }
$$

$-0,02528-1,01591 \mathbf{G}+0,00353-0,00680+0,04634=$ $-\left(4^{\alpha+1}\right)^{2} \mathrm{G}+0,14473+0,03027+0,00337+0,02776$

$$
\text { feu } \quad 6,43486 F=-3,75359
$$$$
21,40769 \mathrm{G}=+0,18534
$$

8. Iro: Hinc prodeunt fequentes valores correcti pro $\mathbf{F}$ et $\mathbf{G}$,

$$
\begin{aligned}
& F=-0,58360 . .4 \mathrm{~L}=9,7661 \mathrm{I2} \\
& \mathbf{G}=+0,00866 \text {. . } l \mathrm{G}=7,937400
\end{aligned}
$$


Ex formula autem fexta hinc leui calculo colligitur fore:

fore:

$$
y-f=1,58161 \text { et } y=2,67537
$$

Valores autem ex $F$ et $G$ deriuati erunt

$$
\begin{aligned}
& \mathrm{F}^{\prime}=-0,49919 \text { et } \mathrm{G}^{\prime}=0,01107 \\
& \frac{2 x \mathrm{~F}+g}{m}=-0,00772 \quad \mathrm{~s} \frac{(2 x \mathrm{~F}+f)}{m}=7,887828 . \\
& \frac{2 x G+G}{m}=0,00017 \quad, \frac{2 x G+G}{* 11}=6,232305
\end{aligned}
$$

§. I13. Nunc procedamus ad valores littexarum a et $\dot{b}$ qui erunt

$8,867676 \mathrm{~A}=-3,75000 m 0,68827-0,13148-0,037646$ $+0,02776$

$3,735352=-0,57467-0,04973-0,39807-0,018840$ $+0,04611$

- vnde reperitur:

$$
a=-2,42686 \text {. . } l a=0,385044
$$$$
b=-0,24899 \cdot \cdot 4 b=9,396182
$$

huncque porro

$d=29+0,037686-4,86420+0,16048$

$H=4 a b+0,0,1884+0,16887+0,64373$

$$
+0,01450-0,01744
$$

cos

$$
d=206+0,03768 b-4,70372
$$

$w=4 a b+0,03334 * 0,79516$

6. II4 Aequationes IV et $V$ hine induent to quentes formas: rum iv.

IV. $+3,75000-1,01591$ \& $50,32200-0,36363=40228$ $+4,89730)$

$-0,07034^{b}+8,78034+4,70500-0,36551-0,00125$ 새 $-0,14067 b$ $-0,02996$

V. $+0,50246-1,0159 \times 6-0,87350+0,28239-0,95732$ $-0,02155 a$

$=-16 \cos b-0,12447 a-2,96860-0,00142 b$

$-0,03517$ a 0,17723

$-0,14354$

$-0,91659$

Hine fit

$2,47355 a=-0,21101 b-46,21580$ $12,93836 b=-0,13809 \div-2,80553$

et $\quad a=-18,64200 \cdots \ln a=1,270493$ $b=-0,01794$. . $l-b=8,253822$

ex quibus oriuntur:

$d^{\prime}=-39,52164 \quad \cdots \quad b^{\prime}=+0,10663$

et $\quad \frac{2 n a+a}{m n}=-0,22790 \mathrm{~lm} \frac{(2 n a+a)}{n \text { s. }}=9,357746$

valor autem ipfius $\frac{2 n b+b}{n n}$ nullius plane exit momenti, vnde eum praetermittimus.

5. 115. Ex prima autem aequatione f. 106.colligitur $\frac{x}{y}=y-f+0,02285$

fupra autem inuenimus effe $\gamma-f=1,58161$, ficque eric $\frac{\pi}{2} \delta=1,60446$.atque

$$
\delta=3,20892: \cdot 68=0,506358
$$

Nune cum fit proxime: $\hat{\mathrm{S}}=-0,123 ; \mathrm{H}=\mathrm{I}, 033$ ideoque $\frac{2 \& H+W}{88}=-0,0126 ;$ ob $\&=041,453$ et $\mathrm{L}=+6,252 \%$ habebimus 
$-0,132324 \$=-3,75000+0,00882-0,09088$ $+0,05336-0,01012$ $-0,52839+0,05474$

$+3,867676 \Omega=3,75000+0,00882-0,09088$ $+0,01012$

Hine reperitur $-0,15987+0,00917$

$\xi=32,05945 .+\quad \mathcal{S}=1,505956$

$S=-1,02714 \cdot 2 . \Re=0,011629$

§. 116. Hinc vltèrius progrediendo habebimus.

$J^{\prime}=2(\alpha-1) J+0,2857 x-4,94924+0,23502=$ $\begin{array}{ll}-0,01591 & -0,08015\end{array}$ $2(a-1) J-4,52457$

$K^{\prime}=2(\infty+1) K+0,28571+0,08507-0,00317=$ $-0,01591$

$2(\alpha+1) \mathrm{K}+0,35170$

- vnde aeguationes XII et XIII fiunt

$+3,75000-64,69550-x, 01591 J+51,28180$

. $-0,94292+0,00321-0,36999=$ $-0,0044 \mathrm{I})$

$-4(w-1)^{2} \mathrm{~J}-\frac{0,00441)}{-0,5987^{1}}-0,26690+4,32164$ $+0,02972-0,47004$

$+3,75000+2,07275-1,01591 \mathrm{~K}=0,88006$ $-0,94292+0,00321+0,00636=$

$-4(\alpha+1)^{2} \mathrm{~K}=\frac{0,0044 \mathrm{I})}{\mathrm{I}, 36026-0,26690-0,21665}$

ex quibus colligitur fore

$$
+0,02972+0,00810
$$

$$
\begin{aligned}
& \mathrm{J}=-\mathrm{I} 4,09600 \ldots . \mid \mathrm{I}-\mathrm{J}=\mathrm{I}, \mathrm{I49096} \\
& \mathrm{K}=-0,41676 \text {. . } \operatorname{lm} \mathrm{K}=9,619888
\end{aligned}
$$

$C A B U T$ VII.

Hine $J^{\prime}=-2,65933 . . K /=-1,26020$

atque $\frac{24 J+9}{m}=+0,02057 \cdot \frac{2 n J+\Phi}{m}=8,3 \pm 3172$

$$
\frac{2 x \mathrm{~K}+\Re}{m n}=-0,01063 \cdot \ln \frac{24 \mathrm{~K}+\Omega}{m}=8,026598
$$

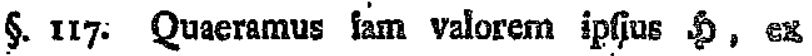
aequatione

$2 \mathfrak{B}=-0,45434-0,39771-0,01652+0,62330$ erit $\mathfrak{S}=-0,12264 . . \quad 1-\mathfrak{S}=9,088632$

hineque reperitur: $\quad \mathrm{H}=2 \mathrm{H}+0,0801$

Ynde aequatio XI praebet:

$2,04688+0,24748-1,0159 \mathrm{IH}+0,27805-3,06199$ $+0,36275+0,00118-0,00623+0,02224+0,03552$

$-0,62485-0,33247-0,83753+0,49710=$

$-4 \mathrm{H}-0,16022-0,0485 \mathrm{~L}-0,53944-0,37802$

feu $\quad 2,98409 \mathrm{H}=-2,68053$

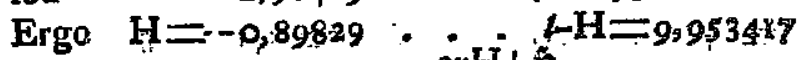

$\mathrm{H}=-1,1,1.647 ; \frac{24 \mathrm{H}+5}{\mathrm{~m}}=-0,01102$

\$. 118; Tandem fuperfiunt litterae \& en

$2(20-1) \cdot \&=-0,45434-0,05280-0,01652-1,31570$ $+0,00158$ $-0,00015$

$2(2 a+1) M t=+0,00158+0,00368+0,02935$. $-0,00015$

Hinc $f=+1,40715:, I f=0,148340$ Mn:=+0,00426 . - Mn:7,629896

- Deinde 
Deinde vero habebitur:

$\mathrm{L}^{\prime}=2(2 a-1) \mathrm{L}-0,00213+0,17162 \cdots 12,31170-0,02596$ $+0,00013$

$-0,26555$

$M /=2(2 a+1) \quad M-0,00213-000254-0,00742$

$$
+0,00013
$$

feu $\quad L^{\prime}=2(2 \alpha-1) L-12,43359$

$$
M^{\prime}=2(2 a+1) M-0,01196
$$

\$. 319. Numo denique aggrediamur sequationes XIV et XV

XIV. $-2,83960-1,0159$ I L $0,88774+0,00702+0,36275$. $+0,28733 \quad-0,06016+0,03675+7,60650=$ $-3,0$ ir $144 \mathrm{~L}+21,57666+0,00255-0,1+680+10,75272$

XV. $+0,00860-1,01591 \mathrm{M}+0,01317+0,00702-0,00623$ m0,00494 … : - $0,00176+0,00336+0,01360=$ ': m32,894is M+0,06859+0,00255+0,00325 ex quibus eruitur

$L=+13,86720 \ldots t L=1,141988$
$M=+0,00131 \ldots t M=7,117165^{\circ}$ hincque $U=11,3090$. et $M t=-0,00445$.

$$
\begin{aligned}
& \frac{2 x \mathrm{~L}+\mathrm{g}}{n n}=0,75125 \cdots \cdot \frac{2 \times \mathrm{L}+\mathrm{g}}{n n}=9,179684 . \\
& \frac{2 x \mathrm{M}+\mathrm{M}}{n n}=0,00004 \quad \frac{2 x \mathrm{M}+2 n t}{n n}=5,59277^{\circ} .
\end{aligned}
$$

Ex his valoribus nouac correctiones inueniri poffent, fed differentiae prodirent tam exiguae, vt operae pre: tium non fis tas ineftigare.
8. 20. His : igitur valoribus inuentis, denotante iam a diftantiam Lune mediam a Terra, et eivs da

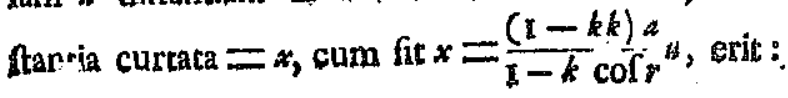

$$
\begin{aligned}
& \begin{array}{l|l}
3=1-0,0074991 \operatorname{col} 2 \% & \begin{array}{l}
\text { log. coefficient: } \\
7,875009
\end{array}
\end{array} \\
& +0,0000532 \operatorname{cof} 4 \\
& +0,191557 k \operatorname{cof}(2 \eta-r) \\
& -0,003293 k \operatorname{cor}(2 \eta+r) \\
& -0,003321 k \operatorname{cof}(4 \eta-r) \\
& +0,000049 k \operatorname{cor}(4 \eta+r) \\
& -0,00511 k k \cdot \operatorname{cof} 2 \gamma \text { : } \\
& -0,08022 k k \operatorname{col}(2 \eta-2 r) \\
& \therefore \quad-0,00237 k k \text { cof }(2 \eta+2 r) \\
& \therefore \cdots+0,0 \text { \% } 892 k \cdot \operatorname{cof}(4 \eta-2 r) \text {. } \\
& \text {. } \quad+0,00001 k k \operatorname{cor}(4 y+2 r) \\
& 5,735912 \\
& \text { 9, } 28229 \% \\
& 7,517525 \\
& 7,521296 \\
& 5 ; 692584 \\
& 7,70860 \mathrm{r} \\
& 8,904280 \\
& 7 ; 375072 \\
& \text { 8;897172 } \\
& \therefore \quad \\
& 4,8 \% 2349^{\circ}
\end{aligned}
$$

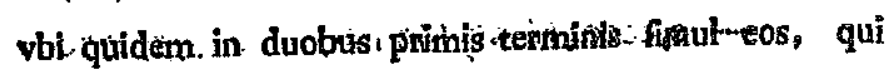
per $k k$ erant affeti, fumus complexi, potito $k=0,05445$. Exiamfi enim hic valor non omnino effer iuftus, tamen inde in his terminis minimis nullus error nafei poteris. $\therefore \because$

$\mathrm{O}_{2}$

§. $12 \mathrm{k}$, 
6. 121. Porro quoque hinc ex $\S$. Ii6. valorem ipfus $\frac{d \varphi}{d r}$ determinabimus, quatenus a fola excentricicate orbi-

1 ipfius

z orbi-

rae lumaris pendet.

$$
\begin{aligned}
& \frac{d \varphi}{d r}=\quad 1,009276 \\
& +0,0195144 \text { cor } 2 \text {. } \\
& -0,0000322 \operatorname{cof} 4 y \\
& \text { - 0,001231k col } r \\
& -0,366103 k \operatorname{cor}(2 . y-r) \\
& +0,012832 k \operatorname{cof}(2 \eta+r) \\
& +0,002829 k \operatorname{cof}(4 y-r) \\
& -0,000171 k \operatorname{cof}(4 \eta+r) \\
& +0,01182 k k \text { col } 2 r \\
& -0,02057 k k \operatorname{cof}(2 y-2 r) \\
& +901063 k k \operatorname{cof}(2 \eta+2 r) \\
& -0,09883 k k \operatorname{cor}(4 \eta-2 r) \\
& -9,00004 k \operatorname{cor}(4 y+2 \pi)
\end{aligned}
$$

oeff.
§. 122. Cum nunc fit $\frac{d \theta}{d r}=\frac{d s}{d s}=\frac{r+2 e q}{s}+\frac{2}{8} k \cot \theta$ $+\frac{3}{2 n} k k \operatorname{col} 2 r$, erit

$$
\begin{aligned}
& \begin{array}{l|l|l}
\frac{d y}{d r}=0,933838 & 00 \mathrm{eff} \\
9,970272
\end{array} \\
& \begin{array}{l|l}
+0,0195144 \operatorname{col} 2 \% & 8,290355
\end{array} \\
& \begin{array}{l|l}
-0,0000322 \operatorname{col} 4 \text { i } & 5,507856
\end{array} \\
& \begin{array}{l|l}
-0,152101 k \operatorname{cof} r & 9,182132
\end{array} \\
& \begin{array}{ll}
-0,366103 k \operatorname{cor}(2 \pi-r) & 9,563604
\end{array} \\
& \begin{array}{l}
+0,012829 k \operatorname{cor}(2 y+r) \quad 8,108292 \\
\hline
\end{array} \\
& +0,002829 k \operatorname{cof}(4 \eta-r) \quad 7,451633 \\
& \text { - } 0,000171 k \operatorname{cor}(4 y+r) \quad 6,232305 \\
& \begin{array}{l|l}
-0,10133 k k \operatorname{col} 2 r & 9,005738
\end{array} \\
& -0,02057^{k k} \cos (2 y-2 r) \quad 8,313178 \\
& +0,01063 k k \operatorname{cor}(2 \eta+2 r) \quad 8,026598 \\
& -0,09883 k k \operatorname{co}(4 \eta-2 r) \quad 8,994889 \\
& -0,00004 k k \operatorname{cor}(4 \eta+2 r) \quad 5,592770
\end{aligned}
$$

quae formulae ad motum Lunae horarium tam sblolus tum quam a fole adhiberi poffune, quemadmodum illa diftantiam definiens diametro apparenti et parallaxi hocizontali inueftigandae infervis.

$0_{3}$ 
8. 23. Quaeramus nunc valorem integralem pro longitudine Lunae $\varphi$, quatenus a fola', excencricitate orbitae lunaris pendet, ac ponamus.

$\varphi=0 r+\mathfrak{A}^{\prime} \operatorname{fin} 2 \eta+\mathfrak{a}^{\prime} k k$ fin $2 \eta+\mathfrak{B}^{\prime} \operatorname{fin}_{4} \eta+\mathfrak{b}^{\prime} k$ fin $_{4} \eta$

$+\mathbb{C}^{\prime} k$ fin $r+D^{\prime} k$ fin $(2 \eta-r)+\mathfrak{f}^{\prime} k$ fin $(4 \eta-r)$

$: \eta-r)$

$\eta+r)$

$+\mathbb{E}^{\prime} k$ fin $(2 \eta+r)+(\mathrm{H} / k$ fin $(4 \eta+r)$

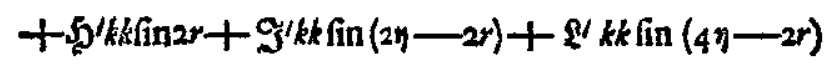

$+\mathfrak{R} / k k$ in $(2 y+2 r)+\mathfrak{M} / k k$ in $(4 y+2 r)$

$+2 r$

atgue fequentes obtinebimus formulas:

$$
\begin{aligned}
& +0,0188387=2 a \mathfrak{A}^{\prime}-\frac{\mathscr{A}^{\prime}(2 x \mathrm{~B}+\mathfrak{B})}{n n}-\frac{2 \mathfrak{B}^{\prime}(2 \mu \mathrm{A}+\mathfrak{A})}{n n} \\
& \text { - }-0,0000370=4 \alpha \mathfrak{B}^{\prime}-\frac{\mathfrak{A N}^{\prime}(2 n \mathrm{~A}+\mathfrak{A})}{n n} \\
& -0,001231=C^{\prime}-\frac{2 \mu(2 n \mathrm{D}+\mathfrak{D})}{n n}+\frac{2{ }^{\prime}(2 x \mathrm{E}+\mathrm{G})}{n n} \\
& -\left(D^{\prime}+\mathrm{CS}^{\prime}\right)(2 \dot{\mathrm{A}} \mathrm{A}+\mathscr{4}) \\
& -0 ; 366 i 03=(2 a-i) D^{\prime}-\frac{q^{\prime}(2 n+(E)}{n \pi} \\
& B * \\
& +0,012832=(2 a+i) e_{-1}-\frac{2(2 n+\mathcal{E})}{n n}
\end{aligned}
$$

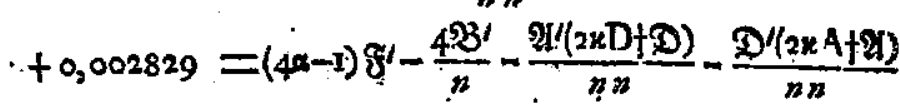

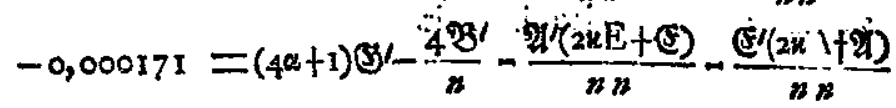

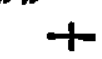$$
+0,22790=2 \alpha a^{\prime}-\frac{\left(D^{\prime}+B^{\prime}\right)(2 n+C)}{n s}-\frac{2 g^{\prime}(24 D+D)}{n}
$$$$
-\frac{2 b^{\prime}(3 n A+g)}{n B}
$$$$
+0,00163=4 x-\frac{\mathfrak{a}^{\prime}(2 x A+P)}{n n}-\frac{\left(E^{\prime}(2 x D+D)\right.}{n n}
$$$$
-\frac{2\left(g^{\prime}+(j)(2 n+6)\right.}{n n}
$$$$
+0,01182=2 b^{\prime}-\frac{C^{\prime}(2 n D+D)}{n n}-\frac{2 M^{\prime}(2 x J+\Phi)}{n}
$$$$
-\frac{\left(\mathcal{N}^{\prime}+\Re\right)(2 n \mathrm{~A}+2)}{n n}-\frac{\mathfrak{D}^{\prime}(2 n \mathrm{E}+\mathcal{E})}{n n}
$$$$
-0,02057=2(\alpha-1) \mathbb{J}^{\prime}-\frac{3 \#}{2 n}-\frac{\mathfrak{D}^{\prime}(2 n+\mathfrak{C})}{n n}-\frac{\mathfrak{N}^{\prime}(2 n \mathrm{H}+\mathfrak{S})}{n n}
$$$$
-\frac{2 g^{\prime}(2 x A+g)}{n n}
$$

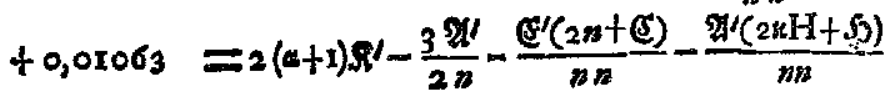$$
-\frac{2 S^{\prime}(2 x D+D)}{n}
$$

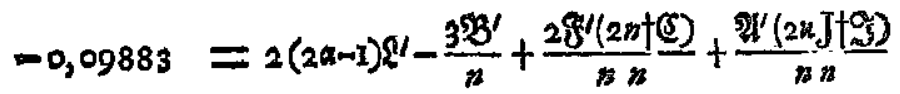$$
-\frac{S^{\prime}(2 \times A+F)}{n}-\frac{D^{\prime}(2 \times D+D)}{n}
$$ 
8. 124. Ex his oliciumtur valores fequentes:

$\mathrm{M}=+0,0100887-|\mathfrak{A}=8,003837| \mathfrak{a}^{\prime}=0,09140$

$\mathfrak{Y}=-0,0000409 \cdot \mid-\mathfrak{B}^{\prime}=5,611723 \mathrm{a}^{\prime}=8,960934$

$\mathfrak{d}^{\prime}=+0,010146 \cdot t \mathrm{f}^{\prime}=8,006295 \mathrm{~b}^{\prime}=0,00089$

$D^{\prime}=-0,420226-h D^{\prime}=9,623483 / b^{\prime}=6,949340$

$\mathrm{E}^{\prime}=+0,004992-t \quad \mathrm{C}^{\prime}=7,698261 / 21+\mathrm{a}^{\prime} k \mathrm{k}=0,0103597$

$\mathrm{F}^{\prime}=+0,005286-4 \mathrm{f}^{\prime}=7,723163 \mathfrak{B}^{\prime}+\mathrm{b}^{\prime} k \mathrm{k}=0,0,00003$

$\mathfrak{G}^{\prime}=-0,000086-h \mathbb{O}^{\prime}=5,935307 l\left(\mathfrak{A}^{\prime}+\mathrm{A}^{\prime} / k k\right)=8,015347$

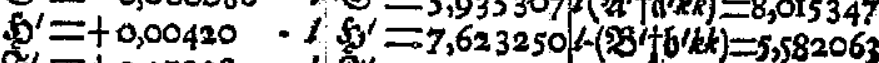

$\Phi^{\prime}=+0,57328 \cdot 1 \mathbf{J}^{\prime} \equiv 9,758367$

$\mathbb{R}=40,00318 \cdot 1 \mathbb{S}^{\prime}=7,502427$

$q=-0,15083-1-2=9,178488$

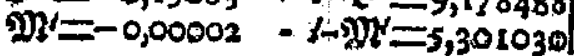

6. 125. Pro longitudine ergo Luane habemus haetetus hanc formulam

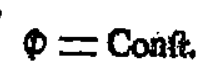

log. coeff.

$$
\begin{aligned}
& +1,0085272 \text { r } \\
& +0,0103597 \text { fin } 2 \eta \\
& +0,0000382 \text { fin } 4 \eta \\
& +0,010146 k \text { fin } \\
& +0,400226 k \text { fin }(2 \eta-r) \\
& +0,004992 k \text { fin }(2 \eta+r) \\
& +0,005286 k \text { fin }(4 \eta-r) \\
& +0,000086 k \text { fin }(4 \eta+r) \\
& +0,00420 k k \text { fin } 2 r \\
& +0,00318 k k \text { fin }(2 \eta+2 r) \\
& +0,15083 k k \text { fin }(4 \eta-2 r) \\
& +0,00002 k k \text { fin }(4 \eta+2 r)
\end{aligned}
$$

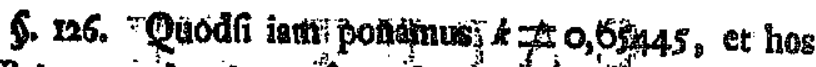
coefficientes \&d minuta lecunda cum partibus decimallio bus reducamus, longitudo $\Phi$ ita exprimetur vé hat:

$$
\begin{aligned}
& =\text { Conft }+r, 0085272 \\
& +213641 / 8 \mathrm{gin} 2 \mathrm{i} \\
& \text { - 7, } 8 \text { fin } 4 \text { y } \\
& + \text { 213, } 9 \text { fin. } \\
& \text { - 4719, } 6 \text { fin }(2 y-r) \\
& +56,1 \ln (2 \%+r) \\
& +59,4 \text { fin }(4 y-r) \\
& \text { - is. } 0 \operatorname{fin}(4)+r) \\
& +\quad 2 \text {; } 5 \text { fn } 2 \text {; } \\
& +350,6 \text { fin }(2 y-2 y) \\
& +\quad \therefore \text {, sfinfenter) } \\
& \longrightarrow \quad: 92,2 \text { (in }(4)-2) \\
& \text { - } \quad 0,0 \text { fin }(4 y+2 r) \text {. }
\end{aligned}
$$

Hisque formulis praecipuae inaequaltigkes, quibis mo tus Lunae perturbatur, continentur. 
: MPATT: VII

\section{DE MOTU APOGEI LUNAE.}

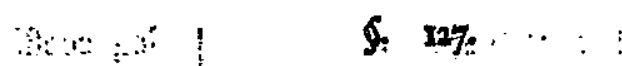

To Tis inuentis iam apduam illam de matu apogei $\mathrm{La}$ nae quaseftionem examinare, atque adeo decidere licebii. . Quanquam enim in praecedentibus calculis ybique verum apogei monum, quem obferuationes oftendunt , introduxi, ita, vt.jd jpfum, quod in controverfia eft, aflumfife videar; tamen quoniam in hune ipfuim fitiem terrae vim, qua luna vrgetur, indefinitam fum contemplatus, dum rationi diftantiarum reciprocae duplicatae terminum indefinitum adiunxi, vnde littera $\mu$

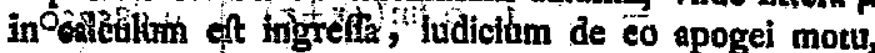
quit-Theopiae Neutoniansel offet:confentaneus, non erit

- difficile, Quodf enim valor litterae $\mu$ nihilo aequalis reperiacuir; hinc concludendum erit Theoriam Neutoni

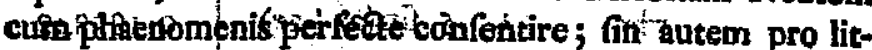
tere $A$ nowbilis prodeat Malor, Theoris ifta infufficiens exit centenda

6. 128. Motus atutem apogel, quoniam huius rei in calculo nusguam mentio ef fage, in co continetur proportione, quam motus lutase medius ad motum anomaliae tenere eft potitus. Cứn enim remotis lunae inasqualitatibus, quae regulae Keplerianse aduerfantur, longicudo lunae vera obeineatur, fi eius anomalia vera $r$ ad longitudinem apogei addatur: denotet $\%$ longitudinem apogei, eritque longitudo vern $\varphi=v+r$, vnde

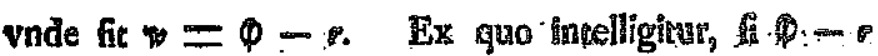
quantitatem defignet contantem is ...pogeum : ir: quigte relinqui, fin autem $\phi-r$ valorem variabilem obcineat, tum apogeum quoque lunae motum effe habiturumo

§. 129. Cum autem terminos illos omnes, qui io nus angulorum implicant, ideoque inaequalitates periodim cas continent, quibus apogei motus non afficicur, omise timus, per integrationem deducimur ad builusmodí fosmulam $\phi=$ Conf. + Or, vnde propterea habetor longitudo apogei $=$ Conft $+(0-1) \%$. Hine son fequimúr fequentés proportiones

1. Vt I ad $0-I$, ita motus anomaliae lunae ad mo tum apogei.

11. -Vc 0 ad 1 , ita mótis lunge medius ad motura anomaliae.

II. Vt $\mathrm{O}$ ad $\mathrm{O}$ - $\mathrm{Y}$, ita motuș lạne medius ad mgo sum apogei,

6. 130. :Si obferuationes confulamus; valor litte-

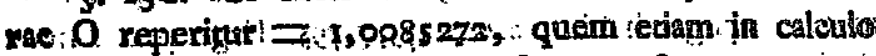
vbique adhibui; propterea quod propofiturn' nat not tam in iftum valorem a priori inquirere, quam ipfans pocius Theoriam ita intituere, atque $f$ opus fueri, emendare, vt motus inde apogei experteatiae confente.

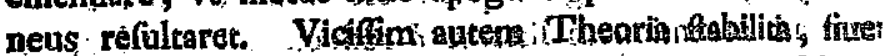
Neuroniana fue alia, quae ex determinato pro fu fublio tuto valore oriatur, facile erit valorem ipfius 9 a prios. ti eruere, quem deinceps sum valore pero, $1,0085273_{1}$

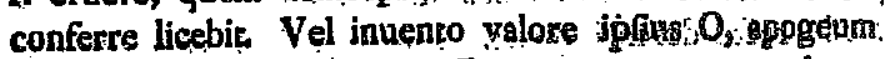

$\mathbf{p}_{2}$ lunes 


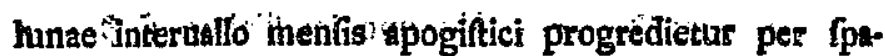
tum $(\mathrm{O}-1) 360 \%$, intervallo autem mentis periodici per fpatium $\left(1-\frac{T}{0}\right) 360 \%$. Secundum obferuationes autem apogeum promouetur

vịo menfe apogiftico per (patium $3^{\circ}, 4^{\prime}, \mathrm{II}^{\prime \prime}$

vno menfe periodico per fpatium $3,2,38$

5. I31. Ex calculo antem 9. 10\%. expofito (valor litterae $\mathbf{O}$ ex elementis ante affumtis ita definitur, vt fit $0+0,000649=x+0,000285$ fiue $O=x-0,000364$ Etfi enim heec exigua particula 0,000364 jam ex va? lore ipfius . veritati confentanee affumto eft orta, tamen perficuum eft, letaem differentiam nullius hic momenti futuram fưiffe Verum littera $*$ per Theoriam ita erat affumta, ve effet

$$
\therefore \quad \because \quad, \quad{ }^{4}=\left(\frac{3+4 \beta+2}{2 n \pi}\right)
$$

- vbi quidem valor ipfius $n x$ ex motu medio lunae ad motum folis, zelato habetur; ;ita: ro : fit fine refpebu ad

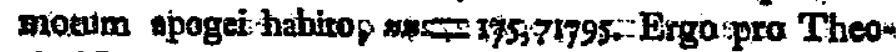

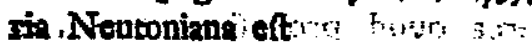

$$
x=V\left(1+\frac{3+\delta}{2 m}\right) \cdots \text { et } \delta=V\left(1+\frac{3+\delta}{2 m x}\right)-0,000364
$$

6. 132. Iflic igitur patet tom hane inueltigatio-

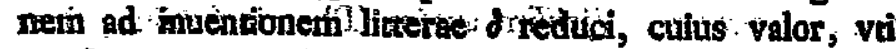
ex fuperiori calculo matiifeftum eft, a pluribus litteris et coefficientibus terminorum; quos ante eruere oportebat; pendet, it ve tlegledta hat hittera d moris apogei nullo modo, reate definiri queat. Ifilte quidem vbi hanc lic

$C A D T$ DIII.

ser fog.

Jici per

s autem

o ivalor

$r$, ve fit

200364

ex va-

tta, ta-

lic mo.

เeoriam

nae ad

eहta ad

Theo-

30364 .

tigatioor, vii litteris rtebat; nullo ine lit. teram teram in calculum induximus, quod factum eff $\$ 44_{5}$ haec res leuis momenti eft vifa; cum enim pro $C C$, quge erat conftans per integrationem in calculum ingreffa, $\mathrm{v} / \mathrm{O}$ lorem vero proximum inuenifemus $1+\frac{3+4 \beta_{5}}{2}$, quonian facile erat praeuidere, reliquis adhibitis elementis at mo? tum Iunae pertinentibus, hunc valorem sliquanturn insomurari poffe, pro vero valore ipfus $\frac{C C}{10 k k}$ pofainus $1+\frac{3+4 p+\delta}{2 x y}$. Deinde antem valor ipfius d potifimumition pendet a valore litterae $\gamma$, qua vfi fumus ad verum vo lorem conftantis $\frac{m}{m}=\mathrm{I}+\frac{2+3 m+\gamma}{m}$ obtinendum, cum $\mathrm{p}^{\mathrm{s}}$ : xime vetus effet inuentus $=x+\frac{2+3 \mu}{n}$.

5. 133. Ab his ergo litteris $\gamma$ et $\delta$, quae initio nullius fere vfus effe videbantur, determinatio motus apo gel potifimum pendet, quae cum ex pluribus atque adeo omnibus inaequalitattbus hunae ab excentricitate opa tis detetminari debeant, inirumi fatie non eft, quod leglo tima motus apogei defignatio, curn tantis implicata fie difficultaribus, tam dudium sueric abfcondita. Plerique enim, qui motum apogei ex. fola Theoria conchdere funt annifi, ad omnes has inaequalitates non refpexerume, atque salculum porinde adininiftrauerunt, ac fi hic litesras $y$ et $\delta$ neglexiftemus. Ac fi non defuere, qui fibi perfuaferunt, motum apogei cum Theoria Neutoniana confentire, if plerumque per errorem calculi feduki ad veritatem perueniffe fabi funt vif. Quin etiam Ipfe NTeuP 3
ธดมแม 
tonus Theoriae fuae in moku apogei determinando parum tribuifle videtur.

5. 134. Hinc ex negleatu harum litterarum $y$ et $d$, feu ex alia omifione eodem recidente, factum eft, vt Theoria Neutoni obferuationibus circa motum apogei lunae inftitutis plane non fatisfacere fit putata; quae opinio etiam ita inualuit, ve perlpicacifimus quisque hanc Theoriam infufficientem pronunciaret, Atque fagacifimus Clairaltius huic opinioni vehementiffime erat addiQus, antequam publice in contrarias partes difcefferat. Eadem féilicer ratione ob neglectum minutarum illarum particularum erat deceptus, qua et ego fateri cogor, me per complures annos conftanter effe opinatum, ex Theoria Neutoni pro motu apogei Lunae non vlera femiffern prodire, ita vt error vitra femifem exfurgens commitceretur;

\$. 135. Fors itaque huius erroris, qui nifi fumma - circumfpectio adhibeatur, vix euitatur, in eo latet, quod in calcule debica illa conftantium determinatio, pro qua equidem hic litteras $\gamma$ et $\delta$ adhipui, negligatur. Quemadmodum per hanc omiffionem dimidius tantum apogei mo: tus eliciatur, oftendige iuuabic, Sir igitar $\delta=0$, atque littera illius $\mathbf{O}$ fecundum Theoriam Neutonianam, qua eft $\mu=0$, valor erit $O=V\left(I+\frac{3}{2 m}\right)-0,000364$; qui evolutus fit: $O=1,0043592-0,000364$. Quare etiamfi particula 0,000364 vtpote ex profundiori indagine nata pretermitetur, tamen ifte valor pro $\mathrm{O}=1,0042592$, fi cum vero per obferuationes cognito $O=1,0085272$ comparetur, exake fere dimidium motum apogei prae- nando pa.

um $\gamma$ et $\delta$,

$\mathrm{m}$ eft, ve

$n$ apogei

quae opi-

que hanc

fagaciffi.

zrat addi-

ifcefferat.

n illarum

Jgor, me

sTheoria

?m prodi-

itteretur.

fi fumma

tet, quod

pro qua

Quemad.

ogei mo

$o$, atque

am, qua

364; qui

e etiamfi

gुine na-

1042592,

5085272

ei prae.

bet; bet; atque adeo haec tam accurata medietas rion parum digna viderur.

\$. 136. Jam videamus, quam prope valoren life terae $\delta$ adhibendo ad veritatem perducamus. Inuesío mus autem (II5) $\delta=3,20892$, vnde prodit

$$
V\left(I+\frac{3+\delta}{2 n n}\right)=1,0087947 \text {. }
$$

qui valor iam maior eft quam verus 1,0085272 , fed rescordandum eft inde fubtrahi debere 0,000364 , ficque relinquetur $\mathrm{O}=\mathrm{I}, 0084307$, ex quo motus progreffuus apogei pro interuallo menfis apogiftici prodibit = $3^{\circ} 2^{\prime} 9^{\prime \prime}$ et pro interuallo menfis periodici $=$

$3^{\circ} \mathrm{O}^{\prime} 37^{\prime \prime}$, qui numeri duobus tantum minutis a vero dece. ficiunt. Ad hunc defeftum fupplendum litterae $\mu$ tribui poterit valor conueniens ex formula $\mu=\frac{x}{2}(\mu s-\Sigma)$ s 38 $-\frac{3}{4}-\frac{x}{2} \delta$, vnde reperitur $\mu=0,03782$, qui valor tantillus eft, ut nifi de motu apogei fit quaeftio, femper pro nihilo haberi pofit.

\$. 137. Verum nullo modo affirmare poffumus, v2lores illos pro $\gamma$ et $\delta$ inuentos ita effe abfolutos, $v s$ nulla amplius correctione indigeant. Quin potius, fi formulas fupra exhibitas attentius perpendamus, aneum abeft vt eas pro completis habere poffimus, vt potius manifeftum fie, omnes reliquas inaequalitares motus luo ae perinde ac eas quas iam definiuimus, terminos qquo que in eas fuppeditare. Qui etfi admodum erunt papvi, tamen omnino fufficere poterunt ad exiguum iftud fup plementum, quo adhuc a vero diftamus, conficiendum.

Curs 
Cum enim fola fere inaequalitas ab angulo 2 i $-r$ pendens motum apogei a dimidie tantopere anxiftet, $v t$ valor ipfius $O$ ab 1,0042592 vsque ad 1,0084307 increviflet, nullum fere eft dubium, quin leuis defectus hu. ius numeri a vero valore 1,0085272 a reliquis inaequalitatibus proficifcatur.

\$ 138. Hinc igitur concludere debemus, Theoriam Neutonianam cum motu apogei obferuato tam exsEte conuenire, vt aberratio, fi guidem vlla locum hobeat, tam fit exigua, ve merito pro nihilo reputari pos. fit: neque etiam calculi ope ob fummam paruitatem eam certo definire licebit. Cum itaque hoc palto Theoria Neutoniana a forcifima obiectione fic vindicata, gloria huivs infignis inventi cum induftriae tum candori excellentiffimi Clairalti dehetur, qui primus egregi- um hunc Theoriae confenfum cum vericate detexit et publice eft profeffas: cui ea re co maiores debemus gratias, quod fine eius fudio fummo, quod in hac inveltigatione confumfit, Theoria Neutoniana fortafe vix vnẹam ab hac fufpicione infufficientiae effet liberats. Arque nuno demuen pleno lumine veritas iftius Theoriae, cui vni Aftronomiae Theoria vniuerfa innititur, fulgere eft cenfenda, cum antee non mediocribus tenebris fuiffet inuoluta. -r peniflet, ve 17 incre:etus hu. inaeque-

, Theotam ex. sum haari pos* suitatem : pacto indicata, candoegregitexit et ebemus hac inafte vix liberata.

Theonititur,

s tene-

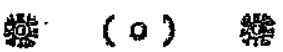

12음

\section{CAPUT $\mathrm{IX}$}

\section{INUESTIGATIO INAEQUALITATUM LUNAE A SOLA EXGENTRICITATE ORBITAE SOLIS RENDEMTIUR}

\section{§. 139.}

Tuoniam in hac inueftigatione excentricitas orbitac Q. lunaris non in cenfum venit, inaequalitates quas fcrutamur partim ab anomalia vera folis s partin ab angulo 2 pendebunt. Cum igitur fic

$$
\begin{aligned}
\frac{d s}{d r}=\frac{d \theta}{d r}=\frac{x+2 e s}{n} & +\frac{2}{n} k \operatorname{cor} r-\frac{2}{n} e \operatorname{col}\left(s-\frac{2}{z} e k \operatorname{cor}(r-s)\right. \\
& +\frac{3}{2 n} k k \cos 2 r+\frac{1}{2 n} e e c 0\left\{2 s-\frac{2}{z} e t \operatorname{cof}(r+s)\right.
\end{aligned}
$$

hinc differentiale $d s$ ad differentiale $d r$ reducitur. At que hoc quidem capite, quia ad excentricitatem Lunas non attendimus, erit

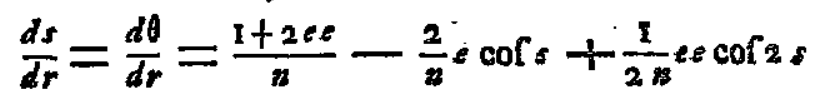

§. 140. Incipiamus ergo a fortanulis $f \mathrm{R} d r$ et quas omiffis terminis ab angulo $r$ pendentibus po. namus

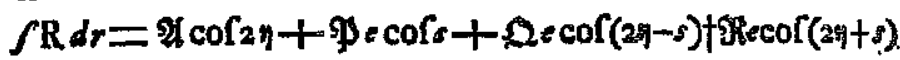

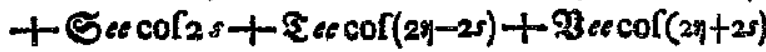

$=\mathrm{Acor} 2 \eta+\mathrm{Pecor} s+\mathrm{Q} e \operatorname{cor}(2 \eta-s) \dagger \operatorname{Recor}(2 \eta+s)$

$+\mathrm{S} e \varepsilon \operatorname{co}\left[2 s+\mathrm{Tes} \operatorname{cof}(2 \eta-2 s)+V_{e s} \operatorname{col}(2 y+2 s)\right.$

Q

\section{CARUT}


vbi. quidem pro 4 et $A$ valores fupra inuentos com-

pletos accipi oporter, ita $v t$ in is termini akk et akk

:omfint comprebenfi; erit ergo

$$
\begin{array}{ll}
\mathfrak{A}=-0,81033 & 1-\mathfrak{A}=9,908662 . \cdot \\
\mathrm{A}=-1,31773 & 1-\mathrm{A}=0,119826
\end{array}
$$

Valores autem hinc deriuati erunt:

$$
\begin{aligned}
& \frac{2 \times \mathrm{A}+M}{n \prime}=-0,0 \times 9744, \frac{-(2 \times \mathrm{A}+\mathscr{A})}{\#}=8,29544^{2} \\
& \mathrm{~A}^{\prime}=-2,47576 \quad /-\mathrm{A}^{\prime}=0,393708
\end{aligned}
$$$$
\text { : } 24=+0,01036 \quad l 2 x^{\prime}=8,015347
$$

Terminos autem angulum guadruplum $4 \eta$ inuoluente's hic ob fummam paruitatem omifi, quoniam in combinatione cum angulo s plane fierent imperceptibiles.

§. 14t. Hine iam primo colligitur:

$$
\begin{aligned}
& \frac{d \varphi}{d r}=x-\frac{(2 x A+P)}{\eta n} \operatorname{cof} 2 y-\frac{(2 x P+Y)}{n n} e \operatorname{cor} s \\
& -\frac{(2 x Q+S)}{n n} e \cot (n y-s)-\frac{(2 x R+\xi R)}{n n} i \operatorname{col}(2 n+s) \\
& \left.-\frac{(2 x S+5)}{n \pi} \times \cos 2\right)^{\circ} \\
& -\frac{(2 x T+F)}{n n} \operatorname{aeco}(2 y-2 s)-\frac{(2 x V+23)}{n n} \operatorname{eccof}(2 y+2 s)
\end{aligned}
$$

atque porro

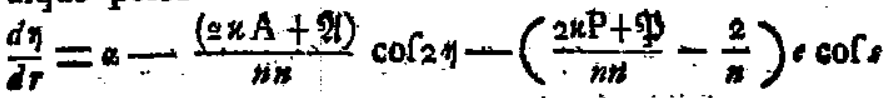

$$
\begin{aligned}
& -\frac{(2 k Q+\beta)}{n z} \cdot \operatorname{cof}(2 y-s)+\frac{(2 k \mathrm{R}+9 \mathrm{k})}{n n} \cdot \operatorname{cof}(2 \eta+s) \\
& -\left(\frac{(2 k S+\phi)}{n n}-\frac{t}{2 n}\right) e \cot 2, \cdots \cdots
\end{aligned}
$$

Deinde quia eft proxime $k k=9 e e$, exic

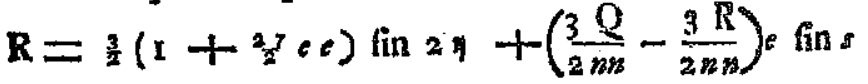

$$
\begin{aligned}
& -\left(\frac{3}{4}-\frac{3 \mathrm{P}}{2 m n}\right) e f \mathrm{fn}(2 \eta-s)-\left(\frac{3}{4}-\frac{3 \mathrm{P}}{2 n n}\right) e \mathrm{fin}(2 \eta+s) \\
& +\left(\frac{3 \mathrm{~T}}{2 n n}-\frac{3 \mathrm{~V}}{2 n n}\right) e e \text { fin } 2 s \\
& +\left(\frac{g}{8}+\frac{3 S}{2 n n}\right) e e f\left(n n(2 \eta-2 s)+\left(\frac{8}{8}+\frac{3 S}{2 n n}\right)=e \ln (2 \eta+2 s)\right.
\end{aligned}
$$

atque omiffis terminis, quibus nọn eft opus

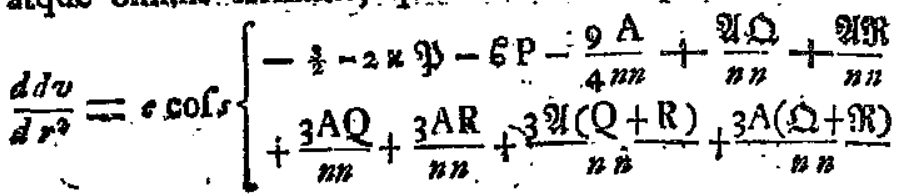

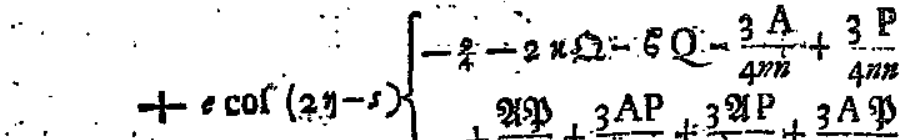

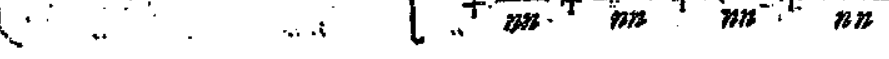

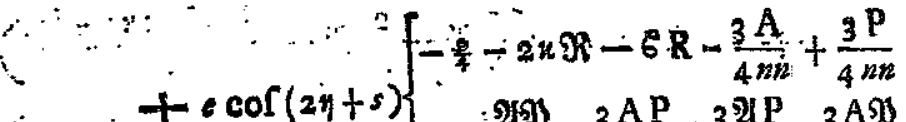

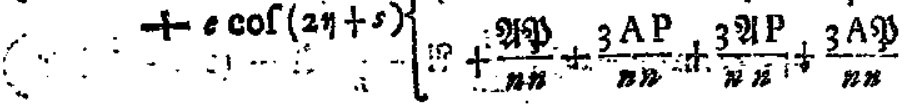

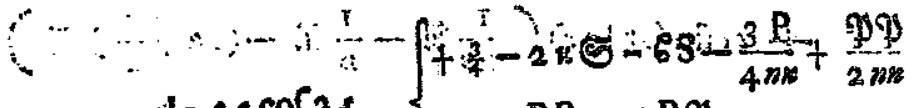

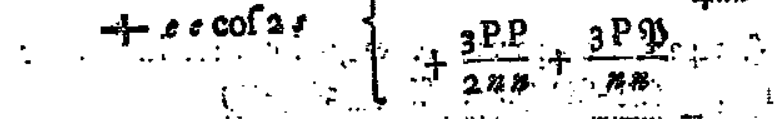

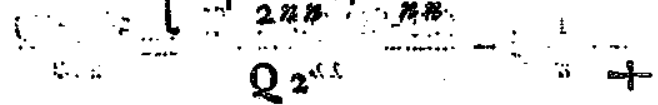


$+x \cos (2 \pi-2 s)\left\{\begin{array}{l}+8-2 x \xi-6 \mathrm{~T}-\frac{9 \mathrm{P}}{8 m}+\frac{9 \mathrm{~S}}{n} \\ +\frac{3 \mathrm{AS}}{n n}+\frac{34 \mathrm{~S}}{n n}+\frac{3 \mathrm{AS}}{n n}\end{array}\right.$

$+\operatorname{cocol}(2 n+2 s)\left\{\begin{array}{l}+8-2 \pi 9-6 V-\frac{9 P}{8 n}+\frac{\pi S}{n} \\ +\frac{3 A S}{m}+\frac{32 S}{n}+\frac{3 A S}{n}\end{array}\right.$

§. 142. Quodif iam forme pro $/ \mathbf{R} d r$ affumta differentietur, orietur :

$R=-2 \times \mathfrak{A}$ fin $2 \pi$

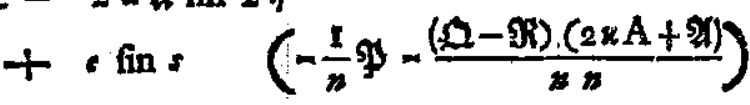

$+\ln (2 \eta-s)\left(-\frac{2 \pi}{n} \pm \frac{9(2 x \mathrm{P}+9)}{n \pi},\left(2 x-\frac{1}{n}\right) \mathbb{Q}\right)$

$+e$ fin $(2 \pi+s)\left(-\frac{2 \mathfrak{A}}{n}+\frac{\mathfrak{A}(2 x \mathrm{P}+\mathfrak{P})}{n \pi}-\left(2 a+\frac{1}{n}\right) \mathfrak{R}\right)$

$+c \sin 2+\left(\frac{1}{n} g_{3}-\frac{2}{n} S-\frac{(2-2)(2 n A+\pi)}{n n}\right)$

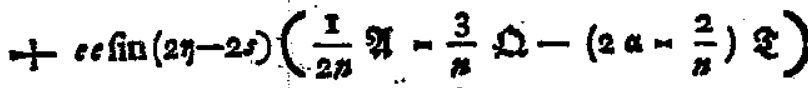

$+\operatorname{ecfin}(2 y+2 s)\left(\frac{I}{2 \pi} x-\frac{I}{x} g-\left(2 a+\frac{2}{\pi}\right) 23\right)$

6. 143. Comparatione ergo inftituta habebitur $-\frac{1}{n} \mathfrak{p}-\frac{(2-9 R)(2 \times A+9)}{m}=\frac{3(Q-R)}{2 m}$
$C A P B T I S$.
$\frac{\mathrm{P}}{m}+\frac{\mathscr{H S}}{n s}$

4 S

$\frac{p}{n}+\frac{\mathscr{S}}{n}$

巨

mea dif-

II)

$-\left(\frac{1}{n}\right)(2)$

$\left.\left.\frac{x}{n}\right) \Re\right)$

$(\underline{A+2})$

$\left(\frac{2}{2}\right)$ ह)

) $)$

tur

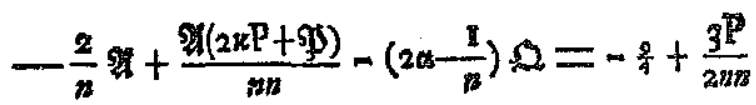

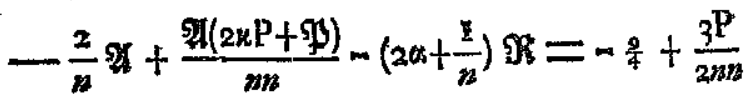

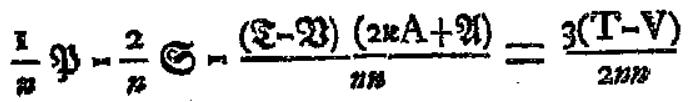

$\frac{1}{2 x} \mathfrak{x}-\frac{3}{n} \mathfrak{Q}-\left(2 a-\frac{2}{n}\right) \mathfrak{z}=\frac{2}{8}+\frac{3 S}{2 n n}$

$\frac{1}{2 n} \mathfrak{A}-\frac{1}{n} \mathrm{R}-\left(2 a+\frac{2}{7}\right) \mathfrak{B}=\frac{2}{8}+\frac{3 \mathrm{~S}}{2 m n}$

vnde deinceps valores litterarum germanicarum $;$ $\mathfrak{R}, \mathfrak{S}, \mathfrak{Z}, \mathfrak{B}$ fumus inueltigaturi.

5. 144. Differentietar fimili modo quantitas $v$, $2 \dot{9}$ ponatur :

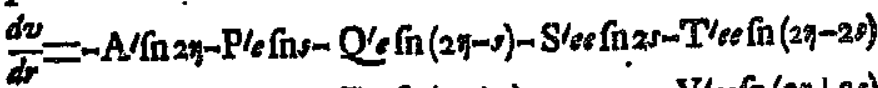

eritque

$-\mathrm{R} / \epsilon \ln (2 \eta+s) \quad-\mathrm{V}^{\prime} e e \mathrm{fn}(2 \eta+2 s)$

$A^{\prime}=2 a 54$, cuius quidem valor iam fupra habetur

$\mathrm{P}^{\prime}=\frac{1}{n} \mathrm{P}+\frac{(\mathrm{Q}-\mathrm{R})(2 \mathrm{~A}+\mathrm{M})}{m}$

$\mathrm{Q}=\left(2 m-\frac{1}{n}\right) \mathrm{Q}+\frac{2}{n} \mathrm{~A}-\frac{\mathrm{A}(2 \times \mathrm{P}+9)}{m}$

$\mathbf{R}^{\prime}=\left(2 a+\frac{1}{n}\right) \mathbf{R}+\frac{2}{n} \mathrm{~A}-\frac{\mathrm{A}(2 u \mathrm{P}+\Omega)}{m}$

$S^{\prime}=\frac{2}{n} S-\frac{1}{m} P+\frac{(T-V)(2 n A+F)}{Q}$

Q 3 
$\mathrm{T}=\left(2 \alpha-\frac{2}{n}\right) \mathrm{T}+\frac{3}{n} \mathrm{Q}-\frac{\mathrm{r}}{2 n} \mathrm{~A}$

$V^{\prime}=\left(2 a+\frac{2}{\pi}\right) \mathrm{V}+\frac{\mathrm{I}}{B} \mathrm{R}-\frac{\mathrm{I}}{2 x} \mathrm{~A}$

$-\frac{9}{4}-2 k 5 \pi-6 \mathrm{R}-\frac{3 \mathrm{~A}}{4 m n}+\frac{{ }_{3} \mathrm{P}}{4 n n}+\frac{(2 \mathrm{~A}+3 \mathrm{~A}) \mathrm{9}}{n n}+\frac{(35 \mathrm{~A}+3 \mathrm{~A}) \mathrm{P}}{m n}=$ $-\left(2 a+\frac{1}{3 z}\right) R^{\prime}-\frac{2}{n} A^{\prime}+\frac{A^{\prime}(z n P+P)}{n n}$

vnde denuo differentiando eruitux.

$\frac{d d v}{d r^{2}}=c \cos s\left(-\frac{1}{n} \mathrm{P}^{\prime}+\frac{\left(\mathrm{Q}^{\prime}+\mathrm{R}\right)(2 \times \mathrm{A}+\mathrm{m})}{n \dot{x}^{2}}\right.$

e $\operatorname{cor}(2 x-5)\left(-\left(2 x-\frac{1}{8}\right) Q^{\prime}-\frac{2}{8} A^{\prime}+\frac{A^{\prime}\left(2 x P^{P}+9\right)}{2 n}\right.$

$-\operatorname{coc}(2 y+s)\left(-\left(2 \alpha+\frac{1}{n}\right) \mathrm{R}^{\prime}-\frac{2}{n} \mathrm{~A}^{\prime}+\frac{\mathrm{A}^{\prime}(2 x \mathrm{P}+\mathrm{N})}{m n}\right.$

$e c \operatorname{col} 2 s\left(-\frac{2}{n} \mathrm{~S}^{\prime}+\frac{\mathrm{r}}{z} \mathrm{P}^{\prime}+\frac{\left(\mathrm{T}^{\prime}+\mathrm{V}^{\prime}\right)}{n n} \frac{(2 x \mathrm{~A}+\mathrm{m})}{n n}\right.$

ce $\operatorname{cor}(2 n-2 s)\left(-\left(2 a-\frac{2}{n}\right) \mathrm{T}^{\prime}+\frac{1}{2 n} \mathrm{~A}^{\prime}-\frac{3}{n} \mathrm{Q}^{\prime}\right.$

$x \operatorname{col}(2 y+2 s)\left(-\left(2 x+\frac{2}{n}\right) V^{\prime}+\frac{I}{2 n} A^{\prime}-\frac{I}{n} R^{\prime}\right.$

4. 145. Sequentes ergo aequationes refóluendà occurrent

$-\frac{3}{2}-2 k \mathfrak{S}-6 \mathrm{P}-\frac{9 \mathrm{~A}}{4^{n}}+\frac{(\mathfrak{W}+3 \mathrm{~A})(\mathrm{Q}+\mathrm{R})}{m}+\frac{(3 \mathcal{P}+3 \mathrm{~A})(\mathrm{Q}+\mathrm{R})}{n n}=$

$\begin{aligned}+\frac{3}{4}-2 x S-6 S-\frac{3 P}{4^{m}}+\frac{2}{2 n n} & -\frac{2}{n} S^{\prime}+\frac{I}{n} P^{\prime}+\frac{\left(T^{\prime}+V^{\prime}\right)(2 n A+P)}{m}=\end{aligned}$

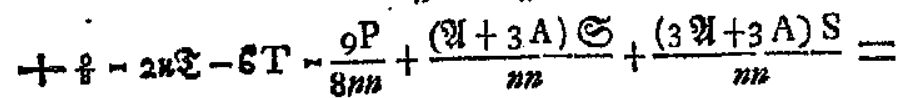
$-\left(2 a-\frac{2}{n}\right) \mathrm{T}^{\prime}+\frac{\mathrm{I}}{2 n} \mathrm{~A}^{\prime}-\frac{3}{n} \mathrm{Q}^{\prime}$

$+8-2 k 23-6 \mathrm{~V}-\frac{9 \mathrm{P}}{8 n+3}+\frac{(\mathfrak{A}+3 \mathrm{~A}) \mathrm{S}}{n n}+\frac{(3 \mathfrak{M}+3 \mathrm{~A}) \mathrm{S}}{n n}=$

$$
-\left(2 \theta+\frac{2}{n}\right) V^{\prime}+\frac{I}{2 n} A^{\prime}-\frac{1}{n} R^{\prime}
$$

Neglectis primo terminis minimis, qui adhuc funt incogniti, reperitur :

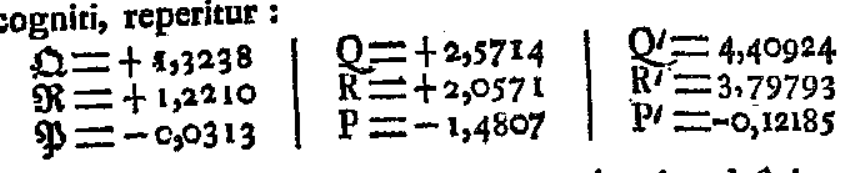

$\therefore-\frac{I}{m}+\frac{\left(Q^{\prime}+R\right)(2 x A+2)}{m}$

§. 146. Ex his autem accuratius ita definientur ie fic:

$$
\begin{aligned}
& \alpha=+1,33859 \cdot \therefore \cdot 19=0,126649 \\
& \Re=+1,23468 \cdots, l \mathfrak{R}=0,0.91545 \\
& \mathrm{Q}=+2,60087 \ldots . t \mathrm{Q}=0,415119 \\
& \mathbf{R}=+2,00590 \text {. . } \quad l \mathrm{R}=0,302308 \\
& Q^{\prime}=4,4480 t ; R^{\prime}=3,6758 \pi
\end{aligned}
$$


$\$ 28$

$$
\text { CAPUT IX }
$$

et $P=-0,04010 \cdot \cdot \ln =8,603 \times 33$ $\mathrm{P}=-1,46488 \cdot \ldots \ln =0,165801$

Deinde reperitur

$$
P=-0,12222
$$

Deinde vero erit

log. coeffi

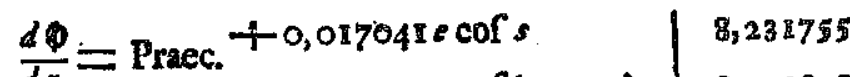

$$
\begin{aligned}
& -0,037487 e \operatorname{cof}(2 \text { y }-s) \quad 8,573878 \\
& -0,030062 \in \operatorname{cor}(2 \eta+4) \quad 8,478023 \\
& \text { - - } 0,00722 e e \cot 25 \quad 7,858166 \\
& +0,03470<e \operatorname{cor}(2 y-2 s) \quad 8,540319 \\
& +0,01533 * \operatorname{cor}(2 y+2 s) \cdot 8,185614
\end{aligned}
$$

$\mathrm{T}=-2,60380 \ldots .4 \mathrm{~T}=0,465615$

$V=-1,02720$. . \& $4 \mathrm{~V}=0,01 \times 672$

Pro Rquentibus vero calculis eft

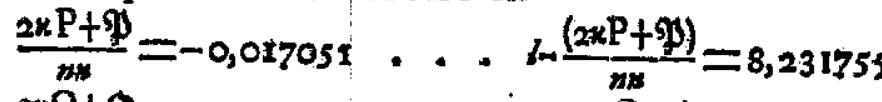
$\frac{2 k \mathrm{Q}+\mathrm{Q}}{m n}=+0,037487 \cdots+\frac{2 k \mathrm{Q}+\mathrm{Q}}{n n}=8,573878$ $\frac{2 \times R+\Re}{m}=+0,030062 \cdot . \cdot 4+\frac{2 \times R+9 R}{m n}=8,478023$

§. 147. Hine igitur pro diftantia lúnae a terra curtata $*=\frac{(I-k k) a s}{I-k \operatorname{cor} r}$, pars quantitatis $*$ ab excentricitate orbitae folaris tancum pendens erit

$n=$ Praeced. - 0,008336e cols log. coeff. $+0,014801$ e cor $(2 \eta-s)$ $+0,0$ rryise col $(2 y+s)$ 7,920985 + 0,00364 ese corzs 8,170303 8,057492 $-0,01482 e t \operatorname{col}(2 y-2 s)$ 7,539670 $-0,00584$ ce $\operatorname{cor}(2 y+2 x)$ 8,170799 7,766856 Deinde

6. 148. Ponatur nunc intograle

$$
\begin{aligned}
& \phi=\text { Praec. }+ \text { Hin } 2 \eta+y^{\prime} e \text { fin } \\
& +\mathfrak{Q}^{\prime} \text { efin }(2 \eta-s)+\mathfrak{R}^{\prime} \text { e fin }(2 y+r) \\
& +5^{\prime} e f\left(i n 2 s+\mathfrak{S}^{\prime} e \operatorname{efin}(2 x)-2 s\right)
\end{aligned}
$$

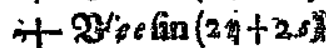

erit differentiatione peracta

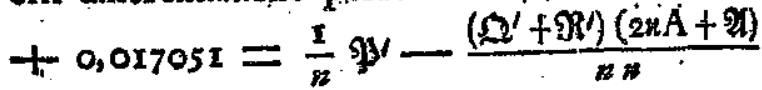

$$
\begin{aligned}
& -0,037439=\left(i x-\frac{1}{x}\right) \alpha^{\prime}+\frac{2}{x}-\frac{m^{\prime \prime}(2 x P+Y)}{n}
\end{aligned}
$$

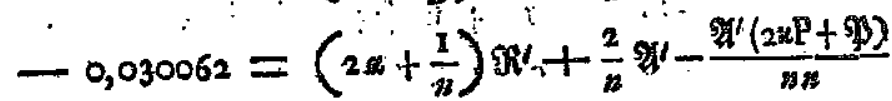

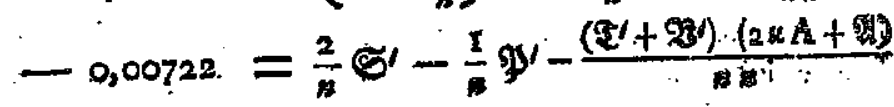

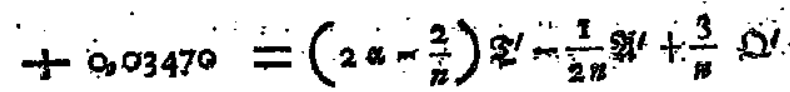

$$
\begin{aligned}
& \rightarrow 0,07533=\left(2 a+\frac{2}{n}\right) 23-\frac{1}{2 n} \mathbb{F}+\frac{1}{m} \\
& \therefore \\
& \text { R } \\
& \text { fietque }
\end{aligned}
$$


fietque his valoribus determinatis

$$
\begin{aligned}
& \varphi=\text { Praec. }+0,2360346 \text { fin } \\
& -0,021889 \cdot \operatorname{fin}(2 y-s) \\
& -0,016368 \text { fin }(2 y+s) \\
& \text { + } 0,06615 \text { ee fin } 2 s \\
& +0,02332 \text { ef in }(2 y-2 s) \\
& +0,00840 \text { ie fin }(2 \eta+2 s) \mid 7,924429
\end{aligned}
$$

log. coeff.

8. 149. Reducamus has ingequalitates etiam ad m?nuts fecunda, ponendo excentricitatem oxbitae folaris $c=0,01680$, atque habebimus

log. coeff.

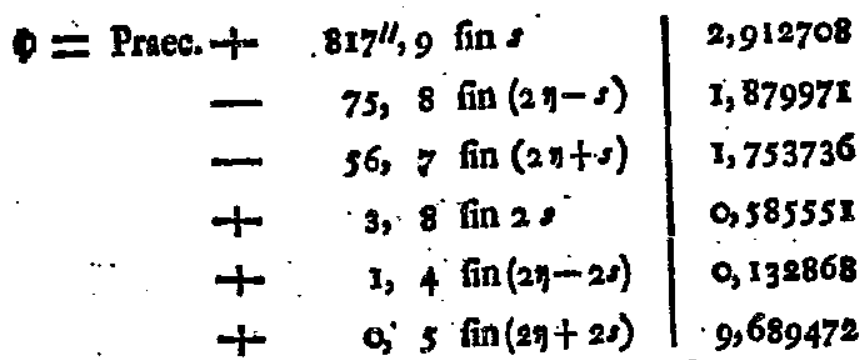

Denotat hic s anomaliam veram folis; vnde patet eam Lunae inaequalitatem, quae finui huitis anomaliae eft proportionalis, admodum effe notabilem, dum ad $13^{\prime}, 38^{\prime \prime}$ exfurgit: Tabulae. autern Aftronomicae, vbi haes inaequalitas aequatio folaris nominatur, eam multo minorem faciunt, cuius rei caufam inueftigari adhue canveniet,

6. 150.

$$
\text { CAET IR }
$$

6. 150. Quodfi enim lizteram accuratius defini. re velimus, habebimus has formulas refoluendas:

coeff.

772974

40237

14002

20508

67825

24429

1 ad ml-

I folaris

coeff.

12708

79971

$\$ 3736$

35551

;2868

39472

tet eam

liae eft

13', 38"

aec in-

mino-

c con-

6. 550.

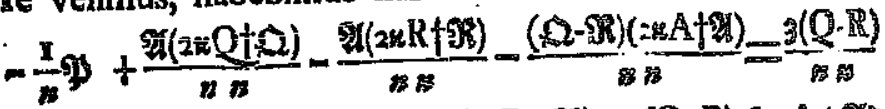

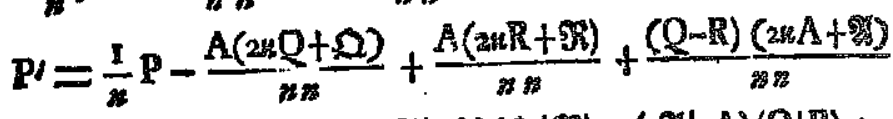

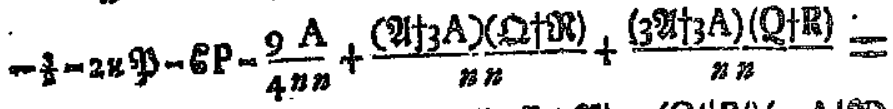

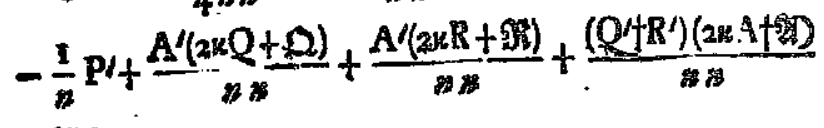

tnde elicimus:

$P=-0,1183 ; P=-x_{2} 1356 ; \quad \frac{P}{n z}=-0,0064$

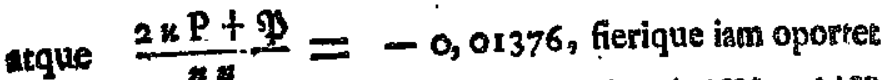

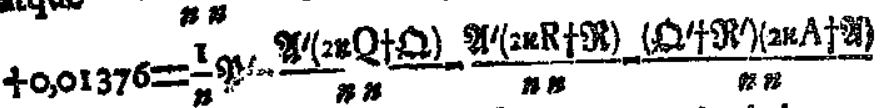
vnde oritur $\mathfrak{F}^{\prime}=+0,201385$. Quare accuratius habernus - = Praec. - $0,006400<\operatorname{cof} \delta \quad 7,806180$ $d \Phi=$ Prace. $+0,013760$ ecols 8,138688 $\phi \equiv$ Praec, $+0,201385+6 \mathrm{in} . \quad 9,304026$ Cu

$\varphi=$ Prace +70 III $^{\prime \prime}$ in $\&$ 2,845780 Ergo aequatio finui Angthi - proportionalis eft 1I', 41". 


\section{A P U T $\mathrm{X}$.}

INUESTIGATIO INAEQUALITATVM LUNAE

AB VTRIUSQUE ORBITAE EXCENTRICITATE

SIMUL PENDENTIUM.

\section{151.}

Q oniam praeuidemus inaegualitates huius generis; quae altiores litcerarum $k$ et $e$ poteftates fimul complectuntur, minimas effe futuras, alios terminos non fcrutabimur, nifi qui producto fimpliei ct fint affecti. Habebimus ergo

$\frac{d s}{d r}=\frac{d \theta}{d r}=\frac{1}{n}+\frac{2}{n} k \cos r-\frac{2}{n} e \operatorname{cof} s-\frac{2}{n} e k \operatorname{cof}(r-s)$

$$
-\frac{2}{n}+k \operatorname{cor}(r+s)
$$

Cum jgitur ad hanc inneftigationem opus non fit illis terminis ex praecedentibus, gui vel per $k^{2}$ vel per $e^{2}$ erant affeeti, quia litterae alphabethi deficere incipiunt, litteris S, T tet feguentibas denuo utemur; quare cervendum, ne iftae liteerae cum ante adhibitis confun dantur.

8. 152. Aftumtis ergo ex terminis iam ante definitis, iis qui in cos, quos iam inueftigamus, vim exfe-

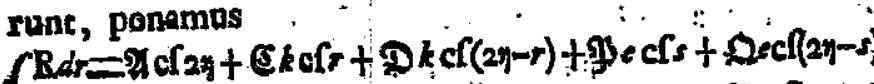

$$
+\operatorname{ekcr}(2 y+r) \quad+\operatorname{Rec}(2 \eta+\gamma)
$$

$+S e k \operatorname{co}\left[(r-s)+23\right.$ ek cor $\left.\left(2 \eta_{i}-r+s\right)+9\right) \cos (2 \eta-r-s)$

$+2 e k \operatorname{cof}(r+s)+2 a k \operatorname{cof}(2 n+r+s)+3 e k \operatorname{cof}(2 n+r+s)$

\section{$C A P T \quad X$}

尌的

$\nu=\mathrm{A} \operatorname{cor} 2 \mathrm{t} \ldots+\mathrm{D} k \operatorname{cor}(2 r-r)+\mathrm{Pecor} s+Q e \operatorname{cor}(2 \eta-s)$

$$
+\mathrm{E} k \operatorname{cor}(2 y+r) \quad+\mathrm{R} e \operatorname{cor}(2 y+s)
$$

INAE

TE

ineris;

fimul

$s$ ter-

ici ẹt

s)

s)

$t$ illis

er $e^{2}$

junt,

:e car-

nfun-

defi-

exfe-

$(2 \eta-5)$

$(\eta+s)$

$-r-s)$

$r+s)$

$v=$

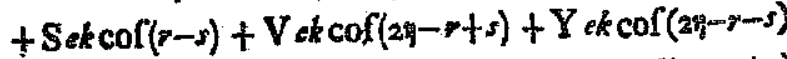

$+\mathrm{T}$ ek $\operatorname{col}(r+s)+\mathrm{X}$ ek cof $(2 n+r-s)+\mathrm{Z} e k \operatorname{cor}(2 r i+r+s)$

erikque ex praecedentibus

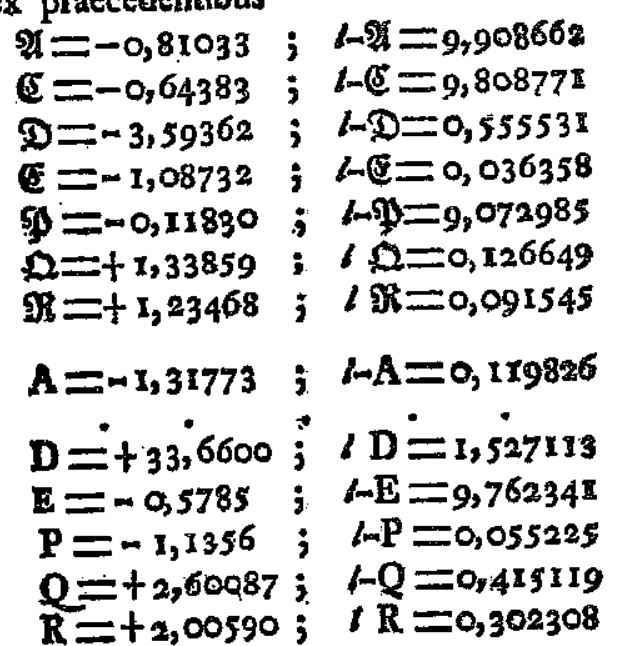

5. 153. Reliqui vero valores hinc derivati, guibus opus habemus, fune:

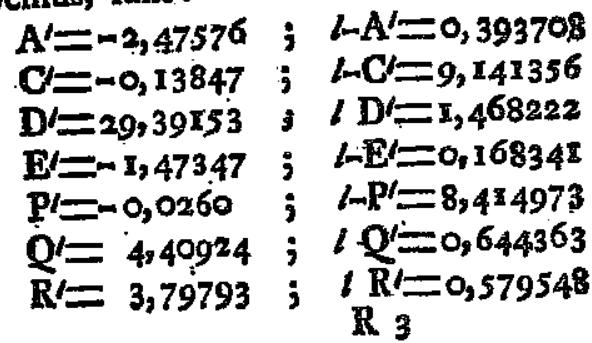

留 


$$
\begin{aligned}
& w^{\prime}=+0,01036 ; \quad \mid W^{\prime}=8,0 \times 5347 \\
& \mathfrak{C}^{\prime}=+0,01015 ; \quad l \mathbb{E}^{\prime}=8,006295 \\
& D=-0,42023 ; \quad L D=9,623483 \\
& \text { CE }=+0,00499 ; \quad b-E I=7,698261 \\
& y=+0,20138 ; l y=9,304016 \\
& \mathfrak{Q}^{\prime}=-0,02189 \cdot ; \quad \ln =8,340237 \\
& \mathfrak{W}=-0,01637 ; l-\mathfrak{W}=8,214002
\end{aligned}
$$

Sit breuitatis gratia

$$
\begin{aligned}
& I^{\prime}=\frac{2 n \mathrm{~A}+4}{n n}=-0,019744 ; \quad \ln \frac{(2 n \mathrm{~A}+2 n)}{m n}=8.295442 \\
& y=\frac{2 \times \mathrm{D}+9}{m}=+0,366 \mathrm{Ir} ; \quad l \frac{2 \times \mathrm{D}+9}{m}=9,563604 \\
& I^{\prime}=\frac{2 \times E+E}{m}=-0,01283 ; \quad l-\frac{(2 \times E+(e))}{m}=8,108292 \\
& \cdot f=\frac{2 \times P+9)}{m}=-0,0,1376 ; \quad t-\frac{(2 \times P+93)}{m}=8,138618 \\
& x^{\prime}=\frac{2 k Q+0}{25}=+0,03749 ; \quad \frac{2 k Q+Q}{m}=8,573878 \\
& N=\frac{2 \times R+15}{n}=+0,03006: \quad l \frac{2 \times R+9 R}{\infty}=8,478023
\end{aligned}
$$

5. 154. Si fimili modo viterius ponatur:

$$
\begin{aligned}
& s=\frac{2 x S+E}{2 x} ; \mu=\frac{2 x T+q}{m} ; v=\frac{2 x V+2}{m} ; \\
& x=\frac{2 x X+x}{x} ; y^{\prime}=\frac{2 x Y+9}{m} ; x^{\prime}=\frac{2 x Z+3}{m}
\end{aligned}
$$

habe. habebimus :

$\frac{d P}{d r}=$ Praec. $-s^{\prime} \mathrm{cf} 2 \eta-\frac{\mathbb{C}}{m n} k \mathrm{cfr}-d^{z} k \mathrm{cf}(2 \eta-r)-p^{\prime} e \mathrm{cfr}-q^{q} e \mathrm{cl}(2 \eta m s)$ $-\sec (2 y+r) \quad-r^{\prime} \mathrm{c}[(2 y+s)$

- slek $\operatorname{cof}(r-s)-v i e k \operatorname{cof}(2 \eta-r+s)-y^{\prime}$ ek $\operatorname{cof}(2 \eta-r-s)$

$-t^{\prime}$ ek $\operatorname{cor}(r+s)-x^{\prime} \operatorname{ckcof}(2 \eta+r-s)-z^{\prime} e k \operatorname{col}(2 \eta+r+s)$ atque pofito $\frac{2}{y}+\frac{c}{z y}=d=0,147 \times 97 ; l d=9,167900$

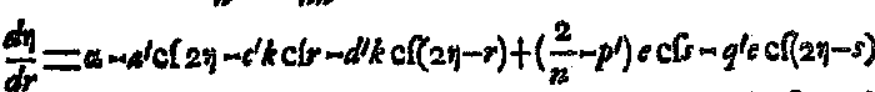
$-c^{\prime} k \mathrm{c} \int(2 \eta+r)$ $\rightarrow p(t) \mathrm{c}(2 \eta+s)$

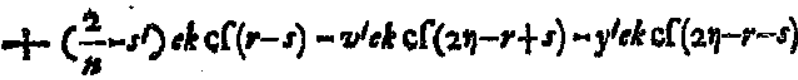
$+\left(\frac{2}{z}-k\right) e k \mathrm{c}(r+s)-x x^{\prime} e k \mathrm{c}(2 \eta+r-s)-z^{\prime} e k \mathrm{c}(2 y+r+s)$ vi cum fit $\frac{2}{n}=0,150876$, exit $\frac{2}{m}-p^{\prime}=0,16464$

6. 155. Nunc termini coefficiente o $k$ affeti, gui in formis $\mathrm{R}=\mathrm{et} \frac{d d v}{d r^{2}}$ infunt, colligantur: eritgue

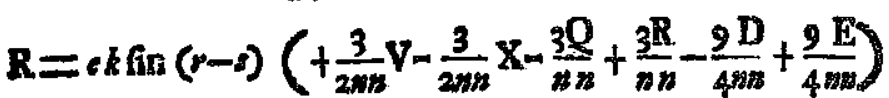

$$
\begin{aligned}
& i k \text { fin }(r+s)\left(+\frac{3}{2 m m} Y-\frac{3}{2 m} \mathrm{Z}-\frac{3 \mathrm{R}}{3 m}+\frac{3 \mathrm{Q}}{\pi n}+\frac{9 \mathrm{E}}{4 m}-\frac{9 \mathrm{D}}{4 m}\right) \\
& \text { ok fin }(2 y-r+s)\left(-\frac{3}{2}+\frac{3}{2 \pi n} S+\frac{3 P}{n n}\right) \\
& \text { es } \sin (2 \pi+r-s)\left(-3+\frac{3}{2 m s} s+\frac{3 P}{3 n}\right)
\end{aligned}
$$


ek [in $(2 n-r-p)\left(-2+\frac{3}{2 * n} \mathrm{~T}+\frac{3 \mathrm{P}}{n n}\right)$

ek fin $(2 n+r+p)\left(-2+\frac{3}{2 m n} \mathrm{~T}+\frac{3 \mathrm{P}}{n n}\right)$

et $\quad \frac{d d v}{d r^{2}}=$

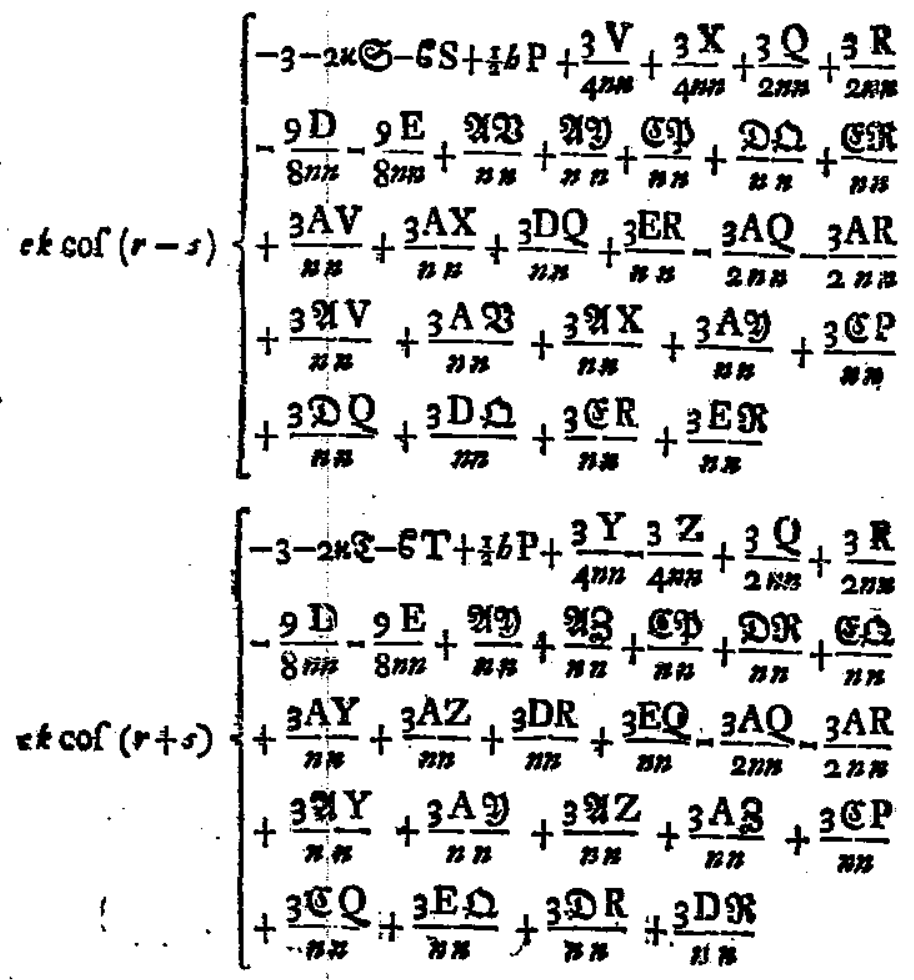

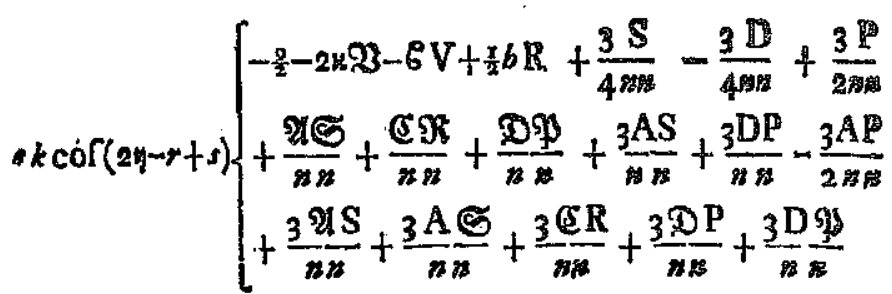

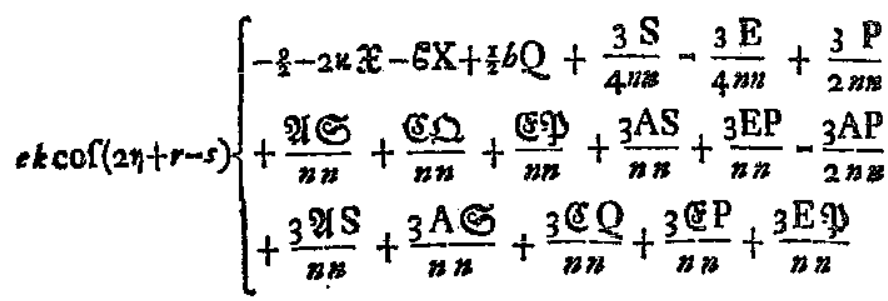

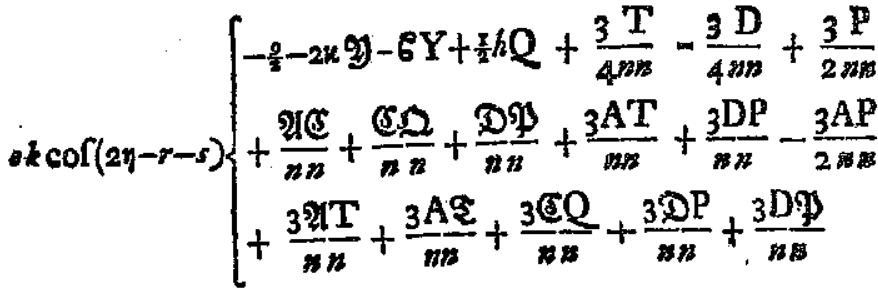

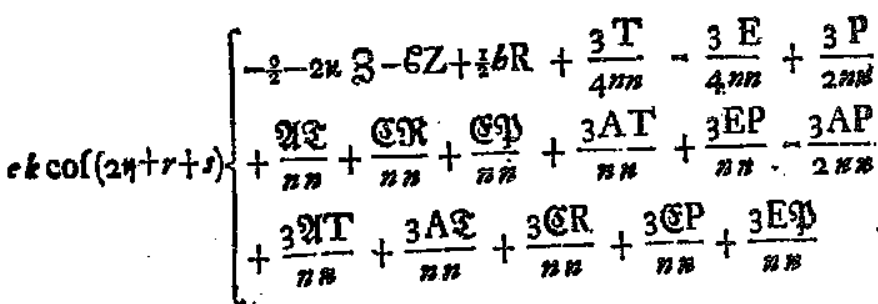
s 
§. 156. Quseramus ergo quoque ex formula asfumta $\int \mathrm{R} d r$ differentiale, quod exit

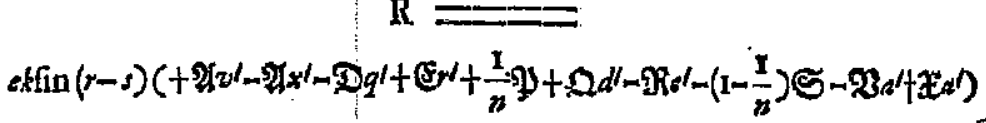

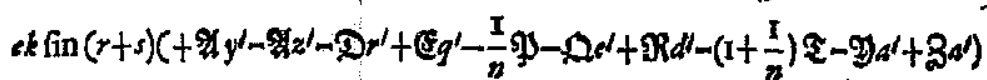

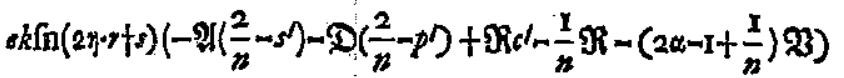

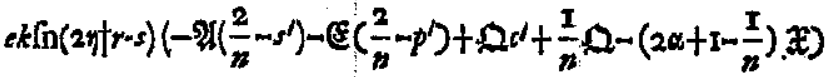

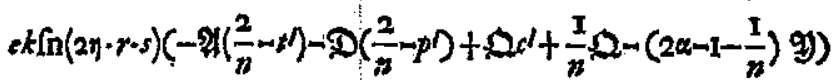

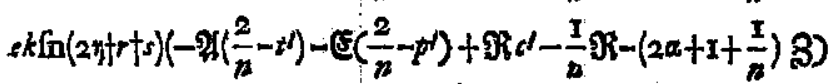

5. 157. Ponatur ex differentiatione formae $v$; $\mathrm{S}^{\prime}=\left(1-\frac{\mathrm{T}}{n}\right) \mathrm{S}-\mathrm{A}\left(\mathrm{v}^{\prime}-x^{\prime}\right)+\mathrm{D} q^{\prime}-\mathrm{E} r^{\prime}-\frac{1}{n} \mathrm{P}-\mathrm{Q} d^{\prime}+\mathrm{R}^{\prime}+(\mathrm{V}-\mathrm{X}) \mathrm{al}^{\prime}$ $\mathrm{T}^{\prime}=\left(\mathrm{I}+\frac{\mathrm{I}}{n}\right) \mathrm{T}, \mathrm{A}\left(\mathrm{g}^{\prime}-z^{\prime}\right)+\mathrm{D} \boldsymbol{r}^{\prime}-\mathrm{E}_{q^{\prime}}+\frac{\mathrm{I}}{n} \mathrm{P}+\mathrm{Q}^{\prime}-\mathrm{R} d^{\prime}+(\mathrm{Y}-\mathrm{Z}) \mathrm{a}^{\prime}$ $V^{\prime}=\left(2 \alpha-1+\frac{1}{n}\right) V+A\left(\frac{2^{\prime}}{n}-s\right)+D\left(\frac{2}{n}-p^{\prime}\right)-R d^{\prime}+\frac{I}{n} R$ $X^{\prime}=\left(2 a+I-\frac{1}{n}\right) X+A\left(\frac{2}{n}-M\right)+E\left(\frac{2}{n}-p\right)-Q c^{\prime}-\frac{1}{n} Q$ $Y^{\prime}=\left(2 a-I-\frac{1}{n}\right) Y+A\left(\frac{2}{n}-p^{\prime}\right)+D\left(\frac{2}{n}-p^{\prime}\right)-Q^{\prime}-\frac{T}{n} Q$ $\mathrm{Z}^{\prime}=\left(2 a+\mathrm{I}+\frac{\mathrm{I}}{n}\right) \mathrm{Z}+\mathrm{A}\left(\frac{2}{n}-b_{n}\right)+\mathrm{E}\left(\frac{2}{n}-p\right)-\mathrm{R} \mathrm{c}^{\prime}+\frac{\mathrm{I}}{n} \mathrm{R}$ ila as- vt habeatur

$\frac{d v}{d r}=-\mathrm{A}^{\prime} \sin 2 y-\mathrm{C}^{\prime} k$ fin $r-\mathrm{D}^{\prime} k \operatorname{fin}(2 y-r)-\mathrm{E}^{\prime} k \sin (2 \eta+r)$

- $\mathrm{P}^{\prime} e \operatorname{fin} s-\mathrm{Q}^{\prime} \operatorname{efin}(2 y-s)-\mathrm{R}^{\prime} e \operatorname{fin}(2 y+s)$

$m S^{\prime} e k f i n(r-s)-V^{\prime} e k f i n(2 \eta \eta-r+s)-Y^{\prime} e k f i n(2 y-r-s)$

$-\mathrm{T}^{\prime}$ ekfin $(r+s)-\mathrm{X}^{\prime}$ ekfin $(2 \eta+r-s)-\mathrm{Z}^{\prime} e^{\prime} \mathrm{k}^{\prime} \mathrm{in}(2 \eta+r+s)$

6. 158. Haec iam forma denuo differentiata dabit $\frac{d d v}{d r^{2}}=$ Praec. .

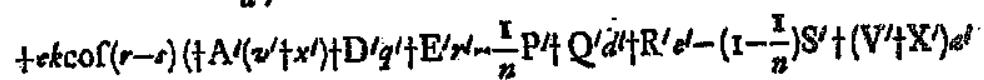

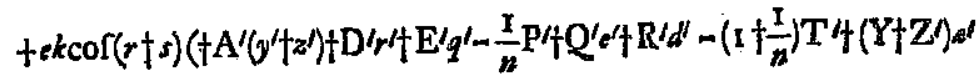
tekcr $(2 \eta-r+s)\left(-\mathrm{A}^{\prime}\left(\frac{2}{n}-s\right)-\mathrm{D} /\left(\frac{2}{n}-p \eta+\mathrm{R}^{\prime} c^{\prime}-\frac{\mathrm{I}}{n} \mathrm{R}^{\prime}-\left(2 \alpha-i \dagger \frac{\mathrm{I}}{n}\right) \mathrm{V}^{\prime}\right)\right.$ $+\operatorname{ckc}(2 n+r-s)\left(-\mathrm{A}^{\prime}\left(\frac{2}{n}-s^{\prime}\right)-\mathrm{E}^{\prime}\left(\frac{2}{n}-p^{\prime}\right)+\mathrm{Q}^{\prime} c^{\prime}+\frac{1}{n} \mathrm{Q}^{\prime}-\left(2 \alpha+1-\frac{1}{n}\right) \mathrm{X}^{\prime}\right)$ $+e k=(2 y-r m s)\left(-\mathrm{A}^{\prime}\left(\frac{2}{n}-t^{\prime}\right)-\mathrm{D}^{\prime}\left(\frac{2}{n}-p^{\prime}\right)+\mathrm{Q}^{\prime} c^{\prime}+\frac{\mathrm{I}}{n} \mathrm{Q}^{\prime}-\left(2 \alpha+x-\frac{1}{n}\right) \mathrm{Y}^{\prime}\right)$ tekc( $(2 n+r+s)\left(-\mathrm{A}\left(\frac{2}{n} z^{\prime}\right)-\mathrm{E}^{\prime}\left(\frac{2}{n}-p^{\prime}\right)+\mathrm{R}^{\prime} c^{\prime}-\frac{\mathrm{I}}{n} \mathrm{R}^{\prime}-\left(2 \alpha+1+\frac{\mathrm{I}}{n}\right) \mathrm{Z}^{\prime}\right)$

§. 159. Priores autem expreffiones, fi litterarum cognitarum valores fubftituantur, fequenti modo prodibunt. $\mathrm{R}=e^{k}$ fin $(r-s)(-0,44856+0,00854(\mathrm{~V}-\mathrm{X}))$

$c k$ fin $(r+s)(-0,42826+0,00854(\mathrm{Y}-\mathrm{Z}))$ ekfin $(2 \eta-r+s)(-4,51938+9,00854 S)$ ekfin $(2 \eta+r-s)(-4,51938+0,00854 \mathrm{~S})$ ekfin $(2 \eta-r-s)(-4,51939+0,00854 \mathrm{~T})$ $c k f i n(2 y+r+s)(-4,51938+0,00854 T)$ 
\$. 160. Altera vero forma pro $\frac{d d v}{d r^{2}}$ fit

$$
\frac{d d v}{d r^{2}}=
$$

ckcf $(r-5)(-2,83505-2 k$ S - $6 S-0,02711(23+X)-0,03207(V+X))$

$3207(\mathrm{~V}+\mathrm{X})$

$13207(\mathrm{Y}+\mathrm{Z}))$

$.0,03207 \mathrm{~S})$

$0,03207 S$

$0,03207 \mathrm{~T}$

$0,03207 \mathrm{~T}$

ck $\operatorname{cr}(2 y+r+s)\left(-\mathrm{r}, 43912-2 \times 3-6 \mathrm{Z}-0,02711 \mathrm{z}-0,03207^{\mathrm{T}} \mathrm{T}\right)$

6. 161. Deinde fimili modo alterae formulae per differentiationem erueae, fubftitutis valoribus cognitis ita fe habebiant.

$$
\begin{aligned}
& \text { re habebunt, } \mathbf{R}= \\
& k \operatorname{lin}\left(r-N^{\prime}\right)\left(+0,59883+24\left(w^{\prime}-x^{\prime}\right) \cdot\left(1-\frac{1}{n}\right) S\right)+0,01974(23-x) \\
& \text { ekfin }(r+5)\left(+0,54556+2\left(y^{\prime}-8\right)-\left(1+\frac{1}{n}\right)(2)+0,01974(9)-3\right) \\
& \text { ekfin }(2 \pi++s)\left(+0,80251+24 \sin \left(2 \alpha-1+\frac{1}{n}\right) 93\right) \\
& \text { ekfin }(2 h+r-s)\left(+0,59936+2 s^{\prime}-\left(2 a+1-\frac{1}{n}\right) x\right) \\
& \text { ekfin }(2 \eta-r-s)\left(+1,01199+x t^{\prime}-\left(2 \alpha-1-\frac{1}{n}\right) y\right) \\
& \text { ekfin }\left(2 n+\frac{t}{4} r+s\right)\left(+0,38988+24 k m\left(a+1+\frac{x}{n}\right) 3\right)
\end{aligned}
$$

mulae per cognicis ita

974(2)-

:974(9)-3)

(2)

(x)

(y)

(3)

Porro
Porro reperiemus fequentes valores

$$
\begin{aligned}
& S^{\prime}=\left(I-\frac{1}{n}\right) S-A\left(v^{\prime} / x^{\prime}\right)+0,38693 \sim 0,01974(V-X) \\
& T^{\prime}=\left(I+\frac{T}{z}\right) \mathrm{T}-A\left(y^{\prime}-z^{\prime}\right)+0,18088 m 0,01974(Y-Z) \\
& \mathrm{V}^{\prime}=\left(2 x-\mathrm{x}+\frac{\mathrm{I}}{n}\right) \mathrm{V}-\mathrm{A} \mathrm{s}^{\prime}+5,19902 \\
& X^{\prime}=\left(2 a+1-\frac{I}{n}\right) X-A s^{\prime}-0,87311 \\
& Y^{\prime}=\left(2 a-I-\frac{1}{g z}\right) Y^{\prime}=A t^{\prime}+4,7639 \mathrm{I} \\
& Z^{\prime}=\left(2 \alpha+1+\frac{1}{n}\right) \mathrm{Z}-\mathrm{A} \boldsymbol{A}^{\prime}-0,43800
\end{aligned}
$$

ac denique $\quad \frac{d d z}{d y^{2}}=$ Praec.

tekc $\left((r-s)\left(-\left(1-\frac{1}{x}\right) S^{\prime} \nmid A^{\prime}\left(v^{\prime}+x^{\prime}\right)+2,62592-0,01974\left(V^{\prime}+X^{\prime}\right)\right)\right.$ tekef(r+s)(-(it $\left.\left.\left.\frac{T}{n}\right) \mathrm{T} / \mathrm{A} / \mathrm{y}^{\prime} \mathrm{f}^{\prime}\right)+2,16417=0,0 \times 974\left(\mathrm{Y}+\mathrm{Z}^{\prime}\right)\right)$ $+a k \operatorname{cor}(2 y-r+s)\left(-\left(2 x-I+\frac{I}{n}\right) V^{\prime}+A^{\prime} y^{\prime}-4,19296\right)$ $+d k \operatorname{cof}(2 \eta+r-s)\left(-\left(2 a+1-\frac{1}{n}\right) \mathrm{X}^{\prime}+\mathrm{A}^{\prime} s^{\prime}+1,59777\right)$ $+e k \operatorname{cof}(2 y-r-s)\left(-\left(2 \omega-1-\frac{1}{n}\right) Y^{\prime}+A^{\prime} s^{\prime}-3,48384\right)$ $+e k \operatorname{cof}(2 y+r+s)\left(-\left(2 x+I+\frac{I}{g}\right) Z^{\prime}+A^{\prime} t^{\prime}+0,88865\right)$

$\mathbf{s}$ 
6. 162. Hinc ergo pro determiandis coefficientibus fequentes obtinemus aequationes

$\left(1-\frac{1}{*}\right) S=1,04739-0,00854(V-X)+\mathscr{H}(z / m-x)$ fo,01974(23-x)

$\left.\left(1+\frac{I}{s}\right) \mathfrak{s}=0,97382-0,00858(Y-Z)+2\left(y^{\prime}-x^{\prime}\right)+0,01974(9)-3\right)$

$\left(2 x-1+\frac{1}{n}\right) 2 B=5,32189-0,00854 S+A s$

$\left(2 x+1-\frac{1}{n}\right) \tilde{x}=5,11874-0,00854 \mathrm{~S}+x$ u

$\left(2 \alpha-1-\frac{1}{n}\right) \geqslant=5,53137-0,00854 \mathrm{~T}+24$

$\left(2 \alpha+1+\frac{1}{n}\right) 3=4,90926-0,00854 T+9 *$

Deinde

$+5,46097=\left(t-\frac{I}{n}\right) S^{\prime}-2 x S-E S-0,027 \mathrm{n}(2 \mathfrak{2}+\mathfrak{X})-0,03207(V+X)$ $-A^{\prime}\left(v^{\prime}+x^{\prime}\right)+0,01974\left(V^{\prime}+X^{\prime}\right)$

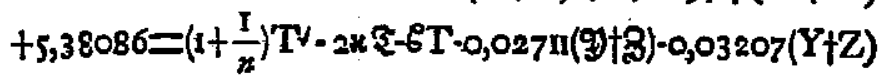
$-A^{\prime}\left(y^{\prime}+2^{\prime}\right)+0,01974(Y / Z Z)$ $-1,92: 371=\left(2 \alpha-1+\frac{1}{n}\right) V_{1}^{\prime}-2 x 23-6 V \cdot 0,027110 \cdot 0,03207 S \cdot A / d$ $+2,13218=\left(2 a+1-\frac{x}{n}\right) X^{\prime} \cdot 2 k x-6 X-0,027 i r$ S-0,03207S.A/N

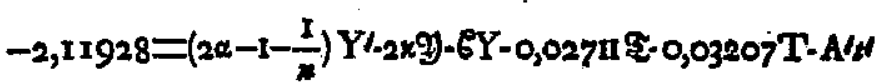

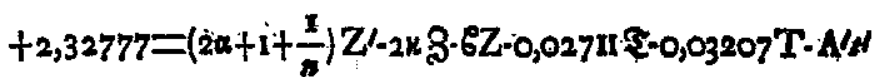

5. 163.
5. I63, Pro vlteriorit calculo elt

$3-\frac{x}{n}=0,924562 \ldots \cdot\left(1-\frac{1}{n}\right)=9,965935$

$1+\frac{1}{n}=1,075438 \ldots . . \cdot\left(1+\frac{1}{m}\right)=0,031570$ $2 \alpha-1+\frac{1}{n}=0,942914 \quad . \quad . \quad l\left(20-1+\frac{I}{n}\right)=9,965935$ $2 \alpha+1-\frac{1}{z}=2,792038 \ldots \cdot l\left(2 \alpha+1-\frac{1}{2}\right)=0,445915$ $2 a-1-\frac{1}{n}=0,792038 \quad . \quad i\left(20-1-\frac{1}{-n}\right)=9,898747$ $208+1+\frac{1}{n}=2,942914 \cdot \cdot \cdot\left(2 \alpha+1+\frac{1}{n}\right)=0,468775$ Hine in aequationibus pofterioribus valores litterarum $S^{\prime}, T^{\prime}, V^{\prime}$ fabitituantur, et ob $E=1,0159 \mathrm{x}$, exit $\left.0,16110 S=-5,10323 \div 2 k S+A^{\prime}\left(z^{\prime}+x^{\prime}\right)-0,02711(3)+3\right)$ $+0,01974\left(\nabla^{\prime}+X\right)$ $+1,21835\left(\nu^{\prime}-x^{\prime}\right)-0,03207(V+X)$ $-0,01825(\mathrm{~V}-\mathrm{X})$

$0,14059 \mathrm{~T}=+5,18709+2 z^{3}+\mathrm{A}^{\prime}\left(\mathrm{y}^{\prime}+z^{\prime}\right)+0,027 \mathrm{II}(\mathrm{g}+3)$

$-0,01974\left(\mathrm{Y}^{\prime}+\mathrm{Z}^{2}\right)$

$-1,41710\left(y^{\prime}-2 /\right)+0,03207(Y+Z)$ $+0,02124(\mathrm{Y}-\mathrm{Z})$

$0,12683 \mathrm{~V}=+6,8259 \mathrm{I} \sim 29.23+3,718.51-0,027 . \mathrm{I}$ I $\because \cdot \cdots$

$-0,03207 \mathrm{~S}$

$6,77934 x=+4,56988+2 x+6,1548 s+0,02711$ S $+0,03207 \mathrm{~S}$

$0,38858 \mathrm{Y}=+5,89253-249+3,5194 t^{\prime}-0,027$ I I $0,0320 \% \mathrm{~T}$

- $7,64474 \mathrm{Z}=+3,61676+248-6,3536 z^{\prime}+0,02711$ $+0,0320 \% 1$ 6. 364 . 
§. 164. Commodifime hi coefficientes inueniri videntur, fi primo $\boldsymbol{B}, \mathfrak{X}, \mathfrak{Y}, \boldsymbol{Z}$ et $\mathrm{V}, \mathrm{X}, \mathbf{Y}, Z$ proxime quaerantur, quod fiet terminos minimos negligendo:

$$
\begin{aligned}
& 93=+5,7560 \text {. . } 193=0,760125 \\
& \mathfrak{X}=+1,8334 . ., l \mathfrak{X}=0,263245 \\
& y=+6,9837 \cdot . \text {. } \quad y=0,844088 \\
& B=+1,6643 . .+13=0,221240 \\
& \mathrm{~V}=-37,7650^{\circ}, \cdots 4-\mathrm{V}=\mathrm{I}, 577086 \\
& \mathrm{X}=+\mathrm{I}, 2 \mathrm{r98} \text {. . . X }=0,086293 \\
& Y=-21,1040 \text {. . I } I-Y=1,324360 \\
& \mathrm{Z}=+0,9125 \ldots . l \mathrm{Z}=9,960209 \\
& V=-29,7170 . .: 21=-0,398 \\
& X^{\prime}=+2,5326 \cdots x^{\prime}=+0,024 \\
& \boldsymbol{Y}=-\mathrm{i} 1,9510 . \cdots y^{\prime}=-0,201 \\
& Z=+2,2472 \text { : . . } \quad z=+0,020
\end{aligned}
$$

\$. 165. Hic autem valores pro $V^{\prime}$ et $Y^{\prime}$ tam Gunt magnt. vt vicifim poit inuentas litteras $S, T$ nimịum valores modo erutos afficiant, ynde neceffe erit refolutionem harum aequationum ordinario modo inftriere - Reperitur ergo

$$
\begin{aligned}
& 9=5,7560-0,0193 \text { S-0, 0050' } \\
& x=t, 83.33-0,0064 S-0,016.6 \\
& \text { y) }=6,9837-0,0225 \mathbf{T}-0,0058 \mathfrak{R} \\
& \because B=1,6643-0,0067 T-0,0016 \mathrm{R} \\
& 2 \times 23=11,6155-0,0390 \mathrm{~S}-0,0100 \mathrm{~S} \\
& 24 \hat{X}=3,6996-0,0129 \mathrm{~S}-0,0033 \mathrm{G} \\
& \text { 2* })=14,0930-0,0455 \mathrm{~T}-0,0118 \mathfrak{T} \\
& \text { - } 23=3,3586-0,0132 \mathrm{~T}-0,0032 \mathrm{~K}
\end{aligned}
$$

ri vi-

vime

:

im

ni-

rit

n- qui valores fubftituti dant :

$$
\begin{aligned}
& V=-37,7647+0,3912 \mathrm{~S}+0,0031 \mathrm{~S} \\
& \mathrm{X}=+1,2198-0,0076 \mathrm{~S}-0,0016 \mathrm{~S} \\
& \mathrm{Y}=-21,1038+0,1385 \mathrm{~T}+0,0121 \mathrm{~S} \\
& \mathrm{Z}=+0,9125-0,0069 \mathrm{~T}-0,0016 \mathrm{~S} \\
& 2 \mathrm{~V}=-76,2085+0,7893 \mathrm{~S}+0,0064 \mathrm{~S} \\
& 2 \mathrm{X}=+2,4615-0,0153 \mathrm{~S}-0,0033 \mathrm{~S} \\
& 2 \mathrm{Y}=-42,5870+0,2794 \mathrm{~T}+0,0244 \mathrm{~S} \\
& 2 \mathrm{Z}=+1,8413-0,0140 \mathrm{~T}-0,0033 \mathrm{~S}
\end{aligned}
$$

\$. 166. Hinc porro valores deriuaci erunt

$$
\begin{aligned}
& V^{\prime}=-29,7167+0,3767 \mathrm{~S}+0,0094 \mathrm{~S} \\
& \mathrm{X}^{\prime}=+2,5326-0,006 \mathrm{I}+0,0029 \mathrm{~S} \\
& \mathrm{Y}^{\prime}=-11,951 \mathrm{I}+0,1248 \mathrm{~T}+0,016 \mathrm{I} \\
& \mathrm{Z}^{\prime}=+2,2472-0,0054 \mathrm{~T}+0,0028 \mathrm{~S} \\
& v^{\prime}=-0,4009+0,0044 \mathrm{~S}+0,0001 \mathrm{~S} \\
& x^{\prime}=+0,0244 \\
& y^{\prime}=-0,2026+0,0015 \mathrm{~T}+0,0001 \mathrm{~T} \\
& z^{\prime}=+0,0200
\end{aligned}
$$$$
\text { ac porro }
$$

$$
\begin{aligned}
& \text { 23- } \mathrm{x}=3,9227-0,0129 \mathrm{~S}-0,0034 \mathrm{~S} \\
& \text { 9) }-3=5,3194-0,0164 \mathrm{~T}-0,004^{2} \mathrm{~S} \\
& \mathrm{~V}-\mathrm{X}=-38,9845+0,3988 \mathrm{~S}+0,0047 \mathrm{~S} \\
& \mathbf{Y}-\mathbf{Z}=-22,0163+0,1454 \mathrm{~T}+0,0137 \\
& \mathfrak{B}+\mathfrak{X}=7,5893-0,0257 \mathrm{~S}-0,0066 \text {; } \\
& \text { 2) }+8=8,6480-0,0286 \mathrm{~T}-0,0074 \mathrm{~T} \\
& \mathrm{~V}+\mathrm{X}=-36,5449+0,3836 \mathrm{~S}+0,0015 \mathrm{C} \\
& \mathbf{Y}+\mathbf{Z}=-20,1913+0,1316 \mathrm{~T}+0,0105 ?
\end{aligned}
$$

$\mathbf{T}$

$V^{\prime}+X^{\prime}$ 
146 $C A P U T$

$V^{\prime}+X^{\prime}=-27,184 \mathrm{I}+0,3706 \mathrm{~S}+0,0123$ ह $Y^{\prime}+Z^{\prime}=-9,7039+0,1194 \mathrm{~T}+0,0189 \mathrm{E}$ $v^{\prime}-x^{\prime}=-0,4253+0,0044 S+0,0001$ S $y^{\prime}-z^{\prime}=-0,2226+0,0015 \mathrm{I}+0,0001 \mathfrak{S}$ $v^{\prime}+x^{\prime}=-0,3765+0,0044 S+0,0001 \mathrm{~S}$ $y^{\prime}+z^{\prime}=-0,1826+0,001 s^{\prime} \Gamma+0,0001 s$

6. 16\%. His valoribus fubftitutis reperitur

$$
\begin{aligned}
& \left(\mathrm{I}-\frac{1}{n}\right) \mathrm{S}=+\mathrm{I}, 8024-0,0070 \mathrm{~S} \\
& \left(\mathrm{I}+\frac{\mathrm{I}}{n}\right) \mathrm{F}=+\mathrm{I}, 4453-0,0027 \mathrm{~T}
\end{aligned}
$$

vnde concluditur

$$
\begin{array}{c|c}
S=1,9495-0,0075 \mathrm{~S} & 2 x \mathcal{S}=3,9340-0,0160 \mathrm{~S} \\
\mathrm{I}=\mathrm{I}, 3440-0,0025 \mathrm{~T} & 2 \times \mathrm{R}=2,7121-0,0053 \mathrm{~T} \\
0,1401 \mathrm{~S}=-9,2444 & 0,1479 \mathrm{~T}=+7,9767
\end{array}
$$

§. 168. Nunc iginur habebimus

$$
\begin{aligned}
& S=-66,6980, \quad \text {. } 4 \mathrm{H}=1,824119 \\
& \mathrm{~T}=+53,9330 ., \quad \cdot \mathrm{T}=1,731855 \\
& \text { S }=+2,4497, \cdot l \mathbb{S}=0,389113 \\
& \mathfrak{S}=+1,2092 \text {. . } / \mathfrak{E}=0,082498 \\
& s^{\prime}=-0,75204 \text {. . } l m s^{\prime}=9,87624 \mathrm{x} \\
& t^{\prime}=+0,62626 . t^{\prime}=9,796755 \\
& v^{\prime}=-0,6942 \quad \cdot \quad \cdot \quad l-v^{\prime}=9,841484 \\
& x^{\prime}=+0,0244 \text {. . } \quad x^{\prime}=8,387390 \\
& y^{\prime}=-0,1216 \quad \text {. } \quad \cdot \quad l-y^{\prime}=9,084933 \\
& z^{\prime}=+0,0200 \text {. . } \quad z^{\prime}=9,301030
\end{aligned}
$$

$C A B U T$

$$
\begin{aligned}
& 2=+7,0311 \text {. . } l 3=0,847029 \\
& \mathfrak{X}=+2,256 \mathrm{II} \cdot l \mathfrak{X}=0,353358 \\
& y=+5,7659 \cdot \cdots, t y=0,760867 \\
& B=+1,3339 \text {. . } 13=0,825123 \\
& V=-63,8498, \quad, \quad l-V=1,805160 \\
& \mathrm{X}=+\mathrm{I}, 745 \mathrm{I} \cdot \because l \mathrm{X}=0,241820 \\
& Y=-13,6222, \cdot, l-Y=1,13424 \\
& \mathrm{Z}=+0,535^{\circ} \cdot . \cdot \mathrm{Z}=9,72884^{\circ}
\end{aligned}
$$

§. I69. His iam valoribus inuentis pro diftantia lunae $x=\frac{(1-k k) a u}{1-k c u, r}$ erif valoris ipfius $*$ porcio ab his terminis pendens:

$n=$ Praec, $-0,3796$ ek $\operatorname{cof}(r-s)$

$$
\begin{aligned}
& +0,3069 \text { ek cor }(r+s) \\
& -0,3634 \text { ek cor }(2 y-r+s) \\
& +0,0099 \text { ek cor }(2 y+r-s) \\
& -0,0775 \text { ek cor }(2 y-r \rightarrow s) \\
& +0,0030 \text { ek cor }(2 y+r+s)
\end{aligned}
$$

Log. coeff.

et pro longitudine lanae

$\frac{d \Phi}{d r}=$ Praecs $+0,7520$ ek $\cot (r-s)$

$-0,6263 t k^{\prime} \operatorname{cof}(r+s)$

$+0,6942 e k^{\prime} \operatorname{cor}(2 \eta-r+s)$

- 0,0244 et cof $(2 \eta+r-s)$

$+0,12 \pm 6$ ak cof $(2 y-r-s)$

- 0,0200 ek col $(2 y+8+s)$

$$
\mathbf{T} 2
$$

cuius

\section{9,579297}

9,487093

9,560344

7,997004

$.8,889425$

7,484024 
148

$C A P U T X$

cuius integrale fi ponatur:

$\Phi=$ Praec. $+\mathcal{S}^{\prime} e k \mathrm{fn}(r-s)+\mathfrak{B}^{\prime} \mathrm{ekfn}(2 \eta-r+s)+\mathfrak{Y}^{\prime} e k \mathrm{fn}(2 \eta-r-s)$

erit

$$
+\mathfrak{S}^{\prime} e k \mathrm{fn}^{\prime}(r+s)+\mathfrak{X}^{\prime} e k \mathrm{fn}(2 \eta+r-s)+8 e k \mathrm{fn}(2 \eta+r+s)
$$

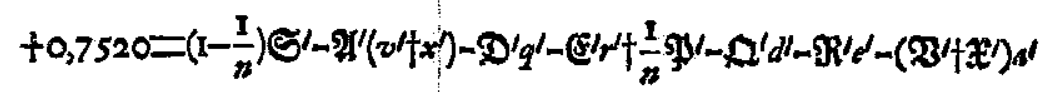

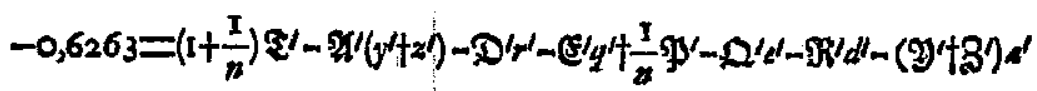
$+0,6942=\left(2 \alpha-1+\frac{\pi}{n}\right) \mathfrak{P}^{\prime}+\mathfrak{P}^{\prime}\left(\frac{2}{n}-s^{\prime}\right)+\mathfrak{D}^{\prime}\left(\frac{2}{n}-p^{\prime}\right)-\Re^{\prime}\left(c^{\prime}-\frac{1}{n}\right)$ $-0,0244=\left(2 a+1-\frac{1}{n}\right) \mathfrak{X}^{\prime}+\mathscr{A}^{\prime}\left(\frac{2}{n}-1\right)+\left(\mathbb{S}^{\prime}\left(\frac{2}{n}-p^{\prime}\right)-\mathfrak{Q}^{\prime}\left(c^{\prime}+\frac{1}{n}\right)\right.$ $+0,1216=\left(2 \alpha-1-\frac{\pi}{n}\right) g+P^{\prime}\left(\frac{2}{n}-t\right)+D^{\prime}\left(\frac{2}{n}-p^{\prime}\right)-Q^{\prime}\left(d^{\prime}+\frac{1}{n}\right)$ $m 0,0200^{\circ}=\left(2 \alpha+1+\frac{1}{n}\right) 3+2\left(\frac{2}{n}-t\right)+(1)\left(\frac{2}{n}-p\right)-N\left(d-\frac{1}{n}\right)$

6. 170. Hinc autem reperitur

$$
\begin{aligned}
& \mathrm{S}^{\prime}=+0,7467 \ldots . \quad \mathrm{S}^{\prime}=9,87316 \mathrm{~s} \\
& \xi^{\prime}=-0,6185 \text {. . } 1-R^{\prime}=9,791317 \\
& \mathfrak{B}^{\prime}=+0,81_{43} \text {. . } \quad \mathbf{R B}^{\prime}=9,910800 \\
& X^{\prime}=-0,0142 \cdot \text {. } 4 X^{\prime}=8,150690 \\
& y^{\prime}=+0,2396 \text {. . } y^{\prime} y^{\prime}=9,379550 \\
& B^{\prime}=-0,006 x \text {. . Im } B^{\prime}=7,788910
\end{aligned}
$$

$C A P U T X$

§. 17x. Quatenus ergo longicudo Lunae ab exsentricitate orbitae folis pendet, exic

$(r+s)$

$\left.\mathfrak{X}^{\prime}\right) \mathrm{a}^{\prime}$

$\varphi=$ Praec. $+0,201385$ fin s

log. coeffo

$-0,02.1889 e$ fin $(2 \eta-s)$

$-0,016368$ fin $(2 y+s)$

$+0,06615$ ee fin $2 s$

$+0,02332$ ee fin $(2 y-2 s)$

$+0,00840$ ee fin $(2 \eta+2 s)$

$+0,7476 e k$ fin $(r-s)$

- $0,6185 e k$ fin $(r+s)$

$+0,8143=k$ fin $(2 y-r+s)$

$-0,0142 \in k \operatorname{lin}(2 y+r-s)$

$+0,2396 e k$ fin $(2 y-r-s)$

9, 304026

8,340237

$8,21,4002$

8,820508

8,367825

7,924429

9, $873 \pi 65$

9.791317

9,910800

8,150690

9,379550

- 0,006I ek fin $\left.(2 y+r+s)\right|_{7,788910}$ 
§. 172. Hae autem fingulae inaequalitates ad numerum minutorum fecundorum reductae dabunt :

$$
\begin{aligned}
& \varphi=\text { Praec, }+70 \mathrm{l} / \mathrm{x} \text {, fin } \\
& \text { - 75, 8 fin }(2 \eta-s) \\
& \text { - 56, } 7 \text { fin }(2 \eta+s) \\
& +3 \text {. } 8 \text { in } 2 \text { s } \\
& +\quad 4 \text { fin }(2 \eta-2 s) \\
& +0,5 \text { fin }(2 y+2 s) \\
& +140,9 \text { fin }(r-s) \\
& \text { - I16, } 7 \text { fin }(r+s) \\
& +153, z \text { fin }(2 y-r+s) \\
& \text { - } 23 \text { fin }(2 \eta+r-s) \\
& +45,2 \text { in }(2 y-r-s)
\end{aligned}
$$

log. coeff.

Hic fcilicet et inaequalitates, guas in capite praeçedente inuenimus, et iftas in hoc capite erutas fimul fum complexus, vt coniunetim confpetui exponerentur,
CAPUT

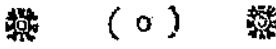

\section{CAPUT XI.}

\section{INUESTIGATIO INAEQUALITATUM LUNAE}

A PaRALLAXI SOLIS PENDENTIUM.

\section{\$. 173.}

Tam in formulis noftris primariis ad eos quoque ter minos progrediamur, qui littera $\nu$ funt affecti, et quoniam eft $1: \nu$ vt diftancia Solis media ad diftan. tiam Lunae mediam a Terra, erit $x: y$ ve parallaxis Lunae media ad parallaxin folis : ex quo inaequalitates Lunae, quae hine oxiuntur, a parallaxi folis pendere dicuntur. Quoniam vero valor ipfius $v$ eft valde paruus, quippe $\frac{x}{280}$ propemodum, alios terminos non contemplabimur, nifi qui per $v$ ac per $v k$ et $y \in$ funt multiplicati, propterea quod magis compofiti fiant minimi.

6. 174. Ex terminis ergo jam inuentis hic retineamus eos, qui funt alicuius momenti, et cum is no: vos determinandos coniungamus; fit ergo:

$$
\begin{aligned}
& \int \mathrm{R} d r=2 \mathrm{cC} 2 \eta+6 k \mathrm{cr}+\mathfrak{D} k \mathrm{cl}(2 \eta-r)+5 \mathrm{CeC}+\operatorname{Sec}(2 \eta-s) \\
& +\mathbb{C} k \mathrm{cC}(2 \eta+r)+\operatorname{Rec}(2 \eta+s) \\
& +8 y \operatorname{cor} v+\$ v k \operatorname{cor}(\eta-r)+\Re v e \operatorname{cor}(\eta-s) \\
& +(8) \operatorname{cor} 3 \eta+9 y k \operatorname{cor}(\eta+r)+\& y \in \operatorname{cor}(\eta+s) \\
& v=\mathrm{A} \operatorname{cor} 2 \eta \ldots+\mathrm{D} k \operatorname{cor}(2 \eta-r)+\mathrm{Pecol} s+\mathrm{Q}_{e} \operatorname{cor}(2 \eta-s) \\
& +\mathrm{E} k \operatorname{cor}(2 \eta+r)+\mathrm{R} z \operatorname{cor}(2 \eta+s) \\
& +\mathrm{F} y \operatorname{cor} \eta+\mathrm{H} v k \operatorname{cor}(\eta-\eta)+\mathrm{K} v e \operatorname{cor}(\eta-s) \\
& \text {. }+\mathrm{G} y \operatorname{cof} 3 \eta+\mathrm{J} v k \operatorname{cor}(\eta+r)+\mathrm{L} y \in \operatorname{cor}(\eta+s)
\end{aligned}
$$


Non difficulter enim praeuidere licet, terminos, quit angulos $r$ et $s$ cum angulo 3 y habeant coniunetos, fore tam exiguos, vt fine errore praetermitti queant.

\$. 175. Quodfi iam retentis litterarum \$. 153. inductarum valoribus, praeterea ponamus:

$f^{\prime}=\frac{2 x \mathrm{~F}+\mathcal{f}}{n n} ; g^{\prime}=\frac{2 x \mathrm{G}+\mathcal{G}}{n n} ; \quad b=\frac{2 x \mathrm{H}+\mathfrak{S}}{n n}$

$i=\frac{2 x \mathrm{~J}+9}{n n} ; k=\frac{2 x \mathrm{~K}+\mathrm{S}}{n n} ; y=\frac{2 x \mathrm{~L}+\mathrm{g}}{n n}$

habebimus :

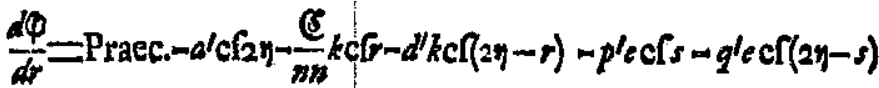
$-e / k c f(2 \eta+r) \quad-r l e c f(2 \eta+s)$

$-f^{\prime} v \operatorname{cor} \eta+b^{\prime} v k \operatorname{cor}(\eta-r)-k^{\prime} v \in \operatorname{cor}(\eta-s)$ $-g^{\prime} y \operatorname{col} 3 \eta-z^{\prime} v k \operatorname{col}(\eta+r)-v y \in \operatorname{col}(\eta+s)$ $\frac{d y}{d r}=\alpha-\alpha^{\prime} \mathrm{c}\left[2 y-d^{\prime} k \mathrm{c}\left(r-d / k \mathrm{c}(2 y-r)+\left(\frac{2}{z}-p^{\prime}\right) e c\left[s-q^{\prime} \operatorname{cc}[(2 y-s)\right.\right.\right.$ $-c\left(k c\left\{(2 \eta+r) \quad-r_{e} c(2 \eta+s)\right.\right.$ $-f^{\prime} y \operatorname{cor} \eta-b^{\prime} y k \operatorname{cor}(\eta-r)-k^{\prime} y e \operatorname{cor}(\eta-s)$

$-g^{\prime} y \operatorname{col} \eta-i^{\prime} y k \operatorname{cor}(\eta+s)-\| y e \operatorname{cor}(\eta+s)$

\$. 176. Jam vero pro his terminis ab v pendenti. bus fequentes colligemus aequationes.

$$
\begin{aligned}
& R= y \cdot \operatorname{fin} \eta\left(\frac{3}{8}+\frac{3 F-3 G}{2 n n}\right) \\
& y \sin 3 y\left(\frac{\pi 5}{8}+\frac{3 F}{2 n n}\right)
\end{aligned}
$$

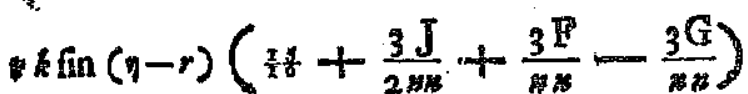

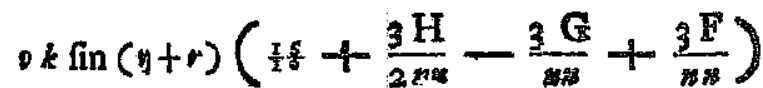

$$
\begin{aligned}
& e \operatorname{efin}(y-s)\left(-3+\frac{3 L}{2 m n}-\frac{9 F}{4 m s}+\frac{9 G}{4 m n}\right) \\
& y \in \operatorname{fin}(y+s)\left(-3+\frac{3 K}{2 m}+\frac{9 G}{4 m}-\frac{9 F}{4 m}\right)
\end{aligned}
$$

$$
\frac{d d v}{d r^{2}}=
$$

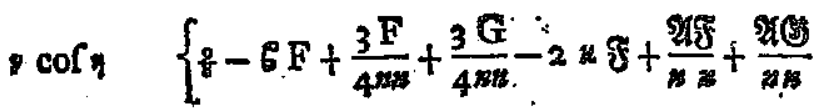

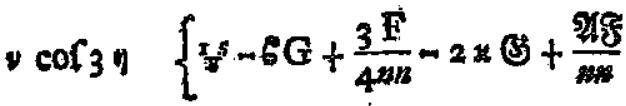

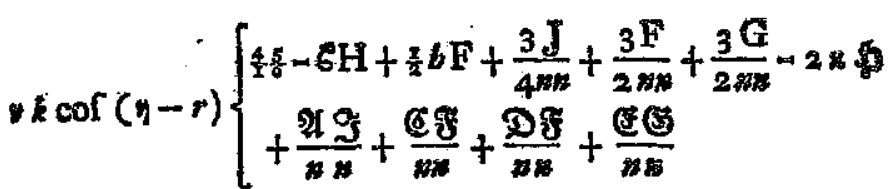

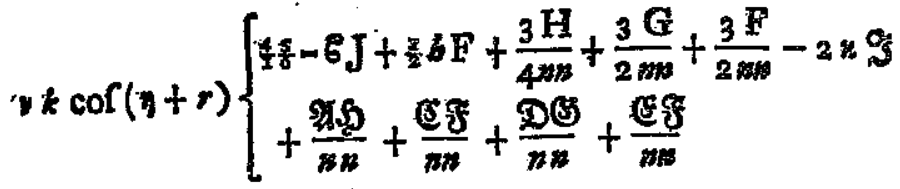




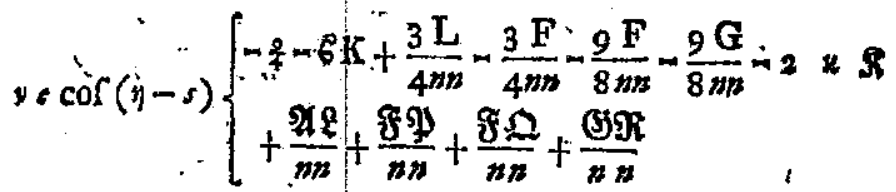

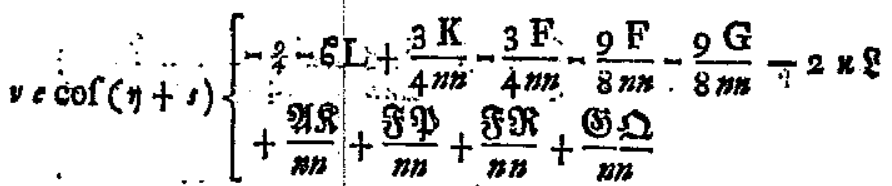

fequentes partes feorfim exponamús:

$y \cot n \quad\left\{\begin{array}{l}+\frac{39 \mathrm{~F}}{n n}+\frac{39 \mathrm{G}}{n n}+\frac{3 \mathrm{AF}}{n n}+\frac{3 \mathrm{AOS}}{n n}+\frac{3 \mathrm{AF}}{n n}+\frac{3 \mathrm{AG}}{n n} \\ +\frac{9 \mathrm{~A}}{8 m}+\frac{15 \mathrm{~A}}{8 n}\end{array}\right.$

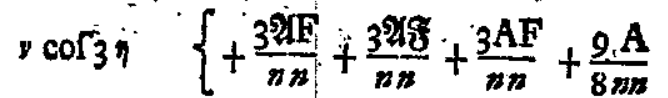

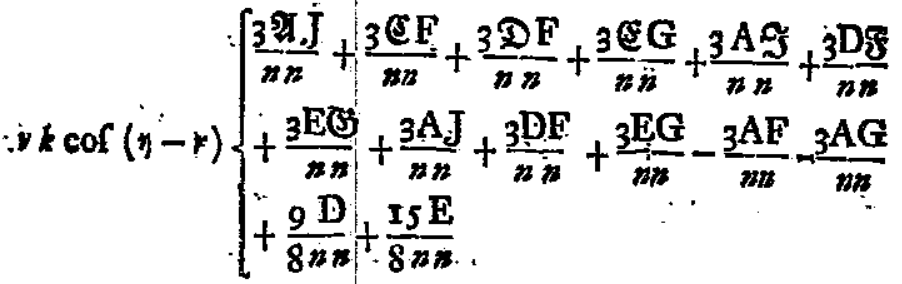

$\therefore \quad \therefore \quad \therefore \quad \frac{3 \text { AH }}{n n}+\frac{3 \mathbb{C E}}{m n}+\frac{3 \mathfrak{D S}}{n n}+\frac{3 \mathbb{E}}{n n}+\frac{3 \mathbf{A S}}{n n}$

$y k \operatorname{cor}(\eta+r)\left\{+\frac{3 \mathrm{DS}}{n}+\frac{3 \mathrm{Eg}}{n n}+\frac{3 \mathrm{AH}}{n n}+\frac{3 \mathrm{DG}}{n n}+\frac{3 \mathrm{EE}}{n n}\right.$ $-\frac{3 \mathrm{AF}}{2 n n}-\frac{3 \mathrm{AG}}{2 m n}+\frac{9 \mathrm{E}}{8 n n}+\frac{\mathrm{r} 5 \mathrm{D}}{8 n n}$

vecor

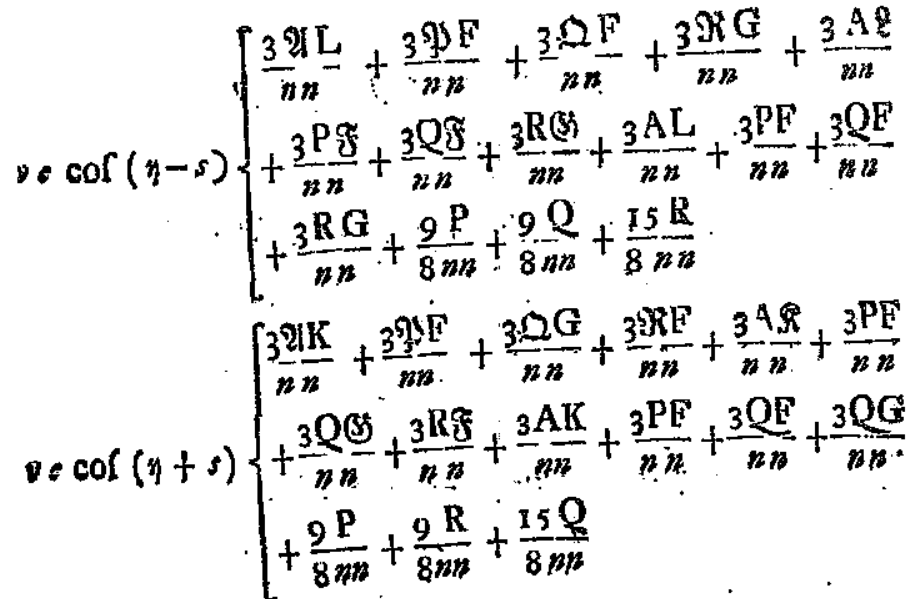

5. 177. Verum differenciando nancifcemur

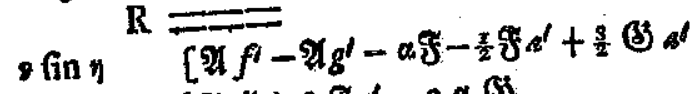

$\nu$ fin 3 i [ $\left\{f^{\prime}+\frac{1}{2} g^{\prime}-3 \alpha\right.$ (S)

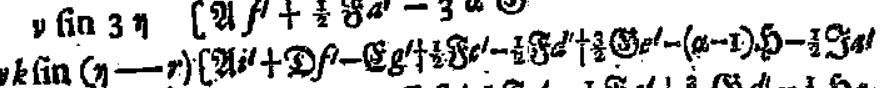

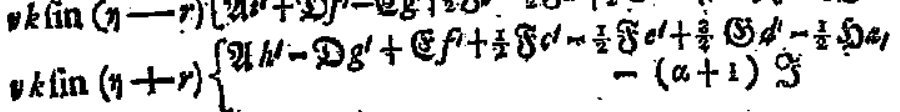

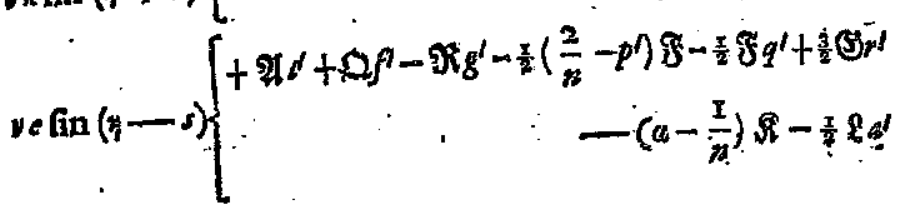

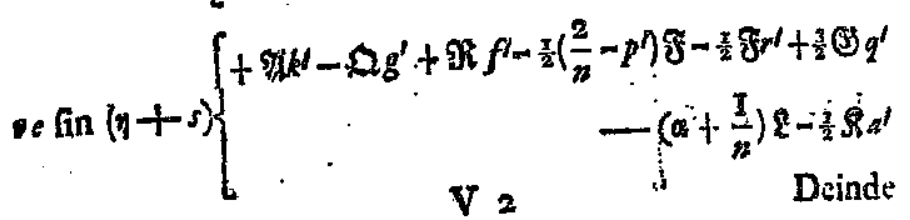




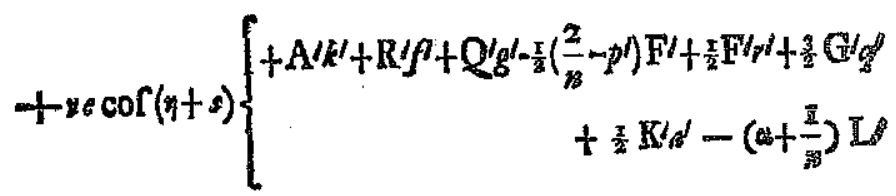

qui valorés cum antecedentibus comparaxi debent, vis inde valores coefficientium eliciantur.

\$. 179. Sumamus primo duos valores ab inisio pofitos, quoniam hi a fequentibus non pendent, asque

habebimus,

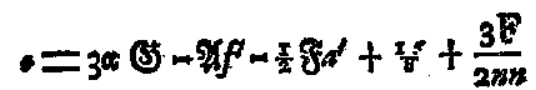

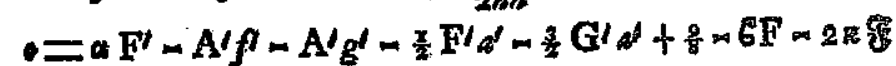

$$
+\frac{3(F+G)}{4^{m}}+\frac{(2 A+3 A)}{m}\left(\xi i+(B)+\frac{(3 A+3 A)}{m m}(F+G)+\frac{3 A}{m}\right.
$$

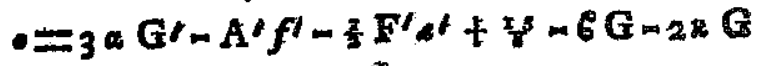

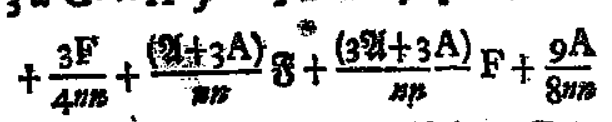

et $F^{\prime}=a \dot{F}-A\left(f^{\prime}-g^{\prime}\right)+\frac{x}{2} F_{d^{\prime}}-\frac{3}{2} G_{d}$

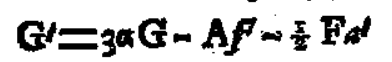

att eff $f=\frac{2 x \mathrm{~F}+F}{m} g=\frac{2 x \mathrm{G}+\mathrm{B} \text {. }}{m}$

Hic igitur primun litterarum, quae funt sognitae, viga lores in numeris fubftituantus, exitque

$v_{3}$

$y=$ 
$F^{\prime}=0,93905 F+0,00753 \mathfrak{F}+0,01443 G-0,00753$ (8)

$\mathbf{G}^{\prime}=2,80121 \mathbf{G}+0,02505 \mathrm{~F}+0,00753 \%$

$0=0,92847 \mathfrak{F}+0,01776 \mathrm{~F}-0,01776 \mathrm{G}-0,02501 \mathrm{~B}$ $+0,37500$

$0=2,80: 21$ (O) $+0,01447 f+0,01776 \mathrm{~F}+x, 87500$ hincque

$$
\begin{aligned}
& \mathfrak{F}=-0,01930 \mathrm{~F}+0,01913 \mathrm{G}-0,42145 \\
& \mathfrak{G}=-0,00623 \mathrm{~F}-0,00010 \mathrm{G}-0,66717
\end{aligned}
$$

§. 180. Inde porro colligemus

$$
\begin{aligned}
& \mathrm{F}^{\prime}=0,93895 \mathrm{E}+0,01457 \mathrm{G}+0,00185 \\
& \mathrm{G}^{\prime}=0,0249 \mathrm{~F}+2,80135 \mathrm{G}-0,00317 \\
& f^{\prime}=0,01138 \mathrm{~F}+0,0001 \mathrm{G}-0,00240 \\
& \mathrm{~g}^{\prime}=0,00004 \mathrm{~F}+0,01148 \mathrm{G}-0,00380
\end{aligned}
$$

quibus valoribus fubftitutis peruenimus ad has aequationes: vnde fit:

$$
\begin{aligned}
& 0,09337 \mathrm{~F}=+0,05421 \mathrm{G}+\mathrm{I}, 96867 \\
& 6,83135 \mathrm{G}=\frac{1}{0,08830 \mathrm{~F}-3,20944}
\end{aligned}
$$

$$
\begin{aligned}
& \mathrm{F}=20,65700 \text {. . . } / \mathrm{F}=\mathrm{r}, 315067 \\
& \mathrm{G}=-0,7368 \mathrm{I} \cdot \text {. . } \mathrm{L} \mathrm{G} \equiv 9,867356 \\
& f=-0,83418 \text {. . } l-f=9,921260 \\
& \text { (S) }=-0,79580 \text {. . . } 1-3=9,900804 \\
& F=+19,35712 . . \quad L 1=1,287512 \\
& \mathrm{G}=-1,55266 \text {. . . } 1-\mathrm{G}=0,191075 \\
& f^{\prime}=+0,23259 . \text {. } t f^{\prime}=9,366501 \\
& g^{\prime}=-0,01309 \text {. . . } / g i=8,116940
\end{aligned}
$$

\$. 181. His valoribus, qui ad inaequalitates abfolutas pertinent, expeditis, progrediamur ad eos, qui ab excentricitate orbitae lunaris pendent, ac his aequation nibus continentur :

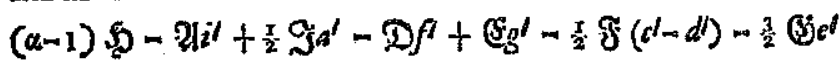

$$
\begin{aligned}
& +\frac{x}{Y} \frac{5}{0}+\frac{3 J}{2 n n}+\frac{3(\mathrm{~F}-\mathrm{G})}{m n}=
\end{aligned}
$$

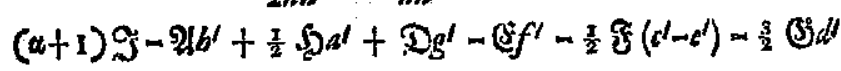

$$
\begin{aligned}
& +\frac{15}{5} \frac{3}{6}+\frac{3 \mathrm{H}}{2 n n}+\frac{3(\mathrm{~F}-\mathrm{G})}{n n}=0 \\
& \mathrm{H}^{\prime}=(\alpha-1) \mathrm{H}-\mathrm{A} i^{\prime}+\frac{1}{2} J a^{\prime}-\mathrm{D} f^{\prime}+\mathrm{E} g^{\prime}-\frac{7}{2} \mathrm{~F}\left(c^{\prime}-d l\right)-\frac{9}{2} \mathrm{G} c^{\prime} \\
& \mathrm{J}^{\prime}=(a+1) \mathrm{J}-\mathrm{A} b^{\prime}+\frac{\mathrm{T}}{2} \mathrm{H} a^{\prime}+\mathrm{D} g^{\prime}-\mathrm{E} f^{\prime}-\frac{1}{2} F\left(d^{\prime}-e^{\prime}\right)-\frac{1}{2} \mathrm{G} d^{\prime} \\
& (a-\mathrm{I}) \mathrm{H}^{\prime}-\mathrm{A}^{\prime} \mathrm{i}^{\prime}-\frac{\mathrm{T}}{2} \mathrm{~J}^{\prime} \mathrm{A}^{\prime}-\mathrm{D}^{\prime} f^{\prime} \sim \mathrm{E}^{\prime} g^{\prime}-\frac{\mathrm{T}}{2} \mathrm{~F}^{\prime}\left(d^{\prime}+d^{\prime}\right)-\frac{3}{2} \mathrm{G}^{\prime} e^{\prime} \\
& +\frac{4}{3} \frac{5}{0}-6 \mathrm{H}-2 n 5+\frac{\tau}{2} b \mathrm{~F}+\frac{3 \mathrm{~J}}{4 m n}+\frac{3(\mathrm{~F}+\mathrm{G})}{2 m n}+\frac{(5)}{n m}+\frac{3(5 \mathrm{~F}}{n m} \\
& +\frac{(\mathfrak{A}+3 A)}{m n} \mathfrak{J}+\frac{(3 \mathfrak{A}+3 A)}{m n} \mathrm{~J}+\frac{(\mathfrak{D}+3 \mathrm{D})}{m n} \mathfrak{J}+\frac{(3 D+3 D)}{m n} \mathrm{~F} \\
& +\frac{(\mathbb{E}+3 E)}{n 3}\left(5+\frac{(3 E+3 E)}{n n} G-\frac{3 A(F+G)}{2 n n}+\frac{9 D+15 E}{8 n n}=0\right. \\
& (a+1) \mathrm{J}^{\prime}-\mathrm{A}^{\prime} b^{\prime}-\frac{\mathrm{x}}{2} \mathrm{H}^{\prime} a^{\prime}-\mathrm{E}^{\prime} f^{\prime}-\mathrm{D}^{\prime} g^{\prime}-\frac{\pi}{2} \mathrm{~F}^{\prime}\left(c^{\prime}+e^{\prime}\right)-\frac{3}{2} \mathrm{G}^{\prime} d d^{\prime} \\
& +\frac{4}{5} 5-6 \mathrm{~J}-2 n \mathrm{~J}+\frac{r}{2} b \mathrm{~F}+\frac{3 \mathrm{H}}{4 n n}+\frac{3(\mathrm{~F}+\mathrm{G})}{2 n n}+\frac{\mathrm{CE}}{n n}+\frac{3 \mathrm{CF}}{n n}
\end{aligned}
$$

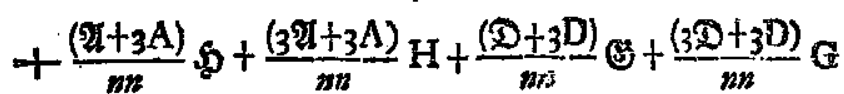

$$
\begin{aligned}
& +\frac{(\mathbb{E}+3 \mathrm{E})}{m} \mathfrak{F}+\frac{(3 \mathbb{E}+3 \mathrm{E})}{m} \mathrm{~F}-\frac{3 \mathrm{~A}(\mathrm{~F}+\mathrm{G})}{2 m}+\frac{9 \mathrm{E}+\mathrm{I} 5 \mathrm{D}}{8 m}=0
\end{aligned}
$$

\$. 188. 
f. 182. In his aequationibus fiablicuantur vilores iam cogniti, atque ohtinebimus, $-0,06626$ Sto,81033 $3 /-0,00987 \$+0,00854 J \pm 2,04620=0$ $1,833749+0,81033$ bl-0,00987 $5+0,00854 \mathrm{H}+2,10646=0$ $H^{\prime}=-0,06626 \mathrm{H}+\mathrm{r}, 31773 i /-0,00937 \mathrm{~J}-5,57458$ $\mathrm{J}^{\prime}=\mathrm{I}, 83374 \mathrm{~J}+\mathrm{I}, 31773^{\prime \prime}-0,00987 \mathrm{H}-\mathrm{I}, 55427$ $\left.-0,06626 \mathrm{H}^{\prime}+2,47576 \mathrm{j}^{i}+0,00987 \mathrm{~J}-\mathrm{I1}, 86106\right]$ $-\mathrm{I}, 0159 \mathrm{H}+0,00427 \mathrm{~J}-0,02711 \mathrm{~J}+2,81250$ $-2,017.98 \$-0,03634 \mathrm{~J} \quad+31,31044=$ $+10,60834 \mathrm{~J}$

I, $83374 \mathrm{~J}^{\prime}+2,47576 \boldsymbol{b}^{\prime}+0,00987 \mathrm{H}^{\prime}+0,27762$ $-1,01591 \mathrm{~J}+0,00427 \mathrm{H}-0,027115 \mathrm{D}+2,81250$ $-2,017989-0,03634 \mathrm{~Hz} \quad+35,81380=$ $-0,81380$

5. 183. Subftituamus primo loco $b^{\prime}$ et is valores, atque noftrae aequationes reducentur ad formas fequentes,

$-0,066265+0,01785 \mathrm{~J}-0,00526 \mathrm{~g}+2,04620=0$ $+1,833749+0,01785 \mathrm{HI}-0,00526 \mathrm{~S}+2,10646=0$ $\mathrm{H}^{\prime}=-0,06626 \mathrm{H}+0,00526 \mathrm{~J}+0,00750 \mathrm{~J}-5,57458$ $J^{\prime}=+\mathrm{I}, 83374 \mathrm{~J}+0,00526 \mathrm{H}+0,00750 \mathrm{~W}-1,55427$ qui valores in fequentibus fubftituti dant

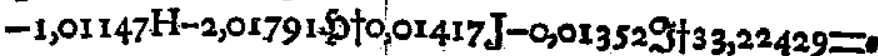
$+2,34674 \mathrm{~J}-2,0179195+0,00535 \mathrm{H}+0,0007390+30,68116=$ vnde elicimus :

$\mathfrak{W}= \pm 0,00077 \mathrm{H}+0,26935 \mathrm{~J}+30,96780$

$\$=-0,00974 \mathrm{H}+0,00077 \mathrm{~J}-1,05989$
6. 184. Hi autem valores in pofterioribus aeguam tionibus fubltituti producent

$\mathrm{I}, 01290 \mathrm{H}+0,52937 \mathrm{~J}+39,25137=0$

$2,34539 \mathrm{~J}+0,02500 \mathrm{H}+32,84282=0$ hincque tandem concluditur :

$$
\begin{aligned}
& H=-21,68110 . . \quad L H=x, 33608 \pi \\
& J=-x_{3,77206} \cdot \cdot \operatorname{dm} J=x_{0} 138999 \\
& \mathfrak{S}=+27,24155 \text {. . } l \mathfrak{S}=\mathrm{d}, 435232 \\
& 9=-1,25933 \cdots l-\$=0,100140 \\
& b^{\prime}=-0,09396 \cdot . \quad l-b^{\prime}=8,972943 \\
& i^{\prime}=-0,16533 . .2 \ln j^{\prime}=9,218352
\end{aligned}
$$

6. 185. Nunc pro excentricitate orbitae folaris hae reftant aequationes,

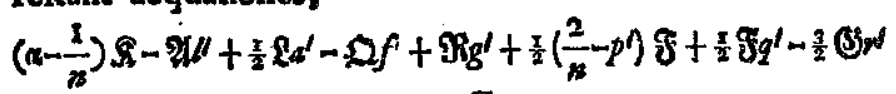$$
-\frac{3}{4}+\frac{32}{2 m n}-\frac{9 F}{4 m}+\frac{9 G}{4 n s}=
$$

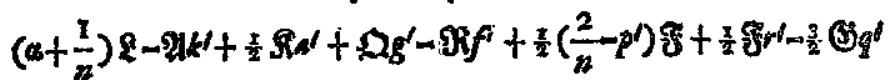

$$
-\frac{3}{4}+\frac{3 \mathrm{~K}}{2 m m}-\frac{9 \mathrm{~F}}{4 m}+\frac{9 \mathrm{G}}{4 m m}=0
$$

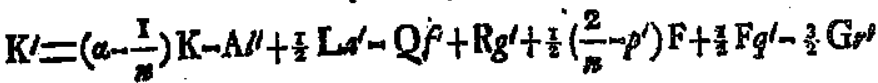
$\mathrm{L}^{\prime}=\left(\alpha+\frac{\mathrm{x}}{n}\right) \mathrm{L}-\mathrm{A} k^{\prime}+\frac{\mathrm{x}}{2} \mathrm{~K} a^{\prime}+\mathrm{Q} g^{\prime}-\mathrm{R} f^{\prime}+\frac{\mathrm{T}}{2}\left(\frac{2}{n}-p^{\prime}\right) \mathrm{F}+\frac{\mathrm{x}}{2} \mathrm{~F} r^{\prime}-\frac{3}{2} \mathrm{G} q^{g}$ $\left(\alpha-\frac{1}{n}\right) K^{\prime}-A^{\prime} / d-\frac{1}{2} L / d^{\prime}-Q^{\prime} f^{\prime}-R^{\prime} g^{\prime}+\frac{1}{2}\left(\frac{2}{n-p^{\prime}}\right)^{\prime} F^{\prime}-\frac{1}{2} F^{\prime} q^{\prime}-\frac{3}{2} G^{\prime} r^{\prime}$

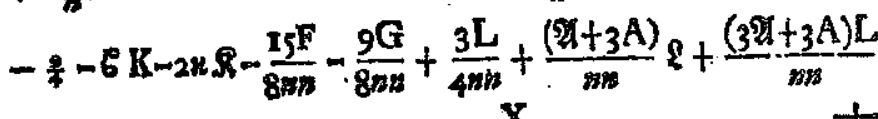


164.

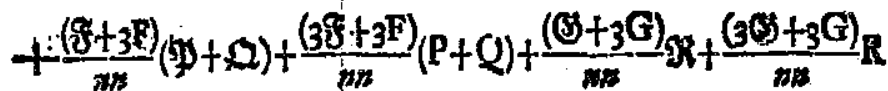

$$
\therefore+\frac{9 \mathrm{P}}{8 m}+\frac{9 \mathrm{Q}}{8 m}+\frac{15 \mathrm{R}}{8 m n}=0
$$

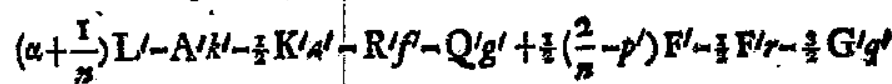

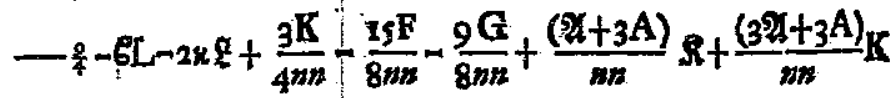

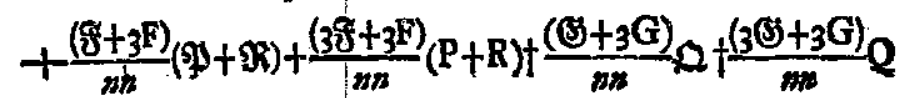

$$
+\frac{9 \mathrm{P}}{8 m}+\frac{9 \mathrm{R}}{8 m}+\frac{15 \mathrm{Q}}{8 n}=0
$$

\$.186. Hic autem obferuo, hanc determinationem me. xime effe lubricam, cum coefficiens litterse $L$, quem poftremo eft habitura, admodum fiat paruus; vnde is a terminis, ques omifimus, non mediocrem mutationem perpeti poffet. Hanc ob caufam confultum iudico, in calculum quoque terminos $3 \eta-s$ et $3 \eta+s$ introduce. re, quia praeuideo ab is coefficientes terminorum, quos quaerimus, non leuiter affici Sequenti ergo modo calculum redintegro.

\$. 187. In hunc finem quogue rationem habea. mus angulorum $3 \eta-s$ et $3 .+s$, fitque

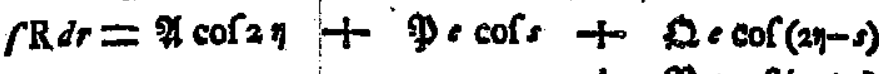

$+\pi e \operatorname{cor}(2 \eta+s)$

$+8 v \operatorname{cor} \eta+\AA v e c o r(\eta-s)+\mathfrak{m u e c o r}(3 \eta-s)$

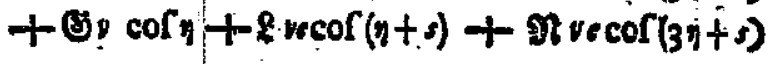

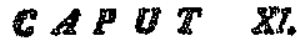

$=1 \operatorname{col} 2 \eta+2 e \operatorname{cor}+Q e \operatorname{col}(2 \eta-5)$

$+\operatorname{Recor}(2 \eta+s)$

$+F y \operatorname{cof} \eta+K v e c o f(\eta-s)+M y e \operatorname{col}(3 q-s)$

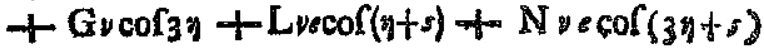

8. 188. Quodfi iam ponamus:

$$
\begin{aligned}
& \frac{2 x \mathrm{~K}+\Re}{n n}=k^{\prime} ; \frac{2 x \mathrm{~L}+\mathfrak{n}}{n n}=n \\
& \frac{2 x \mathrm{~V}+\mathrm{M}}{n}=m \quad \frac{2 x \mathrm{~N}+\mathfrak{M}=n}{n}=n
\end{aligned}
$$

erit

$\frac{d \varphi}{d r} \doteq$ Prace. $-e^{\prime} \operatorname{cof} 2 \eta-p^{\prime} e \operatorname{col} s-q^{\prime} e \operatorname{col}(2 \eta-s)$

in ma.

quem

de is a

tionem

judico,

oduce.

jorum,

i ergo

habea.

$(2 y-s)$

$(2 y+$.

$(y-5)$

$x+s)$

$v=$ $-f v \operatorname{cor} \eta-k^{\prime} v e \operatorname{cor}(\eta-s)-m^{\prime} v e \operatorname{col}(3 \eta-s)$

$-g^{\prime} v \operatorname{col}_{3} y-v e \operatorname{col}(\eta t s)-n^{\prime} v e \operatorname{col}(3 y+s)$

atquie ob $\frac{d s}{d r}=\frac{1}{n}-\frac{2}{3}+\operatorname{cof} s$ eric

$\frac{d \eta}{d r}=a-A^{\prime} \operatorname{cof} 2 \eta+\left(\frac{2}{2}-p^{\prime}\right) e \operatorname{cor} s-q^{\prime} \cos (2 \eta-s)$

$-f^{\prime} y \operatorname{cor} \eta-k^{\prime} y+\operatorname{cor}(y-s)-m^{\prime} y e \operatorname{cof}(3 \eta-s)$

$-g^{\prime} \cdot v \operatorname{col}(3 \eta-\| y \operatorname{cor}(\eta+s)-4 y e \operatorname{co}\{(3 \eta+s)$

18. 189. Formulas nuric affumtas differentiemus, folosque terminos, quibus opus habemus, in leado exprimamus ac reperiemus : $\quad \ldots+, \ldots$.

$\mathbf{R}=$ Praec.

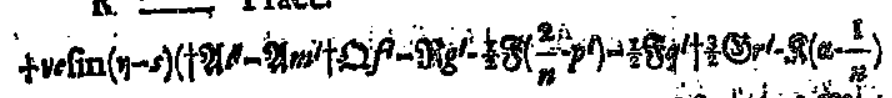

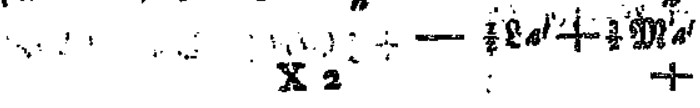

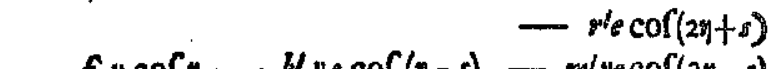


264

$$
\text { C.AP.U. } \boldsymbol{X}
$$

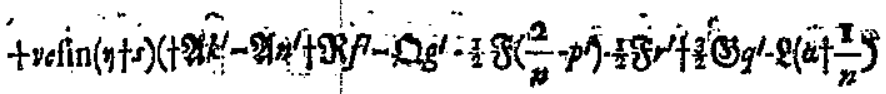
- $\frac{x}{2} \Re^{\prime}+\frac{3}{2} \mathfrak{S}^{\prime}$

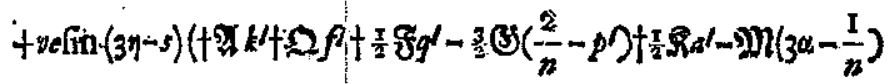

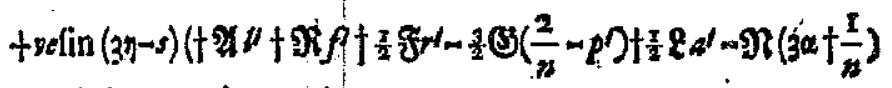
Ac fi breuitatis gratia ponamus:

$\mathrm{K}^{\prime}=\left(\alpha-\frac{1}{n}\right) \mathrm{K}-\mathrm{A}\left(t^{\prime}-m^{\prime}\right)-\mathrm{Q} f^{\prime}+\mathrm{R} g^{\prime}+\frac{3}{2} \mathrm{~F}\left(\frac{2}{n}-p^{\prime}\right)+\frac{1}{2} \mathrm{~F} q^{\prime}-\frac{3}{2} \mathrm{G}{ }^{\prime}$ $+\frac{3}{2} \mathrm{~L} d-\frac{3}{2} \mathrm{Md}$ $\mathrm{L}=\left(\omega+\frac{\mathrm{I}}{n}\right) \mathrm{L}-\mathrm{A}\left(k^{\prime}-n^{\prime}\right)-\mathrm{R} \rho+\mathrm{Q} g^{\prime}+\frac{x}{2} \mathrm{~F}\left(\frac{2}{n}-p\right)+\frac{x^{2}}{\mathrm{~F}} \mathrm{~F}-\frac{3}{2} \mathrm{G} q$ $+\frac{1}{2} \mathrm{~K} A^{\prime}-\frac{3}{2} \mathrm{~N} d$ $M^{\prime}=\left(3^{a}-\frac{T}{n}\right) M-\dot{A} k^{\prime}-Q f^{\prime}-\frac{\pi}{2} q_{q^{\prime}}+\frac{3}{2} \mathbf{G}\left(\frac{2}{n}-p\right)=\frac{x}{2} \mathrm{Kal}$ $N^{\prime}=\left(3 \alpha+\frac{x}{n}\right) N-A \mu_{-} R f^{\prime}-\frac{x}{2} \mathrm{~F} \dot{H}+\frac{3}{2} \mathrm{G}\left(\frac{2}{n}-p\right)-\frac{x}{x} \mathrm{~L} d$ erit

$$
\frac{\partial v}{d r}=-\mathrm{A}^{\prime} \text { fin } 2 \eta \perp \mathrm{P}^{\prime} \text { ifins }-\mathrm{Q}^{\prime} \text { efin }(2 \eta-3)
$$$$
\text { - } \mathrm{F}^{\prime} / \mathrm{fin} \eta-\mathrm{K}^{\prime} \mathrm{vefin}(\eta-s) \longrightarrow \mathrm{R} / \mathrm{e} \text { fin }(2 \eta+s)
$$
- M/yefin $(3 y-s)$

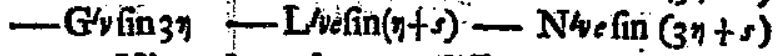
6. 190. Hinc jam denno differéritiando nancis: cemur

$$
\frac{d d v}{d r^{2}}=\text { Praec: }
$$

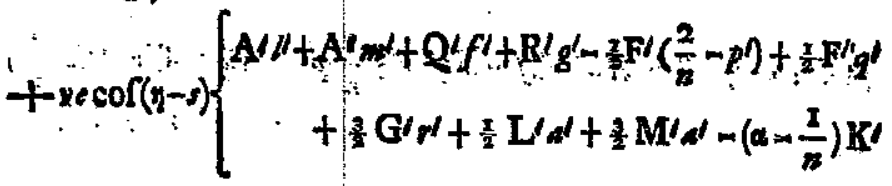

$\triangle A P U T \quad Z I$.

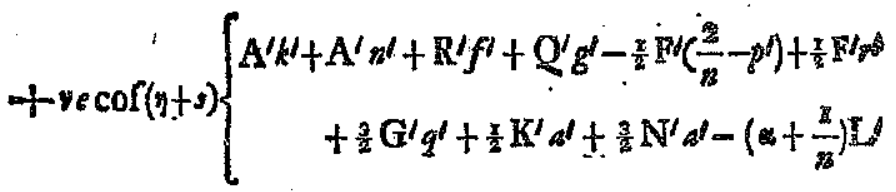

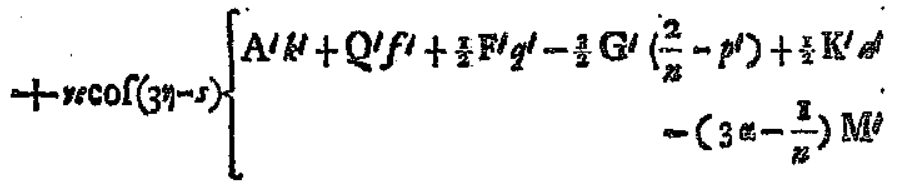

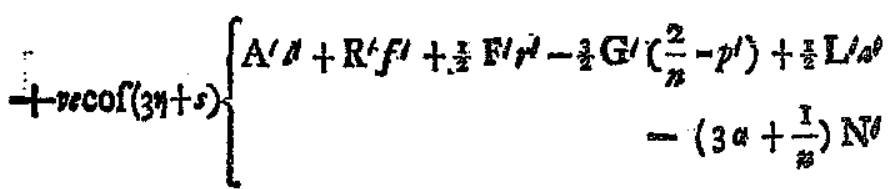

\$. 191. Quodf antem valores iam inuenti fubttic tuantur, habebitur

$$
\begin{aligned}
& \mathbf{R}=\text { Praec. } \\
& \operatorname{trefn}(y-s)\left\{\begin{array}{c}
-0,85830 \AA+0,005262-0,025002 M+0,37592 \\
-0,00931 \mathrm{~L}+0,00931 \mathrm{M}
\end{array}\right\} \\
& \operatorname{tvefn}(\eta+s)\left\{\begin{array}{c}
-1,00918 \&+0,00526 \Omega(-0,025009 R+0,34115 \\
-0,00931 \mathrm{~K}+0,0093 \mathrm{IN}
\end{array}\right\} \\
& +\operatorname{vefn}(3 \eta-5)\left\{\begin{array}{c}
-2,72578927-0,014489+0,49223] \\
-0,00931 \mathrm{~K}
\end{array}\right] \\
& \operatorname{tvefn}(3 \operatorname{tits})\left\{\begin{array}{c}
-2,87666927-0,01448 \&+0,47116 \\
-0,00931 L
\end{array}\right] \\
& x_{3} \\
& x=
\end{aligned}
$$


$C A P U T \mathbb{Z}$

487

$\mathbf{K}^{\prime}=0,85830 \mathrm{~K}+0,00527 \mathrm{~L}+0,01447 \mathrm{M}+\mathrm{I}, 48970$ $+0,00750 \mathfrak{l}-0,00750 \mathrm{M}$

$L^{\prime}=1,00976 \mathrm{~L}+0,00527 \mathrm{~K}+0,01_{447} \mathrm{~N}+1,55183$ $+0,00750 \Omega-0,00750 \mathfrak{R}$

$M^{\prime}=2,72528 \mathrm{M}+0,02501 \mathrm{~K}-\mathrm{I}, 17408$ $+0,00750 \Omega$

$N^{\prime}=2,87666 \mathrm{~N}+0,02501 \mathrm{~L}-0,95901$

$$
\frac{d d v}{d r^{2}}=\text { Praec. }
$$

tved(y-s) $\left\{\begin{array}{l}-0,85830 \mathrm{~K} /-0,02843 \mathrm{~L}-0,02843 \mathrm{M}-0,32674 \\ -0,00987 \mathrm{~L}-0,014098-0,01409 \mathrm{M} \\ -0,02961 \mathrm{M} /\end{array}\right\}$

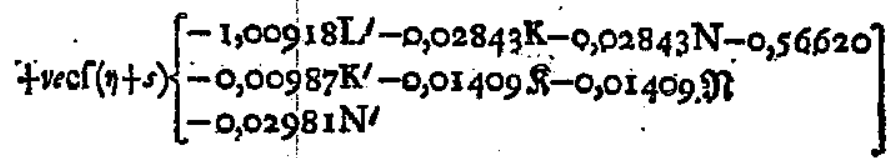

tvec $(3 y-5)\left\{\begin{array}{l}-2,72578 M^{\prime}-0,02843 \mathrm{~K}+0,74683 \\ -0,00987 \mathrm{~K} / \\ -0,01409 \mathrm{~A}\end{array}\right]$

tvec $\left\{(3 y+s)\left\{\begin{array}{l}\left\{, 8766 \mathrm{~N} \mathrm{~N}^{\prime}-0,02853 \mathrm{~L}\right. \\ -0,00987 \mathrm{~L}-0,01409 \mathrm{~L}\end{array}+0,67486\right\}\right.$

6. 192. Valores; autem litterarum commate notatarum hic fubftitutac dabunt : :

$\frac{d d v}{d r^{2}}$

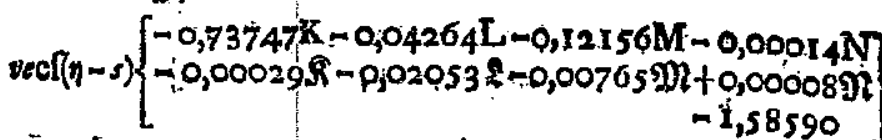

8970

5183

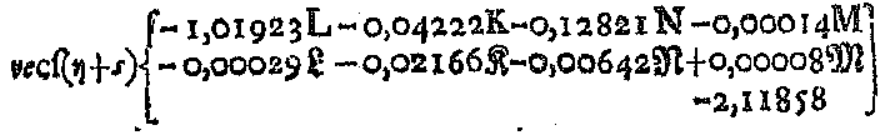

$\operatorname{vec}(37-5)\left\{\begin{array}{l}-7,43058 \mathrm{M}-0,09507 \mathrm{~K}-0,00005 \mathrm{~L}+3,93357 \\ +0,00008 \mathrm{M}-0,03453 \mathrm{R}-0,00007 \mathrm{~g}\end{array}\right]$

$\operatorname{vec}\left\{(3 y+s)\left\{\begin{array}{l}-8,27529 \mathbb{N}-0,11338 L=0,00005 K+3,41830 \\ +0,00008 \mathfrak{N}-0,03566 \mathbb{R}-0,00007 \AA\end{array}\right]\right.$

674

$520]$

193. His expreffionibus ita euolutis atque ad calculum numericum praeparatis, quaeramus easdem expreffiones ex formulis fupra traditis pro $\mathbb{R}$ et $\frac{d d v}{d r^{2}}$, quae continentur in $\$ .52$ et 54 . Inde autem omitrendis terminis, quos iam tractauimus, confequemur.

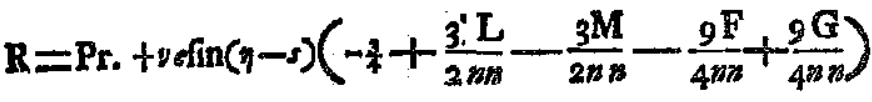

$$
\begin{aligned}
& +v e \operatorname{fin}(n+r)\left(-\frac{3}{4}+\frac{3 \mathrm{~K}}{2 m}-\frac{3 \mathrm{~N}}{2 m B}+\frac{9 \mathrm{G}}{4 m}-\frac{9 \mathrm{~F}}{4 m}\right)
\end{aligned}
$$

20ta-

$$
\begin{aligned}
& \text { trefin }(3 y-s)\left(-\frac{\pi s}{4}+\frac{3 K}{2 m n}-\frac{2 F}{4 n n}\right) \\
& +\operatorname{vefin}(3 n+s)\left(-\frac{x^{5}}{4}+\frac{3 L}{2 m n}-\frac{9 F}{4 m}\right)
\end{aligned}
$$


5. 194. Introducantur hic quoque valoses im cogniuti, ac prodibic

$\mathbf{R}=\operatorname{Pr} .+\nu e$ fin $(y-s)[-\mathrm{T}, 02394+0,00854[-0,00854 \mathrm{M}]$ $+v e f i n(4+s)[-1,08394+0,00854 \mathrm{~K}-0,00854 \mathrm{~N}]$ $+v e$ fin $(3 y-s)[-4,01450+0,00854 \mathrm{~K}]$ $+y_{e}$ fin $(34+s)[-4,01450+0,00854 \mathrm{~L}]$

$\therefore \quad \frac{d d v}{d r^{2}}=$ Praec.

$+\operatorname{vecl}(y-s)\left\{\begin{array}{l}-1,01591 \mathrm{~K}-0,03207 \mathrm{~L}-0,03207 \mathrm{M}-1,16867 \mathrm{I} \\ -2,01798 \Re-0,027112-0,02711 \mathrm{MI}\end{array}\right]$

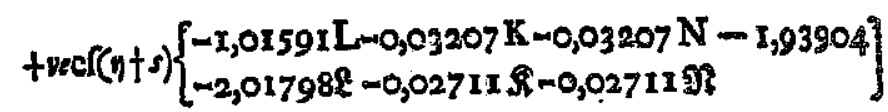
$+y_{k} c(3 \%-s)\left\{\begin{array}{l}-1,01591 \mathrm{IM}-0,03207 \mathrm{~K}-2,49545 \\ -2,01798 \mathrm{Mm}-0,027 \mathrm{II} \AA\end{array}\right]$ trecI(13ts $)\left\{\begin{array}{l}-1,01591 N-0,03207 \mathrm{~L}-2,78450 \\ -2,0179892-0,02711 \mathrm{I}\end{array}\right\}$ 

refulsabunt

L $0,85830 R=+0,00526 \mathfrak{R}-0,02500 \mathfrak{M}+\mathrm{x}, 39986$ $-0,01785 \mathrm{~L}+0,03785 \mathrm{M}$

Il. $1,00918 \mathrm{R}=+0,00526 \mathrm{M}-0,02500 \mathfrak{N}+1,36509$ $-0,01785 \mathrm{~K}+0,01785 \mathrm{~N}$

III. $2,72578 M=-0,01448 \Omega+4,50673$ $-0,01785 \mathrm{~K}$

IV. $2,87666 \Re=-0,01448 \&+4,48566$ $-0,01785 \mathrm{~L}$

V. $f 0,27844{ }^{K} \cdot 0,01057 L-0.08949 M-0,0001_{4} N+0,10081=$ t2,01769 ifto,00658 \&-0,01946Mito,00008:?

V1.-0,00332L-0,01015K-0,09614 N-0,00014 M-0,17954=。

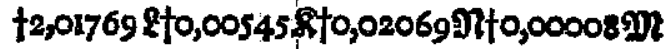

VII, $-6,41487 \mathrm{M}-0,0,6300 \mathrm{~K}-0,00005 \mathrm{~L}+6,42802=$ $+2,01806 \mathfrak{M}-0,00742 \mathrm{~S}-0,00007$ \&

Vill, $-7,25938 \mathrm{~N}-0,08131 \mathrm{~L}-0,00005 \mathrm{~K}+6,20280=$

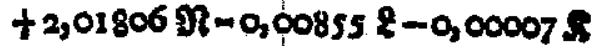

6. 196. Ex sequationibus III et IV fatim elioina: wi hi valores

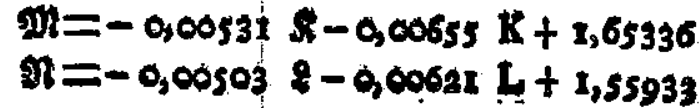

$$
\text { CADT } X I_{s}
$$

qui in I et II lubtituti praebent:

$0,85817 R=1,35853+0,00526 \&-0,01785(L-M)+0,00016 \%$

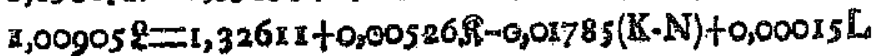
vnde obtinetur:

$\Re=+1,59116-0,02080(\mathrm{~L}-\mathrm{M})+0,00008 \mathrm{~K}+0,00010 \mathrm{~N}$ $2=+1,32251-0,01769(K-N)+0,00005 \mathrm{~L}+0,0001 \mathrm{M}$ $\mathfrak{M}=+\mathbf{r}, 6449 \mathrm{x}-0,00655 \mathrm{~K}+0,00010(\mathrm{~L}-\mathrm{M})$ $\mathfrak{n}=+1,55268-0,0 \$ 62 \mathrm{~L}+0,00009(\mathrm{~K}-\mathrm{N})$

8. 197. His valoribus fubftituris caeterae aequatio nes abibunt in formas fequentes:

$0,27862 \mathrm{~K}-0,05252 \mathrm{~L}-0,04754 \mathrm{M}+0,00017 \mathrm{~N}+3,35313=6$ $-0,00346 \mathrm{~L}-0,04584 \mathrm{~K}-0,06045 \mathrm{~N}+0,00020 \mathrm{M}+2,83002=$ $-6,41502 \mathrm{M}-0,07622 \mathrm{~K}+0,00030 \mathrm{~L}+9,73587=0$ $-7,2597 \mathrm{IN}-0,09384 \mathrm{~L}+0,00028 \mathrm{~K}+9,32499=0$

ex quarum binis poftremis ftatim obtinetur:

$\mathrm{M}=-0,01188 \mathrm{~K}+0,00005 \mathrm{Li}+\mathrm{\pi}, 5176 \mathrm{~g}$

$\mathrm{N}=-0,01293 \mathrm{~L}+0,00004 \mathrm{~K}+\mathrm{I}, 2844^{\circ}$ ynde colligitur :

$+0,27918 \mathrm{~K}-0,05252 \mathrm{~L}+3,28120=$ $-0,00268 \mathrm{~L}-0,04584 \mathrm{~K}+2,45267=0$ ac denique

$\mathrm{K}=+40,447 \mathrm{10}$. . . l K $\mathrm{K}=1,606887$

$L=+368,40200 . . \quad l L=2,566322$

$\mathrm{M}=+\mathrm{t}, 05555$. . $6 \mathrm{M}=0,02347^{8}$

$N=3,47730$ : . l $L N=0,541242$

qul 
\$. 198. Litterarum germanicarum valores hine erunt:

$$
\begin{aligned}
& \Re=-5,04677 \text {. . . } L-R=0,703013 \\
& g=+0,56302 \text {. . } l \mathfrak{l}=9,750524 \\
& \mathfrak{M}=+1,41672, \cdots, / \mathfrak{M}=0,151283 \\
& \mathfrak{N}=-0,73119 \text {. . . } \quad \text { l } \mathfrak{l}=9,864030
\end{aligned}
$$

ac litterarum hinc deripatarum:

$$
\begin{aligned}
& k^{\prime}=+0,43578 \text {. . } t k=9,639267 \\
& \|=+4,23401 \quad \cdot \quad l\|=0,626752 \\
& m^{\prime}=+0,02018 \text { : . } l m^{\prime}=8,304921 \\
& n=-0,04409 \text {. . . . } \operatorname{lm} m=8,644340
\end{aligned}
$$

§. 199. Nunc iginur intelligimus inaegualicates ab angulis $3 y_{-} s$ et $3 y+s$ pendentes tam effe paruas, ve fine vllo errore reiici queant, etiamfi valores $\mathbf{K}$ et $\mathbf{L}$ aliquantum immutauerine. Diftantia ergo lunse strtata a terra $x=\frac{(1-k k) a n}{1-k \operatorname{cof} r}$ ita ab his inaequalitatibus paral. lacticis perdebit, ve fit

$\omega=$ Prace. $+0,11756$. cort

$$
-0,004 \pi 9 . \operatorname{cor} 3 y
$$$$
-0,1234, k \cos (\eta-r)
$$$$
-0,0784 \text { th } \operatorname{cor}(\eta+r)
$$$$
+0,2302 v \operatorname{cor}(\eta-s)
$$$$
+2,0965 y \operatorname{cor}(p+s)
$$

\section{Log. coeff.}

9,070249

7,622540

9,091265

8,894183

9,362071

0,321506
Motus autem momentaneus ita hine afficieture vt fis $\frac{d \phi}{d r}=$ Praec.

$$
\begin{aligned}
& 0,23259 \vee \operatorname{cor} y \\
& +0,01309 \times \operatorname{cor}_{31} \\
& \text { + 0,0939 vecof }(y-r) \\
& +0,1653 \text { skcof }(y+r) \\
& \text { - } 0,4358 \text { yecor }(y-s) \\
& -4,2340 \text { vecor }(y+s)
\end{aligned}
$$

5. 200. Quodí iam ipfam longizudinem. lunae, quatenus ab his inaequalitatibus parallacticis pendet, ponamus:

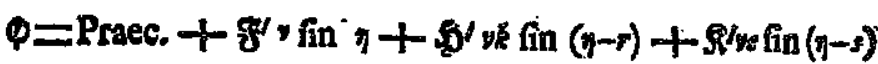
$+\sigma^{\prime} y$ fin $3 y+9 / y k$ fin $(y+r)+q^{\prime} y e$ fin $(\eta+s)$

fequentes obtinebimus aequationes pro horum coefficientium determinatione:

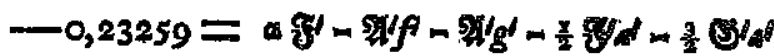

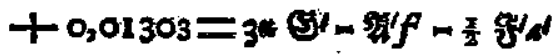

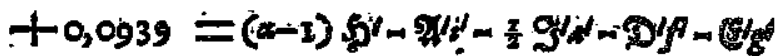

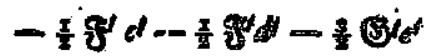

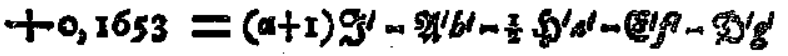

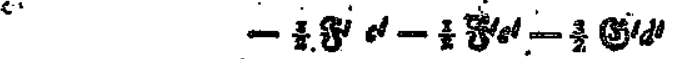

$$
\begin{aligned}
& x_{3}
\end{aligned}
$$




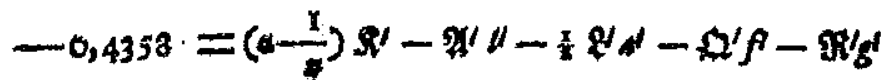

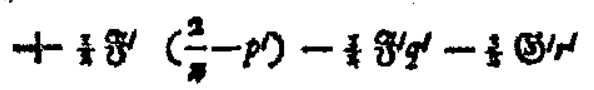

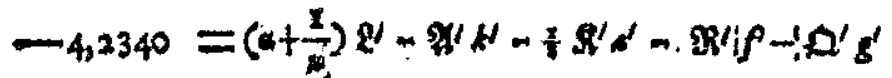

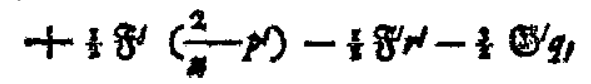

5. 202. Valoribus autem iam cognitis hic fubtitu. Atitu. tis, aequationes iftae in fequentes abibunt formas;

$-0,2303 x=+0,94361 z+0,0296 x$ or

$+0,01550=+2,801223+0,00987 \mathrm{gH}$

$-0,0056=-0,06626,940,00987 \mathrm{~J}^{\prime}-0,25665 \mathrm{~g}^{\prime}+0,01926 \mathrm{BJ}^{\prime}$

$+0,1710=f r, 93374940,00987 \oint^{\prime}-0,06717 \mathrm{~J}^{\prime}-0,54918$ es

- $-0,3968=\nmid 0,85830 \Omega^{\prime}+0,009878 / 40,06357 z^{\prime \prime}-0,04509$ or

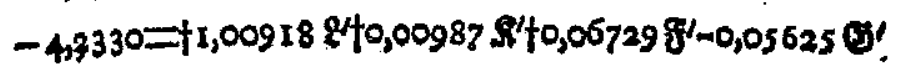

rnde colligitur fore

$$
\begin{aligned}
& g=-0,244^{27} \cdot \cdots, F^{\prime}=9,387868 \\
& O H=+0,00639 . .2 B=7,805991 \\
& \$=+1,1959 \quad \therefore \quad t \Phi^{\prime}=0,077694 \\
& g^{\prime}=+0,0757 \text {. . } l g=8,879096 \\
& S^{\prime}=-0,3959 \quad \cdots \quad M B^{\prime}=9,597508
\end{aligned}
$$

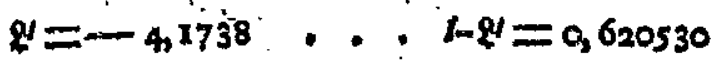

5. 202. Hine ergo habebimus fequentes partes pro longitudine Lunae, quas firmul ope valorum proxime cogritorum pro $y_{2} k_{2}$, ad minuta fecunda reducamus:

$$
\text { Log.coeff. Val, coeff. }
$$

$$
\begin{aligned}
& \varphi=\text { Praec. }-0,24427 \text { fin } 4 \quad 9,387868 \mid-175^{\text {1d }} \\
& +0,00039 v \text { in } 34 \text { 7, } 305991+4^{\prime \prime} \\
& +1,1959 \text { ok fin }(y-r) \quad 0,077694+59^{\prime \prime} \\
& +0,0757 \text { vk fin }(\eta+r) \quad 8,879096-4^{\prime \prime} \\
& -0,3959 \text { ve fin }(\eta-s) \quad 9,597508-5^{\prime \prime}
\end{aligned}
$$

Sicque omnes iam adeptì fumús motus lunae in. zequalitates, quae quidem ab inclinatione eius orbitae ad eclipticam non pendent. Intexim tamen non diffiteor, darl aliquas infuper inacgualitates, quae alicuius forte fint momenti, quas in hac inueftigatione practeri. imus, cuiusmodi funt eac, quae ab angulis $2 \eta y-3 r$ et $2 y-2 r+s$ pendent, quae ad plure minuta fecunda ago furgere pofie videntur. Verum earum determinacio tarib eft cediofn, vt malim eam obferuationibus relinquere.

6. 203. Quac ergo hakteras invenimus, in vaurm colligamus ac primo pro diftantia lunie a terca curtato $s=\frac{(1-k t)}{1-k \cos }$ erit 
$\because A B U T$ XI.

4.

8. 204. Pro mocu autem lunae momentaneo, $+0,0000532 \mathrm{col} 41 \quad 5,725912+0,000053$

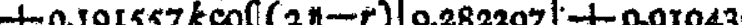
$-0,003293 k \operatorname{col}(21+r) 7,517525-0,000179$ $-0,003321$ keor $(41-r)$ 7,521296 $-0,000181$ $+0,000049 k \operatorname{col}(4 \eta+r)[5,692584+0,000005$ $-0,00511 k k \cos 2 r$ $7,708601-0,000015$ $-0,08022 k k \operatorname{cor}(2 n-2 r) \mid 8,904280-0,000238$ $-0,00237 k k \operatorname{cor}(2 y+2 r)$ 7,375072. $-0,000007$ $+0,07892 k k \operatorname{cor}(4 y-2 r) \mid 8,897172]+0,000234$ $+0,00001 k k \operatorname{cor}(4 t+2 x)+4,872349+0,000000$ $-0,006300<\mathrm{cor} s$ $+0,014801 / \cos (21-5)$ 8,170303 $+0,000249$ $+0,021415$ ecor $(2 y+s) 8,057492+0,000192$ $+0,00364$ ecof $2 s \quad 7,539670+0,000001$ $-0,01482 \times e \operatorname{cor}(2 \eta-2 s)] 8,170799-0,000004$ $-0,00584$ ecor $(2 \eta+2 s)] 7,766856-0,000001$ $-0,37957$ ekdor $(r-s) \quad 0,579297-0,000347$ $+0,30693$ ekcor $(r+s) \quad 9,487039+0,000281$ $-0,36337$ ekcor $(2 x-r+s)[0,560344-0,000332$ $+0,00993$ skcor(2n+r-s) $7,097004+0,000009$ - 0,077.52 ck cof(2y-r-s) 8,889425 $-0,00007 \mathrm{x}$ $+0,00305$ e $k \operatorname{col}(24+r+s) \quad 7,484024+0,000003$ $+0,11756$ cof $9,070249+0,000408$ $-0,00419$ cor 3 7 7,622540 $=0,000015$ $-0,1234$ (1) $\operatorname{co}(\eta-r) \quad 0,091265-0,000024$ $-0,0784 v k \operatorname{cof}(y+r) \quad 8,894183-0,000015$ $+0,2302$ ve cof $(y-s) \quad 9,362071+0,000013$ $+2,0965 x=\operatorname{cor}(y+s)|0,321506|+0,000122$

Hic ad latus adiunxi valores coefficientium integrorum in numeris abfolutis expreflos, ponendo $k=0,05445$, $s=0,01680$ et $y=1+1$; quos proinde, $f$ hi valores aliter per obferuationes determinentur, frcile erit emen. dare. quo eius motus horarius definiri poterit, habebimus:

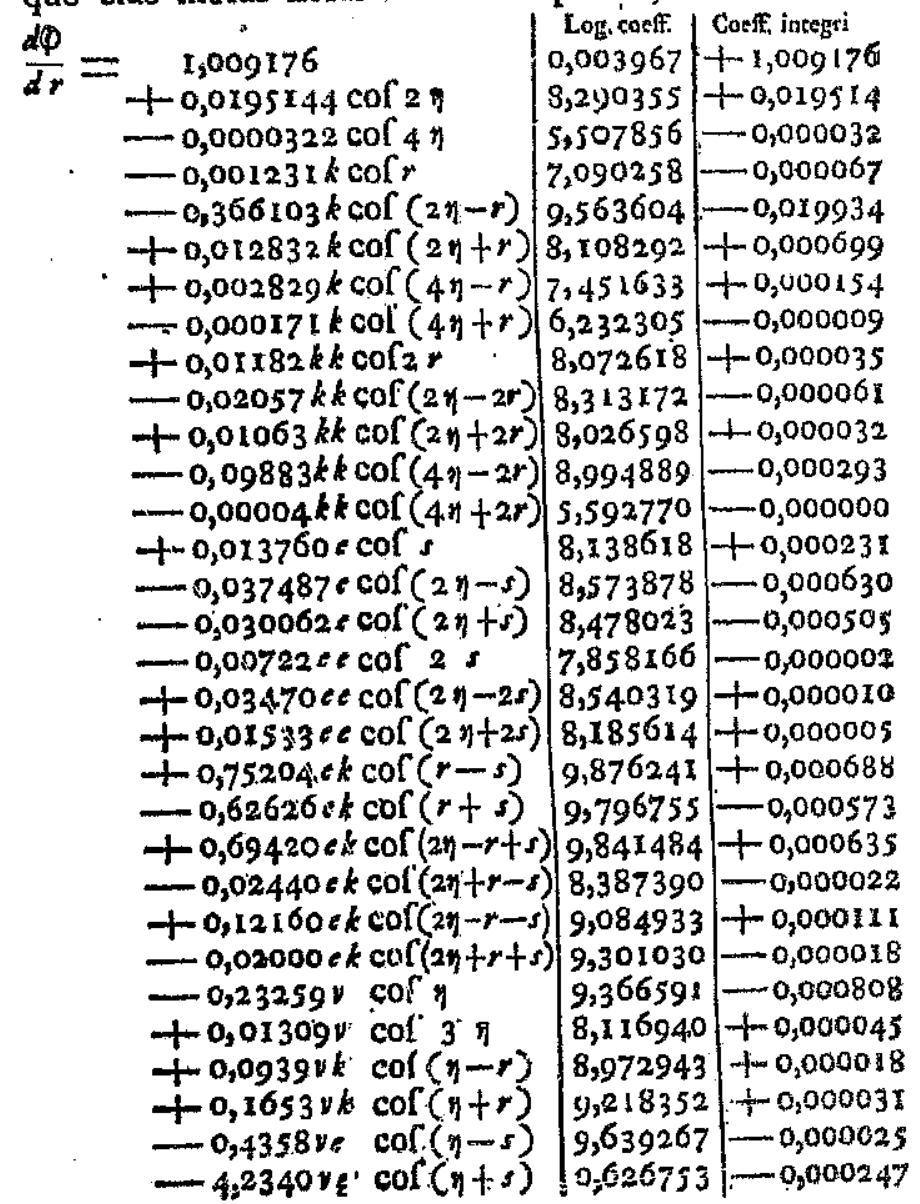

$\mathbf{Z}$ 
\$. zo5. Sj iam longitudo Lunae per folam excentricita. tem fecundum regulas Keplerianas determinate ponatur $=\zeta$, ita ve pofita eius anomalia vera $=r$, futurum lit $\zeta=\mathbf{C}$ $+1,0085272$ r, erit longitudo vera per haltenus inuentas inaequalitates.

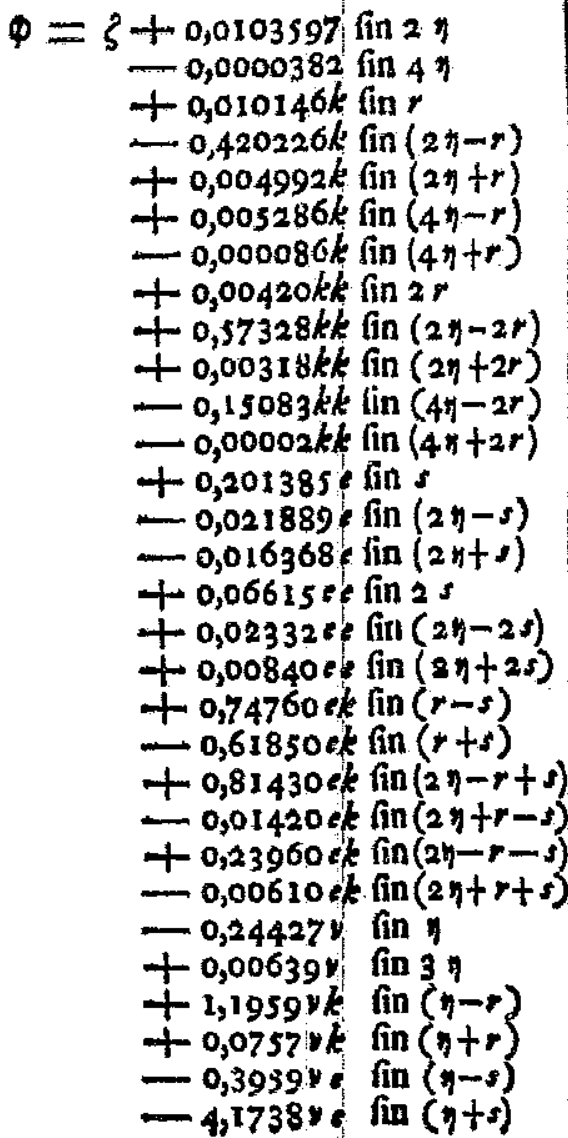

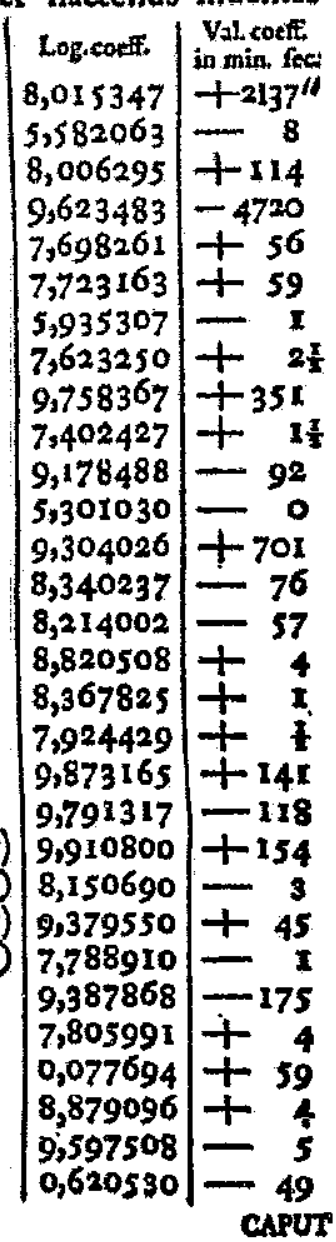

ericita-

onatur

\section{A PUT XII.}

\section{INUESTIGATIO INAEQUALITATUM MOTUM} LINBAE NODORUN ARPICIENTIUM,

6. 206 .

A ntequam reliquas motus Lunae inaequalitates, quae ab inclinatione eius orbitae ad eclipticam peno dent, definire licer, cum variationes, quae in motu lineae nodorum Lunae, tum eas, quae in ipfa inclinatione eius orbitae ad eclipticam deprehenduntur, inueftigari oportet. Refidua enim pars aequationis noftrae principalis, qua omnes motus Lunae inaequalita tes continentur, litteras $x$ et $g$ implicat, quarum illa longitudinem nodi afcendentis, haec vero $\ell$ inclinationem ad eclipticam defignac. Nifi igitur ytriusque hu. ius quantizatis incrementa vel decrementa ad differen tiale $d r$ reduxerimus, refiduas moths Lunpe inaequalitates determinare non poterimuss.

207. Aequatio autem fupra (55) pro moth lineac nodorum tradita, cum fit $\frac{2 k v+\int R d r}{n}=n+\frac{1+2 e e}{n n}-\frac{d \phi}{d r}$

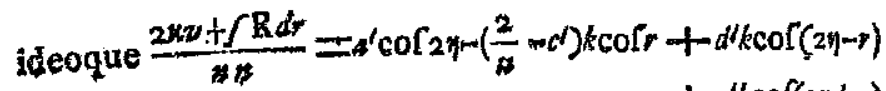
$+\epsilon^{\prime} k \cos (2 \eta+r)$ $+p^{t} e \cos s+q^{\prime} e \cos (2 \eta-s)$ $+\operatorname{sic}(2 \theta+s)$

黑 2 \$i 
(i ponamus breulatetis gratia $\frac{3(1+2 k k+g e c)}{x k+z}=i$, vt fit $i=$ 0,0168918 , induet formam fequentem:

$\frac{d \pi}{d r}=-1\left(x+\alpha \cos 2 n-\left(\frac{2}{n}-4\right) k \operatorname{cor} r+d k \operatorname{col}(2 n-r)+e t c\right)$

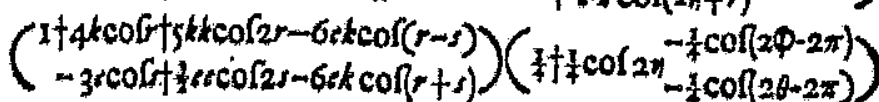

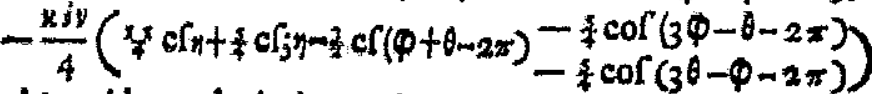
vbi quidem plurimi termini tam funt parui, ve facile negligi queant.

6. 208. Productum sutem ex duobus prioribus: factoribus, quonism id in formula pro inclinatione recurrit, feorfin exlibesmus : fiet id surem reießtis terminis, qui prae reliquis admodum funt parui vt feguitur:

$$
\begin{aligned}
& \text { ' }-x i+2 i\left(\frac{2}{n}-\infty\right) k k-i j p^{\prime}(r] \quad-0,017043 \\
& \operatorname{col}^{21} \quad\left(-i^{2} s^{2}-2 i \alpha^{4} k k\right. \\
& k \operatorname{cor} r \quad\left(i\left(\frac{2}{n}-x\right)-4 k i\right. \\
& k \operatorname{col}(2 \eta-r)\left(-i d^{4}-2 i k^{\prime}\right. \\
& k \operatorname{cof}(2 \eta+r)(-i d-2 i n t \\
& \text { kt cor } 2 r \quad\left(-5 x i+2 i\left(\frac{2}{2}-r^{\prime}\right)\right. \text {. } \\
& \text { cort } \quad\left(-i p^{\prime}+3 x i\right. \\
& \text { ecor }(2 y-s)\left(i q^{\prime}+i \alpha\right.
\end{aligned}
$$

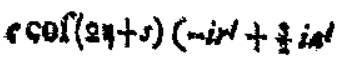

$$
\begin{aligned}
& m \operatorname{cor} 2 s \quad\left(-\frac{3}{2} x i+\frac{3}{2}\right) \\
& +0,000162 \text { cor } 2 h \\
& -0,068110 \text { k colr } \\
& -0,005791+\operatorname{cor}(2 \eta-r) \\
& =+0,000828 * \operatorname{cor}(2 y+r) \\
& -0,085092 \text { ktcor } 2 r \\
& +0,051362 \text { e cors } \\
& -0,000929+\operatorname{cor}(2 q-s) \\
& -0,000804 \text { e cor }(2 y+s) \\
& -0,025914 \mathrm{eecol} 2 \mathrm{~s}
\end{aligned}
$$

\$. 209. rit: $=$

facile

ioribus

ne re-

th ter-

ve fe-

$1 n$
§. 209. His valoribus fubttitutis prodibit

$$
\begin{array}{cc}
\frac{d r}{d r}=- & 0,004261+0,000020 \\
\operatorname{cof} 2 \pi & (+0,000040-0,004261 \\
k \operatorname{cor} r \quad & (-0,0 \pi 7043-0,000620
\end{array}
$$

$k \operatorname{cor}(2 \eta-r)(-0,001448-0,0085 \pi 4)-0,010636 k k \mathrm{cf}(2 \gamma-2 r)$

$k \operatorname{cor}(2 \eta+r)(+0,000207-0,008514)-0,010036 k k c(2 \eta j+2 r)$

kk col $2 r \quad(-0,021273$

e cors $\quad(+0,012840 m 0,000217$

e $\operatorname{cor}(2 y-s)(-0,000232+0,0064,20)-0,003239 \operatorname{cec}(2 y-2 . s)$

- $\operatorname{cor}(2 y+s)(-0,00020 x+0,006420)-0,003239 \operatorname{eec}\{2 y t 2 s)$

se cor $2 s \quad(-0,006479)$

$\operatorname{cor} 2(\phi-\pi)(+0,00326 \operatorname{Im} 0,000020)-0,00004 \mathrm{r} \operatorname{cof}$ i

$+0,000019 \cos (3 \theta-\theta-2 \pi)$

$\operatorname{cor}_{2}(\theta-\pi)(+0,004261-0,000020)-0,000019 \operatorname{cor} 34$ $+0,000019 \operatorname{cor}(3 \theta \ln \varphi-2 \pi)$

$k \operatorname{cf}(29-2 \pi-r)(+0,008514+0,000724)$

$+0,000022 \cos (\phi+\theta-2 \pi)$

$k \operatorname{cr}(2 \varphi-2 \pi+r)(+0,008514-0,000103)$

$+0,010636 k k c 0\left\{2\left(\varphi_{-y-\pi)}\right)\right.$

$k \operatorname{cr}(2 \theta-2 \pi-r)(+0,008514-0,000103)$

$k \mathrm{cf}(2 \theta-2 \pi+r)(+0,008534+0,000724)$

$\operatorname{ccf}(24-2 \pi-s)(-0,006420+0,000116)$

$\operatorname{ccf}(2 \varphi-2 \pi+s)(-0,006420+0,000100)$

eff $\left(2^{\theta}-2 \pi-s\right)(-0,006420+0,000100)$

$\operatorname{col}(2-12 \pi+s)(-0,006420+0,000136)$

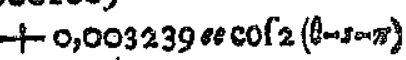

$\mathrm{Z}_{3}$

§. Iro. 
d. 210. Habebimus ergo

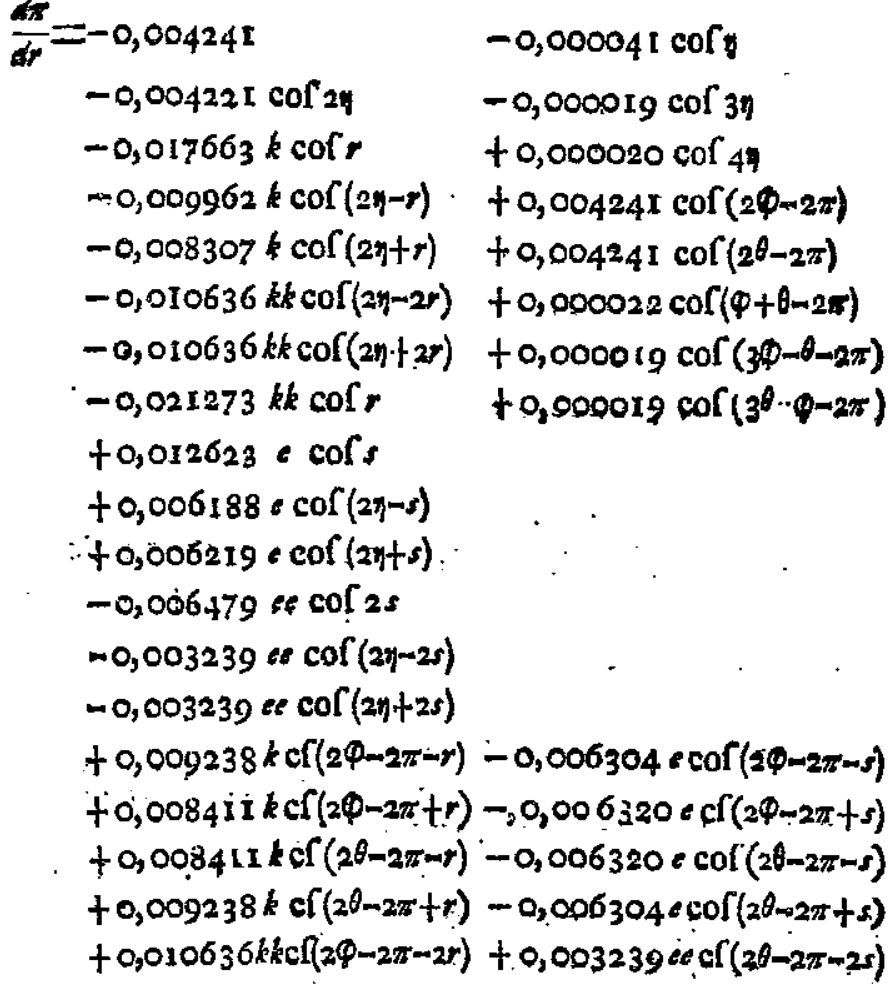

6. 211. Quanquam plurimi horum terminorum tam funt parui, ve in fe fpectati tuto reiicl poffent, ta men quidam per integrationem ad magnitudinem fatis notabilem excrefcere pofunt. Hujus autem indolis funt illi termini, qui eiusmodi compleçłunnur angplọs, qua- rum differentialia ad $d r$ admodum paruam senént atio nem, cuiusmodi funt anguli is, $25,2 \theta-2 \pi, 2 \theta-2 \pi s, s$, $2 \theta-2 \pi+s, 2 \varphi-2 \pi-2 r$ et $2^{\theta}-2 \pi-2 s$; quorum natura differentialium ex fequentibus formulis colligi poreft:

$\frac{d \eta}{d r}=a-a^{\prime} \operatorname{cor} 2 \eta-c^{\prime} k \operatorname{cor} r-d^{\prime \prime} k \operatorname{cor}(2 \eta-r)-e^{\prime} k \operatorname{cor}(2 \eta+r)$ $+\left(\frac{2}{n}-p^{\prime}\right) e \operatorname{cor} s-q^{\prime} e \operatorname{cor}(2 \eta-s)-r^{\prime} e \operatorname{cor}(2 y+s)$

$\frac{d \varphi}{d r}=a+\frac{1+2 \varepsilon e}{n}-a^{\prime} \operatorname{col} 2 \eta+\left(\frac{2}{n}-c^{\prime}\right) k \operatorname{cor} r$

$$
-d^{\prime} k \operatorname{cor}(2 \eta-r)-p^{\prime} t \operatorname{cor} s-q^{\prime} e \operatorname{cof}(2 \eta-s)
$$

$$
-e(k \operatorname{col}(2 \eta+r) \quad-\quad-r l e \operatorname{col}(2 \eta+r)
$$

$\frac{d s}{d r}=\frac{d \theta}{d r}=\frac{1+2 e e}{n}+\frac{2}{n} k \operatorname{cor} r-\frac{2}{n} e \operatorname{cor} s$

\$. 2 I 2. Quaeramus primo inaequalitares motus nodorum, quae neque ab excentricitate orbitae luna. ris neque folaris pendent, fitque :

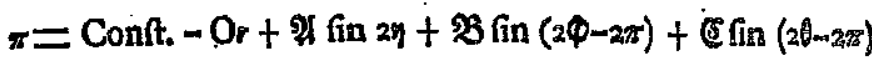
reie@tis reliquis terminis, quos praeuidemus fore minimos, ac differentiando obtinebimus :

$$
\begin{aligned}
& \frac{d \pi}{d}=-0-2 A^{\prime}-0,00424193-0,00424 i \\
& +\operatorname{cof} 2 \eta(20 \%-0,0042413-0,0042416
\end{aligned}
$$

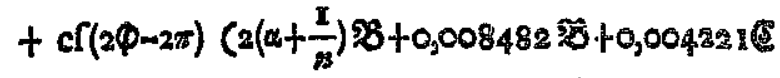

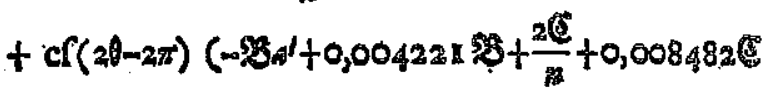


vnde orinur:

O- 0,019744 A +0,004241 $\mathfrak{B}+0,004241 \mathcal{C}=0,00424 \mathrm{E}$ $1,867476 \mathfrak{A}-0,004241 \mathfrak{B}-0,00424 \mathrm{I} E=-0,00422 \mathrm{I}$

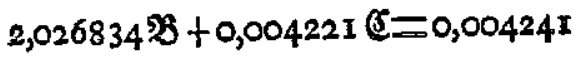
$0,023965 \mathfrak{B}+0,159358 \mathfrak{C}=0,004241$

6. 213. Valores hinc igitur prodibunt fequentes :

$$
\begin{aligned}
& 0=+0,004078 \text {. . } 10=7,610447 \\
& \mathfrak{A}=-0,002596 . .1-\mathfrak{A}=7,341634 \\
& \mathfrak{B}=+0,002037 \cdot . \quad 1 \mathfrak{B}=7,30899 \mathrm{I} \\
& \mathfrak{E}=+0,026307 \text {. . } l \mathfrak{C}=8,42008 \mathrm{I}
\end{aligned}
$$

Vbi primum obferuo valorem ipfius $O$ iam proxime accedere ad motum medium iunae nodorum, vti per obfervationes conftat; inde enim effe deberes $\mathrm{O}=0,004053$ facile antem intelligitur, hunc exiguum defectum per - reliquas inaequalitates fuppleri poffe. Quocirca hinc erit

$$
\begin{aligned}
& \pi=\text { Conft. }-0,004078 r \quad \text { Valones is min. fee } \\
& -0,002196 \text { fin } 2 \\
& +0,002037 \text { fin }(24-2 \pi)+453 \\
& +0,0263.07 \text { fin }(20-2 \pi) \mid+5426
\end{aligned}
$$

quae inaequalitates mirifice conueniunt cum obferuatio. nibus. His addi poteft terminus :

$$
+0,00033^{6} \text { fin }\left(4^{\theta-4 \pi}\right)
$$

cuius in minutis fectundis valor eft $+6 g^{\prime \prime}$, qui terminus aypir poftemo illo facile conituris poteft.

5. 21 4. Quxisramus jam feorfim insequalitates, quae ab excentricitate orbitae tumaris pendent, litque

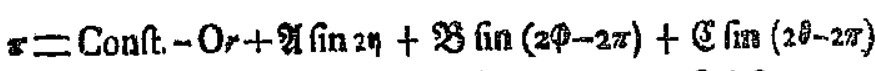
$+\mathfrak{D} k$ fin $(2 y-r)+\mathbb{E} k \operatorname{fin}(2 y+r)+g k$ fin $r$ + @s $k k \ln (2 n-2 r)+5 k k$ fin $2 r+9 k \ln (2 \varphi-2 r-r)$ $+\Re k \operatorname{fn}(2 \varphi-2 \pi+r)+\{k \ln (2 \theta-2 \pi-r)+M k f n(2 \theta-2 \pi+r)$ $+9 k k$ fin $(2 \hat{\varphi}-27 \pi-2 P)$

eritque differentiando

$\frac{d \pi}{d r}=\mathrm{Pr} . \dagger k \mathrm{cf}(2 y-r)\left(-2 \pi c^{\prime}-0,009238 \Re-0,0092386+(2 a-1)\right)$

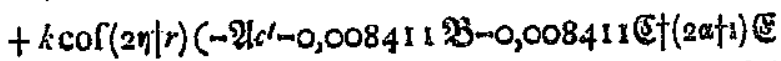

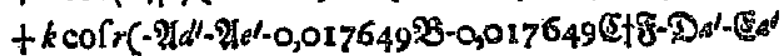

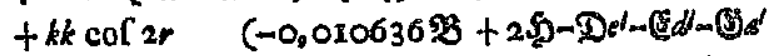
$+k k \operatorname{cor}(2 y-2 r)(-0,010636 c+2(\alpha-1)(3)-D d$

$+k \operatorname{cof}(2 \theta-2 \pi-r)\left\{\begin{array}{r}-3 e^{\prime}+0,008307 \mathfrak{B}+\frac{2}{8} \mathbb{C}+0,017663 \mathfrak{C} \\ +\left(\frac{2}{2-1}\right)+0,008482\end{array}\right.$

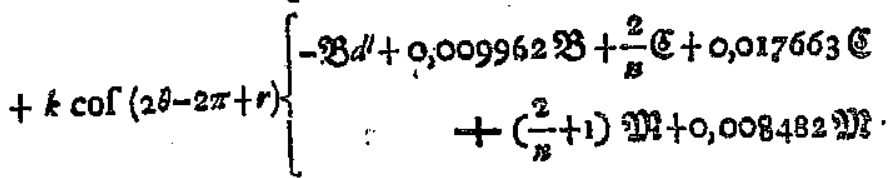

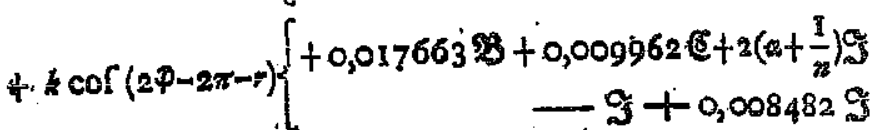

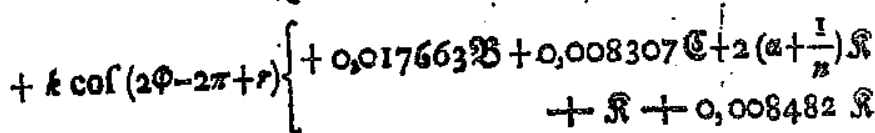

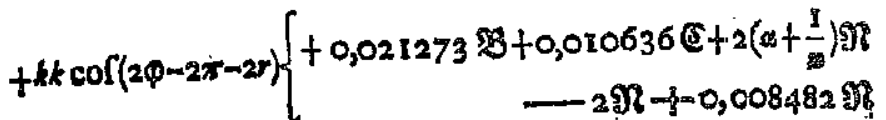


\$. 215. Superfluum foret maiorem curam in his differentialibus adhibere, guia vero proxime tantum rem determinare fufficit; erit ergo :

Hineque

$$
\begin{aligned}
& 0,867476 \mathfrak{D}=-0,009962 \\
& 2,867476 \text { \& }=-0,008307 \\
& \text { et in min fec. }
\end{aligned}
$$

$$
\begin{array}{l|l}
\mathfrak{P}=-0,011480 & D_{k}=-129^{\prime \prime} \\
-0,002900 & \mathbb{E} k=-33^{\prime \prime}
\end{array}
$$

quae inaequalitates in loco nodi vix alicuius funt momenti, vnde eas exactius determinare non eft opus.

§. 216. Calculo autem etroluto eris

$$
\begin{aligned}
& \pi=\operatorname{Pr} .-0,0 \times 1480 k \text { fin }(2 \pi-r) .\left|8,05994^{\circ}\right|-129^{\prime \prime} \\
& -0,002900 k \text { fin }(2 y+r) \quad 7,462400-33 \\
& -0,017663 k \text { fin } \text { r } \\
& +0,090497 k k \text { fin }(2 y=0 r) \\
& -0,011978 k k \text { in } 2 r \\
& +0,008707 k \text { fin }(2 \phi-2 \pi-r) \mid 7,939851+98 \\
& +0,002701 \ddot{k} \text { fin }(2 \varphi-2 \pi+r)|7,43151 \sigma|+30 \\
& -0,004680 k \text { fin }(2 \theta-2 \pi-r) \quad 7,670224-53 \\
& +0,004685 k \text { fin }(2 \theta-2 \pi+r) \mid 7,670680+53
\end{aligned}
$$

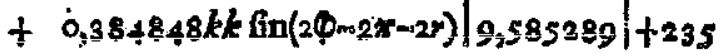

6. $2 \times 7$. Simili modo inueftigemus inaequalitates motus nodorum, quae pendent ab excentricitate orbitae folaris fitgue;

$$
C A P U T X I \text {. }
$$

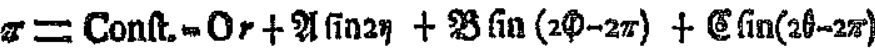

+ Defins + Eefin $(2 y-s)+\operatorname{gefin}(2 y+s)$

+ Geefin2s $+\operatorname{sinefn}(2 \varphi-2 \pi-s)+\operatorname{Sefn}(2 \theta-2 \pi-s)$

$+\operatorname{sefn}(2 \varphi-2 \pi+s)+s e \ln (2 \theta-2 \% t s)$

+2 eefn $(2 \theta-2 \pi-2 s)$

vade differentiando pro terminis quaefitis erit:

$$
\begin{aligned}
& \frac{d \pi}{d r}=\text { Praec. }
\end{aligned}
$$

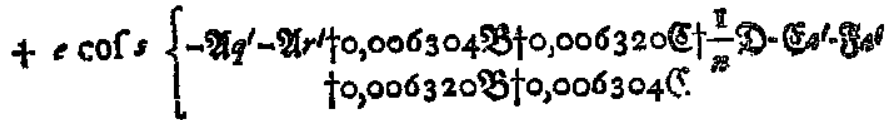

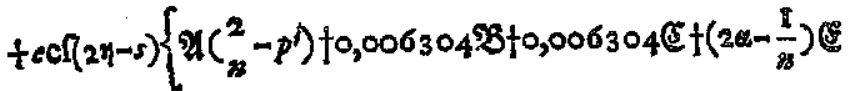

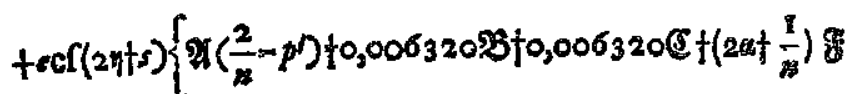

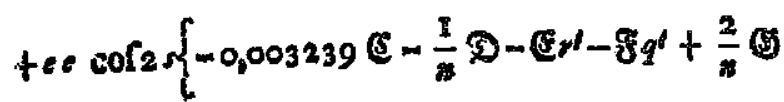

$$
\begin{aligned}
& +e \cos (2 \phi-2 \pi-s)\left\{\begin{array}{l}
-9 \$ p^{\prime}-0,01262393-0,006188( \\
+\left(2 \pi \pm \frac{1}{8}+0,008482\right) \$
\end{array}\right. \\
& +\cos (2 \varphi-2 \pi+5)\left\{\begin{array}{l}
-3 p^{\prime}-0,012623 \Re-0,0062196 \\
+\left(2 \pi+\frac{3}{3}+0,008482\right) \%
\end{array}\right.
\end{aligned}
$$

A. 2 


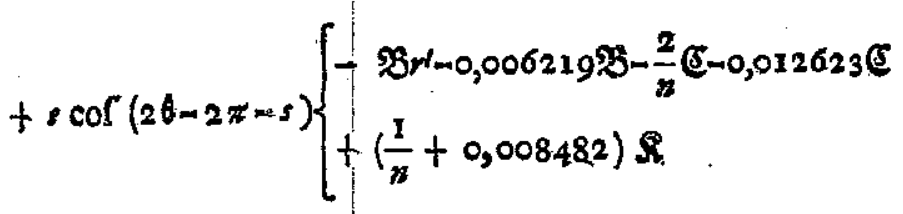

§. $x$ 19. Colligamus ergo has inaegualitates in vnam fummam, atque obsinebimus longitudinem veram nodi afcendentis

$+e \operatorname{cof}(24-2 \pi+s)\left\{\begin{array}{l}-3 q^{\prime}-0,0061883 B-\frac{2}{x}(5-0,012623 \mathfrak{C} \\ +\left(\frac{3}{3}+0,008482\right) \mathfrak{g}\end{array}\right.$

$z=$ Conft $-0,004053$ r alor. in minus, fes. $+e c \cos (2 \theta-2 \pi-2 s)\left\{\begin{array}{l}+0,003239 \Re+\frac{1}{2 B} C+0,006479 \mathbb{C} \\ +0,008482 \mathfrak{M}-\frac{1}{3} \Re-0,012623 \mathbb{R}\end{array}\right.$

\$. 218. Hinc reperiuntur fequentes valores

- $D=0,159070 . / D=9,201585 ; D C=551 / l$

E $=0,003562 \cdot l \mathbb{E}=7,551680 ; \mathbb{E}=\mathrm{x}_{2}^{\frac{\pi}{2}}$

$g=0,003301$ ol $l=7,518677 ; g e=11 x$

$0,03 \times 650 . t O=8,587191 ; 04=2^{\prime \prime}$

$\mathfrak{W}=-0,003 \times 53$. $4 \mathfrak{\$}=7,498692 ; \mathfrak{\$}:=-\mathrm{II}^{\prime \prime}$

$9=-0,002932$. $49=7,467118 ; 9 e=-10$ th

$\Omega=-0,025750$. $4 \Omega=8,410784 ; \Omega_{C}=-90$

$z=-0,009076$. $42=7,957885 ; \& e=-32$

At valor ipfiue im tam fit paruas, $r t$ merito pro nihilo haberi poffit

$-0,002196$ fin $2 \%$ $+0,002037$ fin $\left(2 \varphi_{-2 \pi}\right)$ $+0,026307$ fin $(2 \theta-2 \pi)$ $+0,000370$ fin $\left(4^{6-4 \pi}\right)$ $-0,01766 k$ fin $r$

$$
\begin{aligned}
& -453^{1 k} \\
& +\quad 420 \\
& +5426 \\
& +\quad 75 \\
& -\quad 198
\end{aligned}
$$$$
-0,01148 k \text { fin }(2 \eta-r) \quad-122 \text {. }
$$$$
-0,00290 k \text { fin }(2 \pi+\psi)-3
$$$$
+0,0905 k k \text { fin }(2 \eta-2 r)+35
$$$$
-0,0120 k k \text { fin } 2 \% \text { r }
$$$$
+0,00871 k \text { fin }(2 \varphi-2 \pi-r)+98
$$$$
+0,00270 k \text { in }\left(2 \varphi_{-2 \pi+r}\right)+30
$$$$
-0,00468 k \text { fin }(2 \operatorname{lom} 2 \pi-r)=53
$$$$
+0,00468 k \text { fin }\left(2 b_{2} 2 \pi+p\right)+53
$$$$
+0,3848 k k \ln (29 \cdot 2 \pi-2 x)+3
$$$$
+0,15907 \text { e fin s }+551
$$$$
-0,02575 \text { e fin }(2 \theta-2 \pi-s) \text {. } \quad 9^{\circ}
$$$$
-0,00907 \cdot \operatorname{fin}(2 \theta-2 \pi+s)-32
$$

omiffis fcilicet is inaequalitatibus, quae non fupsa $30 / t$ exfurgunt.

CAPUT 


\section{A P U T XIII.}

\section{INUESTIGATIO INCLINATIONIS ORBITAE} LUNARIS AD ECLIPTICAM.

\section{0.}

Dro inclinatione orbitae lunaris ad eclipticam inuenienda, forma \$. 208. euoluta multiplicari debet per $-\frac{x}{4}$ fin $2 \eta+\frac{\pi}{4}$ fin $2(\varphi-\pi)+\frac{x}{4}$ fin $2(\theta-\pi)$, ac produktum erit $=\frac{d . \text { tange }}{d r}$ : Hine ergo habebitur:

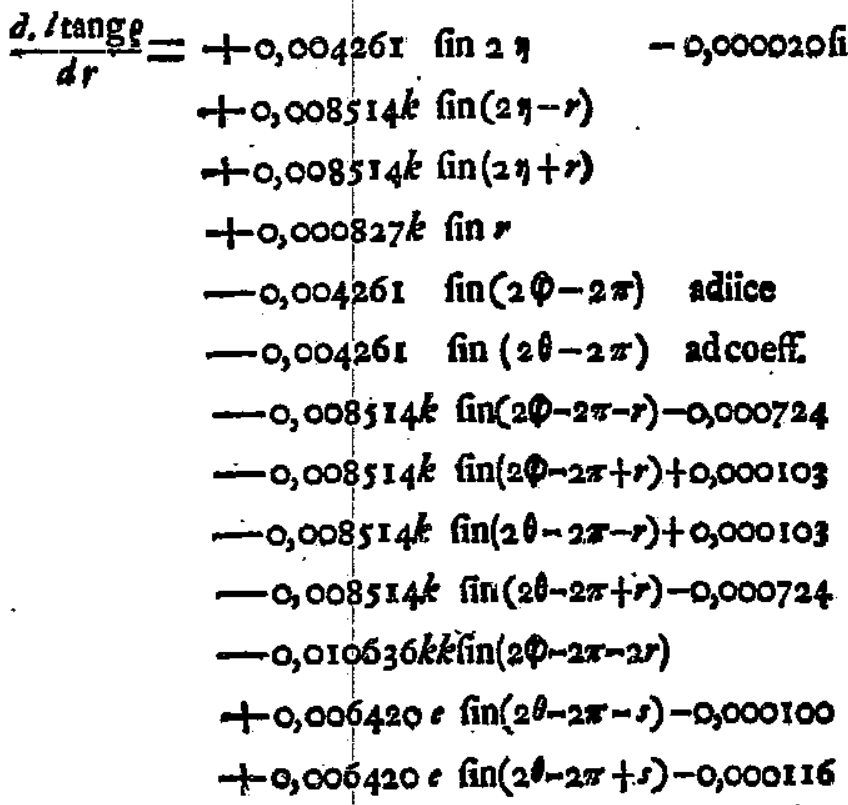

6. 22I. Quaeramus primo cerminos. ㄸำ excencricitate pendene, fitque

$$
\text { t } \frac{\text { tang } e}{\text { tang }}
$$

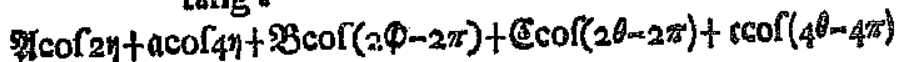
eritque differentiando:

$$
\frac{d \text { tange }}{d r}=\text { fin } 2[-2 \alpha \mathfrak{A}+0,0042412 \delta-0,004241 C
$$$$
\text { fin } 4 \eta[-4 \alpha \mathfrak{a}+2 \text { ad }
$$

$$
\begin{aligned}
& \text { fin }(2 \phi-2 \pi)\left\{-2\left(\alpha+\frac{I}{n}\right) \Re-0,0084822 B \sim 0,004227\right. \text { (E } \\
& \text { fin }(2 \theta-2 \pi)\left\{-\frac{2}{13}\left(\mathcal{C}+B A^{\prime}-0,00422153-0,008482 \mathbb{C}\right.\right. \\
& \text { fin }\left(4 ^ { 8 - 4 \pi } \left\{\left\{+0,004241 \text { (E) }-\frac{4}{8}\right.\right.\right.
\end{aligned}
$$

9. 222. Ex his iam reperitur :

$$
\begin{aligned}
& \text { 解 }=-0,002630 \text {. . l- } 4=7,419914 \\
& \mathfrak{B}=+0,002037 \text {. . } \mathfrak{B}=7,30899 \mathrm{I} \\
& c=+0,026307 \text {. . . l } c=8,42008 \mathrm{I} \\
& a=+0,000019 \text {. . . } l a=5,278753 \\
& c=+0,000370 . . \quad l c=6,567938
\end{aligned}
$$

it vt hinc fit:

$$
\begin{aligned}
t \frac{\text { tang }}{\text { tang: }}= & -0,002630 \operatorname{col} 21 \\
& +0,000019 \operatorname{cof} 41 \\
& +0,002037 \operatorname{cof}(2 \varphi-2 \pi) \\
& +0,026307 \operatorname{cof}(2(-2 \pi) \\
& +0,000370 \operatorname{cor}(4 \theta-4 \pi)
\end{aligned}
$$


6. 223. Quaeramus iam feorfim terminos ab excentricitate Lanae pendentes: fitque

$<\frac{\text { tang } \varphi}{\text { tang } \varepsilon}=\mathscr{A} \operatorname{cor} 2 \eta+3 \operatorname{cor}(2 \varphi-2 \pi)+\mathcal{C o r}(2 \theta-2 \pi)$

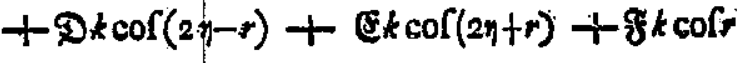
$+0 k \operatorname{col}(2 \varphi-2 \pi-r)+9 k \operatorname{cor}(2 \theta-2 \pi-r)$ $+\$ k \cos (2 \phi-2 \pi+r)+\Re k \cos (2 \theta-2 \pi t r)$ $+2 k^{2} \cos (2 \Phi-2 \pi-2 r)$

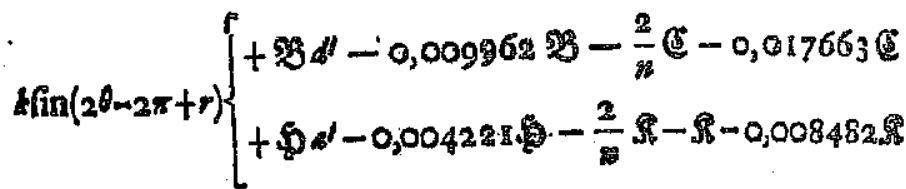

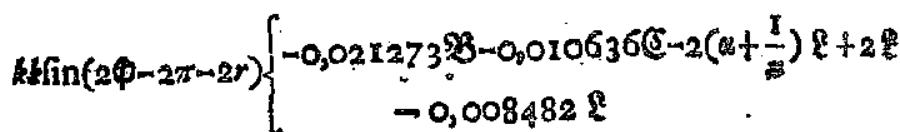
hincque reperitur:

vnde differentialibus fumendis habebitur: $\frac{d . \text { tang } \rho}{d r}=$ $D=0,010487$. . $l \mathfrak{D}=8,02063$

$E=0,003166 \ldots . .1 E \pm 7,500435$

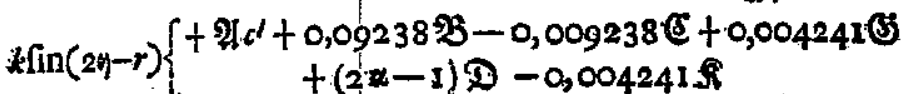
$f=-0,001600 \ldots \cdot \cdot 1-f F=7,204120$

(S) =十0,008719 . . . l $\$=7,940484$

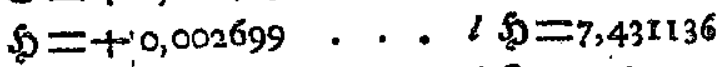

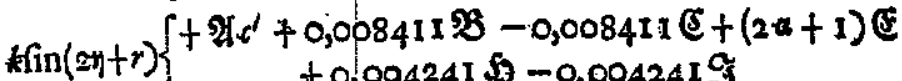
$\pm 0,0042419-0,0042419$

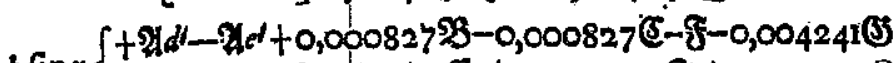
$\mathfrak{S}=-0,004460$. . $1-\mathfrak{I}=7,649305$ $\Re=+0,004717$. . . l $\$ 7=7,623628$

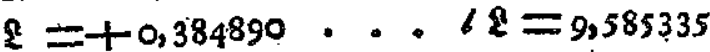

6. 224. Nunc derigque pro inaequalitatibus ab

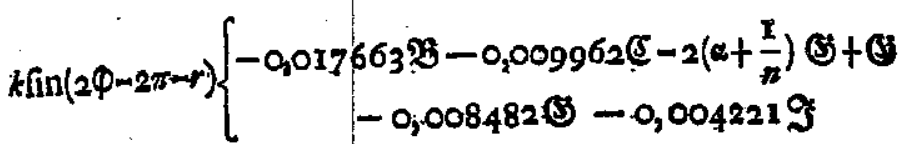
centricicate orbitae folaris pendentibus ponatur.

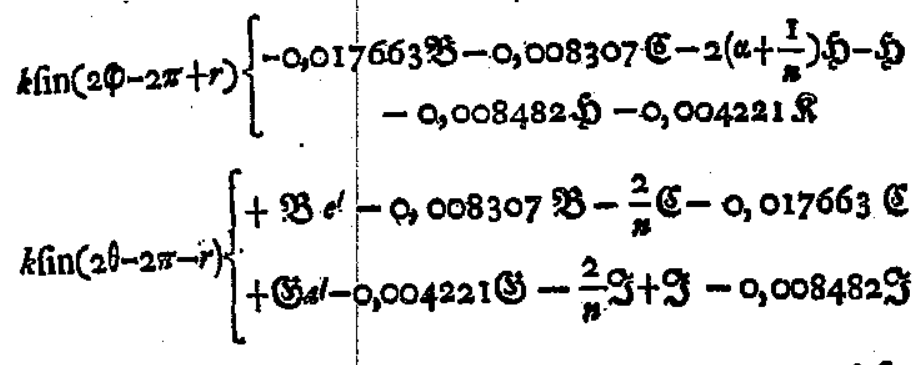

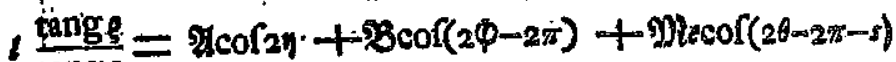

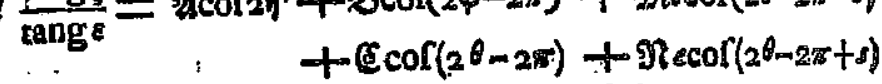
acidifferenisindo prodibit : $\frac{d . \text { lange }}{d r}=$

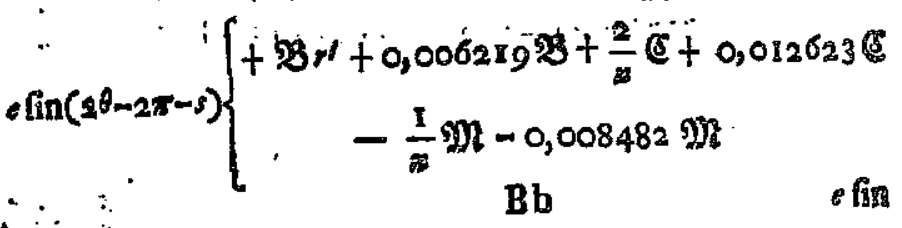


$\operatorname{efin}\left(2^{\theta-2 \pi}+s\right)\left\{\begin{array}{c}+9 q^{\prime}+t 0,06 \mathrm{r} 88 \mathfrak{B}+\frac{2}{n} \mathfrak{C}+0,012623 \mathfrak{C} \\ -\frac{3}{n} \mathfrak{R}-0,008482 \mathfrak{N}\end{array}\right.$

vade reperitur

$$
\begin{aligned}
& \mathfrak{M}=-0,024034: \therefore \quad l \mathbb{M}=8,380835 \\
& \mathfrak{N}=-0,008519
\end{aligned}
$$

S.-225. Si- ergo denotet inclinationem mediam orbitae lunaris ad eclipticam, es $\rho$ inclinationem veram, exit

$$
\begin{aligned}
& \text { tang } g=-0,002630 \text { cor } 2 \eta \\
& \begin{array}{l}
+0,000019 \operatorname{cof} 47 \\
+0,002037 \operatorname{cof}(20-2 \pi)
\end{array} \\
& +0,002037 \text { cor }(20-2 \pi) \\
& +0,02630^{\circ} \text { cor }(20-2 \pi) \\
& +0,01049 k^{\prime} \operatorname{cof}(2 j-r) \\
& +0,00317 k \text { cor }(2 y+r) \\
& \text { - } 0,00160 \text {. cor } r \\
& +0,00872 k \operatorname{cor}(2 \phi-2 \pi-r) \\
& +0 ; 00270 k \cdot \operatorname{cor}(2 \varphi-2 \pi+r) \\
& 0,00446 k \cos (2 \theta-2 \pi-r) \\
& +0,00472 k \text { cor }(2 \theta-2 \pi+r) \\
& +0,3849 k k \text { cor }(2 \varphi-2 \pi-2 r)
\end{aligned}
$$

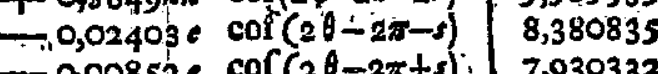

$$
\begin{aligned}
& 7,419915 \\
& 5,278753 \\
& 7,308993 \\
& 8,42008 \mathrm{I} \\
& 6,567931 \\
& 8,020638 \\
& 7,500439 \\
& 7,20,4120 \\
& 7,940484 \\
& 7,4.31136 \\
& 7,649305 \\
& 7,673628 \\
& 9,58.5335 \text {. } \\
& 7,930332
\end{aligned}
$$

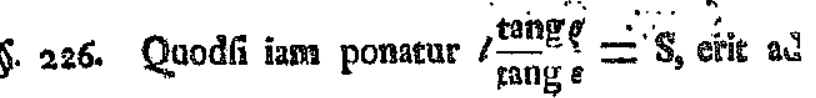

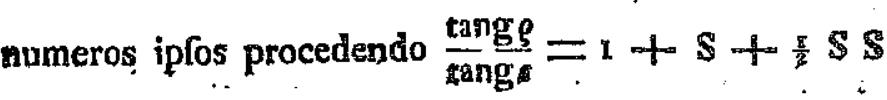
Hinc igitur negligendo terminos minimos, confso quemur:

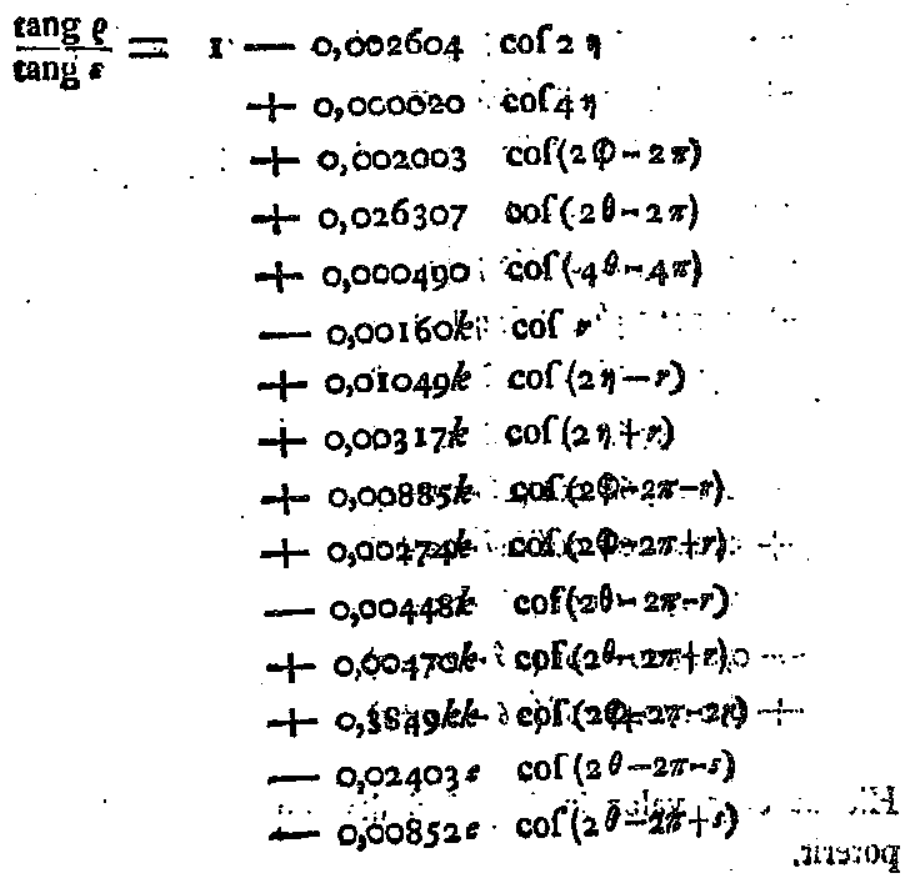

Bb 2 
5. 227. Cum in aequatione noftra principali, quae motum Lunae continet, infit terminus $\frac{\operatorname{tang} \rho^{2}}{\operatorname{tang} \tau^{2}}$, huiusguoque valorem euolui conueniet : erit ergo

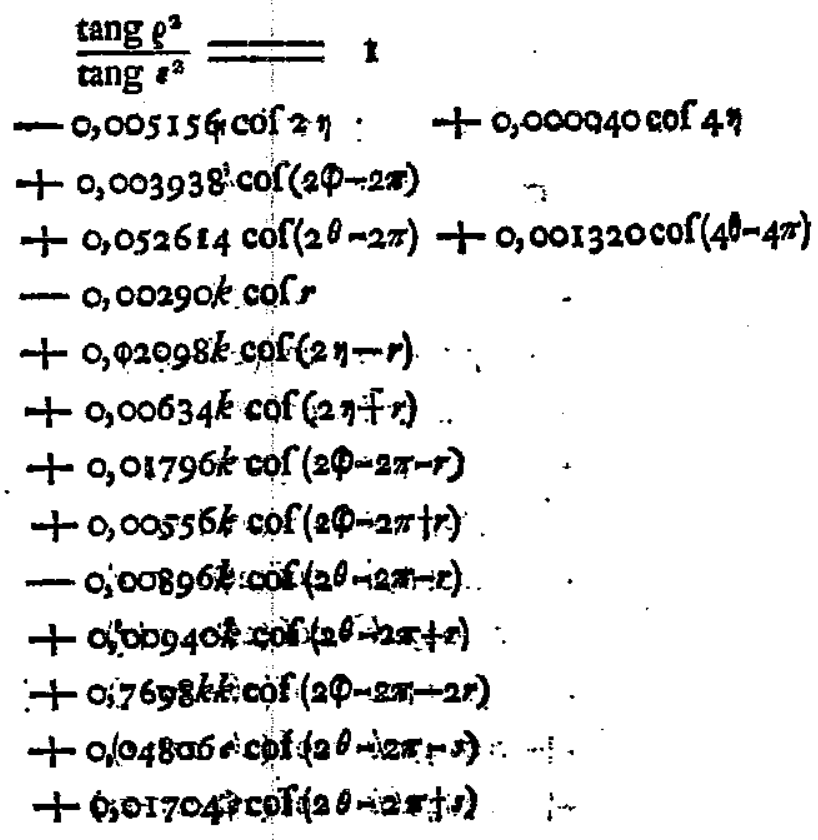

Hicque ergo valos in fuperiori illa aequatione fubtitui poterit.

$$
C A P U T \text { XIME }
$$

\$. 228. Celeb. autem Clairaut conclufi inclinationem mediam " ex obferuationibus exquifitifimis $5^{\circ} 8^{\prime} g^{\text {t" }}$, ex qua igitur ad quoduis tempus inclinationem veram elicere licebit. Sir enim $\rho=\varepsilon+\omega$, erit sang $\rho=\frac{\operatorname{tg} g+\omega}{1-\omega \operatorname{tg} \frac{t}{g}}$ $=\operatorname{tang}+\frac{\omega}{\operatorname{col} \varepsilon^{2}}=V \operatorname{tang} \varepsilon$, posendo $V$ gro expres. fone ipfius $\frac{\operatorname{tang} \xi}{\operatorname{tang} \varepsilon}$. Hinc erit $\omega=(V-1)$ fin $\varepsilon \operatorname{cof} \varepsilon=$ $\frac{x}{2}(V-1)$ fin 2s $=0,08915\left(V_{-1}\right)$ : vnde reperitur in minusis fecundis

$$
\begin{aligned}
& e=e-48^{\prime \prime} \operatorname{col} 2 \text { भ } \\
& +36 \operatorname{cor}\left(2 \varphi_{-2 \pi} \pi\right) \\
& +484 \operatorname{cor}(2 \theta-2 \pi) \\
& +\quad 9 \cos \left(4^{\theta-4 \pi}\right) \\
& -2 \operatorname{cor} r \\
& +\quad 11 \operatorname{cor}(2 i-r) \\
& +3 \operatorname{cof}(2 \pi+r) \\
& +9 \cos (20-2 \pi-r) \\
& +3 \operatorname{cor}\left(2 \varphi_{-2 \pi+x)}\right. \text {. } \\
& \text { - } 5 \operatorname{cor}(2 \theta-2 \pi-\gamma) \\
& +5 \operatorname{cof}(2 \theta-2 \pi+r) \\
& +23 \operatorname{cof}(2 Q-2 \pi-2 r) \\
& \text { - } 7 \operatorname{cor}\left(2^{\theta-2 \pi m s}\right) \\
& \text { - } 3 \cot (2 \theta-2 \pi+s)
\end{aligned}
$$

$\mathrm{Bb} 3$ 


$$
C A P U T \text { XIII. }
$$

§. 229. Hic notandum eft, etiamfi valor inclinationis mediae aliquantillum immutetur, aequationes has tamen inde vix alterari, ita vt eae femper caedem fint manfurae. Perfpicuum quoque eft in calculo aftronomico fufficere tres inaequalitaces primores, et reliquas omnes fine errore fenfibili praetermitti poffe; nifi for: te sequatio $23 \operatorname{col}(2 \varphi-2 \pi-2 r)$ retinenda cenfeatur, quae inter reliquas eft maxima. Exprimic autem angulus $29-2 \pi m 2 r$ duplam diftantiam apogei Lunae ab eius nodo, a quo angulo quoque locum nodi non mediocriter affici vidimus, cum correctio hine oriunda pro loco nodi vsque ad $235^{\prime \prime}$ afturgere poffit.

\section{CAPUT}


§. 231 . His valoribus fubftitutis in formula \$. 52 . orietur

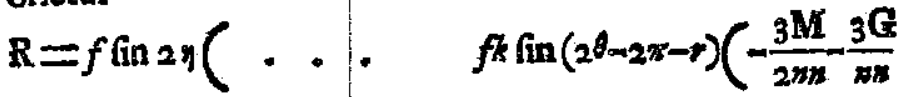

$$
\begin{aligned}
& f \operatorname{fin}(2 \varphi-2 \pi)\left(+\frac{3 \mathrm{H}}{2 m m} \ldots f k \operatorname{fin}(2 \pi-2 \pi+r)\left(-\frac{3 N}{20 m}-\frac{3 G}{\pi x} .\right.\right. \\
& f \sin (2 \theta-2 \pi)\left(\frac { 3 G } { 2 n \pi } \ldots f e \operatorname { f i n } ( g \theta - 2 \pi x - s ) \left(+\frac{9 G}{4 m s}\right.\right. \\
& f k \text { in } r\left(+\frac{3 \mathrm{~K}}{2 n n}-\frac{3 \mathrm{~L}}{2 n x} \cdot \cdot f \in \operatorname{lin}\left(2^{\theta-2 \pi}+s\right)\left(+\frac{9 \mathrm{G}}{4 m s}\right.\right. \\
& f k \sin (2 \eta-r)\left(+\frac{3 I}{2 \pi r}\right. \\
& f k \operatorname{lin}(2 \eta-r)\left(+\frac{3 I}{2 m m}\right. \\
& f k \sin (2 \Phi-2 \pi-r)\left(+\frac{3 \mathrm{~S}}{2 \pi n}+\frac{3 \mathrm{HI}}{n \pi}\right. \\
& f k \sin (2 \varphi-2 \pi+r)\left(+\frac{3 \mathrm{~T}}{2 m n}+\frac{3 \mathrm{H}}{m \pi}\right. \\
& f k \text { fin }(2 \phi-2 \pi-2 r)\left(+\frac{3 S}{x+\pi}\right.
\end{aligned}
$$

§. 232. Altera vero aequatio fuadamentalis induet formam fequentem:

$\frac{d d v}{d r^{2}}=$ Praec. $+f$ co $[2 y[-6 \mathrm{~F}-2 x \cdot \delta-0,005156-0,026307$

$+f \operatorname{col}(2 \varphi-2 \pi)[-6 G-2 x)+0,003938-1$

$+f \operatorname{col}\left(2^{\theta} \theta_{-2 \pi}\right) \cdot[-6 H-2 x+5+0,052614+0,002578$
$C A P D T$ XIV.

20

$+f k \operatorname{col} P[-g]-28.9 ;-0,00290-0,00893$

$-0,00098-0,00278-0,0009 \%$

$+f k \operatorname{col}(2 \eta-r)\left[-6 \mathrm{~K}+\frac{\pi}{2} 6 \mathrm{~F}-238+0_{2} 02098-0_{8} 00470\right.$

$-0,00258-0,01315$

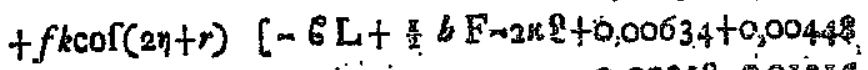

$-0,00258-0,01315$

$+f k \operatorname{co}(2 \phi-2 \pi-m)\left[-6 \mathrm{M}+{ }_{1} b G-219270,01796+0,00145\right.$.
$+0,00197-\frac{\pi}{2}$

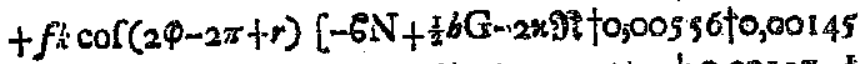

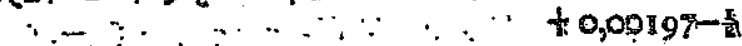

$+f k k \operatorname{cof}(2 \varphi-2 \pi-2 r)\left[-60+\frac{\pi}{2} h M+\frac{3}{4} \mathrm{G} \cdot 2 \mu \$+0,7698 \dagger 0,0089\right.$

$\therefore \quad \because \quad \ldots+0,0010+0,0007-\frac{\pi}{4}$

$+f k \operatorname{cor}(2 \theta-2 \pi-r)\left[-6 S+\frac{3}{2} b H-2 y-0,0,00896-0,00318\right.$

$+0,02631+0,00129$

$+f k \operatorname{cor}(2 \theta-2 \pi+r)\left[-6 \mathrm{~T} f \frac{x}{2} 6 \mathrm{H}-2 \mathrm{x}\{+0,00940-0,01049\right.$

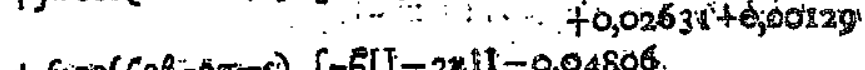

$+f \in \operatorname{col}(2 \theta-2 \pi-r)(-6 U-2 x+41-0,04806$

$+f e \operatorname{cor}(2 \theta-2 \pi F s)\left[-6 V-2 x^{2}\right\}-0,01704$

6. 233. Quoniam manifeftum eft, coefficientes $F_{8}$

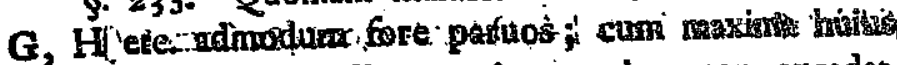
generis inaequalitas aliquor minura prima non excedat

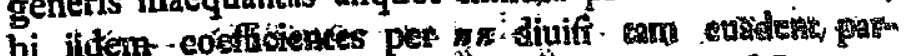
vi, ve fine errore reiici queant. "Hos autem facto quo" que litrera habendae, ex quo fola pofterior aequatio differentio-difa ferentiatis refoluenda fapererit; in qua ob eandtut tho tionem terminos ex diuifrone coefficientium ner oriundos omingius, cun in tan operofo calculo for

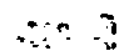

C c ciat 
ciat correatones -inde refultantes proxime Gltem determinaffe; praefertim cum haec practermiffio vix ad ali-

deter. quot minuta fecunda fit afcenfura.

\$. 234. Ob eandem rationem licebit in valoribus differentialium $\frac{d \varphi}{d r}$ et $\frac{d y}{d r}$ parciculas $a b$ inclinatione pendentes negligere, vnde erit: : $\frac{d v}{d r}=$ $f$ fin $2 \eta[-2 \alpha F=-F\}$ $f$ fin $(2 \varphi-2 \pi)\left[-2\left(\alpha+\frac{I}{n}\right) \mathrm{G}-0,008482 \mathrm{G}=-\mathrm{G}\right]$ $f$ fin $\left.(2 \theta-2 \pi)\left(+\mathrm{G}_{8} /-\frac{2}{n}\right) \mathrm{H}-0,008482 \mathrm{H}=-\mathrm{H}\right]$ $f k$ fin $r\left[\mathrm{~F} d-\mathrm{F} e^{\prime}-\mathrm{J}=-\mathrm{J}^{\prime}\right]$

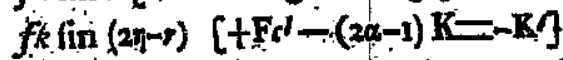

$f(k \sin (2 n+x) \cdot[+F d-(2 a+r) L=-L]$

fin $\operatorname{fin}(2 \varphi-2 \pi m-r)\left[-2\left(\omega+\frac{1}{\pi}\right) M+M-0,008482 M=-M n\right.$

$f f \operatorname{fin}(2 \varphi-2 \pi+r)\left[-2\left(\omega+\frac{\pi}{\pi}\right) \mathrm{N}-\mathrm{N}-0,008482 \mathrm{~N}=-\mathrm{N} /\right.$

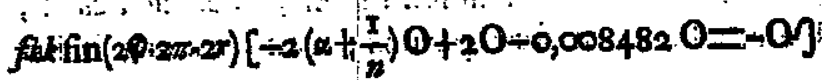

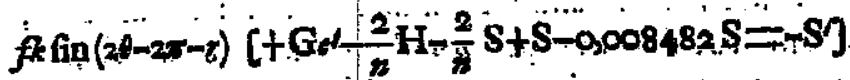

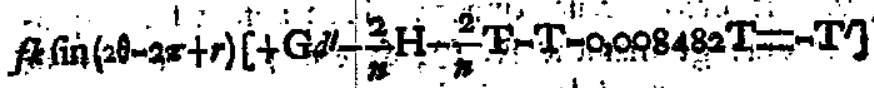

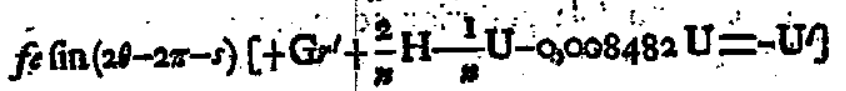

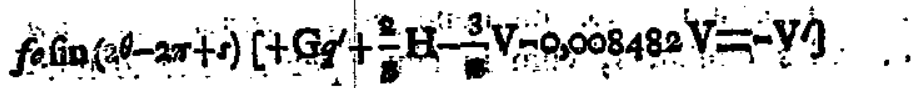
$\therefore \therefore$

5. $235^{\circ}$

ribus
C. A $U$ U T...

293

5. 35. Si nune fumili modo denue difererefemus, prodibit:

$f \operatorname{col} 2 \pi[-2 a \mathrm{~B}]$ $\frac{d d v}{d} r^{2}=$ $f \operatorname{cor}(2 \varphi-2 \pi)\left[-2\left(\alpha+\frac{i}{i j}\right) G-0,008482 G\right]$

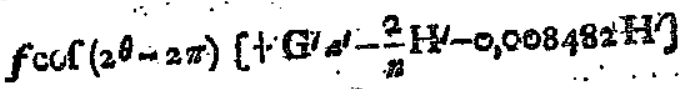
$f k \cos \%\left[\mathrm{~F} / d^{\prime}+\mathrm{F} e^{\prime}-\mathrm{J}^{\prime}\right]$ $f k \operatorname{cor}(2 y-r)\left[F^{\prime} d-(2 \theta-1) k\right]$ $f k \operatorname{cor}(2 \eta+n)\left[\mathrm{F} i c^{-r}-(2 a+i) L\right]$

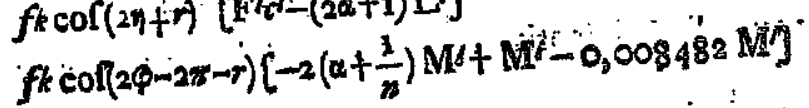
$f k \operatorname{cof}(2 p-2 \pi+r)\left[-2\left(\alpha+\frac{1}{n}\right) N^{\prime}-N^{\prime}-0,008482 N^{\prime}\right]$ $f(k)\left[\left(2 \phi_{1}-2 \pi-2 x\right)\left[-2\left(a+\frac{1}{n}\right) \mathrm{O}^{\prime}+2 \mathrm{O}^{\prime}-0,008482 \mathrm{O}^{\prime}\right]\right.$ $f k \operatorname{co}(20-2 \pi-7)\left[G^{\prime} / d-\frac{2}{\pi} H^{\prime}-\frac{2}{\pi} S^{\prime}+S^{\prime}-0,008482 S\right]$ $\int k \operatorname{co}(2 \theta-2 \pi+r)\left(\mathrm{G} / d^{\prime}-\frac{2}{2} \mathrm{H}-\frac{2}{n} \mathrm{~T}^{\prime}-\mathrm{T} / 0,008482 \mathrm{~T} J\right.$

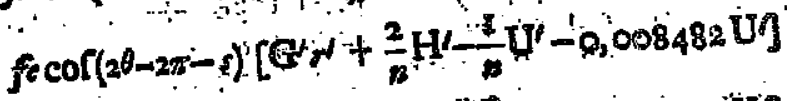

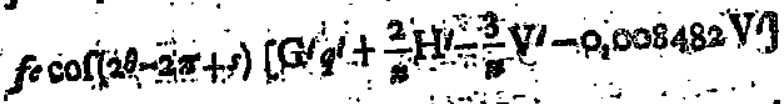

\$. 236.1 Hine aucem fequentes eliciumar valores $\mathrm{F}=0,01273 \ldots . t \mathrm{~F}=8,104833$

$\mathrm{G}=0,32213 \cdot \cdots, \mathrm{G}=9,508032$ $H=0,06976 . .1 \mathrm{H}=88_{2} 843599 \ldots \ldots$ $\mathrm{H}=-1,87800 \cdots \because \mathrm{J}=0,2737 \mathrm{10} \quad \mathrm{X}=$

$$
\mathrm{C}{ }_{2}
$$$$
x=
$$ 
204

CAPUT XIV:

$$
\begin{aligned}
& \mathrm{x}=+0,05615 \therefore \cdot \mathrm{l}=8,749352 \\
& L=-0,00077 \text {. . } L L=6,88890 \text { A } \\
& M=-0,29638 \quad \text {. . . } 7-M=9,471854 \\
& \mathrm{~N}=+0,0,0012 \ldots, \quad, \mathrm{N}=6,089109 \\
& 0=+0,32287 \text {. . } 1.0=9,509034 \\
& \mathrm{~S}=+0,3909.1 .+. \quad \text { l } \mathrm{S}=9,592073 \\
& \mathrm{~T}=+0,69579 \quad . \quad . \quad / \mathrm{T}=0,84^{2475} \\
& \mathrm{U}=-0,07922 \text {. . } \quad \mathrm{U}=8,898830 \\
& V=-0,05141, \quad \angle-V=8,7 \cdot 1093
\end{aligned}
$$

6. 287. Pro diftantio ergo luną a fole çurtata $x=$ $\frac{(1-k k) \text { ait }}{1-k \cos t}$ exit

$$
w=\text { Praec. }
$$

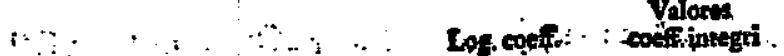

$+0,000072 f$ cor $2 \eta$

$+0,001833 f$ cor $(2 \phi-2 \pi x)$

$+0,000397 f \operatorname{cor}(2 \theta-22 i)$

- osple6gft. coft

$+0,00032 f k$ cof $(2 y-r)$

- a,00000 ofk cof $(2 n+4)$

$|5,860017|+0,000079$

$7,263216+0,002005$

$6,598774+0,000434$

$8,028894 \div 0,000634$

$6,504536+0,000019$

$4,644088 \div 0,000010$

- 0500169fk cor $(2 \varphi-2 \pi-r)$. 7,227038-0,000100

$+0,0000 f_{k} \operatorname{cof}(20-2 \pi+r) \cdots 3,834293+0,000000$

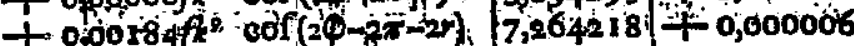

$+0,00223 f k \operatorname{cor}(20-2 \pi-r) \quad 7,347257]+0,000132$

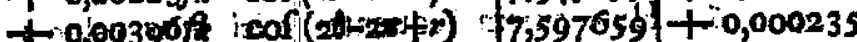

$-0,00045 f_{t} \operatorname{col}(3 \theta-2 \pi-s)$. $6,6540: 4-0,000000$

- $0,00029 f_{6} \operatorname{cof}(2 \theta-2 \pi t s) \quad|6,466277|-0,000000$

vbi notanduin eft effe $f=1,093756 ;$; et $f=0,0,38921$.

\section{$C A P U T X I F$.}

205

\$. 338. Deinde pro motu momentaneo habebieus

$$
\frac{d \varphi}{d r}=\text { Praec. }
$$

- $0,000146 f \operatorname{cor} 2$ m

- $0,003700 f \operatorname{cor}(2 \phi-2 \pi)$

- 0,0008 if $\operatorname{cor}(2 \theta-2 \pi)$

$+0,02157 f k$ cor $r$

$\pm 0,00065 f k$ cor $(2 \eta-r)$

$+0,00000 f_{k}$ cof $(2 \eta+r)$

$+0,00340 f k \operatorname{cof}(2 \varphi-2 \pi-r)$

$+0,003400 \mathrm{fk} \operatorname{col}(2 \varphi-2 \pi+r)$

$-0,00371 f^{2} \operatorname{col}(2 \phi-2 \pi t-2 r)$

- $0,00449 f k$ cor $(2 \theta-2 \pi m+r)$

$-0,00799 f$ cof $2 \theta-2 \pi+r)$

$+0,00091 f_{e} \quad \operatorname{co}(2 \theta-2 \pi-s)$

$+0,00091 f f e$
$+0,00059 f e$
$\operatorname{cof}(2 \theta-2 \pi t s)$

20 coeff. in namaris

$|6,164934|-0,000160$ $7,568133-0,004946$ $6,903691-0,000876$

8,3338 II $+0,001286$

$6,809453-0,000038$

$4,949005+0,000005$

$7,531955+0,000203$

$4,139210-0,000000$

$7,569135-0,000012$

$7,652174-0,000267$

$7,902576-0,000476$

$6,9589.31+0,000000$

$|6,77 \pm 194|+0,000000$

§. 239. Pro correctione longitudinis verae hinc oriunda ponatur,

$$
\varphi=\text { Praec. }
$$

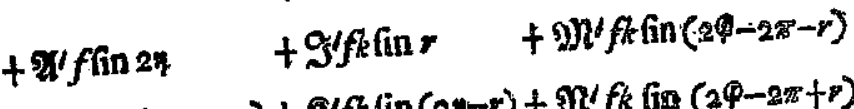
$+\left(\sigma^{\prime} f f \operatorname{in}(2 \varphi-2 \pi)+\Omega^{\prime} f k\right.$ in $(2 \eta-r)+N^{\prime} f k$ fin $(2 \varphi-2 \pi+p)$ $+\Phi^{\prime} f \operatorname{fin}(2 \theta-2 \pi)+\mathbb{N}^{\prime} f k \operatorname{fin}(2 \pi+r)+\Phi^{\prime} f k k$ fin $(2 \varphi-2 \pi-2 r)$ $+\mathcal{E}^{\prime} f k$ fin $(2 \theta-2 \pi-r)+\mathfrak{u}^{\prime} f e$ fin $(2 \theta-2 \pi-s)$ $+z^{\prime} f k$ fin $(2 \theta-2 \pi+r)+3^{\prime} f \in \operatorname{fin}(2 \theta-2 \pi+s)$

Cc 3 


\section{$C A P U T$ XIV.}

$20 \%$.

\$. 24I. Haec omnia fatis conuenione cum notis inaequalitatibus motus lunäe, nifi quod inaequalitas ab angulo $2 \theta-2 \pi$ pendens plane aduerfari videacur, cunt nullum eius veftigium in rabulis aftronomicis occurrat; quod quidem eo magis eft miranduin, cum correctio inde oriunda ad $18^{\prime}, 3^{\prime \prime}$ exfurgat. Lubens equidem agnofco, in hoc calculo non omnem curam effe adhibitam, vt hanc aequationem tanquam omnibus numeris abfolutam fpectare liceat, quoniam ad plurimos terminos, quos formulae noftrae fuppeditant, non refpexi. Interim tamen calculum repetenti mox patebit, non admodum enormiter effe aberratum, praefertim cumaequa tio ab angula $2 \phi-2 \pi$ pendens, quae pari paffu procedit, vericati perquam confentanea prodierit, cum ea reduetio lunae ad eclipticam contineatur. Ac is quidem haec inaequalitas ad femifem vsque diminuatur, tamen tanca remanet, vt meritp dubitare debeamus, eius effe Gum ab Aftonomis non effe animaduerfum; cum eius omifio vix per aliam aequarionem compenfari queat. Hancobrem fiue omiffio terminorum neglectorum tit in caufa, five etiam in calculo numerico error fueris admiffus, quod facile euenire potuit, iftam inueftigarionem in capite fequenti accuratius fufcipiamus. 


\section{$C A P U T R$}

209

6. 243. His valoribus in formulis principaltbus fubftitutis habebimus has aequationes:

UALL-

ACCURATIOR INUESTIGATIO INAEQUALITATUM LUNAE AB INCLINATIONE EJUS ORBITAE PENDENTIUM.

5. 242 .

uonism praecipuum dubium circa inaequalitatem ab angulo $2 \hat{\theta}-2 \pi$ pendentem verfatur, noftram inuertigationem ab is inaequalitatibus, qquae fimul ab alterutra excentricitate pendent, abftrahamus. Po-' namus ergo:

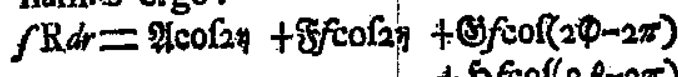

$+5 f \operatorname{col}(2 \operatorname{lo-2\pi })+9 f \operatorname{col}\left(4^{\theta}-4 \pi\right)$ et $w=A \operatorname{col} 2 y+\mathrm{F} f \operatorname{col} 2 y+\mathrm{G} f \operatorname{cof}(2 \phi-2 \pi)$

$+\mathrm{H} f \mathrm{col}(2 \theta-2 \pi)+\mathrm{J} f \operatorname{cor}\left(4^{\theta}-4 \pi\right)$

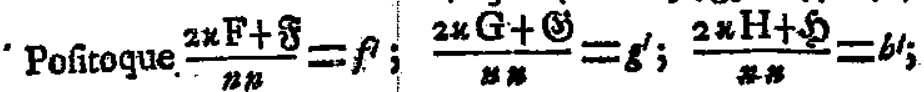
$\frac{2 \times \mathrm{J}+\mathrm{I}}{m *}=j$ erit:

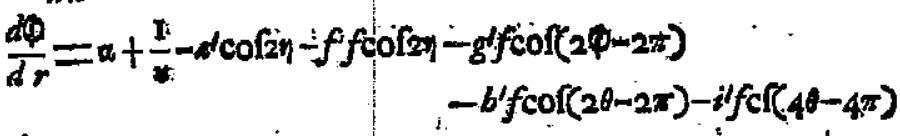

$\frac{d \eta}{d r}=a-\alpha^{\prime} \operatorname{co}\left[2 \eta-f^{\prime} f \operatorname{cor} 2 \eta-g^{\prime} f \operatorname{col}(2 \varphi-2 \pi)\right.$

$\frac{d \theta}{d r}=\frac{1}{n}$ et $\frac{d \pi}{d r}=-\mid \begin{gathered}-b / f \operatorname{cor}(2 \theta-2 \pi r)-i f f(4 \theta-4 \pi) \\ 0,004241-0,004221 \cdot \operatorname{col} 2 t\end{gathered}$

$+0,004241 \operatorname{cof}(2 \varphi-2 \pi)$

$+0,004241 \cos (2 \theta \mathrm{m} 2 \pi)$

§. 243.

litatem

oftram

: fimal

Po.

$(\theta-4 \pi)$

$: \theta-4 \pi)$

$1=b$

$(-4 \pi)$
$\mathrm{R}=-\frac{3 \mathrm{G}}{2 m n} f$ fin $(2 \theta-2 \pi)+\frac{3 \mathrm{H}}{2 m} f$ fin $(2 \varphi-2 \pi)$

$\frac{d d v}{d r^{2}}=f \cos 2 \eta\{-6 \mathrm{~F}-2 x$

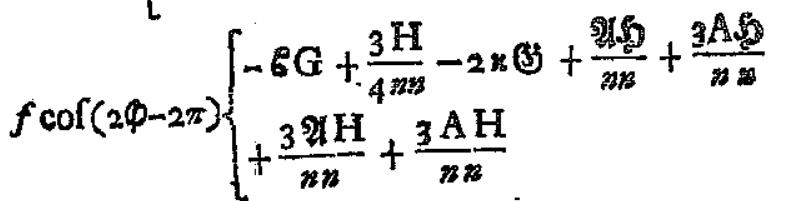

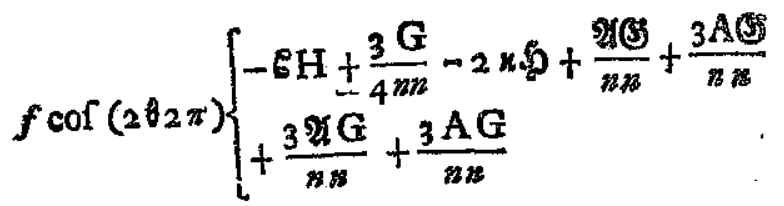

$f \operatorname{cor}(4-4 \pi)[-6]-245$

$f \operatorname{cor} 2 \pi\left(-0,005156-0,026307+0,001969 \frac{A}{n n}\right)$

$f \operatorname{cor}(2 \varphi-2 \pi)\left(+0,003938 m 1-0,052614 \frac{\mathrm{A}}{m \mathrm{~B}}-0,002578 \frac{\mathrm{A}}{m \mathrm{~m}}\right)$

$f \operatorname{cor}(2 \theta-2 \pi)\left(+0,052614+0,002578-0,001969 \frac{2 A}{n \pi}+\frac{A}{2028}\right)$

$f \operatorname{cof}\left(4^{\theta}-4 \pi\right)\left(+0,001320+0,026307 \frac{\mathrm{A}}{\mathrm{mB}}\right)$

6. 244. Vel in numeris erit

$R=0,008540 \mathrm{H} f \mathrm{fin}\left(2 \varphi_{m-2 \pi}\right)-0,008540 \mathrm{G} f \mathrm{fin}(2 \theta-2 \%)$

Dd 


$$
\begin{aligned}
& 210 \\
& C A P U T X V \\
& \frac{d d v}{d r^{2}}==
\end{aligned}
$$

$f \cos 2,4[-x, 01591 \mathrm{~F}-2 x f-0,031478$

$f \subset(2 \phi-2 \pi)\left\{\begin{array}{c}-\mathrm{I}, 01591 \mathrm{G}-2 \times \mathrm{O})+0,000636 \mathrm{H} \sim 0,845648 \\ -0,0271105 \mathrm{~g}\end{array}\right.$ $f \circ f(2 \theta-2 \pi)\left\{\begin{array}{c}-1,01591 H-2 x \$+0,000636 \mathrm{G}+0,047722 \\ -0,027110 \text { (S) }\end{array}\right.$

$f \mathrm{f}\left(4^{\theta-}-4 \pi\right)[-1,01591]-249+0,001123$

Nunc autem ex formulis affumtis erit

$\mathrm{R}=f$ tin $2 \eta \quad[-2 \propto f+0,00424 \mathrm{I}(\mathrm{S}-0,004241 \mathfrak{W}]$

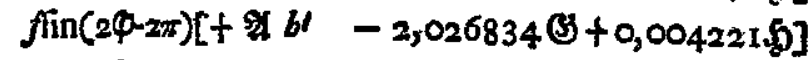
$f$ fin $(2 \theta-2 \pi)\left[-\mathbb{A} g^{\prime}-0,023965(0)-0,159358 @\right]$ at eft $f \operatorname{fin}(4 \theta-4 \pi)[+0,004245 \$-0,318716 \$]$

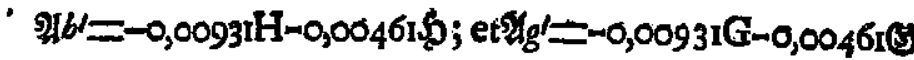

vnde fit: $1,867476 \mathfrak{f}=0,004241(3-\$)$

$$
\begin{aligned}
& 2,026874 \mathrm{G}=-0,01785 \mathrm{H}-0,00039 \mathfrak{W} \\
& 0,159358 \mathfrak{W}=+0,01785 \mathrm{G}-0,01935 \mathfrak{G} \\
& 0,318716 \mathrm{~S}=+0,004241 \mathfrak{W}
\end{aligned}
$$

6. 245 . Tum fimili modo differentiando valorem ipfius $\approx$ ponatur

$$
\begin{aligned}
& \mathrm{F}^{\prime}=1,867476 \mathrm{~F}-0,00424 \mathrm{G}+0,00424 \mathrm{H} \mathbf{H} \\
& \mathrm{G}^{\prime}=2,026834 \mathrm{G}+0,0109 \mathrm{H}+0,00750 \mathrm{H} \\
& \mathrm{H}^{\prime}=0,159358 \mathrm{H}+0,03909 \mathrm{G}-0,00750 \mathrm{H} \\
& \mathbf{J}^{\prime}=0,318716 \mathrm{~F}-0,004241 \mathrm{H}
\end{aligned}
$$

vt fit

$$
\frac{d v}{d r}=
$$

$A^{\prime}$ fin2y $-\mathrm{F}^{\prime} f$ fin2 $\eta-\mathrm{G}^{\prime} f$ fin $(2 \hat{\varphi}-2 \pi)$

eritque

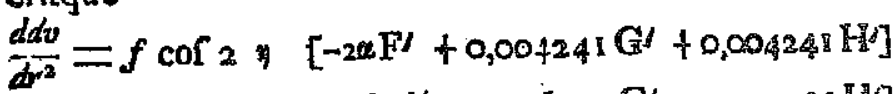
$f \operatorname{col}(2 \varphi-2 \pi)\left[+\mathrm{A}^{\prime} b^{\prime}-2,026834 \mathrm{G}^{\prime}-0,004221 \mathrm{H}^{\prime}\right]$ $f \operatorname{cof}\left(2 \Phi-2 \pi\left[+\mathrm{A}^{\prime} g^{\prime}-0,159358 \mathrm{H}^{\prime}=0,023965 \mathrm{G}\right]\right.$ $f \operatorname{col}\left(4^{\theta}-4 \pi+\left[+0,004241 H^{\prime}-0,318710\right]\right]$

feu $\frac{d d v}{d r^{2}}==$

$f \operatorname{cof} 24\left\{\begin{array}{r}-3,48745 \mathrm{~F}+0,01668 \mathrm{G}-0,00721 \mathrm{H}-0,00003\} \\ +0,00007\end{array}\right.$ $f \in(2 \phi-2 \pi) \cdot\{-4,10320 \mathrm{G}-0,0512 \mathrm{IH}-0,029295+0,0000$, (I) $f \mathrm{f}(\mathrm{f}: 6 \mathrm{m-2 \pi})[-0,02565 \mathrm{H}-0,08323 \mathrm{G}-0,0129000,0,000185$ $f \mathrm{cl}\left(4^{\theta-4 \pi}\right)[-0,10158 \mathrm{~J}+0,00016 \mathrm{G}+0,00202 \mathrm{H}-0,00003$ (6)

\$. 246. Hine pro $f$ et $\$$ fubftituris valoribus $\xi=0,00227(\$-\$)$ et $\$=0,01331$

habebimus has aequationes

$+2,47 \times 54 \mathrm{~F}-0,01668 \mathrm{G}+0,0072 \mathrm{IH}=0,0,3147$

$+3,09229 \mathrm{G}+0,05185 \mathrm{H}+0,002185900,095648$

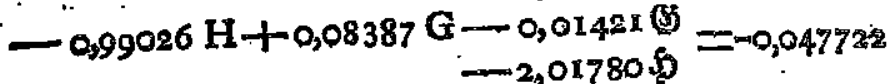

$=0,001123$

Dd 2

\$. $247 \%$ 
6. 247. Deinde reperitur

$$
\begin{aligned}
& \mathfrak{S}=-0,00881 \mathbf{H}-0,00002 \mathbf{G} \\
& \mathfrak{S}=+0, \mathbf{1}=1201 \mathbf{G}+0,00107 \mathbf{H}
\end{aligned}
$$

qui valores fubiticuti praebent :

$+2,47154 \mathrm{~F}-0,01618 \mathrm{G}+0,00725 \mathrm{H}=0,031478$

$+3,09256 \mathrm{G}+0,06964 \mathrm{H}=0,995648$

$+0,99229 \mathrm{H}+0,14239 \mathrm{G}=0,047722$

$-0,91430 J-0,00297 \mathrm{G}-0,00205 \mathrm{H}=-0,001123$

vide tandem reperitur

$$
\begin{aligned}
& \mathrm{F}=+0,014830 . . \quad \mathrm{l} F=8,171166 \\
& \mathrm{G}=+0\} 321910 \text {. . . } / \mathrm{G}=9,507734 \\
& \mathrm{H}=+0,00190 \mathrm{r} . . \quad \mathrm{H}=7,278927 \\
& \mathrm{~J}=+0,000180 \text {. . : } \quad \mathrm{J}=6,25638 \mathrm{~B} \\
& \text { atque } \\
& \mathfrak{f}=-0,000080 . \cdot \operatorname{lf} f=5,903090 \\
& \text { E }=-0,000023 \cdot . \quad 4 \text { OS }=5,361728 \\
& \$=+0,036056 \text {. . } l \$=8,556977 \\
& \$=+0,000480 \text {. . . } \quad \$=6,681241
\end{aligned}
$$

5. 248. Hine ergo pro diftantia $x=\frac{(1-k k)}{1-k \operatorname{cof} r}$ reperitur

log.coeff. val.coeff. $5,926350 \mid+0,000092$ $+0,001832 f \operatorname{cof}(2 \phi-2 \pi)(7,262918+0,002004$ $+0,000011 f \operatorname{cor}(2 \hat{2}-2 \pi)(5,034111+0,000012$ $+0,000001 f_{1} \operatorname{cor}\left(4^{\theta}-4 \pi\right)|4,011565|+0,000001$
Ae pro motu momentaneo eris

log.coeff. valstoef

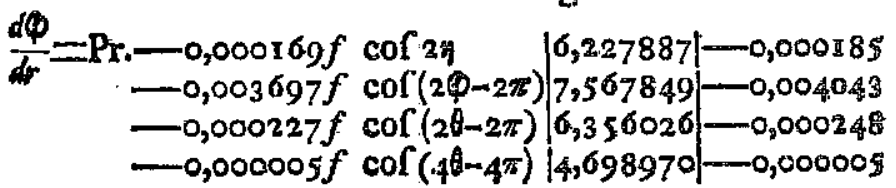
vnde quidem iam paret inaequalitatem ab angulo $26-278$ pendentm multo fore minorem, quarr fupra inueneras mus, in quo non partum veritacis criterium cernitur.

\$. 249. Pro ipfa iam longitudine lume ponamus:

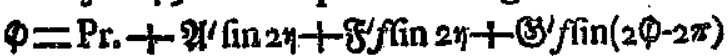

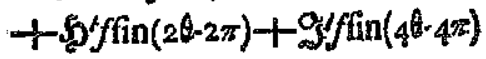

atque obtinebimus has aequationes:

$24 . g^{\prime}-0,004241\left(\mathrm{~S}^{\prime}+\mathfrak{W}\right)=-0,000169$

$2,02683407+0,004221 W^{\prime}-0,000227$ W $^{\prime}=0,003697$

$0,159358 \mathrm{D}^{\prime}+0,023965 \mathrm{OS}^{\prime}-0,003697 \mathrm{H}=0,000228$ $0,318716 \mathrm{~J}^{\prime} \div 0,004241 \mathrm{~g}^{\prime}=-0,000005$ vnde erit
Pater ergo zevera aequationem ab angulo $26-2 \pi$ ortam multo effa minorem, quam capite praecedente inuens ramus; atque nunc quidem non vlera $205^{\prime \prime} \mathrm{Seu} 3 l_{2} 25^{\prime \prime}$ afcendere. Nullum igitur eft dubium, quin haec aequasto tabulas lunares ad multo maiorem perfectionem fic elrectura.

§. 250. 
§. 250. Cum igitur neglętus terminorum, minimorum tantum errorem pepererit in aequatione $a b$ angulo $2 \theta-2 \pi$ pendente, operae erit pretium, etiam aequationes infuper ab excentricitate orbitae lunaris pendentes curatius inueftigare, guae guidem alicuius videntur effe momenti, In hanc finem popamus.

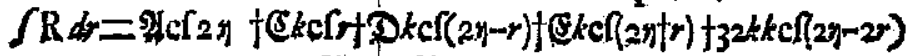
+ (S) $f \operatorname{cor}(2 \theta-2 \pi)+\$ f f \operatorname{cor}(2 \theta-2 \pi)$ $+\$ j f k \operatorname{cog} r+\mathfrak{M} f k \operatorname{co}(2 \Phi-2 \pi-r)+\mathfrak{S} f k \operatorname{col}(2 \theta-2 \pi-r)$ $+0 f k k c(2 \phi-2 \pi m 2 r)+2 f k \operatorname{col}(2 \theta+2 \pi+r)$ et $v=A \mathrm{c}(2 y+\mathrm{Dkcf}(2 y-r)+\mathrm{Ekcol}(2 y \mid r)-14 k k \operatorname{col}(2 \eta-2 r)$ $+G f \operatorname{col}(2 \theta-2 \pi)+\mathrm{H} f \operatorname{col}(2 \theta-2 \pi)$ $+\mathrm{J} f k \cos +\mathrm{M} f k \operatorname{col}(2 \varphi-2 \pi-r)+\mathrm{S} f k \operatorname{col}(2 \theta-2 \pi-r)$ $+0 j k k \operatorname{cof}(2 \varphi-2 \pi-2 r)+T f k \operatorname{col}(2 \theta-2 \pi+r)$

6. 25 t. His valoribus in aequacionibus noltris principalibus fubftitutis habebimas:

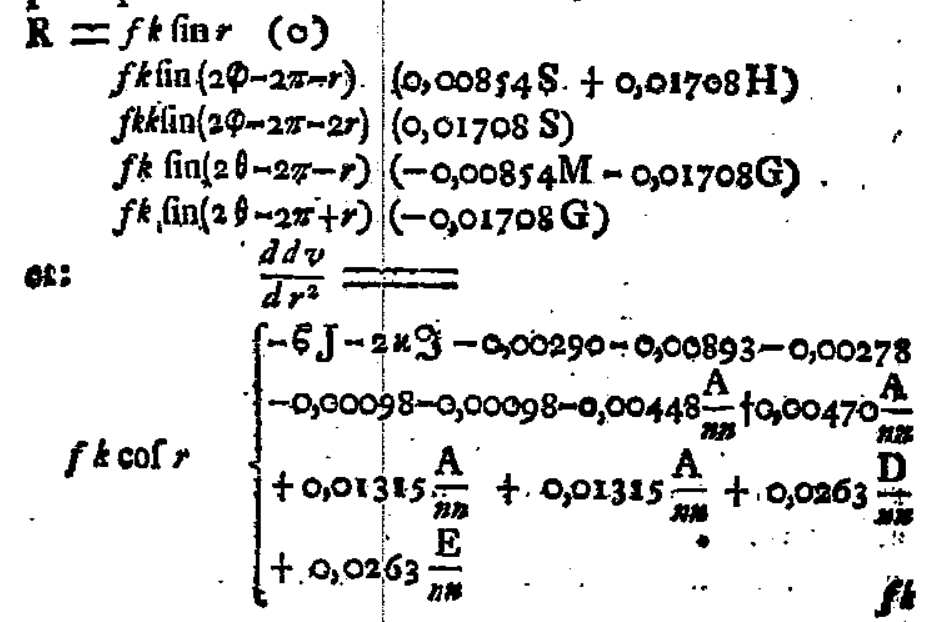

$f k \cos r$

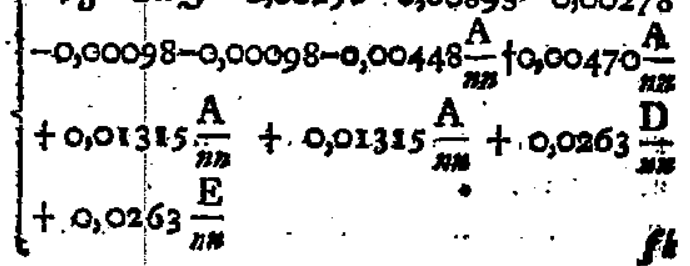




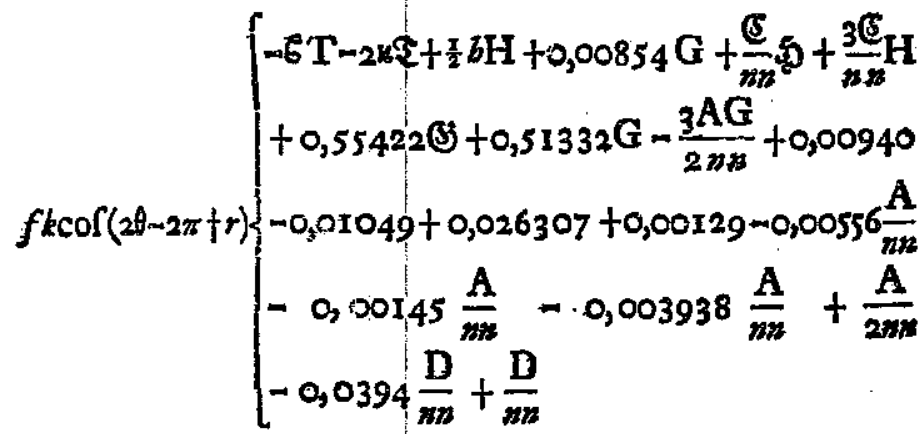

\$. 252. Hae autem formulae intricatae reducuntur ad fequentes

$\frac{d d v}{d r}=\operatorname{Pr}_{0}+j k \operatorname{cof} r(-5 J-2 \times \Phi-0,01182)$

$f k \operatorname{col}(2 \varphi-2 \pi-r)\left(\begin{array}{c}-6 \mathrm{M}-2 k M 7-0,03207 \mathrm{~S} \nmid 0,53619 \\ -0,02711 \text { S }\end{array}\right)$

fkkcor $(2 \varphi-2 \pi-2 r)(-60-2 k x+1,52115 \mathrm{M} \nmid 0,76615)$

$f k \operatorname{col}(2 \theta-2 \pi-r)\left(\begin{array}{c}-E S-2 * S-0,03207 \mathrm{M}+0,00286 \\ -0,027 \mathrm{IIMl}\end{array}\right)$

$f k \operatorname{col}(2 \theta-2 \pi+r)(-6 \mathrm{~T}-2 x i f 0,38147)$

6. 253. Nunc eosdem valores ex formulis aftumtis eruamus, ac pofito more adhuc recepto $\frac{2 x J+\Phi}{n B}=i$, $\frac{2 n M+M}{m}=m^{\prime}$ etc. $\quad$ erit

$\frac{d \varphi}{d r}=\alpha+\frac{1}{n}-a^{\prime} \operatorname{cor} 2 \eta-d l k \operatorname{cor}(2 \eta-r) \quad-g f f \operatorname{cor}(2 \varphi-2 \pi)$.

$-a k \operatorname{cof}(2 \eta+r)-b^{\prime} f \operatorname{cof}\left(2^{\theta-2 \pi x}\right)$

$-i / f k \operatorname{co}\left[r-m^{\prime} f k \operatorname{cof}(2 \varphi-2 \pi-r)-s^{\prime} f k \operatorname{col}(2 \theta-2 \pi-r)\right.$ $-n l f k k f(2 \Phi-2 \pi-2 r)-k f f k \operatorname{cor}(2 \theta-2 \pi+r)$
$C A B U T$ IIV.

종y

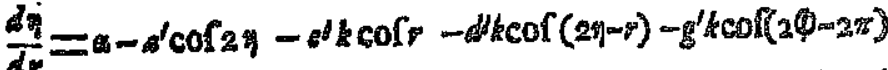

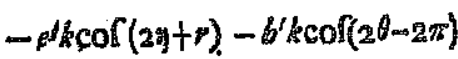

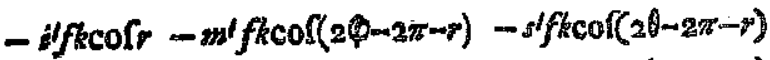

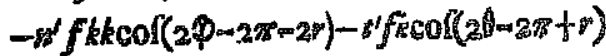

$\frac{d \theta}{d r}=\frac{1}{x}+\frac{2}{n} k \operatorname{col} r+\frac{3}{2 n} k k \operatorname{col} 2 r$ agque

$$
\frac{d \pi}{d r}=
$$

$-0,00424 \mathrm{x} \quad f 0,00424 \mathrm{x}(2 \phi \cdot 2 \pi)$

$-0,004221 \operatorname{co} 2 \pi f+0,004241 \mathrm{cf}\left(2^{\theta}-2 \pi\right)$

$-0,01766 k \operatorname{col} r-0,00996 k \operatorname{col}(2 y-r)=0,010636 k k \mathrm{cl}(2 \eta \eta \cdot 2 r)$ $-0,00831 k \operatorname{col}(2 \eta+r)-0,021273 k k \operatorname{cof} 28$ $+0,00924 k \operatorname{cof}(2 \varphi-2 \pi-r)+0,00841 k \operatorname{col}(2 \theta-2 \pi-8)$ $+0,0084 \pi k \operatorname{cof}(2 \varphi-2 \pi+r)+0,00924 k \cos (2 \theta-2 \pi+\nu)$ $+0,01064 k$ cor $(20-2 \pi \cdots 20)$

6. 254. His iam valoribus introducendis differetsotiemus formulas noftras affumtas pro $f \mathbb{R} d r$ o $w_{i}$ que obtinebimus primo:

$$
\mathbf{R}=\text { Praec. }
$$

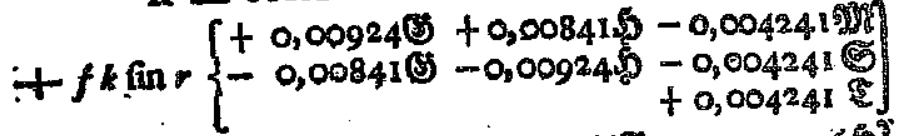

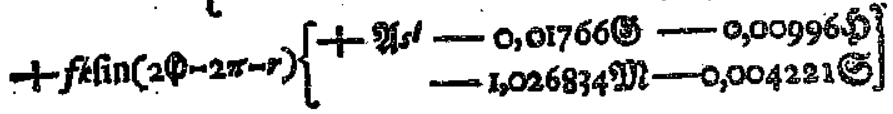

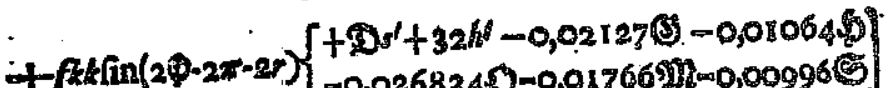

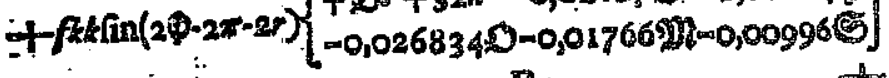

Ee 


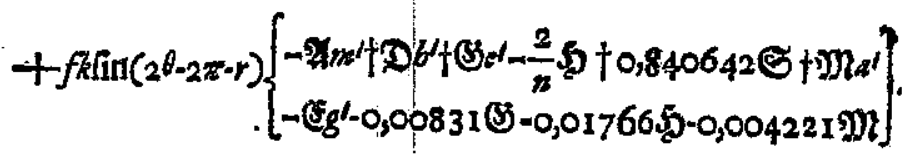

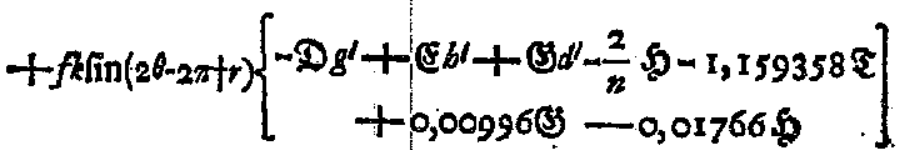

§. 255. Hine igitur confequimur iftas aequationes $0,00083(\mathfrak{S}-\mathfrak{W})-\mathbf{\$}-0,004241(\mathfrak{D}+\mathfrak{S}-\mathfrak{S})=0$

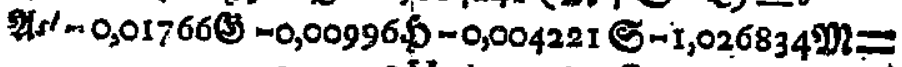
$0,01708 \mathrm{H}+0,00854 \mathrm{~S}$

$\mathfrak{D s}^{\prime}+32 b^{\prime}-0,002127$ (S-0,01064 $\mathfrak{W}-0,026834 \mathfrak{D}-0,01766 \mathfrak{M l}$ $-0,00996 \mathrm{~S}=0,01708 \mathrm{~S}$

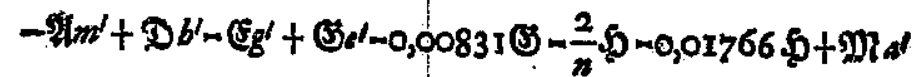
$-0,004221$ M $+0,840642$ S $=-0,01708 G-0,00854 M$

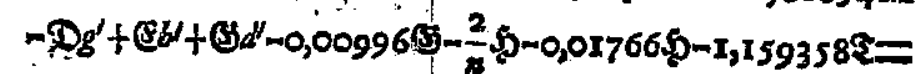
- $-0,01708 \mathrm{G}$

\$. 296. Ponatur nunc vlterius :

$J^{\prime}=\$-0,00083(\mathrm{G}-\mathrm{H})+0,004241(\mathrm{M}+\mathrm{S}-\mathrm{T})$

$M^{\prime}=1,026834 \mathrm{M}-A s^{\prime}+0,01766 \mathrm{G}+0,00996 \mathrm{H}+0,004221 \mathrm{~S}$ $\mathbf{O}^{\prime}=0,026834 \mathrm{O}-\mathrm{D}^{\prime}+14 \mathrm{w}^{\prime}+0,02127 \mathrm{G}+0,01064 \mathrm{H}$ $\therefore . . \quad+0,01766 M+0,00996.5$ $F^{\prime}=-0,840642 \mathrm{~S}+\mathrm{A} / g^{\prime} / \mathrm{D}^{\prime}+\mathrm{Eg}^{\prime} \sim \mathrm{G}_{e}^{\prime}+0,00831 \mathrm{G}+\frac{2}{p} \mathrm{H}$ $+0,01766 \mathrm{H}-\mathrm{MA}_{\mathrm{A}}+0,00422 \mathrm{M}$

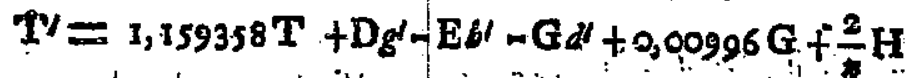
$+0,01766 \mathrm{H}$ eritque eritque $\frac{d d v}{d r}=$ Prace.

$+f k \operatorname{col} r\left[+0,01766\left(\mathrm{G}^{\prime}+\mathrm{H}^{\prime}\right)-\mathrm{J}^{\prime}+0,00424 \mathrm{I}\left(\mathrm{MH}^{\prime}+\mathrm{S}^{\prime}+\mathrm{T} /\right.\right.$ $t f k \operatorname{cor}\left(2 \varphi_{-2 \pi} \pi r\right)\left\{\begin{array}{r}\mathrm{A}^{\prime} \mathrm{s}^{\prime}-0 ; 01766 \mathrm{G}^{\prime}-0,00996 \mathrm{H} / \mathrm{M} \\ -0,004221 \mathrm{~S}^{\prime}-1,026834 \mathrm{M} /\end{array}\right.$

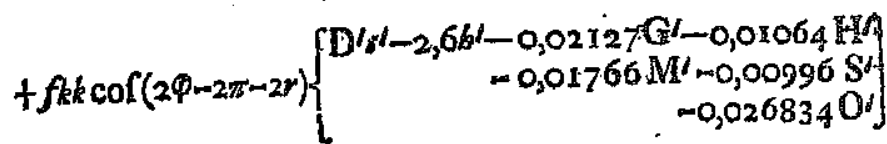

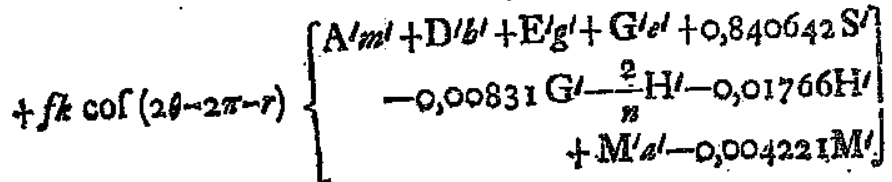
$+f k \operatorname{cor}(2 \theta-2 \pi+r)\left\{\begin{array}{r}\mathrm{D}^{\prime} g^{\prime}+E^{\prime}\left(b^{\prime}+\mathrm{G}^{\prime} d^{\prime}-0,00996 \mathrm{G}-\frac{2}{13} \mathrm{H}^{\prime}\right. \\ \left.-0,01766 \mathrm{H}^{\prime}-1,259358 \mathrm{~T}\right)\end{array}\right]$

\$. 257. Prioris ordinis aequationes huc reducuntur: $\sigma^{\prime}=-0,00424 \mathrm{r}(\mathfrak{M}+S+\mathfrak{S})$ ,026834 $\mathrm{M}=-0,01785 \mathrm{~S}-0,00883$ S $-0,00039$ $0,026834 \mathrm{O}=0,05835 \mathrm{~S}-0,03041 \mathrm{~S}$ $0,01766 \mathrm{MI}+0,00690$

$-0,840642$ S $=+0,01785 \mathrm{M}=0,0.9359 \mathrm{M}+0,00263$ $x, \times 59358=+0,01247$

Hinc fit

$G=+0,00007 \mathbf{S}+0,00008 \mathrm{M} \neq 0,00006$ fou $\%=0$ $\mathfrak{M l}=-0,01738 \mathrm{~S}+0,00018 \mathrm{M}-0,000.3 .5$ $D=-2,16266 \mathrm{~S}+0,02394 \mathrm{M}+0,26092$ $\mathcal{S}=-0,00040 . S-0,02123 \mathrm{M}-0,00313$ $z=-0,01076$

Ee ? 
§. 258. Porro reperietur

$$
\begin{aligned}
& J^{\prime}=J+0,004241(M+S-T)-0,00026 \\
& M^{\prime}=1,026834 M+0,01935 S-0,00016 M+0,00568 \\
& \mathbf{O}^{\prime}=0,026834 \mathrm{O}-0,37652 \mathrm{~S}+0,01805 \mathrm{M}+0,01063 \\
& S \cdot=0,840642 S+0,00013 S+0,00883 M-0,00167 \\
& \mathbf{T}^{\prime}=\mathbf{1}, 159358 \mathrm{~T}+0,01023 \\
& \text { ac fuccinctius habebitur } \quad \frac{d d v}{d r^{2}}=\text { Praec. } \\
& +f k \cos r\left[-\mathrm{J}^{\prime}+0,004241^{1}\left(\mathrm{M}^{\prime}+\mathrm{S}^{\prime}+\mathrm{T}^{\prime}\right)+0,01175\right] \\
& +f * \operatorname{cof}(24-2 \pi-r)\left\{\begin{array}{r}
\left.-1,026834 M^{\prime}-0,00422 S^{\prime}-0,02842 S\right\} \\
+0,00030 \mathrm{M}-0,01160
\end{array}\right\} \\
& +f k k \operatorname{co}\left\{(2 \varphi-2 \pi-2 r)\left\{\begin{array}{r}
-0,0268340 /-0,01766 M^{\prime}-0,00354 M \\
-0,01511-0,00996 S^{\prime}+0,33746 S
\end{array}\right\}\right. \\
& \cdot+f k \operatorname{col}\left(2 \theta_{-2 \pi m r)}\left\{\begin{array}{r}
+0,840642 \mathrm{~S}^{\prime}-0,02396 \mathrm{M}^{\prime}-0,02843 \mathrm{M} \\
-0,0147^{2}+0,00025 \mathrm{~S}
\end{array}\right\}\right. \\
& +f k \operatorname{cor}(2 \theta-2 \pi+r)\left[-1,159358 T^{\prime}+0,33856\right]
\end{aligned}
$$

5. 259. Hine tandem/valores guaefiti eliciunerr

$$
\begin{aligned}
& \mathrm{J}=-0,81144 \text {. . . I J J }=9,909256 \\
& M=-1,25325 \text {. . . L M } M=0,098046 \\
& O=-2,12630 \text {. . . } 1-0=0,327624 \\
& S=-0,13490 \ldots \text {. . }-S=9,130012 \\
& T=\sim 0,10080 \ldots . \quad M T=9,003441
\end{aligned}
$$

$+0,00568$

$+0,01063$

$-0,00167$

$0,01175]$

\begin{tabular}{|c|c|c|}
\hline 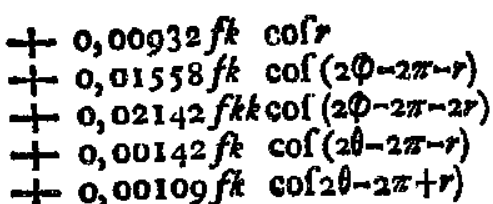 & $\begin{array}{l}7,969416 \\
8,192568 \\
8,330819 \\
7,152288 \\
7,037426\end{array}$ & $\mid \begin{array}{r}+0,000555 \\
+0,000928 \\
+0,000069 \\
+0,000085 \\
+0,00006\end{array}$ \\
\hline
\end{tabular}

$.02842 \$ ?$

0,01160

$10354 \mathrm{M}$

$33746 \mathbf{S}$

$2843 \mathrm{M}$

0025 S

tar ac

$$
\begin{aligned}
& 9=-0,00005 \cdot .609=5,698976 \\
& \text { In=+0,00177 . . . } 691=7,247973 \\
& D=+0,52266 \text {. . } t S=9,718219 \\
& \mathrm{~S}=+0,02355 . . \quad \cdot \mathrm{S}=8,37199 \mathrm{I} \\
& z=+0,01076 \text {. . } 1 \text { s }=8,031812
\end{aligned}
$$

5. 260. Ex his iam pro diftancia Lunae a terma erit:

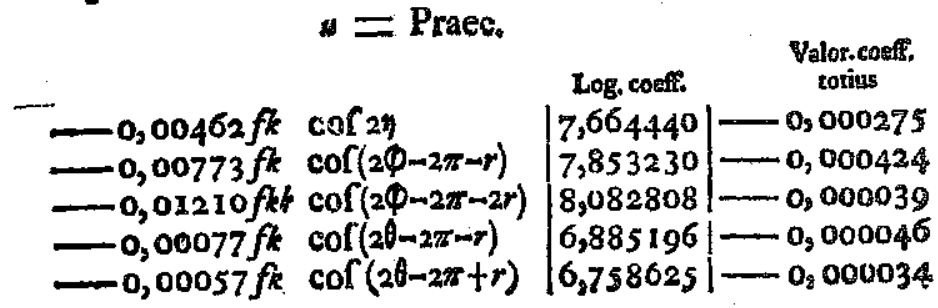

et pro motu momentaneo

$$
\frac{d p}{d r}=\text { Praec. }
$$

5. 26!. Pro lungitudine autem luna fequentes sefolui debent aequationes:

$+0,00932=\$^{\prime}-0,01766\left(S^{\prime}+5\right)=0,00424 \mathrm{I}\left(92 /+S^{\prime}+W^{\prime}\right)$ $+0,01558=1,0268342 M^{\prime}-2 / s^{\prime}+0,017665^{\prime}+0,00996 b^{\prime}$

Ee 3 
$+0,02142=0,0268340 !-D^{\prime} s^{\prime}+2,6 b^{\prime}+0,021270$ $+0,01064 \mathfrak{g}^{\prime}+0,01766 \mathfrak{D M}^{\prime}+0,00996 \mathrm{~S}^{\prime}$

$+0,00142=-0,840642 \mathfrak{S}^{\prime}-\mathfrak{I}^{\prime} / \mathrm{m}^{\prime}-\mathfrak{D}^{\prime} / b^{\prime} \mathfrak{d} / g^{\prime}+0,02114 \mathbb{S}^{\prime}$ $+0,16853, \mathfrak{j}^{\prime}+0,02396 \mathrm{M}^{\prime}$

$+0,00109=1,159358 \mathfrak{F}^{\prime}-\mathfrak{D}^{\prime} g^{\prime}-\mathbb{C}^{\prime} b^{\prime}-0,35615 \mathfrak{G}^{\prime}$

hincque prodit pro longitudine vera : $+0,16850$ \$!

$\varphi=$ Pr. $+0,00932 f k$ fin $r$ $+0,01521 \mathrm{fk}$ fin $(2 \varphi-2 \pi-r)$ $+0,79079 f k k$ fin $(2 \varphi-2 \pi-2 r)[9,898060+529$ $-0,0012 \mathrm{r} f k$ fin $(2 \theta-2 \pi-r) \quad 7,083939$ - 15 $-0,00082 f k$ fin $(2 \theta-2 \pi+r)|6,913527|-10$

\$. 262. Ob inclinationem ergo orbitae lunaris ad eclipticam omnes correetiones huc redeunt, ve fit

- I. Pro diftantia lunae a terra :

$$
y=\text { Praec. }
$$

$+0,000084 f \operatorname{col} 2 y$ $+0,001832 f \operatorname{cor}\left(2 \Phi_{-2 \pi}\right)$ $+0,00001$ if $\operatorname{cor}(2 \theta-2 \pi)$ $+0,00000$ If $\operatorname{col}(4 \theta-4 \pi)$ $-0,00462$. $-0,00773 f k \operatorname{cor}(2 \phi-2 \pi-r)$ - $0,01210 f k k \operatorname{cor}(2 \varphi-2 \pi-2 r)$ $-0,0007 f^{\prime}$ cor $(2 \theta-2 \pi-r)$ $-0,00057 f^{k} \cos (2 \theta-2 \pi+r)$
Log, coeff. Val, coeff. $|5,926350|+0,000092$ $7,262918+0,002004$ $.5,034111+0,000012$ $4,011565+0,000001$ $7,664440-0,000275$ $7,853230-0,00042$ $8,082808-0,000039$ $6,885 \times 9^{6}=0,000046$ [i 758625] $-0,000034$

$$
\text { CAPUT XP. }
$$

II. Pro modu momentaneo:<smiles>C[14CH3]</smiles>

6

14 (3)

6 פMI

$15 \mathrm{BS}^{\prime}$

$0 \mathfrak{S}^{\prime}$

L. coeff.

nin.fece

$-115$

I 87

529

15

Io

ad

III. Pro longitudine lunae vera

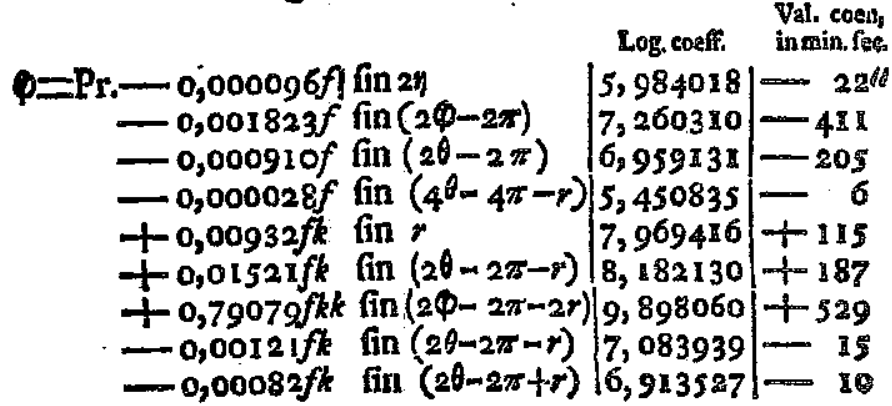

Log. coeffR Val. coeff. $|6,227887|-0,000185$ $7,567849-0,004043$ $6,356026-0,000248$ $4,698970-0,000005$ $7,969416+0,000555$ $8,192568+0,000928$ $8,3308 \times 9+0,000069$ $\left|\begin{array}{l}7,1522.88 \\ 7,037426\end{array}\right|+0,000085$ $+0,00142 f k \operatorname{cor}(2 t-2 \pi-r)$

$+0,00109 f_{k}$ cor $(2 \theta-2 \pi t r)$
$-0,003697 f \operatorname{col}(2 \varphi-2$

$0,000227 f \operatorname{col}(20-2 \pi)$

$0,000005 f \mathrm{cor}\left(4^{\theta-4}\right.$

$+0,0.2932 f k$ col $r$

H. Pro 


\section{263 .}

Q

uas igitur inuenimus hactenus lunae inaequalitates eae primum, fi originem earum fpectemus, ad fex claffes reducuntur. Quatenus enim luna in motu fuo a regulis Keplerianis, in quibus quidem mo. tum apogei compectimur, recedic, eius errores vel primo a folo lunae afpectu, feu eius diftantia a fole pendent, feu quod codem redit, per angulum y tantum definiuntur, quibus variacio lunae continetur. Ad fecundam claffem refero eas lunae inaequalitates, quae infuper ab excentricitate eius orbitae pendent. Terth claffis eas compleetitur inaequalitates, quae ab excen-

- tricitate orbitae lolis ortum trahunt, Quarta vero eas, quae per vtramque excentricitarem coniunktim determinantur. Quintae porro clafi annumeramus ess inaequalitates, quae parallaxin folis inuoluunt, atque errores quatuor ante memoratorum generum implicant. Sex. to denique claffis fuppeditat eas inaequalintes, quae praeterea ab inclinatione orbitae lunaris ad eclipticam pendent.

\$. 264. Quodfi vero ad vfum harnm inaequalimtum attendamus, prouti eae, ad lunam accommodari debent, tum eae in quinque claffes commodiffime discribuuntux. Primo enim perpendendae funt eae insequali- qualitates, quarum ope vera diftantia lunae a terra de terminatur, vt inde porro tam lunae diameter appa. rens, quam eius parallaxis horizontalis aftignari poftic. Secundo loco formulae erunt collocandae illae, quae motui momentaneo definiendo inferuiunt, ex quibus deinceps motus lunae horarius acsurate exhiberi pote. rit. Tertium locum occupabunt eae inaequalitases, quas veram longitudinem lunac ad eclipticam relatam prae bent. Quarto vero poficio lineae nodorum linae, fets longieudo nodi afcendentis; ac quinto vera inclinatio orbitae lunaris ad eclipticam inueniri debebit; vo deinde vera lunae latitudo concludi pofit. Manifertum autem eft, has inaequalitates plurimum inter fe permifceri, ita vt vix vllum habeatur genus, cuius inaequalitas tes non a reliquis generibus pendeant; cui tamen in. commodo facile medela adhibetur.

§. 265. Quanquam numerus inaequalitatum, gaas fumus confecuti, tantopere increuit, vt calculus fine maxima moleftia expediri nequeat, tamen iam monui, non omnes inaequalitates, quibus motus Lunae perturbatus, effe definitas, fed potius earum numerum omnino effe intinitum. Facile quidem intelligitur, plerasque has praetermiffas inaequalitates vullius fere effe momenti, atque fine notabili errore is fuperfederi poffe: verum tamen funt inter eas nonnuliae, quae ad plura minuta fecunda affurgere videntur, quarum argumensa fupra iam innui ; ex quo omnino operae effet pretium in eas omni cura inquirere. Sed earum inueftigatio tam eft lubriet incerts, vt leuiffima omiffio in calculo facta eas Ff maxime 
maxime afficiac. Cum igitur in calculo plurimos terminos reiicere cogamiur, iftam inueftigationem fruftra plane fufciperemus, quamdiu fcilicet rem fine appropinquatione exequi non licet. Cuius defectus eximium habemus exemplum in inaequalicatibus pottremo loco inuentis, quae fatim atque in negligendo minus largi fueramus, mirum quantum prodierunt immutatae; ac nulIum plane eft dubium, fi calculum adhuc accuratius profequi liceret, quin valores inuenti notabilem infuper mutationem fint fubiturae. Imprimis autem aequatio ab angulo $2 \varphi-2 \pi-2, r$ fen a dupla diltancia apogei a nodo pendens, eft fúpecta, ac minime pro certa haberi poteft, cum leuiflima circumfantia eam magnopere peruurbare valeat.

\$. 266. Si enim in canfan inquiramus, cur analyfis pofterior tam diuerfos valores pro his insequalizatibus fuppeditauerit, primo quidem fratim patet, negleAum litterarum germanicarum $\mathfrak{S}, \mathfrak{M}, \mathfrak{O}$, etc. in calculo priosi potifimum hoc difcrimen produxiff: ingens enim valor litterae $\$$ imprimis aequationem ab angulo $20-2 x-2 r$ pendentem tantopere auxit. Proterea vero etiam non parum augmenti haec aequatio inde eft nakba, quod in calculo pofteriori rationem quoque ha. buimus sermini $\operatorname{cor}(2 \eta-2 r)$, qui tam in valore $\frac{d \varphi}{d r}$ quam $\frac{d \eta}{d r}$ inefte eft deprehenfus; vnde tuto colligere licet, fi alios quoque terminos fimiles veluti ${ }_{2} \theta_{-2 \pi}-2 r$, etfi per fe fune minimi, in calculum introduxiffemus, co- efficientes terminorum $2 \varphi-2 \pi-2 r$ non mediecrenti inde mucationem fubituros fuiffe. Quamobrem plus hime colligere non poffumus, nifi inaequalitatem Lunae ah angulo hoc $2 \varphi-2 \pi-2 r$ pendertem aninime effe contemnendam, etiamfi fortaffe tanta non fie, quam inue nimus. Vera autem eius quantiras certius ex obferuationibus quam ex Theoria colligi polfe videtur.

5. 267. Quoniam vero hae inaequalieates omnes ad anomailiam Lunae veram referuntur, antequam eas ad vfam adhibere liceat, modum tradi conveniet ad quodvis tempus propofitum anomaliam Lunae veram determinandi. Cognita autem excentricitate orbisac lunatis $k$ et motu anomaliae mediae, inde ad quodvis tempus facile anomalia media $p$ colligitur. Verum ex anomaliọ media $p$ et excentricitate $k$ anomalia vera definiri debet ope huius aequationis $d p=\frac{(1-k k)^{\frac{3}{2}} d p}{\left(i-k \operatorname{cor}(k)^{2}\right.}$; rnde quidem non difficulter, fi not effer anomalia verst $r$, vicifirm inveniri poffet anomalia media fo Calcula enim peralto, fi bretikatis gratia ponarus

$$
\delta=\frac{1-V(I-k k)}{k}=\frac{i}{k} k+\frac{1.1}{2.4} k^{3}+\frac{1.4 .3}{2.4 .6} k^{s}+\text { ete. }
$$

reperitur:

$p=r+2 k$ in $r+2 \delta\left(k-\frac{\pi}{2} \delta\right)$ fin $2 s+2 \delta\left(k-\frac{2}{3} \delta\right)(8) 3 x$ $+2 \delta^{3}\left(k-\frac{3}{4} \delta\right)$ Sin $4 s$ etc. cuius feriei progreffio primo intritu patet. Cum als. tem ac proinde \& fit valde parvum, eric fatid exage: FE 2 
$p=r+2 k \operatorname{fin} r+\left(\frac{3}{4} k k+\frac{7}{5} k^{4}+\frac{3}{5} k^{6}\right)$ fin2 $r+\left(\frac{2}{3} k^{3}+\frac{3}{8} k^{5}\right)$ fingr

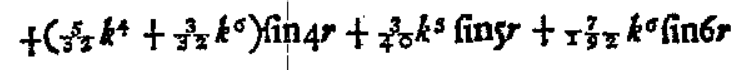

6. 268. Data ergo anomalia media Lunae $p_{2}$ eius anomalia vera $r$ elici debebic ex hac aequatione

$r=p-2 k$ inir $-\left(\frac{3}{4} k k+\frac{5}{3} k^{4}+\frac{3}{6} k^{5}\right) \operatorname{fin} 2 r-\left(\frac{\pi}{3} k^{3}+\frac{5}{3} k^{5}\right) \operatorname{lin} 3 r$

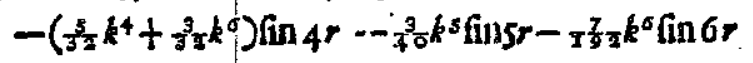

cuius quidem ope, fi cognita fiuerit excentricitas $k$, calculus non fdifficulter expedietur. Quoniam enim termini finus insoluentes funt admodum parui, in is ftaim poni poterit $r=p$, vade valor verior pro $r$ eruetur, qui deinde iterum in his terminis adhibitus, iuftiorem valorem pro $r$ fuppeditabit 'Atque học moda poit aliquot operationes verus tandem valor pro anomalia vera $r$ obtinebitur. Interim tamen, quo ifte cal- colus facilius perfici queat, aequatio haec ita poteft tranisformari, vt loco finuum anomaliae verae $r$, finus anomaliae mediae introducantur; id quod fequent modo praeftabitur.

5. 269 Ponatur breuitatis gratia:

$$
\frac{3}{4}+\frac{x}{8} k k+\frac{3}{4} k^{4}=\alpha ; \frac{3}{3}++\frac{7}{2} k k=6 ; 3+\frac{3}{3} k k=y
$$

ve fit:

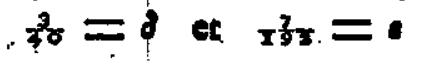

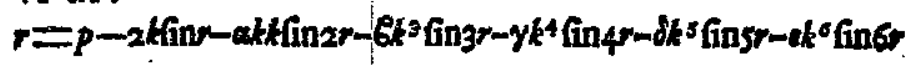
ac ponatur denuo

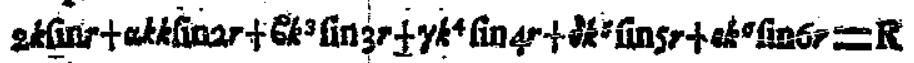

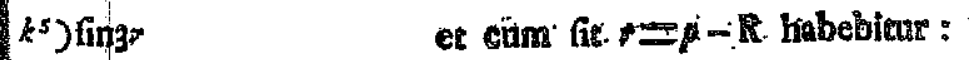


fupra (206) per $?$ indicanimus, ita exprimetur; , th fit

vt fit $i=\mathrm{C}+1,0085272 \mathrm{P}$

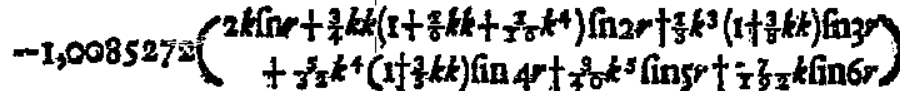

vbi $\mathrm{C}+1,0085272$ exhibet longitudinem Lunne mediam; quae fi vocetur $=\frac{\hbar}{\text {; }}$ atque in coefficientium partibus minimis pro $*$ fcribatur valor proximus 0,0545 , erie

$$
\begin{aligned}
& \text { erite } \\
& \xi=\xi-2,0170544^{k} \text { fin }+0,304718 \mid 22675^{\prime \prime}=6^{\circ}, 17^{\prime}, 55^{\prime \prime} \\
& -0,756770 k k \text { fin2 } \mid 9,878964{ }_{464}=7,44 \\
& -0,336551 k^{3} \text {. fingrip 9,5\%7.05: } \\
& -0,15786 \text { * findris.198282 } 5
\end{aligned}
$$

vade patet fuperfluum futurum fuife, fi fuperiores expreffiones vlise quartam poreftatem ipfus $t$ extendere roluiftemitis:

$$
\because \quad ;+\cdots: \div
$$

a. foc.

, I7? $15 s^{\prime \prime}$

7,44

res extendere
艘 $(0)$

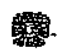

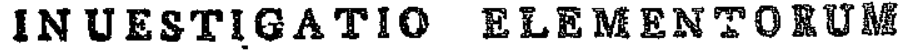
MOTUS IUUA

5. 272.

Tnuentis iam per Theoriam hisce inaequatizatibus, quigo bus motus Lunae perixubatur, antequam eas ad computam aftronomicum accommodare 'iceat; ele menta, quae in eas ingrediuntur, per obferuationes des terminari oportet. Primo fcilicet ad datan epochams cum longitudo Lunae media, eum eius anomalie mo dia, as locus nodi medius conftitui debebit, vt eaeders res inde ad quoduis aliud tempus affignaxi queant. Deinde quoque ex obferuationibus verus valor excentriciso tis lunaris colligi debet, a quo potifimum quanicas praecipuarum inaequalitatum pendet. Excentricitas aus tem orbitae folaris pro fatis certa haberi poteric, cum fit $e=0,0168$. Lunae vero excentricizas tam prope iam conftat, $v t$ inde fine errore ad quamibet anomato liam mediam vera fatis exalte affignari pofit Eti enim in anomalia vera error aliguot minutorum primorum commitritur, inaequalitates Lunae inde non vies alloguot minute fecunda afficiuntus.

6. 273. Quodf autem ftarim quasuis tunse oba feruationes ad hune finem adhibere velimus, ob tam ingentem inaequalimatum numerum, inueftigstio le menrorum maxime molefts redideremur. Quoeinen obrer-

\section{CAPUT}


1430

CAP $P T$ XFII.

obferuationjíts eas eligi conueniet; pró quibus numerus inaequalitatum multo fiat minor; dum fcilicet difantia Lunae a fole feu angulus $\eta$ datum obtinet valorem. Commodifimae ergo erune eae obferuationes, quae in ipfis momentis coniunationis vel oppofitionis funt inftitutae. Accuratas itaque obleruationes eclipfium lunarium ad boc negotium adhibebo, quoniam praeter haec rempora, vera vel coniunctionis vel oppofitionis momenta nos fatis certo ex.obferuationibus colligi licec.

6. 274. Momento autem oppofitionis verae Lunac et Solis, longitudo Lunae fex fignis diftat a longitudine folis, tia we fit $\theta=\varphi \pm 180^{\circ}$, ideoque angulus $1=180^{\circ}$. Pofito autem pro a hoc valore longitudi Lunae vera $\phi$ ex media $\xi$ per fequentes formulas definieur, in quas formulae hadtenus inuentae abeunt:

$\varphi=\xi-2,0170544 k$ fin $-0,75677^{\circ}$ okfinar $-0,33655 k^{3} \mathrm{fn} 3 r$ $+0,0101460+0,004200$

$+0,4202260-0,573280$

$+0,0049920+0,003180$

$-0,0052860+0,150830$

$-0,000860$

$+1,1959$.

$-0,0757^{\circ}$

$+0,00932 f$

$+(0,201385+0,021889-0,016368-0,39598+4, \sqrt{73} 38)$ - fin $+(0,06645-0,02332+0,00840)$ ee fin2s

$+(0,74760-0,81430-0,01420)$ ekfin $(r-1)$

$+(-0,61850-0,23960-0,00610)$ ekfin $(r+s)$
$C A D U T Z Z$

$-(0,002823+0,000910) f \mathrm{fin}(2 \mathrm{2}-2 z)$

$-0,000028 f$ fin $(4 \varphi-4 \pi)$

$+(0,01521-0,0012 x) f t \sin (2 \varphi-2 x-\theta)$

$+0,79079 f k k$ in $(2 \phi-2 \pi-2 r)$

6. 275. Cum igitur lit $f=x, 09375$, $y=\frac{7}{2}=$ erit has formulas colligende:

$\phi=\xi-1,572993 k$ fin $r-1,17186 k k$ fin $2 r-0,3365^{k 3} \mathrm{fin}_{3}$ "

$+0,21998$ efins $+0,05123=e$ fin $2 s-0,0809 e k$ in $(r-s)$

$-0,8642$ ek $\sin (r+s)$

$-0,002989 \operatorname{fin}(2 Q-2 \pi)+0,01531 k \operatorname{lin}(2 \varphi-2 \pi-r)$

$-0,00003 \mathrm{sin}(4 \phi-4 \pi)$

$$
+0,86493^{k k f i n}\left(2 \varphi_{\infty 2 \pi} 2 \pi r\right)
$$

et cum fit $e=0,0168$, erit hoc valore fubttituto:

$\varphi=\xi-1,572993 k$ fin $-1,17186 k k$ finar $-0,3365 k^{3}$ fing

$+0,003697$ fins $+0,000014$ fin2s $-0,001359 k$ in $(r-s)$

$-0,014523 k \operatorname{lin}(p+s)$

$-0,002939 f i n(2 \varphi-2 \pi)+0,01531 k \operatorname{fin}(2 \varphi-2 \pi \cos r)$.

$-0,00003 \operatorname{Ifin}(2 \varphi-4 \pi)$

$+0,86493^{h k f a}\left(2 \varphi_{-2 \pi}-2 r\right)$

8. 276. Affumta iam hypothefi quaniam non nimis a vero aberrante, vnde ad datum guoduis tess- 
pus definiri poffit tam longitudo lunae, quam eius ano malia media, ex qua praeterea ope excentricitatis proxime cognitae : anomalia vera affignari queat: haec elementa correctione indigebunt, quam ex obferuationibus elici oporteat. Ponamus ergo longitudinem mediam ex tabulis defumtam angeri debere $m$ minutis fecundis. Tum vero excentricitas fuppofita, quae fit $=0,0545$, augeri debeat $\frac{n}{10000}$, vt fit $k=0,0545+\frac{n}{10000}$ : ipfa vero anomalia vera tabularis, quae fit $=v$, augmentum requirat $\mu$ minutorum fecundorum;, vt fit $r=v+\mu^{\prime \prime}$ : exitque fin $r=$ fin $v+\mu^{\prime \prime} \operatorname{col} v$; fin $2 r=$ in $2 v+2 \mu \prime \operatorname{cor} 2 v$; et fin $3 r=$ fin $3 v$ in terminis enim minimis haec correttio praetermitti poteric.

6. 277. Quod ti hace omnia in minuta fecunda eonuertantur, prodibit longitudo lunae vera

$$
\varphi=\text { Long. med }+w^{\prime \prime}
$$

- $17682 \|$ fin $v-32,445 z^{\prime \prime}$ fin $v-0,085728 \mu^{\prime \prime} \operatorname{cor} \theta$

- 718 , fin $2 v-.2,635 w^{\prime / f i n} 2 v-0,006962 \mu / \operatorname{cor} 2 v$

- $\quad 11^{\prime \prime \text { fin }} 3 v+762^{\prime \prime}$ fin $s+3^{\prime \prime}$ fin $2 s$

- $15^{\prime \prime}$ fin $(r-s)-163^{\prime \prime}$ fin $(r+s)$

- 616"f fin $(2 \varphi-2 \pi)+172^{\prime \prime} \operatorname{fin}(2 \varphi-2 \pi-r)$

- $\quad 6^{\prime \prime} \operatorname{fin}(4 \varphi-4 \pi)+530$ fin $(2 \varphi-2 \pi-2 r)$
Cum autem poltemus terminus fic fufpertus, loco eius coefficientis 530 malumus ponere coefficientem indefnitum rooy, atque ex obferuationibus valorem ipfius y indagare. Deinde fit error anomaliae verae $i$ minusorum primorum, vt calculus commodior reddacur, atgue ob $\mu=60 i$, neglectis terminis minimis erit:

$$
\begin{gathered}
\varphi=\text { Long. med. }+w^{\prime \prime} \\
-17682^{\prime \prime} \text { fin } v-32,445 n^{\prime \prime} \text { fin } z-5,143 i^{\prime \prime} \operatorname{col} v \\
-718^{\prime \prime} \text { fin } 2 v-2,635 n \text { fin } 2 v-0,417 i \operatorname{col} 2 v \\
+762 \text { fin } s-15 \text { fin }(r-s)-163 \text { fin }(r+s) \\
-616 \text { fin }(2 \varphi-2 \pi)+12^{\prime \prime} \text { fin }(2 \varphi-2 \pi-r) \\
+100 y \text { fin }(2 \varphi-2 \pi-2 r) \\
\vdots
\end{gathered}
$$

6. 278. Oblata autem obferuatione ecliptis luna, quaeratur primum momentum medium huius ecliplis, pro quo colligatur longitudo folis; itemque longitedo nodi afcendentis. Punctum autem foli oppofitum nondum erit longitudo lunae vera in ecliptica; verumta. men longitudo lunae pro hoc momento eclipfis medio inueniri poterit ope fequentis tabellae : 
Subtrahatur longitudo nodi a longitudine folis, et ae. quatio tabulae fecundum ticulos adfcriptos applice. tur puntto foli oppofito in ecliptica.

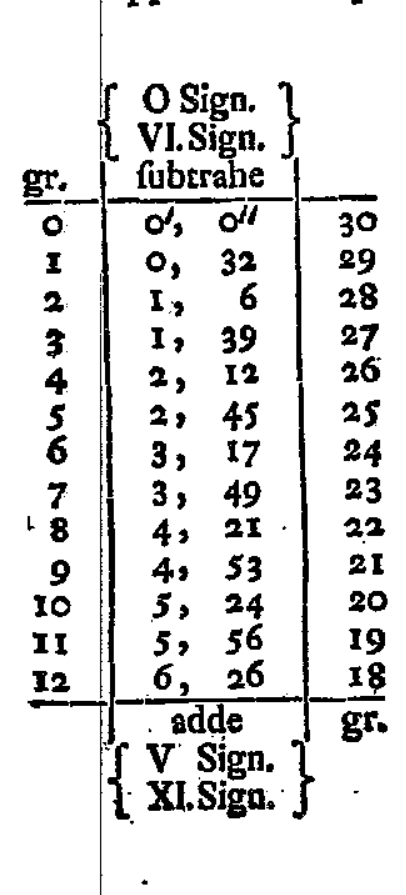

5. 279. Quanquam autem hoc momento, ad quod lunae longitudinem hinc colligimus; non vera lunae oppofitio exiftit, fed luna fecundum longitudinem a puncto foli oppofito diftat particula, quam haec tabula monitrat; tamen tuto pro hoc momento ex formula noftra longitudinem lunae inueftigare pocerimus, vifuri, lunae inuertigare poterimus, vifur,
quam quam exacte ea conueniat cum longitudine eilus ad hoc rempus ex obleruatione conclufa. Cum enim luna hoc tempore nunquam vltra $s^{\prime}$ a vero oppofitionis loco dis ftet, fi formula noftra generali vti vellemus, foret ano gulus $\eta$ minor 5 minutis primis; vnde facile peripicinur difcrimen in loco lunae inde orinndum vix vaguams 12" effe iuperaturum. Quoniam irague medium cuinsque eclipfis momentum ipfum tam accurate definimi ne quit, ve non error dimidii minuti primi fit percimeo fcendus, fuperfluum fane foret in calculo ad ifiusmodi minutias attendere.

\$. 280. Hanc ob caufam quoque ex calculo, quem inibo, non fummam praecifionem expetari conuenies: quia ipfae obferuationes, quibus vtar, non plenae accuracionis funt capaces. plus igitur me non effecturum confido, quam vt fatis prope tam excentricitatem or bitae lunaris, quam longitudinem et anomaliam Iunae mediam ad datam epocham definiam. Quod cum fuerit faltums maiori confidentia theoriam ad quasuis alias obferuationes transferre licebit; quae fi nullis erroribus fuering inquinacae, non admodum erit difficile reliquas elemen. torum corrętiones, quibus formulae noftrae funs innixae, inde concludere. Imprimis autem hic calculus yeram excentricitatem orbitae lunaris fatis exacte manis fettabit, vt deinceps accuratius pro quauis anomalia me. dia conuenientem anomalam veram definire valeamus. Hunc igitur in finem nonnullas eclipfes lunares Parifim inftitutas calculo fubiiciam.

Gg 3

8. 288 
8. 281. Primae igitur eclipfis medium contigiffe reperio Parifiis A. 1712. Jan. 23, $7^{b}, 55^{t}, 16^{\prime \prime}$ temp. medio. Pro quo momento colligitur:

Longitudo folis 0 . . . 10, $3^{\circ}, .0^{\prime}, 54^{\prime \prime}$

Anomalia vera folis s. . . 6, 24, 25, .13

Deinde ex tabulis meis -

Longitudo lunae media $.4,7,18,55$

Anomalia lunae media $\cdot 2,0,18,20$

Anomalia lunae vera $v=1,25,6,27$

Longitudo nodi vera $\pi=9,24,34,32$

Dift. nodi a fole $\theta-\pi=0,8,26,22$

Hinc aequatio loci lunae $,-\quad 4,33$

Ergolongitudo lunae vera $\varphi=4,2,56,21$

5. 282. Hinc calculus fequenti modo infituetur :

$v=1,25,6,27 ;$ fin $v=t$ fin $55^{\circ}, 6^{\prime}, 27^{\prime \prime}$ $\operatorname{cor} v=+$

$2 v=3,20,12,54 ;$ fin $2 v=t$ fin 69,$47 ; 6$ $\operatorname{col} 2 v=-$

$s=6,24,25,13 ;$ fin $s=-$ fin $24,25,13$ $\nu=7,0,41,14 ;$ fin $=-$ fin $30,41,14$ $v+s=8,19,31,40$; fin $=-$ fin $79,31,40$ $\varphi-\pi=6,8,21,49$

$2 \varphi_{-2}=0,16,43,38 ;$ fin $=t$ fin $16,43,38$ $r=\mathrm{x}, 25,6,27$

$29-2 \pi \cdot 8=10,21,37,11 ;$ fin $=-$ fin $38,22,49$ $29-2 \pi \cdot 2 r=8,26 ; 30,44 ;$ fin $=-$ fin $86,30,44$

:igifle

emp.

$27^{\prime \prime}$

6

13

14

40

38

49

44

$+$
CAPUT XYII

มิริา

$$
\begin{aligned}
& \begin{array}{l}
+9,91393+9,9139+9,7575 \\
=4,24753-1,511-0,7104 \\
\hline 4,16146-1,42501-0,46795
\end{array} \\
& \begin{array}{l}
+9,9724+9,9724-9,5385 \\
=2,8561-0,4208-9,6201 \\
\hline 2,8285-0,393218+9,15868
\end{array} \\
& -9,6163-9,7078-9,9927 \\
& \begin{array}{l}
+\quad 2,8819 \\
+2,4982 \\
+20,8839+2,2049
\end{array}
\end{aligned}
$$

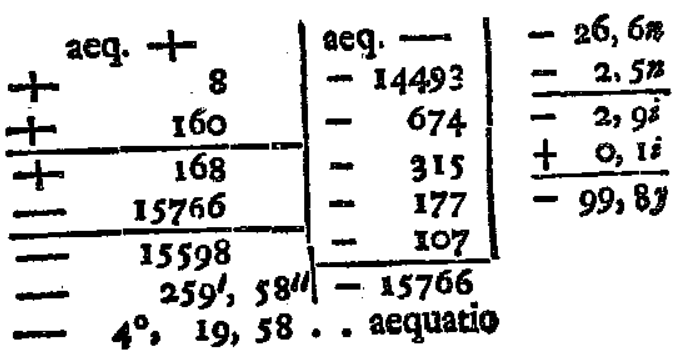

Long.med. 4,7, 18,55+m

aeq. $\frac{-4,19,58}{4,2,58,57}-29,18-2,8 i-99,8 y=4,2,56,2 \pi$ $4,2,56,21$

Eigo $0=2,36-29,18-2,8 i-99,8 y+3$ 
§. 283. Secundae eclipfi medium contigit: Pariliis A, 1713. Dec. $1^{b}, 15^{b}, 261,34^{\prime \prime}$ temp. med. Pro quo momento colligitur

Longitudo Solis $\theta=$. 8, 9, 53,40 Anomalia vera Solis $s=$. 5, 1, 46, 43 Longitudo Lunne media . 2, 5, 2, 26 Anomslia Luare media * 9, 12, 27, 42 Anomalia Lunae vera $v=09,18,24,49$ Longitudo nodi $\pi=.8,17,46,10$ Diftantia Solis a nodo . II, 22, 7, 30 Aequatio loci Lunae * + 4, 17 Longitudo Lunae vera $\varphi=2,9,57,57$

8. 284. Hine calculus fequens infituatur:

$$
\begin{aligned}
& \text { v }=9,18,24,49 ; \text { fin } v=- \text { fin } 71,35, \mathrm{II} \\
& \operatorname{cor} v=+ \\
& 2 v=7,6,49 ; \text { finzo }=- \text { fin } 36,49 \\
& s \quad=5, x, 46 \quad \text {; fin } s=+ \text { fin 28, } 14 \\
& \text { z:s }=4,16,39 \text {; fin }=+ \text { fin } 43,21 \\
& v+s=2,20,11 \text {; fin }=+ \text { fin 80, } 11 \\
& \varphi-\pi .=11,22,12 \\
& 24-2 \pi=11,14,24 ; \text { in }=-\sin 1 \text { 15, } 36 \\
& r \neq 9,18,25 \\
& 29-2 x-r=\frac{1,25,59}{T} ; \text { fin }=+ \text { Iin } 55,59 \\
& 2 \varphi-2 \pi-2 r=4,7,34 ; \text { fin }=+ \text { fin } 52,26
\end{aligned}
$$

CAPUT ZYPR.

2유ㅁㅛㅛ

$$
\begin{aligned}
& \begin{array}{l}
-9,97717-9,9772+9,4996 \\
=4,24753-1,5112-0,7104 \\
\hline 74,22470+1,488310-0,2100
\end{array} \\
& \text { - } 9,7776-9,7776-9,9034 \\
& \frac{-2,8561}{-0,4208-9,6201} \\
& +9,6749+988366+9,9936 \\
& \begin{array}{r}
+2,8819-1,1761-2,2122 \\
\hline+2,5568-1,0127-2,2058
\end{array} \\
& -9,4296+9,9185+79,2 y \\
& \begin{array}{r}
-2,7866 \\
+2,2192 \\
+2,2355 \\
\hline 2,1530
\end{array}
\end{aligned}
$$

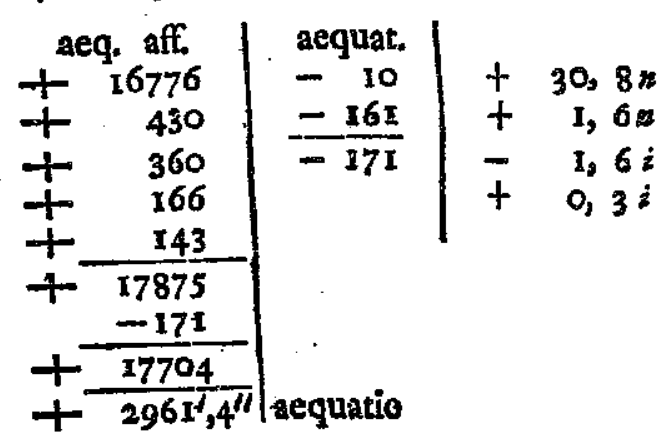

Long. media $=2,5,2,26+m$ aeq. $\frac{1,4,55,4}{2,37,30,+28}$

Long. ver

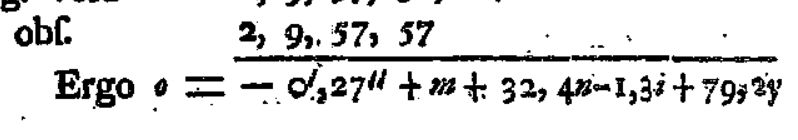


242

$$
C A P U T X V I I .
$$

\$. 285. Tertiae eclipfis medium contigit Parifis A. 1717 Marc. 26, $15^{b}, 21^{\prime}, 20^{\prime \prime}$ temp. med. Pro quo tempore colligitur

Longitudo folis vero $=0^{\circ}, 6^{\circ}, 19^{\prime}, 56^{\prime \prime}$

Anomalia folis vera $s=8,28,0,17$

Longitudo media lunae . . 6, $1,37,2$

Anomalia media lunae .. 8,24, 7, 21

Anomalia lunae vera $v=9,0,19,10$

Longitudo nodi vera $\pi=6,13,30,22$

Diftantia nodi a fole . . $5,22,49,29$

aeq. pro loco lunae $+\quad 3,57$

Ergo longitudo lunae vera $\varphi=6,6,23,53$

5. 286. Calculus igitur ita fe habebit

$v=9,0,19,10$; fin $v=-$ fin $89^{\circ}, 40^{\prime}, 50^{\prime \prime}$ col. $=+$

$2 v=6,0,38, \quad ;$ fin2v $=-$ fin $0^{\circ}, 38^{\prime}$ col $=-$

$s=8,28,0 \quad ;$ fin $s=-$ in $88^{\circ}$, o

$v-s=0,2$, 19 ; fin $=+$ fin $2^{\circ}$, 19

$v+s=5,28$, Ig ; fin $=+$ fin 1,42

$\varphi-\pi=5 ; 22,53$

$2 \phi-2 \pi=11,15,46 ;$; fin $=-$ fin 14,14

$r=9,0,19$.

$29 m 2 \pi m r=2,15,27 \quad$; fin. $=+$ fin 75,27

$2 Q-2 \pi-2 r=5,15,8,6 \mathrm{fn}=t \operatorname{fin} 14,52$
$C A B U T \quad X Y Z I_{*}$

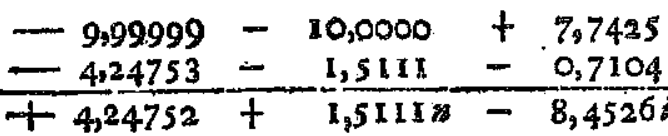

$-8,0435-8,0435-9,9909$

$\frac{2,8561}{+0,8996+0,4208-9,6201}$

$\begin{array}{r}9,9997+8,6066+8,8,4680 \\ +2,8819-1,1761-2,2122 \\ \hline 2,8816-9,7827 \cdot-0,0802\end{array}$

$\begin{array}{r}9,3907+9,9858 \\ -2,7893+25,659 \\ \hline\end{array}$

$+2,1893+2,2213$

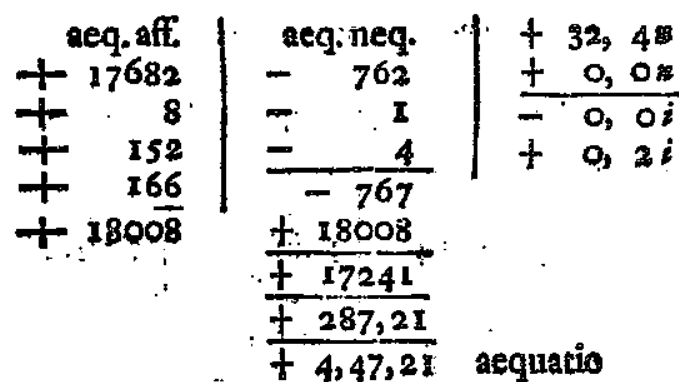

Long. media $i=6, i_{3}, 37,2$

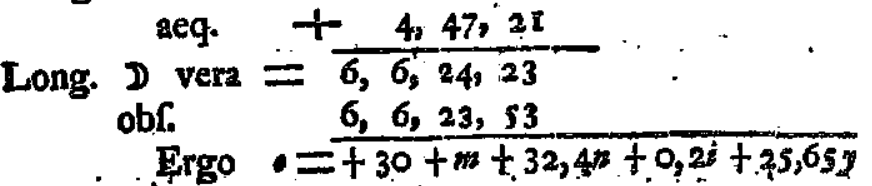

Hh 2

\$. 287 
244

\section{CAPUT XVII.}

3. 287. Quartae eclipfis medium erat Parifiis A. 1718 Sept. 9a, $8^{b}, 1$, $x^{\prime \prime}$ temp. medio Pro guo tempore colligitur

Longitudo folis vera $\theta=5,16,40,58$

Anomalia folis vera $s=2,8,19,59$

Longitudo lunae media $11,17,25,16$

Anomalia lunae media $0,10,41,28$

Anomalia lunae vera $v=0,9,36,52$

Longimdo nodi vera $\pi=5,15,59,35$

Diftantia nodi a fole

$0,0,4 x, 23$

aeq. pro loco lunae

Longitudo lunae obf $\emptyset=\overline{11,16,40,36}$

6. 288. Calculus ergo fequens habebitur. $v=0,9,36$, s2 ; fin $v=+$ fin $9,36,52$

$$
\operatorname{cor}=t
$$

$2 v=0,19,14 \quad ;$ fin.2v=tifin $19 ;$ is $\operatorname{cof}=+$

$s=2,8,20 \quad ;$ fin $s=t$ fin 68,20

$v-s=10,1,17 \quad$; fir $=-$ fin 58,43

$v+s=2,17,57$. ; fin $=+$ fin 77,57 .

$\varphi-\pi=0,0,41$

$2 \varphi-2 \pi=0,1,22$

$$
r=0,9,37
$$

$2 \varphi-2 \pi-r=11,21,45$

$29-2 \pi-2 r=11,12,8$
; fin $=+$ fin 1,22

;.fin $=-$ fin 8,15 ; fin $=-$ fin 17,52
$C A P U$ XVIS

$+9,22274+9,2227+9,9938$

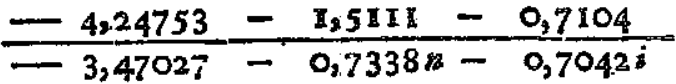

$+9,5177+9.5177+9,9750$

$\frac{+2,8561-0,4208}{-2,3738-9,93858-9,520518}$

$+9,9682-9,9318+9,9903$.

$\begin{array}{r}+2,8819-1,1761=2,2122 \\ \hline+2,8501+1,1079-2,2025\end{array}$

$+8,3775-9,1568-30,68 y$

$=2,7896+2,2355$

$$
\begin{array}{c|c|c}
\text { aeq. aff. } & \text { 2eq.neg. } & -5,4 n \\
\pm 708 & -2953 & -0,812 \\
\pm 13 & -237 & -5,18 \\
\hline 721 & -159 & -0,48 \\
\hline 3389 & -15 & \\
\hline \pm 2668 & =25 &
\end{array}
$$

Long. \& med. II, 17, 25, 16

seq. $\quad 44,28$

- Long. vera II, 16, 40,48

obf $I 1,16,40,36$

Ergo $\doteq+12+m=6,2 m-5,5 i m 30,68 y$

Hh 3

ร. 289. 
5. 289. Quintae eclipfis medium erat:

Parifis A. 1719 Aug. $29^{d}, 8^{b}, 33^{\prime}, 19^{\prime \prime}$ temp. med, Pro quo tempore colligitur:

Longitudo folis vera $\theta=5,5,47,14$

Anomalia folis vera $s=I, 27,25,24$

Longitudo lunae media - II, 2, 9, $4^{\circ}$

Anomalia lunae media . . 10, 15, 59, 25

Arlomalia lunae vera $\nu=10,20,5,19$

Longitudo nodi vera $\pi=4,27,44,39$

Diftantia nodi a cole $=0,8,2,35$

Aequ pro loco lunae $\cdots-\quad 4,22$

Long. lunae obferuata . . I1, 5, 42,52

6. 290. Calculus ergo ita fe habebit:

$$
\begin{aligned}
& v=10,20,5,19 ; \text { fin }=- \text { fin } 39,54,41 \\
& 2 v=9,10,11 \quad ; \text { fin } 2 v=- \text { fin } 79,49 \\
& v=10,20, s \\
& s=x, 27,25 \\
& v-s=8,22,40 \\
& \operatorname{col}=+ \\
& \text {; fin }=+ \text { fin } 57,25 \\
& \text {; fin }=- \text { fin } 82,40 \\
& v+s=0,17,30 \\
& \text {; } \text { in }=+ \text { fin } 17,30 \\
& \varphi-\pi=0,7,58 \\
& 2 \varphi-2 \pi=0,15,16 \\
& r=10,20,5 \\
& \text { : } f \text { in }=t \text { fin } 15,56 \\
& \text {; } \text { in }=+ \text { fin } 55,51 \\
& 2 \varphi-2 \pi-2 r=3,5,46 \quad ; \text { fin }=+ \text { fin } 84,14
\end{aligned}
$$

\begin{tabular}{|c|c|c|c|c|c|}
\hline & $\begin{array}{r}9,80726 \\
4,24753 \\
\end{array}$ & $\overline{-}$ & $\begin{array}{l}9,8073 \\
1,5111 \\
\end{array}$ & \pm & $\begin{array}{r}9,8849 \\
0,7104 \\
\end{array}$ \\
\hline & 4,05479 & $t$ & 1,31847 & - & $0,5953^{i}$ \\
\hline & $\begin{array}{l}9,9931 \\
2,8561 \\
\end{array}$ & $\overline{-}$ & $\begin{array}{r}9,993 \mathrm{I} \\
0,4208 \\
\end{array}$ & \pm & $\begin{array}{r}9,2475 \\
9,6201 \\
\end{array}$ \\
\hline & $2,849^{2}$ & $t$ & 0,4139 & - & $89.867^{6 i}$ \\
\hline & $\begin{array}{r}9,9256 \\
2,8819 \\
\end{array}$ & - & $\begin{array}{r}9,9964 \\
x, 1761 \\
\end{array}$ & \pm & $\begin{array}{l}9,4781 \\
2,2122 \\
\end{array}$ \\
\hline & 2,8075 & $t$ & 1,1725 & $\div$ & 1,6903 \\
\hline & $\begin{array}{r}9,4386 \\
2,7896 \\
\end{array}$ & $\begin{array}{l}t \\
t \\
\end{array}$ & $\begin{array}{l}9,9178 \\
2,2355 \\
\end{array}$ & $t$ & 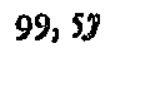 \\
\hline & 2,2282 & . & 2,1533 & & \\
\hline
\end{tabular}

CAPUT XYII.

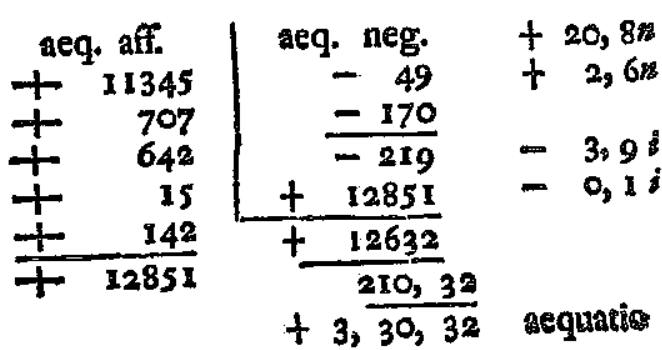

Long. lunae med. $11,2,9,40$

aeq. $\quad+3,30,32$

Long, lunae vera $11,5,40,12$ obf 
§. 29I. Sextae eclipfis medium crat

Parifis A. 1722. Jun. $28^{d}, 13^{b}, 58^{\prime}, 4 x^{\prime \prime}$ temp. med.

Pro quo tempore habetur:

Longitudo folis vera $=3,6,31,7$

Anomalia folis vera $=11,28,26,56$

Longitudo lunae media . 9, 9, 31, 50

Anomalia lunae media $.4,28,8,18$

Anomalia lunae vera $v=4,24,39,53$

Longiaudo nodi vera $x=3,2,36,2$

Diftantia nodi a fole . . $0,4,15,5$

Aequatio loci lunae $\cdots \quad$ 2, 20

Longitudo lunae abferuata $9,6,48,47$

6. 292. Calculus engo ita fe habebit

$$
\begin{aligned}
& v=4,24,39,53 \text {; fin } v=+ \text { fin } 35^{\circ}, 20^{\prime}, 7^{\prime \prime} \\
& \operatorname{col} v=- \\
& 2 v=9,19,20 ; \text { fin } 2 v=- \text { fin } 70,40 \\
& \operatorname{col} 2 v=4 \\
& r=4,24,40 \\
& s=\mathrm{Ir}, 28,27 \\
& \text { r.s }=4,26,13 \\
& r+s=4,23,7
\end{aligned}
$$

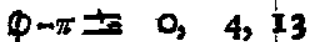

$$
\begin{aligned}
& 2 \varphi-2 \pi=0,8,26 \\
& r=4,24,40 \\
& 2 \phi-2 \pi-r=7,13,46 \\
& 2 \varphi-2 \pi-2 r=2, x 9,6
\end{aligned}
$$

$C \& U T$ ZPH.
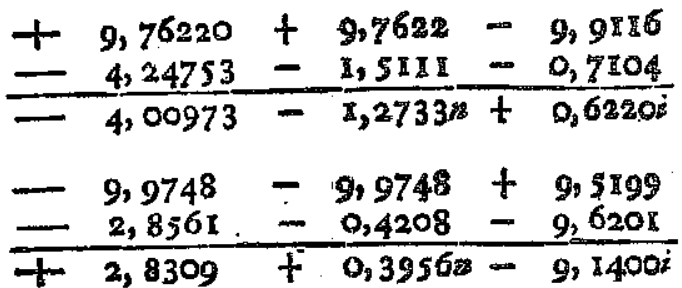

$\begin{array}{r} \pm, 4321+9,7451 \pm 9,7783 \\ \pm \quad 2,8819-1,8761-2,2122 \\ \hline 1,3140-0,9212-1,9909\end{array}$

$+9,1663-9,8399+98,27$

$=2,7896+2,2355$

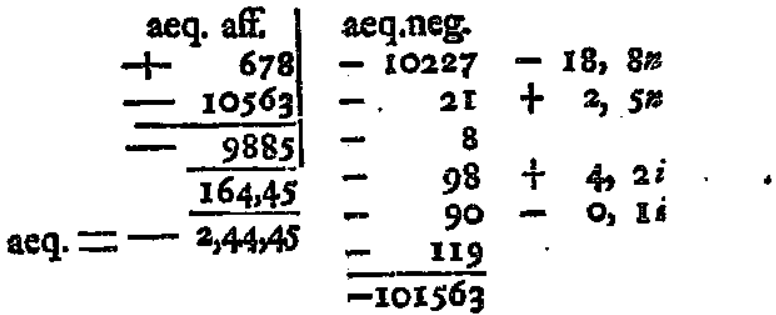

Long. lun. med. 9, 9, 31, 50

Long. lun. $\quad \frac{-2,44,45}{9,6,47,5}$

obf. $\quad 9,6,48,47$

Ergo $0=-1,42+m-16,3 z+4,1 i+98,9 y$

Ii

\$. 293. 
8. 293. Septimase eclipfis medium obferuatum eft Parifiis A. 1724 . OA. $31^{\prime}, 15^{b}, 34^{\prime}, 17^{\prime \prime}$. temp. med. Pro quo tempore colligitur

Longieado folis vera $=7,8,56$, I

Anomalia folis vera $s=4,0,29,44$

Longitudo lunae media

Anomalia lunee medis I, 9, 23, 59

Anomalia lunae vera $v=5,21,46,51$

Longitudo nodi vera

$1,16,36,22$

Diftantia nodi a fole $5,22,19,39$

aequatio loci lunae

$\frac{+4,10}{1,9,0,11}$

5. 294. Calculus ergo ita ineatur:

$v=5,21,46,51$; fin $v=+$ fin $8^{\circ}, 13^{\prime}, 9^{\prime \prime}$

$2 v=11,13,34$ cor $=-$

$3^{\prime}, 9^{\prime \prime}$

$r=5,21,47$

$s=4,0,30$

$r-s=\mathrm{x}, 21, \mathrm{17}$

$r+s=9,22,17$

$\varphi-\pi=5,22,24$

$2 \varphi-2 \pi=11,14,48$

$r=5,21,47$

$29-2 \pi-r=5,23,1$

$29-2 \pi-2 r=0, \mathrm{i}, 24$
; fin2v $=-$ fin $16^{\circ}, 26^{\prime}$

cor $=t$

$;$ fins $=+\operatorname{fin} 59^{\circ}, 301$

; fin $=+$ fin 5,17

; fin $=-$ fin 67,43

; fin $=-$ fin 15,12

; fin $=+$ fin 6,59

ifin $=+$ in 1,14 n eft

ed.

261

301

17

13

12

19
CAPUT XVII.

$\begin{array}{r}+9,75520+9,3552-9,9955 \\ +\quad 4,24753-1,5116-0,7504 \\ \hline 3,40273-0,666328+0,70598\end{array}$

\begin{tabular}{l}
$-9,4516-9,4516+9,9819$ \\
$=\quad 2,8561-0,4^{208}-9,6201$ \\
\hline$+2,3077+9,87242-9,60203$
\end{tabular}

$+9,9353+9,8922-9,9663$

$+2,8819-1,1761-2,2122$
$+2,881,0683+2,1785$

$-9,4186+9,0849+2,15 y$

$\mp \quad 2,7896$
$+2,2082$
$+1,3024$

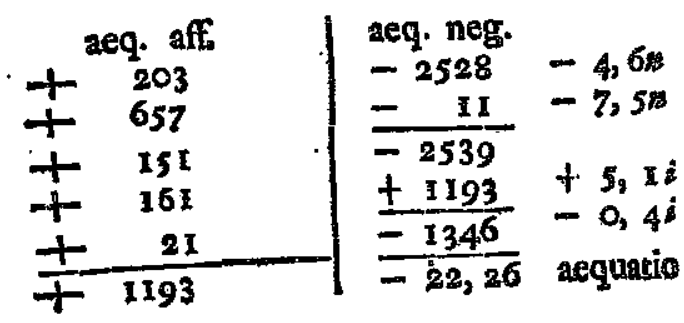

Long. 2 med. $1,9,23,59$

Long. calc. $1,9,1,33$.

Long. obC 1, 9, 0, II $0=+1,22+8 x-3,9 n+4,7 i+2,15 y$. 
§. 295. Oatauae eclipfis medium obferuatum eft Parifis A. 1729. Febr. $13^{d}, 9^{b}, 61,56^{\prime \prime}$ temp. med. Pro quo tempore colligitur :

Longitudo folis vera $\theta=10^{\prime}, 25^{\circ}, 13^{\prime}, 23^{\prime \prime}$ Anomalia folis vere $s=7,16,43,34$ Longitudo Iunae media . $5,0,5,27$ Anomalia lunae media $\cdot 3,18,53,24$ Anomalis lunae vera $v=3,12 ; 54 ; 9$ Longieudo nodi vera $\pi=10,24,4,30$ Diftantia nodi a fole $=0,1,8,53$ aequatio pro long. lunae . Longitudo lunae obferuata $\frac{-0,37}{4,25,12,46}$

§. 296. Calculus ergo ita fe habebit:

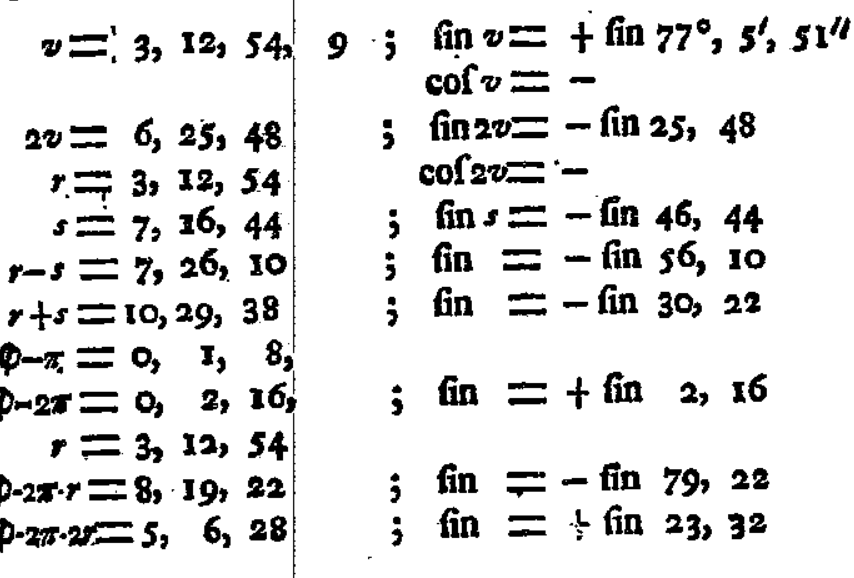

CAPUT XVII.

8)

Long. lunae medis $=5,0,5,27$

aeq.

Long, lunae calc. Long. lunae obr.

$$
\begin{aligned}
& -4,52,57 \\
& \hline 4,25,12,30 \\
& 4,25,12,46
\end{aligned}
$$

$=-16+m-30,6 n+1,4 \hat{b}+39,9 y$ 
CAPUT XYII

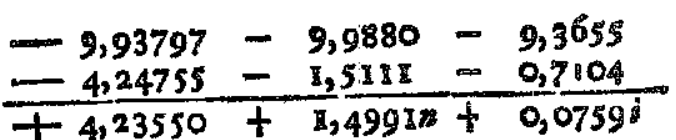

$+9,6548+9,6548-9,9505$

$\frac{ \pm 2,8561-0,4208-9,6201}{-2,5109-0,0756 \%+9,57068}$

$=4,16,17,29$

Anomalia folis yera $s=1,7,47,12$

Longitudo lunae media $10,11,23,57$

Anomalia lunae media $8,10,36,19$

Anomalia lunae vera $v=8,16,34,40$

Longitudo nodi vera $\pi=10,24,58,21$

Diftantia-nodi a fole $6,7,19,{ }^{\prime} 8$

Aequatio pro loco lunae

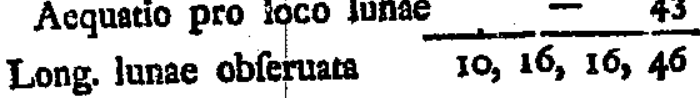

\$. 298. Calculus ergo in inftituetur:

$v=8,16,34,40 ;$ fin $v=m$ fin $76,34,40$ $\cot v=-$

$2 v=5,3,9 ; \operatorname{fin} 2 v=+\operatorname{fin} 26,51$ $\operatorname{cof} 2 v=-$

$r=8,16,35$

$s=I, 7,47 \quad ; \operatorname{fin} s=+\operatorname{fin} 37,47$

$r-s=7,8,48$; fin $=-\operatorname{rin} 38,48$

$r+s=9,24,22^{\circ}$; fin $=-$ fin 65, 38

$9-\pi=6,1,19$

$2 \varphi-2 \pi=0,2,38$; fin $=+$ fin 2,38

$r=8,16,35$

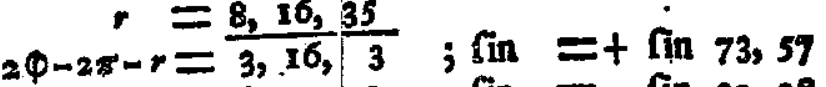

$20-2 \pi-2 r=6,29,28 \quad$; fin $=-$ fin 29,28
$+9.7872-9,7970-9,9595$

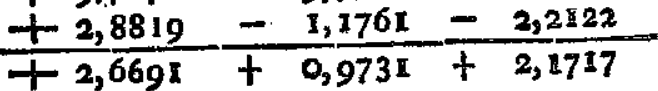

$=2,7896+2,2355$

\begin{tabular}{|c|c|c|}
\hline $\begin{array}{r}\text { aeq.aff. } \\
+\quad 17199 \\
+\quad 467\end{array}$ & $\begin{array}{r}\text { aeg. neg. } \\
-\quad 324 \\
-\quad 28 \\
\end{array}$ & $\begin{array}{l}+31,6 \% \\
-\quad 1,28\end{array}$ \\
\hline $\begin{array}{r}9 \\
148\end{array}$ & $\begin{array}{r}352 \\
+\quad 17988 \\
\end{array}$ & +0, \\
\hline+17988 & 293,56 & \\
\hline
\end{tabular}

Long. 7 med. $=10,11,23,57$ seq. $+\quad 4,53,56$

Long. 7 calc. $=10,16,17,53$ Long. obch
$+8,6622+9,9827-49,2 y$.

$$
1=+1,7+m+30,4+1,68 \times 49,28
$$

ริ. $29 \%$ 
§. 299. Decimae eclipis medium obferuatum eft

Parifis A. 1731. Jun. 19, $13^{b}, 55^{\prime}, 13^{\prime \prime}$, semp. med.

Pro quo tempore colligitur

Longitudo folis vera $\theta=2^{\prime}, 28^{\circ}, 5^{\prime}, 41^{\prime \prime}$

Anomalia folis vera $s=. \mathrm{II}, 19,48,47$

Longitudo lunae media . 9, 1, 45, 1

Anomalia lunse media . .4, 15, 9,43

Anomalia lunse vera $v=4,10,34,28$

Longirudo nodi vera $x=9,8,6,38$

Diftantia nodi a fole : $5,5,19,59,3$

Aequatio pro loco lunae $\cdot \frac{t}{8,28,11,5}$

5. 300. Calculus engo ita initituetur

$$
\begin{aligned}
& v=4,10,34,21 \text {; fin } v=+ \text { fin } 49,25,39 \\
& \operatorname{col} v=- \\
& 2 v=8,21,9 \quad \text {; fin } 2 v=- \text { in } 8 \mathrm{r}, 9 \\
& r=4,10,34 \\
& s=11,19,49 \\
& \operatorname{col} 2 x= \\
& r \cdots s=4,20,45 \quad \text {; fin }=+ \text { fin } 39,15 \\
& r+s=4,0,23 \quad ; \text { fin }=+ \text { fin } 59,37 \\
& \phi-\pi=5,20,4 \\
& { }_{2 \varphi-2 \pi}=11,10,8 \\
& \dot{r}=4,10,34 \\
& \text {; fin }=- \text { fin } 29,52 \\
& \text {; fin }=- \text { fin } 29,34 \\
& 2 \varphi-2 \pi-r=6,29,34 \\
& \text {; } \text { fin }=+ \text { fin } 79,0
\end{aligned}
$$

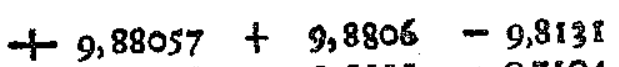


\$. 301. Eclipfis undecimae medium obferuatum eft Parifiis A. 1732 Dec. $x^{\prime}, 9^{b}, 48^{\prime}, 23^{\prime \prime}$ temp. med. Pro quo tempore colligitur:

Longitudo folis vera $\theta=8,10,3,6$ Anomalia folis vera $s=5,1,29,50$

Longitudo lunae media . 2, 6, 8, 19

Anomalia lunae media . : $7,19,24,12$

Anomalia lunae vera $v=7,24,19,39$

Longitudo nodi vera $\pi=8,10,41,14$

Diftantia nodi a fole $==11,29,21,52$

Aequ. pro loco lunae * $\frac{+\quad 2 I}{2,10,3,27}$

5. 302. Calculus ergo ita fe habebit:

$$
\begin{aligned}
& v=7,24,19,39 ; \text { fin } v=- \text { in } 54,19,39 \\
& 2 v=3,18,39 \quad ; \text { fin } 2 v=+ \text { fin } 7 x, 21 \\
& r=7,24,20 \\
& s=5,1,30 \\
& r-s=2,22,50 \\
& r+s=0,25,50 \\
& \varphi-\pi=11,29,22
\end{aligned}
$$

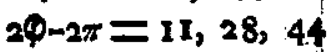

$$
\begin{aligned}
& r=7,24,40 \\
& 2 \varphi-2 \pi-r=4,4,4 \\
& 2 \varphi-2 \pi-2 r=8,9,24
\end{aligned}
$$

um eft
CAPUT XYII.

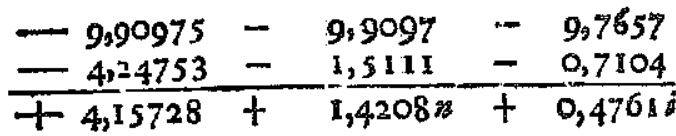

\begin{tabular}{l}
$+9,9766+9,9766-9,5048$ \\
$=2,8561-0,4208-9,6201$ \\
\hline \hline $2,8327-0,39748+9,1249$
\end{tabular}

$\begin{array}{r}+9,6787+9,9969+9,6444 \\ +2,8819-1,1761-2,2122 \\ \hline+2,5606-1,1730-1,8506\end{array}$

$\begin{array}{r}-8,3445+2,9182 \\ -2,7896+2,2355 \\ \hline+1,1341+93,6 y\end{array}$

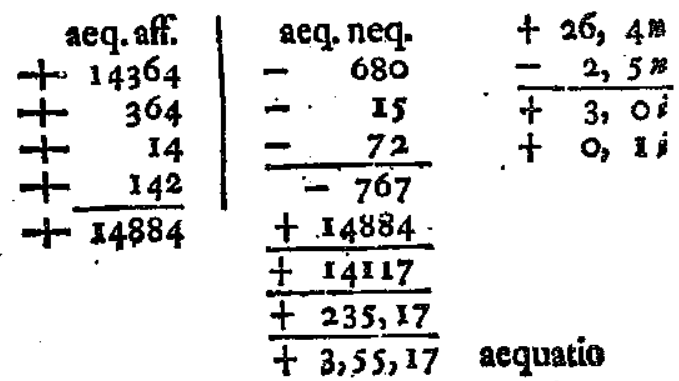

Long. lunse media $\quad 2,6,8$, 19

Long. lunae cale. $+\frac{3,55,-17}{2,10,3,36}$

obl.

. $\frac{2,10,3,27}{+9+m+23,9 n+3,18-93,6 y}$

KK 2

6. 303 

Pariliis A. 1736 Mart. $26^{d}, 12^{b}, 14^{\prime}, 3^{\prime \prime}$ temp. med.

sum eq med. Pro quo tempore colligitur

Longitudo folis vera $\theta=0^{\prime}, 6^{\circ}, 35^{\prime}, 4^{\prime \prime}$

Anomalia folis vera $s=8,27,58,24$

Longitudo lunae media

Anomalia lunae media $6,-4,5,0$

$7,3,25,43$

Anomalia lunae vera $v=7,7,2,56$

Longitudo nodi vera $\pi=6,6,24,31$

Diftantia nodi a fole 6,0, II, II

aeq. pro long. lunae

Lopgitudo lunae obf.

$\frac{6}{6,6,35,36}$

§. 304. Calculus ergo ita infituatur.

$v=7,7,2,56$; fin $v=-$ fin $57,2,56$ $\operatorname{col}=-$

$2 v=2,14,6 ; \operatorname{fin} 2 v=+\operatorname{fin} 74,6$ $\operatorname{cor}=t$

$r=7,7,3$

$s=8,27,58$.

$r-s=10,925$

; fin $s=-$ fin 87,58

; fin $=-$ fin 50,55

$r+s=4,5, i$ ifin $=+$ fin 54,59

$\varphi-\pi=6,0,11$

$2 \varphi_{-2 \pi}=0,0,22$; fin $=+$ fin 0,22

$r=7,7,3$

$2 \varphi-2 \pi-r=4,23 ; 19$; fin $=$ fin 36,42

$20-2 \pi-2 \pi=9,16,16$

; fin $=-$ fin 73,44

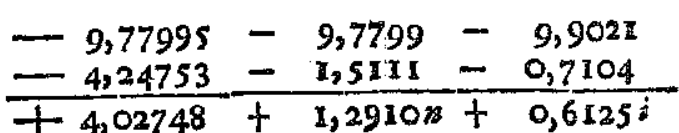

$+9,9831+9,9831+9,4377$

$\frac{-2,8561-0,4208-0,6201}{-2,8392}=\frac{9,03}{9,05786}$

$\begin{array}{r}-9,9997-9,8900+9,9133 \\ +2,8819-1,1761-2,2122 \\ \hline 2,8816+1,0661-2,1255\end{array}$

$+7,8061+9,7763-96$, oy

$=2,7896+2,2355$

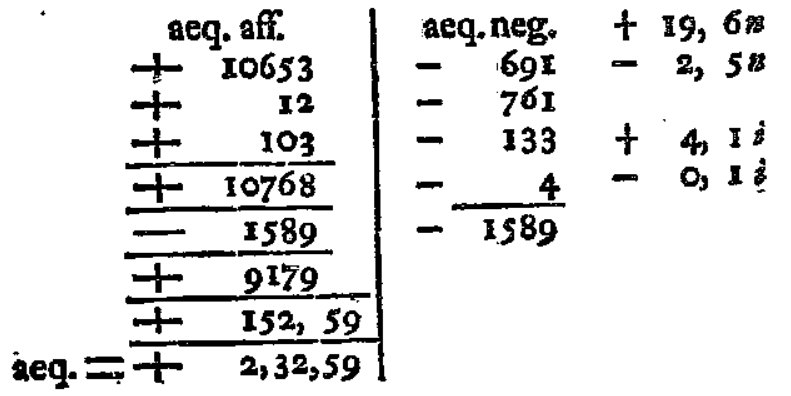

Long. 6 med. $6,4,5,0$

aeq. $+2,32,59$

Long. $D$ calc. $\overline{6,6,37,59}$

obf: $6,6,35,36$

- $=+23^{\prime}, 23^{\prime \prime}+m+17,1 n+4,0 i m 96,09$ 
§. 305. Elipfis decima tertiae medium obferúatum eft

atum eft med.

Pro guo tempore colligitur

Longitudo folis vera $\dot{\theta}=55^{\prime}, 27^{\circ}, 21^{\prime}, 39^{\prime \prime}$, Anomalia folis vera $s=21,18,43,5 \mathrm{I}$

Longirudo lunae media . . II, 27, 48, 53

Anomalia lunae media .. $0,7,25,42$

Anomalia lunae vera $v=0,6,40,44$.

Longitudo nodi vera

$5,27,15,4$

Difaniag nodi a fole $\cdot 0,-0,6,35$

aeq. pro long. lunae

Longitudo lunae obferuata $I I, 27,2 I, 35$

5. 306 . Calculus ergo ita inftituetur

$$
\begin{aligned}
v=0,6,40,44 ; \text { fin } v & =+ \text { fin } 6 ; 40,50 \\
\text { cor } v & =+
\end{aligned}
$$

$2 v=0,13,2$

; fin2v $=t$ fin $13,3 x$ $\operatorname{cof} 20=+$

$r=0,6,4 x$

$s=2,18,44$ :

$r-s=9,17,57$

$r+s=2,25,25$

$\varphi-\pi=0,0,6$

$2 \varphi-2 \pi=0,0,12$ $r=0,6,41$

; fin $s=+$ fin 88,44

; fin $=-$ fin 72, 3

; fin $=+$ fin 85,25

; fin $=+$ fin 0,12

; fin $=-$ fin 6,29

; fin $=-$ fin 13 , 10 $2 \varphi-2 \pi-2 r=I I, I 6 ; 50$
$+9,06561+9,0656+9,9970$

$=4,24753-1,5111=0,7104$

$\begin{array}{r}+9,3634 \pm 9,3634+9,9881 \\ =2,8561-0,4208-9,6201 \\ \hline 2,2195-9,78423-9,60828\end{array}$

$\begin{array}{r}+9,9915-9,9783+9,9986 \\ +2,8819-1,1751-2,2122 \\ \hline+2,873.4+1,1534-2,2108\end{array}$

$+7,5429-9,0527-22,6 y$

$=2,7896+2,2355$

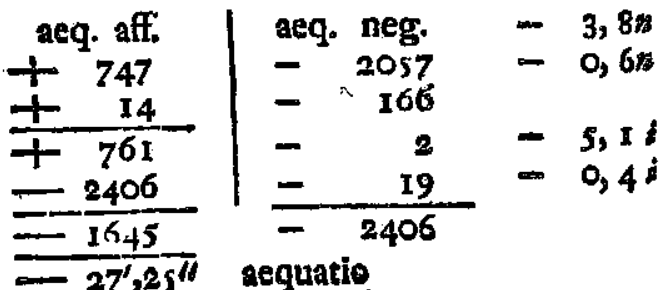

Long. 2 med. II, 27, 48, 53

aeq.

Long. $D$ calc. $11,27,21,28$

Long. 3 .obC

Ix, 27, 2x, 35

$=0,7^{d 1+m}-4,43+5,5 i-22,6 y$ 
\$. 307. Ex his ergo tredecim eclipfibus nacti fu-

racti firquibus onis ab mus aequationes, ex quibus cum tabularum, quibus fum vfus, correctiones, tum verus valor aequationis ab angulo $20-2 \pi-2 r$ pendentis definiri debebit:

Aequaciones autem inde ortae funt fequentes.
I. $=+156^{\prime \prime}+m-29,1 n-2,8 i-99,89$
II. $0=-27+p+32,4 n-1,3 i+79,2 j$
III. $0=+30+m+32,4 n+0,2 i+25,6 y$
IV. $0=+12+m-6,2 n-5,5 i-30,7 y$
V. $=-160+m+23,4 n-4,0 i+99,59$
vl. $\cdot=-102+m-16,3 n+4,1 i+98,2 y$
VII. $=t 82+n-3,9 n+4,7 i+2,1 y$
VIII. $=-16+m-30,6 n+1,4 i+39,93$
IX. $0=+67+m+30,4 z+1,6 i-49,27$
X. $\cdot=-47+m-22,2 n+3,4 j-98,19$
XI. $:=+9+m+23,9 n+3,1 i-93,6 y$
XII. $\cdot=+\mathrm{r}_{43}+m+\mathrm{r}, \mathrm{I} n+4,0 i-96,0 y$

XIIl. $:=-7+m-4,4 n-5,5 i-22,6 y$
5. 308. Hic fratim commode enenit, ve errores calculi ab obferuationibus infra tria minute prima fubfiftant, qui autem infra resquiminutum primum deprimuntur, fimul ac liecerae $y$ valor tribuitus vnitati fere aequalis. Hincqiae ergo cognolcimus va. lorem ipfius $y$, guem quinario maiorem inueneramus, merito nobis fuiffe lufpeetum, cum iam perfpiciamus, eum vnitatem fuperare non poffe. Quamobrem pona. mus $y=1$, feu in formula noftra pro longitudine lunae feribamus terminum Ioo"l fin $(2 \varphi-27-2 r)$. Quod autem ad litteras $m, n$ et $;$ attinet, tentanti mox pate. bit, quoscunque ipfis valores tribuamus, errores inde non admodum poffe diminui; interim tamen decem circiter minutis fecundis diminuentur, fi ponatur $y=\frac{4}{5}$; $n=\frac{x}{2} ; i=-3$ et $m=-4 ;$ quo facto errores vis vnum minutum primum fuperabunt.

1 
I RUM

\section{CONSTITUTIO ELEMENTORUM PR.O TABULIS LUNARIBUS.}

\$. 309.

Tabulae autem, guibus in praecor $:$ calculo fum vfus, praebent pro meridianc "arifin. ad epocham i'or feu ad meridiem diei v.simi anni 3700 tempore medio

Longitudinem Lunae mediam $5^{\prime}, 20^{\circ}, 19^{\prime}, 47^{\prime \prime}$

et Anomaliam Lunae mediam $6,13,26,51$

Hinc accuratius habebimus haec elementa pro eodem tempore eodemeque loco feilicet

Longitudinem Lunae mediam $5^{\prime}, 20^{\circ}, 19^{\prime}, 43^{\prime \prime}$

Anomalian Lunse mediam $6,13,24,0$

unde Longirado Apogei $11,6,55,43$

6. 310. Si haec elementa comparemus cum Tabulis aftronomicis Cel. Caffini et Monnierii, reperiemus pro eodem tempore et loco

\begin{tabular}{l|l|l} 
Long. mediam Lunae & $5,20,18,19$ & $5,20,19,28$ \\
Anom. mediam Lunae & $6,13,10,48$ & $6,13,13,2$
\end{tabular} \begin{tabular}{l|r|r} 
Anom. mediam Lunae & $6,13,10,48$ \\
Long. Apogei & $6,13,13,2$ \\
$11,7,7,27$ & $11,7,6,26$
\end{tabular}

Hic quidem longizudo media fatis convenit cum ea, quam ex obferuationibus conclufimus; verum anomalia media inuenta fuperat Caffinianam 13', 12", Monnierianam aurem $3 I^{\prime}$, quoci difcrimen fatis eft notabile. culo fum

ad epo-

imi anni

, $47 !$.

, 51

, codem

', 43 '

, 0

, 43

Tabulis

nus pro

nier:

19,28

13,2

6,26

um eq,

nomalia

Uonnie.

cotabile.

Verum

Verum fi perpendamus motum lunae a tam multis va. riisque inaequalitatibus perturbari, mirum fâne non en, anomaliam mediam per folas obferuationes accuratius definiri non potuiffe; praefertim cum error $15^{\prime}$ in ano. malia media commifus in loco lunae ad fummum extorem $1^{\prime}, 45^{\prime \prime}$ gignere valeat.

\$. 31 1. Excentricitatem autem orbitae lluarais,

quam ftatueram $=0,0545$ iam $\frac{\pi}{0} \frac{x}{0} 00$ vel 0,00005 augeri oportet, ita vt nunc fir excentricitatis valos $k=0,05455 ;$ qui a fupra affumto tam parum difcre. par, ve anomalia vera inde ex media collecta pro facis exacta haberi poffic: aequationes aucem ab excentricitate pendentes aliquod augmentum capient, quod nuac quidem diligentius definiri oportet. Primum ergo fogmulam pro longitudine lunae inuentam bine corriga mus; deinde vero etiam formulas pro diftancia lunae a terra, pro eius motu momentaneo, et pro loco nodi veraque inclinatione orbitar lunaris ad eclipticam hing euoluamus,

5. 312. Ante omnia autem oportebit formulam exhibere. cuius ope ex data quauis anomalia lunae media $p$ elicere licear, conuenientem anomaliam veram . Ac fubltituto quidem pro $k$ vero eius valore nune in vento, coefficientibusque in minuta fecunda conuerfis, formula fupra (\}. 306) exhibita fequentem induet fornam:

$$
\begin{aligned}
& r=p-22495^{\prime \prime} \text { fin } p+766^{\prime \prime} \text { fin } 2 p-36^{\prime \prime} \text { fin } 3 p \\
& 4,3.52086 \quad 2,884229 \quad \square, 55630 \\
& \text { Ll } 2 \text { Huivs }
\end{aligned}
$$

Verum 
Huius ergo formulae ope haud diffirulter tabula com. putabitur, quae ad fingulos anomaliae mediae gradus exhibeat valores anomaliae verae.

§. 3I3. Inuenta autem anomalia vera $r$, fi habea: tur quoque anomalia vera folis $s$, vna cum angulo et longitudinibus $\phi, \theta, \pi$ faltem proxime, formula longitudinem veram $\phi$ datae mediae $\xi$ refpondentem exhibens, fequenti modo habebitur expreffa:

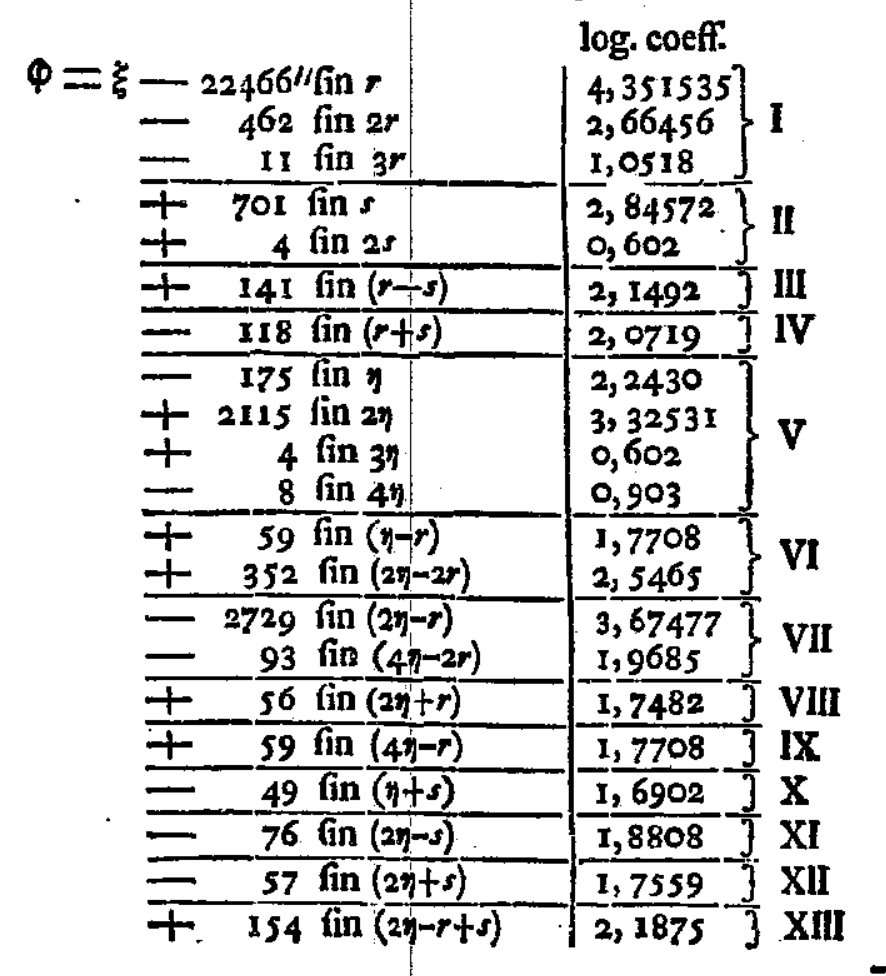

ula com-

\& gradus

Gi habea: ingulo $n$ ula lonzm exhi1

II
$C A P U T X I I I$. 869

\begin{tabular}{|c|c|c|}
\hline+ & 45 fin $(29-r m s)$ & 1,6532 \\
\hline & $41 \times$ fin $(2 \varphi-2 \pi)$ & $2,6 \longdiv { 3 8 }$ \\
\hline & $\begin{array}{r}205 \text { fin }(2 \theta-2 \pi) \\
6 \text { fin }\left(4^{\theta}-4 \pi\right)\end{array}$ & $\begin{array}{l}2,3117 \\
0,778\end{array}$ \\
\hline+ & 187 fin $(2 \varphi-2 \pi-r)$ & 2,2718 \\
\hline 7 & 80 fin $(2 \varphi-2 \pi-2 r)$ & 1,9031 \\
\hline & I5 fin $(2 \theta-2 \pi 5-r)$ & $I, 176$ \\
\hline & 10. in $(2 \theta-2 \pi+r)$ & $I_{2}, 000$ \\
\hline
\end{tabular}

§. 3 I 4. Inaequalitates has ita difpofui, vi eas, quae vna tabula comprehendi poffunt, coniunctim expofaerim, quo facilius calculus expediri queat. Hinc jgitur patet omiffis iis inaequalitatibus, quae 10 " non fuperant, locum lunae per viginti inaequalitates $\cos ^{\text {- }}$ rigi debere, antequam vera eius longitudo obtineatur.

5. 3 I5. Haec autem expreffio adhue ifto defeetus laborat, quod pleraegue inaequalitates ipfam lunae longitudinem veram $\varphi$, quae tamen demum quaeritur, in voluant, ideoque calculus, cum longicudo lunae etiamnunc eft incognita, commode expediri non poffit. Quoniam tamen fufficit longitudinem lunae proxime tantum nofle, cum longitudo media per quatuor priores inaequalitates fuerit correcta, ea pro requentibus inaequalitatibus loco longitudinis verae vfurpari poterit ficque tandem longitudo lunae multo exactior reperietur. Quo falto fi accuratior defideretur, omnes in2equalitates poft 4 priores denuo ad calculum revocari conueniet, isque euoluxis longitudo lunae vera prodibit, quae nulla amplius correctione indigebit. Interim L1 3 เameti 
tamen ne calculum per fe fatis taediofum bis repetere opus fit, non difficulter hanc expreffionem ita transformare licet, vt locus lunae per quatuor tantum priores inaequalitates correctus fine errore in fequentibus loco Q adhiberi poffit.

§. 3 I 5. Cum autem longindo lunae iam per obferuationes faerit cognita, haec expreffo fine vlla immutatione ad calculum accommodabitur, ve hoc modo confenfus theoriae cum veritate exploretur. In inaequalitatibus enim determinandis pro littera $\phi$ vbique longitudo lunae obferuata introducetur, calculoque per-. acto parebit, quantum locus lunae per calculum definitus etiatnnunc difcrepet ab eius loco vero obferuato. Atgue fi hoc modo plurimae obferuationes calculo fubiiciantur, ex aberrationibus a veritate non folum ele. - menta, quibus haec formula innicitur, accuratius definire licebir, fed etiam inaequalitates, quae nondum fatis cerrae videntur, inde emendari poterunt. Quin etiam nouae inaequalitates, quas per Theoriam determinare non licuerat, hoc modo forte cerrius colligi poterunt.

\$. 317. Antequam autem huiusmodi calculi fpecimen exhiberi queat, neceffe eft ve aequationem pro loco nodi vero inueniendo ad calculum accommodemus. Formulae autem fupra ( 2 I 9 ) exhibitae, fi pro $r$ fubtituamus valorem inuentum $r=p-2 k$ fin $r$ $-\frac{3}{4} k k$ fin $2 r$, pars: Conft $-0,004053 p$ indicabit longikudinem nodi medjam. Hincque longitudo nodi vera erit repetere transfor. priores us loco

per ob-

illa im-

c modo

in inae-

vbique

ue per.

im defi-

feruato.

lo fub-

In ele.

us defi-

ium fa-

Quin

deter-

colligi

uli fpetionem ommo:

, fi pro

fin $r$

dicabit:

o nodi

$\pi$.
Log.coeffo

\begin{tabular}{|c|c|c|}
\hline & 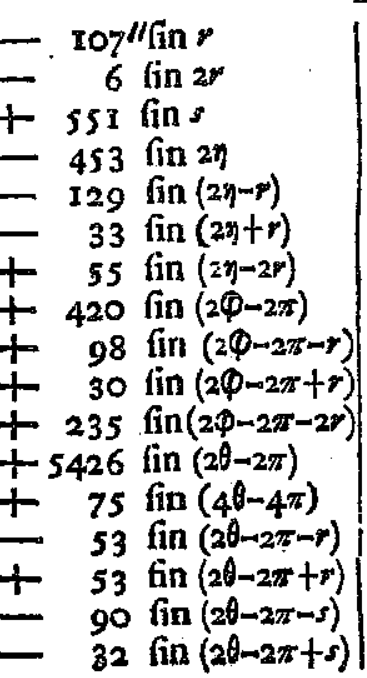 & $\begin{array}{l}2,0294 \\
0,778 \\
2,741 \mathrm{I} \\
2,656 \mathrm{I} \\
2,1106 \\
1,518 \\
1,740 \\
2,6232 \\
1,991 \\
1,477 \\
2,3711 \\
3,73448 \\
1,875 \\
1,724 \\
1,724 \\
1,954 \\
1,505\end{array}$ \\
\hline
\end{tabular}

6. 318. In hoc calculo plerasque inaequalicares omittere licet, fiquidem tantum longitudinem lunae in. veftigare fit propofitum: manifeftum cnim eft, esiamif in loco nodi error plurium minutorum primorum combmittatur, inde vix errorem aliquot minutorum fecus: dorum in longitudinem lunae redundare. Quodfi vero eclipfis cuiuspiam omnia phaenomena diligenter deffo nire velimus, tum locum nodi exactir $T_{\text {? }}$ le cognitum effe oportec. Praeterea vero pro latitudine affignanda vera inclinatio orbitae lunaris ad eclipeicam ex media s accuratifime exit definienda ope huius formulke: 


$$
\begin{aligned}
& 722^{\circ} \\
& \text { CAPUT XVII. }
\end{aligned}
$$

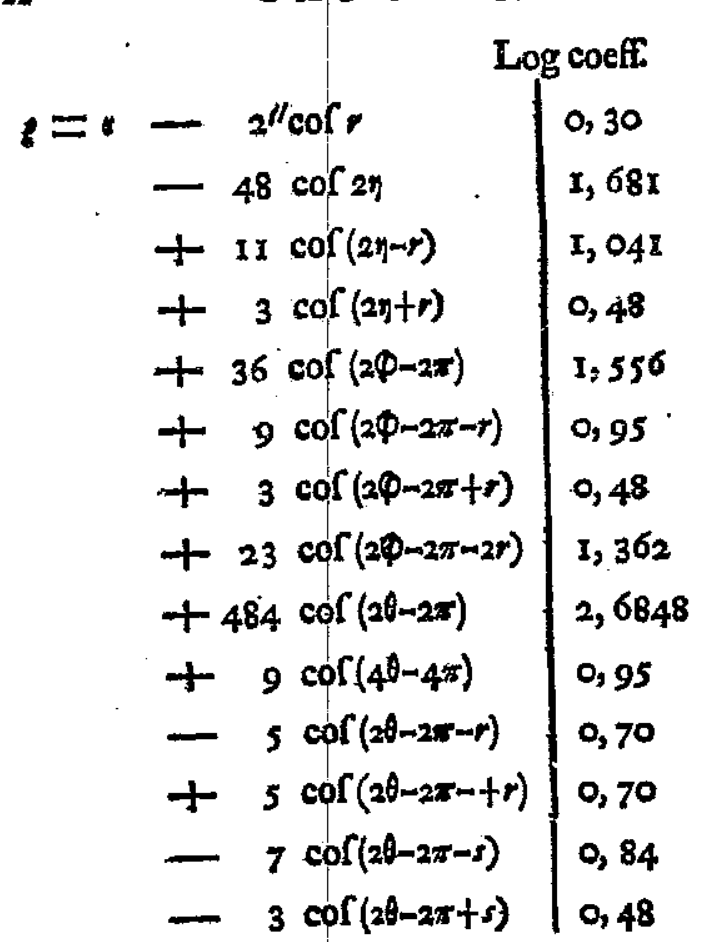

Tabula autem pro diftantia lunae a terra, vnde eius parallaxis et diameter apparens definianr, ex formulis fupra exhibitis facile conftruecur.

\section{茞 (0) 筑 \\ ADDITAMENTUM
CONTINENS ALIAS METHODOS
INUESTIGANDI MOTUS LUNAE
INAEQUALITATES.

243

$\mathrm{Q}$ ui methodum ante defcriptam azcuratius euolueri, earn quidem in fe fpectatam fatis bonam asque plexisque lunae inaequalitatibus deciniendis aptam deprehendet; interim tamen fateri cogor, eam non folum maxime effe operofam, fed etiam ita comparalam, ve plures inaequalitates, quae tamen motum lunae imprimis afficere videntur, non latis exacte exhibear, et quafi in dubio relinquat. Caufa huius incertitudinis ma. nifefto in hoc eft fita, quod omnes inaequalitates ita inter fe funt connexae, vi nullius valor verus accurate definiri poffit, quin fimul reliquae innequalitates omnes fuerint cognitae. Cum igitur einsmodi methodo approximandi fim vius, ve primo guasdam inaequalitates tanquam cognitas affumferim, ex quibus deinceps reliquas

Ide eius formulis

D D 1 definiuerim, probe notandum eft ab his inuentis iterum priores, guae erant affumtre, leuem quandam mutacionem pati; quae fi ftatim ab inicio nota fuiffet, etiam reliquarum valures aliquantillum mutati prodiifent: at quaedam inaequalitates adeo funt lubricae, vt fact vel minima mutatione in is, a quibus pendent, inde non exiguam alterationem trahant. Huc imprimis pertinet mo- tus apogei, cuius inueftigatio omnes omnino inaequa. M m litates 
litates implicat, ita vt fine harum cognitione neutiquam accurate definiri queat.

Cum iginur haec methodus iftis tantis incommodis fir obnoxie, aliam maxime diuerfam tẹntaui viam, quae ab is effet libera, etiamfi negare nequeam, etiam hanc fuis non carere incommodis, quae ramen prorfus alius funt generis, Ex quo confido his duabus diuerfis methodis combinandis haud exiguum fructum in veram motuum lunarium cognitionem effe redundaturum. Prae* cipuum autem difcrimen verfatur in electione anomaliae, quae in fuperiore methodo non ita eft affumta, vt diftantia lunae a terra fierec vel maxima vel minima, fi anomalia vel $=0$ vel $=180^{\circ}$ ftatuatur : neque enim differentiale diftantiae $d x$ euanefcit, guando finus anomaliae in nihilum abit, fed praeterea etiamnunc ab elongatione folis a luna feu angulo $\eta$ pendet. Ita fecundum hanc methodum neque apogaeum lunae neque perigaeum ibi ftacuitur, ubi angulus, quem motus lunae direEio cum radio veetore facic, eft rętus; fed plerumque in alia punet incidunt, quae ab is locis, vibi luna terrae vel eft proxima, yel ab ea maxime remota, notabiliter fint diueria. Etfi autem in hoc calculo non verae lineae abfidum pofitio confideracur, hinc tamen methodus minime vitiola eft reputanda; propterea quod non eft quaetio, quo nomine quaepiam orbitae lunaris puncta appellentur, dummodo cunctae inzequalitates reCe exprimantur. Sed quoniam circa has ipfas inaequalitates nonnulla grauiora dubia funt orta, haud abs re fore arbitror, et alteram methodum hic proponere.
- Sit igitur vt ante : Maffa folis $=0$; terrae $=\frac{7}{6}$ et lunae $=D$; atque vis ateractiua terrae in diftantia $d v \mathrm{vc}$ $\frac{1}{d d}-\frac{1}{b b} ;$ manente vi folis quadrato diftantiae exalte proportionali. Tum vero fic

Longitudo lunae $=\varphi$; latitudo $=\psi$; et diftantia curtata $=x$

Longitudo folis $=\theta$; eiusque a terra diftancia $=y$ Longitudo nodi afcendentis lunae $\approx \pi$ et inclinatio ad eclipticam $=\rho$ ac ponatur breuitatis ergo elongatio lunae a fole $\varphi_{-} \theta=$ = et diftantia $V\left(x x\right.$ fec. $\left.\psi^{2}-2 x y \operatorname{cor} y+y y\right)=z$.

Quibus pofitis fupra S. 20: vidimus motum lunat his quatuor aequationibus contineri :

L $2 d x d \varphi+x d d \varphi=-\frac{x}{2} d t^{2} \cdot \circ\left(\frac{y}{e^{3}}-\frac{1}{y y^{2}}\right)$ fin $y$

Il. $d d * m * d \varphi^{2}=-\frac{x}{2} d i^{*}\left(\frac{x}{b}+2\right) \operatorname{col} \psi^{3}\left(\frac{1}{x x}-\frac{1}{b 6}\right)$

$-\frac{1}{2} d t^{*} \cdot O\left(\frac{x-y \operatorname{cor} \eta}{2^{3}}+\frac{\operatorname{cof} \pi}{y y}\right)$

III. $d \pi=-\frac{x}{3} d t^{2} \cdot \odot\left(\frac{y}{x^{3}}-\frac{x}{y y}\right) \frac{\sin (\varphi-\pi) \sin \left(\theta_{m} \pi\right)}{x d \phi}$

IV. $d$ tang $\rho=\frac{d \pi}{\text { tang }(\varphi-\pi)}$, et tang $\psi=\operatorname{tg} \rho \operatorname{cof}(\varphi-\pi)$ vbi elementum temporis $d t$ fumtum eft pro conftante.
$M m 2$
II. Qua- 
Quatenus hic motus folis ingreditur, is pro regilari atque regulis Kepleri conformi haberi poterit: ha. bebimus ergo

$$
2 d y d \theta+y d d \theta=0 . \text { et } d d y-y d \theta^{2}=-\frac{x}{2} d t^{2} \cdot \frac{\text { O+t }}{y y}
$$

vnde fi ponamus orbitae folaris:

femiparametrum $=i ;$ excentricitatem $=e$ et ano-

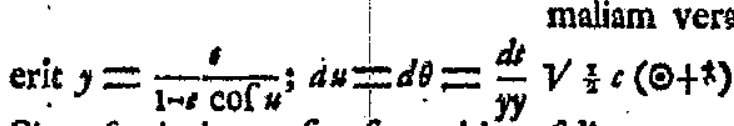

Sit a femiaxis tranfuerfus orbitae folis, ac tempore $=$ fol motu medio abfoluat angulum $=\omega$, quo pro menfura termporis $t$ vtamur: eric ergo $d \omega=\frac{d t}{d A} V \frac{x}{2} a(0+t)$ ideoque $\frac{z}{2} d t^{2}=\frac{d^{3} d \omega^{2}}{O+\delta}$. At eft $A=\frac{c}{1-6 \epsilon^{2}}$. Hine ergo fit

$$
d u=d \theta=\frac{a d \omega}{y y} V a c=\frac{a e d \omega}{y y} V(I-c e)=\frac{d \omega(1-c \operatorname{cof} t \theta)^{2}}{(I-c e) V(I-c t)}
$$

ficque tam $d x$ quam $d \theta$ per elementum dw loco tempo. ris jintroductum expreffimus. Quia aturem maffé folis 0 maffam terrae $\$$ eam enormiter excedit, fine crrore pro $\frac{x}{3} d t^{2}$ fcribi poterit $\frac{a^{3} d \omega^{2}}{0}$, eruntque noftrae aequationes pro luna:

$$
\begin{aligned}
& \text { I. } 2 d x d \varphi+\alpha d d \varphi=-\alpha^{3} d \omega^{2}\left(\frac{y}{z^{3}}-\frac{1}{y y^{2}}\right) \text { fin } \\
& \text { II. } d d x-x d \Phi^{2}=-\frac{a^{3}(t+D) d \omega}{0} \operatorname{cor} \psi^{3}\left(\frac{1}{x x}-\frac{1}{b t}\right) \\
& -\sin ^{3}\left(\frac{x-y \operatorname{cor} \eta}{x^{3}}+\frac{\operatorname{col} \eta}{y}\right)
\end{aligned}
$$

ro rega-

rit: ha

$\underline{0+8}$

yy

ano:

$=$

sre $=t$

o men-

$(0+6)$

ergo fit

of $u)^{2}$

I-ee)

tempo.

ílis o

ore pro

dationes

$\left(-\frac{1}{x}\right)$

)

III. ds
III. $d \pi=-\frac{a^{3} d \omega^{2}}{x d \phi}\left(\frac{y}{z^{3}}-\frac{1}{y y}\right)$ fin $(\varphi-\pi)$ fin $(\theta-\pi)$

IV. $d . l \operatorname{tang} \rho=\frac{d \pi}{\operatorname{tg}(\hat{\phi}-\pi)}$; atque ob tg $\psi=\operatorname{tg} \rho \mathrm{cr}(\varphi-\pi)$, habebitur proxime cor $\psi^{3}=1-\frac{3}{4} \operatorname{tg} \varphi^{2}-\frac{3}{4} \operatorname{tg} g^{2} c[2(\varphi-\pi)$.

III.

Incipiamus a duabus aequationibus prioribus, as ponamus breuitatis gratia

$$
a^{3}\left(\frac{y}{z^{3}}-\frac{1}{y y}\right) \text { fin } y=M \cdot \text { et }
$$

$\frac{A^{3}(b+D)}{O} \operatorname{cof} \psi^{3}\left(\frac{I}{x z^{2}}-\frac{1}{b b}\right)+a^{3}\left(\frac{x-y \operatorname{cof} y}{z^{3}}+\frac{\operatorname{cof} y}{y y}\right)=\frac{A}{x x^{2}}+N$ quandoquidem haec pofterior expreffo terminum in. voluit formae $\frac{A}{x x}$ prae ceteris incomparabiliter maiorem; atque habebimus has duas aequationes:

$2 d x d \varphi+x d a \varphi=-\mathrm{M} d \omega^{2}$ et $d d x+x d \phi^{2}=-\frac{A d \omega^{2}}{x}-\mathrm{N} d(\omega)^{2}$ quarum prior per $2 x^{3} d \varphi$ multiplicata ob $d \omega$ conftans habebit integrale :

$$
x^{4} d \phi^{2}=-2 d \omega^{2} \int \mathrm{M} x^{3} d \phi
$$

Tum prior multiplicata per $2 x d \Phi$ addatur ad pofteriorem per $2 d x$ multiplicatam, eritque, aggregatum:

$2 x d x d \varphi^{2}+2 x x d \varphi d d \varphi+2 d x d d x=-2 M x d \omega^{2} d \varphi$ $\frac{-2 A d \omega^{2} d x}{x x}-2 N d \omega^{2} d \omega$

Cuius integrale erie :

$$
d x^{2}+x x d \phi^{2}=+\frac{2 A d \omega^{2}}{x}-2 d \omega^{2} /(M x d \phi+\mathrm{N} d x)
$$

$\operatorname{Mm} 3$

IV. 
IV.

Ponantur formulae integrales, quae in his express fionibus infunt:

$-\int \mathrm{M} x^{3} d \varphi=\mathrm{p}$ et $-\int(\mathrm{M} x d \varphi+\mathrm{N} d x)=\mathbf{Q}$ vt habeamus has duas aequationes:

$x^{4} d \Phi^{2}=2 \mathrm{P} d \omega^{2}$ et $d x^{2}+* x d \Phi^{2}=\frac{2 A d \omega^{2}}{*}+2 Q d \omega^{2}$ vade cum fit $x+d \varphi^{2}=\frac{2 \mathrm{P} d \omega^{3}}{* \pm}$ erit

$d x^{2}=2 d \omega^{2}\left(Q+\frac{A}{x}-\frac{P}{x x}\right)$ et $d x= \pm d \omega v_{2}\left(Q+\frac{A}{x}-\frac{P}{x x}\right)$ fieque differentiale $d s$ per $d w$ exprimirur. Deinde ve. ro habetur

$$
d \varphi=\frac{d \omega}{x x} V_{2} \mathrm{P}
$$

eftque per hypothefin:

$d \mathrm{P}=-\mathrm{M} x d \omega V_{2} \mathrm{P}$ et $d \mathrm{Q}=-\frac{\mathrm{M} \delta \omega}{*} V_{2} \mathrm{P}+\mathrm{N} d \omega V_{2}\left(\mathrm{Q}+\frac{\mathbf{A}-\mathbf{P}}{x \neq}\right)$ vbi quidem fignorum ambiguorum inferius locum ba bere ftatuamus, quia motum ab apogeo numerare in nimo eft, it ve hinc exeundo diftentia $*$ minustur.

\section{V.}

Cum igitur differentiale $d x$ in apogeo et perigeo euanefcat, neceffe eft ve his locis formula irracionalis $V\left(\mathrm{Q}+\frac{\mathrm{A}}{\mathrm{x}}-\frac{\mathrm{P}}{\mathrm{X} *}\right)$ in nihilum abeat, in' reliquis autem locis valorem fortianr realem. Commodiffime ergo hacc formula per finum cuiuspiam anguli $v$ exhibebitur, qui cum in apogeo euanefcat, in perigeo autem duobus re. ixpres-

$1=Q$

$Q d \omega^{2}$

$\left.-\frac{\mathbf{p}}{x+x}\right)$

le ve

$\left(\frac{1}{x+}\right)$

$\mathrm{m}$ he.

are in

ar.

erigeo

ionalis

Im lo-

, haec

, qui

us re-

atis
Ctis aequalis fiat, anomaliam lunae referet: idque fenfu vero, ita ve diftantia $x$ in apogeo prodeat maxima, in perigeo vero minima. Sit igitur vt forman motus regula ris fequamur :

femilatus rectum orbitae lunaris $=p$ excentricitas orbicae $=q$ et anomalia vera lunas $=v$ eritque hinc per eandem legem diftantia $x=\frac{p}{I-q \operatorname{co} \gamma{ }^{\circ}}$. Verum hic quantitates $p$ et $q$, quae in motu regulari esd fent conftantes, nunc pro variabilibus funt habendae, earumque variabilitas per variabilitatem quantitatum $P$ et $Q$, quae in motu regulari itidem fupt confantes, dererminari debebit.

VI.

Subftituamus ergo valorem affumtum $x=\frac{p}{1 m g \operatorname{co}[y}$ in formula irrationali $V\left(Q+\frac{A}{x}-\frac{p}{x x}\right)$, quae abibit in $\frac{I}{p} V\left(Q p p+\operatorname{Ap}(1-q \operatorname{cor} w)-\mathbf{P}(\mathbf{I} \sim q \cos p)^{2}\right)$ et euoluta dabir

$\frac{1}{p} V\left(\mathrm{Q} p p+A p-\mathrm{P}-\mathrm{A} p q \operatorname{col} w+2 \mathrm{P} q \operatorname{co}\left(v-\mathrm{P} q q \cos \left(p^{2}\right)\right.\right.$ quae ve reducatur ad formam $V$ fin $v$, ftatuatur primo $2 \mathbf{P}-\mathbf{A}_{p}=0$ tum vero $\mathrm{Q} p p+\mathrm{A} p-\mathrm{P}=\mathrm{P} q q$ ac noftra formula fier $=\frac{1}{q} V_{q q}$ fin $v^{2}=\frac{q \text { fin } v}{l} V \mathrm{P}$, habebimusque 


$$
\begin{aligned}
& d x=-\frac{q d \omega \sin v}{p} V_{2} \mathrm{P} \text { ex } \\
& d \mathrm{Q}=-\frac{\mathrm{M} d \omega}{\nu} V_{2} \mathrm{P}+\frac{N_{q} d \omega \text { fin } v}{P} V_{2} \mathrm{P}
\end{aligned}
$$$$
\text { VII. }
$$

Cum iam fit $2 \mathrm{P}-\mathrm{A} p=0$; erit $\mathrm{P}=\frac{\mathrm{x}}{2} \mathrm{Ap}_{\mathrm{P}}$ : que valore in altera formula fubltituto orietur :

$$
Q p p+\frac{x}{2} A p=\frac{3}{2} A p q q \text { feu } Q=-\frac{A}{2 p}(1-q q)
$$

Sumantur nunc differentialia; eritque $d \mathrm{P}=-\mathrm{M} x d \omega \gamma_{2} \mathrm{P}=\frac{\pi}{2} \mathrm{~A} d p$, quae ob $2 \mathrm{P}=\mathrm{A} p$ abit in hane

$-\mathrm{M} x d \omega \vee \mathrm{A}_{p}=\frac{x}{2} \mathrm{~A} d p$, fiue $d p=-\frac{2 \mathrm{M} x d \omega}{\mathrm{A}} \vee A_{p}$ vel etiam $V A_{p}=-\int \mathrm{M} x d w$

Simili modo erit

$d Q=+\frac{A d p(\mathrm{I}-g q)}{2 p p}+\frac{A q d q}{p}=-\frac{M x d \omega(\mathrm{I}-q q)}{p p} \vee A p+\frac{\Delta q d q}{p}$ ideoque

$\frac{\mathrm{A} q d q}{p}=\mathrm{Md \omega}\left(\frac{x(\mathrm{I}-q q)}{p p}-\frac{\mathrm{I}}{x}\right) V \mathrm{~A}_{p}+\frac{\mathrm{N} g d \omega \mathrm{fin} v}{p} V_{\mathrm{A}_{p}}$ Ateft $\frac{x(1-q q)}{p p}-\frac{1}{x}=\frac{x}{p p}\left(1-q q+\frac{p p}{x x}\right)=\frac{*}{p p}\left(1-q q-1+2 q \mathrm{c}\left(x-q q \mathrm{cfv} v^{2}\right)\right.$ five $\frac{x(1-q q)}{p p}-\frac{1}{x}=\frac{q x}{p}\left(2 \operatorname{cor} v-q-q \operatorname{cor} v^{2}\right)$

Hinc ergo colligitur:

$d q=\frac{M x d v)}{A p}\left(2 \operatorname{cof}\left(v-q-q \operatorname{cof} v^{2}\right) V A p+\frac{1 \mathrm{~N} d \omega \text { fin } v}{A} V A p\right.$ fiue $d q=d \omega\left(\frac{\mathrm{M}}{\mathrm{A}}\left(2 \operatorname{cof} v-\frac{q \operatorname{fin} v^{2}}{1-q \operatorname{col}(z)}\right)+\frac{\mathrm{N}}{\mathrm{A}}\right.$ fin $\left.v\right) v A p$

\section{VIIII.}

Inuenta iam relatione differentialium $d x, d p$ ef $d q$ ad differentiale temporis dis feilicet:

$$
d x=-\frac{q d \omega \mathrm{fin} v}{q} V A p ; \quad d p=-\frac{2 \mathrm{M} x d \omega}{\mathrm{A}} V \mathrm{~A}
$$

Ap: que

$1-99)$

$p$ abit in

$-V A$

$p+\frac{A q d q}{p}$

$\because Y A$

$\left(v \cdot g 9 c v^{2}\right)$

)

$V A t$ fine Ap et $d q=d \omega\left(\frac{\mathrm{M}}{\mathrm{A}}\left(2 \operatorname{cof} v-\frac{q \sin v^{2}}{\mathrm{i}-q \operatorname{cof} v}\right)+\frac{\mathrm{N}}{\mathrm{A}}\right.$ fin $\left.v\right) V \mathbb{A} p$

fupereft, vt quoque relationem elementi amonaliae $d v$ definiamus. Cum igitur fit

$x=\frac{p}{1-q \text { cof } v}$, erit $1-q \operatorname{col} v=\frac{p}{x}$, hincque differemiando $q d v \sin v=d q \operatorname{cof} v+\frac{d p}{x}-\frac{p d x}{x x} ;$

fubftituantur valores pro $d q, d p$ et $d x$ inuenti; ac disi fione facta per $q$ fin $v$ prodibie

$d v=\frac{d \omega}{x x} V A \cdot p-\frac{d \omega}{q}\left(\frac{M}{A}\left(2 f i n v+\frac{q \text { finvcofv }}{\mathrm{I}-q \operatorname{co} \delta}\right)-\frac{\mathrm{N}}{\bar{A}} \operatorname{cof} v\right) V A P$ Pro elemento autem longitudinis $d \Phi$ ob $2 \mathrm{P}=\mathrm{Ap}$, ex antecedentibus habemus:

$$
d \varphi=\frac{d \omega}{x x} V A p=\frac{d \omega(1 \sim q \operatorname{cor} v)^{2}}{p p} V A p .
$$

$$
\text { IX. }
$$

Ex his formulis ftatim fe offert motus apogei; cum enim longitudo apogei fit $=\varphi-\nu$, erit eius differentiale pro tempuículo $d \omega:$

$d \phi-d v=\frac{d \omega}{q}\left(\frac{M}{A}\left(2 \sin v+\frac{q \operatorname{fin} v \operatorname{cor} v}{1-q \cos v}\right)-\frac{N}{A} \operatorname{cof} v\right) v A q$. cuius ergo integrale praebebit verum motum apogei cum omnibus inaegualitatibus, quibus perturbatur. Vnde $\mathrm{N} \mathbf{n}$ 
quidem perficitur, quod per fe eft manifetum, $f$ quanticates $M$ et $N$ euanefcerent, motum apogei fore nullum, feu apogeum perpetuo in loco fixo effe permanfurum. Deinde etiam iuuabit notaffe has formulas:

$$
\begin{aligned}
& \text { d. } q \operatorname{cof} v=-q d \varphi \sin v+\frac{2 \mathrm{M}}{\mathrm{A}} d \omega V \mathrm{Ap} \\
& \text { d. } q \text { fin } v=+q d \phi \operatorname{cof} v+d \omega\left(\frac{\mathrm{N}}{\mathrm{A}}-\frac{\mathrm{M}}{\mathrm{A}} \cdot \frac{q \operatorname{cin} v}{\mathrm{I}-q \operatorname{cof} v}\right) V \mathrm{Ap}
\end{aligned}
$$

im, $\mathbf{f}$

i fore

e per-

unlas:

$V A_{p}$

Tandem quoque habemus ex motu folis $d w=d \theta=$ $\frac{d(u)(1-e \cos u)^{2}}{(I-e c) \sqrt{(1-e e)}}$ ideoque

$d \eta=d \varphi-d \theta=d \omega\left(\frac{(\mathrm{I}-q \operatorname{cof} v}{p p} V A p-\frac{(\mathrm{I}-e \operatorname{cof} u)^{2}}{(1-c e) V(1-\epsilon \epsilon)}\right)$

$\mathbf{X}$.

Inuentis nunc omnitim differentialium relationibus - ad elementum temporis $d \omega$, euoluamus valores litterar ram $M$ et $N$, ac primo guidem cum fit

$z=V\left(y y-2 x y\right.$ cof $y+x x$ (ec. $\left.\phi^{2}\right)$; quoniam quantitas $z$ nonnifi in terminis minimis occurrit, pro fec. $\psi$ tuto unitas fcribi poterit, et quia $y$ tantopere excedit $*$, erit proxime

$$
\begin{aligned}
& \frac{1}{z^{3}}=\frac{1}{y^{3}}+\frac{3 x}{y^{4}} \cos \eta+\frac{3 x x}{2 y^{5}}\left(5 \operatorname{col} y^{2}-1\right) \text { five } \\
& \frac{1}{z^{3}}=\frac{1}{y^{3}}+\frac{3 x}{y^{4}} \cos \eta+\frac{3 x x}{4 y^{5}}(3+5 \cos 2 \eta)
\end{aligned}
$$

Ideogue hinc habebinur:

$$
\frac{y}{z^{3}}-\frac{1}{y y}=\frac{3^{x}}{y^{3}} \cos y+\frac{3 x x}{4 y^{4}}(3+5 \cos 2 y)
$$

Vnde obtinemus :

$$
\begin{aligned}
& M=a^{3}\left(\frac{3 x}{2 y^{3}} \text { in } 2 y+\frac{3 x x}{8 y^{4}}(\text { fin } y+5 \text { (in } 3 y)\right)
\end{aligned}
$$

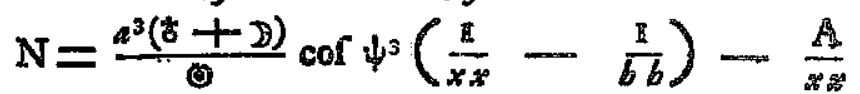

$$
\begin{aligned}
& -a^{3}\left(\frac{x^{*}}{2 y^{3}}(1+3 \cos 2 \eta)+\frac{3 x^{2} x}{8 y^{4}}(3 \operatorname{cor} \eta+5 \operatorname{cof} 3 \eta)\right)
\end{aligned}
$$

XI.

Cum fit proxime cof $\psi^{3}=-\frac{3}{4} \operatorname{tang} \rho^{2}-\frac{3}{4} \operatorname{tang} \rho^{2} \operatorname{cof} 2(\phi-\pi)$, eius valor vnitate erit minor, atgue ex parte confante, et parte variabili conftabit, quae illa mulco erit minor. Ponatur ergo $\operatorname{cof} \psi^{3}=\lambda+\Pi$; vt fit $\left.\Pi=\pi-\lambda-\frac{3}{4} \operatorname{tang} \xi^{2}-\frac{3}{4} \operatorname{tang} \rho^{2} \operatorname{cof} 2\left(\varphi_{-\infty}\right)^{2}\right)$ vbi $\lambda$ denotat partem conftantem vaitate proxime as qualem, II vero partem variabilem.

Erit ergo :

$$
\begin{aligned}
& N=\frac{\lambda a^{3}(t+D)}{0}\left(\frac{1}{x+x}-\frac{1}{b b}\right)-\frac{A}{x+2}+\frac{a^{3}(b+D)}{0} \Pi\left(\frac{1}{x+x}-\frac{1}{b b}\right) \\
& -a^{3}\left(\frac{x}{2 y^{3}}(1+3 \cos (2 y))+\frac{3 x x}{8 y^{4}}(3 \cos y+5 \operatorname{co}(3 y))\right.
\end{aligned}
$$

Statuatur nunc $A=\frac{\lambda_{A^{2}}(\gamma+2)}{0} ;$ it fiat

$$
\begin{aligned}
& \mathbf{N}=-\frac{\mathbf{A}}{b b}+\mathbf{A} \Pi\left(\frac{1}{x+s}-\frac{1}{b b}\right) \\
& -B^{3}\left(\frac{x}{2 y^{3}}(I+3 \cos 2 y)+\frac{3 x+5}{8 y^{4}}(3 \operatorname{col}(y+5 \operatorname{col} 3 y))\right.
\end{aligned}
$$


ac ponatur breuitatis gratia : $p=b(1+\xi)$

exit $\frac{V A p}{p p}=V \frac{A}{b^{3}(\mathrm{I}+\xi)^{3}}=\left(1-\frac{3}{2} \xi+\frac{x}{8} \xi^{2}\right) V \frac{A}{b^{3}}$

ob $\xi$ prae I vehementer paruum, firque porro:

$$
V \frac{A}{b^{3}}=V \frac{\lambda^{3}(\delta+D)}{Q}=m,
$$

atque habebitur $d \varphi=m d \omega\left(1-\frac{3}{2} \xi+\frac{3}{z^{5}} \xi^{2}\right)(1-q \operatorname{cof} v)^{2}$

\section{XII.}

Subftituantur nune pro $x$ et $y$ valores $\frac{p}{1-q \operatorname{cof} v}$ et $\frac{c}{1-e \operatorname{cor} u}$, exitque

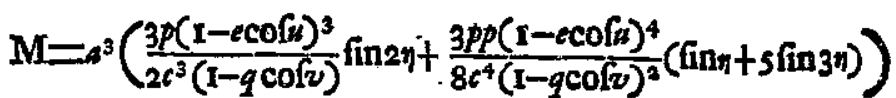

- $\mathbf{N}=-\frac{\mathrm{A}}{b b}+\mathrm{A} \Pi\left(\frac{(\mathrm{I}-q \operatorname{cor} v)^{2}}{\not p}-\frac{\mathrm{I}}{b b}\right)$.

$-n^{3}\left(\frac{p(I-e \cos u)^{3}}{2 c^{3}(1-q \operatorname{cof}(v)}(1+3 \cos 2 \eta)+\frac{3 p p\left(1-e \operatorname{cof}(u)^{4}\right.}{8 c^{4}\left(I-q \operatorname{cof}(v)^{2}\right.}\left(3 \cos \eta 5 c f_{3 \eta)}\right)\right.$

vbi quidem quoque terminus $\frac{A \Pi}{b \bar{b}}$. prae termino $\frac{A}{b b}$

omitri poteft. Nunc vt hinc valores $\frac{M}{A} V A P$ et $\frac{N}{A} V A P$

commode exprimantur, erit

$$
\frac{a^{3} b}{\bar{A} c^{3}} V A b=\frac{a^{3}}{m c^{3}}=\frac{I}{m(I-c c)^{3}}=\frac{I+3 e c}{m}
$$

quonism in his terminis minimis pro. 1-ee feribere licet $x$ Tum vero fit $\frac{b}{c}=n$, eritque $n$ fractio valde parus.

\section{XllI.}

Factis ergo his fubtitutionibus, ob $p=6(1+\xi)$ habebimus :

$$
\begin{aligned}
& \frac{M}{A} V A p=\frac{3(1+3 e \epsilon)}{2 m} \frac{\left(1-e \operatorname{co}(n)^{3}\right.}{I-q \cos v}\left(1+\frac{3}{2} \xi\right) \text { fin } 2 \eta \\
& +\frac{3 n}{8 m} \frac{\left(1-e \operatorname{cof}(v)^{4}\right.}{(1-q \cot v)^{2}}\left(1+\frac{5}{2} \xi\right)(\sin \theta+5 \sin 3 \pi)
\end{aligned}
$$

Pro altera valore $\frac{\mathrm{N}}{\mathrm{A}} V \mathrm{Ap}$ ftatuatur terminus minimus:

$$
\frac{V A b}{b b}=V \frac{\lambda A^{3} b(b+D)}{O b^{4}}=; ; \text { eritque }
$$

$\frac{\mathrm{N}}{\mathrm{A}} \dot{V} \mathrm{~A} p=-\frac{(1+3 e e)}{2 m} \frac{(\mathrm{1}-e \operatorname{cof} u)^{3}}{1-q \operatorname{co} \gamma}\left(1+\frac{3}{2} \xi\right)(1+3 \operatorname{col} 2 \eta)$

$-\frac{3 n}{8 m} \frac{\left(1-e \operatorname{co}(u)^{4}\right.}{(1-q \cos v)^{2}}\left(1+\frac{5}{2} \xi\right)(3 \cos y+5 \operatorname{col}(3 y)$

$$
+m\left(1-q \operatorname{co}(v)^{2}\left(1-\frac{3}{2} \xi\right) \Pi-i\right.
$$

vbi notari oportet, terminos per $*$ multiplicatos racio ne praecedentium efle minimos; tum vero quantitates $\xi$ et $I$ atque multo magis $i$ effe fractiones prae vnotate fere euanefcentcs.

\section{XIV.}

Quoniam hi ipfi termini quantitates $M$ et $N$ involuentes funt valde parui, in is fine errore altiores pateftates veriusque excentricitatis $q$ et e negligi pos. funt. In terminis ergo primis fimpliciter per m diutfis excentricitates tantum ad duas dimenfiones intro.

$$
N \mathbf{n}_{3}
$$


ducantur, in terrainis autem per $\frac{n}{m}$ multiplicetis penisus omittantur, quia fraetio $n$ iam fere quadrato excentricitatis $q$ sequiulet In termino autem littera minime II affetto, quia is per numerum $m$ hatis magnum, vtpoke 13 fere, eft muliplicatus, excentricicas $q$ vnius dimenfionis retinestur.

His obferuatis habebimus:

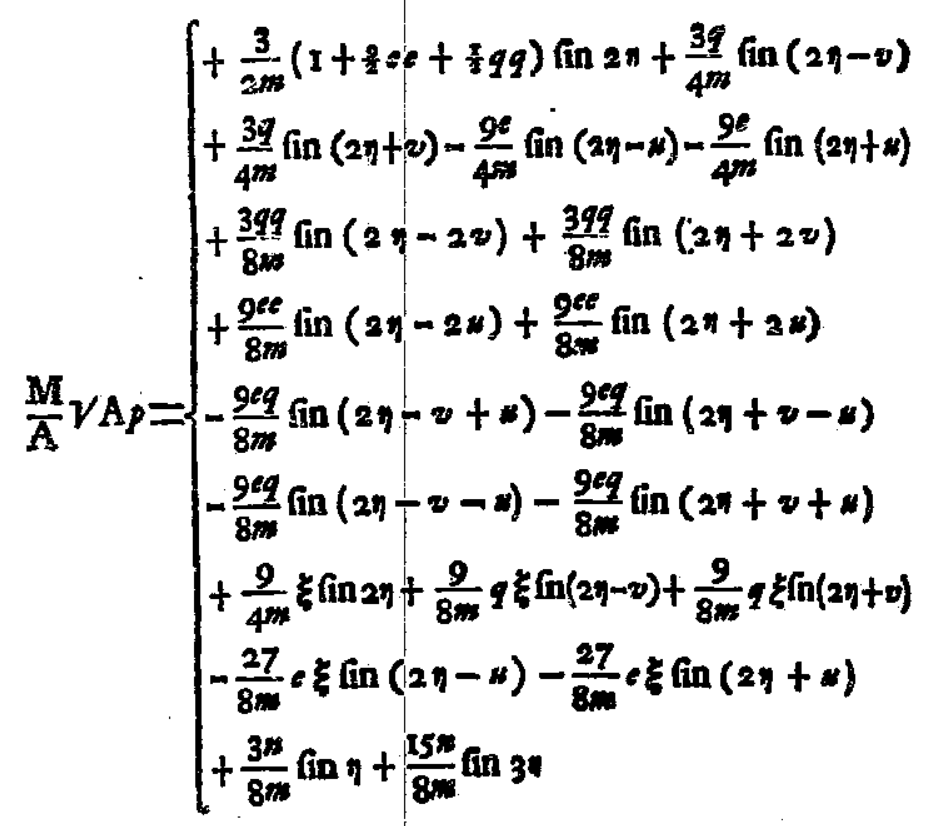




\section{XV.}

Quaeramus igitur valores evolutos noftrorum aijfferentialium ad elementum temporis applicatorum : ac primo quidem habebimus;:

$\frac{d \varphi}{d \omega}=m\left(1+\frac{\pi}{2} q q\right)-2 m q \operatorname{co} \int v+\frac{x}{2} m q q \operatorname{co} \int 2 v-\frac{3}{2} m\left(1+\frac{\pi}{2} q q\right) \xi$ $+3 m q \xi \operatorname{cor} v-\frac{3}{4} m q q \xi \operatorname{col} 2 \pi+j^{x} s m \xi \xi$

$\frac{d u}{d \omega}=\frac{d \theta}{d \omega}=1+2 e e-2 e \operatorname{co} \int u+\frac{\pi}{2} e e \operatorname{co} \int 2 u ;$ vnde concludimus $\left.\frac{d \eta}{d w}=m\left(1+\frac{\pi}{2} q\right]\right)-1-2 e e-2 m g \operatorname{co}\left(v+2 e \operatorname{co}\left[u+\frac{\pi}{2} m q q \operatorname{co} 2 v\right.\right.$ $-\frac{1}{\xi} \operatorname{ce} \operatorname{col} 2 u-\frac{3}{2} m\left(1+\frac{\pi}{2} g q\right) \xi+3 m g \xi \operatorname{co} 2 v$ $-\frac{3}{4} m g q \xi \operatorname{cor} 20+\frac{\pi 5}{8} m \xi \xi$

Deinde cum fit $\frac{d p}{d \omega}=-2 x \cdot \frac{M}{A} V A p=-\frac{2 b(I+\xi)}{I-q c \sqrt{t}} \cdot \frac{M}{A} V A_{P}$, ob $p=b(1+\xi)$ exit $\quad \frac{d \xi}{d \omega}=$ $\left(-2\left(x+\frac{\pi}{2} q q\right)-2 q \operatorname{co}(v-q q \operatorname{co} 2 v-2 \xi-2 q \xi \operatorname{col} v) \frac{\mathrm{M}}{\mathrm{A}} V A p\right.$ ac valorem pro $\frac{M}{A} V A p$ inuentum fubftituendo obtinebi. mus fequences formulas: lif-

$$
\begin{aligned}
& -\frac{3}{m}\left(1+\frac{2}{2} \cot +\frac{3}{2} q q\right) \operatorname{tin} 2 q-\frac{3 q}{m} \operatorname{fin}(2 \eta-v)-\frac{3 q}{2} \operatorname{lin}(2 n+v)
\end{aligned}
$$

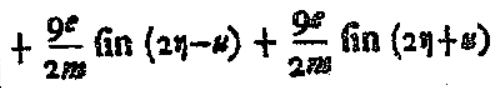

$$
\begin{aligned}
& -\frac{9 g 9}{4 m} \text { fin }(2 \eta-2 v)-\frac{99 q}{4 m} \text { fin }(2 v+2 v) \\
& -\frac{9^{e e}}{4^{m}} \text { fin }(2 \eta-2 H)-\frac{9^{e e}}{4^{m}} \ln (2 \eta+2 H) \\
& \frac{d \xi^{\circ}}{d w}=\left\{+\frac{9 e q}{2 m} \mathrm{fin}(2 \pi-v+w)+\frac{9 q q}{2 m} \sin (2 \eta+v-m)\right. \\
& +\frac{5 e q}{2 m} \mathrm{fin}(2 \eta-v-4)+\frac{9 e q}{2 m} \mathrm{fin}(2 y+z+\xi) \\
& -\frac{15}{2 \pi z} \xi \operatorname{fin} 2 \eta-\frac{15 q}{2 m} \xi \operatorname{fin}(2 y-v)-\frac{15 q}{2 m} \xi \sin (2 \eta+v) \\
& +\frac{45 e}{4 m} \xi \operatorname{fin}(2 \eta-x)+\frac{45 e}{4 m} \xi \operatorname{fin}(2 \eta+B) \\
& -\frac{3^{n}}{4^{m}} \text { fin } n-\frac{15 n}{4^{m}} \text { in } 3 n
\end{aligned}
$$

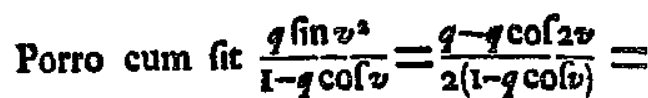
$\frac{1}{2} q-\frac{\pi}{2} q \mathrm{cr} 2 v+\frac{x}{4} q q \operatorname{cor} v-\frac{2}{4} q q \operatorname{cor} 3 v ;$ erit

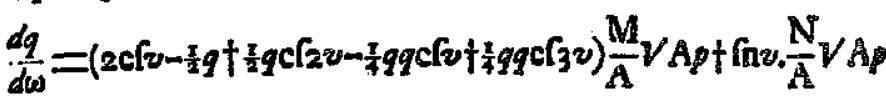
Facta ergo fubctitutione valorum pro $\frac{M}{\mathrm{~A}} V A p$ et $\frac{\mathrm{N}}{\mathrm{A}} V \mathrm{Ap}_{p}$ inuentorum, habebitur:

Oo $\frac{d a}{d(t)}=$ 


$$
\begin{aligned}
& +\frac{9 q}{8 m} \xi\left(\operatorname{in} 2 \eta+\frac{9 q}{4 m} \xi \ln (2 \eta-2 v)+\frac{9 q}{8 m} \xi \ln (2 q+2 v)\right. \\
& -\frac{39}{8 m} \xi \sin 2 v+\frac{9 e}{8 m} \xi \operatorname{in}(\nu-u)+\frac{9 g}{3 m} \xi \operatorname{Sin}(v+w) \\
& -\frac{81 e}{16 m} \xi \operatorname{fin}(2 \eta-v-z)-\frac{81 e}{16 m} \xi \operatorname{lin}(2 \eta-v+v) \\
& -\frac{27^{e}}{16} \xi \operatorname{fin}(2 v+v-x)-\frac{27^{2}}{16 m} \xi \sin (2 v+v+4 v) \\
& +\frac{15 n}{16 m} \text { fin }(\eta-v)-\frac{3 n}{16 m} \text { fin }(\eta+v) \\
& +\frac{45 n}{16 m} \operatorname{fin}(3 y-v)+\frac{15 n}{16 m} \sin (3 y+v) \\
& +m \Pi \text { fin } v-m g \text { II (in } 2 v-\frac{3}{2} m \xi \Pi \text { IIn } v-i \text { ใin } v
\end{aligned}
$$

\section{XVII.}

Deinde cum fit $\frac{q \operatorname{fin} v \operatorname{cof} v}{1-q \operatorname{cof} v}=\frac{q \operatorname{lin} 2 v}{2(1-q \operatorname{cor} v)}=$ $=\frac{x}{2} q$ in $2 v+\frac{x}{4} q q$ fin $v+\frac{x}{4} q 9$ fin $3 v$; erit pro motu elementari apogei :

$\frac{q(d \varphi-d v)}{d \omega}=\left(2 \sin v+\frac{x}{2} q \operatorname{fin} 2 v+\frac{x}{4} q q\right.$ in $v$

$$
\left.+\frac{z}{4} q \text { fin } 3 v\right) \frac{\mathrm{M}}{\mathrm{A}} V A p-\operatorname{cor} v \cdot \frac{\mathrm{N}}{\mathrm{A}} V A p
$$

ac falta fubltitutione obtinebitur:

$$
002 \quad \frac{q(d q \cdot d v)}{d(u)}=
$$


292

$A D D I T A B E N T U M$.

$\int+\frac{9}{4^{m}}\left(1+\frac{2}{2} e c+\frac{7}{x^{3}} 99\right) \operatorname{cor}(2 y-9)-\frac{3}{4^{m}}\left(1+29 e+\frac{1}{4} 99\right)$

$\operatorname{col}(2 \eta+v)+\frac{1}{2 \pi}\left(1+\frac{3}{2} e c+\frac{3}{4} 9 q\right) \operatorname{cor} v$

$+\frac{3 q}{4 x} \cos 2 y+\frac{3 q}{2 m} \operatorname{cor}(2 y-2 z)-\frac{3 q}{4 x} \operatorname{cor}(2 y+2 z)$

$+\frac{9}{4^{m}} \operatorname{cor} 2 v+\frac{q}{4^{m}}-\frac{3 e}{4^{m}} \operatorname{cor}(v-w)-\frac{3 e}{4^{m}} \operatorname{cor}(v+w)$

$-\frac{27 e}{8 w} \operatorname{cor}(2 y-v-x)-\frac{27 e}{8 m} \operatorname{cor}(2 y-v+x)$

$+\frac{9 e}{8 m} \operatorname{cor}(2 y+v-n)+\frac{9 e}{8 m} \operatorname{cof}(2 n+v+n)$

$+\frac{1599}{16 m} \operatorname{cor}(2 \eta-3 v)-\frac{999}{16 m} \operatorname{cor}(2 \eta+3 v)+\frac{99}{8 m} \operatorname{cor} 3 v$

$-\frac{9 e q}{8 m} \operatorname{cor}(2 y-\mu)-\frac{9 e q}{8 m} \operatorname{cor}(2 y+x)-\frac{3 e q}{4 m} \operatorname{cor} *$

$-\frac{3 e q}{8 m} \operatorname{col}(2 v-x)-\frac{3 e q}{8 m} \operatorname{cor}(2 v+x)$

$-\frac{9 e q}{4 m} \operatorname{cor}(2 \eta-2 v+\mu)-\frac{9 e q}{4 m} \operatorname{cor}(2 \eta-2 v-x)$

$+\frac{9 e q}{8 m} \operatorname{cor}(2 x+2 v-x)+\frac{9 e q}{8 m} \operatorname{cor}(2 x+2 v+x)$

$+\frac{27 e e}{16 m} \operatorname{cor}(2 y-v-2 w)+\frac{37 e e}{16 m} \operatorname{cor}(2 \eta-v+2 w)$

$-\frac{9 e e}{16 m} \operatorname{cor}(2 \eta+v-2 v)-\frac{9 e e}{16 m} \operatorname{col}(2 \eta+v+2 v)$

$+\frac{3 e c}{8 m} \operatorname{cor}(v-2 w)+\frac{3 e e}{8 m} \operatorname{cor}(v+2 n)$
$A D D I T A M E N T U M$.

293

$$
\begin{aligned}
& 1+\frac{27}{8 m} \xi \operatorname{cof}(2 y-v)-\frac{9}{8 m} \xi \operatorname{cor}(2 \eta+v)+\frac{3}{4 m} \xi \operatorname{col} v \\
& +\frac{9 q}{8 m} \xi \operatorname{cor} 2 y+\frac{9 q}{4 m} \xi \operatorname{cl}(2 y-2 z)-\frac{9 g}{8 m} \xi \operatorname{cr}(2 y+2 z) \\
& +\frac{39}{8 m} \xi+\frac{3 q}{8 m} \xi \operatorname{cor} 2 v \\
& -\frac{81 \epsilon}{16 m} \xi \operatorname{cor}(2 y-v-u)+\frac{27 \epsilon}{16 m} \xi \operatorname{cor}(2 y+v-n) \\
& -\frac{81 \epsilon}{16 m} \xi \operatorname{cor}(2 y-v+\mu)+\frac{27 \epsilon}{16 m} \xi \cos (2 y+v+\mu) \\
& -\frac{9 e}{8 m} \xi \operatorname{cor}(v-u)-\frac{9 e}{8 m} \xi \operatorname{cof}(v+u) \\
& +\frac{15 n}{16 m} \operatorname{col}(y-v)+\frac{3 n}{16 m} \operatorname{col}(y+v) \\
& +\frac{45 n}{16 m} \operatorname{co}\left((3 n-v)-\frac{15 n}{16 m} \operatorname{col}(3 n+v)\right.
\end{aligned}
$$
XVIII.

Euoluamus fimili modo valorem differentialium $d x$ et et cum fit $\frac{y}{z^{3}}-\frac{x}{y y}=\frac{3 x}{y^{3}} \operatorname{cint} \frac{3 x x}{4 y^{4}}\left(3+5 c(2 \eta)\right.$ et $d \varphi=\frac{d \omega}{x x} v$ A $p$; eris $d x=-\frac{\pi^{3} x d \omega}{V A p}\left(\frac{3 x}{y^{3}} \cos \eta+\frac{3 x x}{4 y^{4}}(3+5 \cos (2 \eta)) \operatorname{lin}(\theta-\pi) \operatorname{fin}(\varphi-\pi)\right.$

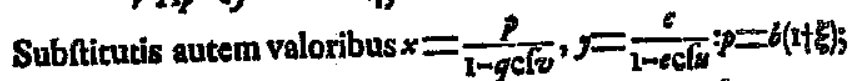
$V A p=m V b^{3} p=m b b\left(1+\frac{x}{2} \xi\right), \frac{a^{3}}{c^{3}}=\frac{1}{(1-c e)^{3}}=x+3 e c \mathrm{et} \frac{b}{c}=s$, erit

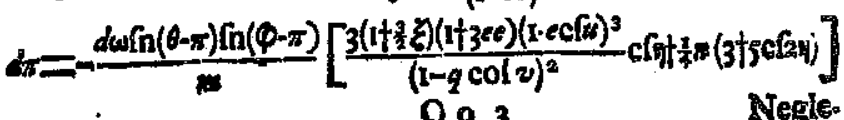


Neglectis igitur terminis, qui nullum valorem fenfibi-

Gibi.

lem conrinent, habebimus

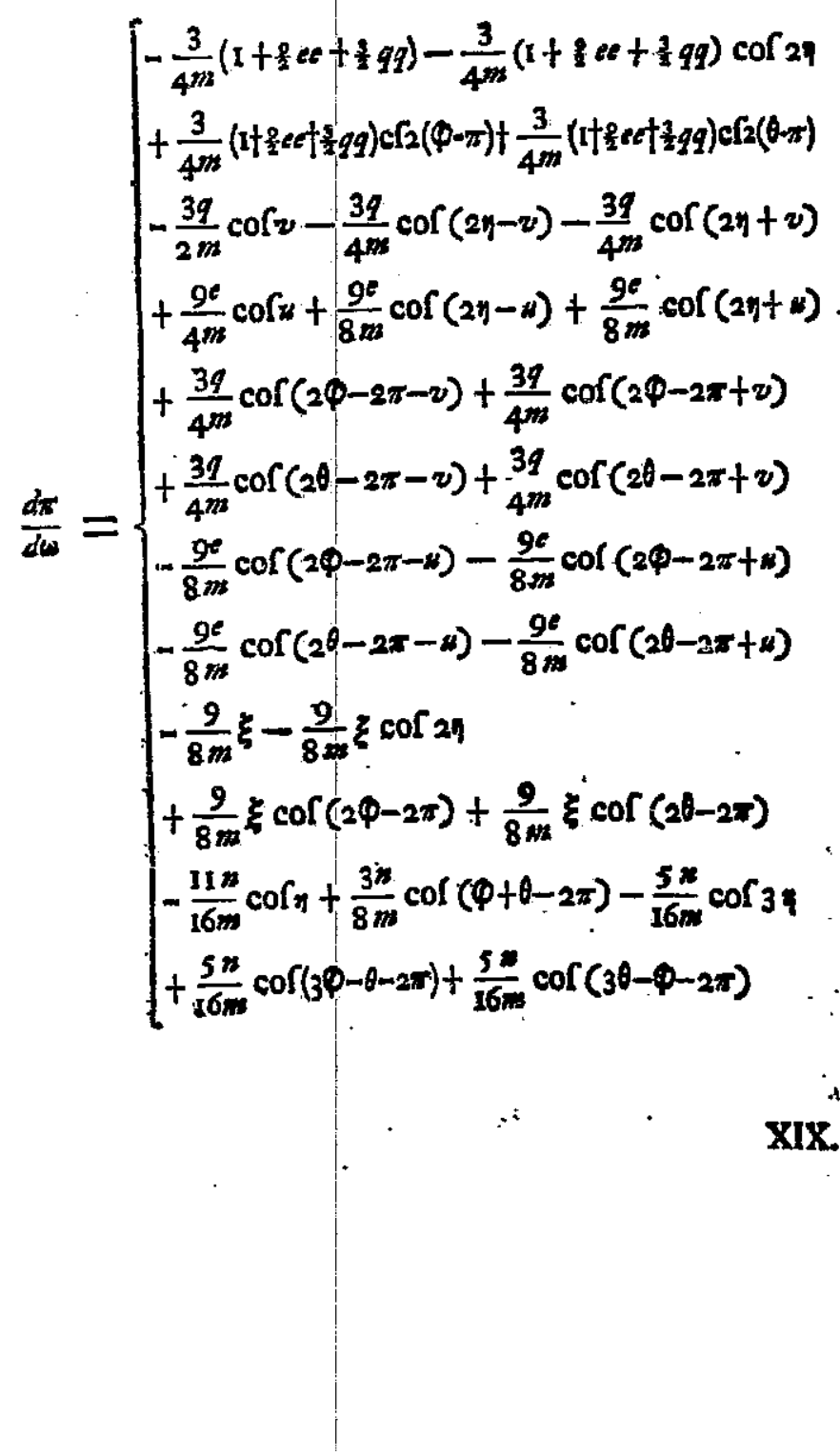

\section{XIX.}

Simili autem modo praecedentem valorem per tang $(\phi-\pi)$ diuidendo prodibit differentiale logarithmi tangentis inclinationis $\rho$, erit enim

$$
\begin{aligned}
& \int+\frac{3}{4^{m}}\left(1+24 e+\frac{3}{2} g q\right) \text { fin } 2 m-\frac{3}{4^{m}}\left(1+2 y+\frac{3}{2} q 9\right) \\
& \text { fin } 2(\varphi-x)-\frac{3}{4^{m}}\left(1+\frac{2}{2} e c+\frac{3}{2} g q\right) \text { fin } 2(\theta-\pi) \\
& +\frac{3 q}{4^{m}} \text { fin }(2 y-v)+\frac{3 q}{4^{m}} \text { fin }(2 y+v) \\
& -\frac{9 e}{8 m} \text { in }(2 \eta-w)-\frac{9 e}{8 m} \text { fin }(2 \eta+w) \\
& -\frac{39}{4^{m}} \text { fin }(2 \varphi-2 \pi r-v)-\frac{3 q}{4^{m}} \text { fin }(2 \varphi-2 \pi+w) \\
& \frac{d . \operatorname{tang} g}{d w}=\left\{-\frac{39}{4 m} \text { in }(2 \theta-2 \pi-x)-\frac{39}{4 m} \text { fin }(2 \theta-2 \pi+\infty)\right. \\
& +\frac{9 e}{8 m} \text { fin }(2 \varphi-2 m-n)+\frac{9 e}{8 m} \operatorname{lin}(2 \varphi-2 m+m) \\
& +\frac{g^{e}}{8 m} \text { fin }(2 \theta-2 \pi-x)+\frac{g^{e}}{8 m} \text { in }(2 \theta-2 \pi+m) \\
& +\frac{9}{8 m} \xi \operatorname{lin} 2 \eta-\frac{9}{8 m} \xi \ln (2 \varphi-2 \pi)-\frac{9}{8 m} \xi \lim \left(2 \operatorname{lom}^{2} 2 \pi\right) \\
& +\frac{n}{16 m} \text { in } \eta-\frac{3 x}{8 m} \text { in }(\varphi+\theta-x)+\frac{5 \pi}{16 m} \text { in } 3 \text { \% } \\
& -\frac{5 n}{16 m} \text { in }(3 \varphi-\theta-28)-\frac{5 x}{16 m} \text { in }\left(3^{\theta+\infty} \varphi-2 \pi\right)
\end{aligned}
$$


XX.

Quo iam facilius has formulas admodum complicatas euolvere queamus, quadruplicis generis terminos diftingui conuenit. Primum fcilicet genus eos compleEitur terminos, qui tantum ab excentricicate orbitae lunaris pendent, neque excentricitatem folis, neque pa. rallaxin folis feu literam , neque inclinationem orbine lunaris feu litteram $\Pi$ inuoluune. Ad fecundum genus refero terminos, qui ad primum genus infuper excentricitatem folis adiungunt. Ad tertium autem eos, qui praeterea parallaxin folis fea litteram quarto autem eas inaequalitates, quae infuper ab obliguicate orbitae lunaris proueniunt, complexurus fum. $A b$ inaegualiatibus ergo primi generis exardiar, ideoque cum excentricitatem folis e, tum eius parallaxin, tum quoque obliquicatem orbitae lunaris reiiciam

\section{INUESTIGATIO INAEQUALITATUM LUAAE PRIMI GENERIS. \\ XXI.}

Negleetis ergo excentricitate folis cum eius parallaxi et obliquitate orbitae lunaris, has habebimus ae. quationes :

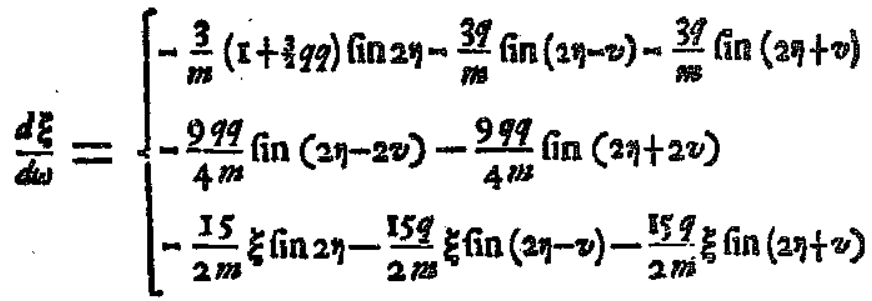

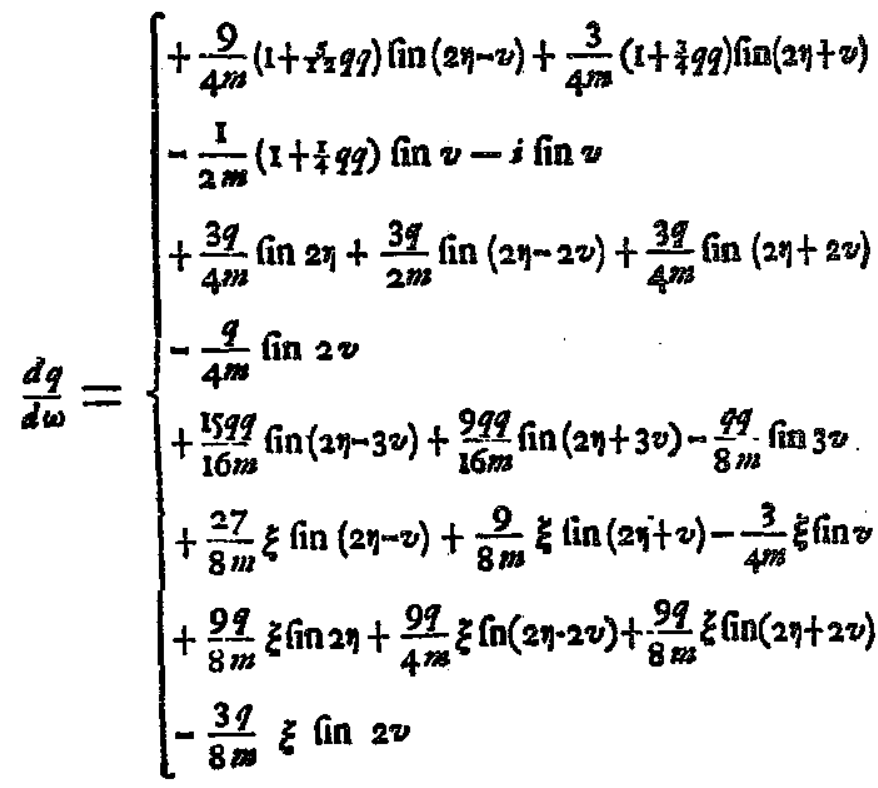

$\mathbf{P}_{\mathrm{p}} \frac{(d \theta+d y)}{d y}=$ 


$$
\begin{aligned}
& +\frac{9}{4^{m}}\left(1+x^{3} 299\right) \operatorname{cor}(2 y-v)-\frac{3}{4^{m}}\left(1+\frac{x}{2} 99\right) \operatorname{cor}(2 y+z) \\
& +\frac{1}{2 x}\left(1+\frac{3}{499}\right) \operatorname{cor} v+i \operatorname{cor} v \\
& +\frac{3 q}{4^{m}} \operatorname{col} 2 \eta+\frac{3 q}{2 m} \operatorname{col}(2 y-2 v)-\frac{3 q}{4 m} \operatorname{col}(2 \eta+2 v)
\end{aligned}
$$

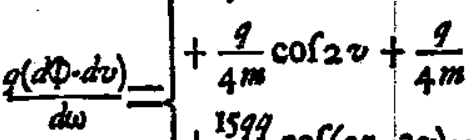

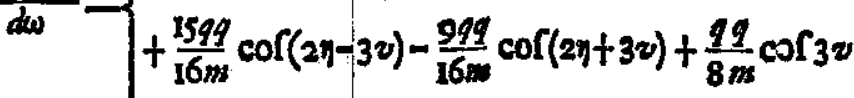

$$
\begin{aligned}
& +\frac{27}{8 m} \xi \operatorname{cor}(2 \eta-v)-\frac{9}{8 m} \xi \operatorname{cor}(2 \eta+v)+\frac{3}{4 m} \xi \operatorname{cor} v \\
& +\frac{9 q}{8 m} \xi \mathrm{c}\left(2 \eta+\frac{9 q}{4 m} \xi \mathrm{cr}(2 \eta-2 v)-\frac{9 q}{8 m} \xi \mathrm{cl}(2 \eta+2 v)\right. \\
& +\frac{3 q}{8 m} \xi+\frac{3 q}{8 m} \xi \operatorname{cor} 20
\end{aligned}
$$

$\frac{d \varphi}{d w}=m\left(1+\frac{1}{2} q q\right)-2 m q \operatorname{cof} v+\frac{1}{2} m g q \operatorname{cof} 2 v-\frac{1}{2} m\left(1+\frac{z}{2} q q\right) \xi$ $+3 m q \xi \operatorname{col} v-\frac{3}{4} m q q \xi \operatorname{col} 2 v+3 y \xi \xi$ $\frac{d \eta}{d \omega}=m\left(1+\frac{x}{2} q q\right)-1-2 m g \operatorname{co} \int v+\frac{\pi}{2} m g q \operatorname{co} \int 2 v-\frac{3}{2} m\left(1+\frac{\pi}{2} q q\right) \xi$ $+3 m g \xi \operatorname{col} v-\frac{3}{4} m g g$ ह $\operatorname{cor} 2 v+x^{2} m \xi \xi$

XXII.

Hic autem primo patet valores litterarum $z$ et 9 fine cognitis rationibus $\frac{d \eta}{d \omega}$ et $\frac{d v}{d \omega}$ definiri non pofte, has autem viciffim ipfas quantitates $\xi$ et $q$ inuoluere. Cum autem ad valores $\xi$ et $g$ inueniendos non opus fit ratones tiones $\frac{d y}{d \omega}$ et $\frac{d v}{d \omega}$ eo praecifionis gradu noffe, quo iph illi valores defiderantur; patet fi valores $g$ et prope tantum veri conftent, is in rationibus $\frac{d v^{\circ}}{d \omega}$ et $\frac{d v}{d v^{2} \mathrm{dh} \text {. }}$. bitis, eosdem multo exatiores repercum iri. Cum igitur, fi motus eflet regularis, foret $z=0$ g $q=$ cos. ftanti, hinc primam hypothefin confticuamus. Sit ergo

$$
\xi=0 \text { es } q=8
$$

et negleçis terminis, qui ob harum litterarum errores aftici poffent, vtpore valde paruis prae reliquis, habebimus proxime

$\frac{d \Phi}{d \omega}=m\left(1+\frac{1}{2} g g\right)-2 m g \operatorname{cor} v ; \quad \frac{d \eta}{d \omega}=\left(1+\frac{\pi}{2} g g\right)-I-2 m g \operatorname{cof} v$ et $\frac{d \varphi-d v}{d w}=\frac{9}{4 m g}\left(1+x^{\frac{7}{2}} g g\right) \operatorname{cor}(2 x-v)-\frac{3}{4 m g}\left(I+\frac{\pi}{4} g g\right) \operatorname{cof}(2 y+v)$ $+\frac{1}{2 m g}\left(1+\frac{3}{4} g g\right) \operatorname{co} z u+\frac{i}{g} \operatorname{co}\left(v+\frac{1}{48 B}\right.$

ideoque $\frac{d v}{d w}=m\left(1+\frac{\pi}{2} g s\right)-\frac{1}{4 m}-\left(2 m g+\frac{1}{2 m g}+\frac{3 g}{8 m}+\frac{i}{g}\right) \operatorname{cof} v$ $-\frac{9}{4^{m g}}\left(1+1^{\frac{7}{2} g g}\right) \operatorname{cor}(2 y-v)+\frac{3}{4^{m g g}}\left(1+\frac{r}{4} g g\right) \operatorname{cof}(2 y+v)$

XXIII. 
et neglectis quadratis $g g$ in reliquis terminis, habebimus has formulas fimpliciores:

$\frac{d \eta}{d \omega}=\alpha-\gamma \operatorname{cof} v$
$\frac{d v}{d w d}=6-\delta \operatorname{cor} v-\frac{9}{4 m g} \operatorname{cor}(2 \eta-v)+\frac{3}{4 m g} \operatorname{cor}(2 \eta+v)$
Tum vero pro valoribus $\xi$ et $q$ propius inueniendis Tum vero pro

$$
\begin{aligned}
& \frac{d \xi}{d \omega}=-\frac{3}{m}\left(1+\frac{3}{2} g g\right) \operatorname{lin} 2 \eta-\frac{3 g}{m} \text { in }(2 \eta-v)-\frac{3 g}{m} \text { fin }(2 \eta+v) \\
& \frac{d g}{d b^{2}}=+\frac{9}{4 m}\left(1+y^{s} g g\right) \text { fin }(2 r-v)+\frac{3}{4 m}\left(1+\frac{g}{4} g g\right) \text { in }(2 y+v) \\
& -\frac{1}{2 m}(1+3 g g) \text { fin } v-i \text { fin } v \\
& +\frac{38}{4 m} \text { in } 2 \pi+\frac{3 g}{2 m} \text { in }(2 q-2 v)+\frac{3 g}{4 m} \sin (2 y+2 x)-\frac{g}{4 m} \text { fin } 2 v \text {. }
\end{aligned}
$$$$
\text { XXIV. }
$$

Fingamus ergo primo:

$$
\xi=\hat{A} \operatorname{cor} 2 \xi+3 \operatorname{cor}(2 \xi-v)+C \operatorname{cor}(2 \eta+v)
$$

vbi notandum eft terminos binos pofteriores, vei in differentiali, multo effe minores primo. Quare cum etiam in differentialibus et $d v$ duplicis generis termini occurrant, guorum pofteriores prae primis fint valde parui, in differentiatione folius primi termini totum differentialis $d n$ valorem pono, in duobus vero religuis tantum valorem principalem; fic prodibie

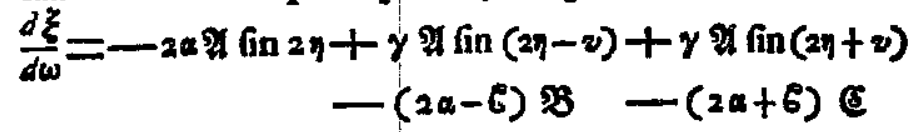

Collato ergo hoc differentiali cum forma propolita obtinetur:

ADIT

bimus

iendis

$$
\begin{aligned}
& x=\frac{3}{2 m \alpha}\left(1+\frac{3}{8} g g\right) \\
& (2 \alpha-6) B=\gamma \alpha+\frac{38}{m} \text { ergo } s=\frac{3(\gamma+2 u g)}{2 m a(2 u-6)} \\
& (2 c+6) c=y+\frac{3 g}{d} \text { ergo } c=\frac{3(y+2 a g)}{2 m a(2 a+b)}
\end{aligned}
$$

Simili modo fingatur :

\section{XXV.}

$y=g+\mathrm{A} \operatorname{cof}(2 \eta-v)+\mathrm{B} \operatorname{cor}(2 n+v)+\mathrm{C} \operatorname{col} v$

$+\mathrm{D} \operatorname{col} 2 \eta+\mathrm{Ecor}(2 \eta-2 v)+\mathrm{E} \operatorname{cor}(2 \eta+2 v)+\mathrm{G} \operatorname{cof} 2 v$ $+\mathrm{H} \operatorname{cor} 4 \eta+\mathrm{J} \operatorname{cor}(4 \eta-2 v)+\mathrm{K} \operatorname{cor}\left(4 \eta \frac{1}{2} 2 v\right)$

bi linea prior continet terminos multo maiores, quam binae inferiores. Hinc ergo fit differenciando fecua dum regulam fupra datam:

$\frac{d y}{d}=-(2 \alpha-6) \mathrm{A}$ fin $(2 y-v)-(2 x+6) \mathrm{B} \operatorname{fin}(2 \eta+v)-\operatorname{secin} v$

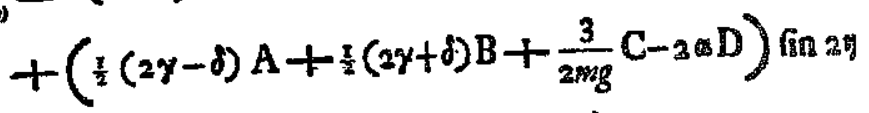$$
+\left(\frac{r}{2}(2 \gamma-\delta) A-\frac{9}{8 n g} C-2(\alpha-6, E) \text { fin }(2 x-2 x)\right.
$$$$
+\left(\frac{\pi}{2}(2 \gamma+\delta) B-\frac{3}{8 m g} C-2(a+6) F\right) \operatorname{lin}(2 y+2 w)
$$$$
+\left(\frac{x}{2} 8-\frac{3 A+9 B}{8 m g}-26 G\right) \text { fin } 2 v
$$

$$
+\left(\frac{3 A+9 B}{8 m g}-4 \pi H\right) f(n 4 \pi
$$

$+\left(-\frac{9 A}{8 m g}-2(2 \alpha-6) J\right) \mathrm{fin}(4 \eta-2 v)$

$+\left(-\frac{3 B}{8 m g}-2(2 a+6) K\right)_{\mathrm{Pp}_{3}}$ in $(4 m+2 v)$ 
Hincque elicientur fequentes coefficientium valores :

$$
\begin{aligned}
& (2 \alpha-6) A=-\frac{9}{45}\left(1+x^{5} g g\right) \\
& (2 a+6) B=-\frac{3}{4 m}(1+39 g) \\
& \mathrm{CC}=\frac{\mathrm{I}}{2 m}\left(\mathrm{I}+\frac{x}{4} g g\right)+i \\
& 2 \alpha \mathrm{D}=\frac{\pi}{2}(2 \gamma-\delta) A+\frac{\pi}{2}(2 \gamma+d) B+{ }_{2 m g}^{3} C-\frac{38}{4 m} \\
& 2(\alpha-E) \mathrm{E}=\frac{x}{2}(2 y-\delta) \mathrm{A}-\frac{9}{8 m g} \mathrm{C}-\frac{3 g}{2 m} \\
& 2(\alpha+5) \mathrm{E}=\frac{\mathrm{x}}{2}(2 \gamma+\delta) \mathrm{A}-\frac{3}{8 \mathrm{mg}} \mathrm{C}-\frac{38}{4^{23}} \\
& 26 \mathrm{G}=-\frac{3 \mathrm{~A}+9 \mathrm{~B}}{8 m g}+\frac{x}{2} \delta \mathrm{C}+\frac{\mathrm{g}}{4^{2 m}} \\
& { }_{4} \mathrm{H}=\frac{3 \mathrm{~A}+9 \beta}{8 \operatorname{mg} g} ; 2(2 \alpha-6) \mathrm{J}=-\frac{9}{8 m g} A \\
& 2(2 \alpha+6) \mathrm{K}=-\frac{3}{8 m g} \mathrm{~B} \\
& \text { XXVL. }
\end{aligned}
$$

Cum igitar his inuentis valoribus fit multo verius: Porro ob $\left.\frac{1}{q}=\frac{\mathrm{I}}{\mathrm{g}}-\frac{\mathrm{A}}{\mathrm{gg}} \operatorname{cor}(2 \mathrm{~g}-v)-\frac{\mathrm{B}}{\mathrm{gg}} \operatorname{cor}(2 \eta+v)\right)^{\prime-\frac{C}{g g}} \operatorname{col} v$ erit

$$
\begin{aligned}
& \frac{d \varphi-d v}{d w}=\frac{9}{4 m g}\left(1+\frac{7}{2} g g\right) \operatorname{cor}(2 y-v)-\frac{3}{4 m g}\left(1+\frac{5}{4} g g\right) \operatorname{cof}(2 y+v) \\
& +\frac{1}{2 m g}\left(\mathrm{I}+\frac{3}{2} g g\right) \mathrm{cfv}+\frac{i}{g} \mathrm{cf}+\frac{1}{4^{m}}\left(\mathrm{I}-\frac{9 \mathrm{~A}+3_{3} \mathrm{~B}-2 \mathrm{C}}{2 g g}\right) \\
& +\frac{1}{4^{m}}\left(3-\frac{\mathrm{A}-\mathrm{B}-3 \mathrm{C}}{g g}\right) \mathrm{cf} 2 \eta+\frac{1}{4 m}\left(6-\frac{2 \mathrm{~A}-9 \mathrm{C}}{2 g g}\right) \mathrm{Cr}(2 q \cdot 2 v) \\
& -\frac{1}{4^{m}}\left(3+\frac{2 \mathrm{~B}-3 \mathrm{C}}{2 g g}\right) \mathrm{ff}(2 n+2 v)+\frac{1}{4 m}\left(1+\frac{3 \mathrm{~A}-9 \mathrm{~B}-2 \mathrm{C}}{2 g g}\right) \mathrm{d}(2 v \\
& +\frac{3 A-9 B}{8 m g g} \operatorname{cof} 4 i-\frac{9 A}{8 m g g} \operatorname{co}(4 y-2 v)+\frac{3 B}{8 m g g} \operatorname{co} \int(4 y+2 v)
\end{aligned}
$$

XXVII.

Ponatur ad abbreuiandum:

$$
\begin{aligned}
& m\left(1+\frac{x}{2} 8 B-C\right)-1=\alpha ; 2 m g=y \\
& m\left(\frac{3}{2} 2+A+B\right)=e ; v t \text { fic }
\end{aligned}
$$

$\frac{d \eta}{d \omega}=a-\gamma \cdot \operatorname{cor} v-\operatorname{cor} 2 \eta$

$-m A \operatorname{cor}(2 v-2 v)-m B \operatorname{cor}(2 \eta+2 v)+m\left(\frac{\pi}{2} g g n C\right) \operatorname{co}(2 \psi$

Porro fit

$\xi=4 \operatorname{cor} 2 y$ et $q=g+A \operatorname{cor}(2 y-v)+B \operatorname{cor}(2 y+v)+C \operatorname{cr} s$

$$
\begin{aligned}
& m\left(1+\frac{1}{2} 8 g-C\right)-\frac{1}{4 m}\left(1-\frac{9 A+3 B-2 C}{2 g g}\right)=6 \\
& 2 m g+\frac{1}{2 m g}+\frac{3 g}{8 m}+\frac{i}{g}=8 \\
& +m\left(\frac{3}{2} 9+A+B\right)+\frac{1}{4 m}\left(3-\frac{A-B m-3}{g g}\right)=8
\end{aligned}
$$


XXIX.

$m A+\frac{1}{4 m}\left(6-\frac{2 A-9 C}{2 g g}\right)=1$

$m B-\frac{1}{4 m}\left(3+\frac{2 B-3 C}{2 g s}\right)=0$

$m\left(\mathrm{C}-\frac{x}{2} g\right)+\frac{1}{4 m}\left(1+\frac{3 \mathrm{~A}-9 \mathrm{~B}-2 \mathrm{C}}{2 g g}\right)=\dot{x}$

ve habeatur

$\frac{d v}{d w}=6-\delta \operatorname{cor} v-\frac{9}{4^{m i g}} \operatorname{cor}(2 y-v)+\frac{3}{4^{m g}} \operatorname{cor}(2 y+v)$

$-\{\operatorname{co}(2 y-y) \operatorname{cof}(2 y-2 v)-\theta \operatorname{cor}(2 y+2 v)-x \operatorname{col} 2 v$

$-\frac{3 A+g B}{8 m g g} \operatorname{cof} 4 q+\frac{9 A}{8 m g g} \operatorname{cor}(4 \eta-2 v)-\frac{3 B}{8 m g g} \operatorname{cor}\left(4^{n+2 v)}\right.$

vbi caueatur, ne coefficientes $\eta, \theta$, cum angulis cognominibus confundantur.

\section{XXVII.}

Opus plane non eft, vt valores litteramm $\varepsilon$ et $q$ accuratius determinemus, atque ad plures terminos, quam ante inuenimus, expediamus; verum hos ipfos terminos, quos ante inuenimus, accuratius obtinebimus, fi litteris $a$ et 6 eos valores tribuemus, quos nunc eis conuenire collegimus. Pluribus autem terminis non indigebimus tam ad longitudinem lunae $\varphi$, quam ad eius anomaliam veram $v$ fatis exake definiendam. Verum ad hoc ipfirm negotium valores differentiales $\frac{d \omega}{d \omega}$ et $\frac{d \omega}{d \omega}$, ac praecipue hunc pofteriorem; quo motus apogei continetur, accuratius euolui oportet, quoniam imprimis in motu medio apogei minimise particulae ingentis mo. menti efle poffunac. $\therefore$

XXIX.
Cum igitur accuratius quam adhuc affumfomus tie

$$
\xi=9 \operatorname{cor} 2 \eta+2 \operatorname{cor}(2 \eta-v)+(5 \operatorname{cor}(2 \eta+v) \quad e r
$$

$q=g+\mathrm{A} \operatorname{cor}(2 y-v)+\mathrm{B} \operatorname{cor}(2 \eta+v)+\mathrm{C} \operatorname{cor} v$

$$
\begin{aligned}
& +\mathrm{D} \operatorname{co} 2 \eta+\mathrm{Ecr}(2 x-2 v)+\mathrm{ECr}(2 \eta+2 v)+\mathrm{Cef} z \\
& +\mathrm{HcoC}_{4 v}+\mathrm{Jer}(4 v-2 v)+\mathrm{Kcr}(4 v+2 v)
\end{aligned}
$$

erit terminus ad quartum vsque ordinem extenfis

I.

11.

$\frac{d \varphi}{d \omega}=m\left(1+\frac{\pi}{2} g g-C\right)-\left(2 m g-\frac{3}{2} m g C+m G\right) \operatorname{col} v$

III.

$-m\left(\frac{3}{2} 2+\mathrm{A}+\mathrm{B}\right) \operatorname{cof} 2 \eta-m \mathrm{~A} \operatorname{cof}(2 \eta-2 v)-m \mathrm{~B} \operatorname{cor}(2 \eta+2 v)$

$$
-2 a\left(\mathrm{C}-\frac{1}{2} 8 g\right) \operatorname{coc} 2 v
$$

\section{IV.}

$+m\left(\frac{3}{2} g \mathcal{X}-\frac{3}{2} \cdot \mathfrak{B}+g \mathrm{~A}+\frac{3}{2} g \mathrm{~B}-\mathrm{D}-\mathrm{E}\right) \operatorname{cor}(2 \eta-z)$

$+m\left(1 g A-\frac{1}{2} C+g B+\frac{1}{2} g A-D-F\right) \operatorname{col}(2 y+z)$

$+m\left(\frac{1}{2} g A-E\right) \cot (2 x-3 v)+m\left(\frac{\pi}{2} g B-F\right) \operatorname{cof}\left(2 y+3 z^{\circ}\right)$ $+m\left(\frac{\pi}{2} g \mathrm{C}-\mathrm{G}\right) \operatorname{col} 3 v$

$-m(\mathrm{H}+J) \operatorname{cof}(4 y-v) \rightarrow m(\mathrm{H}+\mathrm{K}) \operatorname{cor}(4 y+v) m m J \operatorname{col}(4 y-3 v)$

$$
-\pi x \operatorname{cor}(4 y+3 x)
$$

vnde cum effet ante $\gamma=2 m$, nunc accuratius exit

$$
\gamma=2 m g-\frac{3}{2} m g \mathbf{C}+m G
$$

Q9

XXX. 


\section{XXX.}

Deinde cum nunc quoque fit accuratius:

II.

$\frac{1}{q}=\left(\frac{1}{g}+\frac{A A+B B+C C}{2 g^{3}}\right)$

III.

$$
-\frac{\mathrm{A}}{g g} \operatorname{cor}(2 \eta-v)-\frac{\mathrm{B}}{g g} \operatorname{cor}(2 \eta+v)-\frac{\mathrm{C}}{g g} \operatorname{cor} v
$$

IV.

$+\left(\frac{(\mathrm{A}+\mathrm{B}) \mathrm{C}}{g^{3}}-\frac{\mathrm{D}}{g g}\right) \cos 2 \eta+\left(\frac{\mathrm{AC}}{\mathrm{g}^{3}}-\frac{\mathrm{E}}{g g}\right) \operatorname{cor}(2 \eta-2 \eta)$

$+\left(\frac{B C}{g^{3}}-\frac{F}{g g}\right) \cos (2 \eta+2 v)+\left(\frac{2 A B+C C}{2 g^{3}}-\frac{G}{g g}\right) \cos 2 v$

$+\left(\frac{\mathrm{AB}}{g^{3}}-\frac{\mathrm{H}}{g g}\right) \cos 4 \eta+\left(\frac{\mathrm{AA}}{2 g^{3}}-\frac{\mathrm{J}}{g g}\right) \operatorname{cor}(4 \eta-2 v)$

$$
+\left(\frac{\mathrm{BB}}{g^{2}}-\frac{\mathrm{K}}{g 8}\right) \operatorname{cor}(4 \eta+2 v)
$$

Hinc quoque ad terminos guarti ordinis vsque valor formulae $\frac{d \phi-d v}{d \omega}$ definiri polfet, fed expreffio prodiret tantopere complicate, vt eius euolutio fummam requirerec patientiam; neque tamen hic labor vllius foret vfus, nili forte in motu apogei exactius eruendo: iplae enim inaequalitates nullius forent momenti ; propterea quod error in anomalia commiffus multo minorem errorem in longitudine producit.
XXXI.

Ponatur ergo longițudo apogei :

$\varphi-v=$ Cont.

$+\mathrm{A}^{\prime} \mathrm{fin}(2 n-v)+\mathrm{B}^{\prime} \operatorname{fin}(2 y+v)+\mathrm{C}^{\prime} \sin 2 v$ $+\Delta \omega+D / f i n 2 \eta+E /(i n(2 \eta-2 v)+F / \sin (2 \eta+2 z)+G / \sin 2 v$

$+\mathrm{H}^{\prime}$ (in $4 \eta+\mathrm{J}$ fin $(4 \eta-2 v)+\mathrm{K}$ fin $(4 \eta+2 v)$ et eric differentiando:

$$
\frac{d \varphi-d v}{d(0)}=
$$

$(2 a-6) A^{\prime} \operatorname{cor}(2 y-v)+(2 a+6) B^{\prime} \operatorname{cor}(2 y+v)+6 C^{\prime} \operatorname{cor} z$ $+\Delta-\frac{x}{2} \delta C^{\prime}+\frac{9 A^{\prime}}{8 m g}+\frac{3 B^{\prime}}{8 m g}-\epsilon D^{\prime}-(m A-\eta) E^{\prime}$

$$
-(m \mathrm{~B}+\theta) \mathrm{F}^{\prime}-\mathrm{G}^{\prime}-\frac{9 \mathrm{AJ}}{8 m g g}-\frac{3 \mathrm{BK}}{3 m g g}
$$

$$
\cos 2 n\left(-\frac{\pi}{2}\left(2 \gamma-\delta^{8}\right) A^{\prime}-\frac{\pi}{2}(2 \gamma+\delta) B^{\prime}-\frac{3 C^{\prime}}{4 m g}+2 \alpha D^{\prime}\right.
$$

$\operatorname{col}(2 \pi-2 v)\left(-\frac{x}{2}(2 \gamma-\delta) A^{\prime}-\frac{9 O}{8 m g}+2(\alpha-6) E^{\prime}\right.$

$$
\operatorname{cor}(2 y+2 v)\left(-\frac{x}{2}(2 y+\delta) B^{\prime}+\frac{3 C^{\prime}}{8 m g}+2(\alpha+6) F^{\prime}\right.
$$

$$
\operatorname{cof} 2 v\left(-\frac{\mathrm{t}}{2} \delta \mathrm{C}^{\prime}-\frac{3 \mathrm{~A}^{\prime}}{8 m g}-\frac{9 \mathrm{~B}^{\prime}}{8 m g}+26 \mathrm{G}^{\prime}\right.
$$$$
\operatorname{cor} 4 \eta\left(-\frac{3 A^{\prime}}{8 m g}-\frac{9 B^{\prime}}{8 m g}+4 m H^{\prime \prime}\right.
$$$$
\operatorname{cof}(4 \eta-2 x)\left(\frac{9 A^{\prime}}{8 m g}+2(2 \alpha-6) J^{\prime}\right.
$$

$\operatorname{cor}(4 \eta+2 v)\left(\frac{3 B^{\prime}}{8 m g}+2(2 \alpha+6) K^{\prime}\right.$

Qq 2 


\section{XXXIII.}

Calculo autem praccipue in primis terminis accuratius expedito eft :

$$
\begin{aligned}
& \frac{d \varphi-d v}{d \omega}= \\
& +\frac{1}{4^{m g}} \operatorname{cof}(2 y-v)\left(9+{ }^{2 x} g g+\frac{27 \mathrm{AA}+18 \mathrm{BB}+15 \mathrm{CC}}{4 g g}\right. \\
& \left.-\frac{3 \mathrm{AB}+2 \mathrm{AC}+\mathrm{BC}}{g g}-\frac{2 \mathrm{D}-2 \mathrm{E}+3 \mathrm{G}+3 \mathrm{H}-9 \mathrm{~J}}{2 g}\right) \\
& +\frac{1}{4^{n m g}} \operatorname{cor}(2 n+2)\left(-3-\frac{3}{4} g g-\frac{6 \mathrm{AA}-9 \mathrm{BB}+15 \mathrm{CC}}{4 g g}\right. \\
& \left.+\frac{9 \mathrm{AB}+\mathrm{AC}+2 \mathrm{BC}}{g g}-\frac{2 \mathrm{D}-2 \mathrm{~F}-9 \mathrm{G}-9 \mathrm{H}+3 \mathrm{~K}}{2 g}\right) \\
& +\frac{1}{2 m g} \operatorname{cor} v\left(1+3 g g+\frac{2 A A+2 B B+3 C C}{4 g g}\right. \\
& \left.+\frac{A B+6 A C+6 B C}{2 g g}-\frac{3 D-3 E-3 F-G}{2 g}+2 m i\right) \\
& +\frac{1}{4^{m}}\left(1-\frac{9 A+3}{2 g g}\right) \\
& +\frac{1}{4^{m}}\left(3-\frac{A-B-3}{g g}\right) \operatorname{cor} 2 y+\frac{1}{4^{m}}\left(1+\frac{3 A-9 B-2 C}{2 g g}\right) \operatorname{cor} 2 v \\
& +\frac{1}{4 m}\left(6-\frac{2 A-9 C}{2 g g}\right) \mathrm{r}(2 \eta-2 v)-\frac{1}{4 m}\left(3+\frac{2 B-3 C}{2 g g}\right) \mathrm{c}(2 \eta+2 v) \\
& +\frac{3 A-9 B}{8 m g g} \operatorname{cor} 4 i-\frac{9 A}{8 m g g} \operatorname{cor}(4 \eta-2 v)+\frac{3 B}{8 m g g} \operatorname{cor}(4 \eta+2 v)
\end{aligned}
$$

Simili
Comparatione autem infticuta reperitur: $(2 a-6) A=\frac{1}{4 m g}\left(9+3^{2} B g+\frac{27 A A}{3+B 8 B B+15 C C}\right.$ $\left.-\frac{3 A B+2 A C+B C}{g g}-\frac{2 D-2 E+3 G+3 H-9 J}{2 g}\right)$ feu

$$
\begin{aligned}
& (2 a-6) A=-\frac{1}{4^{m}}(9+x g g+: C) \\
& (2 a+6) B=-\frac{1}{4 m}\left(3+\frac{9}{4} g g+3 C\right) \\
& 6 \mathrm{C}=\frac{1}{2 m}\left(1+\frac{x}{4} g g+3+2 m i\right) \\
& \text { XXXIII. }
\end{aligned}
$$

$(2 \alpha-6) A^{\prime}=-\frac{1}{g}(2 a-6) A+\frac{1}{4 m g}\left(\frac{3}{2} g g-\frac{2}{2} C+\frac{27 A A+18 B B+1 g C C}{4 s g}\right.$ $\left.-\frac{3 A B+2 A C+B C}{B g}-\frac{2 D-2 E+3 G+3 H-9 J}{2 g}\right)$

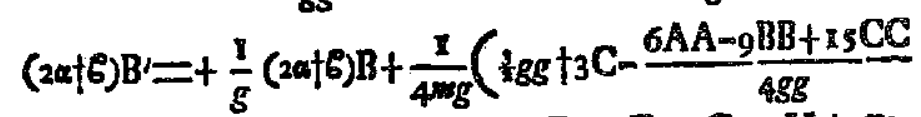
$\left.+\frac{9 \mathrm{AB}+\mathrm{AC}+2 \mathrm{BC}}{g g}-\frac{2 \mathrm{D}-2 \mathrm{~F}-9 \mathrm{G}-9 \mathrm{H}+{ }_{3} \mathrm{~K}}{2 g}\right)$

$$
\begin{array}{r}
6 C^{\prime}=+\frac{1}{g} 6 C+\frac{1}{2 m g}\left(\frac{1}{2} g g-\frac{3}{4} A+\frac{2 A A+2 B B+3 C C}{48 g}\right. \\
\left.+\frac{A B+6 A C}{25 g}+6 B C-\frac{3 D-3 E-3 F-G}{2 g}\right) \\
\text { Qq } 3 \quad \text { Quibas }
\end{array}
$$


Quibus valoribus fubftitutis obtinebitur pro apogei motu medio, qui in termino $\Delta \omega$ continetur:

$$
\begin{aligned}
& \Delta=\frac{1}{4^{m}}+\frac{2 m g \delta-1}{4 m g g} \mathrm{C} \\
& +\frac{f}{46 m g}\left(\frac{x}{2} g g-\frac{3}{4} A+\frac{2 A A+2 B B+3 C C}{4 g g}\right. \\
& \left.+\frac{A B+6 A C+6 B C}{2 g g}-\frac{3 D-3 E-3 F-G}{2 g}\right) \\
& \frac{9}{32} \frac{9}{(2 a-6)}\left(\frac{3}{2} g g-g \mathrm{C}+\frac{27 \mathrm{AA}+18 \mathrm{BB}+15 \mathrm{CC}}{18 g}\right. \\
& \left.-\frac{3 \mathrm{AB}+2 \mathrm{AC}+\mathrm{BC}}{g g}-\frac{2 \mathrm{D}-2 \mathrm{E}+3 \mathrm{G}+3 \mathrm{H}-9 \mathrm{~J}}{2 g}\right) \\
& \frac{3}{32}\left(\frac{3}{2 a+6}\right) \mathrm{mmgg}\left(\frac{3}{2} g g+3 \mathrm{C}-6 \mathrm{AA}=9 \mathrm{BB}+15 \mathrm{CC}\right. \\
& \left.+\frac{9 A B+A C+2 B C}{g g}-\frac{2 D-2 F-9 G-g H+3 K}{2 g}\right) \\
& +{ }^{\prime} D^{\prime}+(m A-y) E^{\prime}+(m B+\theta) F^{\prime}+x G^{\prime}+\frac{9 A_{2} J^{\prime}}{8 m g g}+\frac{3 B^{\prime} K^{\prime}}{8 n g g}
\end{aligned}
$$

Quae expreffio, cum omnino fit fimilis illi, quae methodo praecedente eft inuenta, nullum etiam dubium relinquit, quin et hine motus apogei proditurus fit oblerugtionibus conformis; ideoque littera illa $i$ ounitri poteric.

\section{XXXIV.}

Hine igitur patet ad motum apogei definiendum $\mathrm{r}$. lores litterarum $\mathbf{A}, \mathbf{B}, \mathbf{C}$ et $\mathbf{A}^{\prime}, \mathbf{B}^{\prime}, \mathbf{C}^{\prime}$ fumma accuratione inueftigari debere, qui cum conftent partibus duplicis ordinis, eciam fi partes pofterioris ordinis prae primo primo admodum videaneur paruas, eas temen onni cu. ra euolui oportet, propterea quod pro motu apogei gartes primi ordinis fe deftruunt. Quod cum in determinatione reliquorum coefficientium vfi mon eueniat, igg his quoque non erit opus, vt partes iltae minores in compucum ducantur, fed fufficier partibus principalibus vi. Scilicet etfi determinatio literae $\Delta$ maxime eft lilo brica, atque a reliquorum coeficientium exactifimis va loribus pendet, reliqui tamen coefticientes tantam follertiam minime requirunt, fed facis exalte fine tanta opera detiniri poffunt

\section{XXXV.}

Valores ergo reliquorum cocficientium fequenti modo neglectis exiguis particulis ita fe habebunt,

$$
\begin{aligned}
& (2 a-6) A^{\prime}=\frac{9}{4 m g} ; \quad(2 a+6) B^{\prime}=-\frac{3}{4} ; \quad 6 C^{\prime}=\frac{1}{2 m g} . \\
& 2 a D^{\prime}=\frac{1}{2}(2 \gamma-\delta) A^{\prime}+\frac{x}{4}(2 \gamma+\delta) B^{\prime}+\frac{3 C^{\prime}}{4^{m g}}+\frac{1}{4^{m}}\left(3-\frac{A-B-3 C}{g g}\right) \\
& 2(\alpha-6) E^{\prime}=\frac{7}{2}(2 \gamma-8) A^{\prime}+\frac{9 C^{\prime}}{8 \operatorname{lng} g}+\frac{1}{4}\left(6-\frac{2 A-9 C}{2 g g}\right) \\
& 2(\alpha+6) F^{\prime}=\frac{x}{2}(2 \gamma+\delta) B^{\prime}-\frac{3 C^{\prime}}{8 m g}-\frac{1}{4 m}\left(3+\frac{2 B-3 C}{2 g g}\right) \\
& 26 \mathrm{G}^{\prime}=\frac{3}{2} 8 \mathrm{C}+\frac{3 \mathrm{~A}^{\prime}+9 \mathrm{~B}^{\prime}}{8 m g}+\frac{1}{4 m}\left(1+\frac{3 \mathrm{~A}^{2} \mathrm{~g}-2 \mathrm{C}}{2 g g}\right) \\
& 48 \mathrm{H}^{\prime}=\frac{3 \mathrm{~A}^{\prime}+9 \mathrm{~B}^{\prime}}{8 m g}+\frac{8 \mathrm{~A}-9 \mathrm{~B}}{8 m g}=0 \\
& 2(2 a-6) J^{\prime}=-\frac{9 A^{\prime}}{8 m g}-\frac{9 A}{8 m g g}=0 \mid 2\left(2 \alpha+6, K^{\prime}=-\frac{3 l^{\prime}}{8 m g}+\frac{3}{8 m g g}=\right. \\
& \text { ob } A^{\prime}=-\frac{A}{g} ; B^{\prime}=\frac{B}{g} \text { er } C=\frac{C}{g} \text { prosime. }
\end{aligned}
$$




\section{XXXVI.}

Cum autem fit proxime: $y=2 \mathrm{mg} ; \gamma=2 \mathrm{mg}+\frac{\mathrm{I}}{2 \mathrm{mg}}$; his valoribus quoque fubftitutis fiet:

$(2-6) A^{\prime}=\frac{9}{4^{m g}} ;(2 \alpha+6) B^{\prime}=-\frac{3}{4 m g} ; \quad+C=\frac{1}{2 m g} ;$ fiue:

$$
\begin{aligned}
\mathrm{A}^{\prime} & =-\frac{\mathrm{A}}{g} ; \mathrm{B}^{\prime}=\frac{\mathrm{B}}{g} ; \mathrm{C}^{\prime}=\frac{\mathrm{C}}{\mathrm{g}} ; \\
2 \times \mathrm{D}^{\prime} & =\frac{3}{4 m}-\mathrm{A}+3 m \mathrm{~B} ;
\end{aligned}
$$

$2(\alpha-6) E^{\prime}=\frac{3}{2 m}-m A$

$2(\alpha+6) \mathrm{F}^{\prime}=-\frac{3}{4^{3}}+3 m \mathrm{~B}$

$$
26 \mathrm{G}^{\prime}=\frac{1}{4 m}+m \mathrm{C}
$$

et reliqui coefficientes $H^{\prime}, J^{\prime}, \mathbf{K}^{\prime}$ pro euanefcentibus funt habendi. Valores autem litterarum $A, B, C$, etc. §. 25. funt exhibiti.

\section{XXXVIL}

Quaeramus nunc quoque longitudinem lunse $\varphi$, huscque in finem fingamus:

$$
\varphi=\text { Conit. }
$$

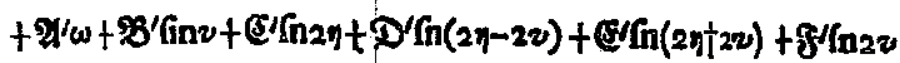

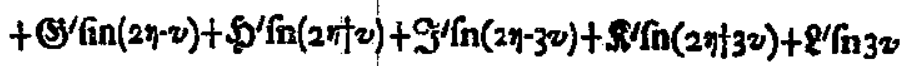

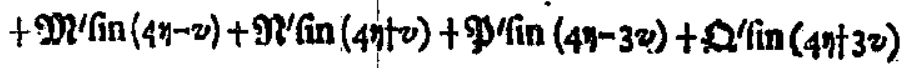

ac differentiatione inftieut obtinebimus:

$\frac{d \varphi}{d \omega}=+2$

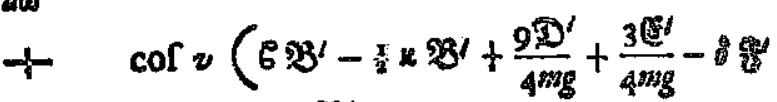

$+\quad \operatorname{cor} 2 \pi\left(-\frac{3 \mathfrak{B}^{\prime}}{4^{m g}}+2 a \mathrm{C}^{\prime}\right.$

$+\operatorname{cor}(2 \eta-2 v)\left(-\frac{9 \mathfrak{B}^{\prime}}{8 m g}+2(z-E) D^{\prime}\right.$

$+\operatorname{col}(2 \eta+2 v)\left(+\frac{3 \mathfrak{B}^{\prime}}{8 m g}+2(\alpha+6) c^{\prime}\right.$

$+\quad \cos 2 v\left(-\frac{1}{2} \delta \xi^{\prime}+26 g^{\prime}\right.$

$+\operatorname{cor}(2 \eta-v)\left(-\frac{\pi}{2} \xi B^{\prime}-\frac{\pi}{2} \eta B^{\prime}-\gamma C^{\prime}-(\gamma-\delta) D^{\prime}+\frac{3 f^{\prime}}{4 m g}\right)(2 u-6) S^{\prime}$

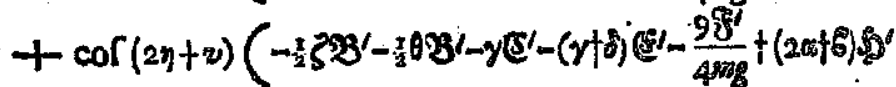

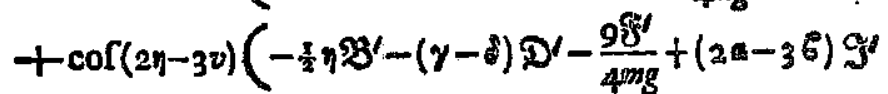

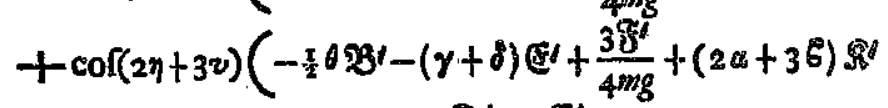

$+\quad \operatorname{cor} 8 v\left(-\frac{\pi}{2} \times \mathfrak{B}^{\prime}-\frac{3 \mathfrak{D}^{\prime}}{4 m g}-\frac{9 G^{\prime}}{4 m g}-\delta g^{\prime}+36\right.$

$+\operatorname{cor}(4 \eta-v)\left(-\frac{3 A+9 B^{\prime}}{16 m g g}+\frac{9 A}{16 m g g}-\frac{3 B^{\prime}}{4 m g}+(40-6) 997\right.$

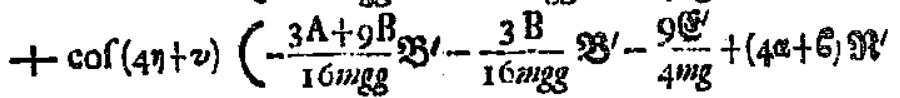

$+\operatorname{cor}(4 \eta-3 v)\left(+\frac{9 \AA}{16 m g g} 3^{\prime}+\frac{9 D^{\prime}}{4 m g}+(4 \alpha-36) y^{\prime}\right.$

$+\operatorname{cor}(4 \eta+3 v)\left(-\frac{3 B}{16 m a g} 3^{\prime}+\frac{3 E^{\prime}}{4 m g}+(4 x+36) D^{\prime}\right.$

XXXVII 
XXXVill.

Comparata iam hac forma cum valore ipfius $\frac{d \varphi}{d \omega}$ in $\$ .29$. exhibito, obtinebitur

$$
\mathscr{A}=\frac{x}{3} \delta \mathscr{B}^{\prime}+m\left(1+\frac{x}{2} g g-\mathrm{C}\right)
$$

$\mathfrak{C B}^{\prime}=\frac{x}{2} \times \mathfrak{B}^{\prime}-\frac{9 \mathcal{D}^{\prime}-3 \mathrm{C}^{\prime}}{4^{m g}}+\delta \mathscr{F}^{\prime}-2 m g+\frac{3}{2} m g \mathrm{C}-m \mathrm{G}$

$2 a \mathfrak{C}^{\prime}=\frac{3 \mathfrak{g} \prime}{4 m g}-m\left(\frac{3}{2} \mathfrak{A}+\mathrm{A}+\mathrm{B}\right)$

$2(\alpha-6) D^{\prime}=\frac{9 \mathfrak{B}^{\prime}}{8 m g}-m \mathrm{~A}$

$2(a+E) E^{\prime}=-\frac{3 B^{\prime}}{8 m g}-m \mathrm{~B}$

$$
26 \mathrm{~g}^{\prime}=\frac{\mathrm{x}}{2} \delta^{2} \mathfrak{B}^{\prime}-m\left(\mathbf{C}-\frac{x}{2} \&\right)
$$

$(2 \alpha-\varepsilon) \mathcal{G}^{\prime}=\frac{3}{2}(\xi+y) B^{\prime}+\gamma \mathbb{C}^{\prime}+(y-8) \mathbb{D}^{\prime}-\frac{3 g^{\prime}}{4 m g}$

$$
+m\left(\frac{3}{2} g \boldsymbol{A}-\frac{3}{2} \mathfrak{B}+g \mathrm{~A}+\frac{z}{2} g \mathrm{~B}-\mathrm{D}-\mathrm{D}-\mathrm{E}\right)
$$

$(2 \alpha+b) \mathfrak{d}^{\prime}=\frac{\gamma}{2}(\zeta+\theta) \mathscr{B}^{\prime}+\gamma\left(\mathbb{E}^{\prime}+(\gamma+\delta) \mathbb{E}^{\prime}+\frac{9 \mathscr{g}^{\prime}}{4 \mathrm{mg}^{\prime}}\right.$

$$
+m\left(\frac{3}{2} g \mathfrak{A}-\frac{3}{2}\left(C+g B+\frac{x}{2} g A-D-F\right)\right.
$$

$(2 \alpha-3 b) S^{\prime}=\frac{1}{2} \eta \mathcal{B}^{\prime}+(\gamma-\delta) D^{\prime}+\frac{9 S^{\prime}}{4 m g}+m\left(\frac{x}{2} g A-E\right)$

$(2 a+3 b) \mathbb{S}^{\prime}=\frac{1}{2} \theta \mathfrak{B}^{\prime}+(\gamma+\delta) \mathbb{g}^{\prime}-\frac{3 \mathbb{F}^{\prime}}{4^{m g}}+m\left(\frac{3}{2} g B-F\right)$

$$
3 E^{\prime}=\frac{x}{2} \times B^{\prime}+\frac{3 D^{\prime}+9 g^{\prime}}{4^{m g}}+\delta g^{\prime}+m\left(\frac{x}{2} g C-G\right)
$$

$\left(4 a-6 ; D 2=-\frac{6 \mathrm{~A}-9 \mathrm{~B}}{16 \mathrm{mgg}^{\prime}} \mathrm{B}^{\prime}+\frac{3 \mathrm{D}^{\prime}}{4^{m g}}-m(\mathrm{H}+\mathrm{J})\right.$

$(4 \alpha+6) \mathfrak{N}^{\prime}=+\frac{3 \mathrm{~A}-6 \mathrm{~B}}{16 \mathrm{~m}^{\prime} g} \mathfrak{B}^{\prime}+\frac{9 \mathrm{E}}{4 m g}-m(\mathrm{H}+\mathrm{K})$

$\left(4^{\alpha-3 E)} \mathfrak{Y}=-\frac{9 A^{\prime}}{16 m g g} \mathfrak{B}^{\prime}-\frac{9 D^{\prime}}{4 m g}-m \mathrm{~J}\right.$

$(4 a+36) Q^{\prime}=+\frac{3 B^{B}}{16 m g} 2 B^{\prime}-\frac{3 \mathbb{E}^{\prime}}{4^{m g}}-m \mathrm{~K}$

$\mathbf{x x x t x}$

\section{XXXIX.}

Inuentis iam valoribus litterarum $p=b(I+\xi)$ et $q$

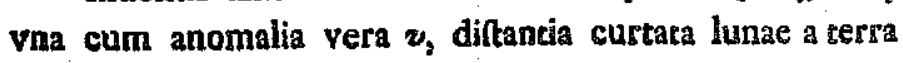
$*=\frac{p}{\mathrm{I}-q \mathrm{co} v}$ cognofcecur : ac fi deinceps latitudinis lu-

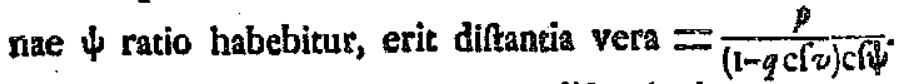
In Aftronomia autem non tam diftantia lunae, quam cius diameter apparens et parallaxis requiri folet; qua rum verague cum fit diftantiae lunae a terra reciproce proporzionalis, erit tam diameter apparens quam parallaxis ve $(1-q$ cof $v)$ cof $\psi$; vnde fi veriusque valor medius ex cbferuationibus fuerit definitus, ad quoduis tempus valor verus affignari poterit. Sit igitur fiue diameeri appa. rentis fiue parallaxis horizontalis valor medius $=\sigma$, eritque is pro tempore dato $=\frac{b \sigma}{p}(1-q \operatorname{cof} v) \operatorname{cor} \psi$. Eft atstem proxime $\operatorname{cof} \psi=1-\frac{5}{4} \operatorname{tang} \rho^{2}-\frac{5}{4} \operatorname{tg} \rho^{2} c \int_{2}(\varphi-\pi)=\frac{2}{3}$. $+\frac{\lambda+\Pi}{3}$, et $\frac{b}{p}=1-\xi+\xi \xi$ : vnde fit diameter (eu parallaxis $=\frac{x^{2}}{3} \cdot(2+\lambda+\Pi)(1-\xi+\xi \xi)(i-q \operatorname{col}(v)$, quae euoluitur in hane expreffionem : ob $\frac{2+\lambda}{3}=$ i proxime: $\frac{x}{3}(2+\lambda) \sigma\left[1-q \cos v-\xi+q \xi \operatorname{cof} v+\xi \xi+\frac{\pi}{3} \Pi-\frac{\pi}{3} q \Pi \cos v\right]$

$\mathrm{XL}$.

Pro praefenti ergo cafu, quo parallsxin folis, eiusque excentricitacem vna cum inclinatione orbitae luna-

$\mathbf{R}_{\mathbf{2}} \mathbf{2}$ 
ris negligimus, eric lunae diameter apparens vel paral-

laxis horizontalis

$$
=\frac{r}{3}(2+\lambda) \sigma \text { mult. per }
$$

$1-\frac{\pi}{2} \mathbf{C}-\left(g+\frac{1}{2} \mathbf{G}\right) \cot v$

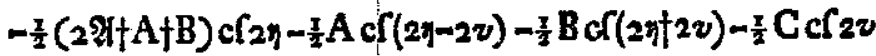

$-\frac{\pi}{2}(2 \mathfrak{B}+\mathrm{D}+\mathrm{E}) \operatorname{cof}(2 \eta-v)-\frac{\pi}{2}(2 \mathrm{C}+\mathrm{D}+\mathrm{F}) \operatorname{col}(2 \eta+v)$

$-\frac{x}{2} \mathrm{E} \operatorname{col}(2 \eta-3 v)-\frac{1}{2} \mathrm{~F} \operatorname{col}(2 \eta+3 v)-\frac{2}{2} \mathrm{G} \operatorname{col} 3 v$

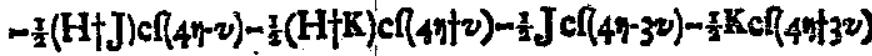

vbi quidem fator conftans ${ }_{3}^{2}(2+\lambda)$ o omitri potet, fiquidem tantum proportio vel diametri apparentis vel parallaxis horizontalis defideretur.

\section{XLL.}

Si nunc hos valores in numeris euoluere velimus, - ex obferuationibus primum colligimus has determinationes:

$\Re=13,3682 ; \Delta=0,1123$ et proxime $g=0,05445$ ac poftremo quidem valore ipfius $g$ tantum in terminis minimis $y t a r$, in maioribus ipfam litteram $g$ relicturus, vt deinceps ex collatione calculi cum obferuationibus accuratius fortaffe determinari poffit. Habemus ergo

$$
13,3682=\frac{x}{2} d 23^{\prime}+m\left(1+\frac{x}{2} 88-C\right)
$$

vnde ob $\mathfrak{B}^{\prime}$, es et $\mathbf{C}$ numeros admodum paruos, ftatim prope colligitur $m=13 ; 3682$. Tựm vero eft prope

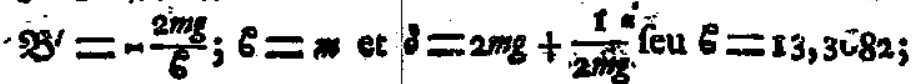

$A D D I T A M E N T U M$

$\delta=2,1419 ;$ hinc $\frac{1}{2} \delta B^{\prime}=-0,1165$, ergo accuratius $m\left(I+\frac{1}{2} g g-C\right)=13,4847=a+1$ et $a=12,4947$

Porro ob $\mathrm{C}=\frac{\mathrm{I}}{2 \mathrm{~mm}}$ erit fatis exacte. $m=13,503 \mathrm{~g}$ vnde ex valoribus AetB proxime colle $\mathrm{tis}$ fit $E=13,0644$ $y=26,95248$.

At vaior ipfius duabus conftat partibus, altera pti $g$ multiplicata altera diuifa, quibus feparatim expreflis erit $d=27,0355 \mathrm{~g}+0,0370 . \frac{1}{g}=2,152 \mathrm{I}$ proxime. XLII.

tert,

i vel
Hinc iam computo infituto fequentes fupra aftumb torum coefficiencium eruuntur valores numerici : $\mathscr{F}=0,008931 ; \mathfrak{B}=0,038958=0,00209 ; \mathfrak{C}=0,01219 g=0,00064$ ideoque

$\xi=0,00893 \mathrm{re}[2 \eta+0,03895 \mathrm{gf}(2 \eta-v)+0,01229 \mathrm{gf}(2 \eta+v)$ Deinde reperitur :

$A=-0,013995 ; B=-0,003460 ; C=f 0,002834$ $\mathbf{D}=-0,001213 \cdot g+0,00002198 \cdot \frac{\mathrm{I}}{\mathrm{g}}=-0,000280$ proxime $E=+0,25834 \cdot g-0,00001889 \cdot \frac{\mathrm{I}}{\mathrm{g}}=+0,014012$ proxime $F=-0,00225 \cdot g-0,00000207 \cdot \frac{\mathrm{l}}{\mathrm{g}}=-0,000161$ proxime $\mathbf{G}=+0,00218 \cdot g+0,00001221 \cdot \frac{\mathrm{I}}{g}=+0,000340$ proxime $H=-0,00001022 \cdot \frac{1}{g}=-0,000184$ $J=+0,00004897 \cdot \frac{1}{g}=+0,000882$

$K=+0,00000053 \cdot \frac{I}{g}=+0,000009$ 


\section{XLIII.}

Hinc porro pro motu apogei eiusque inaequalitatibus colligitur: $\Delta=0,1123$; qui quidem valor ex obferuationibus eft defumtus

$$
\begin{aligned}
& \mathbf{A}^{\prime}=-0,013995 \cdot \frac{1}{g}=-0,25703 \text { proxime } \\
& \mathbf{B}^{\prime}=-0,001460 \cdot \frac{1}{g} \mid=-0,02682 \text { proxime } \\
& \mathbf{C}^{\prime}=+0,002834 \cdot \frac{1}{g} \mid=+0,05205 \text { proxime } \\
& \mathbf{D}^{\prime}=+0,007432 \\
& \mathbf{E}^{\prime}=-0,259170
\end{aligned}
$$

in minutis fecundis

$$
\begin{aligned}
\mathrm{A}^{\prime}=-53018^{\prime \prime} & =-14^{\circ}, 43^{\prime}, 38^{\prime \prime} \\
\mathrm{B}^{\prime} & =-5532 \\
\mathrm{C}^{\prime} & =-10736=-10,32,12 \\
\mathrm{D}^{\prime}=+1533 & =+2,58,56 \\
\mathrm{E}^{\prime} & =-53459=-0,25,33 \\
\mathrm{~F}^{\prime}=-14,50,59 & =-164=-0,7,44 \\
\mathrm{G}^{\prime}=+449 & =-0,7,29
\end{aligned}
$$

Ergo longitudo apogei in minutis fecundis

$$
\varphi-v=\text { Conit. }
$$

$+0,1123 w-53018 \|$ fin $(2 y-v)+5533^{\prime \prime}$ fin 21

$$
\begin{array}{rlll}
-5532 & \text { fin }(2+v) & -53459 & \mathrm{fn}(2 \gamma-2 v) \\
+10736 \text { fin } v & - & 464 & \mathrm{fn}(2 \eta+2 v) \\
& + & 449 \mathrm{fin} 2 v
\end{array}
$$

XLIV.

$A D D I T A M E N T U M$.

1alita-

or ex

XLIV.

lam pro longitudine ipfa inuenienda habentur primo ex \$. 27. valores: $\gamma=\sqrt{1} 4^{17} 50$

$\delta=+2,15210 ; \varepsilon=-0,027791 ; \quad \zeta=+0,07138$ $\eta=-0,06974 ; \theta=-0,039809 ; x=-0,070920$

Deinde cum lit proxime

$$
\mathfrak{E F}^{\prime}=-2 \mathrm{mg} \text { feu } \mathfrak{B}^{\prime}=-0,12256
$$

erit quoque proxime

$$
\begin{aligned}
& C^{\prime}=-0,003485 ; \quad D^{\prime}=-0,014456 \\
& C^{\prime}=+0,001509 \quad ; \quad \mathbb{F}^{\prime}=-0,005334
\end{aligned}
$$

Hinc ergo accuratius elicietur $\mathfrak{B}^{\prime}=-0,11019$, ideo que hic et reliqui conficientes cam abfolute quam in numeris fecundis exunt:

$$
\text { in minutis fecundis }
$$

$$
\begin{aligned}
& 3^{\prime}=-0,11019 . .3^{\prime}=-22728^{\prime \prime}=-6^{\circ}, 18^{\prime}, 4^{\prime \prime} \\
& \boldsymbol{c}^{\prime}=-0,00339 . \mathrm{E}^{\prime}=-700=-0,11,40 \\
& \mathfrak{D}^{\prime}=-0,01742 . . D^{\prime}=-3594=-0,59,54 \\
& \text { (E) }=+0,00149 . \text {. E }=+306=+0,5,6 \\
& \mathrm{~g}^{\prime}=-0,00524 . \mathrm{f}^{\prime}=-108 \mathrm{I}=-0,18, \mathrm{I} \\
& \mathrm{S}^{\prime}=-0,01824 \cdot \mathrm{S}^{\prime}=-3762=-1,2,42 \\
& \text { (5) . } 115=-0,00056=-1,55 \\
& G^{\prime}=+0,01368 \text {. . } g^{\prime \prime}=+2823=+0,47,3 \\
& J^{\prime}=+0,01368 \text {. } \aleph^{\prime}=t \quad 47=+0,0,47 \\
& \Omega^{\prime}=+0,00023 \cdot{ }^{\prime}=-128=-0,2,8 \\
& q^{\prime}=-0,00062 \cdot \mathrm{I}^{\prime}=-246=-0,4,6 \\
& m=-0,00119 \cdot \text {. } M M^{\prime}=-246=-0,4,6 \\
& \mathfrak{N}=+0,00020 \cdot \cdot \mathfrak{N}=+{ }^{41}=+0,6,19 \\
& \text { \$Y }=+0,00: 84 \cdot \cdot \text {. } 379=+0,6,19 \\
& \mathrm{D}^{\prime}=-0,0000 \mathrm{I}, \mathrm{Q}^{\prime}=-\quad 2=-0,0,{ }_{\mathrm{XLV}}^{2}
\end{aligned}
$$


320

\section{$A D D T T A M E N T U M$}

XLV.

Hinc ergo fi ad datum tempus iam cognita fit anomalia Inne vera $v$ cum angulo $\eta$, longitudo lunae per aequationes in minutis fecundis expreffas erit

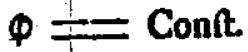

$$
\begin{aligned}
& +13,3682 \omega-22728^{\prime \prime} \text { fin } v-700^{\prime \prime} \text { fin } 27 \\
& \text { - } 1081 \text { in } 2 v-3594 \text { fin }(2 y-2 v) \\
& \text { - } 128 \text { fin } 3 v+306 \text { in }(2 \eta+2 v) \\
& \text { - } 3762^{\prime \prime} \text { fin }(2 y-v)-246^{\prime \prime} \text { fin }\left(4^{\left.\gamma_{i}-v\right)}\right. \\
& \text { - } 115 \text { fin }(2 \eta+v)+4^{1} \text { fin }(4 \eta+v) \\
& +2823 \operatorname{lin}(2 y-3 v)+379 \text { fin }(4 y-3 v) \\
& +\quad 47 \text { fin }(2 y+3 v)-2 \text { fin }(4 y+3 v)
\end{aligned}
$$

vbi Conft $+13,3682 \omega$ denotar longitudinem mediam; in reliquis autem terminis continentur inaequalitates poriodicac pro hac hypothefi.

\section{XLVI.}

Inde iam vicifim anomalia vera lunee $v$ colligitur, $v$ fit

$$
y=
$$

$$
\begin{aligned}
& \text { 13,2559* -33464" fin } v \quad-2233^{\prime \prime} \text { fin } 21 \\
& \text { - } 1530 \text { in } 20 \quad+49864 \text { fin }(2 y-2 v) \\
& \text { - } 128 \text { fin } 3 v+770 \text { fin }(2 y+2 v) \\
& +49256 \text { fin }(2 y-2)-246 \text { fin }(4 i-v) \\
& +5417 \text { in }(2 y+v)+41 \text { lin }(4 y+v) \\
& +2823 \text { fin }(2 y-32)+379 \text { fin }(4 y-32) \\
& +47 \text { fin }(21+3 v)-2 \operatorname{lin}(4 n+3 v)
\end{aligned}
$$

vbi primus terminus 13,2559 w defignat anomaliam me. diam lunae, quae fic $\frac{1}{\neq}$ ?: tum. ex ea primum quaerarur
$A D D I T A M E N T U M$

anomalia Repleriana, quae fcilicet ofola excentriciate pen det, fitque ea $=8$, vt fit

$8=\zeta-33454^{\prime \prime}$ fin $8-1530^{\prime \prime}$ fin $28-128^{\prime \prime}$ ) in $3^{88}$ vnde quidem facile tabulae conftruentur. Tum fartatur $v=8+z$, et quia angulus $z$ eft modicus, inde is fatis prope poterit definiri. Interim tamen expedire vi. detur aliquot operationibus irerandis ifam anomaliam ve. ram $v$ determinari; dum fcilicet primum valor non ni$m i s$ a veritate abhorrens pro $v$ aeftimando affumicur, ex eoque deinceps exactior colligitur; qui fi nimis ab affumto difcrepare reperiatur, ex hoc denuo exactior quaergo cur, donec nulla amplius correctione fuerit opus.

\section{XLVII}

Formula denique, cui tam diameter lunae apparens geocentrica quam parallaxis horizontalis eft propostio nalis, ex $\$ .40$. reperitur

$$
\begin{aligned}
& \text { I }-0,05470 \operatorname{col} v \quad-0,00120 \operatorname{col} 21 \\
& -0,00142 \operatorname{col} 2 z+0,00700 \operatorname{col}(2 y-2 z) \\
& -0,00017 \operatorname{col} 3 v \quad+0,00073 \operatorname{col}(2 \eta+2 v) \\
& -0,00898 \operatorname{cof}(2 y-v)-0,00035 \operatorname{cor}(4 \eta-v) \\
& -0,00042 \operatorname{cor}(2 y+v)+0,00009 \operatorname{col}(4 \eta+v) \\
& -0,00701 \operatorname{col}(2 \eta-3 v)-0,00044 \operatorname{cor}(4 \eta-3 v) \\
& +0,00008 \operatorname{cor}(2 i+3 v)-0,00001 \operatorname{cor}(44+3 v)
\end{aligned}
$$

quorum quidem terminorum plures, qui pro parallaxi infra aliquot minuta fecunda fubfitunt, tuto omitri porerunt. His igitur tribus formulis pro anomalia vera $v$, longitudine $\phi$ et parallaxi feu diametro apparene inuentis monus lune contineretur, is quiden tam folis paral-

zeratur

กno-
S \& 
laxis quam eitus excentricitas et inclinatio orbitae lunaris ad eclipticam negligatur. Hae autem funt inaequalitates praecipuae, quae etiam ad reliquas eruendas adbiberi debent; vnde nunc ad inaequalitates ab excentricitate folis oriundas progrediamur.

\section{INUESTIGATIO INAEQUALITATUM IUNAE SECUNDI GENBRIS SEU AB EXCENTRICITATE SOLIS PENDENTIUM. \\ XLVIII.}

Formulae noftrae differentiales, quatenus ab excentricitate orbitae folaris pendent, omifis terminis, quos iam conftat effe minimos, erunt

$$
\begin{aligned}
& \text {. } \frac{d \xi}{d w}=\text { Praec. }+\frac{9 c}{2 m} \text { fin }(2 y-u)+\frac{9 e}{2 m} \text { fin }(2 y+u) \\
& \frac{d q}{d w}=\text { Praec }+\frac{3 c}{4 m} \text { in }(v-w)+\frac{3^{e}}{4 m} \text { in }(v+w) \\
& -\frac{27 e}{8 m} \operatorname{lin}(2 y-v-x)-\frac{27 e}{8 m} \operatorname{lin}(2 y-v+u) \\
& -\frac{9 c}{8 m} \text { in }(2 \pi+v-w)-\frac{9 c}{8 m} \text { in }(2 y+v+w)
\end{aligned}
$$

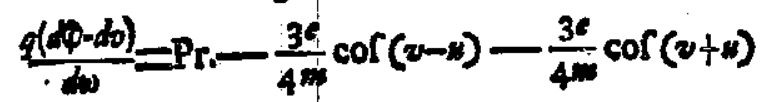

$$
\begin{aligned}
& -\frac{27.6}{8 m} \operatorname{cor}(2 y-y-n)-\frac{27 f}{8 m} \operatorname{cor}(2 x-y+x) \\
& +\frac{9 e}{\sin } \operatorname{cor}(2 n+v-n)+\frac{9 e}{8 m} \operatorname{cor}(2 q+v+n)
\end{aligned}
$$

Quanquam enim nunc tam quam $q$ etiom ab excentricitate e pendeant, tamen in his formulis, in quas hae quanticates ingrediuntur, haec mutatio earum fine erro re pro nihilo haberi poreft; quoniam hi termini per fe funt minimi, et quia iam terminoş ab et et $q$ famul pen. dentes omilimus. Thth vero erit

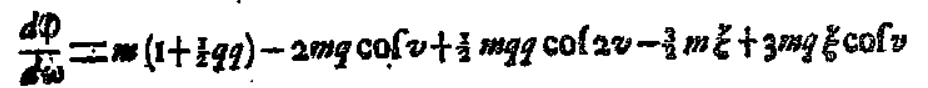

et $\frac{d u}{d \omega}=\frac{d \theta}{d \omega}=I+2 e e-2 e \operatorname{col} \omega$

\section{XLIX.}

Ad formulas has integrandas feu tanmm ad integraliụm partes inueniendas, quae ab excentricitate folis e pendent, opus eft vt formularum $\frac{d \eta}{d \omega}, \frac{d v}{d \omega t}$ et $\frac{d w}{d(\omega t}$ primum habeamus partes principales, tum vero etiam eas quae a fimplici folis excentricitate e pendent: habebimus ergo primo

$$
\begin{aligned}
& \frac{d \eta}{d w}=m\left(I+\frac{\pi}{2} g g\right)-I-2 e x-2 m g \operatorname{col} v+2 \varepsilon \operatorname{col} u \\
& \frac{d v}{d w}=m\left(1+\frac{x}{2} g g\right)-2 m g \operatorname{cor} v+\frac{3 e}{4^{m g} g} \operatorname{col}(v-u)+\frac{3 e}{4^{m g}} \operatorname{col}(v+w) \\
& -\frac{1}{4 m}\left(1-\frac{9 A+3 B-2 C}{2 g g}\right)+\frac{27 \delta}{8 m g} \mathrm{cf}(2 x+v-u)+\frac{27 e}{8 m g} \mathrm{c}(2 \eta-v+x) \\
& -\frac{g e}{8 m g} \mathrm{cr}(2 v+v-x)-\frac{g e}{8 m g} \mathrm{c}(2 v+v+4)
\end{aligned}
$$

feu introducendis, vt fupra $\$ .27$. breuitatis graeia, litteris

$$
\begin{aligned}
& a=m\left(x+\frac{x}{3} 8 g-C\right)-2 e f \quad ; y=2 m g
\end{aligned}
$$

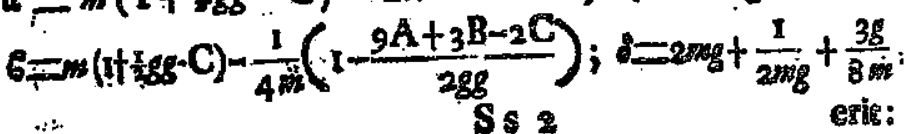


erit: $\frac{d y}{d \omega} a-\gamma \operatorname{cor} v+2 e \operatorname{cor} u$

$\frac{d v}{d v}=6-8 \operatorname{cor} v-\frac{9}{4 m g} \operatorname{cor}(2 y-v)+-\frac{3}{4 m g} \operatorname{cor}(2 y+v)$

$+\frac{3 e}{4 m g} \operatorname{cor}(v-u)+\frac{3 e}{4 m g} \operatorname{cor}(v+u)+\frac{27 e}{8 m g} \operatorname{cor}(2 q-v-x)$

$+\frac{27 e}{8 m g} c f(2 \eta-v+v)-\frac{9 e}{8 m g} c f(2 \eta+v-z)-\frac{9 e}{8 m g} c f(2 v f v f \omega)$

et $\frac{d x}{d w}=1-2 e \cos w$

\section{L.}

Fingamus nune primo:

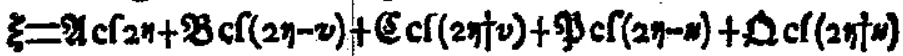
ac differentiando eos cantum fumamus terminos, qui formulae differentiali refpondent, quandoquidem relt. quos ian inuenimus: eritgue

$\cdot \frac{d \xi}{d w}=-2 e x$ fin $(2 y-w)-2 e x$ fin $(2 y+u)$

$$
-(2 a-1) \$-(2 a+1) \mathfrak{Q}
$$

vnde colligicur:

$(2 \alpha-1) 9=-\frac{9 e}{2 m}-2 c \% ;(2 \alpha+1) Q=-\frac{9 e}{2 \pi}-2 e \%$

Cum igitur fit $i=0,0.168$, erit in numeris :

$$
\eta=-0,000247 \text { et } \mathcal{A}=-0,000227 \text {. }
$$

L.

Fingatur porro: $\quad g=8$

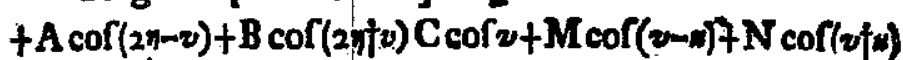

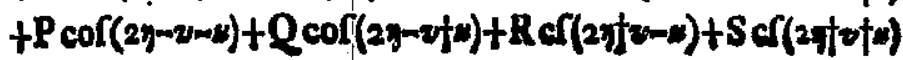

sc differensiando obtinebirus: $\frac{d q}{d \omega}=$

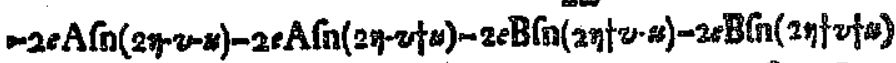
$m(2 a-6-1) P-(2 a-6) Q-(2 a+B-1) R \quad m(2 a+b+1) S$

$-(6-1) \mathrm{M}$ fin $(y-x)-(6+1) \mathrm{H} \sin (y+y)$

Comparatione ergo inftituta reperietur:

$(6-1) \mathrm{M}=-\frac{3^{e}}{4^{m}} ;(c+1) \mathrm{N}=-\frac{3 e}{4^{m}}$

$(2 \omega-6-1) \mathrm{P}=\frac{27 e}{8 m}-2 e \mathrm{~A} ;(2 \omega-6+8) Q=\frac{27 e}{8 m}-2 e \mathrm{~A}$

$(2 a+6-1) \mathrm{R}=\frac{9 e}{8 m}-2 e \mathrm{~B} ;(2 a+6+1) \mathrm{S}=\frac{9 e}{8 m}-2 e \mathrm{~B}$

et in numeris

$\mathbf{M}=-0,00008 ; \mathbf{P}=+0,00042 ; \quad \mathbb{R}=+0,00004$ $\mathbf{N}=-0,00006 ; \mathbf{Q}=+0,00036 ; \mathrm{S}=+0,00004$

\section{LII.}

Hic autem in differentiatione negleximus partes ipfius $\frac{d v}{d \omega} a b$ excentricicace e pendentes, qquarum tamen codem iure ratio haberi debuiffet, arque partis in differentiali $\frac{d \eta}{d \omega}$; inde autem multo plures termini accedent ad valorum ipfius $q$, ponatur ergo ob hos cerminos:

$$
9=g
$$

$+A \operatorname{cof}(2 \eta-v)+B \operatorname{cof}(2 \eta+v)+C \operatorname{cof} v+M \operatorname{cof}(v-\xi z)$ $+\mathrm{N} \operatorname{cor}(v+u)+\mathrm{D} \operatorname{cor}(2 \eta-u)+\mathrm{Ecor}(2 \eta+u)$ $+\mathrm{P} \operatorname{cor}(2 \eta-z-x)+\mathrm{Q} \operatorname{cor}(2 \eta-v+z)+\mathrm{R} \operatorname{cor}(2 \eta+v-z)$ $+\mathrm{S} \operatorname{cof}(2 y+v+n)+\mathrm{K} \operatorname{col}(2 v-w)+\mathrm{L} \operatorname{cof}(2 z+\mu)$

$$
\text { S } 83
$$


326 ADDITAMENTEM.

$+\mathrm{F} \operatorname{cor}(2 \eta-2 v-x)+\mathrm{G} \operatorname{col}(2 \eta-2 v+x)+\mathrm{H} \operatorname{cor}(2 \eta+2 v-x)$ $+J \operatorname{cor}(2 y+2 z+x)+\mathrm{T} \operatorname{co}(4 y-x)+V \operatorname{cor}(4 y+x)$

$+W \operatorname{cor}(4 \eta-2 v-4)+X \operatorname{cor}(4 \eta-2 v+u)+Y \operatorname{cor}(4 \eta+2 v-4)$ $+Z \operatorname{cor}(4 \eta+2 v+\omega)$

et fumto differentiali pleno reperiotur:

$d q=+$ fin $(2 n-z-u)[-2 e A-(2 a-6-1) P]$

$+f(2 n-v+4)[-2 e A-(2 \alpha-6+1) Q]-(6-1) \mathrm{Mfin}(v-w)$

+ in $(2 y \bar{F} v-u)[-2 c \mathrm{~B}-(2 \alpha+6-1) \mathrm{R}]$

$+\operatorname{fin}(2 n+v+x)[-2 e \mathrm{~B}-(2 a+6+1) \mathrm{S}]-(6+1) \mathrm{N}$ fin $(v+u)$

$+f \operatorname{fin}(2 \eta-u)\left(+\frac{3 e \mathrm{~A}}{8 m g}-\frac{3 e \mathrm{~B}}{8 m g}-\frac{27 e \mathrm{C}}{16 m g}-\frac{9 e \mathrm{C}}{16 m g}-(2 u-1) \mathrm{D}\right)$

$+\sin (2 y+u)\left(+\frac{3 e \mathrm{~A}}{8 m g}-\frac{8 e \mathrm{~B}}{8 m g}-\frac{27 e \mathrm{C}}{16 m g}-\frac{9 e \mathrm{C}}{16 m g}-(2 \alpha+1) \mathrm{E}\right)$

$+f i n(2 \eta-2 v+x)\left(+\frac{3 e A}{8 m g}+\frac{27 e \mathrm{C}}{16 m g}-(2 a-2 G+1) \mathrm{G}\right)$

$+f \operatorname{fin}(2 y-2 v-\mu)\left(+\frac{3 e \mathrm{~A}}{8 m g}+\frac{27 e \mathrm{C}}{16 m g}-(2 \omega-26-1) \mathrm{F}\right)$

$+\mathfrak{f n n}(2 \eta+2 v-\mu)\left(-\frac{3 e \mathrm{~B}}{8 m g}+\frac{9 e \mathrm{C}}{16 m g}-(2 \alpha+2 E-1) \mathrm{H}\right)$

$+f i n(2 y+2 v+x)\left(-\frac{3 e \mathrm{~B}}{8 m g}+\frac{9 e \mathrm{C}}{16 m g}-(2 x+26+1) J\right)$

$+\quad$ in $n\left(+\frac{27 e \mathrm{~A}}{16 m g}-\frac{27 e \mathrm{~A}}{16 m g}+\frac{9 e \mathrm{~B}}{16 m g}-\frac{9 e \mathrm{~B}}{16 m g}-\frac{3 e \mathrm{C}}{8 m g}+\frac{3 e \mathrm{C}}{8 m g}\right)$

$+f i n(4 \eta-2 v-w)\left(+\frac{27 e A}{16 m g}-(4 a-26-1) W\right)$

$+\operatorname{fin}(4 z-2 v+w)\left(+\frac{27 e A}{16 \sin g}-\left(4^{a}-26+1\right) x\right)$

ADDITARENTU

$2 \eta+2 v-4)$

$\operatorname{cor}(4 \eta+n)$

$4 \eta+2 v \cdots n)$

$4 \eta+2 v+4)$

$\operatorname{lfin}(z-x)$

$\operatorname{lin}(v+x)$

$(-1)$ D)

+1) E)

i)

)

1)

1)

$\left.+\frac{3 e \mathrm{C}}{8 m g}\right)$ $+f \operatorname{fin}(4 y+2 z-x)\left(+\frac{9 e B}{16 m g}-(4 x+26-3) Y\right)$

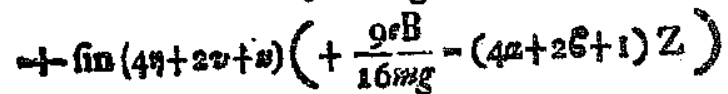

$+f \sin (2 u-4)\left(+\frac{9 e A}{10}-\frac{27 e B}{26 m g}=\frac{3 e C}{8 m g}-(26-1) K\right)$

$+\operatorname{fin}(2 v+u)\left(+\frac{g^{e A}}{16 m g}-\frac{27 e \mathrm{~B}}{16 m g}-\frac{3 e \mathrm{C}}{8 m g}-(26+1) L\right)$

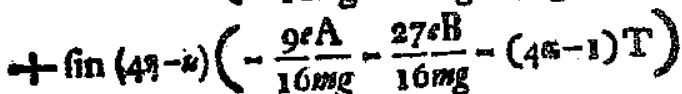

$+\sin (4 \eta+*)\left(-\frac{9 e \mathrm{~A}}{16 m g}-\frac{27 \mathrm{eB}}{16 m g}-(4 a+1) V\right)$

vnde reperitur:

$$
\begin{aligned}
& D=-0,000010 ; \mathrm{H}=+0,000008 \\
& \mathrm{E}=-0,000010 ; \mathrm{I}=+0,000001 \\
& \mathrm{~F}=+0,000005 ; \mathrm{K}=-0,000006 \\
& \mathrm{G}=+0,000069 ; \mathrm{L}=-0,000006 \\
& \mathrm{~T}=+0,000004 ; \mathrm{X}=-0,000022 \\
& \mathbf{V}=+0,000004 ; \mathrm{Y}=-0,000000 \\
& \mathrm{~W}=-0,000023 \cdot ; \mathrm{Z}=-0,000000 \\
& \text { L'Il. }
\end{aligned}
$$

Ponamus nunc etiam pro motu apogel

$$
\varphi-v=\operatorname{Cont}+\Delta
$$

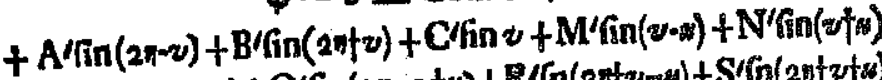

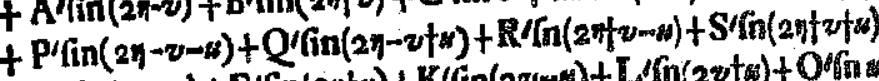

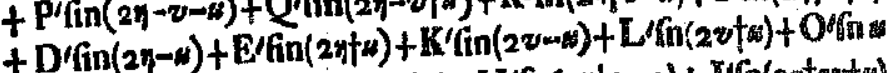

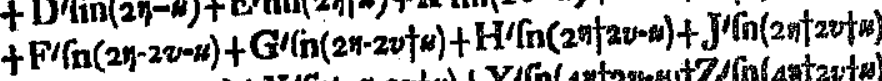

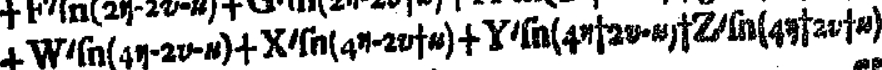
$+T^{\prime} / \sin \left(4 V^{-w}\right)+V^{\prime} \sin (4 \eta+4)$ 
et fumto differentiali pleno reperienar:

$$
\begin{aligned}
& \frac{d \varphi-d v}{d w}=\Delta+\operatorname{cor}(2 \eta-z-\psi)\left[2 e A^{\prime}+(2 \theta-6-I) P\right] \\
& +\operatorname{cor}(2 y-v+u)\left[2 e A^{\prime}+(2 u-6+1) Q\right]+(6-1) M^{\prime} \operatorname{cor}(v-u) \\
& +\operatorname{cor}(2 y+2-x)\left[2 e B^{\prime}+(2 a+6-1) R^{\prime}\right] \\
& +\operatorname{cor}(2 x+v+u)\left[2 \epsilon \mathrm{B}^{\prime}+(2 a+6+1) \mathrm{S}^{\prime}\right]+(6+1) \mathrm{N} / \operatorname{col}\left(v f^{\prime} u\right) \\
& +c r(2 x-2)\left(-\frac{3 e}{8 m g} A^{\prime}+\frac{3 f}{8 m g} B^{\prime}+\frac{27 \varepsilon}{16 m g} C^{\prime}-\frac{9 e}{16 m g} C^{\prime}+(2 x-1) D^{\prime}\right) \\
& +c(2 n+w)\left(-\frac{3 e}{8 m g} A^{\prime}+\frac{3 e}{8 m g} B^{\prime}+\frac{27 e}{16 m g} C^{\prime} \frac{g^{e}}{16 m g} C^{\prime}+(2 a+1) E^{\prime}\right) \\
& -t \operatorname{cor}(2 \eta-2 v-w)\left(-\frac{3 c}{8 m g} A^{\prime}+\frac{27 c}{16 m g} C^{\prime}+(2 a-26-1) F^{\prime}\right) \\
& +\operatorname{cor}(2 v-2 v+u)\left(-\frac{3 e}{8 m g} A^{\prime}+\frac{27 e}{16} \frac{c}{m g} \mathbf{C}+(2 x-26+1) G^{\prime}\right) \\
& +\operatorname{cor}(2 \eta+2 v-x)\left(+\frac{3 e}{8 m g} B^{\prime}+\frac{9 e}{16 m g} C_{+}+(2 a+26-1) H^{\prime}\right) \\
& +\operatorname{cor}(2 \eta+2 v+\alpha)\left(+\frac{3 e}{8 m g} B^{\prime}+\frac{9 e}{16 m g} \mathrm{C}+(2 \alpha+26+1) \mathrm{J}^{\prime}\right) \\
& +\quad \operatorname{cor} *\left(-\frac{27 e}{16 m g} A^{\prime}-\frac{27 e}{16 m g} A^{\prime}-\frac{9 e}{16 m g} B^{\prime}-\frac{9 e}{16 m g} B^{\prime}\right. \\
& +\frac{3 e}{8 m g} C^{\prime}+\frac{3 e}{8 m g}\left(1+O^{\prime}\right)
\end{aligned}
$$

$$
\begin{aligned}
& +\operatorname{cor}(2 v-4)\left(+\frac{9 t}{16 m g} A^{\prime}+\frac{27 e}{16 m g} B \frac{3 e}{2} C+\left(2 b^{2}-1\right) K^{\prime}\right) \\
& +\operatorname{col}(2 v+u)\left(+\frac{9 e}{16 m g} A^{\prime}+\frac{2 a c}{16 m g} B^{\prime}+\frac{3 e}{8 m g} C^{\prime}+(2 b+g) U\right) \\
& +\operatorname{cof}\left(4^{n-n)}\left(+\frac{9 e}{16 m g} \mathrm{~A}^{\prime}+\frac{27 q}{16 m g} \mathrm{~B}^{\prime}+(2 a-1) \mathrm{T}^{\prime}\right)\right. \\
& +\operatorname{cof}(4 y+u)\left(+\frac{9 e}{16 m g} A^{\prime}+\frac{27 e}{16} M^{\prime} B^{\prime}+(2 \omega+1) V^{\prime}\right)
\end{aligned}
$$

IIV.

Singuli iam hi termini multiplicentur per q, cuius valor quidem erit $=g$, quoniam hi cermini in fuo ge. nere iam funt minimi : fed quoniam valor $\frac{d \varphi-d v}{d \omega}$ adhus hos terminos praecipuos continet:

$(2 a-6) A^{\prime} \operatorname{cor}(2 \eta-v)+(2 \alpha+6) B^{\prime} \operatorname{cor}(2 \eta+v)+6 C^{\prime} \operatorname{cor} v$ fi et hi per $g$ uultiplicentur, inde narcentur quogue termini angulum " inuoluentem, erit autem pro his, fumtis parcibus cantum praccipuis :

$$
q=\text { Praec. }+\mathrm{P} \operatorname{cor}(2 \eta-v-w)+\mathrm{Q} \operatorname{cor}(2 q-z+u)
$$

Ergo ad illos terminos per $q$ mulciplicatos infuper acce dent ift: :

$\operatorname{col} \omega\left[\frac{x}{2}(2 \alpha-6) \mathrm{PA}^{\prime}+\frac{x}{2}(2 \alpha-6 \mathrm{QA}]+\frac{1}{2} \mathrm{PCC}^{\prime} \operatorname{col}(2 q-\alpha)\right.$ $\operatorname{col}(4 y-2 v-u)\left[\frac{x}{2}(2 \alpha-6) \mathrm{PA}^{\prime}+\frac{1}{2} 6 \mathrm{PC}\right)+\frac{1}{2} 6 \mathrm{QC}^{\prime} \operatorname{cor}(2 n+u)$

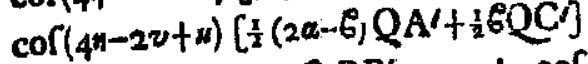
$+\operatorname{cor}(4 y-x)\left[\frac{x}{2}(2 a+6) P B\right.$ $\operatorname{cof}(2 v-u)\left[\frac{x}{2}(2 \alpha+\varepsilon) Q^{\prime}+\operatorname{col}(4 y+B)\left[\frac{x}{2}\left(2 \alpha+6, Q^{\prime}\right.\right.\right.$ T 
Hinc ergo obtinentur fequentes determinationes:

$+\frac{9 e}{16 m} A^{\prime}+\frac{27 e}{16} B^{\prime}+\frac{3 E}{8 m} C^{\prime}+(36-1) g^{\prime} K^{\prime}+\frac{8}{x}(2 a+C) Q^{\prime}=$ $+\frac{9 e}{16 m} \mathrm{~A}^{\prime}+\frac{27 e}{16 m} \mathrm{~B}^{\prime}+\frac{3 e}{8 m} \mathrm{C}^{\prime}+(26+1) \mathrm{L}^{\prime}+\frac{5}{2}\left(2 a+b^{2}\right) \mathrm{PB}^{\prime}=$ $+\frac{9 e}{16 m} \mathrm{~A}^{\prime}+\frac{27^{e}}{16 m} \mathrm{~B}^{\prime}+(4 \alpha-1) g \mathrm{~T}^{\prime}+\frac{5}{2}(2 \alpha+6) \mathrm{PB}^{\prime}=$ $+\frac{9^{e}}{16 m} A^{\prime}+\frac{27^{c}}{16 m} B^{\prime}+(4 s+1) g^{\prime}+\frac{\pi}{8}(2 \alpha+6) Q^{\prime}=0$ $26 g A^{\prime}+(2 \alpha-6-1) g \mathrm{P}^{\prime}=-\frac{27 c}{8 m}$ $2 e g A^{\prime}+(2 a-E+1) g Q^{\prime}=-\frac{27 e}{8 m}$ $2 e g B^{\prime}+(2 a+b-1) g R^{\prime}=+\frac{9 e}{8 m}$ $2 e g B^{\prime}+(2 a+6+1) g S^{\prime}=+\frac{9 e}{8 m}$ $(6-1)_{g} M^{\prime}=-\frac{3^{c}}{4^{m}} ;(6+1)_{g} N^{\prime}=-\frac{3 e}{4^{m}}$ $-\frac{3 e}{8 m}\left(A^{\prime}-B\right)+\frac{9 e}{8 m} C^{\prime}+(2 a-1) g D^{\prime}+16 P C^{\prime}=0$ $-\frac{3 e}{8 m}\left(\mathrm{~A}^{\prime}-\mathrm{B}^{\prime}\right)+\frac{8 e}{8 m} \mathrm{C}^{\prime}+(2 \alpha+1) g \mathrm{E}^{\prime}+\frac{2}{2} 6 \mathrm{QC}^{\prime}=0$ $-\frac{3 e}{8 m} A^{\prime}+\frac{27 c}{10 m} C^{\prime}+(2 a-26-1) g F^{\prime}=0$ $-\frac{3 e}{8 m} A^{\prime}+\frac{27 e}{16 m} C+(2 \phi-26+1) g(G=0$ $+\frac{3 e}{8 m} \mathrm{~B}^{\prime}+\frac{y e}{16 m} \mathrm{C}^{\prime}+(2 a+26-1) g \mathrm{H}^{\prime}=0$ $+\frac{3 e}{8 m} \mathrm{~B}^{\prime}+\frac{9 e}{16 m} \mathrm{C}+(2 a+26+1) g J^{\prime}=0$ $-\frac{27^{e}}{8 m} \mathrm{~A}^{\prime}-\frac{9 e}{8 m} \mathrm{~B}^{\prime}+\frac{3 e}{8 m} \mathrm{O}^{\prime}+g \mathrm{O}^{\prime}+\frac{z}{2}(2 \omega-6)(\mathrm{P}+Q) \Lambda^{\prime}=$ $-\frac{27 e}{8 m} \mathrm{~A}^{\prime}+\left(4^{a-26-1}\right) g \mathrm{~W}^{\prime}+\frac{1}{2}(2 a-6) \mathrm{PA}^{\prime}+16 \mathrm{PC}=0$ $-\frac{27 c}{8 m} A^{\prime}+(4 a-26+1) g X^{\prime}+\frac{1}{2}(2 a 6) Q A^{\prime}+16 Q C^{\prime}=0$ $-\frac{9 c}{16 m} \mathrm{~B}^{\prime}+(4 a+26-1) g^{\mathrm{Y}^{\prime}}=3-\frac{9^{c}}{16}-\mathrm{B}^{\prime}+(4 a+2 b+1) \mathrm{g}^{\prime}=$

\section{LVI.}

Valores ergo horum coefficientium iam ad minuta fecunda reductorum erunt: $0^{\prime}=+310^{\prime \prime}$

$$
\begin{aligned}
& \mathrm{p}^{\prime}=-1285^{\prime \prime} ; \mathrm{M}^{\prime}=-293^{\prime \prime} ; \mathrm{F}^{\prime}=+40 \mathrm{~s}^{\prime \prime} \\
& Q^{\prime}=-1087 ; N^{\prime}=-251 ; \mathrm{G}^{\prime}=+5412 \\
& \mathrm{R}^{\prime}=+148 ; \mathrm{D}^{\prime}=-\mathrm{s2} ; \mathrm{H}^{\prime}=-2 \\
& \mathrm{~S}^{\prime}=+14 \mathrm{I} ; \mathrm{E}^{\prime}=-\mathrm{H}^{\prime} ; \mathrm{J}^{\prime}=- \\
& W^{\prime}=-9 \mathrm{r}^{\prime \prime} ; \mathrm{K}^{\prime}=+6 \mathrm{~s}^{\prime \prime} \\
& X^{\prime}=-95 ; L^{\prime}=+61 \\
& \mathrm{Y}^{\prime}=-\mathrm{i} ; \mathrm{T}^{\prime}=+35 \\
& \mathrm{Z}=-\mathrm{I} ; \mathrm{V}^{\prime}=+3 \mathrm{I}
\end{aligned}
$$

Vnde ob excentricitatem orbitae folaris exit:

$\xi=+0,008931 \operatorname{cor} 2 \eta \quad-0,000247 \operatorname{cor}(24-11$; $+0,002090 \operatorname{cof}(2 y-v)-0,000227 \operatorname{cof}\left(2 y_{1}+11\right)$ $+0,000640 \operatorname{cor}(2 i+\eta)$

$=8-0,013 \%, 95 \operatorname{col}(2 \eta-v)-0,000290$ cof $2 \%$ $-0,001460 \operatorname{cor}(2 \eta+v)+0,01 \neq 012 \mathrm{col}\left(2 y_{i}-2 v_{j}\right.$ $+0,002834 \operatorname{col} v \quad-0,00010201(201+22)$ $-0,000184 \operatorname{cor} 41+0,000+20 \cos (2 \eta-v-\beta)$ $+0.000882 \operatorname{cr}(1 n-2 v)+0,000 ; 60 \operatorname{cor}(2 n-z+u)$ +0,00000; $=01(44+20)$

$\operatorname{Tt} 2$ 
332

\section{ADDITAMENTUM.}

$$
\varphi-v=\text { Cont }+0,1123 \omega
$$

$-53018^{\prime \prime}$ (in $(2 \eta-v)+1533^{\prime \prime}$ lin $2 \eta \quad-1285^{\prime \prime}$ (in $(2 y-v-4)$ -5532 fin $(2 \eta+v)-53549$ fin $(2 \eta-2 v)-1087$ fin $(2 y-v-v)$ +10736 fin $v-464$ fin $(2 y+2 v)+148$ fin $(2 y+v-u)$

+449 fin $2 v+141$ fin $(2 v+v+u)$

$-293^{\prime \prime}\left(\operatorname{in}(v-u)+40 x /\right.$ fin $(2 y+2 v-u)+6 I^{\prime \prime}$ in $(2 v-u)$ -251 fin $(v+u)+5412$ fin $(2 y-2 v+u)+61$ fin $(2 v+u)$

-52 fin $(2 y-x)-9 x$ fin $(4 y-2 v-x)+35$ fin $(4 y-x)$

-45 fin $(2 x+x)-95$ in $(4 y-2 x+x)+35$ fin $(4 y+x)$ +310 fin

neglectis fcilicet terminis minimis.

LVII.

Denique pro longitudine lunae vera $\varphi$ inuenienda; cum fit

$$
\frac{d \varphi}{d \omega}=\text { Praec }
$$

- tmg. $0,00005 \operatorname{cof}(2 y-v-x)-m .0,00005 \operatorname{cof}(2 n-u)$ $+m g .0,00002 \operatorname{cof}(2 n-v+x)-m .0,00002 \operatorname{cor}(2 n+u)$

$+m_{s} \cdot 0,00005 \operatorname{col}(2 \eta+\psi-x)-m .0,00042 \operatorname{col}(2 \eta-2 v-k)$

$+m g \cdot 0,00002 \operatorname{col}(2 \eta+v+u)-m .0,00036 \operatorname{col}(2 n-2 v+u)$

tmg. $0,00042 \operatorname{col}(2 y-3 v-u)$

t mg. $0,00036 \operatorname{cor}\left(2 i-3^{2}+4\right)$

ponatur $\phi=$ Conft.

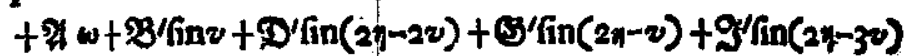
vna cum nouis terminis

$+a^{\prime}$ fin $(2 y-v-x)+e^{\prime}$ in $(2 y-x)+g^{\prime}$ fn $(2 x-2 v-x)+l^{\prime}$ fin $x$

$+b^{\prime}$ in $(2 y-v+w)+f^{\prime} \operatorname{lin}(2 y+w)+h^{\prime}\left(\ln (2 y-2 v+w)+m^{\prime} \ln (2 v-m)\right.$

$+t^{\prime} \operatorname{lin}(2 x+v-x)$.

$+j^{\prime} \ln (2 y-3 v-x)+n / \ln (2 v+x)$

$+b / \sin (2 n+v+w)$
ADDITAMEMTUU

$+q^{\prime} \operatorname{lin}(2 y+2 v-u)+q^{\prime} \operatorname{fin}(z-u)+z^{\prime} \operatorname{lin}(4 y-x)$

$+y^{\prime} \operatorname{fin}(2 \eta+2 v+u)+y^{\prime} \operatorname{lin}(v+x)+t^{\prime} \operatorname{lin}(4 v+z)$

pro reliquorum terminorum, quos forma differencialis requiri, coefficientibus ponamus litteram

\section{LVIII.}

Differentiatione iam per regulas praecedentes inftituta erit: $\quad \frac{d \Phi}{d \omega}=$ Praec.

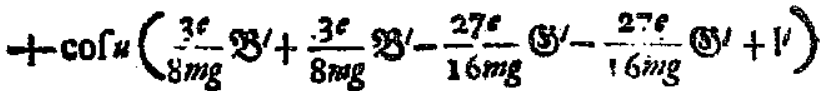

$$
\begin{aligned}
& +\left(x a ! b-i c^{\prime} \operatorname{co}(2 \sin t z-x)\right. \\
& +\operatorname{cor}(2 v-11)\left(\frac{3 e}{b m g} B^{\prime}+\frac{9 e}{16 m g}(b-1)(2 e-1) w^{\prime}\right) \\
& +(2 \alpha+6+1) u^{\prime} c r(2 \eta+v+u) \\
& +\operatorname{cor}(2 v+u)\left(\frac{3 e}{8 m g} x^{\prime}+\frac{9 e}{16 m g}\left(g^{\prime}-\frac{18 e}{16 m g} g^{\prime}+(2 \epsilon+1) n^{j}\right)\right. \\
& +\operatorname{cof}(2 n-u)\left(+\frac{27 e}{16 m g} w^{\prime}-\frac{9 e}{16 m g} w^{\prime}-\frac{3 e}{8 m g} a^{\prime}+(2 a-1) p^{\prime}\right)
\end{aligned}
$$

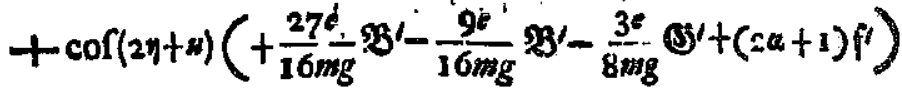

$$
\begin{aligned}
& +\operatorname{cor}(2 y-2 v-4)\left(+\frac{27 c}{16 m g} B^{\prime}+2 m-\frac{9 c}{8 m g} g^{\prime}-\frac{g^{e}}{8 m g} g^{\prime}\right. \\
& \left.+(2 a-26-1) g^{\prime}\right)
\end{aligned}
$$

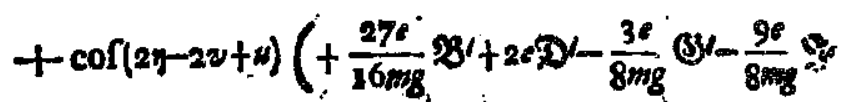

$$
\begin{aligned}
& \left.\therefore \quad+(2 a-2 s+1) b^{\prime}\right)
\end{aligned}
$$


$+\operatorname{col}(4 v-x)\left(+\frac{276}{16 m g} 91+(46-1) 8\right)$

$+\cos (4 v+u)\left(+\frac{276}{16 \pi} g^{\prime}+(46+1) t^{\prime}\right)$

$+\operatorname{cor}(2 y+2 v-x)\left(-\frac{9 e}{16 i n g} B^{\prime}+(2 a+2 b-1) v^{\prime}\right)$

$+\operatorname{cof}(2 v+2 v+\mu)\left(-\frac{9 e}{16 m g} 2^{\prime}+(2 \theta+26+1) \psi^{\prime}\right)$

$+\operatorname{cor}(2 x-x-x)\left(-\frac{3 e}{4 m g} D^{\prime}+2 c 0^{\prime}+(2 x-6-1) a^{\prime}\right)$

$+\operatorname{cor}(2 y-3 v+x)\left(-\frac{3 e}{8 \pi n} D^{\prime}+260 y+(2 x-36+1) w^{\prime}\right)$

$+\operatorname{cor}\left(2 x_{1}-v+2\right)\left(-\frac{3 e}{4 m g} D^{\prime}+2 e \mathcal{O}^{\prime}+(2 \alpha-6+1) b^{\prime}\right)$

$+\operatorname{cof}(4 y-2-y)\left(+\frac{g^{6}}{8 m g} D^{\prime}+(40-6-1) 1\right)$

$+\operatorname{cor}(48-v+4)\left(+\frac{9 e}{8 m g} D^{\prime}+(4 a-6+8) 1\right)$

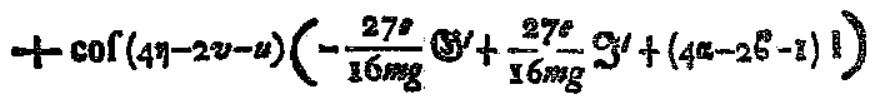

$+\operatorname{cor}(4 y-2 v+4)\left(-\frac{27 e}{16 m g} d+\frac{27 \varepsilon}{16 n g} g^{\prime}+(4 a-2 b+1) 1\right)$

$+\operatorname{cor}(4 u-u)\left(+\frac{98}{1603 g}+(40-1) 1\right)$

$+\operatorname{col}(4 \eta+x)\left(+\frac{9 e}{16 m g}-(1+(4 x+1) 1)\right.$

$+\operatorname{cor}(2 y-4 v-4)\left(-\frac{9 e}{82 y} g^{\prime}+(2 \alpha-461) 1\right)$

$+\operatorname{cor}\left(2 n-4 v+4 ;\left(-\frac{9 e}{8 m g} 9+(20-4 c+1) 1\right)\right.$

$+\operatorname{cor}(4 y-4 v-x)\left(-\frac{81 e}{3 m g} 9^{1}+\left(4^{4}-4^{6}-1\right) 1\right)$

$+\operatorname{cor}(4 n-4 v+x)\left(-\frac{816}{8 m g} 9^{\prime}+(40-46+1) 1\right)$

\section{LIX.}

Colleztis hine valoribus coefficientinn of obtinebitur longirudo lunae vt fequitur: 
gnae, vt fine notabili errore omiti nequean: cuiusmo-

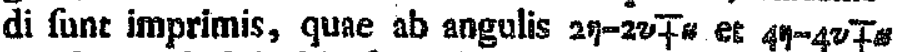
pendent. Sed in his fere idem incommodum vfu venic, quo methodus praecedens premebacus, quod magnitudo harum inaegualitatum per Theoriam non faris accurate definiri queat. Cum enim pro his terminis inueniendis diuifores $2 a-26 F_{1}$ et $46-45 干$ fant perquam exigui, manifeftum eft in diuidendis terminos minimos neglętos non exigui fore momenti : praecipue cum pro litteris $g^{\prime}$ et $h^{\prime}$ termini maiores fere $f e$ mutuo deftru. xiffent. Vnde cum ex valore $\phi$ cantum termini maio. res $\mathfrak{B}^{\prime}, \mathfrak{D}^{\prime}, \mathbb{S}^{\prime}$ et $\boldsymbol{S}^{\prime}$ effent adhibiti, perfpicuum eft etiam reliqui minores fuiftent incroduct, ex is infignem murationem in valore coeficientium $g^{\prime}$ et $q^{\prime}$ oricurans fuife.

\section{LXI.}

De his autem inaequalicatibus cenendum eft, eas per fatis notabile temporis fpatium vix immutari; nam inaequalitates ab angulo $2 y-2 v+w$, ob quantitatera $2 \alpha-26+1=-0,1594$ periodum habent annorum circiter $6 \frac{2}{3}$ annorum, et interuallo 19 annorum ter tantum reuoluuntur: et inaequalizas ab angulo $4 y-4 v+z$ pen. dens fpatio 29 annorum 19 periodos abfoluit. Ex quo cum iftae inaequalicaces per theoriam faltem propemodum fuerint definitee, eas deinceps per obferuationes accuracius definiri conueniet: nifi forte quis laborem in fe fufcipere voluerit, calculum hic adumbratum multo accuratius inftituendi terminorumque hic omifforum rationem habendi; tum vero etiam valores $\xi, q$ et $\emptyset-v$ $\mathbf{v}$ mulso 
multo maiori ftudio, quam hic feci, euolui oporteret, quoniam in horum determinatione multa neglexi, quae in calculo tandem ad notabilem guantitatem excrefcere potuiffent.

\section{LXII.}

Interim tamen hic notari conuenit, hac methodo eas tantum inaegualitates prodire incertas, 'quac fatis longis periodis abfoluunkur; quae incerticudo minus officit, cum per obferuaciones facilius emendari poffit: praecedente vero methodo etiam aliae inaequalimes mi. noribus periodis circumferiptae aliquantum incertae prodierunt, quod fane ingens erat incommodum. Vnde ex hac parte haec methodus polterior priori anteferenda videtur: verum fi ingentem inaequalizatum numerum fpeftemus, quibus non folum lunse longitudo afficitur, fed etiam longitudo apogei, calculus tantopere - fit operofus, ve etiamfi has formulas accuratifime euolverem, tamen in praxi difficillimi foret vfus. Quin etiam plurimae insequalitates in motum apogei ingredi videntur, quarum effectum deinceps per alias longitudinis inaegualiates iterum deftrui oportet, ito vt fatius fuiffer illas penitus omittere.

\section{LXIII.}

Multitudo autem harum ineequalitatum, quibus cam apogei, quam ipfius lunae longitudo turbatur, inde potiffmum originem trahit, quod inaegualitates excentricitatis prae cius quantitate media admodum fint notabiles, atque adeo quadrantem mediae quanticatis fuperent; ita vt prae ea negligi minime queant. Multo plures au- oporteret, lexi, quae excrefcere

methodo quae tatis do minus ari poffit: litates misrtae pro1. Vnde ii antefeatum nulongitudo tantopere ime euol- Quin $i$ ingredi ggitudinis diffet illas

ibus tam inde $\mathrm{po}$ excentrit notabiipperent; ures autem $\triangle D D T A D N T U M$

tem adhuc inaequalitates effens acceflurae, fi escenario: tas luare media adhuc effet minor, quo certe cafu cal. culi difficultates infupersbiles euafiffent hoc vero ipfo cafu methodus prior multo tralabilior redderetur, chas enim plerseque inaequalitates ibi multo minoses prodirent. Atque ob hanc caufan minus expedire videtur, anomaliam lunse ita conficuere, vt eius finus pam pro maximis quam pro minimis diftanciis lunae a serra plane euanefeat, etiamfi haec ratio nacurae rei masime confentanea videacur.

\section{LXIV.}

Cum igitur numerus inaequalitacum iam tantopere increterit, fasile perfpicitur eum adhuc multo magis anetum iri, fi cas inaequalitates, quae cum a parallaxi folis, mm ab eius inclinatone ad eclipticam eftem evo luturus, quo labore propterea, cum eius vfus fere nullus futurus effec, fuperfedebo. Interim tamea hinc tantum colligere licer, inaequaliones $a b$ angulis 2 - $2 v \mathrm{~F}^{*}$ et $4 \eta-4 v \pm$ orcas, minime effe contemsendas; qusae cum methodo praccedente fint vel omiffae vel non fatis caccurate determinacae, fine dubio caufam in fé continent, quod etiam accuratifimae cabulae per obferuationes ement. datae adhuc vitra 4 faepe 8 veritate aberrent.

\section{LXV.}

Sufficiat igitur methodum expoluifle, crius ope inaequalicates lunne am racione apogei, quam longirtudi nis ac latitudinis verae ex anomalia hic adhibita determineri queant: neque propterea laborem calculi roliw

$$
\mathrm{V}_{2} \text { qua }
$$


quarum inaequalicatum, quae vel ex folis parallaxi vel ex inclinatione orbitae lunaris ad eclipticam oriuntur, fufcipio; quippe quarum numerus, fiquidem omnes, quac alicuius momenti effent futurae, perfequi vellem, in immenfum excrefcerec. Non folum aurem mulitudo inee qualitatum hane methodum 'omni vilizate in praxi prius bit, fed etiam ingentes requariones, quas determinatio apogei, atque anomaliae inde pendentis requirit, ita funt com. paratae, vt ipfec iam tatis exattam tam longitudinis quam anomaliae cognitionem requirant; quae res etfi initio fupponi poffent, deinceps iterata eadem operatione accurn. tius definiendae, tamen quia correltio apogel vitra $30^{\circ}$ gradus affurgere poreft, calculus ob inaequalitarum mul. titudinem per fe thediofus, nimis crebro reperi deberet, antequam de conclufione certi effe poffemus.

\section{A P P LIC A T IO \\ FORMULARUM INUENTARUM AD ALIOS CALCULOS LUNARES. \\ LXVI.}

Cum igitur calculus incequalitatum motus lune haEenus duplici modo fit inftixums, dum priori anomalia vera regulis Keplerianis conformis eft affumb, pofteriori vero ita conftinta, vt eius finus com pro maximis lunze a tern difantiis quam pro minimis prorfos euanefes. ret, quorum vterque vti vidimus incommodis non ctret: ite etiom infinitis aliis modis lunae inaequalimates repraefenturi poterunt, quos brevicer expofviffe haud abs

re fore arbitror. Nullum enim eft dubium, quin inter hos infiniros modos quidam reperiantur, qui ipfi naturae rei magis fint confentanei, neque is incommodis laborent, quibus vtrumque expofitum non mediocriter impediri comperimus; etiamfi adhuc difficilimum videacur, inter hanc infinitam multitudinem modum conuenientifimum eligere.

\section{LXVII.}

Poftquam autem inueftigationem ab aequationibus differentio-differentialibus ad aequationes fimpliciter differentiales produximus, etiamfi ad hoc anomalia vera $v$ cuius finus in maximis ac minimis lunae a terra diftaritiis euanefcat, fimus vfi, ramen haec conditio iam ite rum exui poteft. Cum enim tam finus quam cofinus iplius $v$ vbique per quanciratem $q$ fit multiplicarus, loco harum duarum variabilium $q$ et $v$ iam alias duas variabiles in calculum introducere pocerimus, quod commo diffime fiet ponendo $q \operatorname{cof} v=r$ et $q$ fin $v=s$, vs fie $19=r+s s$ et $\operatorname{tang} v=\frac{s}{r}$, tum enim vi formularum §. IX. exhibitarum habebimus iftas aequationes:

$$
\begin{aligned}
& d=-\operatorname{si\varphi }+\frac{2 M}{\Lambda} d \omega V A_{p} ; \\
& s=r d \varphi+\left(\frac{N}{A}-\frac{M s}{A(I-r)}\right) d v A_{p}
\end{aligned}
$$ \\ V $33 \quad$ LXVHLL}


342 ADBITAMENTVM.

\section{IXVIII.}

Hinc autem porro erit: $:=\frac{p}{1-r}$;

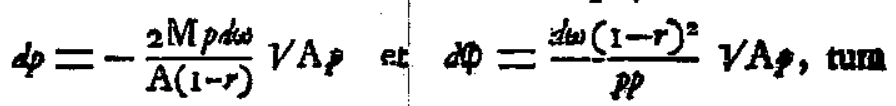
vero vt ante $d u=d \theta=\frac{d \omega(1-e \operatorname{cof} u)^{2}}{(1-\theta) \sqrt{(1-\theta t)}}$.

Beinde vero fi ftatuamus $p=b(1+\xi)$, reliquasque denominariones in $f(11,12,13$. fretas adhibeamus, obtinebimus:

$$
\begin{aligned}
& \frac{M}{A} V A P=\frac{3(1+3 e c)}{2 m} \frac{\left(1-e \operatorname{co}(u)^{3}\right.}{1-+}(1+3) \text { in } 24 \\
& +\frac{3 n}{8 m} \cdot \frac{\left(\mathrm{x}-\mathrm{eco}(u)^{4}\right.}{(1-x)^{2}}\left(\mathrm{x}+\frac{5}{2} \xi\right)(\text { fin } n+5 \text { fin } 3 y) \\
& \frac{N}{A} V A p=-\frac{(1+3 e t)}{2 m} \cdot \frac{\left(I-c \operatorname{cof}(x)^{3}\right.}{i \rightarrow}\left(I+\frac{3}{2} \xi\right)(1+3 \operatorname{col} 2 \eta) \\
& -\frac{3 n}{8 m} \cdot \frac{\left(1-\operatorname{cor}(x)^{4}\right.}{(1-r)^{2}}\left(1+\frac{5}{2} \xi\right)(3 \cos n+5 \cos 3 y) \\
& +(1-)^{2}\left(1-\frac{3}{2}, \xi\right) \pi-i \\
& x=\frac{b(I+\xi)}{I \rightarrow r} ; d \xi=\frac{2(I+\xi) d v}{I-r} \cdot \frac{M}{A} V A_{t} ;
\end{aligned}
$$

$A D D I T A M E N T U H$

\section{LXIX.}

Hinc iam omnium differencialium naciones ad habentur, erit enim

$\frac{d \xi}{d \omega}=-\frac{2(I+\xi)}{I-F} \cdot \frac{M}{A} V A_{p}:$

$\frac{d \varphi}{d \omega}=m\left(\mathrm{x}-\frac{3}{2} \xi+\frac{x^{2}}{\xi} \xi\right)(\mathrm{I}-\mathrm{r})^{2}$

$\frac{d \omega}{d \theta}=\frac{d \theta}{d \omega}=\frac{\left(I-e \operatorname{cof}(u)^{2}\right.}{(I-C e) V(I-e c)}$ et $\frac{d \eta}{d \omega}=\frac{d \varphi+d \theta}{d \omega}$.

$\frac{d r}{d \omega}=-m\left(\mathrm{I}-\frac{3}{2} \xi+\frac{x^{s}}{v^{2}} \xi\right)(I-x)^{2}+2 \cdot \frac{M}{\mathrm{~A}} V A$

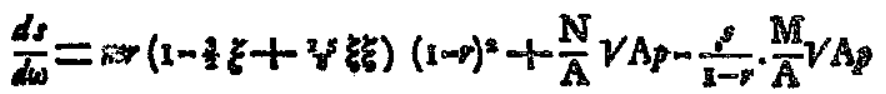

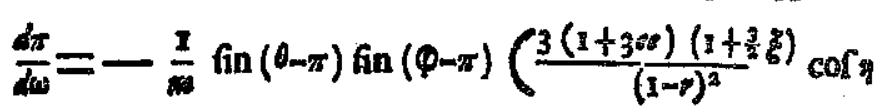
$+\frac{x}{4} n(3+5 \cos (29))$

$\frac{d . \operatorname{tg} \rho}{d \omega}=-\frac{3}{\pi} \operatorname{tin}(l-\pi) \operatorname{cof}(\varphi-\pi)\left(\frac{3(1+3 e e)\left(1+\frac{3}{2} \xi\right)}{(1-\theta)^{3}} \operatorname{cof} \theta\right.$

$$
\left.+\frac{x}{4} n(3+5 \cos 2 \pi)\right)
$$

at eft II $=1-\lambda-\frac{3}{4} \operatorname{tang} \rho^{2}-\frac{3}{4} \operatorname{tang} \rho^{2} \cos 2(\varphi-q)$, vbi pro $\lambda$ affumi poteft $I-\frac{3}{4}$ tang $e^{2}$, denotante $a$ incli-

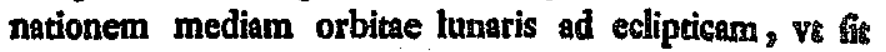
$I=\frac{3}{4} \operatorname{tang} e^{2}-\frac{3}{4} \operatorname{tang} \rho^{2}[x+\operatorname{cor} 2(\varphi-\pi)]$

\section{EXX.}




\section{LXX.}

Quodfi iam pro ftatueretur ifte valor $k \operatorname{col} v$, ita vt $k$ effet quantitas confans, oriretur modus initio traditus inaequalitates lunze repraefencandi, foret enim tum $v$ anomalia vera Kepleri et $k$ denotaret excentricitatem orbitae lunaris. Vnde patet etiam inaequalizates lunae per methodum primam erutas, ex his formulis inueniri poffe, neque ad hof aequationibus fecundi gradus effe opus. Reduceretur autem hoc cafu indoles differentiodifferentialium ad inuentionem quantitatis $s$, quem in finem pro $s$ affumi deberet feries quaedam finuum angulorum $n, v, w$ et $\phi_{-\pi}$ formatorum cum indefinitis coefficientibus, quos deinceps determinare liceret: hoc atstem modo folutio primum tradita effet proditura.

\section{LXXI.}

Cum fit $p=b(1+\xi)$ et $V \mathrm{~A}=m \sqrt{ } b^{3}$, ob $d x=$ $-s^{d} \omega v A_{p}$, fiat $\frac{d x}{d \omega}=-\frac{m b s}{\nu(1+\xi)}=-m b s\left(1-\frac{1}{2} \xi+\frac{3}{8} \xi \xi\right)$, vnde patet fi ciusmodi anomalia $v$ introducarur, vt fit $s=q$ fin $v$, fiue $q$ fit quantiras conftans fue variabilis, tum hanc anomalian tam in maximis quam minimis diftantis finum euanefcentem effe habituram. Ac fi pro q quantias vel conftans vel ex angulis cognitis compofita affumatur, tum inde coefficiences affumi ac praeterea valor litterae $r$ determinabitur. Sin autem pro $q$ eiusmodi quantitas incognita affumatur, vt fit praeterea $r=$ $q$ colv, tum folutio ante expofita refultabic.

LXXII.

\section{LXXII.}

Semper autem vfus aftronomicus exigic, ve anomalia vera quaedam angulo $v$ contenta introducasur, id quod infinitis modis fieri poteft. Quo autem quanticas o vo riabilitatem diftantiarum lanae a terra accuratius exprimat, et valor ipfius $\xi$ quam minimas mutaciones fubeats neceffe eft, vt quantitas $r$ huiusmodi contineat acrainum $k \operatorname{col} v$, vbi $k$ excentricitatem defignet, qui tit quafi eirs pars praecipua; hocque etiam locum habet, fi pro $r$ fumatur $q$ cof $v$, denotante $q$ quanitatem variabilem, quip. pe cuius pars potior excentricitatem * praebere debec. Verum praeterea quantitas $r$ alios terminos concinere poteft, qui ab angulo $v$ vel pendeant vel non pendeant: ita poni poflet: $r=k \operatorname{col} v+\mathrm{A} \operatorname{col} 2 \eta+\mathrm{Bcol}_{4 \eta}+\mathrm{C}$ $\operatorname{cor}(2 \eta-\nu)$ etc. quo valore affume litterae quoque $s$, cum reliquis fuos valores debiros obtinerent.

\section{LXXIII,}

Hoc modo illud incommadum euitari poteft, quo methodum in hoc additameneo traditam laborare vidjmus, fi excentricitas orbitae lunaris eflet nimis parua, vel adeo euanefcens; tum enim diftantiae maximae ec minimae non amplius ab anomalia penderent, fed potius ab angulo $\eta$, atque imprimis quidem a cofinu dupli an: guli $2 \eta$. Calu ergo quo excentricitas plane euanelcie, pro variabili $r$, cuius loco vtique nou variabilis intro. duci debèt, non cónüeniet anomalian u introducere, fed praeftabit affumi feriem colinuum ex folis angulis 2\%, $\mathbf{X} \mathbf{x}$ 
et $\emptyset-x$ conftantem, quorum coefficientes etfi funt conftantes, tamen quia terminorum numerus in infinitum excurrit, vicem novae variabilis fuftinebunt. Tum autem valor ipfius $s$ ex fimili ferie finuum eorundem angulorum conftabit.

\section{LXXIV.}

Quodí ergo rem generatim pro quacunque excentricitate expedire velimus, poterimus ad hos terminos, qui ex hypothefi excentricimis cuanefcentis prodeunt, adhuc adiungere terminos ex anomalia $v$ formatos. Ios negleatis tam inaequalizathus parallaticis, quam its quae tum ab excentricitate orbitae folaris, tum ab inclinatione orbitae lunaris ad eclipticam pendent, poni conueniet:

$$
\begin{aligned}
& =k \operatorname{col} v+A \operatorname{cor} 2 \eta+B \operatorname{cor}(2 y-v)+C \operatorname{cof}(2 y+v) \\
& +D \operatorname{col} 4 \eta+E \operatorname{cor}(4 \eta-0) \text { etc. } \\
& s=\Delta k \text { fin } v+2 \text { fin } 2 y+2 B \text { fin }(2 y-v)+\varepsilon \operatorname{cin}(2 y+v) \\
& +D \text { fin } 4 y+\mathbb{E} \text { fin }(4 y-v) \text { etc. } \\
& z=\ldots \operatorname{cor} v+\ldots \operatorname{cor} 2 v+\ldots \operatorname{cor}(2 y-v)+\ldots \operatorname{cor}(2 y+v) \\
& +\ldots \operatorname{col} 44+. . \operatorname{col}(4 t-v) \text { etc. }
\end{aligned}
$$

Atque fi hoc modo omnes angulorum $2 y$ et $v$ combinationes adhibeantur, hique valores in sequationibus fupra datis fubftituantur, primo iṇde elicietur ratio $d v: d w, a c$ deinceps coefficientes determinationes fues nancifcentur.

\section{LXXV.}

Manebunt sutem coefficientes vnius feriel velus ipfius $\%$ indeterminati, properen guod ipf levies beec conftan.

excurrit,

alor ipti-

onftabit.

e excen-

erminos,

rodeunt,

cos. Ite

1 iis quae

linatione

teniet:

or $(2 \eta+v)$

$\eta-v)$ etc.

$n(2 y+v)$

$n-v)$ etc.

$\int f(2 \eta+v)$

$y-v)$ etc.

mbinatio-

jus fupra

1: d $d \omega, a c$

ifcentur.

iel velut

ries haec ab arbitrio noftro pendes, dum pro vel folum primum terminum $k \operatorname{cof} v$, vel quorquor lubuerit, afturiere po. tuiffemus. Hine auten id commodi confeguemus, ve iftos coefficientes ad fcopum quam conuenientifime de. finire valeamus: feilicet eus ita definiri conuenier, vt primo nullius reliquorum coefficientium determinatio log brica et incerta cuadat, vi in veraque methodo expofita vo venit: deinde vero vt nulli coefficientes fant nimis viu venit: deinde vero vo nulli coetheren effelus per alios terminos iterum deftrui neceffe fit. Fateri quiders cogor calculum hoc modo infticuendun admadum fus turum effe prolixum, verom forcaffe in ipfa operacione non contemnenda fe offerent compendis: vade contido hanc fpeculationem, eciami mili ipfi eam furcipere an vacer, vfu non elle carituram.

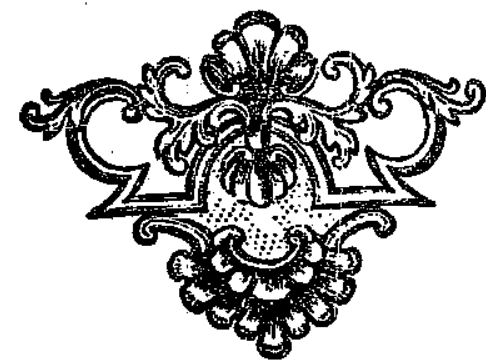

ORROLIN, EX OFEICINA NICRAELTS 
<smiles>CC(C)(C)[Si](C)(C)C12CCCC1CC2</smiles> 